Consortium for Advanced

Simulation of LWRs

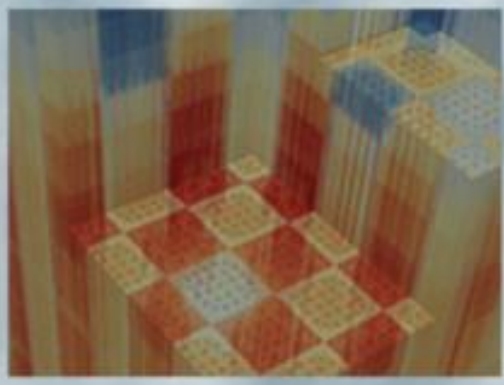

\title{
CTF Validation and Verification
}

Version 4.2

R. Salko ${ }^{1}$, A. Wysocki ${ }^{1}$, A. Toptan ${ }^{2}$, N. Porter ${ }^{2}$, X. Zhao $^{1}$, T. Blyth ${ }^{3}$, J. Magedanz ${ }^{3}$, C. Dances ${ }^{3}$, M. Gergar $^{3}$, C. Gosdin ${ }^{3}$, C. Jernigan ${ }^{4}$, J. Kelly ${ }^{5}$, V.

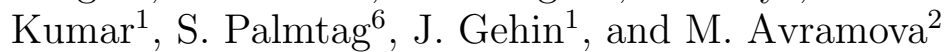

${ }^{1}$ Oak Ridge National Laboratory

${ }^{2}$ North Carolina State University

${ }^{3}$ The Pennsylvania State University

${ }^{4}$ Holtec International

${ }^{5}$ US Nuclear Regulatory Commission

${ }^{6}$ Core Physics

${ }^{7}$ Massachusetts Institute of Technology

$9 / 14 / 2020$

象VER

VERA QA Document - This document was prepared under the ORNL VERA Quality Assurance Program in accordance with procedure VERA-QA-003. The OFFICIAL COPY of this document is the electronic version in the VERA Documents Repository. Before using a printed copy, verify that it is the most current version by checking the Revision ID against the electronic version.

Approved for Public Release
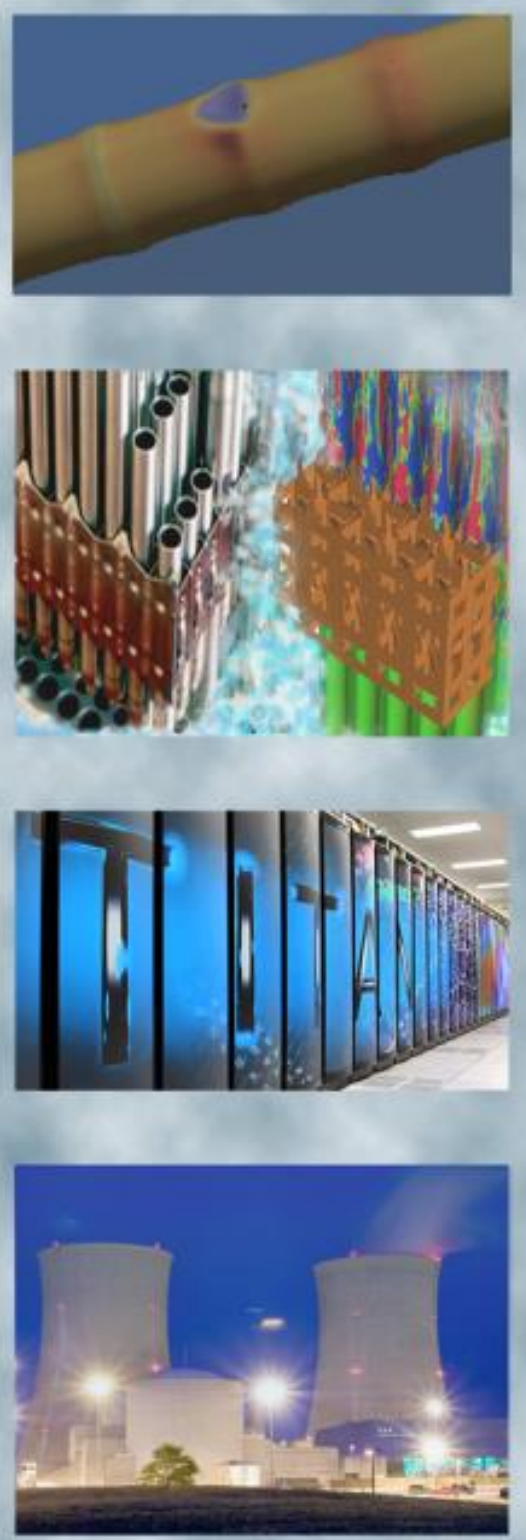


\title{
Revision Log
}

\begin{tabular}{rrrr}
\hline Revision & Date & Affected Pages & Revision Description \\
\hline 0 & $3 / 24 / 19$ & All & Initial Release \\
1 & $11 / 8 / 19$ & All & 4.1 release \\
2 & $9 / 14 / 20$ & All & 4.2 release
\end{tabular}

\section{Document pages that are:}

\author{
Export Controlled: None \\ IP/Proprietary/NDA Controlled: None \\ Sensitive Controlled: None \\ Unlimited: All
}

This document was prepared as an account of work sponsored by an agency of the United States Government. Neither the United States Government nor any agency thereof, nor any of their employees, makes any warranty, express or implied, or assumes any legal liability or responsibility for the accuracy, completeness, or usefulness of any information, apparatus, product, or process disclosed, or represents that its use would not infringe privately owned rights. Reference herein to any specific commercial product, process, or service by trade name, trademark, manufacturer, or otherwise, does not necessarily constitute or imply its endorsement, recommendation, or favoring by the United States Government or any agency thereof. The views and opinions of authors expressed herein do not necessarily state or reflect those of the United States Government or any agency thereof. 
The North Carolina State University

Department of Nuclear EngineERing

Reactor Dynamics and Fuel Modeling Group

NC STATE UNIVERSITY

\section{CTF Validation and Verification \\ Version 4.2}




\section{CTF Validation and Verification}

\section{Version 4.2}

\section{Approvals:}

Robert Salko, CTF Product Software Manager

Belgacem Hizoum, Independent Reviewer
Date

Date 


\section{Executive Summary}

Coolant-Boiling in Rod Arrays- Two Fluids (COBRA-TF) is a thermal/hydraulic $(\mathrm{T} / \mathrm{H})$ simulation code designed for Light Water Reactor (LWR) analysis. It uses a two-fluid, three-field (i.e. fluid film, fluid drops, and vapor) modeling approach. Both sub-channel and 3D Cartesian forms of nine conservation equations are available for LWR modeling. The code was originally developed by Pacific Northwest Laboratory in 1980 and has been used and modified by several institutions over the last several decades. COBRA-TF is also used at the Pennsylvania State University (PSU) by the Reactor Dynamics and Fuel Modeling Group (RDFMG) and has been improved, updated, and subsequently became the PSU RDFMG version of COBRA-TF (CTF). One part of the improvement process includes validating the methods in CTF.

This document seeks to provide a certain level of certainty and confidence in the predictive capabilities of the code for the scenarios it was designed to model - rod bundle geometries with operating conditions that are representative of prototypical Pressurized Water Reactor (PWR)s and Boiling Water Reactor (BWR)s in both normal and accident conditions. This is done by modeling a variety of experiments that simulate these scenarios and then presenting a qualitative and quantitative analysis of the results that demonstrates the accuracy to which CTF is capable of capturing specific quantities of interest. 


\section{Contents}

Executive Summary ................................ . . . . . . .

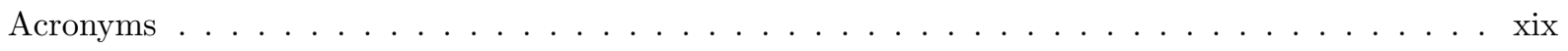

1 Introduction 1

1.1 Organization of the document . . . . . . . . . . . . . . . . . . . 2

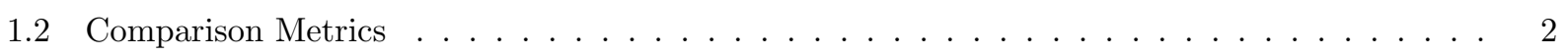

2 Validation Plan 4

3 Test Descriptions 6

$3.1 \mathrm{PSBT} \ldots \ldots \ldots \ldots \ldots \ldots \ldots$

3.2 BFBT $8 \times 8 \ldots \ldots \ldots \ldots \ldots \ldots$

3.3 PNNL $2 \mathrm{x} 6 \ldots \ldots \ldots \ldots$. . . . . . . . . . . . . . . . . . . . . 30

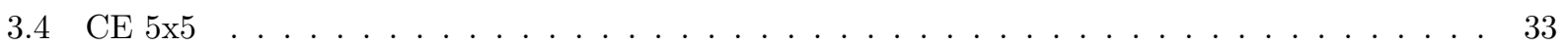

3.5 GE $3 \mathrm{x} 3 \ldots \ldots \ldots \ldots \ldots \ldots \ldots$

3.6 Harwell High Pressure Loop . . . . . . . . . . . . . . . . . . . . . . . . . . . . . . . . . . . . 40

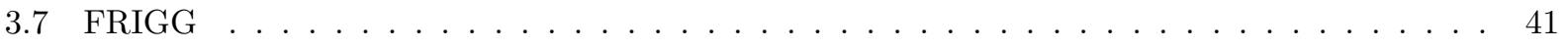

3.7.1 CTF Model of Facility . . . . . . . . . . . . . . . . . . . . . . 43

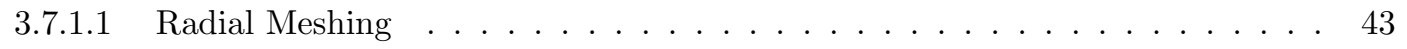

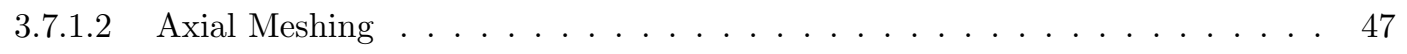

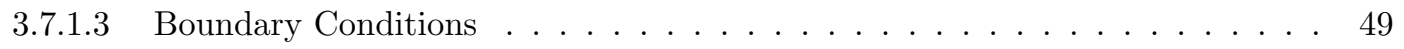

Consortium for Advanced Simulation of LWRs vi visL-U-2019-1887-002 
3.7.1.4 Modeling Choices .......................... 49

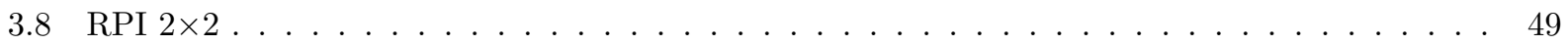

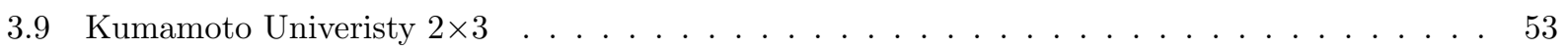

3.10 Halden IFA Test Cases $\ldots \ldots \ldots \ldots \ldots \ldots \ldots$

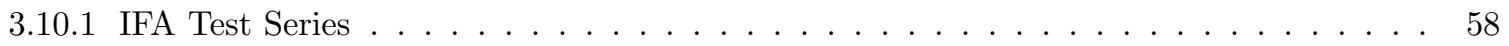

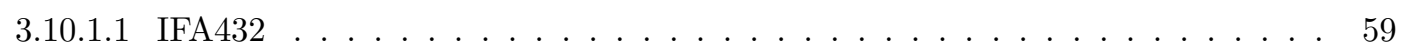

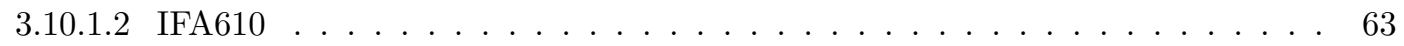

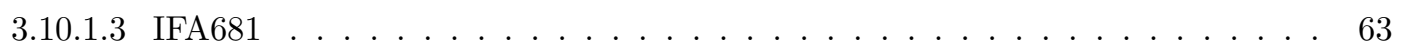

3.11 Nikuradse . . . . . . . . . . . . . . . . . . . . . . . . 64

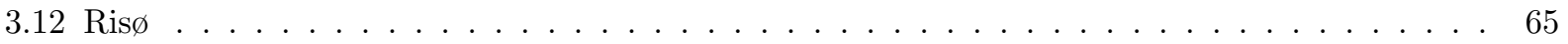

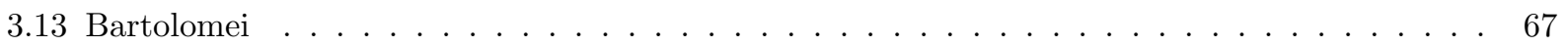

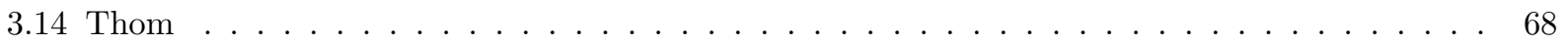

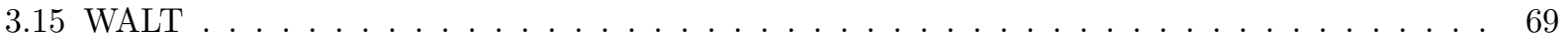

3.16 Rohsenow . . . . . . . . . . . . . . . . . . . . . . . 69

4 Heat Transfer $\quad 70$

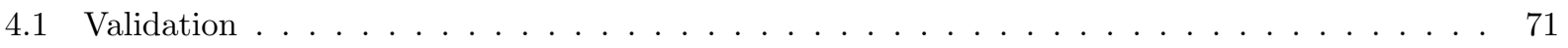

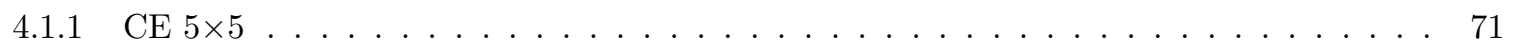

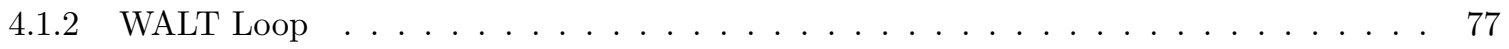

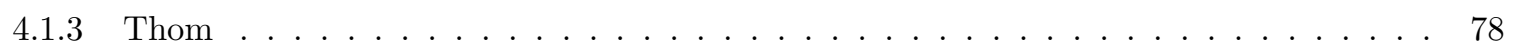

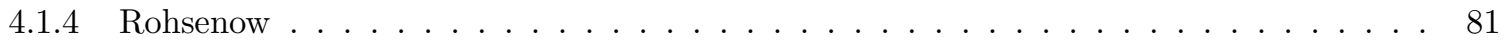

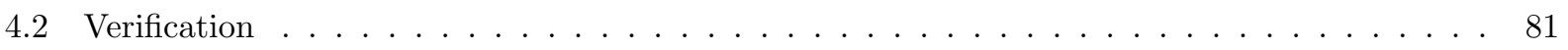

4.2 .1 Heat Exchanger . . . . . . . . . . . . . . . . . . . . . . . . . . . 81

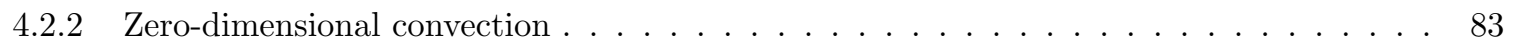

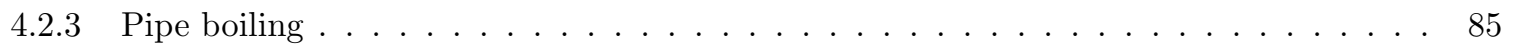

5 Spacer Grid Models $\quad 91$ 


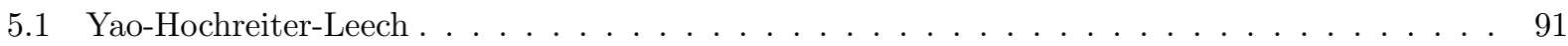

6 Pressure loss $\quad 96$

6.1 Pressure drop in $\mathrm{CTF} \ldots \ldots \ldots \ldots \ldots \ldots \ldots \ldots$

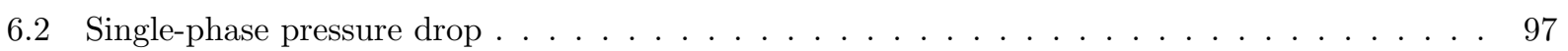

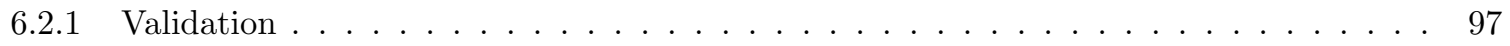

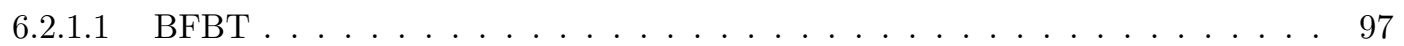

$6.2 .1 .2 \quad$ Nikuradse . . . . . . . . . . . . . . . . . . . . . . . . . . 99

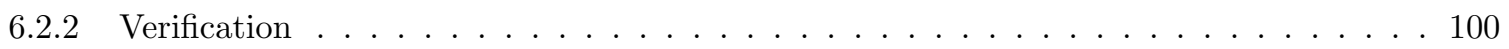

6.2.2.1 Single-Phase Friction Flow Split . . . . . . . . . . . . . . . . . . 100

6.2.2.2 Friction Model Verification . . . . . . . . . . . . . . . . . 105

6.2.2.3 Single-Phase Axial and Lateral Pressure drop Verification . . . . . . . . . . . 113

6.2 .2 .4 Flow expansion . . . . . . . . . . . . . . . . . . 116

6.2.2.5 Single-Phase Axial Pressure Drop Verification . . . . . . . . . . . . . 120

6.3 Two-phase pressure drop . . . . . . . . . . . . . . . . . . . . . . . . . . 124

6.3 .1 Validation . . . . . . . . . . . . . . . . . . . . . 124

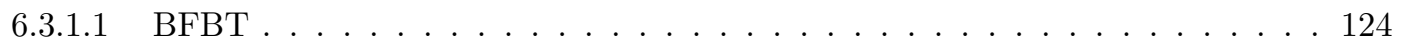

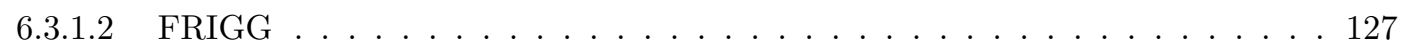

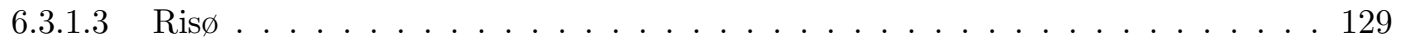

7 Single-Phase Turbulent Mixing $\quad 131$

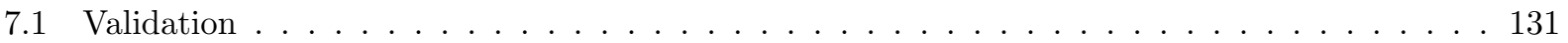

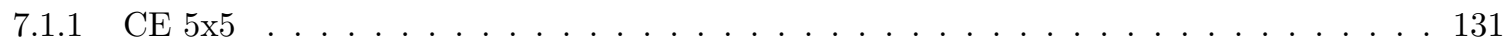

$7.1 .2 \quad$ Kumamoto University $2 \times 3 \quad \ldots \ldots \ldots \ldots \ldots \ldots$

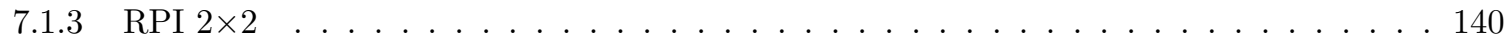

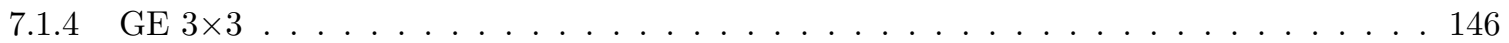

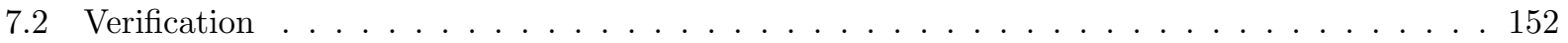

Consortium for Advanced Simulation of LWRs viii $\quad$ CASL-U-2019-1887-002 
7.2.1 Single-Phase Two-Channel . . . . . . . . . . . . . . . . . . . . 152

8 Void Content 159

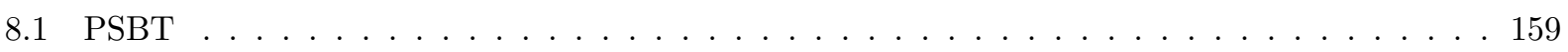

8.1.1 Single Subchannel Facility . . . . . . . . . . . . . . . . . . . . . . 159

8.1.2 Rod Bundle Facility . . . . . . . . . . . . . . . . . . . . . . . . . 164

8.2 FRIGG Facility . . . . . . . . . . . . . . . . . . . . . . . . 168

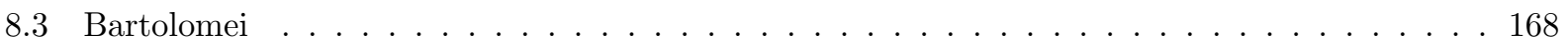

9 Two-Phase Turbulent Mixing and Void Drift 177

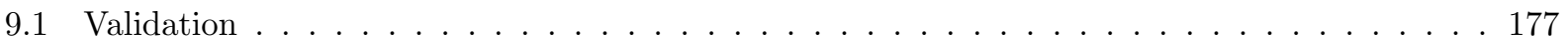

$9.1 .1 \mathrm{GE} 3 \mathrm{x} 3 \ldots \ldots \ldots \ldots \ldots \ldots \ldots$

9.1.2 BFBT $8 \times 8 \ldots \ldots \ldots \ldots \ldots$

10 Dryout 200

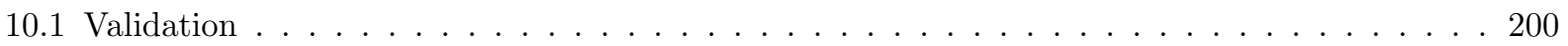

10.1.1 Harwell Facility . . . . . . . . . . . . . . . . . . . . . . . . . . . 200

10.1.2 Takahama Tests ... . . . . . . . . . . . . . . . . . . 203

10.2 Verification . . . . . . . . . . . . . . . . . . . . . 203

10.2.1 Bowring Correlation . . . . . . . . . . . . . . . . . 203

10.2.2 Groeneveld Look-up Table . . . . . . . . . . . . . . . . . . . . . . . . . . . 204

11 Solid Structure Models 208

11.1 Inside Tube Flow . . . . . . . . . . . . . . . . . . . . . . . . . . 208

11.1.1 Direct Heat Input . . . . . . . . . . . . . . . . . . . . . . . . 208

11.1.2 Radial Conduction Test . . . . . . . . . . . . . . . . . . . . 209

11.1 .3 Multiple Inside Channels . . . . . . . . . . . . . . . . . . . . . . . . . . . . 210

12 Natural Circulation 213

CASL-U-2019-1887-002 ix $\quad$ Consortium for Advanced Simulation of LWRs 


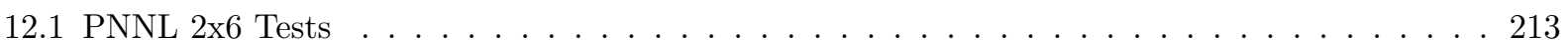

13 Fuel Temperature $\quad 230$

13.1 Fuel Temperature in $\mathrm{CTF} \ldots \ldots \ldots \ldots \ldots \ldots \ldots$

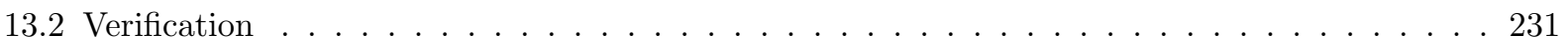

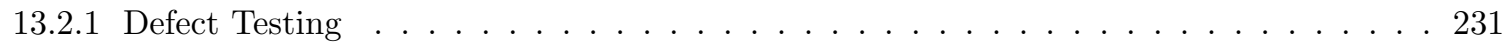

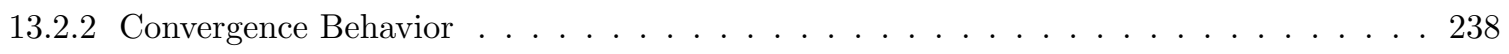

13.3 Validation . . . . . . . . . . . . . . . . . . . . . . 239

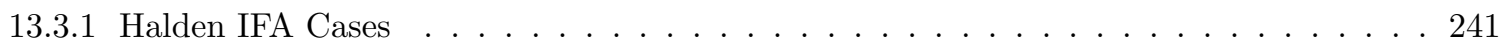

13.3.1.1 Assessment of Fuel Centerline Temperature Predictions at $\mathrm{UO}_{2}$ Fuel . . . . . 241

13.3.1.2 Assessment of Fuel Centerline Temperature Predictions for $\mathrm{UO}_{2}+2 \% \mathrm{Gd}_{2} \mathrm{O}_{3}$

Fuel . . . . . . . . . . . . . . . . . . . . . 241

13.3.1.3 Assessment of Fuel Centerline Temperature Predictions for MOX Fuel . . . . 246

14 Droplet Entrainment $\quad 248$

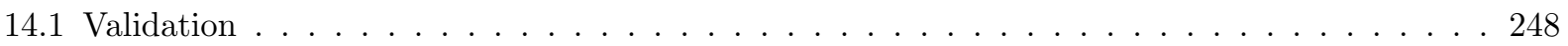

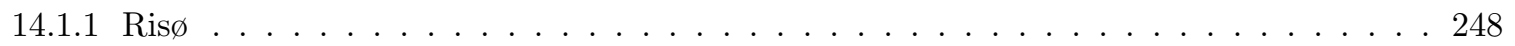

15 Miscellaneous Problems 251

15.1 Water Faucet Problem . . . . . . . . . . . . . . . . . . . . . 251

16 Governing Equation Verification $\quad 259$

16.1 Mass and Energy Equation Advection . . . . . . . . . . . . . . . . 259

16.1.1 Problem Description . . . . . . . . . . . . . . . . . . . . . . . . 259

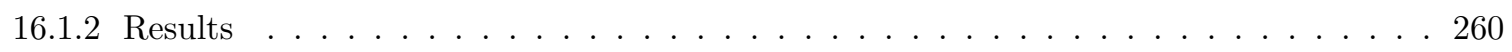

17 Conclusion $\quad 265$

$\begin{array}{ll}\text { Bibliography } & 265\end{array}$

Consortium for Advanced Simulation of LWRs $\quad$ x $\quad$ CASL-U-2019-1887-002 


\section{List of Figures}

3.1 Summary of PSBT single subchannel configurations for CTF validation activities . . . . . . 7

3.2 PSBT single subchannel geometric characteristics . . . . . . . . . . . . . . . . . 7

3.3 PSBT single subchannel cross sectional views . . . . . . . . . . . . . . . . . . . . . 8

3.4 Summary of PSBT rod-bundle configurations for CTF validation activities . . . . . . . . . . 9

3.5 Assembly 0-1, 0-2, and 0-3 description . . . . . . . . . . . . . . . . . . 16

3.6 Assembly 0-1, 0-2, and 0-3 description (continued) . . . . . . . . . . . . . . 17

3.7 Assembly 1, 2, and 3 description ............................ . . . . . . . . . . .

3.8 Assembly 1 power . . . . . . . . . . . . . . . . . . . . . . . . 19

3.9 Assembly 4 power . . . . . . . . . . . . . . . . . . . . . . . . 19

3.10 Assembly 4 description . . . . . . . . . . . . . . . . . . . . . . . . 20

3.11 BFBT bundle pressure tap locations . . . . . . . . . . . . . . . . . . . . . . 21

3.12 BFBT void measurements . . . . . . . . . . . . . . . . . . . . . . . 23

3.13 Assembly $0-1,0-2,0-3$, and 1 flow areas . . . . . . . . . . . . . . 25

3.14 Assembly 4 flow areas ... . . . . . . . . . . . . . . . . . . . . . 25

3.15 Lantern grid . . . . . . . . . . . . . . . . . . . . . . . . . 26

$3.16 \mathrm{C} 2 \mathrm{~A}$ ferrule grid geometry . . . . . . . . . . . . . . . . . . . . . 27

3.17 Lantern grid loss coefficients . . . . . . . . . . . . . . . . . . . . . . . 28

3.18 Ferrule grid loss coefficients . . . . . . . . . . . . . . . . . . . . . . . . 29

3.19 Cross-sectional geometry of the PNNL $2 x 6$ test section . . . . . . . . . . . . . . . . . 30 
3.20 Axial schematic of the PNNL 2x6 facility and CTF meshing (units shown in inches) $\ldots . .31$

3.21 Cross-section diagram of $\mathrm{CE} 5 \times 5$ Test $74 \ldots \ldots \ldots \ldots \ldots \ldots \ldots \ldots$

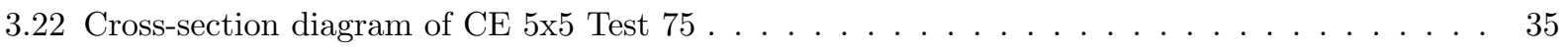

3.23 Axial schematic of the $\mathrm{CE} 5 \mathrm{x} 5$ bundle $\ldots \ldots \ldots \ldots \ldots$

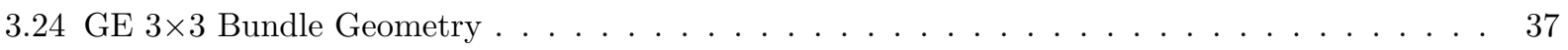

3.25 GE $3 \times 3$ Bundle Pin Spacer Geometry . . . . . . . . . . . . . . . . . . . 39

3.26 CTF model of the Harwell High-Pressure Two-Phase Test Facility . . . . . . . . . . . . . 42

3.27 Cross-section of the FRIGG facility (including labels of CTF model rods and channels) . . . 44

3.28 Definition of functions for the circle that defines the fuel rod and the circle it sits on $\ldots \ldots 45$

3.29 Schematic of FRIGG facility in axial direction . . . . . . . . . . . . . . 48

3.30 Low assembly test section with sinter section shown $[14] \ldots \ldots \ldots \ldots$

3.31 Air/Water Mixing Tee $[14] \ldots \ldots \ldots \ldots \ldots \ldots \ldots \ldots \ldots \ldots$

3.32 NUREG/CR-3373 Experimental Cross Section $[14] \ldots \ldots \ldots \ldots \ldots \ldots$

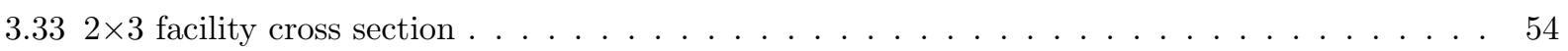

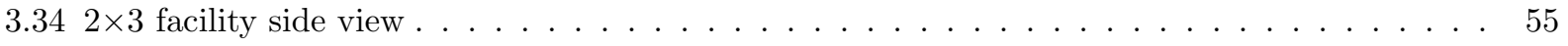

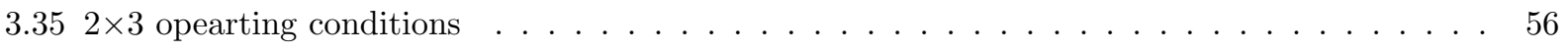

3.36 Arrangement of temperature sensors, neutron detectors, and fuel relative to reference axial thermal flux profile for Instrumented Fuel Assembly (IFA)432[24] . . . . . . . . . . . . . 60

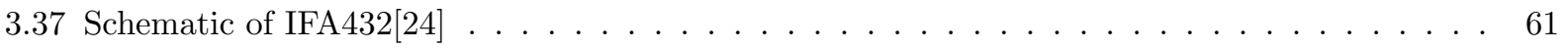

3.38 Location of IFA431 and IFA432 in the Halden Boiling Water Reactor (HBWR) core $\ldots \ldots$

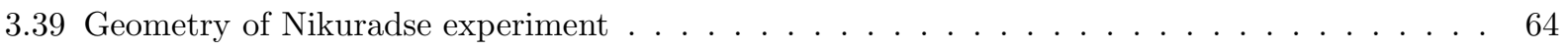

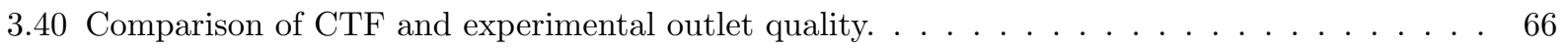

3.41 Difference in CTF versus experimental droplet flow fractions as a function of number of CTF axial nodes. . . . . . . . . . . . . . . . . . . . . 67

4.1 Rod surface measurements and predictions with respect to increasing test heat flux for Rod 25 (central rod) at 77.63 inch axial location . . . . . . . . . . . . . . . 72

4.2 Summary of mean difference between predicted and measured rod surface temperatures for each thermocouple in $\operatorname{Rod} 25$ over all test cases . . . . . . . . . . . . . . . . 73 
4.3 CE 5x5 Test 74 Mixing Vane Grid . . . . . . . . . . . . . . . . . . . . . . . . . 75

4.4 Summary of mean difference between predicted and measured rod surface temperatures for each thermocouple in Rod 24 over all test cases . . . . . . . . . . . . . . . . . . 76

4.5 WALT validation results as a function of heat flux. . . . . . . . . . . . . . . . 78

4.6 Comparison of predicted and experimental data of Thom for the temperature difference between the outer wall and the bulk liquid vs. liquid subcooling. . . . . . . . . . . . . . . 79

4.7 Comparison of predicted vs. experimental data of Thom for the temperature difference between the outer wall and the bulk liquid. . . . . . . . . . . . . . . . . . . . . 80

4.8 Rohsenow validation results as a function of spatial location. . . . . . . . . . . . . . 81

4.9 Diagram of the heat exchanger verificaiton problem . . . . . . . . . . . . . . . 82

4.10 Comparison of the analytical and CTF solution for flow through a heat exchanger . . . . . 84

4.11 Zero dimensional convection temperature vs. time. . . . . . . . . . . . . . . . 86

4.12 Convergence for zero dimensional convection problem. . . . . . . . . . . . . . . . 87

4.13 Results for the pipe boiling verification problem . . . . . . . . . . . . . . . . . 89

4.14 Convergence for pipe boiling verification problem . . . . . . . . . . . . . . . . . 90

5.1 CTF-prediction and curve fit of $L_{2}$ norm of the wall temperature for successive mesh refinements. 93

5.2 CTF-prediction and curve fit of $L_{2}$ norm of the wall temperature for successive mesh refinements. 94

5.3 CTF-prediction and expected axial liquid temperature profile of the channel. . . . . . . . . 94

5.4 CTF-prediction and expected wall temperature with a spacer grid. . . . . . . . . . . . . 95

5.5 CTF-prediction and expected wall temperature with a spacer grid. . . . . . . . . . . . . 95

6.1 BFBT single-phase pressure drop predictions (Series 7) . . . . . . . . . . . . . 98

6.2 Results for Nikuradse smooth and rough pipes . . . . . . . . . . . . . . . . 100

6.3 Diagram of the two-channel flow split problem. . . . . . . . . . . . . . . . 103

6.4 Two-channel flow-split results . . . . . . . . . . . . . . . . . . . . . . . 104

$6.53 \times 3$ rod bundle geometry with (a) rod-centered and (b) channel-centered subchannels. . . . . 106

6.6 CTF-prediction and curve fit of pressure drop for axial pipe flow for successive mesh refinements. 115 
6.7 $L_{\mathrm{inf}}$ norm of CTF-prediction and expected pressure drop for axial pipe flow for successive mesh refinements. . . . . . . . . . . . . . . . . . . . . . . 115

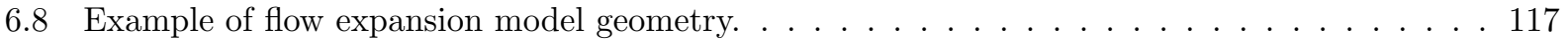

6.9 CTF staggered mesh for flow expansion test near point of expansion. . . . . . . . . . . . 118

6.10 Comparison of expected and calculated velocity in expansion verification test. . . . . . . 119

6.11 Comparison of expected and calculated pressure in expansion verification test. . . . . . . 120

6.12 Verification results for single phase pressure drop with friction and gravity . . . . . . . . 121

6.13 Convergence for single phase pressure drop with friction and gravity $\ldots \ldots$. . . . . . 122

6.14 Ratio of measured-to-predicted pressure drop compared to bundle-average exit quality for BFBT two-phase tests $($ Series P6) . . . . . . . . . . . . . . . 125

6.15 Comparison of measured and predicted bundle-averaged exit quality for BFBT P6 Series . . . 126

6.16 Comparison of experimental and predicted components of pressure drop . . . . . . . . 128

6.17 Measured vs. predicted total pressure drop per unit length at the outlet $\ldots \ldots \ldots$. . . . 130

7.1 Average difference between CTF predicted channel exit temperatures and experimental values

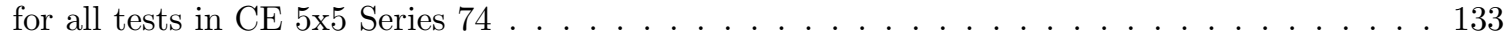

7.2 Average difference between CTF predicted channel exit temperatures and experimental values with measurement error and mean discrepancy . . . . . . . . . . . . . . . . 134

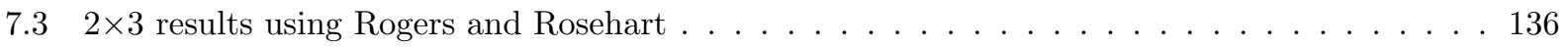

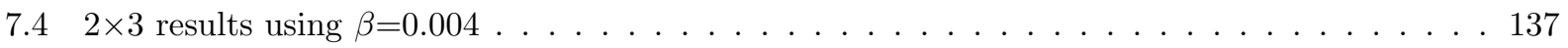

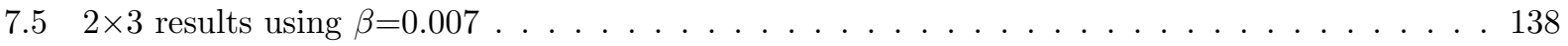

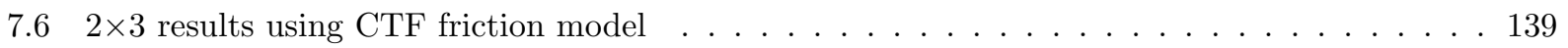

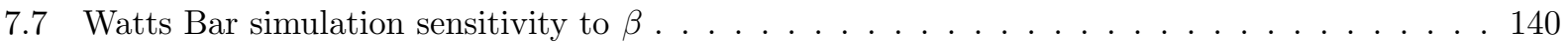

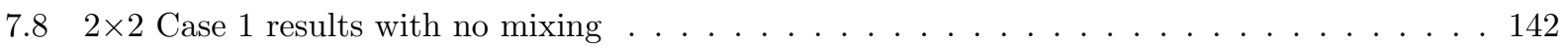

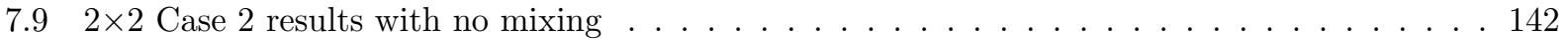

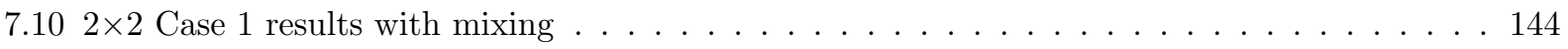

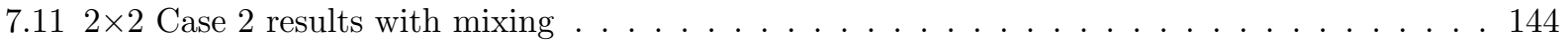

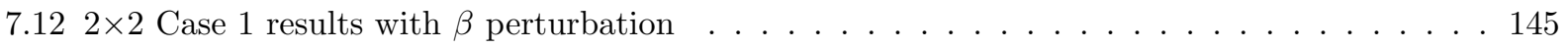

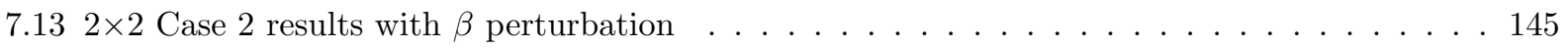

Consortium for Advanced Simulation of LWRs xiv CASL-U-2019-1887-002 
7.14 GE $3 \times 3$ Case 1 B results with mixing . . . . . . . . . . . . . . . . . . . . . . . . 147

7.15 GE $3 \times 3$ Case 1 C results with mixing . . . . . . . . . . . . . . . . . . . . 148

7.16 GE $3 \times 3$ Case 1 D results with mixing . . . . . . . . . . . . . . . . . . . . . . 149

7.17 GE $3 \times 3$ Case 1 E results with mixing . . . . . . . . . . . . . . . . . . . . . . 150

7.18 GE $3 \times 3$ single-phase test summary . . . . . . . . . . . . . . . . . . . . 151

7.19 Model of problem for testing single-phase turbulent mixing of enthalpy. . . . . . . . . . . 153

7.20 Turbulent-mixing problem results . . . . . . . . . . . . . . . . . . . 156

7.21 Mixing problem mass flow rates . . . . . . . . . . . . . . . . . . . . 157

7.22 Mixing problem mass flow rates with no mixing . . . . . . . . . . . . . . . . . 158

8.1 PSBT Series 1 predicted versus measured void fraction . . . . . . . . . . . . . . . 160

8.2 PSBT Series 2 predicted versus measured void fraction . . . . . . . . . . . . . . . . 161

8.3 PSBT Series 3 predicted versus measured void fraction . . . . . . . . . . . . . . . . 162

8.4 PSBT Series 4 predicted versus measured void fraction . . . . . . . . . . . . . . 163

8.5 PSBT Series 5 predicted versus measured void around central rod in bundle . . . . . . . . 165

8.6 PSBT Series 6 predicted versus measured void around central rod in bundle . . . . . . . . 166

8.7 PSBT Series 7 predicted versus measured void around central rod in bundle . . . . . . . . . 167

8.8 Predicted and measured axial bundle-averaged void profile in FRIGG facility . . . . . . . . . 169

8.9 Comparison of $\mathrm{CTF}$ and experimental data of wall temperature, mean liquid temperature, and void fraction for case 1. . . . . . . . . . . . . . . . . . . . . . 170

8.10 Comparison of CTF and experimental data for variation in mass flux at 70.0 bar and heat flux

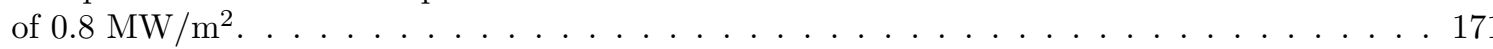

8.11 Comparison of CTF and experimental data for variation in mass flux at 70.0 bar and mass flux of $1000.0 \mathrm{~kg} / \mathrm{m}^{2}-\mathrm{s}$. . . . . . . . . . . . . . . . . . . . . . . . . . . . . . 172

8.12 Comparison of CTF and experimental data for variation in mass flux at 150.0 bar and mass flux of $2000.0 \mathrm{~kg} / \mathrm{m}^{2}$-s. . . . . . . . . . . . . . . . . . . . . . . . . 173

8.13 Comparison of CTF and experimental data for variation in system pressure at mass flux of $2000.0 \mathrm{~kg} / \mathrm{m}^{2}-\mathrm{s}$ and heat flux of $1.1 \mathrm{MW} / \mathrm{m}^{2}$. . . . . . . . . . . . . . . . . . . 174

8.14 Overall comparison of CTF versus experimental void fractions using the subcooled boiling model of Thom. . . . . . . . . . . . . . . . . . . . . . . . . 175 
8.15 Overall comparison of CTF versus experimental void fractions using the subcooled boiling model of Chen. . . . . . . . . . . . . . . . . . . . . . . 176

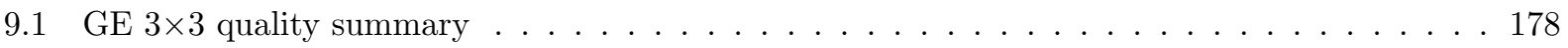

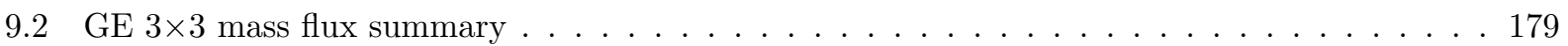

9.3 Comparison of measured and predicted bundle-averaged outlet void. . . . . . . . . . . 181

9.4 Comparison of measured and predicted bundle-averaged outlet thermal equilibrium quality. . 182

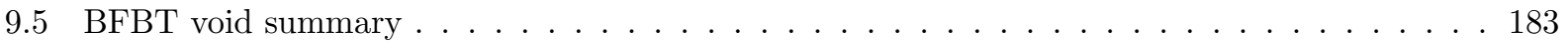

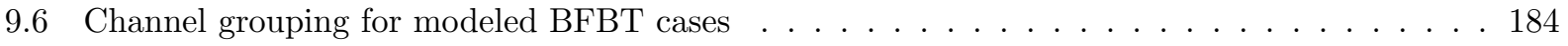

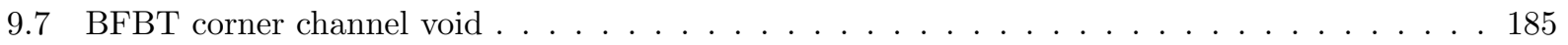

9.8 BFBT side channel void $\ldots \ldots \ldots \ldots \ldots \ldots \ldots \ldots \ldots$

9.9 BFBT inner channel void $\ldots \ldots \ldots \ldots \ldots \ldots \ldots$

9.10 BFBT touching unheated channel void . . . . . . . . . . . . . . . . . 188

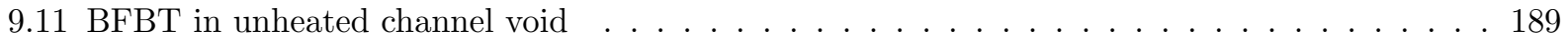

9.12 Example of diagonal lines from where subchannel void data is extracted. . . . . . . . . . . 190

9.13 Measured and predicted void along diagonals of Test 0011-55 . . . . . . . . . . . . . 190

9.14 Measured and predicted void along diagonals of Test $0011-61 \ldots \ldots \ldots$. . . . . . . . 191

9.15 Measured and predicted void along diagonals of Test $0021-16 \ldots \ldots$. . . . . . . . . 192

9.16 Measured and predicted void along diagonals of Test 0021-21 . . . . . . . . . . . 193

9.17 Measured and predicted void along diagonals of Test $0031-16 \ldots \ldots \ldots$. . . . . . . . 194

9.18 Measured and predicted void along diagonals of Test $0031-21 \ldots \ldots \ldots$

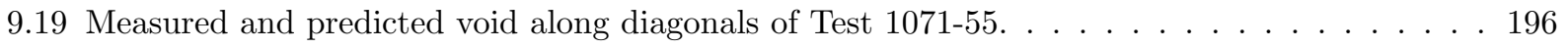

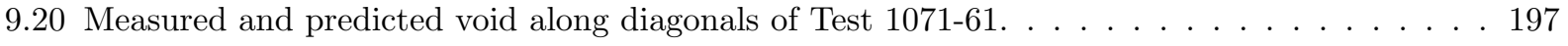

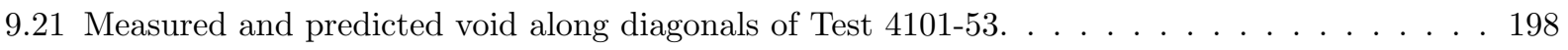

9.22 Measured and predicted void along diagonals of Test 4101-61 . . . . . . . . . . . . 199

10.1 Summary of predicted and experimental dryout locations for Harwell test cases modeled by

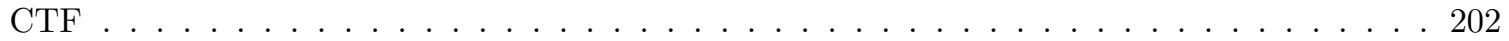


10.2 Groeneveld Hand Interpolation Scheme . . . . . . . . . . . . . . . . . . . . . 206

11.1 CTF predicted wall temperature drop compared to analytical solution for channel on outside versus channel on inside . . . . . . . . . . . . . . . . . . . . . . . 211

12.1 Predicted and measured subchannel velocities for Window 1 at Rake Location $\mathrm{Y}=-0.581$ in in PNNL 2x6 . . . . . . . . . . . . . . . . . . . . . . . . . . . . . . . . . . 214

12.2 Predicted and measured subchannel velocities for Window 3 at Rake Location $\mathrm{Y}=-0.581$ in in PNNL 2x6 . . . . . . . . . . . . . . . . . . . . . . . . . . . . . . . . 215

12.3 Predicted and measured subchannel velocities for Window 5 at Rake Location $\mathrm{Y}=-0.581$ in in PNNL 2x6 . . . . . . . . . . . . . . . . . . . . . . . . . 216

12.4 Predicted and measured subchannel velocities for Window 1 at Rake Location $\mathrm{Y}=0.0$ in in PNNL 2x6 . . . . . . . . . . . . . . . . . . . . . . . . . . . . 217

12.5 Predicted and measured subchannel velocities for Window 3 at Rake Location $\mathrm{Y}=0.0$ in in PNNL $2 \times 6$. . . . . . . . . . . . . . . . . . . . . . . . . . . . . . . . . . 218

12.6 Predicted and measured subchannel velocities for Window 5 at Rake Location $\mathrm{Y}=0.0$ in in PNNL 2x6 . . . . . . . . . . . . . . . . . . . . . . . . . . . . . . . 219

12.7 Predicted and measured subchannel velocities for Window 7 at Rake Location $\mathrm{Y}=0.0$ in in PNNL 2x6 . . . . . . . . . . . . . . . . . . . . . . . . . . . . . 220

12.8 Predicted and measured subchannel velocities for Window 1 at Rake Location $\mathrm{Y}=0.581$ in in PNNL 2x6 . . . . . . . . . . . . . . . . . . . . . . . . . . . . . . 221

12.9 Predicted and measured subchannel velocities for Window 3 at Rake Location $\mathrm{Y}=0.581$ in in PNNL 2x6 . . . . . . . . . . . . . . . . . . . . . . . . . . . . . 222

12.10Predicted and measured subchannel velocities for Window 5 at Rake Location $\mathrm{Y}=0.581$ in in PNNL 2x6 . . . . . . . . . . . . . . . . . . . . . . . . . . . . . 223

12.11Predicted and measured subchannel velocities for Window 7 at Rake Location $\mathrm{Y}=0.581$ in in PNNL 2x6 . . . . . . . . . . . . . . . . . . . . . . . . . . . . . . . . . . 224

12.12Predicted and measured subchannel-center temperatures for Window 1 in PNNL 2x6 . . . . 225

12.13Predicted and measured subchannel-center temperatures for Window 3 in PNNL 2x6 . . . . 226

12.14Predicted and measured subchannel-center temperatures for Window 5 in PNNL 2x6 . . . . . 227

12.15Predicted and measured subchannel-center temperatures for Window 7 in PNNL 2x6 . . . . . 228

12.16Predicted and measured subchannel-center temperatures for Window 9 in PNNL 2x6 . . . . . 229 


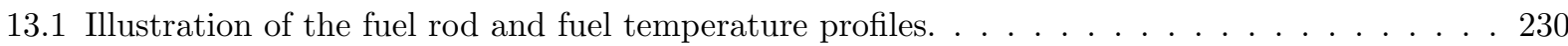

13.2 Comparison of CTF predictions vs. analytical results for fuel centerline temperatures. Grey

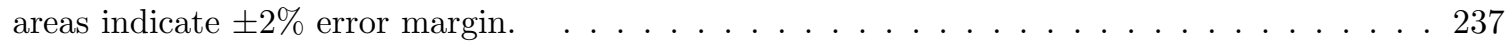

13.3 Comparison of CTF predictions with analytic solution for various $\Delta r$ choices. . . . . . . 239

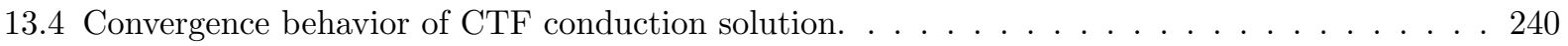

13.5 Predicted and measured fuel centerline temperature predictions for IFA432 Rod-1 at beginning of life (BOL) at lower thermocouple. The light and dark gray areas in the plot correspond to $\pm 5 \%$ relative error and $\pm 20^{\circ} \mathrm{K}$ around the measured data, respectively. . . . . . . . . . 242

13.6 Predicted and measured fuel centerline temperature predictions for IFA432 Rod- 1 at BOL at upper thermocouple. The light and dark gray areas in the plot correspond to $\pm 5 \%$ relative error and $\pm 20^{\circ} \mathrm{K}$ around the measured data, respectively. . . . . . . . . . . . . . 243

13.7 Predicted and measured fuel centerline temperature predictions for IFA432 Rod-1 (at lower thermocouple position). The light, dark gray areas in the plot correspond to $\pm 5 \%$ relative error and $\pm 20^{\circ} \mathrm{K}$ around the measured data, respectively. . . . . . . . . . . . . . . 244

13.8 Predicted and measured fuel centerline temperature predictions at lower thermocouple position for IFA681 Rod-2 (at lower thermocouple position). The light, dark gray areas in the plot correspond to $\pm 5 \%$ relative error and $\pm 20^{\circ} \mathrm{K}$ around the measured data, respectively. . .

13.9 Predicted and measured fuel centerline temperature predictions for IFA610 Rod-2 (at lower thermocouple position). The light, dark gray areas in the plot correspond to $\pm 5 \%$ relative error and $\pm 20^{\circ} \mathrm{K}$ around the measured data, respectively. . . . . . . . . . . . . 246

14.1 Comparison of $\mathrm{CTF}$ and experimental results for film flow rate at the outlet as a fraction of

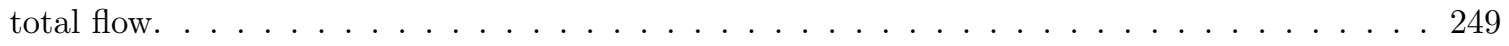

14.2 Comparison of CTF and experimental results for droplet flow rate at the outlet as a fraction of total flow. . . . . . . . . . . . . . . . . . . . . . 250

15.1 Illustration of the Water Faucet Problem. . . . . . . . . . . . . . . . . 252

15.2 Exact and numerical solution of the vapor volume fraction during the transient and at steady state for $P_{\text {out }}=10$ bar $($ run with 120 cells and a CFL of 0.8$) \ldots \ldots \ldots \ldots$

15.3 Convergence plot of the vapor volume fraction in the $L_{1}$ error norm. . . . . . . . . . . 254

15.4 Vapor volume fraction profile for three mesh densities $(30,240$, and 960 cells $)$ at $\mathrm{t}=0.4 \mathrm{~s}$. . 255

15.5 Illustration of the effect of the ICP term on the numerical solution. Vapor volume fraction

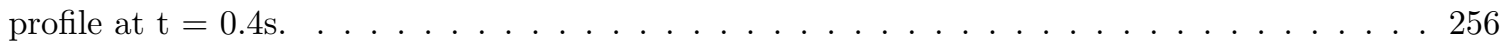

15.6 Effect of the parameter $\delta$ on the steady-state pressure profile $\ldots \ldots \ldots \ldots \ldots \ldots$ 


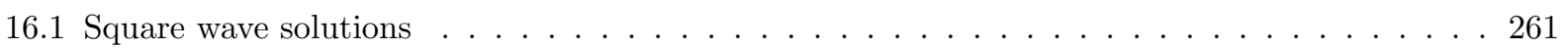

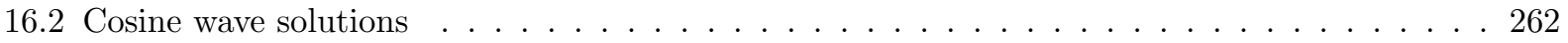

16.3 Hyperbolic tangent wave solutions . . . . . . . . . . . . . . . . . . 262

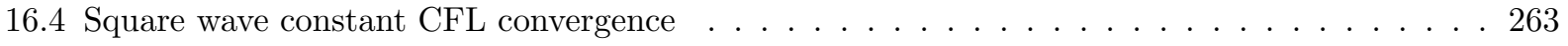

16.5 Cosine wave constant CFL convergence $\ldots \ldots \ldots \ldots \ldots \ldots \ldots \ldots \ldots$

16.6 Hyperbolic tangent wave constant CFL convergence . . . . . . . . . . . . . . . . 264 


\section{Acronyms}

BFBT BWR Full-size Fine-mesh Bundle Tests

BOHL Beginning of Heated Length

BOL beginning of life

BWR Boiling Water Reactor

CASL Consortium for Advanced Simulation of Light Water Reactors

CIPS Crud-induced power shift

COBRA-TF Coolant-Boiling in Rod Arrays- Two Fluids

CTF PSU RDFMG version of COBRA-TF

CHF Critical Heat Flux

DNB Departure from Nucleate Boiling

Gd gadolinia

GE General Electric

HBWR Halden Boiling Water Reactor

HTC Heat Transfer Coefficient

IFA Instrumented Fuel Assembly

JAERI Japanese Atomic Energy Research Institute

LDA Laser Doppler Anemometer

LHS Left-Hand Side

LWR Light Water Reactor

MV Mixing Vane

MVG Mixing Vane Grid 


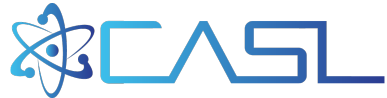

NFUL number of radial nodes in the fuel

NMV Non-Mixing Vane

NRC Nuclear Regulatory Commission

ONB onset of nucleate boiling

PNL Pacific Northwest Laboratory

PSBT PWR sub-channel and bundle tests

PSU Pennsylvania State University

$\mathbf{P u}$ plutonium

PWR Pressurized Water Reactor

QoI Quantity of Interest

RDFMG Reactor Dynamics and Fuel Modeling Group

RHS Right-Hand Side

RIA Reactivity-Insertion Accident

rRMS relative root-mean-square

RMSE Root-mean-square error

TC thermocouple

TD theoretical density

$\mathbf{T} / \mathbf{H}$ thermal/hydraulic

TKE turbulent kinetic energy

$\mathbf{V} \& \mathbf{V}$ validation \& verification

WALT Westinghouse advanced loop tester

YHL Yao-Hochreiter-Leech 


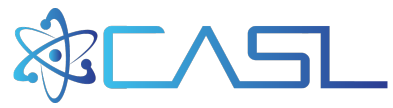

CTF V\&V

\section{Introduction}

Modeling and simulating a physical system or phenomena is a complex process involving several steps. We must formulate a mathematical model that suitably describes the physics we look to model, we need to come up with a way to solve that mathematical model, and finally, we need to then translate all these steps into machine language in the form of a computer program to make the method useful for solving real problems. Additionally, each of these steps - the model selection, assumptions, and coding - must be thoroughly tested and documented so as to instill confidence in the user that the resulting product is useful for its intended purpose. Ideally, good code documentation should inform the user:

1. what the code does,

2. how the code works,

3. how to use the code, and

4. that the code works as advertised.

The CTF documentation suite includes both a User Manual[1] and a Theory Manual[2], which address Item 3 (how to use the code) and Item 2 (how the code works). This document addresses Item 1 (what the code does) and Item 4 (that the code works as advertised). Note that Item 4 covers code testing, which is a broad topic that includes both verification and validation tasks. Whereas verification tasks demonstrate that the mathematical equations are being solved correctly and were implemented into the code in a bug-free way, validation tasks demonstrate that the correct equations are being solved, resulting in good agreement with experimental data. Comparing to experimental data is a necessary part of validation work. This document covers both validation and verification testing that is performed for CTF.

An important aspect of this validation \& verification (V\&V) suite is its tight integration in the CASL version of CTF. All of the tests created as part of this study have been included directly in the CTF code repository. Additionally, many of the tests are used as code regression tests that are run on a regular basis in an automated fashion. Because the testing process is automated, it is possible for the user to re-run the validation suite at the "push of a button". This instills a higher level of confidence in the modeling and simulation capabilities of CTF and helps to keep this validation study relevant, despite ongoing development activities in the code.

Note that while many of these tests have been incorporated as regression tests (meaning tests that are run on a continual basis to demonstrate code results are not changing), this document does not serve as 
a single, comprehensive reference for all regression testing performed on CTF. Many additional regression tests are included in the CTF automated test matrix that do not have experimental results or an analytical solution to compare against; rather, they simply exercise some feature or combination of features that have an effect on the output file, which is checked against a gold version. Documentation for such tests can be found directly in the "CMakeLists.txt" file that drives the automated test matrix (i.e., COBRA-TF/cobra_tf/test_matrix/CMakeLists.txt) or in the corresponding test input files found in COBRATF/cobra_tf/test_matrix.

\subsection{Organization of the document}

Chapter 2 gives the plan that is used to drive CTF V\&V efforts. This document does not seek to "validate CTF"; rather, it attempts to "validate CTF for specific applications". This chapter will outline the intended applications of CTF, discuss the physical phenomena important to these applications, the CTF models used to capture these phenomena, and present the tests used to ensure the correct functioning of these models.

Chapter 3 gives an overview of the experiments that were used for the validation study. Emphasis is placed on a brief overview of the design of the tests and their purpose as well as how CTF was used to model the tests.

The remaining chapters cover the V\&V efforts for each of the intended code applications discussed in Chapter 2 .

\subsection{Comparison Metrics}

In the following analysis, it is necessary to choose some metrics for comparing the accuracy of CTF in predicting the numerous Quantity of Interest (QoI)s. Most of this analysis is rather standard and simple, but for the sake of being clear, some definitions are provided here.

We employ simple averaging to calculate average predicted and measured absolute values and, at times, also the average difference between measured and predicted values. This will be referred to as the "mean" or referenced symbolically as $\mu$ and is calculated as in Equation 1.1. In the equation, $N$ is the number of values in the sample and $x_{i}$ is the sampled value.

$$
\mu=\Sigma_{i=1}^{N}\left(\frac{x_{i}}{N}\right)
$$

The standard deviation, is in reference to the spread of the data that was averaged using Equation 1.1. It is calculated as shown in Equation 1.2.

$$
\sigma=\sqrt{\sum_{i=1}^{N}\left(\frac{\left(x_{i}-\mu\right)^{2}}{N}\right)}
$$


In order to make direct comparisons between the measured and predicted values, the Root-mean-square error (RMSE) is used, which is shown in Equation 1.3. It is a common metric for comparing predicted and measured values and is similar to the standard deviation, except that it compares the distances of the predictions from measured values. In the equation, $x_{\mathrm{ctf}}$ is the predicted value and $x_{\mathrm{m}}$ is the corresponding measured value. A note about this formulation is that it will exaggerate large differences between predictions and measured data due to the squaring of the difference. Outliers are removed where appropriate, but this is clearly noted in the text when done.

$$
\mathrm{RMSE}=\sqrt{\frac{1}{N} \Sigma_{i=1}^{N}\left(x_{\mathrm{ctf}, i}-x_{\mathrm{m}, i}\right)^{2}}
$$

Both the standard deviation and RMSE will retain the units of the sampled value. This can be problematic when we want to compare data sets that were sampled from different operating conditions; in such a case, we expect the magnitude of the differences between measured and predicted results to change, so what we really want is to quantify the average of the relative differences. This is accomplished by taking the relative root-mean-square (rRMS) of the relative errors between measured and computed data. The relative error is defined as shown in Equation 1.4. The rRMS of the relative errors is defined as shown in Equation 1.5.

$$
\begin{gathered}
E_{\text {rel }}=\frac{x_{\text {measured }}-x_{\text {predicted }}}{x_{\text {measured }}} \\
\text { rRMS }=\sqrt{\frac{1}{N} \Sigma_{i=1}^{N} E_{\text {rel }, i}^{2}}
\end{gathered}
$$




\section{Validation Plan}

CTF is being developed in Consortium for Advanced Simulation of Light Water Reactors (CASL) for modeling specific applications. The validation and verification work is meant to demonstrate that the code behaves as expected and produces physically correct, accurate results for these intended applications. Therefore, the intended applications should drive the validation and verification work. This section specifies the intended modeling applications of CTF. A list of high-level code requirements follows the list of applications. The requirements are tasks that the code must be able to perform to be used for its intended applications. The high-level requirements include a link to tests (documented later in this document) that test the requirement.

In addition to high-level requirements, a list of low-level requirements has also been developed and can be found in the CTF Software Requirements, Test Plan, and Test Report. The low-level requirements are more fine-grain requirements (e.g., requirements on individual modules and procedures in the code) that will ultimately feed into the high-level requirements. Each low-level requirement is also linked to a test that ensures the requirement is being met.

The intended CTF applications are as follows:

1. Normal PWR operating behavior

2. Normal BWR operating behavior

3. Departure from Nucleate Boiling (DNB) analysis of PWRs

4. Crud-induced power shift (CIPS) analysis of PWRs

5. Reactivity-Insertion Accident (RIA) analysis in PWRs

It should be assumed that any applications not included in this list are not supported by CTF. A list of high-level requirements is presented as follows.

Table 1: High-level requirements

\begin{tabular}{ll}
\hline Requirement & Tests \\
\hline Single-phase convective heat transfer & 4.2 .1 \\
Subcooled nucleate boiling heat transfer & 4.1 .1
\end{tabular}


Table 1 - Continued from previous page

\begin{tabular}{ll}
\hline Requirement & Tests \\
\hline Near wall vapor generation & 8.1 .1 \\
Single-phase form loss & 6.2 .1 \\
Two-phase form loss & 6.3 .1 \\
Single-phase wall shear & 6.2 .1 \\
Two-phase wall shear & 6.3 .1 \\
Spacer grid heat transfer enhancement & 5.1 \\
Spacer grid turbulent kinetic energy (TKE) enhancement & 5.1 \\
Single-phase turbulent mixing & 7 \\
Two-phase turbulent mixing & 9 \\
Pressure directed cross flow & 6.2 .2 .1 \\
Nucleate boiling heat transfer & 4.1 .1 \\
Void drift & 9 \\
Droplet entrainment and de-entrainment & 14.1 .1 \\
Small bubble interfacial drag and implicit heat transfer & 8.1 .1 \\
Large bubble interfacial drag and implicit heat transfer & 8.1 .1 \\
Annular mist interfacial drag and implicit heat transfer & 9.1 .2 \\
Critical heat flux & 10.1 \\
\hline
\end{tabular}




\section{Test Descriptions}

This chapter presents an overview of all experiments that were used for CTF validation work.

\subsection{PSBT}

The PWR sub-channel and bundle tests (PSBT) experiments include both single-channel and rod-bundle geometries, as well as steady-state and transient operating conditions. The PSBT specifications[3] organize the experiments into two phases with different subcategories.

- Phase I: Void Distribution Benchmark

- Exercise 1: Steady-state single subchannel benchmark

- Exercise 2: Steady-state bundle benchmark

- Exercise 3: Transient bundle benchmark

- Exercise 4: Pressure drop benchmark

- Phase II: DNB Benchmark

- Exercise 1: Steady-state fluid temperature benchmark

- Exercise 2: Steady-state DNB benchmark

- Exercise 3: Transient DNB benchmark

Tests from Phase I, Exercise 1, and Phase I, Exercise 2 were used for these validation cases. Specifically, Test Series 1-7 were used for CTF validation activities in this document. Among these test sets, Test Series 1, 2, 3 and 4 were single subchannels aiming to perform void measurements for each of the four different channel types: S1 for center (typical), S2 for center (thimble), S3 for side, and S4 for corner. Geometry details are presented in Figure 3.1 and Figure 3.2; the cross sectional view of each subchannel type is shown in Figure 3.3 .

A total of 43 single subchannel data points were made publicly available and selected for CTF validation. The test names are summarized in Table 2. Void measurements were made by a gamma-ray CT scanner at $1.4 \mathrm{~m}$ from channel bottom. 


\begin{tabular}{|c|c|c|c|c|}
\hline Item & \multicolumn{4}{|c|}{ Data } \\
\hline $\begin{array}{l}\text { Assembly } \\
\text { (Subjected subchannel) }\end{array}$ & 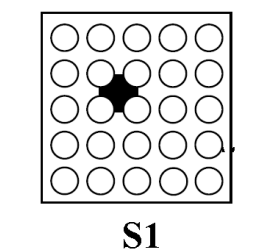 & 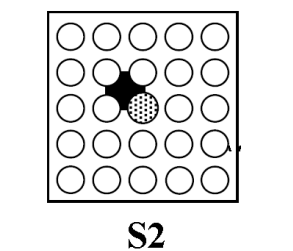 & 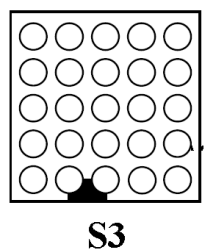 & 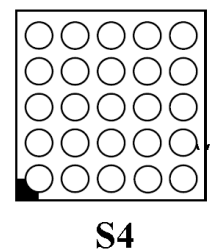 \\
\hline Subchannel type & Center (Typical) & Center (Thimble) & Side & Corner \\
\hline Number of heaters & $4 \times 1 / 4$ & $3 \times 1 / 4$ & $2 \times 1 / 4$ & $1 \times 1 / 4$ \\
\hline Axial heated length $(\mathrm{mm})$ & 1555 & 1555 & 1555 & 1555 \\
\hline Axial power shape & Uniform & Uniform & Uniform & Uniform \\
\hline
\end{tabular}

- : Subjected subchannel $\bigcirc$ : Heated rod ;ik: Thimble rod

Figure 3.1: Summary of PSBT single subchannel configurations for CTF validation activities

\begin{tabular}{|c|c|c|c|c||}
\hline & \multicolumn{4}{|c||}{ Subchannel Type } \\
& Typical (S1) & Thimble (S2) & Side (S3) & Corner (S4) \\
\hline \hline Flow area, $\mathrm{mm}^{2}$ & 107.098 & 107.098 & 68.464 & 42.592 \\
\hline Heated perimeter, $\mathrm{mm}$ & 29.845 & 22.384 & 14.923 & 7.461 \\
\hline Wetted perimeter, $\mathrm{mm}$ & 54.645 & 54.645 & 44.923 & 33.161 \\
\hline
\end{tabular}

Figure 3.2: PSBT single subchannel geometric characteristics

Table 2: PSBT single subchannel tests modeled for the CTF validation study

\begin{tabular}{cccc}
\hline Series 1 & Series 2 & Series 3 & Series 4 \\
\hline 11222 & 21231 & 32231 & 42251 \\
11223 & 21232 & 32232 & 42253 \\
12211 & 21233 & 32451 & 42256 \\
12221 & 23232 & 32452 & 42257 \\
12223 & 23233 & 32453 & 44455 \\
12237 & 24421 & 36431 & 44456 \\
12422 & 24422 & 36432 & 46461 \\
12423 & 24551 & 36461 & \\
14311 & 24552 & & \\
14312 & 26431 & & \\
14325 & 26432 & & \\
14326 & 26433 & & \\
15221 & & & \\
15222 & & & \\
16221 & & & \\
16222 & & & \\
\hline
\end{tabular}




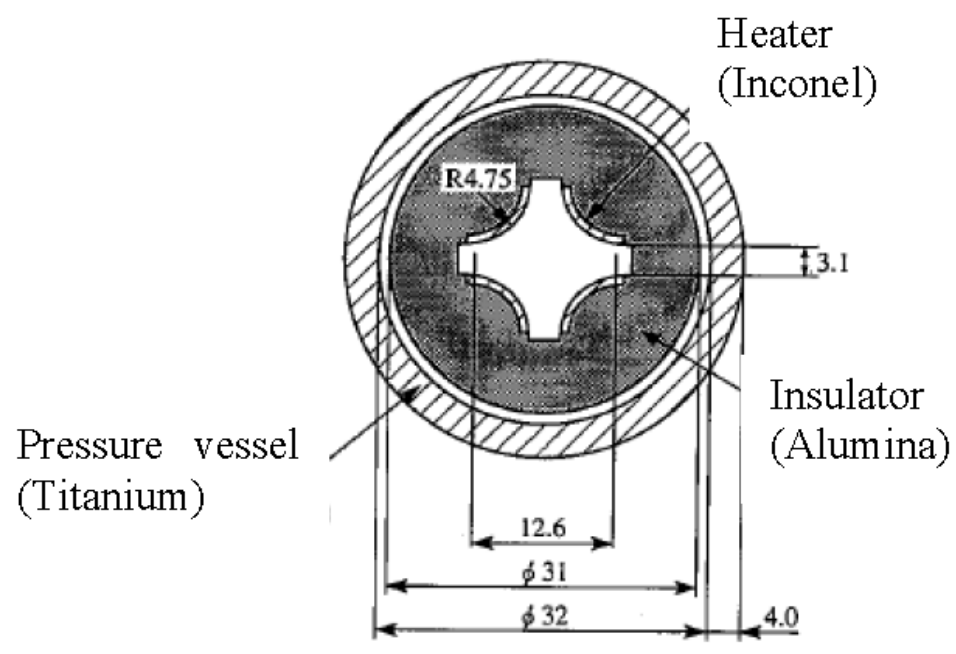

Central (Typical) Subchannel

S1

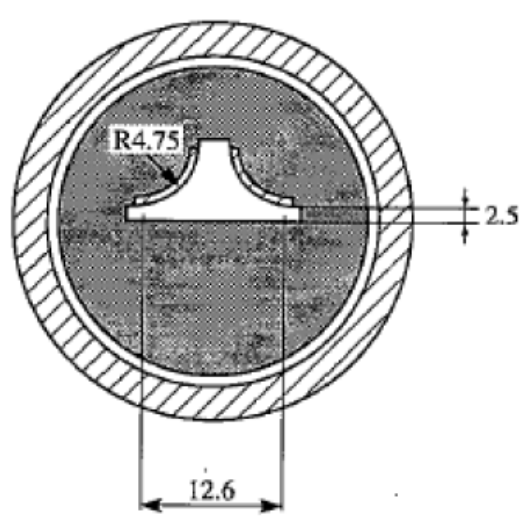

Side Subchannel

S3

\section{Dimensions in $\mathrm{mm}$}

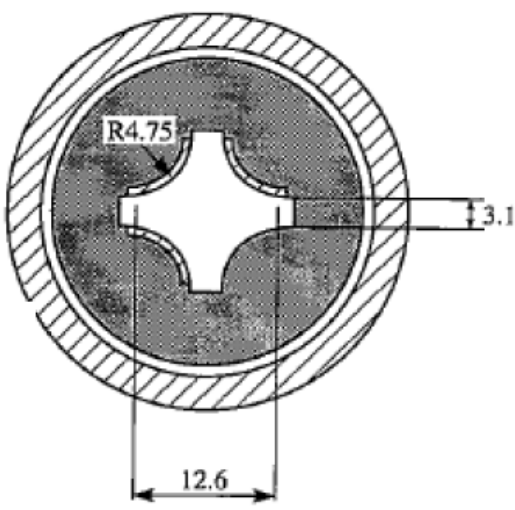

Central (Thimble) Subchannel S2

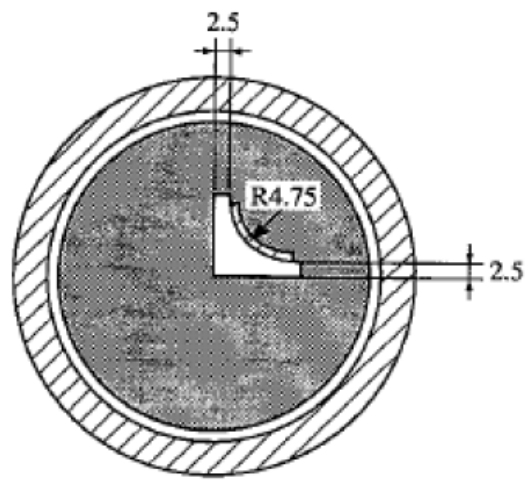

Corner Subchannel

S4

Figure 3.3: PSBT single subchannel cross sectional views 


\begin{tabular}{|c|c|c|c|}
\hline Item & \multicolumn{3}{|c|}{ Data } \\
\hline Assembly & \begin{tabular}{|c|c|}
00000 \\
00000 \\
00000 \\
00000 \\
00000 \\
B5
\end{tabular} & \begin{tabular}{|c|c|}
00000 \\
00000 \\
00000 \\
000000 \\
00000 \\
B6
\end{tabular} & 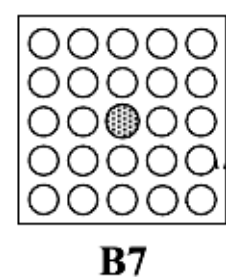 \\
\hline Rods array & $5 \times 5$ & $5 \times 5$ & $5 \times 5$ \\
\hline Number of heated rods & 25 & 25 & 24 \\
\hline Number of thimble rods & 0 & 0 & 1 \\
\hline Heated rod outer diameter $(\mathrm{mm})$ & 9.50 & 9.50 & 9.50 \\
\hline Thimble rod outer diameter $(\mathrm{mm})$ & - & - & 12.24 \\
\hline Heated rods pitch $(\mathrm{mm})$ & 12.60 & 12.60 & 12.60 \\
\hline Axial heated length $(\mathrm{mm})$ & 3658 & 3658 & 3658 \\
\hline Flow channel inner width $(\mathrm{mm})$ & 64.9 & 64.9 & 64.9 \\
\hline Radial power shape & A & A & $\mathrm{B}$ \\
\hline Axial power shape & Uniform & Cosine & Cosine \\
\hline Number of MV spacers & 7 & 7 & 7 \\
\hline Number of NMV spacers & 2 & 2 & 2 \\
\hline Number of simple spacers & 8 & 8 & 8 \\
\hline MV spacer location (mm) & \multicolumn{3}{|c|}{$471,925,1378,1832,2285,2739,3247$} \\
\hline NMV spacer location (mm) & \multicolumn{3}{|c|}{$2.5,3755$} \\
\hline Simple spacer location $(\mathrm{mm})$ & \multicolumn{3}{|c|}{$237,698,1151,1605,2059,2512,2993,3501$} \\
\hline
\end{tabular}

Figure 3.4: Summary of PSBT rod-bundle configurations for CTF validation activities

Test Series 5, 6 and 7 were rod-bundle geometry, with slightly different configurations. These configurations are detailed in Figure 3.4. Main differences included the power shapes and the placement of a central unheated guide tube in Assembly B7. Test Series 5 used the B5 configuration, Test Series 6 used the B6 configuration, and Test Series 7 used the B7 configuration.

The two radial power profile shapes given in Figure 3.4 are shown in Tables 3 and 4. The specification[3] provides an axial power profile table to be used to represent the cosine power shape, which is given in Table 5.

There are three types of spacer grids contained within the PSBT B5 bundle. There are 7 Mixing Vane (MV) spacers, 2 Non-Mixing Vane (NMV) spacers, and 8 simple spacers. Spacer grid locations are shown in Figure 3.4. The grids were modeled using the loss-coefficients provided in the PSBT specification[3]; the simple spacers had a loss coefficient of 0.4, the NMVs had a loss coefficient of 0.7, and the MVs had a loss coefficient of 1.0 .

Among the three rod bundle test series, over 200 experiments were run as part of the experiments. For this 
Table 3: PSBT bundle radial peaking factors: Layout A

\begin{tabular}{|l|l|l|l|l|}
\hline 0.9403 & 0.9403 & 0.9403 & 0.9403 & 0.9403 \\
\hline 0.9403 & 1.1062 & 1.1062 & 1.1062 & 0.9403 \\
\hline 0.9403 & 1.1062 & 1.1062 & 1.1062 & 0.9403 \\
\hline 0.9403 & 1.1062 & 1.1062 & 1.1062 & 0.9403 \\
\hline 0.9403 & 0.9403 & 0.9403 & 0.9403 & 0.9403 \\
\hline
\end{tabular}

Table 4: PSBT bundle radial peaking factors: Layout B

\begin{tabular}{|l|l|l|l|l|}
\hline 0.9838 & 0.9838 & 0.9838 & 0.9838 & 0.9838 \\
\hline 0.9838 & 1.1574 & 1.1574 & 1.1574 & 0.9838 \\
\hline 0.9838 & 1.1574 & 0.0000 & 1.1574 & 0.9838 \\
\hline 0.9838 & 1.1574 & 1.1574 & 1.1574 & 0.9838 \\
\hline 0.9838 & 0.9838 & 0.9838 & 0.9838 & 0.9838 \\
\hline
\end{tabular}


Table 5: PSBT bundle cosine axial power profile

\begin{tabular}{rrr}
\hline & node & ratio \\
\hline (Bottom) & 1 & 0.42 \\
2 & 0.47 \\
3 & 0.56 \\
4 & 0.67 \\
5 & 0.80 \\
6 & 0.94 \\
7 & 1.08 \\
8 & 1.22 \\
9 & 1.34 \\
& 10 & 1.44 \\
& 11 & 1.51 \\
12 & 1.55 \\
& 13 & 1.55 \\
& 14 & 1.51 \\
15 & 1.44 \\
16 & 1.34 \\
& 17 & 1.22 \\
& 18 & 1.08 \\
19 & 0.94 \\
& 20 & 0.80 \\
21 & 0.67 \\
& 22 & 0.56 \\
& 23 & 0.47 \\
24 & 0.42 \\
\hline
\end{tabular}


Table 6: PSBT rod-bundle tests modeled for the CTF validation study

\begin{tabular}{ccc}
\hline Series 5 & Series 6 & Series 7 \\
\hline 51221 & 61121 & 71121 \\
51222 & 61122 & 71122 \\
52111 & 61451 & 71341 \\
52112 & 61452 & 71342 \\
52332 & 62441 & 72221 \\
52442 & 62442 & 73121 \\
53441 & 63452 & 73451 \\
53442 & 64561 & 73452 \\
54562 & 64562 & 74561 \\
56321 & 66561 & 74562 \\
56322 & 66562 & 76321 \\
56552 & & 76322 \\
\hline
\end{tabular}

validation work, only the cases with open, publicly available data were used, which results in 35 tests being used. The test names are summarized in Table 6 .

Operating conditions for the three bundle tests series are given in Tables 7, 8, and 9 .

The average void in the four channels surrounding the central rod was the measured quantity of interest in the steady-state rod-bundle tests (Test Series 5, 6, and 7). These measurements were made via Xray densitometer measurements at three different axial locations; $2.216 \mathrm{~m}, 2.269 \mathrm{~m}$, and $3.177 \mathrm{~m}$ from Beginning of Heated Length (BOHL). The estimated accuracy of the void fraction measurements was $4 \%$ void. Additional information on bundle manufacturing tolerances as well as operating condition uncertainties are given in the test specifications.

\section{$3.2 \quad$ BFBT $8 \times 8$}

Facility Description The BWR Full-size Fine-mesh Bundle Tests (BFBT) are organized into phases and exercises as described in the BFBT specification [4].

- Phase I: Void Distribution Benchmark

- Exercise 1: Steady-state subchannel grade benchmark

- Exercise 2: Steady-state microscopic grade benchmark

- Exercise 3: Transient microscopic grade benchmark

- Exercise 4: Uncertainty analysis of the void distribution benchmark

- Phase II: Critical Power Benchmark

- Exercise 0: Steady-state pressure drop benchmark

- Exercise 1: Steady-state critical power benchmark 
Table 7: PSBT Test Series 5 Boundary Conditions

\begin{tabular}{rrrrr}
\hline $\begin{array}{r}\text { Run } \\
-\end{array}$ & $\begin{array}{r}\text { Pressure } \\
\text { MPa }\end{array}$ & $\begin{array}{r}\text { Mass flux } /\left(\mathrm{m}^{2}-\mathrm{hr}\right) \times 10^{-6} \\
\mathrm{~kW}\end{array}$ & $\begin{array}{r}\text { Power } \\
\text { Inlet temperature } \\
{ }^{\circ} \mathrm{C}\end{array}$ \\
\hline 51221 & 16.50 & 11.00 & 3000 & 292.3 \\
51222 & 16.50 & 10.98 & 2998 & 297.3 \\
52111 & 14.53 & 15.08 & 3296 & 291.9 \\
52112 & 14.52 & 14.98 & 3294 & 296.8 \\
52332 & 14.69 & 7.94 & 2523 & 287.8 \\
52442 & 14.71 & 4.99 & 2000 & 263.0 \\
53441 & 12.28 & 5.00 & 2014 & 247.9 \\
53442 & 12.27 & 5.00 & 2013 & 257.7 \\
54562 & 9.83 & 2.02 & 1016 & 214.3 \\
56321 & 4.82 & 7.87 & 3000 & 173.5 \\
56322 & 4.81 & 7.86 & 3000 & 183.6 \\
56552 & 4.92 & 2.00 & 1028 & 159.1 \\
\hline
\end{tabular}

Table 8: PSBT Test Series 6 Boundary Conditions

\begin{tabular}{rrrrr}
\hline $\begin{array}{r}\text { Run } \\
-\end{array}$ & $\begin{array}{r}\text { Pressure } \\
\text { MPa }\end{array}$ & $\begin{array}{r}\text { Mass Flux } /\left(\mathrm{m}^{2}-\mathrm{hr}\right) \times 10^{-6} \\
\mathrm{~kW}\end{array}$ & $\begin{array}{r}\text { Power } \\
\text { Inlet Temperature } \\
{ }^{\circ} \mathrm{C}\end{array}$ \\
\hline \hline 61121 & 16.43 & 15.16 & 3372 & 301.5 \\
61122 & 16.43 & 15.17 & 3376 & 306.7 \\
61451 & 16.58 & 5.20 & 1914 & 267.4 \\
61452 & 16.58 & 5.20 & 1915 & 272.5 \\
62441 & 14.71 & 5.16 & 2415 & 223.5 \\
62442 & 14.71 & 5.12 & 2412 & 228.4 \\
63452 & 12.28 & 5.00 & 1920 & 262.5 \\
64561 & 9.85 & 2.05 & 973 & 192.6 \\
64562 & 9.85 & 2.05 & 972 & 213.0 \\
66561 & 4.94 & 2.02 & 979 & 144.0 \\
66562 & 4.94 & 2.00 & 979 & 158.5 \\
\hline
\end{tabular}


Table 9: PSBT Test Series 7 Boundary Conditions

\begin{tabular}{rrrrr}
\hline $\begin{array}{r}\text { Run } \\
-\end{array}$ & $\begin{array}{r}\text { Pressure } \\
\mathrm{MPa}\end{array}$ & $\begin{array}{r}\text { Mass flux } /\left(\mathrm{m}^{2}-\mathrm{hr}\right) \times 10^{-6} \\
\mathrm{~kW}\end{array}$ & $\begin{array}{r}\text { Power } \\
\mathrm{knlet} \text { temperature } \\
{ }^{\circ} \mathrm{C}\end{array}$ \\
\hline 71121 & 16.42 & 15.07 & 3385 & 301.8 \\
71122 & 16.42 & 15.07 & 3384 & 306.8 \\
71341 & 16.55 & 7.92 & 2391 & 289.4 \\
71342 & 16.55 & 7.94 & 2391 & 295.3 \\
72221 & 14.64 & 11.01 & 3503 & 272.1 \\
73121 & 12.13 & 15.20 & 3502 & 276.1 \\
73451 & 12.27 & 5.03 & 2023 & 242.8 \\
73452 & 12.27 & 5.03 & 2021 & 260.1 \\
74561 & 9.83 & 2.16 & 1023 & 196.8 \\
74562 & 9.84 & 2.16 & 1023 & 214.9 \\
76321 & 4.89 & 8.10 & 3541 & 153.5 \\
76322 & 4.87 & 8.06 & 3536 & 168.6 \\
\hline
\end{tabular}

- Exercise 2: Transient benchmark

Tests from Phase II, Exercise 0 and Phase I, Exercise 1 are used for validation work. These tests cover single- and two-phase pressure drop as well as void content and distribution. For this validation study, 18 two-phase pressure drop (P6 tests) experiments and 10 single-phase pressure drop (P7 tests) experiments were modeled. Tests are summarized in Tables 10 and 11. Additionally, 16 of the void distribution tests are modeled. Table 12 shows their operating conditions.

The assembly type refers to the placement of heater rods, guide tubes, the water rod, and power configurations. Figures 3.5 and 3.6 show the geometry and rod configuration for Assembly Types 0-1, 0-2, and 0-3. All of these tests have a uniform axial and radial heating. The only difference between the assembly types is that some heater rods are shut off in Assembly Types 0-2 and 0-3. Figure 3.7 shows the geometry and power configuration for Assembly Types 1, 2, and 3. Only tests of Assembly Type 1 are modeled in this study. Assembly Type 1 has a cosine power shape, which is shown in Table 13 . All other assembly types modeled in this study have a uniform axial power shape. Assembly Types 1 and 4 have non-uniform radial power distributions. The Assembly Type 1 radial power distribution is shown in Figure 3.8, and the Assembly Type 4 radial power distribution is shown in Figure 3.9.

Finally, Figure 3.10 shows the rod configuration and power distribution for Assembly Types 4, C2A, C2B, and C3. Tests using Assembly type 4 are used in the void distribution tests. Assembly Type 4 replaces the four central rods with a large water rod. Assembly $\mathrm{C} 2 \mathrm{~A}$ is used in the pressure drop tests.

Table 15 contains the radial power profile for Assembly C2A while Table 14 contains the axial power profile for this bundle. Pressure tap locations are shown in Figure 3.11.

Void measurements were made in the facility using two approaches: 1) an x-ray densitometer was used at several axial locations, and 2) a CT scanner was used to obtain a fine-mesh void distribution measurement $50 \mathrm{~mm}$ above the end of heated length. The CT scanner measurements are used for comparison with CTF in this study. Figure 3.12 shows a schematic of where different void measurements were made in the facility. 
Table 10: BFBT Series P6 Boundary Conditions

\begin{tabular}{rrrrrr}
\hline Test & $\begin{array}{r}\text { Pressure } \\
\mathrm{MPa}\end{array}$ & $\begin{array}{r}\text { Inlet temperature } \\
{ }^{\circ} \mathrm{C}\end{array}$ & $\begin{array}{r}\text { Inlet subcooling } \\
\mathrm{kJ} / \mathrm{kg}\end{array}$ & $\begin{array}{r}\text { Flow rate } \\
\text { ton/hr }\end{array}$ & $\begin{array}{r}\text { Power } \\
\mathrm{MW}\end{array}$ \\
\hline $\mathrm{P} 60001$ & 7.16 & 277.3 & 53.3 & 20.2 & 0.863 \\
$\mathrm{P} 60003$ & 7.16 & 277.8 & 50.8 & 20.1 & 1.521 \\
$\mathrm{P} 60005$ & 7.16 & 277.7 & 51.1 & 20.0 & 2.357 \\
$\mathrm{P} 60007$ & 7.17 & 277.8 & 51.1 & 55.0 & 2.375 \\
$\mathrm{P} 60009$ & 7.17 & 277.8 & 51.1 & 55.0 & 4.197 \\
$\mathrm{P} 60013$ & 7.16 & 278.4 & 47.2 & 69.9 & 3.022 \\
$\mathrm{P} 60015$ & 7.17 & 278.2 & 49.5 & 70.0 & 5.340 \\
$\mathrm{P} 60017$ & 7.16 & 277.8 & 51.0 & 45.1 & 1.919 \\
$\mathrm{P} 60019$ & 7.17 & 278.2 & 49.4 & 45.0 & 3.437 \\
$\mathrm{P} 60022$ & 8.64 & 291.3 & 50.7 & 20.2 & 0.837 \\
$\mathrm{P} 60023$ & 8.63 & 291.0 & 52.3 & 20.2 & 1.464 \\
$\mathrm{P} 60024$ & 8.63 & 290.9 & 52.9 & 20.2 & 2.252 \\
$\mathrm{P} 60025$ & 8.64 & 291.3 & 51.3 & 55.0 & 2.271 \\
$\mathrm{P} 60026$ & 8.64 & 291.0 & 53.0 & 55.1 & 3.975 \\
$\mathrm{P} 60029$ & 8.64 & 291.3 & 51.5 & 70.1 & 2.888 \\
$\mathrm{P} 60030$ & 8.64 & 291.2 & 51.4 & 70.2 & 5.076 \\
$\mathrm{P} 60031$ & 8.64 & 290.9 & 53.0 & 45.1 & 1.869 \\
$\mathrm{P} 60032$ & 8.63 & 291.2 & 51.3 & 45.2 & 3.262 \\
\hline
\end{tabular}

Table 11: BFBT Series P7 Boundary Conditions

\begin{tabular}{rrrrr}
\hline Test & $\begin{array}{r}\text { Pressure } \\
\text { MPa }\end{array}$ & $\begin{array}{r}\text { Inlet temperature } \\
{ }^{\circ} \mathrm{C}\end{array}$ & $\begin{array}{r}\text { Flow rate } \\
\text { ton/hr }\end{array}$ & $\begin{array}{r}\text { Reynolds } \\
\times 10^{-4}\end{array}$ \\
\hline P70027 & 7.15 & 284.9 & 20.30 & 8.07 \\
P70028 & 7.16 & 285.1 & 24.90 & 9.91 \\
P70029 & 7.16 & 285.1 & 29.80 & 11.86 \\
P70030 & 7.16 & 285.7 & 34.70 & 13.82 \\
P70031 & 7.16 & 285.6 & 39.70 & 15.81 \\
P70032 & 7.16 & 285.3 & 44.60 & 17.75 \\
P70033 & 7.15 & 284.7 & 55.00 & 21.86 \\
P70034 & 7.15 & 284.8 & 59.70 & 23.74 \\
P70035 & 7.16 & 284.6 & 64.80 & 25.76 \\
P70036 & 7.15 & 284.8 & 69.90 & 27.79 \\
\hline
\end{tabular}


Table 12: BFBT void distribution test conditions

\begin{tabular}{rrrrrr}
\hline Test & Assembly type & $\begin{array}{r}\text { Pressure } \\
{[\mathrm{MPa}]}\end{array}$ & $\begin{array}{r}\text { Inlet subcooling } \\
{[\mathrm{kJ} / \mathrm{kg}]}\end{array}$ & $\begin{array}{r}\text { Flow rate } \\
{[\text { ton } / \mathrm{h}]}\end{array}$ & $\begin{array}{r}\text { Power } \\
\mathrm{MW}\end{array}$ \\
\hline $0011-55$ & $0-1$ & 7.180 & 52.60 & 54.03 & 1.90 \\
$0011-58$ & $0-1$ & 7.172 & 51.00 & 54.90 & 3.51 \\
$0011-61$ & $0-1$ & 7.210 & 50.90 & 54.79 & 6.44 \\
$0021-16$ & $0-2$ & 7.190 & 54.00 & 54.85 & 1.91 \\
$0021-18$ & $0-2$ & 7.171 & 49.80 & 54.90 & 3.51 \\
$0021-21$ & $0-2$ & 7.179 & 51.40 & 54.90 & 6.45 \\
$0031-16$ & $0-3$ & 7.180 & 52.40 & 54.96 & 1.92 \\
$0031-18$ & $0-3$ & 7.179 & 50.00 & 54.79 & 3.52 \\
$0031-21$ & $0-3$ & 7.171 & 49.40 & 54.90 & 6.45 \\
$1071-55$ & 1 & 7.191 & 52.80 & 54.61 & 1.92 \\
$1071-58$ & 1 & 7.158 & 50.30 & 55.07 & 3.52 \\
$1071-61$ & 1 & 7.200 & 51.80 & 54.65 & 6.48 \\
$4101-53$ & 4 & 7.181 & 52.80 & 54.65 & 1.24 \\
$4101-55$ & 4 & 7.195 & 52.90 & 54.59 & 1.92 \\
$4101-58$ & 4 & 7.152 & 50.60 & 54.58 & 3.52 \\
$4101-61$ & 4 & 7.180 & 52.50 & 54.65 & 6.48 \\
\hline
\end{tabular}

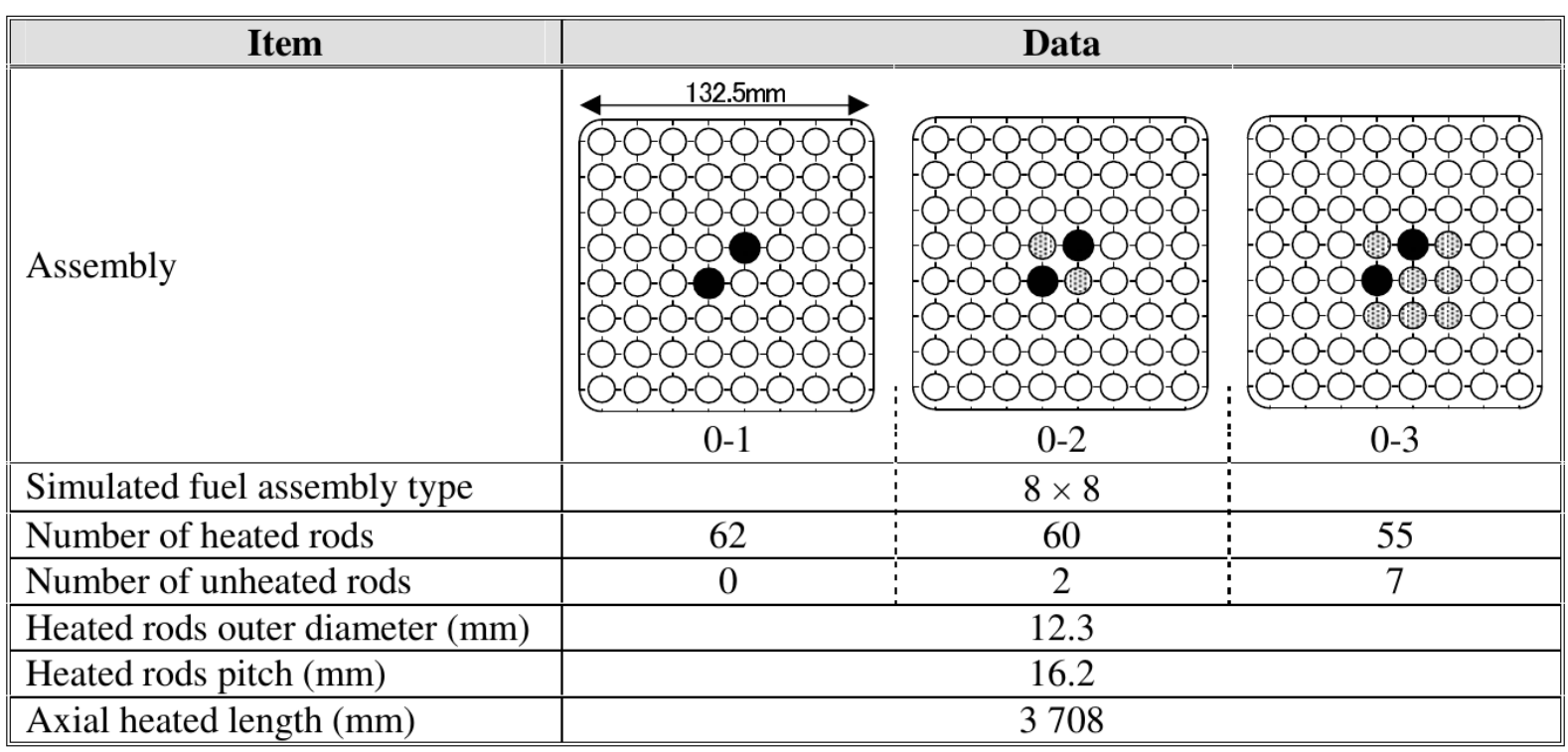

O Heated rod, $($ ) Unheated rod, $\bullet$ Water rod - no flow in water rods

Figure 3.5: Description of BFBT Assembly Types 0-1, 0-2, and 0-3 (reprinted from B. Neykov et al. NUPEC BWR Full-size Fine-mesh Bundle Test (BFBT) Benchmark. Tech. rep. NUCLEAR ENERGY AGENCY, 2006) 


\begin{tabular}{||l|c||}
\hline \multicolumn{1}{|c|}{ Item } & Data \\
\hline \hline Number of water rods & 2 \\
\hline Water rods outer diameter $(\mathrm{mm})$ & 15.0 \\
\hline Channel box inner width $(\mathrm{mm})$ & 132.5 \\
\hline Channel box corner radius $(\mathrm{mm})$ & 8.0 \\
\hline In channel flow area $\left(\mathrm{mm}^{2}\right)$ & 9781 \\
\hline Spacer type & Grid \\
\hline Number of spacers & 7 \\
\hline Spacer pressure loss coefficients & 1.2 \\
\hline Spacer location (mm) & 455, 967, 1 479, 1 991, 2 503, 3 015, 3 527 \\
\hline Radial power shape & Uniform \\
\hline Axial power shape & Uniform \\
\hline
\end{tabular}

Figure 3.6: Description of BFBT Assembly Types 0-1, 0-2, and 0-3 (continued) (reprinted from B. Neykov et al. NUPEC BWR Full-size Fine-mesh Bundle Test (BFBT) Benchmark. Tech. rep. NUCLEAR ENERGY AGENCY, 2006).

Table 13: BFBT Assembly Type 1 axial power distribution.

\begin{tabular}{rr}
\hline Axial level & Power factor \\
\hline 1 & 0.46 \\
2 & 0.58 \\
3 & 0.69 \\
4 & 0.79 \\
5 & 0.88 \\
6 & 0.99 \\
7 & 1.09 \\
8 & 1.22 \\
9 & 1.22 \\
10 & 1.34 \\
11 & 1.34 \\
12 & 1.40 \\
13 & 1.40 \\
14 & 1.34 \\
15 & 1.34 \\
16 & 1.22 \\
17 & 1.22 \\
18 & 1.09 \\
19 & 0.99 \\
20 & 0.88 \\
21 & 0.79 \\
22 & 0.69 \\
23 & 0.58 \\
24 & 0.46 \\
\hline
\end{tabular}




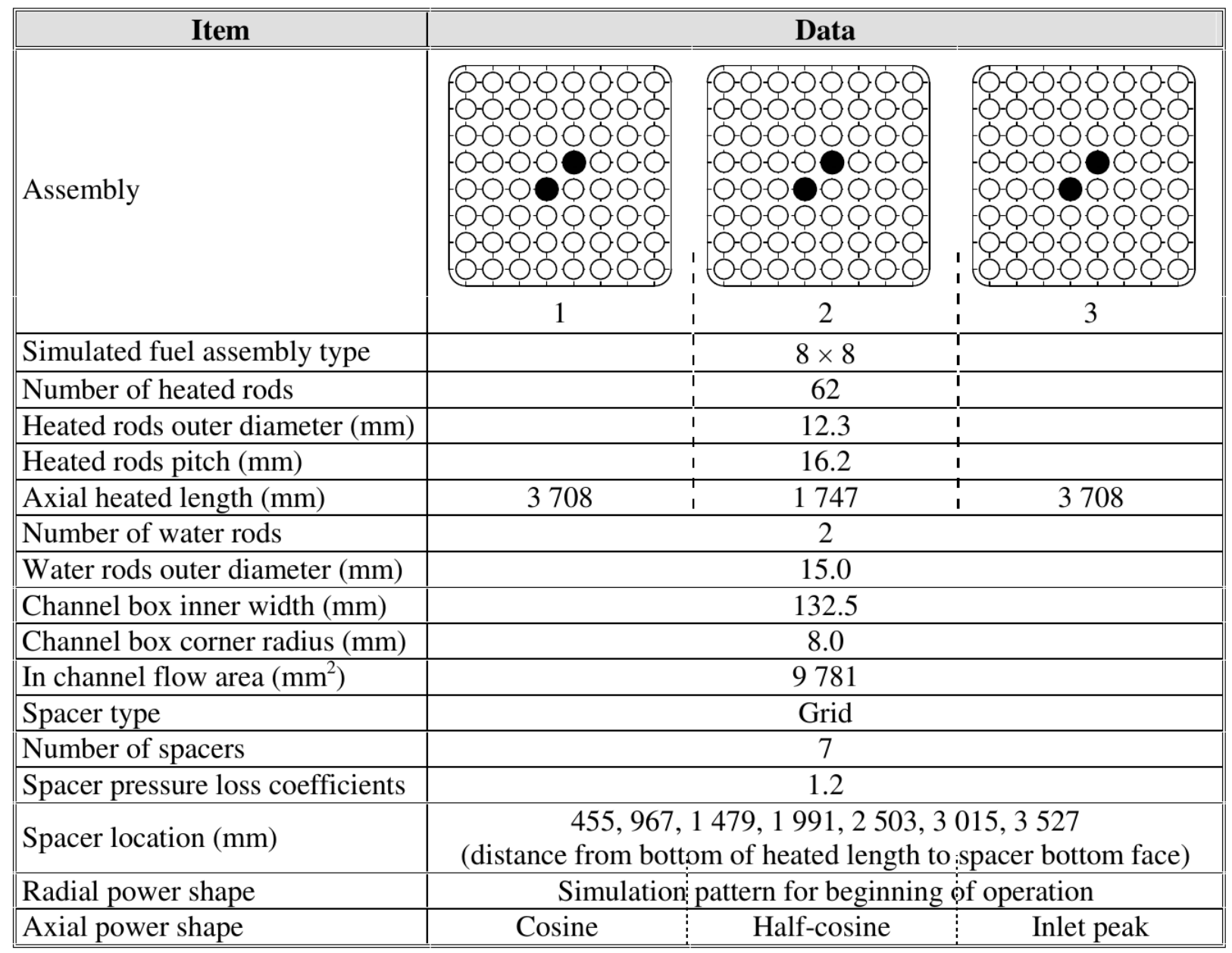

O Heated rod, $\rightarrow$ Unheated rod, $\bigcirc$ Water rod - no flow in water rods

Figure 3.7: Description of BFBT Assembly Types 1, 2, and 3 (reprinted from B. Neykov et al. NUPEC BWR Full-size Fine-mesh Bundle Test (BFBT) Benchmark. Tech. rep. NUCLEAR ENERGY AGENCY, 2006). 


\begin{tabular}{|l:l:l:l:l:l:l:l:l|}
\hline 1.15 & 1.30 & 1.15 & 1.30 & 1.30 & 1.15 & 1.30 & 1.15 \\
\hline 1.30 & 0.45 & 0.89 & 0.89 & 0.89 & 0.45 & 1.15 & 1.30 \\
\hline 1.15 & 0.89 & 0.89 & 0.89 & 0.89 & 0.89 & 0.45 & 1.15 \\
\hline 1.30 & 0.89 & 0.89 & 0.89 & & 0.89 & 0.89 & 1.15 \\
\hline 1.30 & 0.89 & 0.89 & & 0.89 & 0.89 & 0.89 & 1.15 \\
\hline 1.15 & 0.45 & 0.89 & 0.89 & 0.89 & 0.89 & 0.45 & 1.15 \\
\hline 1.30 & 1.15 & 0.45 & 0.89 & 0.89 & 0.45 & 1.15 & 1.30 \\
\hline 1.15 & 1.30 & 1.15 & 1.15 & 1.15 & 1.15 & 1.30 & 1.15 \\
\hline
\end{tabular}

Figure 3.8: Radial power distribution applied to Assembly Type 1 (reprinted from B. Neykov et al. NUPEC BWR Full-size Fine-mesh Bundle Test (BFBT) Benchmark. Tech. rep. NUCLEAR ENERGY AGENCY, 2006).

\begin{tabular}{|l|l|l|l|l|l|l|l|}
\hline 1.15 & 1.30 & 1.15 & 1.30 & 1.30 & 1.15 & 1.30 & 1.15 \\
\hline 1.30 & 0.45 & 0.89 & 0.89 & 0.89 & 0.45 & 1.15 & 1.30 \\
\hline 1.15 & 0.89 & 0.89 & 0.89 & 0.89 & 0.89 & 0.45 & 1.15 \\
\hline 1.30 & 0.89 & 0.89 & & & 0.89 & 0.89 & 1.15 \\
\hline 1.30 & 0.89 & 0.89 & & & 0.89 & 0.89 & 1.15 \\
\hline 1.15 & 0.45 & 0.89 & 0.89 & 0.89 & 0.89 & 0.45 & 1.15 \\
\hline 1.30 & 1.15 & 0.45 & 0.89 & 0.89 & 0.45 & 1.15 & 1.30 \\
\hline 1.15 & 1.30 & 1.15 & 1.15 & 1.15 & 1.15 & 1.30 & 1.15 \\
\hline
\end{tabular}

Figure 3.9: Radial power distribution applied to Assembly Type 4 (reprinted from B. Neykov et al. NUPEC BWR Full-size Fine-mesh Bundle Test (BFBT) Benchmark. Tech. rep. NUCLEAR ENERGY AGENCY, 2006). 


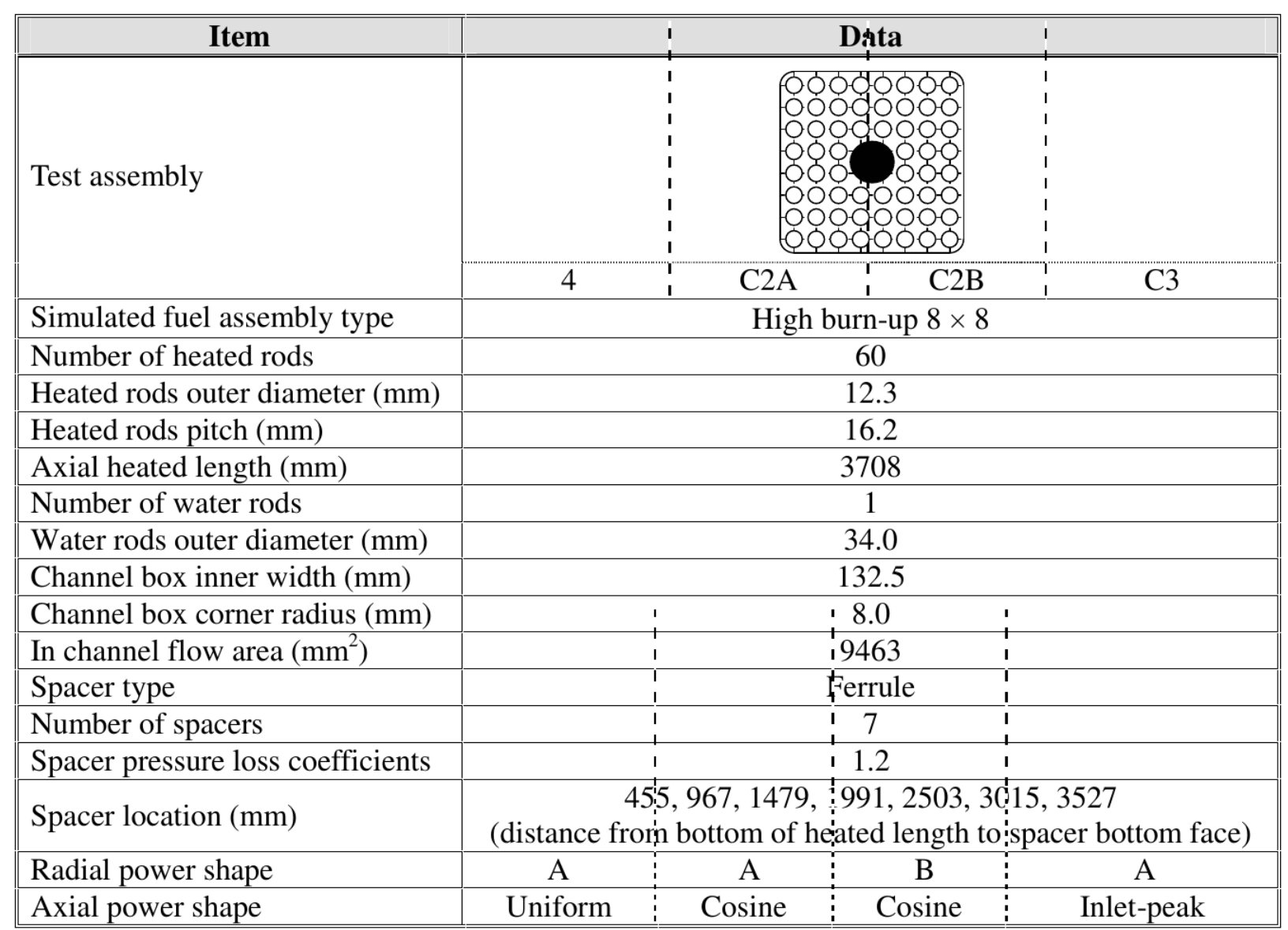

Heated rod, $\bigcirc$ Water rod - no flow in water rods

A: Simulation pattern for beginning of operation.

B: Simulation pattern for middle of operation.

Figure 3.10: Description of BFBT Assembly Type 4 (reprinted from B. Neykov et al. NUPEC BWR Full-size Fine-mesh Bundle Test (BFBT) Benchmark. Tech. rep. NUCLEAR ENERGY AGENCY, 2006). 


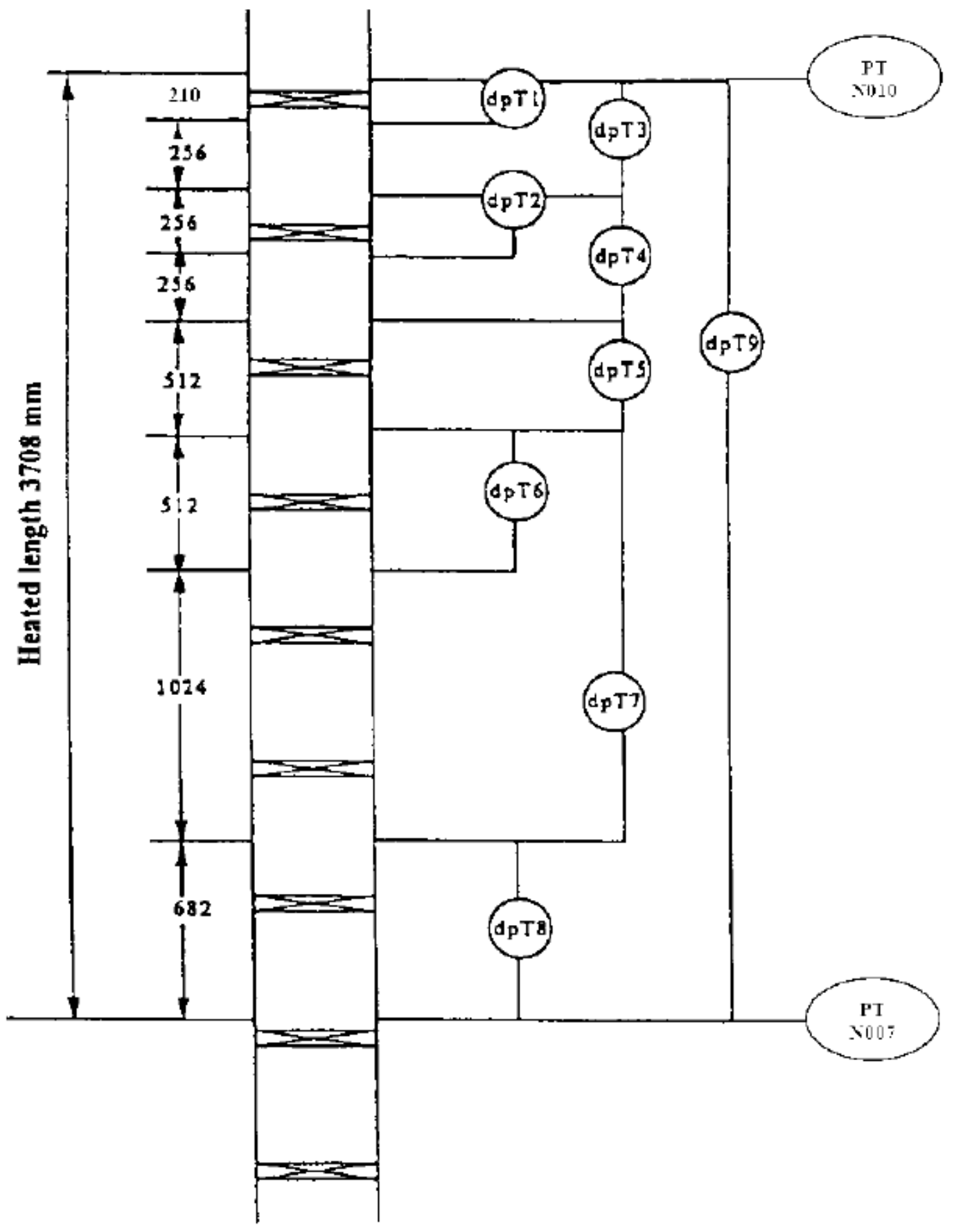

Figure 3.11: BFBT bundle pressure tap locations 
Table 14: BFBT C2A Bundle Axial Power Distribution

\begin{tabular}{ccc}
\hline & node & ratio \\
\hline (bottom) & 1 & 0.46 \\
& 2 & 0.58 \\
3 & 0.69 \\
4 & 0.79 \\
5 & 0.88 \\
& 6 & 0.99 \\
7 & 1.09 \\
8 & 1.22 \\
& 9 & 1.22 \\
& 10 & 1.34 \\
& 11 & 1.34 \\
& 12 & 1.40 \\
13 & 1.40 \\
& 14 & 1.34 \\
15 & 1.34 \\
16 & 1.22 \\
& 17 & 1.22 \\
18 & 1.09 \\
19 & 0.99 \\
20 & 0.88 \\
21 & 0.79 \\
22 & 0.69 \\
& 23 & 0.58 \\
& 24 & 0.46 \\
\hline
\end{tabular}




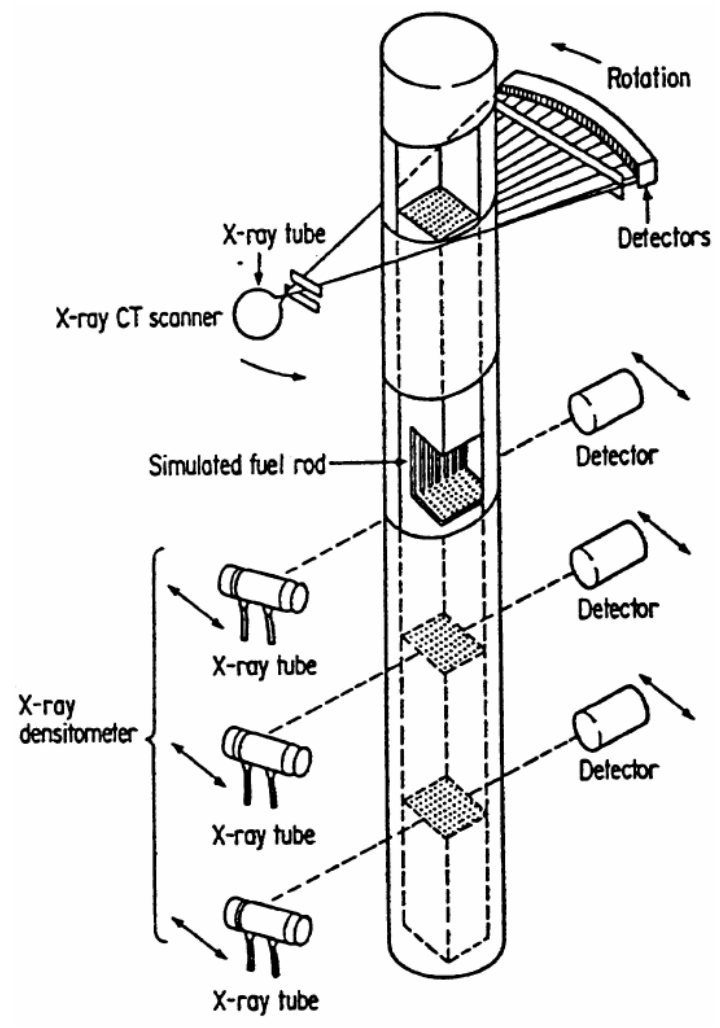

Figure 3.12: Depiction of void measurement locations and techniques in BFBT facility (reprinted from B. Neykov et al. NUPEC BWR Full-size Fine-mesh Bundle Test (BFBT) Benchmark. Tech. rep. NUCLEAR ENERGY AGENCY, 2006). 
Table 15: BFBT C2A Bundle Radial Power Distribution

\begin{tabular}{|l|c|c|c|c|c|c|c|}
\hline 1.15 & 1.30 & 1.15 & 1.30 & 1.30 & 1.15 & 1.30 & 1.15 \\
\hline 1.30 & 0.45 & 0.89 & 0.89 & 0.89 & 0.45 & 1.15 & 1.30 \\
\hline 1.15 & 0.89 & 0.89 & 0.89 & 0.89 & 0.89 & 0.45 & 1.15 \\
\hline 1.30 & 0.89 & 0.89 & - & - & 0.89 & 0.89 & 1.15 \\
\hline 1.30 & 0.89 & 0.89 & - & - & 0.89 & 0.89 & 1.15 \\
\hline 1.15 & 0.45 & 0.89 & 0.89 & 0.89 & 0.89 & 0.45 & 1.15 \\
\hline 1.30 & 1.15 & 0.45 & 0.89 & 0.89 & 0.45 & 1.15 & 1.30 \\
\hline 1.15 & 1.30 & 1.15 & 1.15 & 1.15 & 1.15 & 1.30 & 1.15 \\
\hline
\end{tabular}

The CT scanner worked by sweeping over the bundle at a fixed axial plane to obtain void measurements in small "pixels" as small as $0.3 \times 0.3 \mathrm{~mm}$. The refined measurements have been used for CFD validation work, but the measurements were also post-processed to create a set of subchannel-averaged void measurements for comparison with subchannel codes. The measurements were made several times for the same operating conditions and were time-averaged to avoid the effects of two-phase flow oscillations.

The BFBT specification [4] states that the subchannel accuracy is estimated to be $3 \%$ and the bundle average void is estimated to be $2 \%$ (as will be shown when analyzing symmetry of the results, the measurement error is likely much higher than this).

CTF Model Figure 3.13 shows the flow area and wetted perimeter for the channels in the CTF model for Assembly Types 0-1, 0-2, 0-3, and 1. It also shows the channel indexing scheme employed in the model. Figure 3.14 shows the indexing scheme, flow areas, and wetted perimeters for Assembly type 4, which has a large water rod in the center.

Spacer grid locations are provided in Figures 3.5, 3.6, 3.7, and 3.10. Assembly types 0-1, 0-2, 0-3, and 1 use the lantern-type spacer grid shown in Figure 3.15. Assembly type 4 uses the ferrule-type spacer grid shown in Figure 3.16.

The loss coefficients for this type of spacer grid are calculated using the approach of Shiralkar and Radcliffe [5]. Details of the calculation are shown in [6]. Figure 3.17 shows the radial loss coefficient distribution in the bundle for assemblies using the lantern-style spacer grids and Figure 3.18 shows the radial loss coefficient distribution for assemblies using the ferrule-style spacer grids.

The CTF friction correlation is used [2] along with a single-phase mixing coefficient of 0.007 and a Beus two-phase multiplier, $\Theta_{M}$, of 5.0. The cases are run with and without the droplet field enabled to test the 


\begin{tabular}{|c|c|c|c|c|c|c|c|c|}
\hline 1 & 2 & 3 & 4 & 5 & 6 & 7 & 8 & 9 \\
\hline 10 & 11 & 12 & 13 & 14 & 15 & 16 & 17 & 18 \\
\hline 19 & 20 & 21 & 22 & 23 & 24 & 25 & 26 & 27 \\
\hline 28 & 29 & 30 & 31 & 32 & 33 & 34 & 35 & 36 \\
\hline 37 & 38 & 39 & 40 & 41 & 42 & 43 & 44 & 45 \\
\hline 46 & 47 & 48 & 49 & 50 & 51 & 52 & 53 & 54 \\
\hline 55 & 56 & 57 & 58 & 59 & 60 & 61 & 62 & 63 \\
\hline 64 & 65 & 66 & 67 & 68 & 69 & 70 & 71 & 72 \\
\hline 73 & 74 & 75 & 76 & 77 & 78 & 79 & 80 & 81 \\
\hline
\end{tabular}

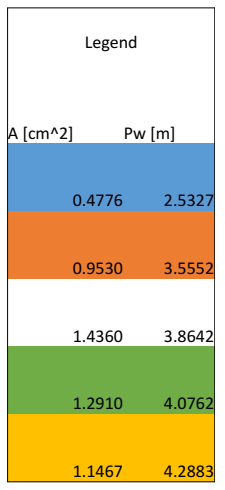

Figure 3.13: Map of the channel flow areas and wetted perimeters for Assembly Types $0-1,0-2,0-3$, and 1.

\begin{tabular}{|c|c|c|c|c|c|c|c|c|}
\hline 1 & 2 & 3 & 4 & 5 & 6 & 7 & 8 & 9 \\
\hline 10 & 11 & 12 & 13 & 14 & 15 & 16 & 17 & 18 \\
\hline 19 & 20 & 21 & 22 & 23 & 24 & 25 & 26 & 27 \\
\hline 28 & 29 & 30 & 31 & 32 & 33 & 34 & 35 & 36 \\
\hline 37 & 38 & 39 & 40 & & 41 & 42 & 43 & 44 \\
\hline 45 & 46 & 47 & 48 & 49 & 50 & 51 & 52 & 53 \\
\hline 54 & 55 & 56 & 57 & 58 & 59 & 60 & 61 & 62 \\
\hline 63 & 64 & 65 & 66 & 67 & 68 & 69 & 70 & 71 \\
\hline 72 & 73 & 74 & 75 & 76 & 77 & 78 & 79 & 80 \\
\hline
\end{tabular}

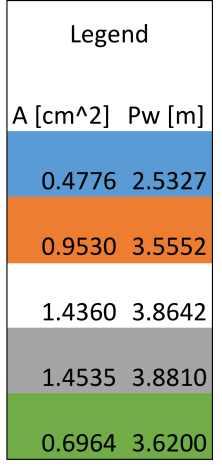

Figure 3.14: Map of the channel flow areas and wetted perimeters for Assembly Type 4. 


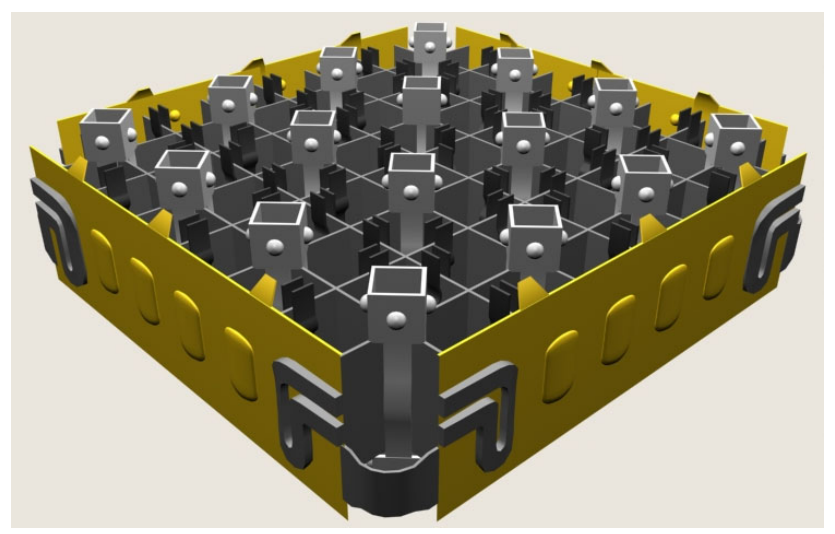

Figure 3.15: Lantern-style grid used in assembly types 0-1, 0-2, 0-3, and 1 of BFBT.

effect on void content and distribution. Results of this study are shown in Section 9.1.2. When the droplet field is disabled, it means that there is no entrainment or de-entrainment, and the interfacial drag and heat transfer between droplets and vapor are eliminated in the solution. The sensitivity study shows that results are improved when the droplet field is disabled, so the remainder of the void-distribution validation work is performed with the droplet field disabled.

The axial mesh is set up so that the height of a spacer grid occupies one full level. The average axial mesh cell height is $7.271 \mathrm{~cm}$. Only heater rods are explicitly modeled; unheated objects, such as guide tubes and the fuel canister, are not modeled, as they have no impact on the solution in a steady-state solve. Furthermore, the power is set as a heat flux boundary condition, which disables the internal rod temperature solution. 

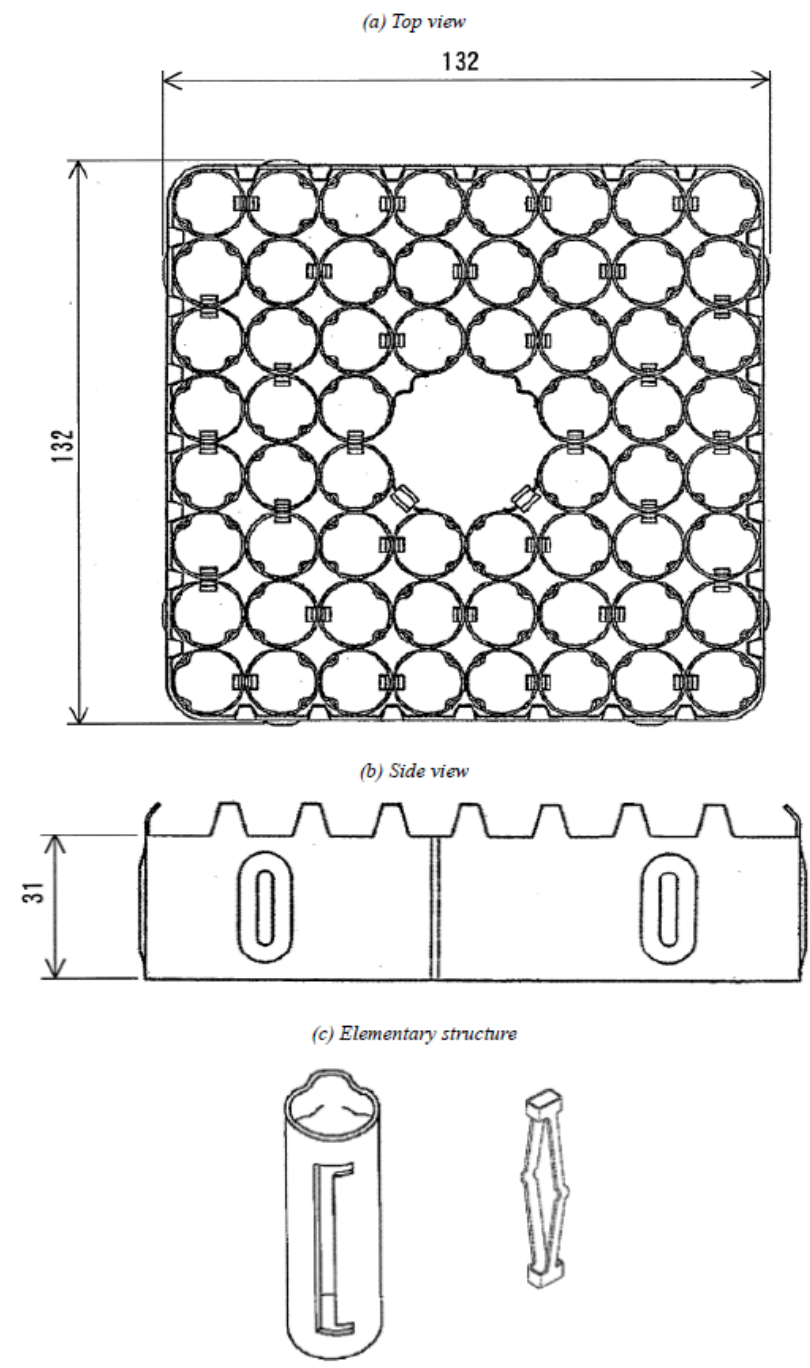

Figure 3.16: BFBT C2A ferrule grid geometry (reprinted from B. Neykov et al. NUPEC BWR Full-size Fine-mesh Bundle Test (BFBT) Benchmark. Tech. rep. NUCLEAR ENERGY AGENCY, 2006). 
Map of channel indices (color coded by grid loss coefficient):

\begin{tabular}{|c|c|c|c|c|c|c|c|c|}
\hline 1 & 2 & 3 & 4 & 5 & 6 & 7 & 8 & 9 \\
\hline 10 & 11 & 12 & 13 & 14 & 15 & 16 & 17 & 18 \\
\hline 19 & 20 & 21 & 22 & 23 & 24 & 25 & 26 & 27 \\
\hline 28 & 29 & 30 & 31 & 32 & 33 & 34 & 35 & 36 \\
\hline 37 & 38 & 39 & 40 & 41 & 42 & 43 & 44 & 45 \\
\hline 46 & 47 & 48 & 49 & 50 & 51 & 52 & 53 & 54 \\
\hline 55 & 56 & 57 & 58 & 59 & 60 & 61 & 62 & 63 \\
\hline 64 & 65 & 66 & 67 & 68 & 69 & 70 & 71 & 72 \\
\hline 73 & 74 & 75 & 76 & 77 & 78 & 79 & 80 & 81 \\
\hline
\end{tabular}

Legend (loss coefficients of color):

\begin{tabular}{|l|l|l|l|l|}
\hline $\mathrm{K}=0.989$ & $\mathrm{~K}=0.888$ & $\mathrm{~K}=1.754$ & $\mathrm{~K}=0.483$ & $\mathrm{~K}=1.110$ \\
\hline $\mathrm{K}=1.266$ & $\mathrm{~K}=1.838$ & $\mathrm{~K}=2.143$ & $\mathrm{~K}=1.963$ & $\mathrm{~K}=0.661$ \\
\hline $\mathrm{K}=0.471$ & $\mathrm{~K}=0.855$ & & & \\
\hline
\end{tabular}

Figure 3.17: Loss coefficient map for BFBT model using the lantern-type grid. 
Map of channel indices (color coded by grid loss coefficient):

\begin{tabular}{|c|c|c|c|c|c|c|c|c|}
\hline 1 & 2 & 3 & 4 & 5 & 6 & 7 & 8 & 9 \\
\hline 10 & 11 & 12 & 13 & 14 & 15 & 16 & 17 & 18 \\
\hline 19 & 20 & 21 & 22 & 23 & 24 & 25 & 26 & 27 \\
\hline 28 & 29 & 30 & 31 & 32 & 33 & 34 & 35 & 36 \\
\hline 37 & 38 & 39 & 40 & & 41 & 42 & 43 & 44 \\
\hline 45 & 46 & 47 & 48 & 49 & 50 & 51 & 52 & 53 \\
\hline 54 & 55 & 56 & 57 & 58 & 59 & 60 & 61 & 62 \\
\hline 63 & 64 & 65 & 66 & 67 & 68 & 69 & 70 & 71 \\
\hline 72 & 73 & 74 & 75 & 76 & 77 & 78 & 79 & 80 \\
\hline
\end{tabular}

Legend (loss coefficients of color):

\begin{tabular}{|l|l|l|l|l|}
\hline $\mathrm{K}=1.348$ & $\mathrm{~K}=1.222$ & $\mathrm{~K}=1.304$ & $\mathrm{~K}=1.475$ & $\mathrm{~K}=0.856$ \\
\hline $\mathrm{K}=1.278$ & $\mathrm{~K}=1.606$ & $\mathrm{~K}=0.748$ & $\mathrm{~K}=0.926$ & $\mathrm{~K}=0.778$ \\
\hline
\end{tabular}

Figure 3.18: Loss coefficient map for BFBT model using the ferrule-type grid. 
Table 16: Subchannel geometry data for the PNNL 2x6 facility

\begin{tabular}{rr}
\hline Parameter & Value \\
\hline Rod diameter & 0.475 in \\
Rod pitch & 0.575 in \\
Rod-wall spacing & 0.350 in \\
\hline
\end{tabular}

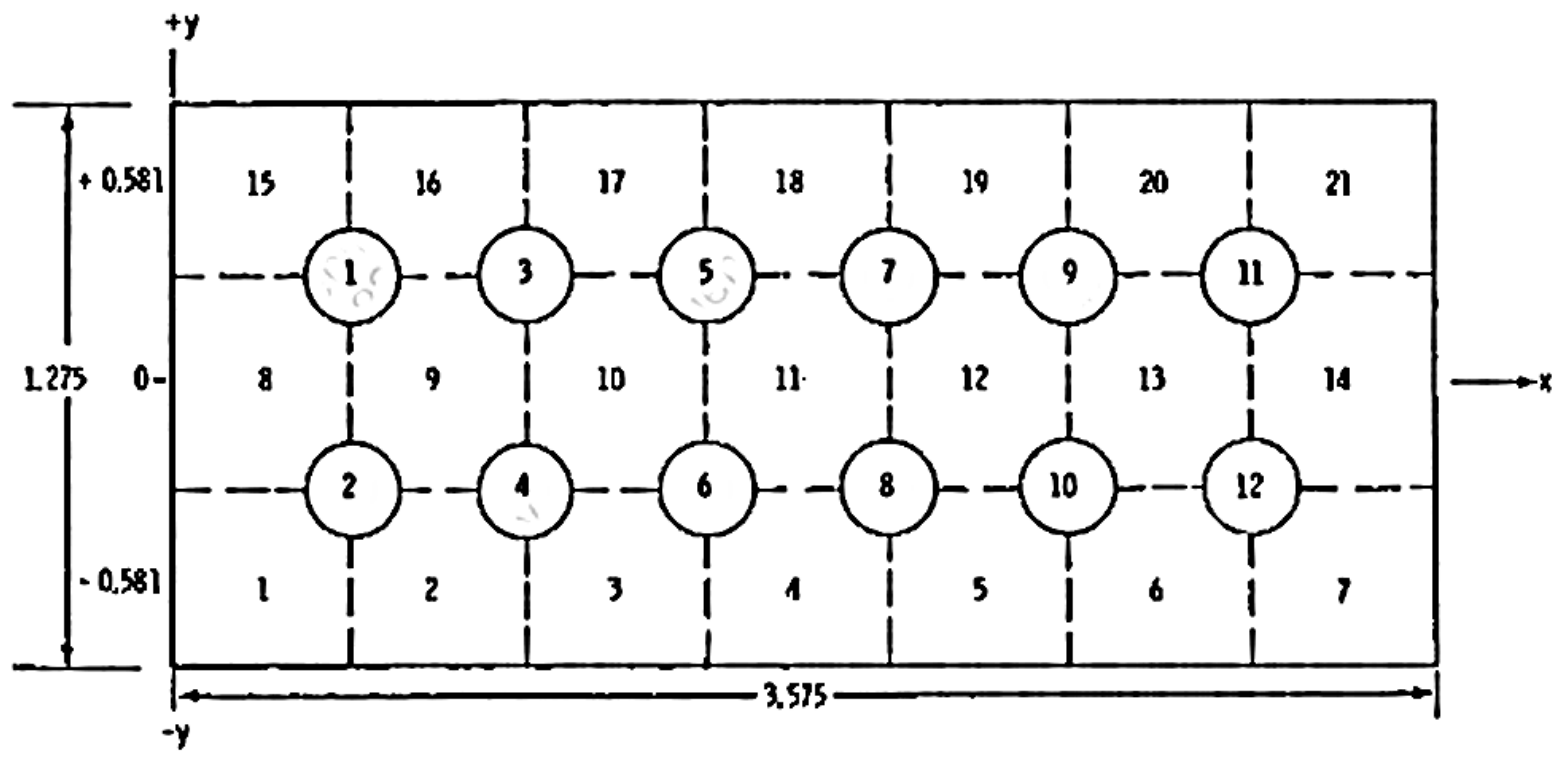

Figure 3.19: Cross-sectional geometry of the PNNL 2x6 test section

\section{$3.3 \quad$ PNNL 2x6}

These two experiments, both made in a 2-by-6 apparatus with the same geometry, were meant to provide benchmark data to evaluate the ability of codes to account for the effects of buoyancy on flow patterns. They contained fixed inlet flow, which was then re-distributed across the bundle by temperature gradients due to differing powers on the two sides. Specifically, for both problems, one half of the assembly (6 rods) was given power while the other half remained unheated. The apparatus contained 9 windows at 6-inch intervals along its 4-ft heated length. At these elevations, an Laser Doppler Anemometer (LDA) was used to measure velocities along lines at 3 locations ( $\mathrm{Y}=-0.581,0.0,0.581)$, which correspond to the three subchannel rows along the 2-rod dimension. Additionally, thermocouple measurements were made in several subchannel centers at these same elevations.

The CTF model contained 12 rods and 21 subchannels arranged as shown in Figure 3.19. The numbering from the steady-state test reference[7] was used, in contrast to that in the transient test reference[8], where the subchannel numbering began in the middle row. The axial meshing of the test section is shown in Figure 3.20. Pertinent geometric information can be found in Table 16. 


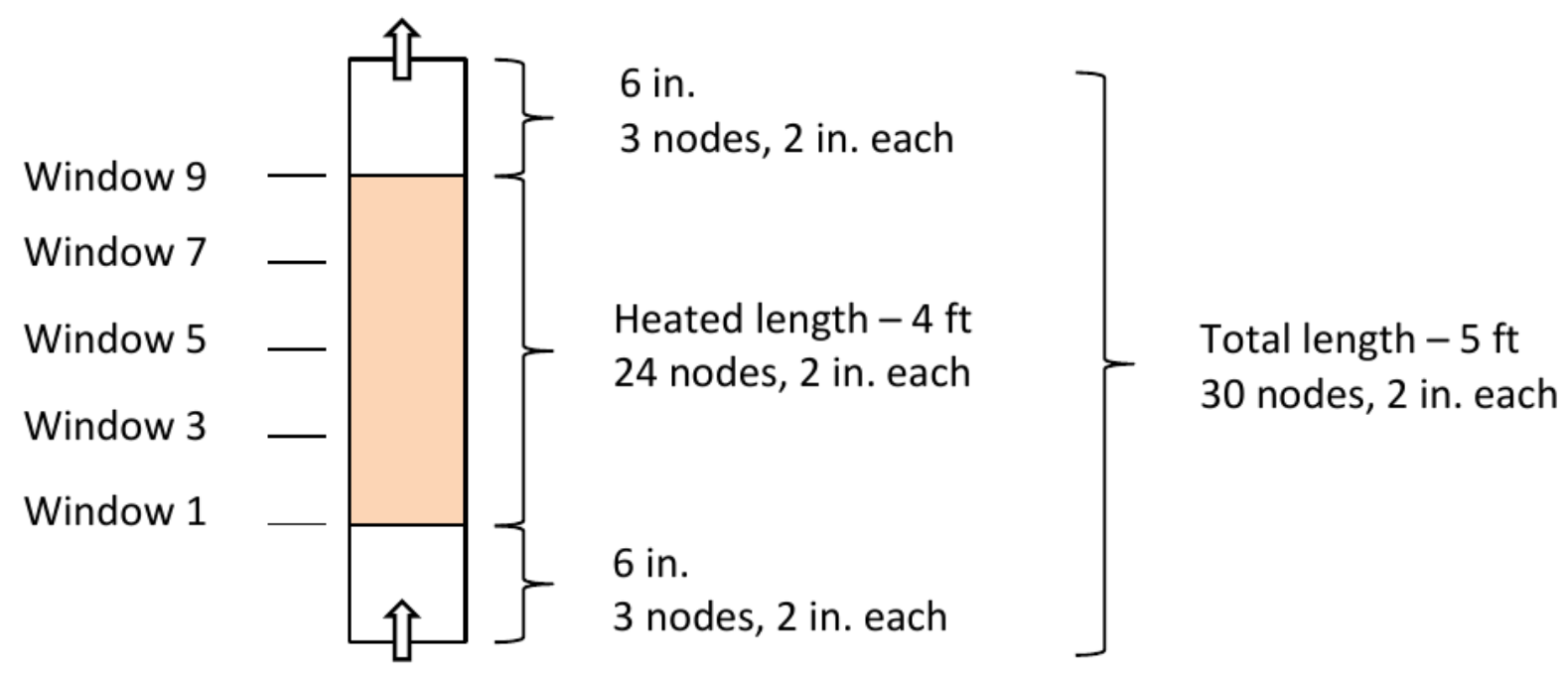

Figure 3.20: Axial schematic of the PNNL 2x6 facility and CTF meshing (units shown in inches)

Table 17: Boundary conditions for PNNL 2x6 steady-state test

\begin{tabular}{rr}
\hline Boundary Condition & Value \\
\hline Pressure $(\mathrm{psi})$ & 60 \\
Inlet temperature $(\mathrm{F})$ & 60.6 \\
Outlet temperature $(\mathrm{F})$ & 93.1 \\
Flow rate $(\mathrm{gpm})$ & 1.25 \\
Power per rod $[\mathrm{kW}]$ & 0.91 \\
\hline
\end{tabular}

Along with the $4 \mathrm{ft}$ heated length of the rods, 6 inches of unheated length above and below were included. There were a total of 30 axial nodes, each 2 inches long. The second friction option was used (irfc $=2$ ), as well as the Rogers and Roseheart single-phase mixing coefficient (imix =2). As these are both single phase cases, options for liquid entrainment, two-phase mixing, and void drift were turned off or set as the defaults.

The steady-state problem was modeled using the boundary conditions found in Table 17 . The transient problem had the boundary conditions shown in Table 18. However, note that convergence problems were experienced for the low pressure case, so the pressure was increased to $60 \mathrm{psi}$ for the transient case. Since the test contained single-phase liquid and low temperature, it is anticipated this change to pressure will have an insignificant impact on results. Additionally, to ensure the case started at steady state, the transient flow ramp was not started until 300 seconds into the CTF simulation. 
Table 18: Boundary conditions for PNNL 2x6 transient test (Case 22)

\begin{tabular}{rr}
\hline Boundary Condition & Value \\
\hline Pressure (psi) & 20 \\
Inlet temperature (C) & 22.9 \\
Outlet temperature (C) & 67.8 \\
Initial flow rate (gpm) & 1.63 \\
Final flow rate (gpm) & 0.55 \\
Flow ramp start (s) & 0.0 \\
Flow ramp end (s) & 45.0 \\
Power per rod (kW) & 0.91 \\
\hline
\end{tabular}


Table 19: Target operating conditions for CE 5x5 Tests 74 and 75

\begin{tabular}{rrr}
\hline Inlet Temperature (F) & Pressure (psia) & Mass Velocity $\left(\mathrm{Mlbm} / \mathrm{hr}_{-} \mathrm{ft}^{2}\right)$ \\
\hline 564 & 2250 & 2.25 \\
583 & 2250 & 2.25 \\
543 & 2250 & 2.97 \\
512 & 1750 & 2.25 \\
\hline
\end{tabular}

\subsection{CE 5x5}

In the early 1980s, Combustion Engineering (CE) teamed up with Columbia University to perform rodbundle experiments to provide insight on the efficacy of single- and two-phase heat transfer models that are typically used for predicting steaming rate and crud buildup[9]. Two $5 \times 5$ rod-bundle tests (named Test 74 and Test 75) were performed at the Columbia University Heat Transfer Research Facility, which were designed to simulate the peripheral region of adjacent CE $14 \times 14$ fuel bundles. These tests were prompted after heavy crud deposits and fuel failures were observed on peripheral rods for bundles in Maine Yankee cycle 4 .

The experiments were run at prototypical PWR pressure, temperature, and heat flux. Target operating conditions are given in Table 19. The rods were 7 feet in length and uniformly electrically heated in the axial direction; rods were non-uniformly heated in the radial direction, as shown in the cross-section diagrams of the facility in Figures 3.21 and 3.22. The grids contained no mixing vanes. Thermocouples were placed at four axial locations in both the central heater tube and one adjacent heater tube. Additionally, pressure measurements were made via four pressure taps. Figure 3.23 shows a simple axial diagram of the assembly.

Pressure drop measurements were made at cold conditions ( 80 and $130{ }^{\circ} \mathrm{F}$ ) at a pressure of 1,000 psia. The inlet mass velocity was varied such that Reynolds-dependent grid-loss correlations could be developed. The Moody friction factor correlation was used to characterize the frictional component of pressure drop. The grid loss coefficients were calculated by the authors on a per-subchannel basis considering the blockage ratio and wetted perimeter of each subchannel (see Table 5.1 of specification[9]). These loss coefficients were used in the CTF model.

Heated tests were run at a wide range of heat fluxes in order to cause single-phase subcooled boiling and saturated boiling regions of the boiling curve in the upper assembly. Transition to boiling was detected by observing a "leveling-off" of the rod surface temperature with increasing heat flux. Additionally, several DNB tests were performed by ramping up the power in the facility until a sharp temperature increase was detected at the thermocouple locations, indicating that a post-CHF heat transfer regime has been entered. CHF results are presented in Table 20. Two of the tests are shown as experiencing CHF in two locations. 


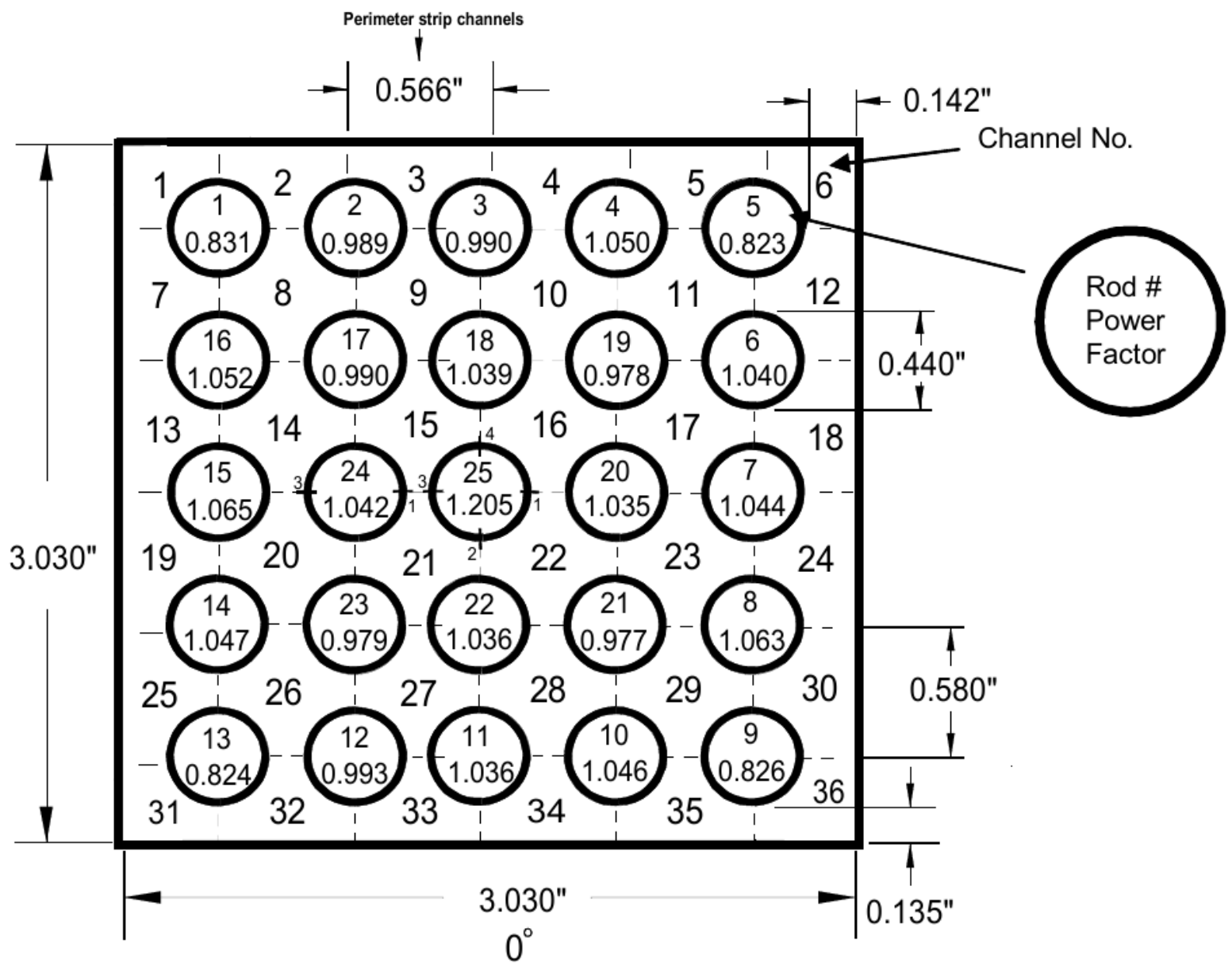

Figure 3.21: Cross-section diagram of CE $5 \times 5$ Test 74

Table 20: CHF results for CE 5x5 Tests 74 and 75

\begin{tabular}{|c|c|c|c|c|c|}
\hline Test & $\begin{array}{r}\text { Inlet Temperature } \\
\text { (F) }\end{array}$ & $\begin{array}{r}\text { Pressure } \\
\text { (psia) }\end{array}$ & $\begin{array}{l}\text { Mass Velocity } \\
\left(\text { Mlbm } / \mathrm{hr}^{-\mathrm{ft}^{2}}\right)\end{array}$ & $\begin{array}{r}\text { Rod CHF } \\
\left(\mathrm{MBTU} / \mathrm{hr}^{-\mathrm{ft}^{2}}\right)\end{array}$ & $\begin{array}{r}\mathrm{T} / \mathrm{C} \mathrm{CHF} \\
\text { Location (in) }\end{array}$ \\
\hline 74 & 562.5 & 2254.7 & 2.259 & 0.652 & 25.43 \\
\hline 74 & 545.7 & 2254.7 & 2.236 & 0.697 & 25.43 \\
\hline 74 & 514.0 & 1749.7 & 2.203 & 0.695 & $\begin{array}{l}25.43 \\
25.41\end{array}$ \\
\hline 74 & 582.0 & 2254.7 & 2.964 & 0.718 & 25.43 \\
\hline 75 & 563.5 & 2249.7 & 2.281 & 0.679 & 25.43 \\
\hline 75 & 547.5 & 2249.7 & 2.211 & 0.708 & 25.43 \\
\hline 75 & 516.0 & 1749.7 & 2.224 & 0.715 & $\begin{array}{l}25.43 \\
25.41\end{array}$ \\
\hline 75 & 584.0 & 2249.7 & 2.927 & 0.743 & 25.43 \\
\hline
\end{tabular}




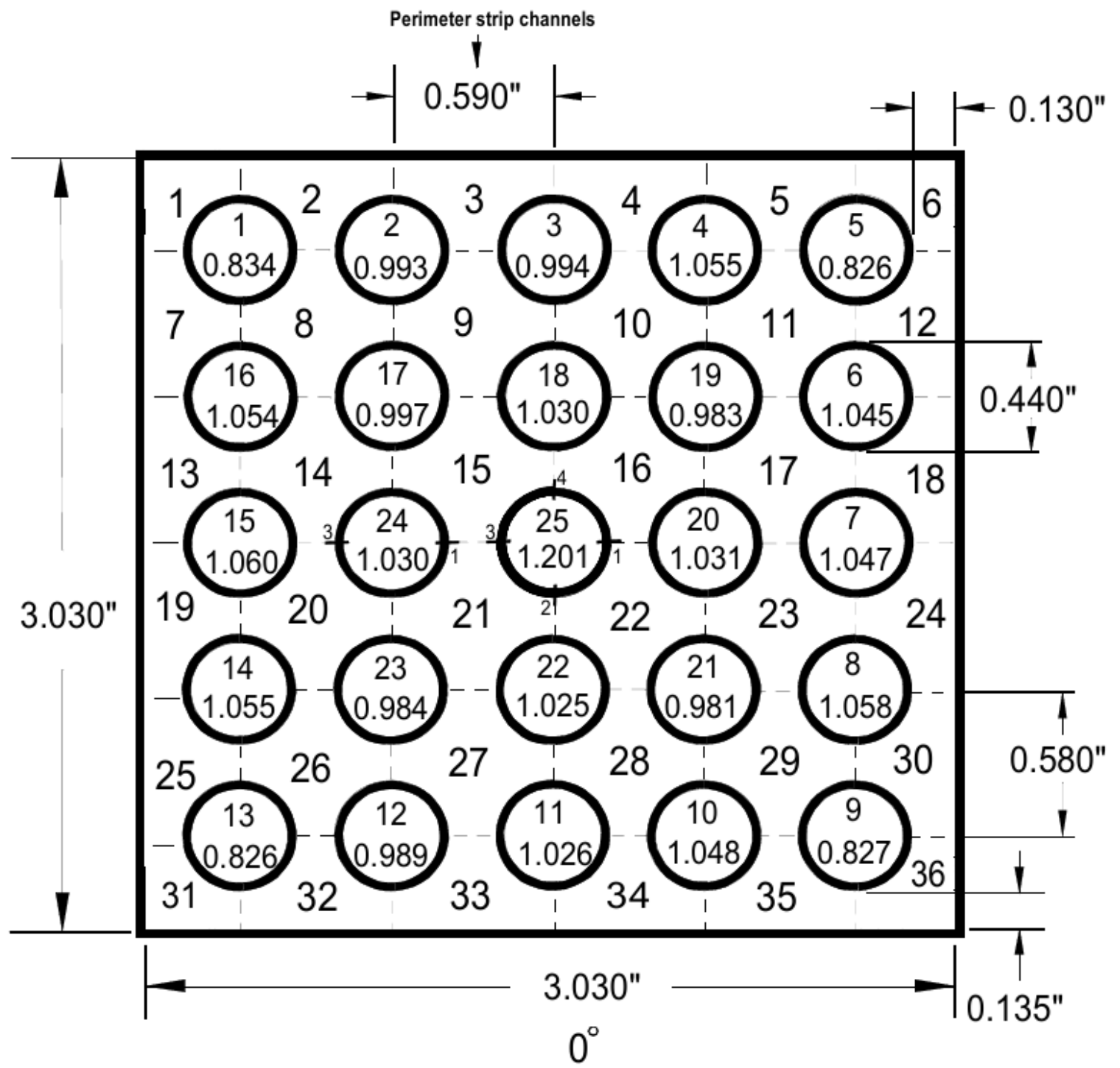

Figure 3.22: Cross-section diagram of CE $5 \times 5$ Test 75 


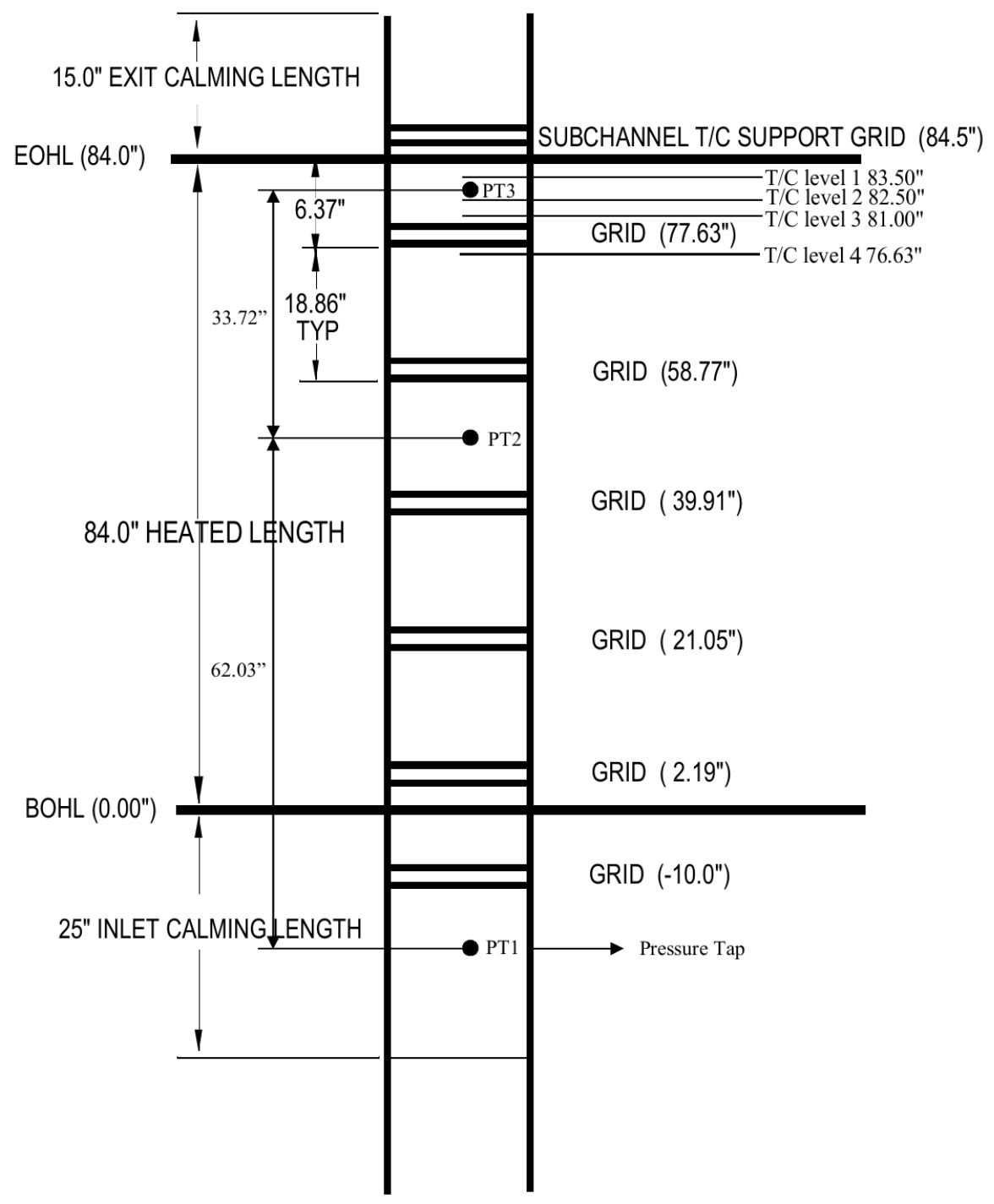

Figure 3.23: Axial schematic of the CE $5 \times 5$ bundle 


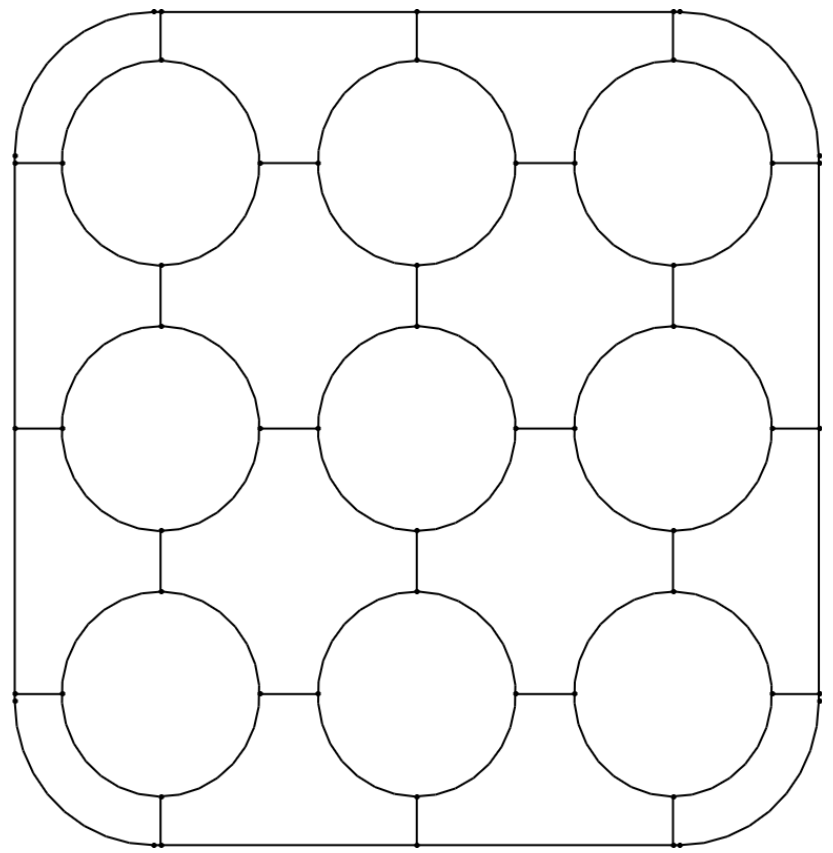

Figure 3.24: GE $3 \times 3$ Bundle Geometry

\subsection{GE $3 \times 3$}

The GE $3 \times 3$ facility is a classic test for assessing inter-subchannel mixing[10] because mass flux and quality measurements could be made for individual subchannel types. A $3 \times 3$ heated tube geometry was used in a BWR-like simulation, with General Electric (GE) rods[11]. The geometry of the bundle is shown in Figure 3.24. The details of this bundle are provided in Table 21. A summary of the geometry of the 16 subchannels is provided in Table 22 .

The axial and radial power profiles were uniform for all of these test cases. The same bundle was used for all the tests. Bundle power, flow rate, and inlet subcooling was varied between different experimental cases.

Subchannel measurements were taken to determine the specifics of coolant flow through different types of subchannels. The target parameters were the flow rate and the enthalpy as compared to the bundle-averaged values. The $2 \mathrm{~B}$ and $2 \mathrm{D}$ series test cases all utilize a uniform radial and axial heat flux distribution. The main difference between the two series is the power level. The subcooling is specified as well, as is shown in Table 23.

There are pins holding the rods in place which act as spacers. These pins are 3.18 mm in diameter and either $3.43 \mathrm{~mm}$ long or $4.27 \mathrm{~mm}$ long, for either rod-rod or rod-wall connections, respectively. There are four pins connected to each rod spaced evenly azimuthally and perpendicular to the shroud surface where they connect. These locations are along the subchannel boundaries which are indicated in Figure 3.25. Pins labeled with either ' $\mathrm{A}$ ' or ' $\mathrm{B}$ ' are $3.43 \mathrm{~mm}$ in length, while pins labeled with either ' $\mathrm{C}$ ' or ' $\mathrm{D}$ ' are $4.27 \mathrm{~mm}$ in length. All pins have the same diameter: $3.18 \mathrm{~mm}$. These pins were fusion-welded to the outside of each pin and to the walls. 


\begin{tabular}{lr}
\hline Item & Description \\
\hline Number of heater rods & 9 \\
Heater rod OD (mm) & 14.5 \\
Heated length (mm) & 1828.8 \\
Rod pitch (mm) & 18.7 \\
Channel box inner width (mm) & 58.83 \\
Channel box corner radius (mm) & 10.2 \\
Flow area (mm ${ }^{2}$ ) & 1890.3 \\
Number of spacers & 6 \\
Spacer type & Pin \\
\hline
\end{tabular}

Table 21: GE $3 \times 3$ Bundle Description

Table 22: GE $3 \times 3$ Bundle Subchannel Geometry

\begin{tabular}{rrr}
\hline Subchannel & $\begin{array}{r}\text { Area } \\
\mathrm{m}^{2}\end{array}$ & $\begin{array}{r}\text { Wetted perimeter } \\
\mathrm{m}\end{array}$ \\
\hline Corner & $5.050 \mathrm{E}-05$ & $2.835 \mathrm{E}-02$ \\
Side & $1.177 \mathrm{E}-04$ & $4.149 \mathrm{E}-02$ \\
Center & $1.868 \mathrm{E}-04$ & $4.548 \mathrm{E}-02$ \\
\hline
\end{tabular}

Table 23: GE $3 \times 3$ Bundle Boundary Conditions by Test Series

\begin{tabular}{rrrr}
\hline Test series & $\begin{array}{r}\text { Power } \\
{[\mathrm{kW}]}\end{array}$ & $\begin{array}{r}\dot{m}_{\text {in }} \\
{[\mathrm{kg} / \mathrm{s}]}\end{array}$ & $\begin{array}{r}\text { Inlet subcooling } \\
{[\mathrm{kJ} / \mathrm{kg}]}\end{array}$ \\
\hline 1B & 0 & 1.231 & 504.6 \\
1C & 0 & 2.538 & 504.6 \\
1D & 0 & 3.871 & 504.6 \\
1E & 0 & 5.054 & 504.6 \\
2B2 & 532 & 1.360 & 348.4 \\
2B3 & 532 & 1.373 & 252.6 \\
2B4 & 532 & 1.373 & 122.7 \\
2C1 & 532 & 2.720 & 132.9 \\
2C2 & 532 & 2.740 & 81.57 \\
2D1 & 1064 & 1.386 & 602.4 \\
2D3 & 1064 & 1.386 & 289.1 \\
2E1 & 1064 & 2.771 & 332.1 \\
2E2 & 1064 & 2.771 & 224.7 \\
2E3 & 1064 & 2.712 & 67.63 \\
2E1 & 1064 & 2.771 & 332.1 \\
2E2 & 1064 & 2.771 & 224.7 \\
2E3 & 1064 & 2.712 & 67.63 \\
2G1 & 1596 & 2.746 & 525.0 \\
2G2 & 1596 & 2.771 & 441.1 \\
2G3 & 1596 & 2.746 & 340.9 \\
\hline
\end{tabular}




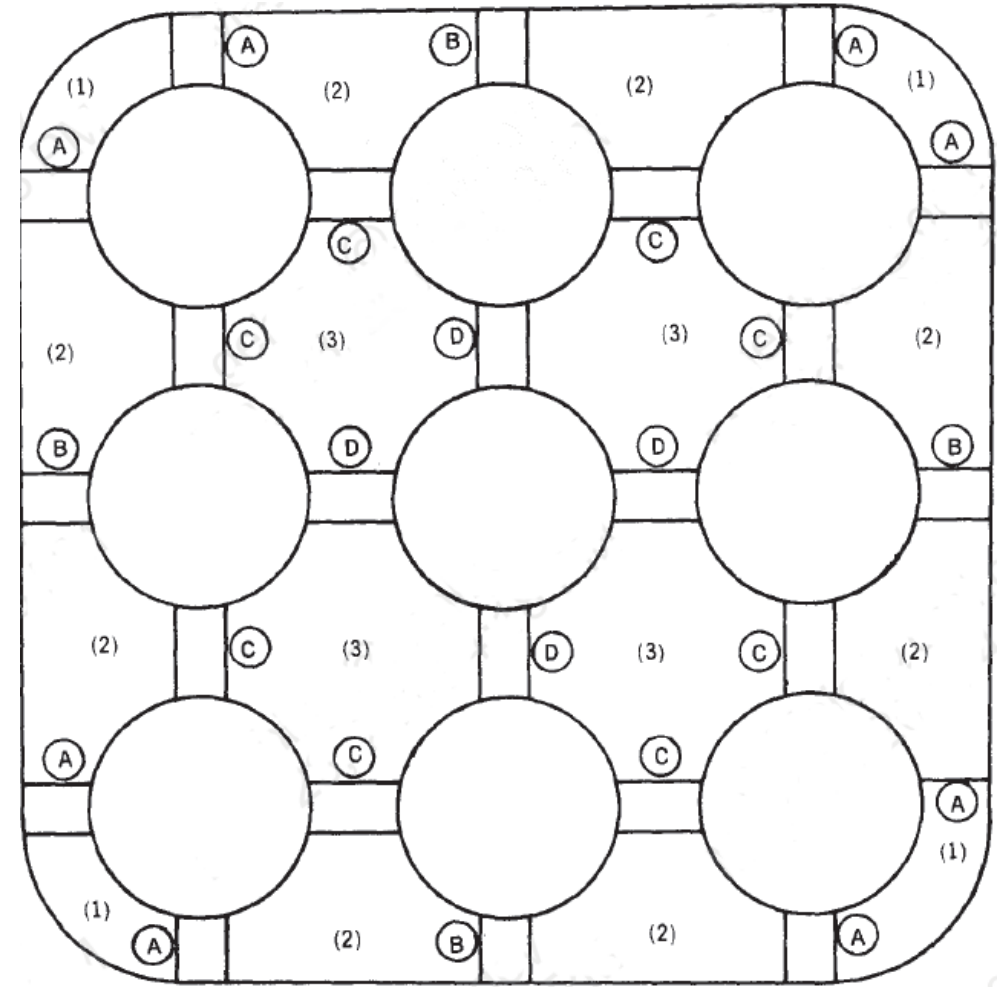

Figure 3.25: GE $3 \times 3$ Bundle Pin Spacer Geometry 
There are six sets of 24 pins spaced every $304.8 \mathrm{~mm}$ starting $50.8 \mathrm{~mm}$ from the bottom of the heated length. The locations of these six pin spacers are 50.8, 355.6, 660.4, 965.2, 1270.0, and $1574.8 \mathrm{~mm}$ from the bottom of the heated length.

Measured values from the experiments include:

- Normalized subchannel mass flux versus bundle-averaged quality

- Normalized subchahnel enthalpy versus bundle-averaged quality

The measurements taken during the experiment were mass flows and enthalpies of specific subchannels. These were grouped by subchannel type: corner, side, or center. The subchannels were isolated using special ducting in order to obtain measurements at only the subchannel type of interest. Once isolated using sheets of metal, the flow rate and enthalpy of the fluid passing through the subchannel are measured using a turbine flow meter (for the flow) and by a heat balance on a calorimeter (for the enthalpy). Pressure loss values were also measured using pressure transducers across the entire bundle [11].

The normalized subchannel mass flux and enthalpy is calculated for each of the three types of subchannels in this bundle. They are normalized in order to gain a comparison of that particular subchannel's flow or enthalpy to that of the entire bundle as a whole. The flow normalization is performed by Equation (3.1). Similarly, the enthalpy normalization is performed as shown in Equation (3.2).

$$
\begin{gathered}
\frac{G_{c h}-\bar{G}}{\bar{G}} \\
\frac{H_{c h}-\bar{H}}{\bar{H}}
\end{gathered}
$$

In these equations, $\bar{G}$ and $\bar{H}$ correspond to the bundle-averaged values of flow rate and enthalpy, respectively, and $\mathrm{G}_{c h}$ and $\mathrm{H}_{c h}$ are the flow rate and enthalpy of the subchannel type.

\subsection{Harwell High Pressure Loop}

Post-dryout (post-CHF) experiments were performed in the Harwell High Pressure Two-Phase Heat Transfer Loop facility[12]. The facility consisted of an electrically heated tube with water flowing in the inside. The tube had a 0.496 inch inner diameter, was $19 \mathrm{ft}$ long, and was vertically aligned. Thermocouples were affixed to the outside of the tube, the spacing being as large as 12 inches and as small as 3 inches near the top of the test section. A series of tests were performed where the flow conditions were such that dryout occurred at some location in the tube. This was achieved by adjusting inlet flow rate and test section power; the outlet pressure was fixed at 1,000 psi for all tests.

The testing procedure involved increasing the power until a sharp temperature increase was detected, indicating that dryout had occurred. Measured temperatures were used to calculate the tube inside surface temperatures, which were summarized for all tests in the report by Bennett, et al.[12]. 
Two varieties of tests were performed; one with a 144 inch active length and another with a 219 inch active length. Only the 219 inch active length tests were modeled in CTF. Therefore, the geometry and problem mesh were the same for all tests modeled. Only the flow conditions were varied from test-to-test. A schematic of the CTF axial mesh is shown in Figure 3.26. The CTF model consisted of one channel and one heater tube. The model was setup as if the flow was on the exterior of the heater tube. The outer diameter of this tube was set equal to the inner tube diameter so that the tube surface area of the rod/fluid interface was consistent with the experiments. The channel flow area and wetted perimeter in the CTF subchannel was set equal to the flow area of the inside of the tube. Only the active region of the experiment was modeled. The axial meshing was done such that the center of the CTF mesh cells were within $4 \mathrm{~mm}$ of the actual measurement location in the experiment.

The axial mesh is laid out in groups of cells all having the same height. In the figure, the green block on the bottom denotes a group of cells having 4 inch heights. From 112 inches and up, the mesh is gradually refined. The blue block represents a region in which the mesh size is 0.5 inches. This was done because this region (112 inches and up) is where dryout occurs, which requires a finer mesh. With the way the mesh was setup, the axial locations of test facility thermocouples always line up with a CTF cell center.

Due to the inclusion of the droplet field entrainment/de-entrainment and the complex physics being modeled, it was difficult to converge these tests to a level of tolerance commensurate with that of simpler single-phase tests. Therefore, the minimum and maximum allowable timestep sizes were set to $1 \mathrm{E}-7 \mathrm{~s}$ and $1 \mathrm{E}-4 \mathrm{~s}$, respectively, and the ratio between the conduction and fluid solution time scales was set to 1.0 (a value we normally increase to 10,000). Even with these extra measures, it was typically only possible to drop the mass and energy storage terms to about $0.1-1 \%$. Mass and energy balance terms were generally more well behaved, dropping to $0.001-0.01 \%$.

Boundary conditions were set to match experimental values; a pressure boundary condition was set at the test outlet and a mass flow rate and enthalpy boundary condition was set at the inlet. The author provided inlet subcooling as a boundary condition, so the tests were run in an iterative fashion to match the inlet subcooling to experimental values. The tube material properties were set to that of Zircaloy, though this

has no impact on the solution value of interest, and the tube surface temperature, because the case is run to steady-state, where all heat generated in the model is being deposited into the fluid.

\subsection{FRIGG}

The FRIGG test facility consists of an electrically heated rod bundle, modeled after the Marviken reactor assemblies[13]. Experiments were run in the facility to verify that the Marviken reactor could be run at the rated power with proper safety margins against burnout. Investigations included single- and two-phase pressure drop measurements, axial and radial void distribution measurements, and burnout in natural and forced circulation. In this study, we model a single uniformly heated, natural convection, two-phase flow test case from the study. 


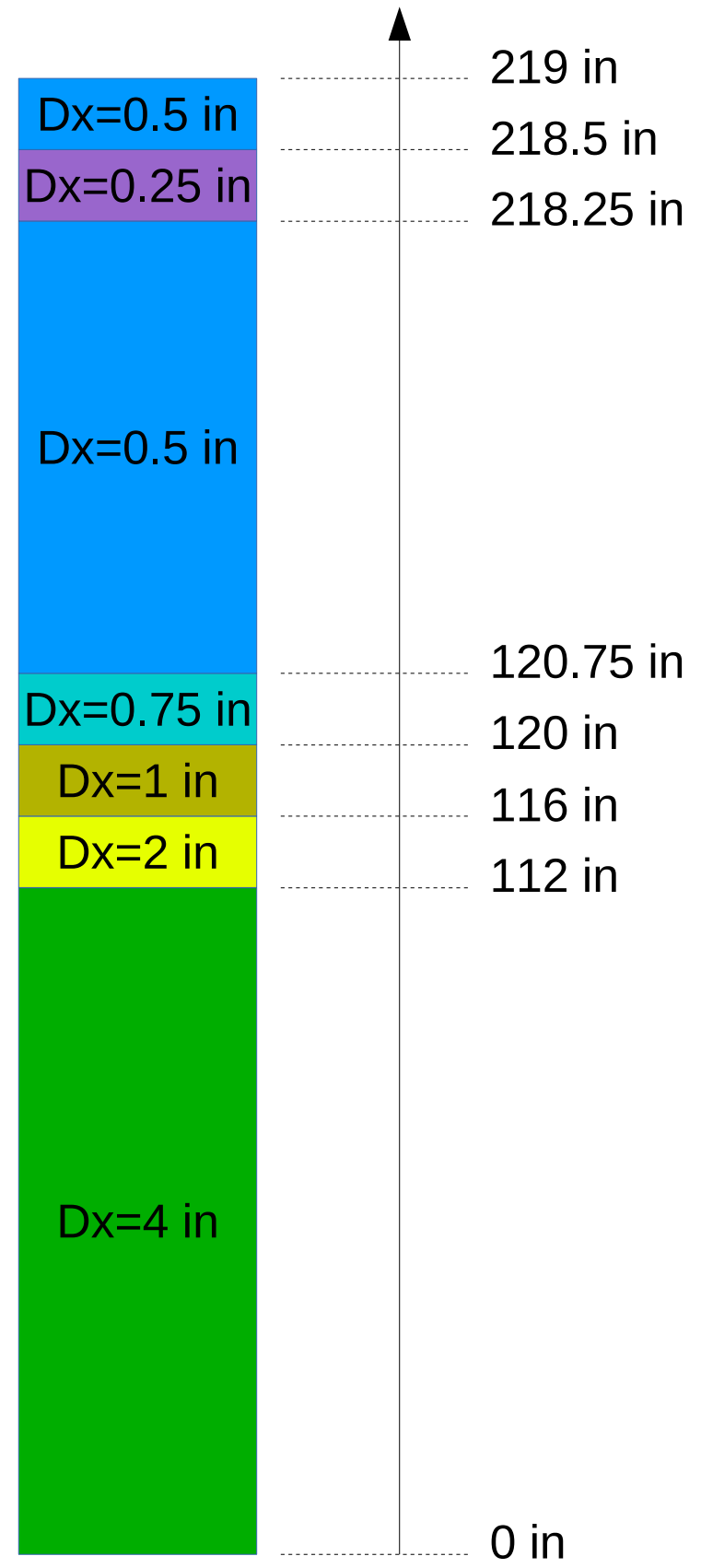

Figure 3.26: CTF model of the Harwell High-Pressure Two-Phase Test Facility 


\subsubsection{CTF Model of Facility}

\subsubsection{Radial Meshing}

The Marviken reactor uses fuel assemblies having lattices much different than that of a typical U.S. PWR. The 36 fuel rods are situated so that their centers lie on rings of increasing size going outwards from the center of the circular shaped bundle. An unheated guide tube lies at the center of the bundle. The first ring of rods occurs at $21.6 \mathrm{~mm}$ from the center and contains 6 fuel rods. The second ring of rods occurs at $41.7 \mathrm{~mm}$ from center and contains 12 fuel rods. The third and final ring occurs at $62.2 \mathrm{~mm}$ from center and contains 18 rods. This leads to a total of 36 fuel rods and one central guide tube. The canister for the fuel assembly has a diameter of $159.5 \mathrm{~mm}$. The gap between rods on the same ring is $7.8 \mathrm{~mm}$.

Figure 3.27 shows the cross-section of the Marviken assembly (and the FRIGG test facility). The CTF modeling approach for this facility is also shown in the figure. To simplify the modeling of the facility, a lumped-subchannel approach was used. The space between the central guide tube and the first ring of rods formed the first subchannel in the CTF model. Likewise, the space between the first and second ring of rods formed the second subchannel, and so on. In addition to lumping the subchannels together, it also made sense to lump fuel rods and gaps, as well. The first ring of rods were lumped into Rod 2, the second ring of rods were lumped into Rod 3, and the third ring of rods were lumped into Rod 4. In this way, there were no gaps modeled in the azimuthal $(\theta)$ direction of the circle; rather, gaps only exist in the radial $(r)$ direction. Since the rod spacing is always $7.8 \mathrm{~mm}$ in the $\theta$ direction, it was possible to lump all gaps on a given ring together.

To create the CTF model, it was necessary to calculate the cross-flow area of the rings as well as their wetted perimeter. Any given fuel rod resides in exactly two rings. In order to obtain the channel area and wetted perimeter, it was necessary to determine how much of the fuel rod is in each of the two rings it occupies. To do this, we first zoom in on a single fuel rod and define an equation for the circle whose circumference passes through the fuel rod center. This is shown in Figure 3.28, where the blue circle represents the fuel rod. If it is assumed that the large circle's center is at the origin, then functions for both circles can be defined.

To obtain the area of the fuel rod in the smaller of the two rings, we can simply integrate the difference of the two functions that define the larger and smaller circles between their intersections. In equation form:

$$
A_{\text {small }}=\int_{x_{0}}^{x_{1}} f_{\text {large circle }}(x)-f_{\text {small circle }}(x)
$$

Here, $A_{\text {small }}$ is the cross sectional area of the rod residing in the smaller channel. Substituting the actual equations of the circles leads to the following:

$$
A_{\text {small }}=\int_{x_{0}}^{x_{1}} \sqrt{R^{2}-x^{2}}-\left(-\sqrt{r^{2}-x^{2}}+R\right)
$$

Here, $R$ is the radius of the large circle and $r$ is the radius of the small circle that represents the fuel rod. It was assumed that the fuel-rod circle lay directly above the center of the large circle, so that its equation is simply shifted up the $y$ axis and requires no shift in the $x$ direction. Since the fuel-rod circle center is situated on the larger circle circumference, the fuel-rod circle equation is shifted up the y-axis by distance 


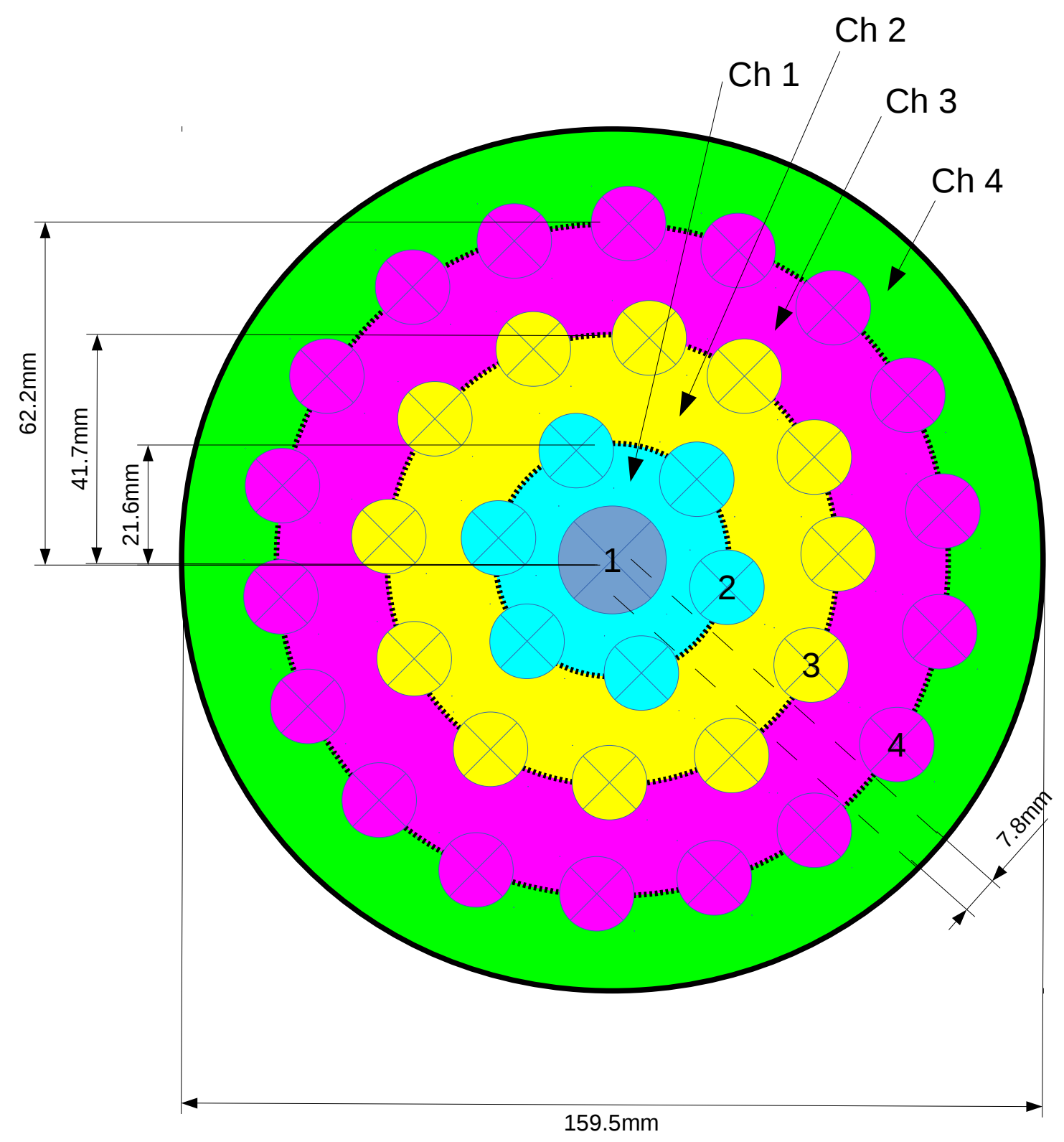

Figure 3.27: Cross-section of the FRIGG facility (including labels of CTF model rods and channels) 


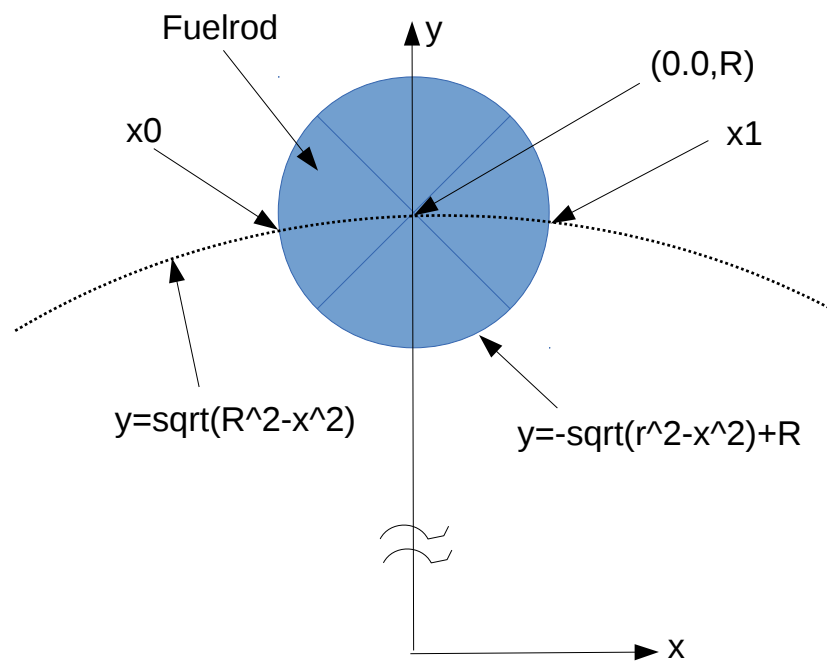

Figure 3.28: Definition of functions for the circle that defines the fuel rod and the circle it sits on

$R$. The intersection points of the two circles can be found by setting the equations equal to one another and solving for $x$. Since the fuel-rod circle is directly above the large circle center, the two intersection points have the same magnitude, but opposite sign:

$$
\begin{gathered}
x_{0}=-\sqrt{r^{2}-\frac{r^{2}}{4 R^{2}}} \\
x_{1}=\sqrt{r^{2}-\frac{r^{2}}{4 R^{2}}}
\end{gathered}
$$

Doing the integration of Equation 3.4 from the bounds given in Equations 3.5 and 3.6 yields the following equation for cross-sectional area of a rod residing in the smaller channel:

$$
A_{\text {small }}=\left[\frac{x}{2} \sqrt{R^{2}-x^{2}}+\frac{R^{2}}{2} \sin ^{-1} \frac{x}{R}+\frac{x}{2} \sqrt{r^{2}-x^{2}}+\frac{r^{2}}{2} \sin ^{-1} \frac{x}{r}-R x\right]_{x_{0}}^{x_{1}}
$$

The area of the rod residing in the larger channel is then simply calculated as the total rod cross-sectional area minus the smaller area given by Equation 3.7.

$$
A_{\text {large }}=\pi r^{2}-A_{\text {small }}
$$


Table 24: Channel area displaced by each rod bank

\begin{tabular}{rrrrrr}
\hline \multicolumn{6}{c}{ Displacement of Channel Area $\left(\mathrm{mm}^{2}\right)$} \\
Rod Bank & Ch 1 & Ch 2 & Ch 3 & Ch 4 & Num Rods \\
\hline 1 & 314.16 & 0.00 & 0.00 & 0.00 & 1 \\
2 & 69.70 & 79.61 & 0.00 & 0.00 & 6 \\
3 & 0.00 & 72.22 & 77.38 & 0.00 & 12 \\
4 & 0.00 & 0.00 & 73.03 & 76.54 & 18 \\
\hline
\end{tabular}

Table 25: Wetted perimeter of each rod in each channel of the model

\begin{tabular}{rrrrrr}
\hline \multicolumn{6}{c}{ Rod Wetted Perimeter $(\mathrm{mm})$} \\
Rod Bank & Ch 1 & Ch 2 & Ch 3 & Ch 4 & Num Rods \\
\hline 1 & 62.83 & 0.00 & 0.00 & 0.00 & 1 \\
2 & 19.46 & 23.89 & 0.00 & 0.00 & 6 \\
3 & 0.00 & 20.53 & 22.82 & 0.00 & 12 \\
4 & 0.00 & 0.00 & 20.91 & 22.44 & 18 \\
\hline
\end{tabular}

The channel cross sectional area displaced by the rods is given in Table 24. Note that this table gives displacement for a single rod in the bank, so the total displacement area of the entire bank must be calculated by multiplying the number of rods in the bank by the displacement of the single rod.

As a sanity check, it is prudent to compare the sum of the values in Table 24 times number of rods in the bank to a simple calculation of the total rod cross-sectional area in the test section. The rod cross-section area can be calculated simply as follows:

$$
A=\frac{\pi}{4} 20^{2}+36 \frac{\pi}{4} 13.8^{2}=5698.7 \mathrm{~mm}^{2}
$$

Doing the math on Table 24 results in a total displacement area of $5697.48 \mathrm{~mm}^{2}$, which agrees with our sanity check to within $0.021 \%$, which can easily be accounted for by roundoff.

To figure out the wetted perimeter, it's necessary to find the angle between the two intersection points, $x_{0}$ and $x_{1}$, and the center of the fuel-rod circle. The trigonometric relation for determining this angle is presented below:

$$
\theta=\pi-2 \cos ^{-1} \frac{x_{0}}{r}
$$

It's then possible to calculate the arc length between the two points with the relationship $\pi \theta$. The wetted perimeter of each rod in each ring is shown in Table 25. Similar to Table 24, the table gives wetted perimeter for a single rod in the bank (not the entire bank).

As a sanity check on the wetted perimeters, the total wetted perimeter of all rods is compared to the sum of the values given in Table 25 multiplied by the number of rods in each bank: 
Table 26: Azimuthal fraction of each rod in each channel of the model

\begin{tabular}{rrrrr}
\hline & \multicolumn{4}{c}{ Azimuthal fraction } \\
Rod Bank & Ch 1 & Ch 2 & Ch 3 & Ch 4 \\
\hline 1 & 1.0000 & 0.0000 & 0.0000 & 0.0000 \\
2 & 0.4489 & 0.5511 & 0.0000 & 0.0000 \\
3 & 0.0000 & 0.4736 & 0.5264 & 0.0000 \\
4 & 0.0000 & 0.0000 & 0.4823 & 0.5177 \\
\hline
\end{tabular}

$$
P_{w}=\pi 20+36 \pi 13.8=1623.6 \mathrm{~mm}
$$

This compares favorable with the total displacement calculated from Table 25, which is $1623.43 \mathrm{~mm}$ (a $0.010 \%$ difference). Note that in the CTF model, the wetted perimeter of the assembly casing is also added to Channel 4.

It is necessary to determine the azimuthal fraction of each rod bank in the channel so that rod heat is properly distributed into the channels. This can be obtained by taking the ratio of rod perimeter in the channel (given in Table 25) to total rod perimeter $(43.4 \mathrm{~mm})$. Azimuthal fractions of rods in given channels are shown in Table 26.

Finally, the gap length and width must be determined to create the 3 gaps that exist between the 4 channels. The width is simply $7.8 \mathrm{~mm}$, which was given in the facility schematic. The length is calculated as the distance between ring centers:

$$
L=\frac{R_{i}-R_{i-1}}{2}+\frac{R_{i+1}-R_{i}}{2}
$$

\subsubsection{Axial Meshing}

A schematic of the assembly in the axial direction is given in Figure 3.29. The figure shows the location of pressure taps with a "P" (e.g. P10, P11), thermocouples with a "T", and gamma densitometers (void measurements) with a "G". Spacer grid locations are labeled "Spacer" in the figure. The CTF model was made to cover the test section from the beginning of heated length (labeled as 0 in the figure) to the location of the P20 pressure tap, located at $4781 \mathrm{~mm}$. As indicated in the drawing, the heated length is $4378 \mathrm{~mm}$. The mesh cell sizing was meticulously set so that scalar cell centers lined up perfectly with the location of a pressure tap, thermocouple measurement, or gamma densitometer measurement. The scalar cell boundaries lined up perfectly with the location of spacer grids (which are placed at the center of momentum mesh cells in CTF). This ensured we could do direct CTF-to-experimental comparisons after running the simulation. A total of 123 mesh cells were used, leading to an average mesh cell height of $38.9 \mathrm{~mm}$. 


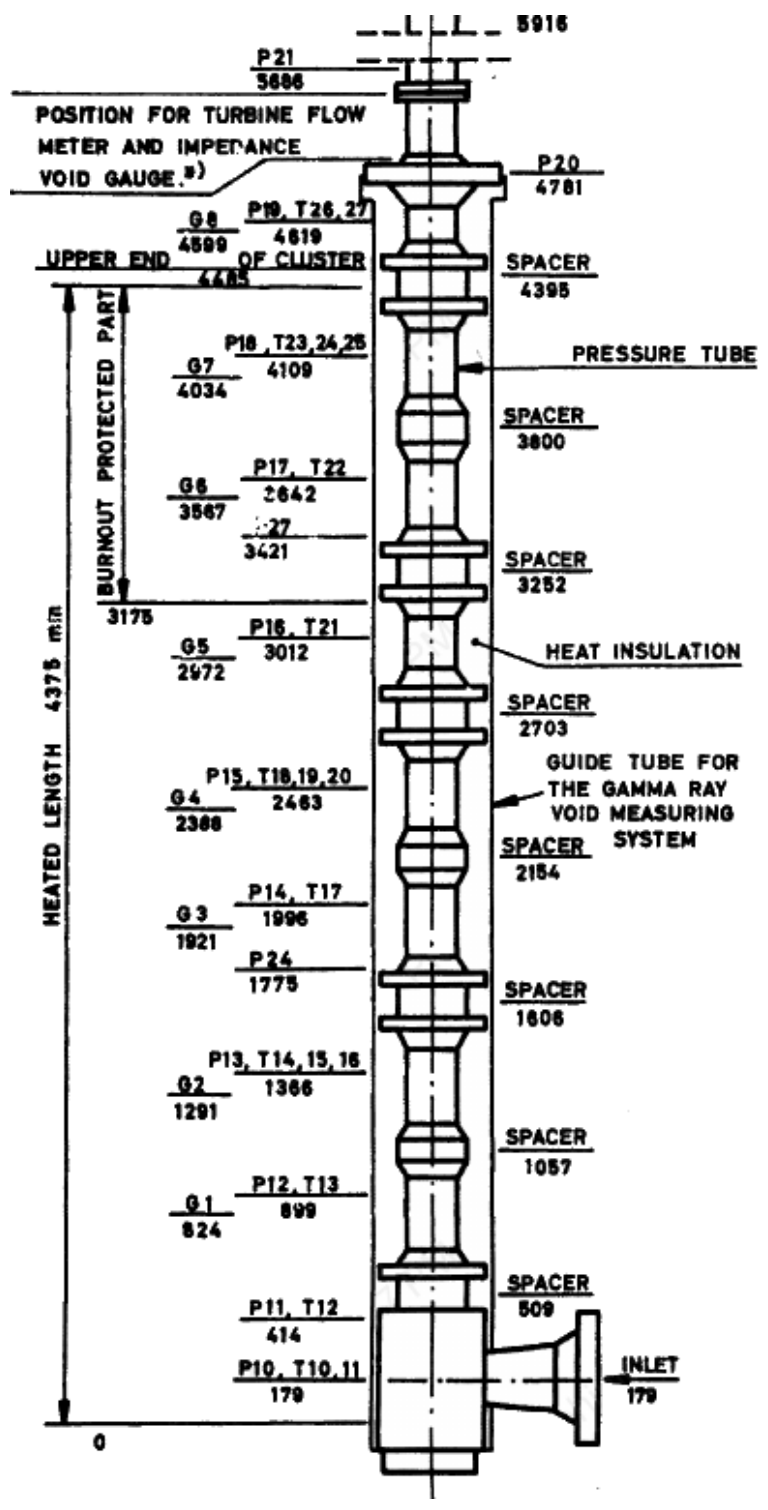

Figure 3.29: Schematic of FRIGG facility in axial direction 


\subsubsection{Boundary Conditions}

Results were to be compared to those presented in Figure 26 of the test report[13], so the boundary conditions of the case presented in that figure were used. The boundary conditions were given in the table in Appendix 1 of the report. The inlet mass flux was $1026 \mathrm{~kg} / \mathrm{m}^{2} \mathrm{~s}$, the outlet pressure was set to 50 bar, and the inlet enthalpy was set to $1122.5 \mathrm{~kJ} / \mathrm{kg}$ in order to achieve the specified inlet subcooling of $4.4{ }^{\circ} \mathrm{C}$. The total test section power was $3000 \mathrm{~kW}$, distributed uniformly throughout the test section. This test was a natural convection case.

\subsubsection{Modeling Choices}

Other important modeling choices included the selection of the grid loss coefficients and turbulent-mixing/ void-drift parameters. Page 26 of the report specifies that the mean spacer grid loss coefficient was 0.58 for a Reynolds number of $2 \times 10^{5}$, so this value was used in the CTF model. The report also mentioned that the friction factor correlation for the test section was $f=0.2 R e^{-0.2}$. The friction correlation used in CTF is $f=0.204 R e^{-0.2}$, so it matches the one specified in the experimental documentation well. The mixing model was set to use Rogers\&Rosehart to determine the single-phase mixing coefficient and Beus to determine the

two-phase multiplier. The equilibrium void distribution weighting factor was set to the suggested value of 1.4 and the $\theta_{M}$ value was set to 5.0 .

\subsection{RPI $2 \times 2$}

The intended purpose of this experiment was to investigate the fully developed two-phase flow distribution in a $2 \times 2$ rod array test section. The test facility includes a 36 -inch long unheated $2 \times 2$ rod bundle with an air-water mixture as the working fluid. With a bundle hydraulic diameter of 0.914 inches, an L/D factor of 39 was calculated, leading to an expected fully developed flow condition at the bundle outlet. Four 0.055-inch thick 314 stainless-steel tubes with 1.0-inch OD were used to simulate the fuel rods. The wall thickness insured a vibration-free environment during the experiment, and a lower tie plate provided support for the rods. No spacer grids were used in this experiment. Two different techniques were used to distribute the air into the bundle inlet, a sinter sections technique, and a mixing tee technique [14].

Uncertainties within this experiment were measured with the theory of propagation of errors, which is based on the assumption of independent errors. The three independent variables in the error analysis were the subchannel liquid flow rate $\left(Q_{l i}\right)$, the air flow rate $\left(Q_{g i}\right)$, and the inlet pressure $\left(P_{i n}\right)$. Uncertainty intervals for each of these three variables were evaluated and used to derive the void fraction error interval, which was calculated for each data point using Eq. (3.13). Using this expression, the errors in the subchannel void fractions were found to be no greater than $\pm 6.0 \%$.

$$
\Delta \alpha_{i}= \pm \frac{\left[2.1 \times 10^{-6} Q_{g i}^{2}+\left(1.16 \times 10^{-4} Q_{l i}+0.075 A_{i}\right)^{2}\right]^{\frac{1}{2}}}{\left\{0.343 Q_{g i}+0.046 Q_{l i}+29.02 A_{i}\right\}^{2}}
$$

The sinter section technique involved feeding the air at the upper tie plate, which was located at the bundle outlet, through the interior of the rods, to sinter sections at the base of each rod. Each rod had its own 
separately controlled sinter section so that any number of the four rods could be supplied with air to simulate boiling. The cross sectional schematic of the sinter section can be found in Figure 3.30.

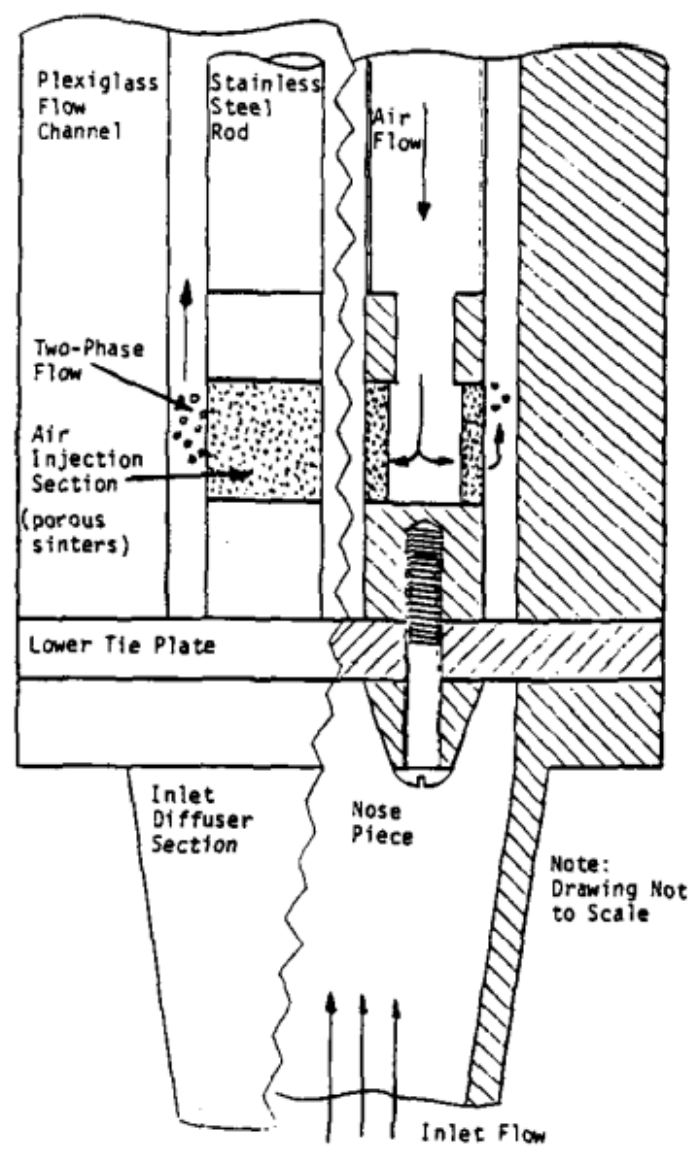

Figure 3.30: Low assembly test section with sinter section shown [14]

The mixing tee technique involved soldering thin slabs of sintered metal over cutouts in the water supply line for the test section. An air annulus was constructed to surround the porous section of the pipe, which allowed the air to be bubbled into the mainline. The cross sectional schematic of the mixing tee setup can be found in Figure 3.31 .

In order to measure the void in each subchannel at the bundle outlet, one of each type of subchannel (corner, side, and center) were physically separated by means of 0.020 -inch thick splitters located two inches from the test section outlet. These isolated subchannels were then simultaneously isokinetically sampled to determine the subchannel flow qualities according to (Eq.) 3.14, where $G_{g}$ and $G_{l}$ refer to the water and air mass fluxes, respectively.

$$
\langle\bar{x}\rangle=\frac{G_{g}}{G_{l}+G_{g}} .
$$




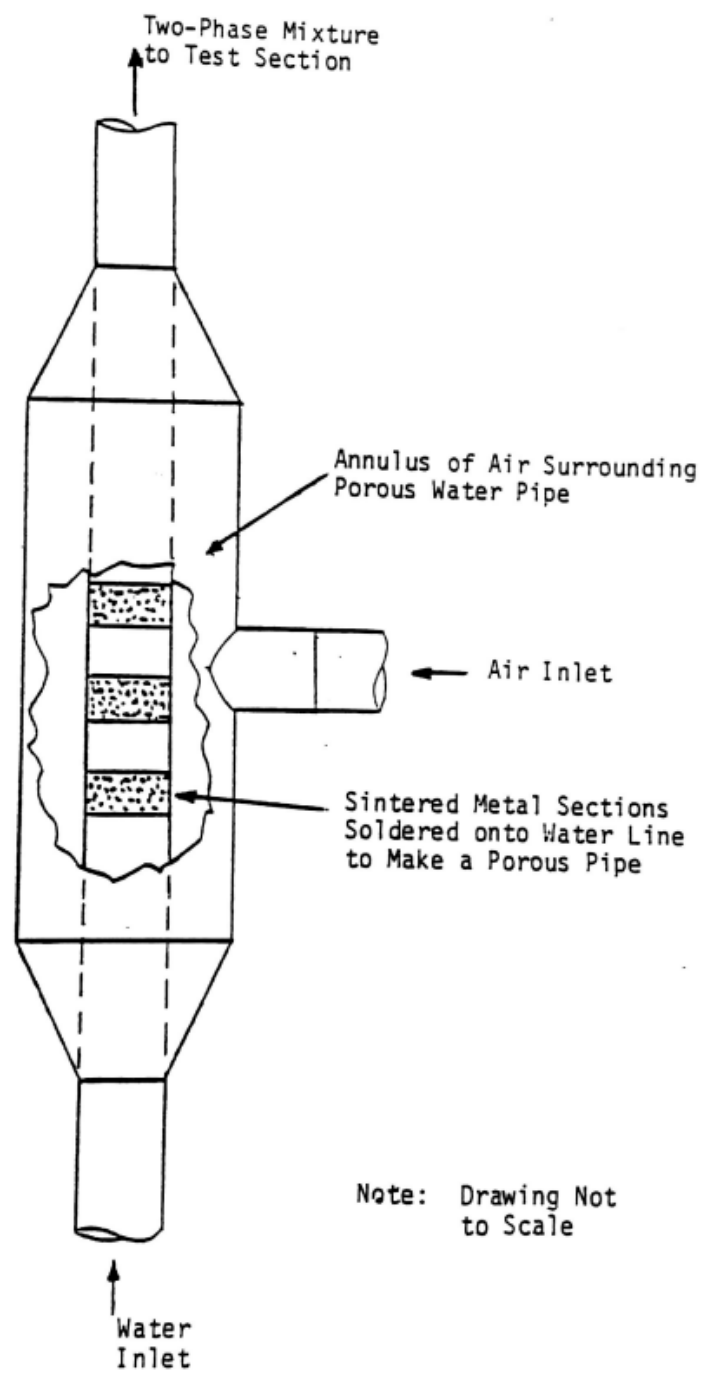

Figure 3.31: Air/Water Mixing Tee [14]

The subchannel void fraction and quality are related by the Zuber-Findlay equation as found in Eq. (3.15), where $\rho_{g}$ and $\rho_{l}, V_{g j}$, and $C_{0}$ refer to the air and water densities, drift velocity, and void concentration parameter, respectively.

$$
\langle\alpha\rangle=\frac{\langle x\rangle}{\left\{C_{0}\left[\langle x\rangle+\frac{\rho_{g}}{\rho_{l}}(1-\langle x\rangle)\right]+\frac{\rho_{g} V_{g j}}{G}\right\}} .
$$

The to-scale cross sectional drawing of the test bundle can be found in Figure 3.32. The dimensions of this test section are twice that of a typical square pitched BWR fuel rod bundle to partially accommodate for the large bubble sizes present with the low pressure air-water experiment. The geometric dimensions used 
for each subchannel in the experiment and CTF simulations are found in Table 27. The different flow area values for these three subchannel types suggest that a below-average void fraction would be measured in the corner, and an above-average void fraction would be measured in the center in the presence of a void drift mechanism. Subchannels 1,2 , and 3 were chosen to be isokinetically sampled to obtain void distribution data for the corner, side, and center subchannel types, respectively.

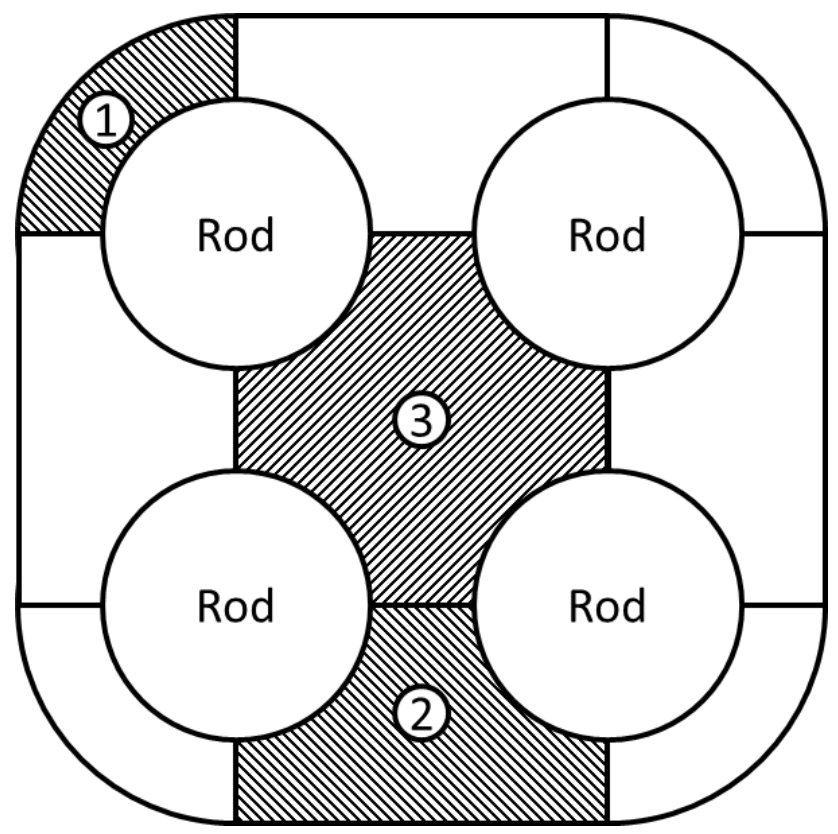

Figure 3.32: NUREG/CR-3373 Experimental Cross Section [14]

Table 27: NUREG Subchannel Flow Dimensions [14]

\begin{tabular}{rrr}
\hline Type & Flow Area $\left(\mathrm{in}^{2}\right)$ & Hydraulic Diameter (in) \\
\hline Corner & 2.08 & 1.59 \\
Side & 4.68 & 2.50 \\
Center & 7.13 & 3.57 \\
Total & 34.14 & 2.32 \\
\hline
\end{tabular}

The operating conditions for these experiments can be found in Table 28, which include the water mass flux $(\mathrm{G})$, bundle averaged void fraction $(\langle\bar{\alpha}\rangle)$, and inlet pressure $\left(P_{i n}\right)$. The fluid for all of the experiments was at ambient temperature. Besides the aforementioned four rod experiments, there was also a set of single phase experiments and two-phase single rod experiments described in the report. The two-phase single rod experiments were conducted to observe the void drift phenomenon while only using the sinter section of one rod. 
Table 28: Experimental Operating Conditions [14]

\begin{tabular}{rrrrr}
\hline Case & $G \times 10^{-6}\left[\frac{l b m}{f t^{2} h r}\right]$ & $\langle\bar{\alpha}\rangle[\%]$ & $P_{i n}[p s i g]$ & Mixing Technique \\
\hline 1 & 0.333 & 0.0 & 6.0 & \\
2 & 0.666 & 0.0 & 15.0 & \\
3 & 0.333 & 20.4 & 6.0 & \\
4 & 0.333 & 32.8 & 6.5 & \\
5 & 0.333 & 46.1 & 8.0 & Mixing Tee \\
6 & 0.666 & 23.6 & 15.5 & \\
7 & 0.666 & 37.5 & 19.0 & \\
8 & 0.666 & 52.7 & 26.0 & \\
9 & 0.333 & 20.4 & 6.0 & \\
10 & 0.333 & 32.8 & 6.3 & \\
11 & 0.333 & 46.1 & 8.0 & 4 \\
12 & 0.666 & 23.6 & 15.5 & \\
13 & 0.666 & 37.5 & 19.0 & \\
14 & 0.666 & 52.7 & 29.5 & \\
\hline
\end{tabular}

\subsection{Kumamoto Univeristy $2 \times 3$}

Facility description The $2 \times 3$ facility is an air/water facility that was operated at Kumamoto University in the early 2000s in order to quantify effects of mixing and void drift [15]. The test section is uniquely designed so that there are only two subchannel types - side and inner-leaving out the additional complexity of the corner type subchannel. Figure 3.33 shows the cross-section of the facility and provides geometric information. The assembly geometry is larger than a typical BWR lattice due to the lower density (and thus larger bubble sizes) of air at atmospheric pressure.

Figure 3.34 shows an axial schematic of the facility. The test section has four axial sections: 1) an entry section, where side and inner channels are separated by a physical barrier; 2) a tracer injection section, where each individual channel is physically separated from one another; 3) a mixing section, where all channel are connected and lateral cross-flow is allowed to take place; and 4) a discharge section, where groups of channels are partitioned and flow is sent to measurement devices.

Because the mixing section is short $(2.25 \mathrm{~m})$, the inlet flow of individual subchannel types is adjusted in the experiment so that flow in the mixing section is in mechanical equilibrium throughout the entire length. In this way, the test eliminates the effects of flow redistribution due to geometry differences between side and inner channels. The facility is used for two different types of tests: single- and two-phase mixing tests [15] and void drift tests [16].

The mixing tests made use of tracer dyes in the facility. The tracer dyes include Acid orange II for the water phase and methane for the gas phase. They are injected into one of the inner subchannels in the tracer injection section and collected and measured at three axial elevations in the mixing section. Measurements are taken by gas chromatograph for the gas phase and spectrophotometer for the liquid phase.

The author derived a tracer-dye conservation equation for each subchannel and used this system of equations to derive the channel mixing term, $w_{i j}^{\prime}$, as a function of tracer dye concentration. Flow conditions for the 


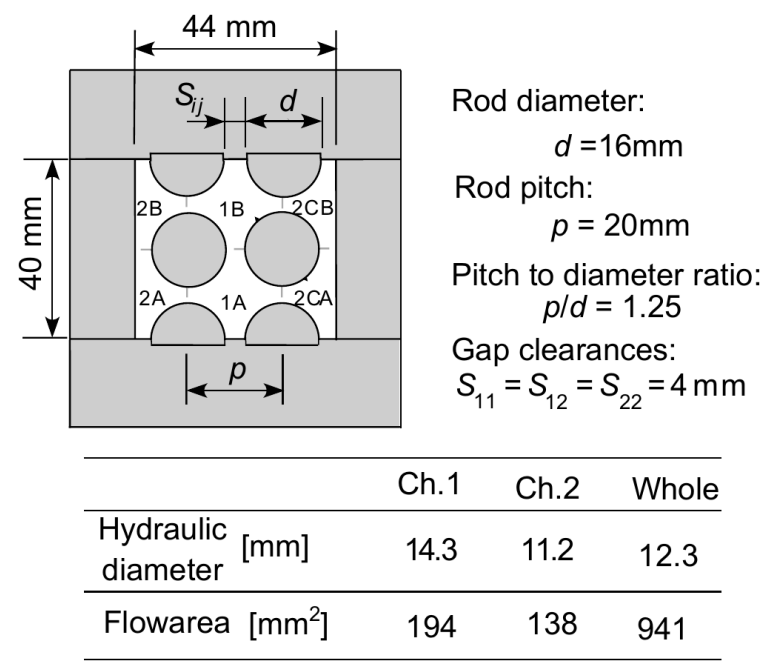

Figure 3.33: Cross-sectional diagram of the $2 \times 3$ facility and relevant geometric information (reprinted from M. Sadatomi et al. "Single- and Two-Phase Turbulent Mixing Rate between Adjacent Subchanels in a Vertical $2 \times 3$ Rod Array Channel". In: International Journal of Multiphase Flow 30 (2004), pp. 481-498.

mixing tests are shown in Figure 3.35 as liquid and vapor superficial velocity. Only the single-phase tests are modeled in this study, which includes four data points. The system is run at room temperature and atmospheric pressure.

CTF Model Description Flow area and wetted perimeter are directly taken from the values of Figure 3.33. An axial mesh size of $2.54 \mathrm{~cm}$ is employed, and only the $2.25 \mathrm{~m}$ mixing section is modeled. Because the experimenters set the inlet flow to equal the equilibrium distribution, a similar approach is used in setting the inlet flow rate in CTF. First, the total injection mass flow rate is determined using the CTF-predicted liquid density, facility flow area, and liquid velocity specified in the experiment. CTF is run, the outlet flow distribution is obtained, and this is used as the inlet distribution for the next simulation. This process is repeated until cross-flow ceases throughout the facility.

The friction correlation will drive the flow distribution, as shown later in Section 6.2.2.1. The author indicates that the Sadatomi friction factor correlation [17] leads to the best agreement with the measured flow distribution; however, its complexity makes it difficult to enter into CTF without significant code changes. The author also shows results of the Blasius equation, which seems to also perform well. Therefore, the Blasius correlation (Equation 3.16) is used as a first step in this study; however, the CTF friction correlation in Eq. (3.17), which is a default model in CTF, is also tested.

$$
\begin{gathered}
f=0.316 R e^{-0.25} \\
f=0.204 R e^{-0.2}
\end{gathered}
$$




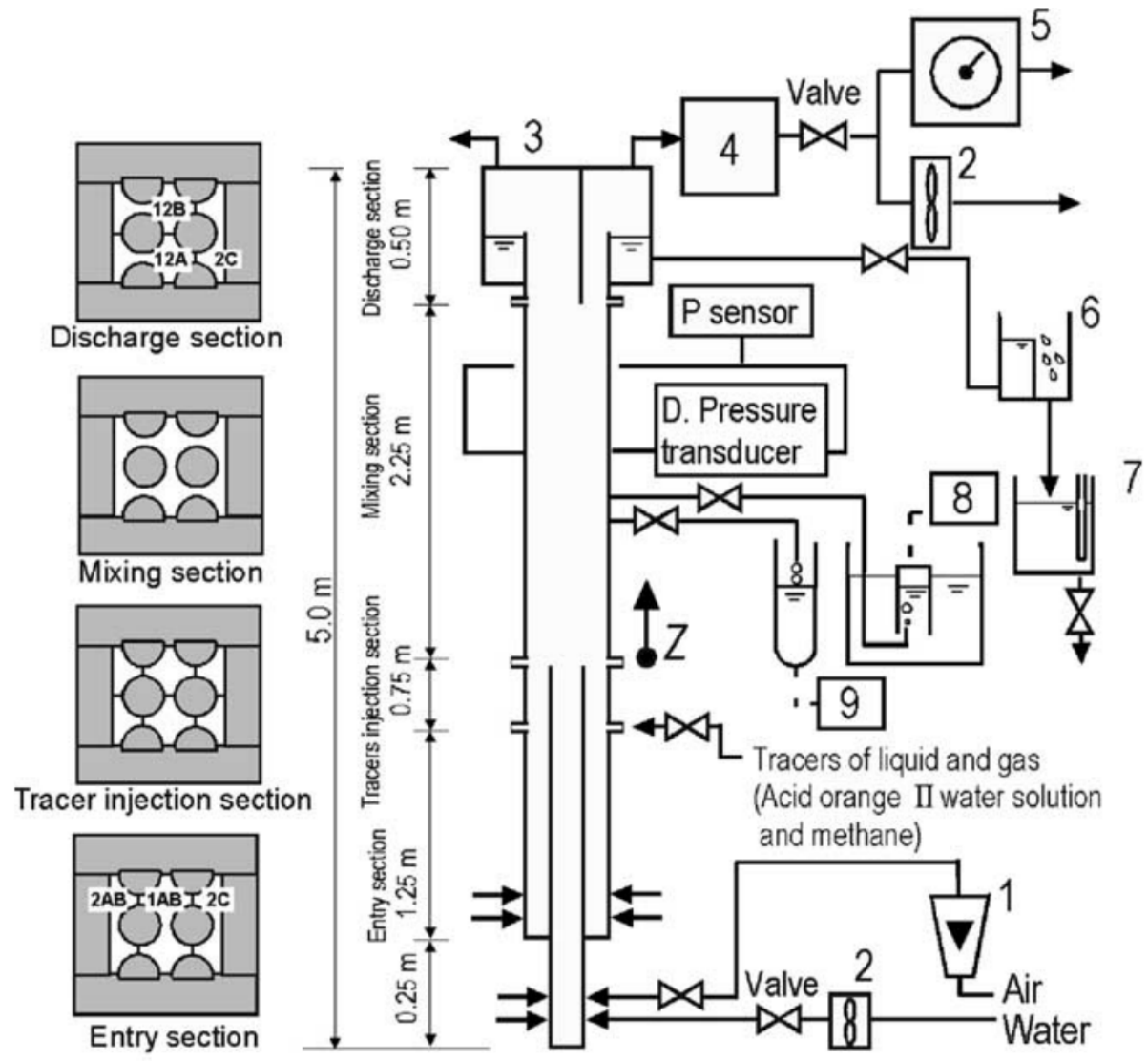

Figure 3.34: Side-view schematic of $2 \times 3$ facility with visualization of channel partitioning in different axial sections (reprinted from M. Sadatomi et al. "Single- and Two-Phase Turbulent Mixing Rate between Adjacent Subchanels in a Vertical $2 \times 3$ Rod Array Channel". In: International Journal of Multiphase Flow 30 (2004), pp. 481-498. 


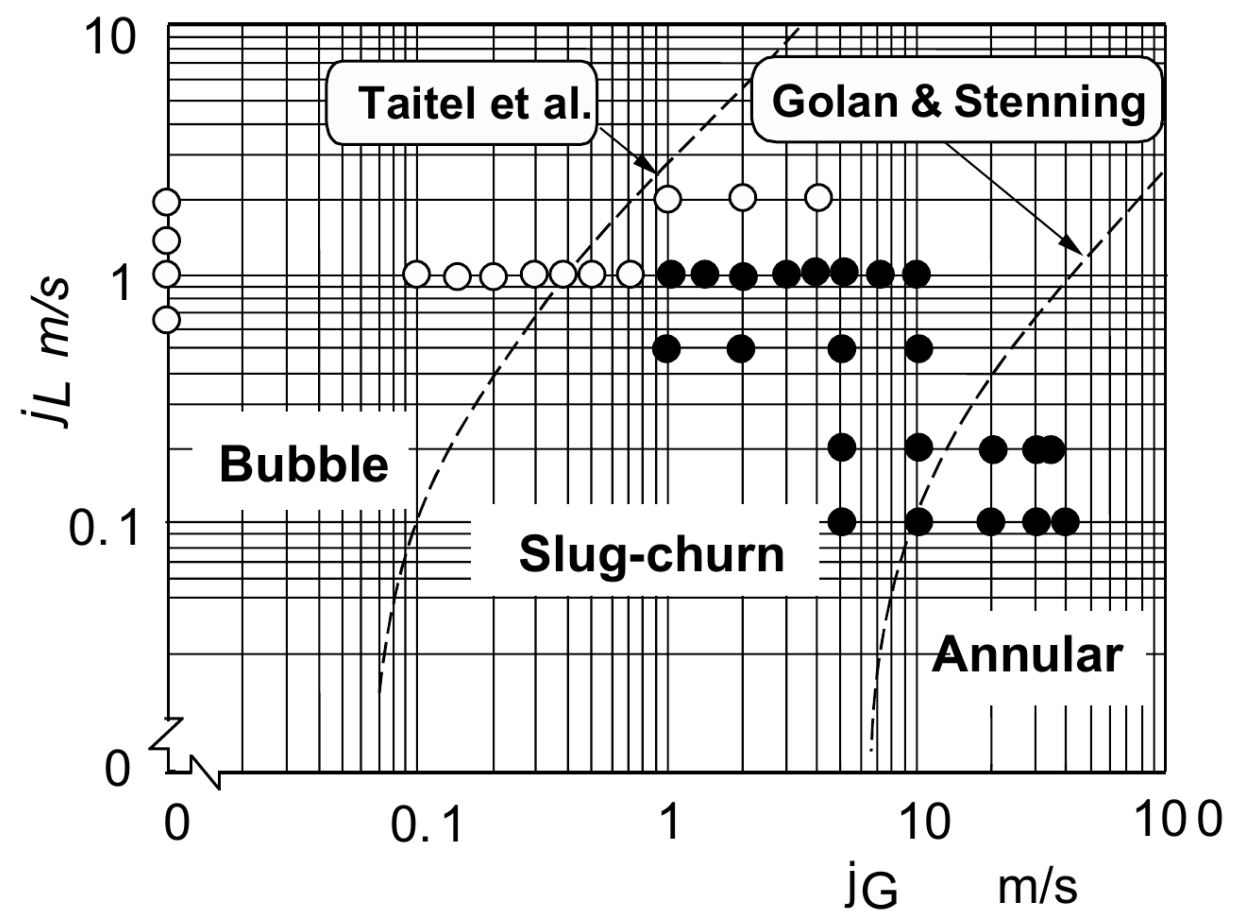

Figure 3.35: Operating conditions for the $2 \times 3$ facility (reprinted from M. Sadatomi et al. "Single- and Two-Phase Turbulent Mixing Rate between Adjacent Subchanels in a Vertical $2 \times 3$ Rod Array Channel". In: International Journal of Multiphase Flow 30 (2004), pp. 481-498. 
The single-phase turbulent mixing coefficient is varied during this study to investigate its impact on mixing results. The inlet temperature is set to $24^{\circ} \mathrm{C}$ and the outlet pressure is set to 1.01325 bar. 


\subsection{Halden IFA Test Cases}

Facility Description The Halden Boiling Heavy Water Reactor (HBWR)[18] is located in Halden, a coastal town in southeast Norway, near the border of Sweden. The reactor hall is situated within a rock hillside on the north bank of the river Tista. HBWR is a natural circulation boiling heavy water reactor. The maximum power is $25 \mathrm{MW}$ (thermal), and the water temperature is $240^{\circ} \mathrm{C}$, corresponding to an operating pressure of 33.3 bar. Pressurization tests are performed at regular intervals using a pressure of 40 bars. The reactor pressure vessel is cylindrical with a rounded bottom. It is made of carbon steel, and the bottom and the cylindrical portion are clad with stainless steel. The flat reactor lid has individual penetrations for fuel assemblies, control stations, and experimental equipment. 14 tons of heavy water act as coolant and moderator. A mixture of steam and water flows upwards by natural circulation inside the shroud tubes which surround the fuel rods. Steam is collected in the space above the water, while water flows downwards through the moderator and enters the fuel assemblies through the holes in the lower ends of the shroud. The steam flows to two steam transformers, where heat is transferred to the light water secondary circuit. Condensate from the steam transformers returns to the reactor by gravity. An external subcooler loop is installed to provide experimental variation of void fraction in the fuel assemblies and the moderator, and it is also used for heating and cooling purposes. In the secondary circuit, two circulation pumps pass the water through the steam transformers, a steam drum, and a steam generator where steam is produced in the tertiary circuit. The tertiary steam is normally delivered as process steam to the nearby paper mill, but it may also be dumped to the river.

Reactor Operating Conditions A fuel charge consists of a combination of test fuel from organizations in member countries and driver fuel assemblies, which provide reactivity for operating the reactor. Light water high pressure loops provide facilities for testing under prototypic BWR and PWR conditions. HBWR uses natural circulation of heavy water for cooling. The reactor operates at the conditions tabulated in Table 29.

Table 29: Operating data for HBWR[18]

\begin{tabular}{|l|c|}
\hline Power level & $12 \mathrm{MW}$ \\
Reactor pressure & $3.4 \mathrm{MPa}(500 \mathrm{psi})$ \\
Heavy-water saturation temperature & $513^{\circ} \mathrm{K}\left(464^{\circ} \mathrm{F}\right)$ \\
Plenum inlet temperature & $510^{\circ} \mathrm{K}\left(459^{\circ} \mathrm{F}\right)$ \\
Thermal flux & $\sim 2 x 10^{16} \mathrm{n} / \mathrm{m}^{2}-\mathrm{s} /(\mathrm{W} / \mathrm{g})$ \\
Fast flux $(>1 \mathrm{MeV})$ & $\sim 5 x 10^{15} \mathrm{n} / \mathrm{m}^{2}-\mathrm{s} /(\mathrm{W} / \mathrm{g})$ \\
Average fuel power density & $14.8 \mathrm{~W} / \mathrm{g}$ \\
\hline
\end{tabular}

\subsubsection{IFA Test Series}

The benchmark studies on fuel centerline temperatures as outlined in [19] between Halden reactor test assembly data ([20], [18], [21], [22]); FRAPCON-3.4 ([22], [23], and CTF [1] predictions are considered in this study with four cases.

Fuel centerline temperature predictions from the current and updated fuel thermal conductivity model in CTF are compared against the Halden experimental test data and FRAPCON-3.4 predictions. The reason 
Table 30: Design parameters and instrumentation for IFA432

\begin{tabular}{|c|c|c|c|c|c|c|c|c|c|c|c|}
\hline \multirow{3}{*}{$\begin{array}{l}\text { Rod } \\
\text { No. }\end{array}$} & \multirow{2}{*}{\multicolumn{2}{|c|}{$\begin{array}{c}\text { Pellet } \\
\text { Diameter }\end{array}$}} & \multirow{2}{*}{\multicolumn{2}{|c|}{$\begin{array}{c}\text { Cold } \\
\text { Diam. Gap }{ }^{1}\end{array}$}} & \multirow{3}{*}{$\begin{array}{l}\text { Fill } \\
\text { Gas }\end{array}$} & \multirow{3}{*}{$\begin{array}{c}\text { Fuel } \\
\text { Dens. } \\
\% T D\end{array}$} & \multirow{3}{*}{$\begin{array}{c}\text { Fuel } \\
\text { Type }^{2}\end{array}$} & \multicolumn{4}{|c|}{ Instrumentation } \\
\hline & & & & & & & & Temp & rature & & Clad \\
\hline & $m m$ & in & $m m$ & in & & & & Upper & Lower & Pressure & Length \\
\hline 1 & 10.681 & 0.4205 & 0.229 & 0.009 & $\mathrm{He}$ & 95 & Stable & $\mathrm{TC}^{3}$ & $\mathrm{TC}$ & $\mathrm{PT}^{4}$ & $\mathrm{EM}^{5}$ \\
\hline 2 & 10.528 & 0.4145 & 0.381 & 0.015 & $\mathrm{He}$ & 95 & Stable & $\mathrm{UT}^{6}$ & $\mathrm{TC}$ & - & EM \\
\hline 3 & 10.833 & 0.4265 & 0.076 & 0.003 & $\mathrm{He}$ & 95 & Stable & $\mathrm{TC}$ & $\mathrm{TC}$ & - & EM \\
\hline 4 & 10.681 & 0.4205 & 0.229 & 0.009 & $\mathrm{Xe}$ & 95 & Stable & $\mathrm{TC}$ & $\mathrm{TC}$ & - & EM \\
\hline 5 & 10.681 & 0.4205 & 0.229 & 0.009 & $\mathrm{He}$ & 92 & Stable & $\mathrm{TC}$ & $\mathrm{TC}$ & $\mathrm{PT}$ & EM \\
\hline 6 & 10.681 & 0.4205 & 0.229 & 0.009 & $\mathrm{He}$ & 92 & Unstable & $\mathrm{TC}$ & $\mathrm{TC}$ & $\mathrm{PT}$ & EM \\
\hline 7 & 10.528 & 0.4145 & 0.381 & 0.015 & $\mathrm{He}$ & 95 & Stable & - & - & - & - \\
\hline 8 & 10.681 & 0.4205 & 0.229 & 0.009 & $\mathrm{He}$ & 95 & Stable & - & - & - & - \\
\hline 9 & 10.732 & 0.4225 & 0.179 & 0.007 & $\mathrm{He}$ & 95 & Stable & - & - & - & - \\
\hline
\end{tabular}

that these rods are selected for comparison to experimental data is that they are representatives for all different conditions, including BOL and burnup conditions, as well as different gadolinia (Gd) concentrations and different fuel rods, $\mathrm{UO}_{2}$ and $\mathrm{MOX}$ rods. The cases are selected from FRAPCON Integral Assessment for comparisons to Halden Reactor experiments, which are listed as:

1. IFA432 Rod-1 at BOL conditions, $\mathrm{UO}_{2}$ fuel

2. IFA432 Rod-1 with achieved a burnup of $\sim 30 \mathrm{GWD} / \mathrm{MTU}, \mathrm{UO}_{2}$ fuel

3. IFA610 Rod-2 with achieved a burnup of $\sim 58 \mathrm{GWD} / \mathrm{MTU}, \mathrm{UO}_{2}+2 \% \mathrm{Gd}_{2} \mathrm{O}_{3}$ fuel

4. IFA681 Rod-2 with achieved a burnup of $\sim 23 \mathrm{GWD} / \mathrm{MTU}, \mathrm{MOX}$ fuel

\subsubsection{IFA432}

Test Description The IFAs were designed by Pacific Northwest Laboratory (PNL) and were irradiated in the HBWR, Halden, Norway. IFA432 test series were sponsored by U.S. Nuclear Regulatory Commission (NRC). IFA432 had a design power of $49 \mathrm{~kW} / \mathrm{m}(15 \mathrm{~kW} / \mathrm{ft})$ and reached its goal burnup of $1720 \mathrm{GJ} / \mathrm{kgU}$ $(20 \mathrm{GWd} / \mathrm{MTU})$ in late 1978. Since most of the instruments in IFA432 were still functioning properly at that time, it was left in the HBWR core to obtain data at higher burnups. IFA432 has provided a vast amount of well-characterized experimental data under conditions that realistically simulate LWR conditions. The design parameters and instrumentation that were used for IFA432 are tabulated in Table 30 [24].

The power profile in the HBWR was also considered during the design, which is shown in Figure 3.36. The tops os the rods were placed at the peak, which forced the bottoms of the rods to operate at 70 to $80 \%$ of peak rod power. To take advantage of the power distribution, thermocouples were placed in the top and bottom end caps[24].

The correct assessment of rod powers and the distribution of power within the rods are of utmost importance to assure the best possible thermal data. Therefore, seven neutron sensors were placed in each assembly as shown in Figure 3.37: one cobalt detector in the center, three vanadium detectors at the top plane of 


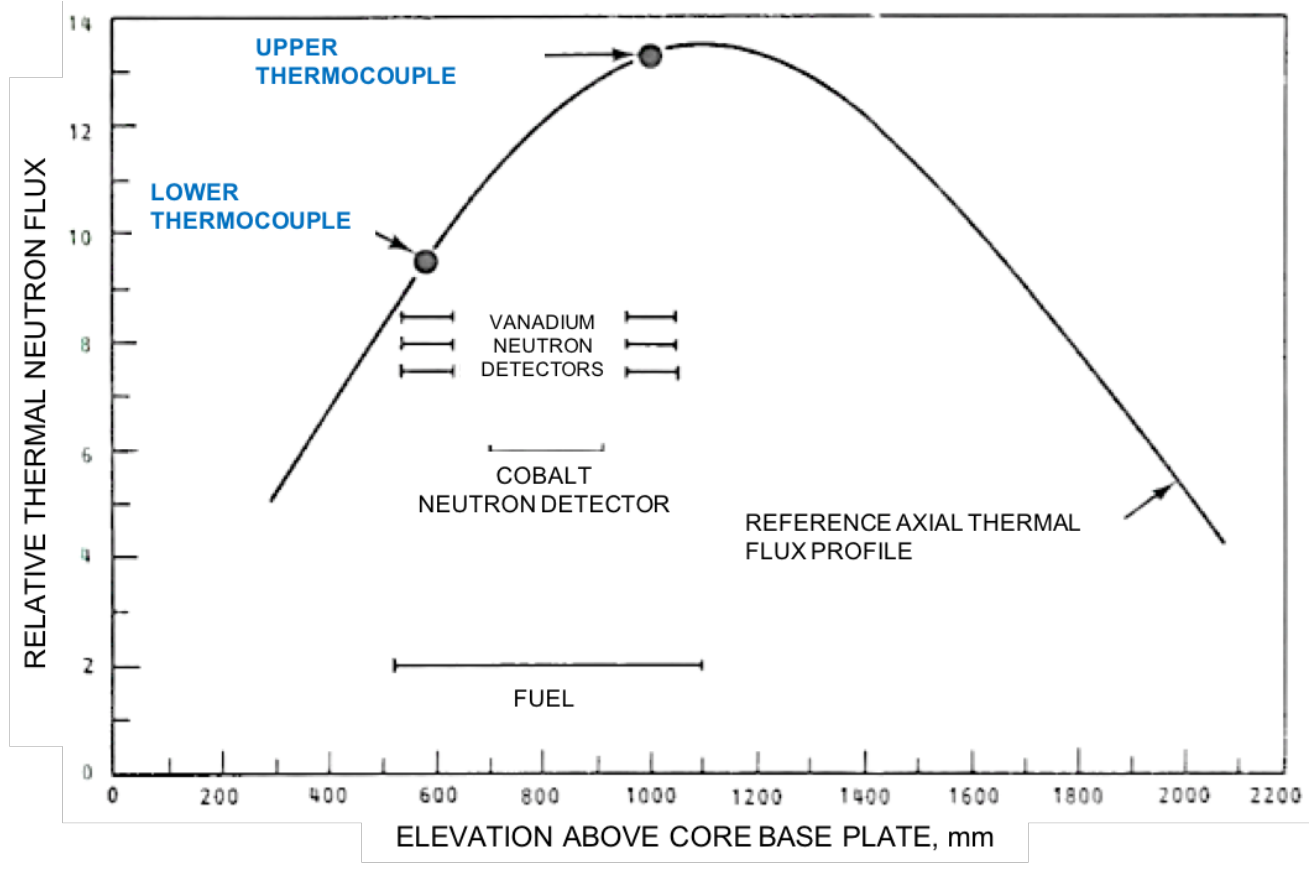

Figure 3.36: Arrangement of temperature sensors, neutron detectors, and fuel relative to reference axial thermal flux profile for IFA432[24]

thermocouple (TC)s, and three vanadium detectors at the bottom plane of the TCs. A cladding elongation monitor was mounted for each rod; rods could be changed without disrupting the elongation sensors[24].

Test Facility The HBWR uses natural circulation of heavy water for cooling. Reactor operating data are shown in Table 29. The schematic of the HBWR core loading in November 1975 is shown in Figure 3.38 with the locations of IFA431 and IFA432.

Irradiation Summary IFA432 was initially charged into the reactor in December 1975, and a total of eight fuel rods have been irradiated in the six fuel rod positions. The assembly reached its goal burnup of $20 \mathrm{GWd} / \mathrm{MTU}$ in late 1978, but since most of the instruments were still operating properly, it remained in the reactor to obtain data at higher burnups. In June 1981, the assembly was removed from the reactor after the original fuel rods $(1,2,3,4,5$, and 6$)$ had attained average burnups of 30GWd/MTU. At this time, the decision was made to remove rods 1 and 6 from the assembly for destructive PIE[24].

CTF Model Description During first ramp to power $\left(\mathrm{UO}_{2}\right)$ at BOL conditions and throughout life $\left(\mathrm{UO}_{2}\right)$ with burnup of 30GWD/MTU for IFA432 Rod-1. The gap conductance values are calculated dynamically in CTF using the dynamic gap conductance method. The gap conductance model is activated by setting IGPC to -1 in Card 9.2 (See CTF User's Manual for details). In pellet-power distribution, burnup and gadolinia, content are read from FRAPCON-3.4 simulations. The geometry description and input parameters are 


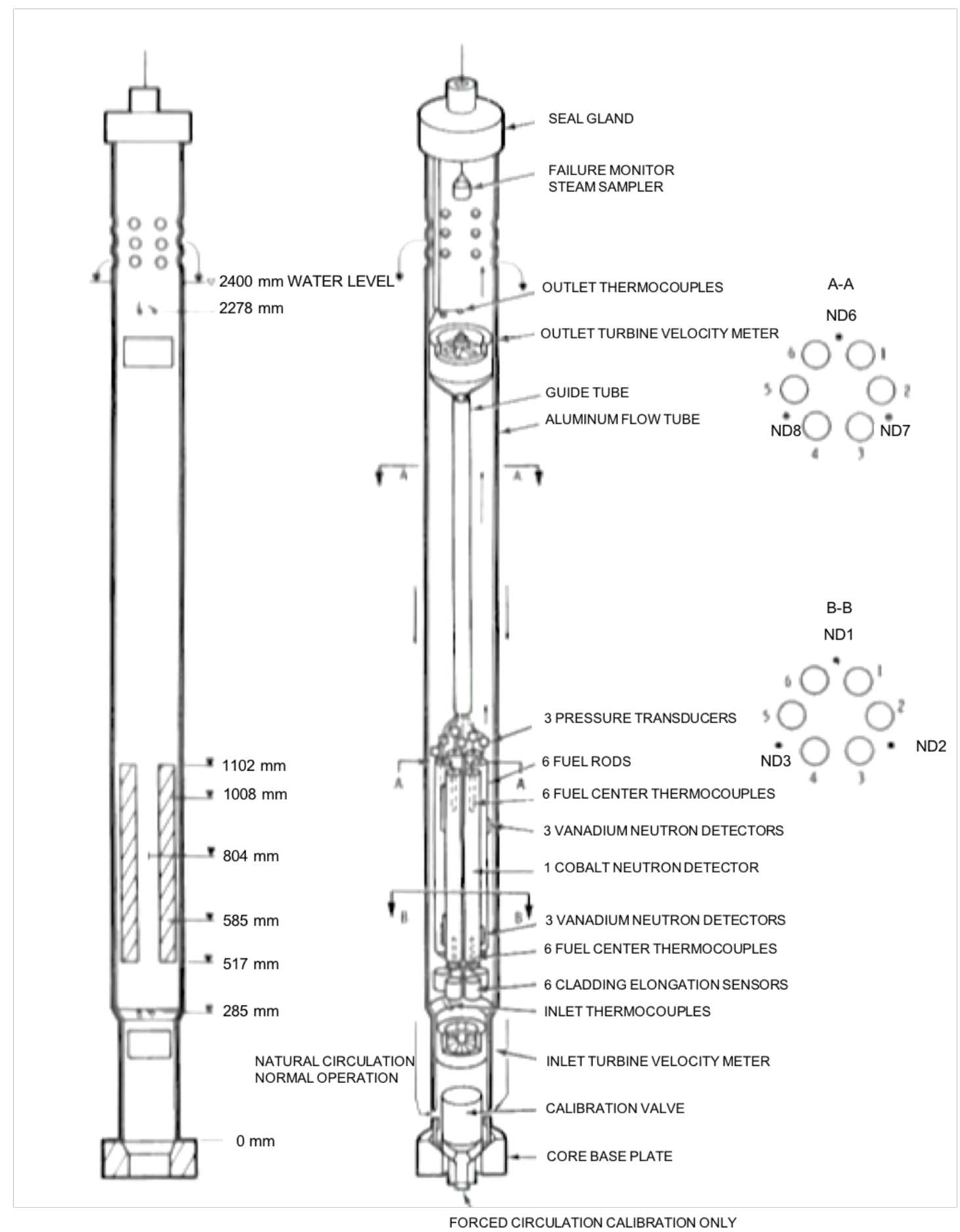

Figure 3.37: Schematic of IFA432[24] 


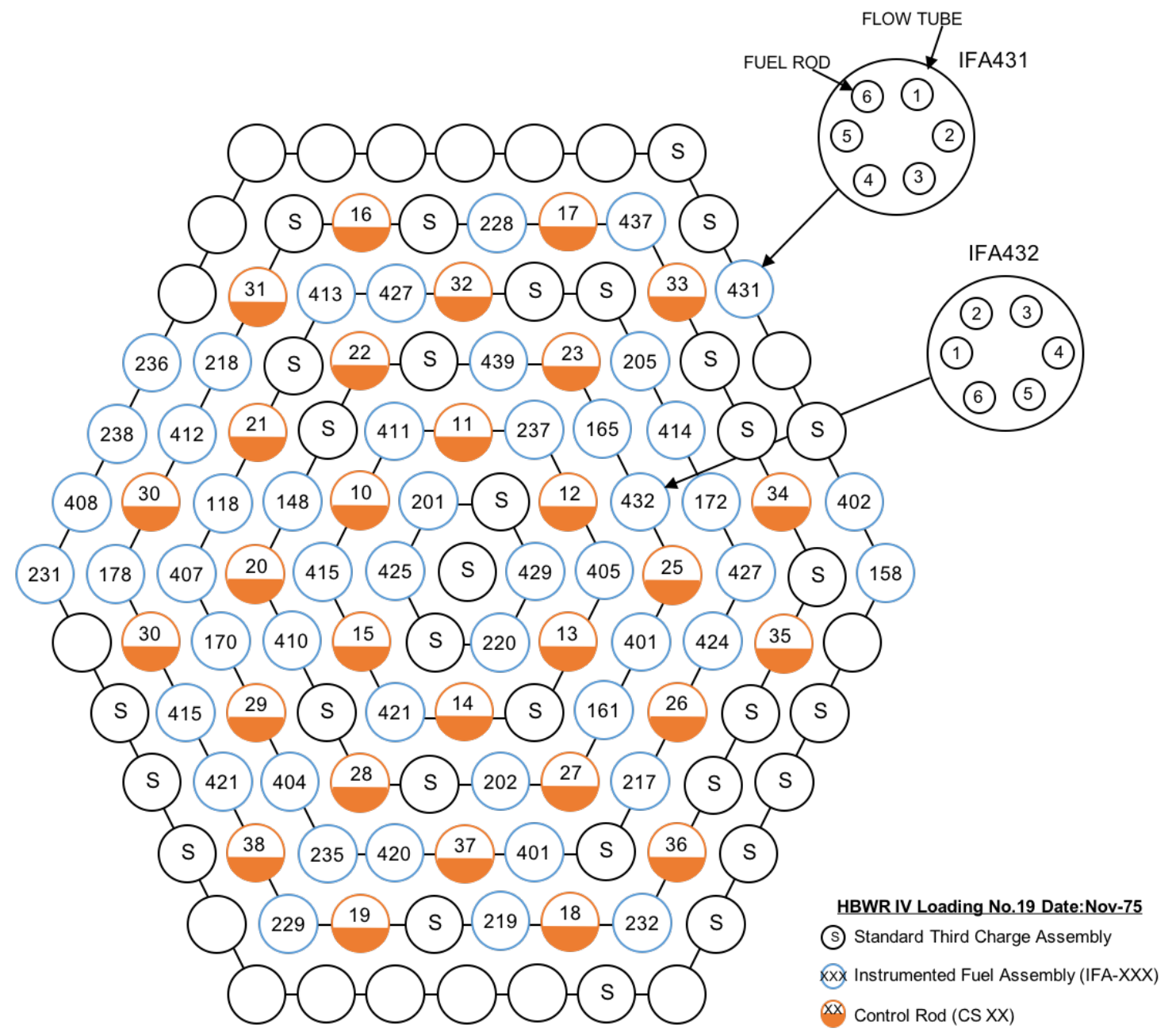

Figure 3.38: Location of IFA431 and IFA432 in the HBWR core 
tabulated for IFA432 in Table 31.

Table 31: CTF input parameters for IFA432

\begin{tabular}{|c|c|c|c|c|c|}
\hline Rod & & & Fuel Pellet & & \\
\hline Fuel stack height & $\mathrm{m}$ & 0.579 & Material & & $\mathrm{UO}_{2}$ \\
\hline Coolant pressure & $\mathrm{MPa}$ & 3.448 & Theoretical density & $\%$ & 95.5 \\
\hline Inlet temperature & ${ }^{\circ} \mathrm{K}$ & 510 & Surface roughness & $\mu \mathrm{m}$ & 2.16 \\
\hline Inlet mass flow rate & $\mathrm{kg} / \mathrm{s}$ & 0.1 & Fuel pellet diameter & & \\
\hline & & & Rod 1 & $\mathrm{~mm}$ & 10.681 \\
\hline Cladding & & & Gap & & \\
\hline Material & & Zr-2 & Fill gas pressure & $\mathrm{MPa}$ & 0.1 \\
\hline Outer diameter & $\mathrm{mm}$ & 12.789 & Fill gas composition & & helium \\
\hline Inner diameter & $\mathrm{mm}$ & 10.909 & Initial radial fuel-clad gap & & \\
\hline Wall thickness & $\mathrm{mm}$ & 0.94 & Rod 1 & $\mu \mathrm{m}$ & 114 \\
\hline Surface roughness & $\mu \mathrm{m}$ & 1.143 & & & \\
\hline
\end{tabular}

\subsubsection{IFA610}

CTF Model Description MOX case with burnup of 58GWD/MTU for IFA610 Rod-2. The gap conductance values are calculated dynamically in CTF using the dynamic gap conductance method. In pellet-power distribution, burnup and gadolinia content are read from FRAPCON-3.4 simulations. The gap conductance model is activated by setting IGPC to -1 in Card 9.2 (See CTF User's Manual for details). In pelletpower distribution, burnup and gadolinia content are read from FRAPCON-3.4 simulations. The geometry description and input parameters are tabulated for IFA610 in Table 32.

Table 32: CTF input parameters for IFA610

\begin{tabular}{|c|c|c|c|c|c|}
\hline \multicolumn{3}{|l|}{ Rod } & \multicolumn{3}{|l|}{ Fuel Pellet } \\
\hline Fuel stack height & $\mathrm{m}$ & 0.579 & Material & & MOX \\
\hline Coolant pressure & $\mathrm{MPa}$ & 3.400 & Theoretical density & $\%$ & 95.2 \\
\hline Inlet temperature & ${ }^{\circ} \mathrm{K}$ & 510 & Surface roughness & $\mu \mathrm{m}$ & 2.16 \\
\hline Inlet mass flow rate & $\mathrm{kg} / \mathrm{s}$ & 0.05 & $\begin{array}{l}\text { Fuel pellet diameter } \\
\text { Rod } 2\end{array}$ & & 8.190 \\
\hline \multicolumn{3}{|l|}{ Cladding } & \multicolumn{3}{|l|}{ Gap } \\
\hline Material & & $\mathrm{Zr}-2$ & Fill gas pressure & $\mathrm{MPa}$ & 0.1 \\
\hline Outer diameter & $\mathrm{mm}$ & 9.500 & Fill gas composition & & helium \\
\hline Inner diameter & $\mathrm{mm}$ & 8.350 & Initial radial fuel-clad gap & & \\
\hline Wall thickness & $\mathrm{mm}$ & 0.57 & $\operatorname{Rod} 2$ & $\mu \mathrm{m}$ & 78 \\
\hline Surface roughness & $\mu \mathrm{m}$ & 1.143 & & & \\
\hline
\end{tabular}

\subsubsection{IFA681}

CTF Model Description Burnup of 23GWD/MTU with $\mathrm{UO}_{2}+2 \% \mathrm{Gd}_{2} \mathrm{O}_{3}$ for IFA681 Rod-2. The gap conductance values are calculated dynamically in CTF using the dynamic gap conductance method. In 
pellet-power distribution, burnup and gadolinia content are read from FRAPCON-3.4 simulations. The gap conductance model is activated by setting IGPC to -1 in Card 9.2 (See CTF User's Manual for details). In pellet-power distribution, burnup and gadolinia content are read from FRAPCON-3.4 simulations. The geometry description and input parameters are tabulated for IFA681 in Table 33.

Table 33: CTF input parameters for IFA681

\begin{tabular}{|c|c|c|c|c|c|}
\hline \multicolumn{3}{|l|}{ Rod } & \multicolumn{3}{|l|}{ Fuel Pellet } \\
\hline Fuel stack height & $\mathrm{m}$ & 0.579 & Material & & $\mathrm{UO}_{2}+2 \% \mathrm{Gd}_{2} \mathrm{O}_{3}$ \\
\hline Coolant pressure & $\mathrm{MPa}$ & 15.514 & Theoretical density & $\%$ & 95.2 \\
\hline Inlet temperature & ${ }^{\circ} \mathrm{K}$ & 510 & Surface roughness & $\mu \mathrm{m}$ & 2.16 \\
\hline Inlet mass flow rate & $\mathrm{kg} / \mathrm{s}$ & 0.245 & $\begin{array}{l}\text { Fuel pellet diameter } \\
\text { Rod } 2\end{array}$ & $\mathrm{~mm}$ & 8.194 \\
\hline \multicolumn{3}{|l|}{ Cladding } & \multicolumn{3}{|l|}{ Gap } \\
\hline Material & & Zr-2 & Fill gas pressure & $\mathrm{MPa}$ & 0.1 \\
\hline Outer diameter & $\mathrm{mm}$ & 9.500 & Fill gas composition & & helium \\
\hline Inner diameter & $\mathrm{mm}$ & 8.350 & Initial radial fuel-clad gap & & \\
\hline Wall thickness & $\mathrm{mm}$ & 0.58 & Rod 2 & $\mu \mathrm{m}$ & 78 \\
\hline Surface roughness & $\mu \mathrm{m}$ & 1.143 & & & \\
\hline
\end{tabular}

\subsection{Nikuradse}

The Nikuradse facility consists of a horizontal pipe through which single phase water flows. The experiments took place in the 1930s $[25,26]$ and use a variety of inflow conditions, geometries, and pipe roughnesses. There are a total of 125 experiments in smooth pipes and 362 experiments in rough pipes. Twenty-eight of the smooth pipe runs and fifty-seven of the rough pipe runs are exluded because of inconsistencies in the data. The pressure drop was experimentally measured, and this will serve as the quantity of interest for comparison to CTF results. The geometry of this test is shown in Figure 3.39.

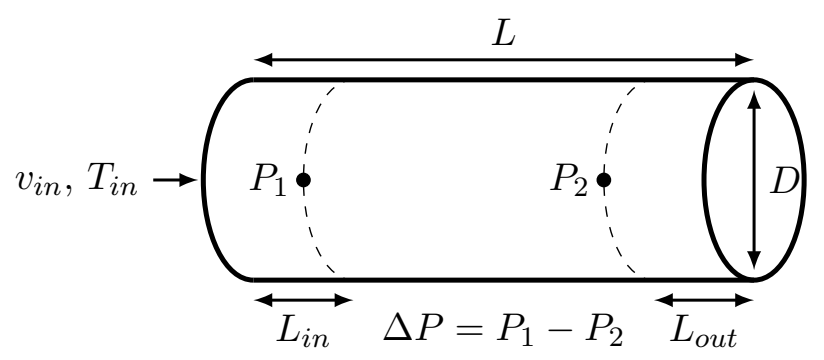

Figure 3.39: Geometry of Nikuradse experiment 
Table 34: Ranges of Operating Conditions for the Riso Test Points

\begin{tabular}{lrrr}
\hline Parameter & Min. Value & Max. Value & Unit \\
\hline Pressure & 30 & 90 & $\mathrm{bar}$ \\
Flow Rate & 0.039 & 0.628 & $\mathrm{~kg} / \mathrm{s}$ \\
Heat Flux & 0.0 & 150.0 & $\mathrm{~W} / \mathrm{cm}^{2}$ \\
Outlet Quality & 8.0 & 80.0 & $\%$ \\
\hline
\end{tabular}

\subsection{Ris $\varnothing$}

The Riso experimental facility and tests are described in Riso Report Number 372 [27]. More than 250 tests were performed in four different types of geometries: a $10 \mathrm{~mm}$ vertical tube, a $20 \mathrm{~mm}$ vertical tube, a vertical annulus facility with $17 \mathrm{~mm}$ inner diameter and $26 \mathrm{~mm}$ outer diameter, and a second annulus facility with the same diameters as the first, but shorter overall length. The tubular geometry test sections were $9 \mathrm{~m}$ long while the annulus facilities were $3.5 \mathrm{~m}$ and $8 \mathrm{~m}$ long. The range of geometric and operating conditions are shown in Table 34. These tests involve upwards flow through a vertical cylindrical pipe, either unheated or with a uniformly applied power over a specified section of the pipe. Seventy-eight of these cases were diabatic (i.e., with a heated test section) and the remaining 102 were adiabatic (i.e., the fluid was heated before entering the test section to achieve a desired constant thermodynamic quality throughout the test section).

Film flow rates were measured by opening a bypass valve which allowed flow to be diverted through perforations in the pipe wall near the test section outlet. A heat balance unit was then used to measure the flow rate of liquid in the diverted flow, with a procedure to correct for any entrained droplet flow that may have been diverted as well. While the heat balance units have a quantifiable amount of measurement uncertainty, it is noted that the data correction procedure has an unspecified, unknown level of uncertainty. Entrained droplet flow rates were then calculated from the measured total flow rate, equilibrium quality, and film flow rate for each case. Pressure drops were measured via differential pressure cells across two locations: the last $1 \mathrm{~m}$ and the last $4 \mathrm{~m}$ of the test section.

Film thicknesses were measured with a needle contact probe inserted into the flow, with film thickness inferred from liquid contact time with the needle at various distances from the wall. Film and droplet flow rate measurements were available for all test points, whereas pressure drop and film thickness data were available for only 102 and 21 of the adiabatic test points, respectively.

CTF Model Datasets used for this study included 180 test points from the two tubular geometry test sections. A script was used to generate the CTF input files automatically for all test points. The CTF models were created using internal flow in a single channel, with the inlet flow rate and outlet pressure for each test point used as boundary conditions. Inlet enthalpy or temperature was not provided for the experiments; however, outlet quality for each case was specified, so the inlet enthalpy to be used as an inlet boundary condition in CTF was calculated based on the total flow rate, total heat rate, and outlet quality for each case. Using the inferred inlet enthalpy values, the resulting outlet quality calculated by CTF matched the intended value from the experiment, as shown in Figure 3.40.

The $9.0 \mathrm{~m}$ (354 in) test section was divided into 320 uniformly spaced axial nodes to give node sizes of 


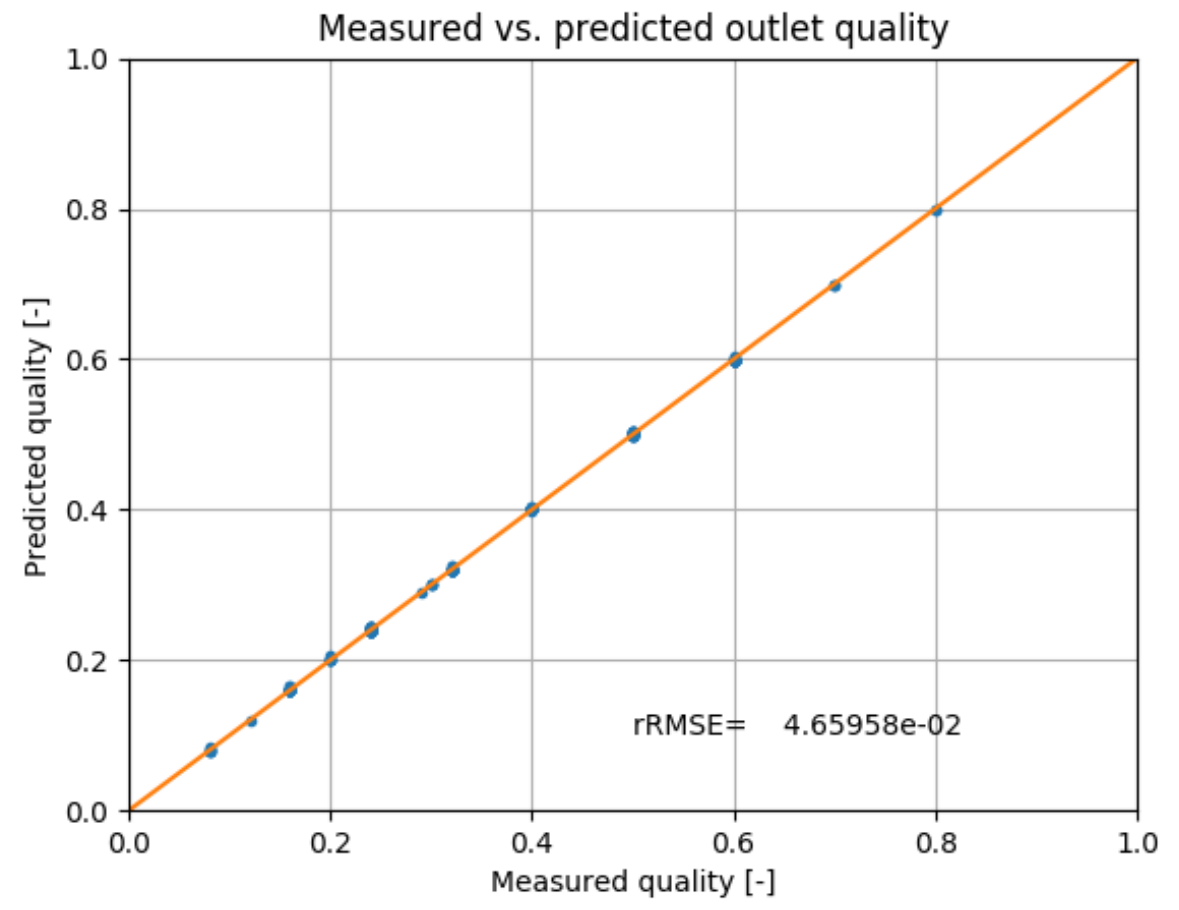

Figure 3.40: Comparison of $\mathrm{CTF}$ and experimental outlet quality. 


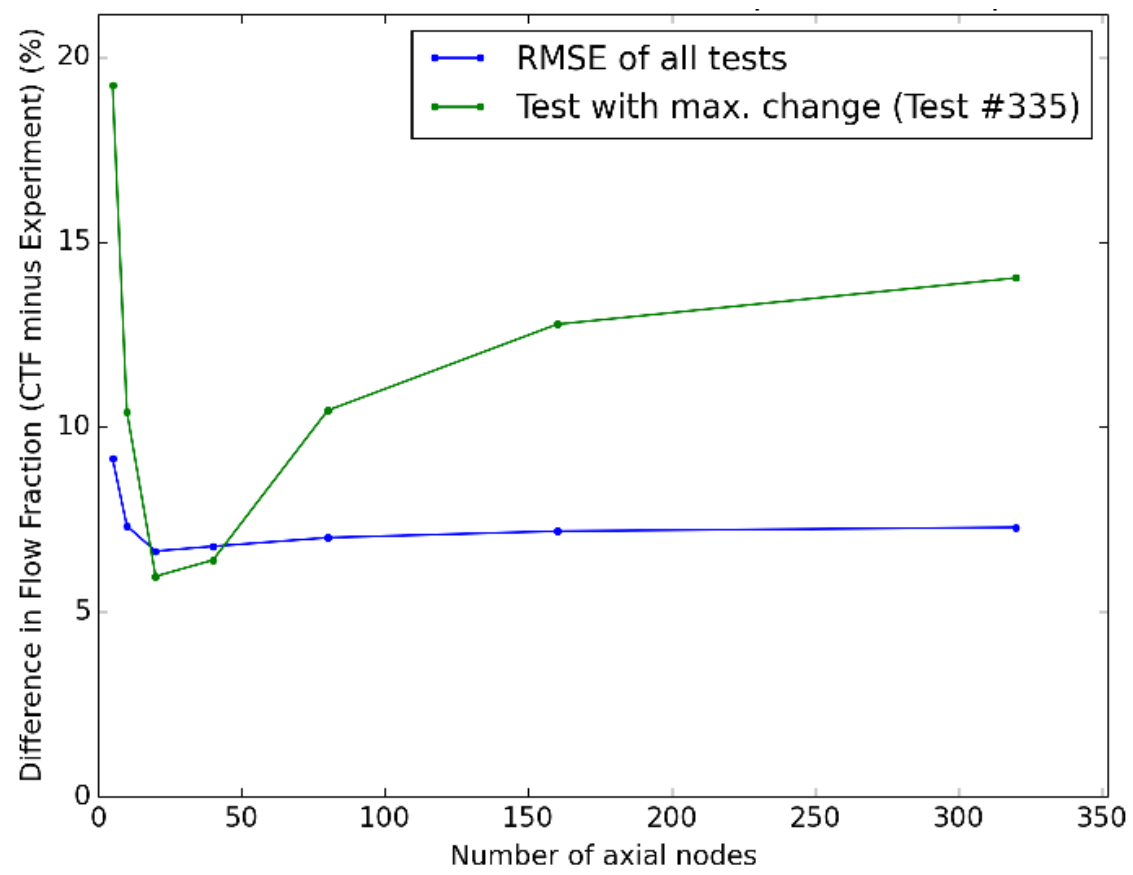

Figure 3.41: Difference in CTF versus experimental droplet flow fractions as a function of number of CTF axial nodes.

roughly one inch, which is consistent with the typically recommended mesh size for CTF calculations. A mesh sensitivity study was performed in to verify that this nodalization was sufficiently spatially converged in terms of outlet flow fractions.

Results of a sensitivity study on the number of uniformly-spaced axial nodes is shown in Figure 3.41. The mesh is well-converged in terms of the RMSE of flow fractions versus experimental values when using 80 or 160 axial nodes; however, a small number of cases still gave a significant change in flow fractions up to 320 nodes and possibly beyond. To maintain reasonable runtimes while ensuring sufficient spatial convergence, 320 axial nodes were used for all remaining calculations in this report.

\subsection{Bartolomei}

The Bartolomei experimental facility and tests are described in the following two references: [28] and [29]. Thirty-eight tests in total were modeled with two different base geometries: a $15.4 \mathrm{~mm}$ vertical tube with a fixed length of $2000.0 \mathrm{~mm}$, and a $20 \mathrm{~mm}$ vertical tube with varying lengths ranging from $800.0 \mathrm{~mm}$ and $1500.0 \mathrm{~mm}$. The range of operating conditions is shown in Table 35. These tests involve upwards flow through a vertical cylindrical pipe, with a constant applied heat flux over the entire pipe. Void fraction measurements are not available for 11 of the 38 cases, so only 27 cases are included. Of these, wall temperature and liquid temperature measurements are available for case 1, in addition to cross sectional void fraction measurements at different axial locations, which are provided for all the 27 cases. 
Table 35: Ranges of operating conditions for the Bartolomei tests

\begin{tabular}{lrrr}
\hline Parameter & Min. value & Max. value & Unit \\
\hline Pressure & 15 & 148 & $\mathrm{bar}$ \\
Mass flux & 405 & 2100 & $\mathrm{~kg} / \mathrm{m}^{2}-\mathrm{s}$ \\
Heat flux & 420 & 2210 & $\mathrm{KW} / \mathrm{m}^{2}$ \\
Inlet subcooling & 11 & 140 & ${ }^{\circ} \mathrm{C}$ \\
\hline
\end{tabular}

The system pressure and inlet liquid flow rate were measured using a manometer and a differential manometerflowmeter. Coolant temperatures at the inlet and outlet of the experimental section were measured using chromel-copel thermocouples. Coolant temperature measurements are also available for Case 1 along the flow axis, as well as radial temperature profile measurements obtained by traversing the thermocouple at several points over the cross section of the flow. The temperature of the outer wall of the tube was also measured using the traversing thermocouples. The mean calometric temperature measurement is used for the Case 1 validation study. Temperature measurements of the external surface of the heated wall tube are also available but not used in the validation study. Heat flux is determined by measuring the electrical power supplied to the heaters. According to the authors, for cases 13-38, the maximum relative errors did not exceed 0.01 for pressure, 0.02 for mass velocity, and 0.03 for heat flux, and the maximum absolute error of temperature did not exceed $1 \mathrm{~K}$. The mean true vapor content (void fraction) was determined by penetrating gamma radiation from a Tu-170 source. Further details on the void fraction measurement, including the calibration process, are given in [28] and [29]. The authors mention that the maximum absolute errors in the void fraction measurements do not exceed 0.04 for cases $13-38$.

\subsection{Thom}

The Thom experimental facility and tests are described in [30]. Seven tests were modeled with the following annulus test section geometry: vertical tube with an inner diameter of $17.78 \mathrm{~mm}$ and an outer diameter of $22.86 \mathrm{~mm}$ and a heated length of $3.66 \mathrm{~m}$. The purpose of this study is to test the implementation of the subcooled wall boiling model of Thom et al. [30], so the simulated tube length is $5.0 \mathrm{~mm}$ using one node. The inlet boundary conditions are set at the subcooled boiling regime, and the CTF wall temperature predictions are compared with data. The range of operating conditions are shown in Table 36 . These tests involve upwards flow with a uniform heat flux applied over the inner tube and the outer tube. The metal thickness was arranged in the test section to obtain nearly equal heat flux on both the inner and outer tubes. Heat flux was determined in the experiments by measuring the electrical power supplied to the heaters. Metal temperatures were measured using thermocouples attached to the outer dry sides of the tubes. The water side metal temperatures were deduced by calculation. The bulk liquid temperatures were measured at the inlet and the outlet to the test section using thermocouples. Liquid temperatures along the test section were estimated assuming a linearly proportional enthalpy gain. The system pressure and mass flux were held constant for all the conditions.

The author does not explicitly state the temperature measurement uncertainty. The temperature is calculated from a one-dimensional heat conduction equation, so uncertainty in material thermal resistance, test voltage, and temperature measurements will contribute to the uncertainty of the values to which CTF is compared. 
Table 36: Ranges of operating conditions for the Thom Tests

\begin{tabular}{lrrr}
\hline Parameter & Min. value & Max. value & Unit \\
\hline Operating pressure & 137.89 & 137.89 & $\mathrm{bar}$ \\
Mass flux & 971 & 1025 & $\mathrm{~kg} / \mathrm{m}^{2}-\mathrm{s}$ \\
Heat flux & 200 & 1400 & $\mathrm{KW} / \mathrm{m}^{2}$ \\
\hline
\end{tabular}

\subsection{WALT}

In 2005, a single-rod thermal hydraulic facility was constructed at the Westinghouse Science and Technology Center in Pennsylvania. This loop has been named Westinghouse advanced loop tester (WALT) and was designed to simulate PWR crud buildup [31]. Water flows through an 18.3 in long annulus with an approximate flow area of $0.6 \mathrm{~m}^{2}$. A ZIRLO heated tube is equipped with four thermocouples located at different azimuthal angles at the same axial location (approximately $10 \mathrm{in}$ from the inlet). The thermocouples measure the inside tube temperature, and a simple heat conduction solution is used to approximate the outside tube temperature.

Though most of the WALT tests were used to create simulated crud and measure its thermal conductivity, a series of clean rod tests was initially performed. These tests are used as a separate effects validation study for CTF. In these tests, the heat flux was sequentially raised, and wall temperature measurements were taken after the system was allowed to reach steady state. This allows the construction of a so-called boiling curve, which can be compared to theory.

Uncertainty of experimental measurements was not stated by the author [31].

\subsection{Rohsenow}

In 1951, Rohsenow published the results of a subcooled boiling experiment [32]. He executed his tests using a 0.1805 in diameter nickel tube with a length of 9.4 in. A copper shield surrounded the tube and was used to heat it. Thermocouples measured the temperature at seven axial locations on the outside of the shield, though the first and last axial locations are not reported due to entrance and exit effects. Rohsenow estimated the inside tube temperature from the thermocouple measurements using a Taylor series, resulting in an estimated error bound of $\pm 3.0 \mathrm{~F}$.

Distilled and degassed water was passed through the heated tube. Inlet and outlet coolant temperatures were measured, and pressure was measured at the inlet. Steady-state measurements were taken at two pressures, three mass fluxes, and a variety of heat fluxes. Therefore, each experiment measured four axial locations in the tube which all had similar conditions but different liquid subcooling. Rohsenow used these results to create a variety of plots and conclusions, which were later incorporated into textbooks [33, 34]. 


\section{Heat Transfer}

When discussing the heat transfer models in the code, what we really care about is the accuracy in the calculation of the Heat Transfer Coefficient (HTC), $h$, that is used in Newton's Law of cooling:

$$
q^{\prime \prime}=h\left(T_{w}-T_{b}\right)
$$

where $q^{\prime \prime}$ is the heat flux from the wall to the fluid, $T_{w}$ is the temperature of the wall surface, and $T_{b}$ is the temperature of the bulk fluid (in the case of a subchannel code, this is the control volume temperature of the fluid).

It is not possible to calculate $h$ directly in experiment but, rather, it must be back-calculated from the experimental measurements of $T_{w}, T_{b}$, and the known rod power of $q^{\prime \prime}$. Since there are difficulties in obtaining values of $T_{b}$ from experiment for the rod-bundle geometry that we are concerned with (associated with holding a thermocouple in a given position of the flow channel and also its impact on the fluid behavior), $T_{b}$ values are typically not available. The $T_{w}$ measurements are much more easily available as the experimenter can insert a thermocouple in the inside of the heater tube, where there is no flow, fix it to the wall, and then post-process the data by solving the 1-D conduction equation for the tube thickness in order to yield the outer tube surface temperature.

In order to actually obtain the experimental value of $h$, this then leaves us with using the subchannel code to estimate values of $T_{b}$ at the $T_{w}$ measurement location so that we can solve Equation 4.1. Of course, this then raises the question of what is the validity of the estimates of $T_{b}$. Rather than introduce this uncertainty into the analysis, we instead directly compare CTF estimates of $T_{w}$ to experimental results. Since the value of the measured wall temperature will be directly related to the magnitude of the convective and boiling heat transfer in the experiment, this is a suitable check on the effectiveness of the employed heat transfer models in CTF. 


\subsection{Validation}

\subsubsection{CE $5 \times 5$}

The CE $5 \times 5$ tests involved running more than 70 tests of varying operating conditions on a $5 \times 5$ electrically heated rod bundle facility. The operating conditions were varied such that heat transfer mechanisms in the bundle ranged from single-phase convection to saturated boiling. Instrumentation included thermocouples fixed to the interior of two of the heater rods.

In Rod 25, the central heater rod, 14 thermocouples were placed at 4 different levels: $76.63,81.00,82.50$, and 83.50 inches from BOHL. One set of thermocouples sat below a mixing vane grid and the other three sets were above the grid. The set below the grid included two thermocouples placed at two different azimuthal locations. The three sets above the grid each included 4 thermocouples placed at 4 azimuthal locations. In Rod 24, the adjacent rod to the west of Rod 25, 4 thermocouples were placed above the grid.

Using the temperature for a given thermocouple over all the testing conditions clearly reveals the incipience of boiling at that position in the bundle with a leveling-off of the temperature profile with respect to bundle heat flux. Figure 4.1 shows this axial profile along with the difference between CTF rod surface temperature predictions and the experimental results. This figure also shows another important thing, which is the existence of outliers in the data. Like for the exit channel temperatures, outliers were eliminated from the data set before doing any data analysis. Again, a discrepancy of greater than $50 \mathrm{C}$ resulted in the data set being removed.

After eliminating outliers, some data reduction was done to generate a more meaningful overview of the predictive capabilities of CTF. First, the merit of interest is the difference between predicted and measured rod surface temperatures. Second, the mean of this discrepancy was calculated for each thermocouple for all tests that were run, leaving us with one mean discrepancy per thermocouple (20 in total). These discrepancies are presented in two figures - one figure for each of the two instrumented rods. Rod 25 results are given in Figure 4.2 and Rod 24 results are given in Figure 4.4 .

In the figures, the $\mathrm{x}$-axis shows the axial location of the thermocouple. The thermocouples, as placed in the figure, are not exactly accurate to their placement in experiment. The red thermocouple (Position 1) is placed at the actual location in the experiment. The following three thermocouples are shifted over to the right slightly to aid in viewing the data. In reality, all thermocouples for a given axial location are at exactly the same axial position in the bundle. The y-axis is the difference between predicted and experimental rod surface temperatures. The two vertical blue lines represent the bottom and the top of the spacer grid. Finally, the error bars do not represent the scatter in the discrepancies for each mean discrepancy. Rather, they represent the $2 \sigma$ scatter of the experimental measurements during the calibration phase of the experiment. For example, in Figure 4.2, the blue thermocouple before the grid has a scatter in measurements of over $5 \mathrm{C}$. The mean discrepancy between predicted and experimental data for that thermocouple is nearly 0 C. Considering the scatter in the thermocouple readings, the actual discrepancy may be anywhere between -4 and $4 \mathrm{C}$.

Note that the grid heat transfer enhancement model was not enabled for these tests. We do see a significant degree of azimuthal variation in the rod surface temperatures, which we should expect given that the test section uses Mixing Vane Grid (MVG)s (see Figure 4.3). It is important to note that CTF has no mixing vane grid models to account for these types of effects. However, some of this scatter must also be related 


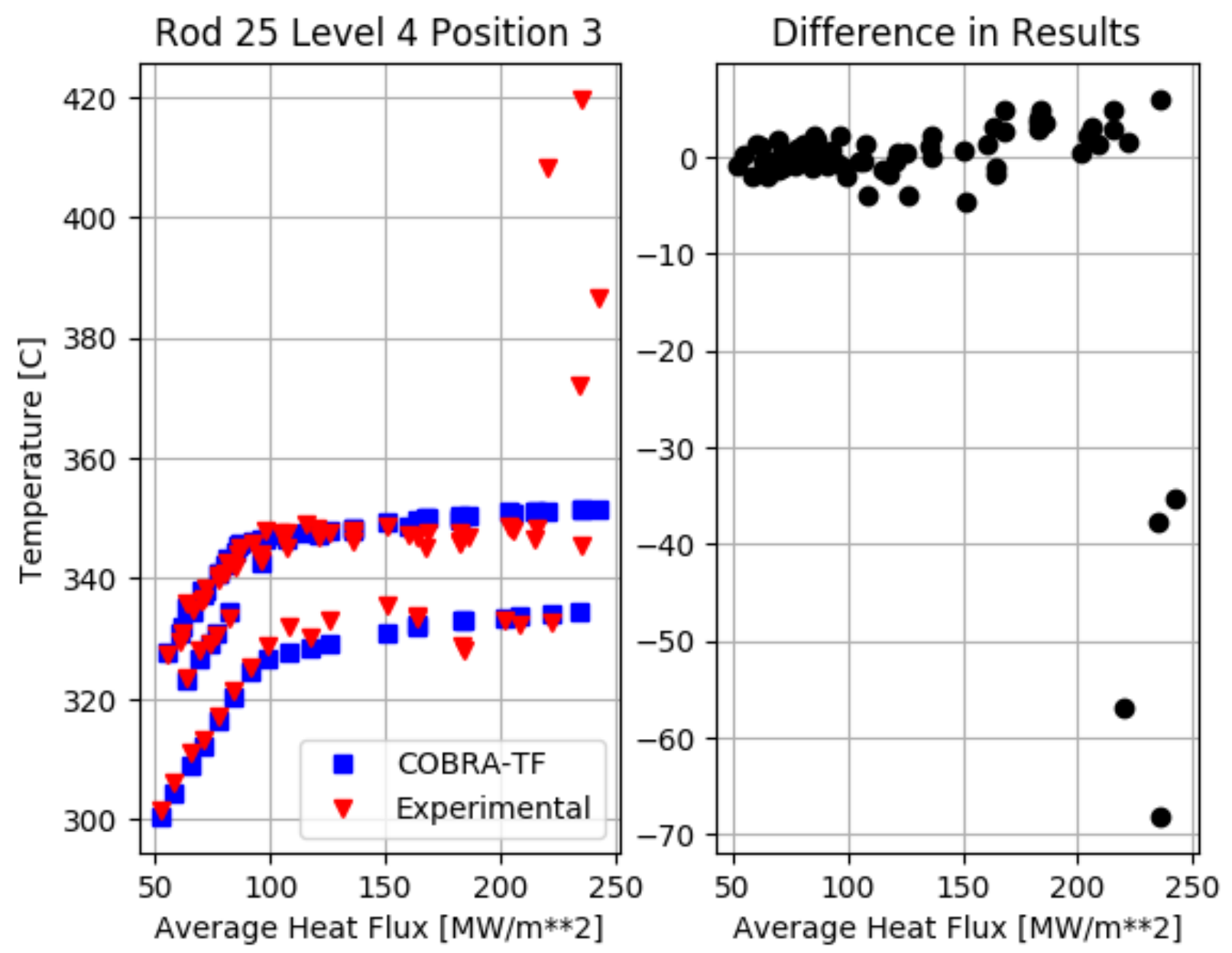

Figure 4.1: Rod surface measurements and predictions with respect to increasing test heat flux for Rod 25 (central rod) at 77.63 inch axial location 


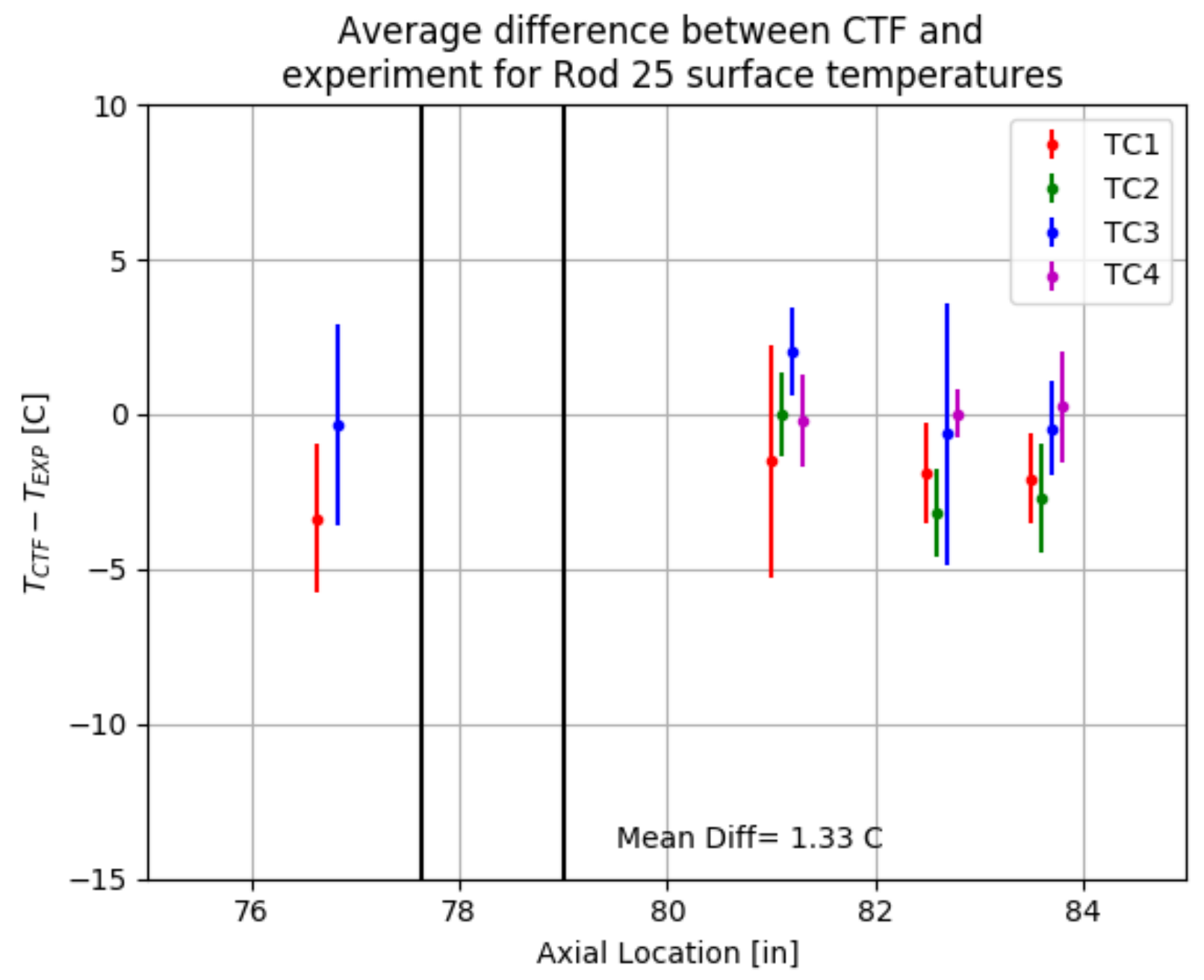

Figure 4.2: Summary of mean difference between predicted and measured rod surface temperatures for each thermocouple in Rod 25 over all test cases 
to uncertainty in the experimental measurements, as the level prior to the grid sees a substantial azimuthal variation. Also, we see that, in many cases, the $2 \sigma$ scatter in experimental measurements is enough to account for this azimuthal variation. 




Figure 4.3: CE 5x5 Test 74 Mixing Vane Grid 


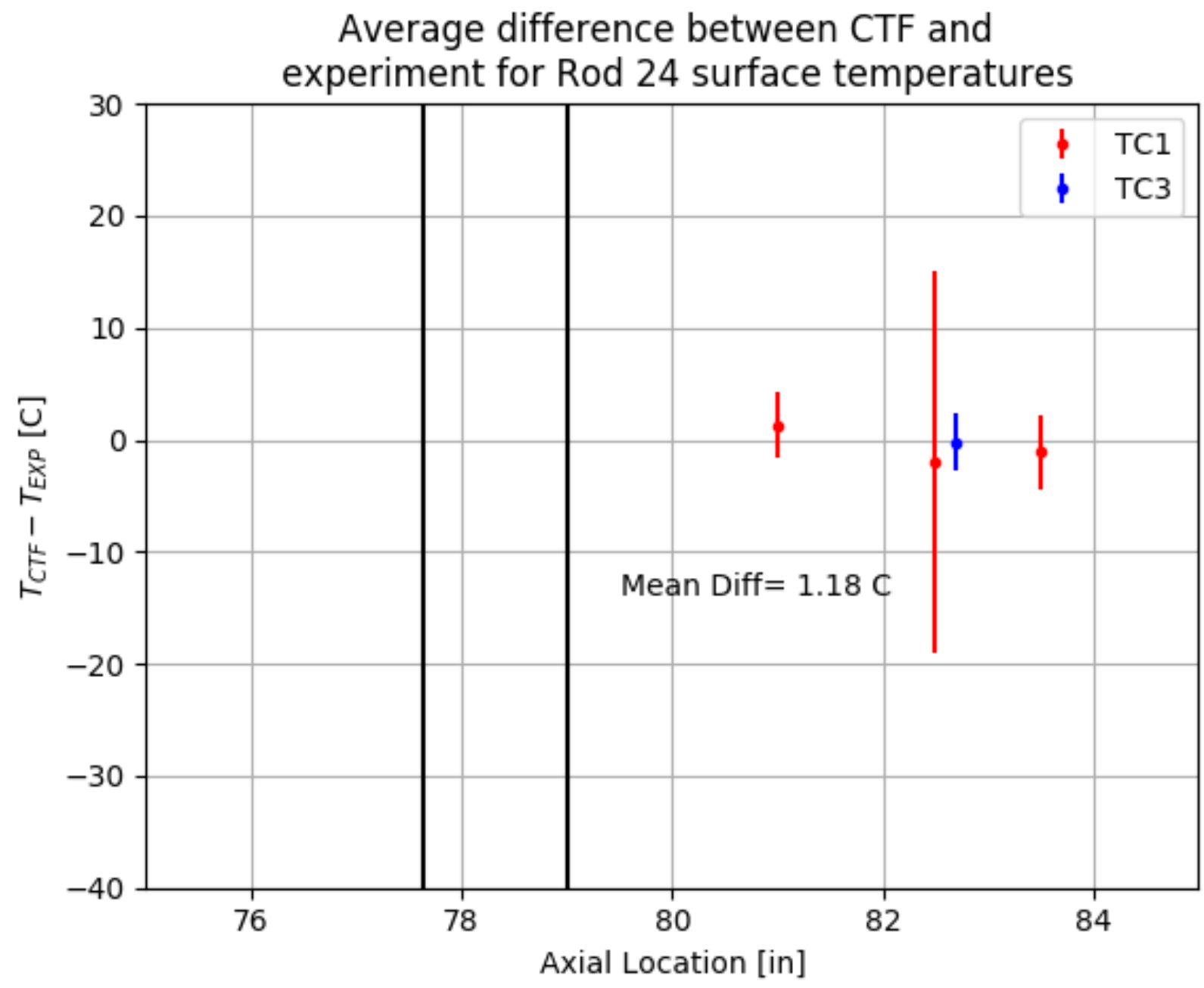

Figure 4.4: Summary of mean difference between predicted and measured rod surface temperatures for each thermocouple in Rod 24 over all test cases 


\subsubsection{WALT Loop}

The WALT report [31] is used to perform a separate effects validation study for CTF heat transfer in the subcooled boiling regime.

The original report does not provide the necessary wall temperatures, so they are approximated. Since the facility and the code input are completely symmetric, the thermocouple results are expected to be approximately the same. Therefore, the average of the four thermocouple measurements is used to calculate the surface temperature. This temperature is approximated as [31, Equation A-7]

$$
T_{\text {out }}=T_{\text {in }}-\frac{q^{\prime \prime \prime}}{4 k}\left[2 r_{\text {in }}^{2} \ln \left(\frac{r_{\text {in }}}{r_{\text {out }}}-r_{\text {in }}^{2}+r_{\text {out }}^{2}\right)\right]
$$

The thermal conductivity of ZIRLO is approximated using a linear function of temperature. (Note that this equation is in British units, the temperature is in Fahrenheit, and the thermal conductivity is in BTU/hr/ft/F [31, Equation 5-11].

$$
k=8.1802+0.0026 T \text {. }
$$

This facility is modeled in CTF using a single rod-centered channel with twenty axial nodes. The Thom correlation is used to determine the boiling heat transfer coefficient. The flow area, wetted perimeter, and rod diameter are set to be consistent with the geometry of the facility. The measured pressure is used as the outlet pressure boundary condition, and the inlet boundary conditions are determined by the experimentally reported inlet temperature and mass flux. For each run, the wall temperature distribution is extracted from the code output. Linear interpolation is used to approximate the wall temperature at the thermocouple location, which is then compared to the value estimated by Eq. 4.2 .

The differences between CTF and experimentally calculated wall temperatures are shown in Figure 4.5. A few cases have very large overpredictions of wall temperature. These cases correspond to case 110a and 111b, which are the only two cases with inlet temperatures that are lower than those in other cases. The overall rms! (rms!) over all cases is $2.893^{\circ} \mathrm{C}$. 


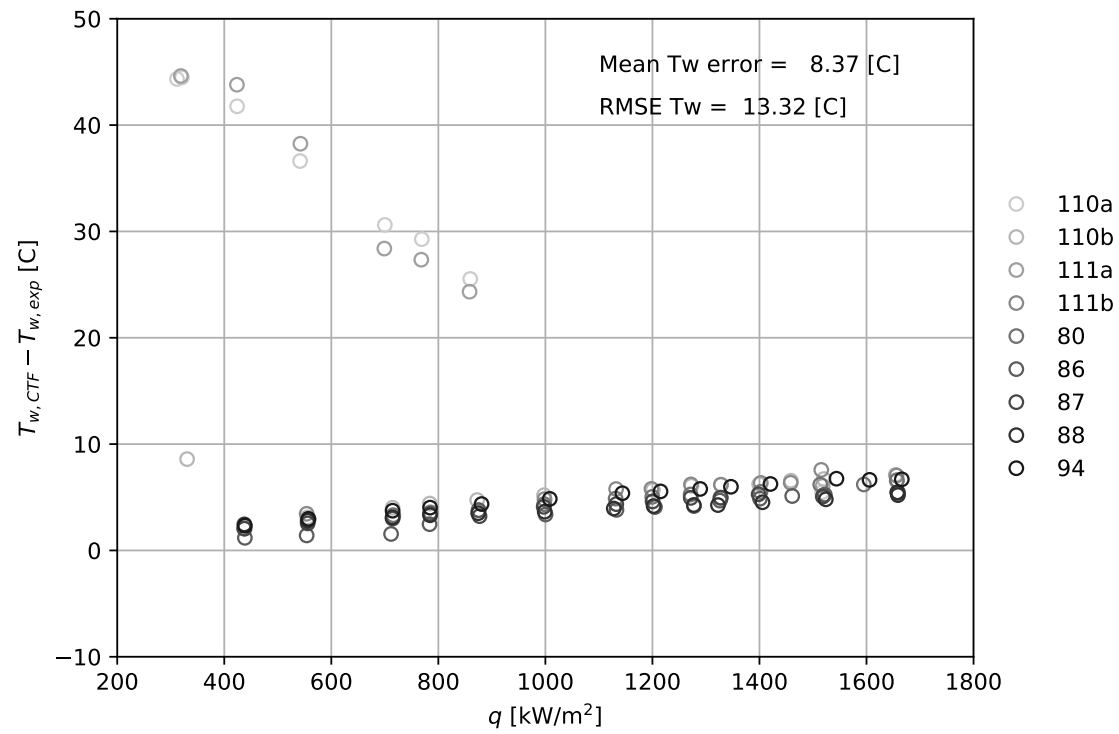

Figure 4.5: WALT validation results as a function of heat flux.

\subsubsection{Thom}

CTF Model Experimental data used for this study includes seven conditions from an annulus geometry test section. A single axial node is used in the simulation with a nodal size of $5.0 \mathrm{~mm}$, which is roughly equal to the hydraulic diameter. The CTF models are created using internal flow in a single channel, with the mass flux and liquid temperature set to match the experimental subcooling for all the datapoints which lie on or close to the subcooled boiling regime. The system pressure is used for the outlet boundary condition. Uniform heat flux is provided at the outer and inner heater wall surface, with the linear heat rate calculated to correspond to the experimental heat flux at the respective surfaces, which are equal.

Results of predicted and experimental delta wall temperatures are plotted in terms of liquid subcooling in Figure 4.6. Additionally, a comparison of predicted and experimental delta wall temperatures is shown in 4.7 , with bounds of $\pm 5.0 \mathrm{~F}$ applied as a reference point. It must be noted that the CTF results are generated using the subcooled wall boiling model of Thom et al. [30]. Since the authors correlated their model to the experimental dataset, CTF predictions are expected to be within model uncertainty. The wall temperatures are overpredicted for a couple of datatpoints and underpredicted for one datapoint. The overprediction occurs close to the transition point to the subcooled boiling regime, and a more accurate solution might be obtained by simulating the entire test section. In summary, it can be concluded that the wall boiling model of Thom et al. [30] is implemented as intended in CTF. 


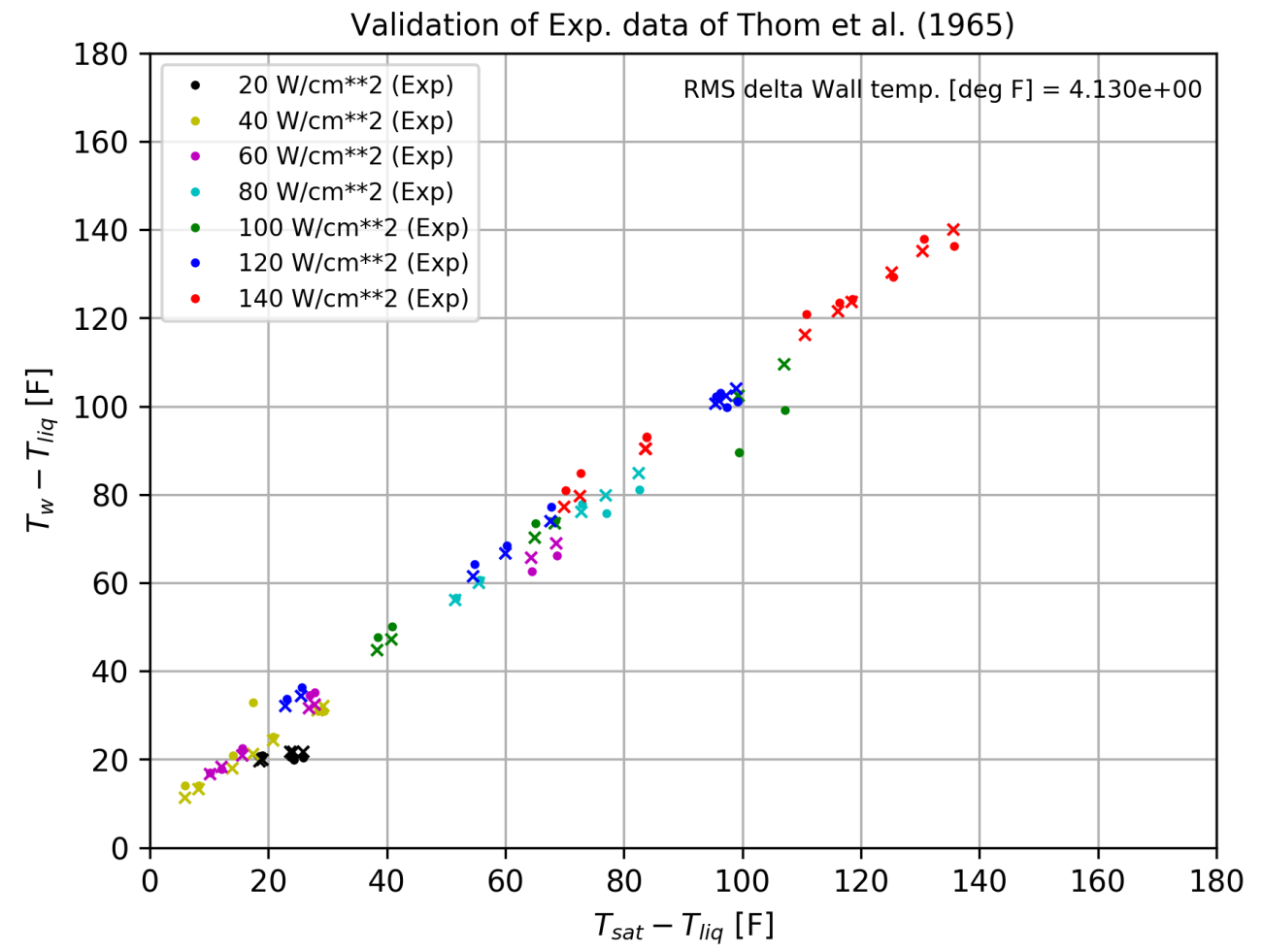

Figure 4.6: Comparison of predicted and experimental data of Thom for the temperature difference between the outer wall and the bulk liquid vs. liquid subcooling. 


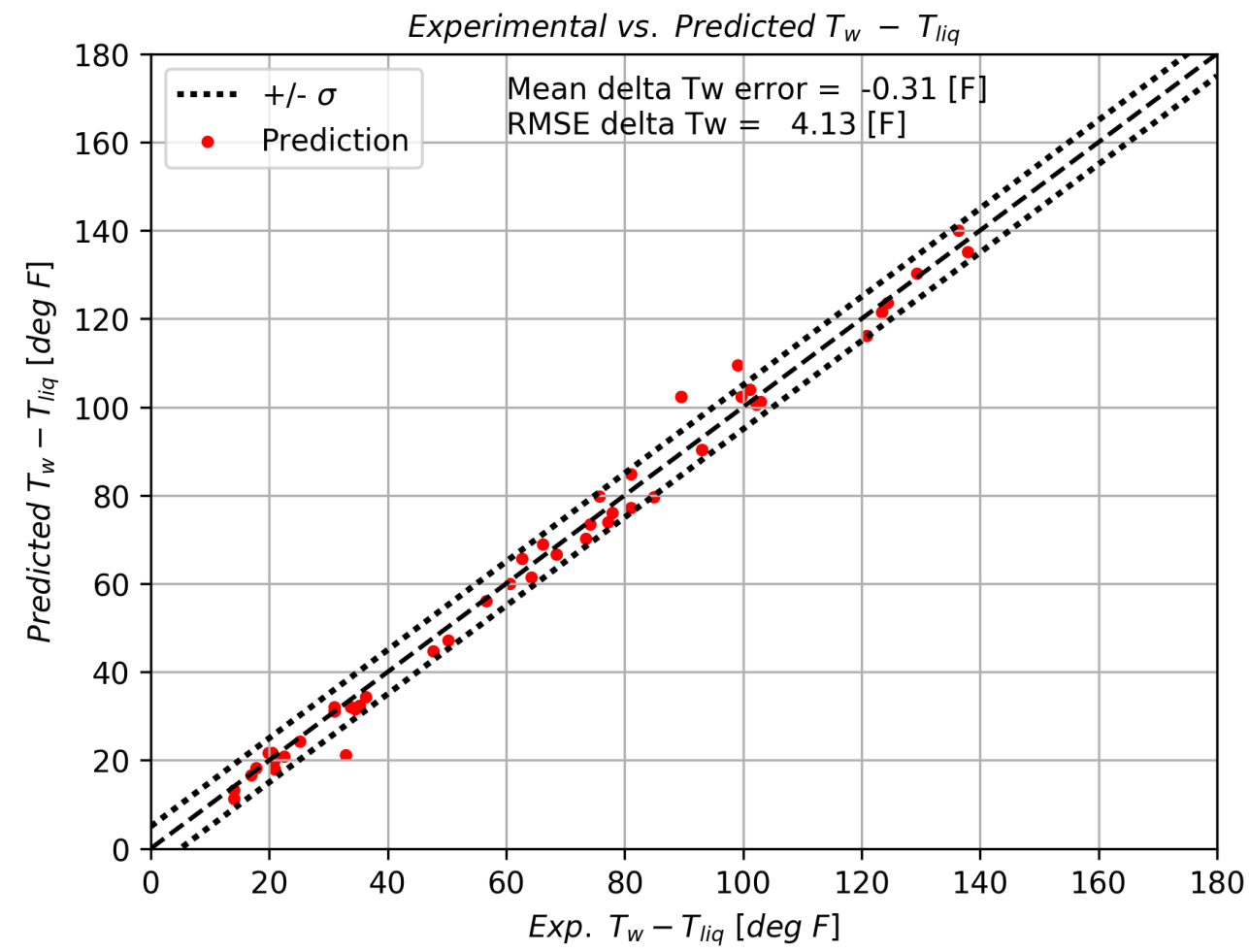

Figure 4.7: Comparison of predicted vs. experimental data of Thom for the temperature difference between the outer wall and the bulk liquid. 


\subsubsection{Rohsenow}

The Rohsenow experiment [32] is used as a separate-effects validation study for subcooled boiling.

This facility is modeled in CTF using a single rod-centered channel with twenty axial nodes. The flow area, wetted perimeter, and rod surface area are set consistently with the tube geometry of the Rohsenow experiment. The approximate pressure reported by Rohsenow is set to the outlet pressure boundary condition; the inlet boundary conditions are determined from the experimentally reported inlet temperature and mass flux. The entire wall temperature distribution is extracted from the output, and linear interpolation is used to approximate the wall temperature at each thermocouple location.

The difference between CTF and experimentally reported wall temperatures is shown in Figure 4.8. The overall RMS over all cases is $0.9445^{\circ}{ }^{\circ} \mathrm{C}$.

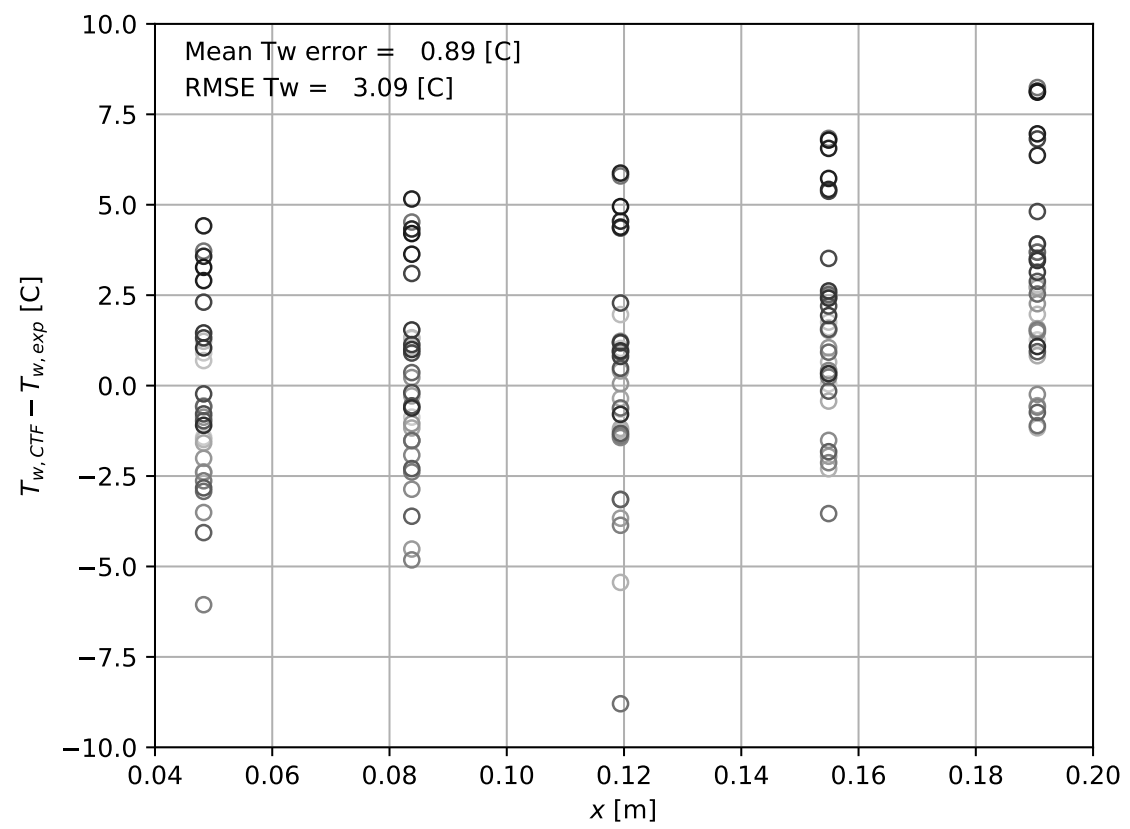

Figure 4.8: Rohsenow validation results as a function of spatial location.

\subsection{Verification}

\subsubsection{Heat Exchanger}

Problem Description This problem verifies the correct functioning of the unheated conductor component that is connected to the ambient. The problem involves a single channel inside of a tube-shaped unheated conductor. The channel contains water flowing upwards. The outside of the tube is a HTC/temperature boundary condition. The temperature of the inlet flow is set to a higher value than the ambient temperature, 


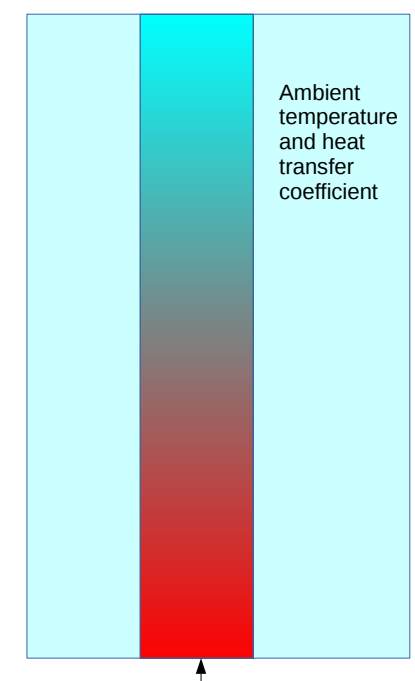

Flow and Temperature

Figure 4.9: Diagram of the heat exchanger verificaiton problem

which causes heat transfer out of the tube as the flow moves upwards. A diagram of this problem is shown in Figure 4.9. It is possible to use First Law and Newton's Law of Cooling to determine the expected temperature drop in the tube.

The boundary conditions in this model include:

1. Inlet temperature of $150^{\circ}{ }^{\circ} \mathrm{C}$

2. Outlet pressure of $10 \mathrm{bar}$

3. Ambient temperature of $100^{\circ}{ }^{\circ} \mathrm{C}$

4. Ambient HTC of $100 \mathrm{~kW} \mathrm{~m}^{-2} \mathrm{~K}^{-1}$

5. Inlet flow rate of $5 \mathrm{~kg} \mathrm{~s}^{-1}$

The tube is $1 \mathrm{~m}$ long and has a diameter of $5 \mathrm{~cm}$. Some assumptions include:

1. Constant specific heat of $4.25 \mathrm{~kJ} \mathrm{~kg}^{-1}$

2. Infinitely thin tube so that conduction through the tube is negligible

3. Infinite HTC between the in-tube fluid and the tube 
The fact that in-tube HTC is infinite and that the tube wall is infinitely thin means that heat transfer is essentially directly from the in-tube flow to the ambient.

The analytical solution is formed by using First Law for an infinitesimal section of the tube.

$$
q^{\prime}=\dot{m} C_{p} \frac{d T}{d x}
$$

Here, $q^{\prime}$ is the linear heat rate, $\dot{m}$ is the mass flow rate in the tube, $C_{p}$ is the specific heat of the coolant, $T$ is temperature, and $x$ is the axial direction in the tube. The heat flux out of the tube at this location is given by Newton's Law of Cooling.

$$
q^{\prime}=P_{w} h_{\infty}\left(T_{\infty}-T\right)
$$

Here, $P_{w}$ is the wetted perimeter of the tube, $h_{\infty}$ is the HTC on the outside of the tube, and $T_{\infty}$ is the ambient temperature. Substituting Equation 4.5 into Equation 4.4 gives us a first-order, linear, ordinary differential equation.

$$
\dot{m} C_{p} \frac{d T}{d x}+h_{\infty} P_{w} T=h_{\infty} T_{\infty} P_{w}
$$

The solution to this equation is:

$$
T=T_{\infty}+\left(T_{\text {in }}-T_{\infty}\right) \exp \left(\frac{h_{\infty} P_{w}}{\dot{m} C_{p}} x\right)
$$

CTF Model Description An axial mesh of $0.01 \mathrm{~m}$ is employed. The tube outside diameter is set to $5.0 \mathrm{~cm}$ and the inside diameter is set to $4.9999 \mathrm{~cm}$ to simulate the tube being infinitely thin. The leading coefficient of the Dittus-Boelter model is set to 1,000 to simulate an infinite HTC.

Discussion of Results A Python script was created for parsing the output of the CTF solution, calculating the analytical solution, and generating a plot comparing the two solutions. Figure 4.10 shows that the comparison between CTF and the analytical solution is good.

\subsubsection{Zero-dimensional convection}

In this problem, heat transfer takes place between a solid and a single phase fluid. The following assumptions are made here: (1) there is only one solid control volume (achieved by setting $k>>0$ in a rod with two control volumes), (2) there is a single fluid control volume (achieved by setting zero inflow and outflow boundary conditions), (3) the fluid and solid are both stationary, (4) all properties are constant, (5) the

heat transfer coefficient is constant, and (6) there is no heat generation. Under these conditions, the coupled ordinary differential equations to be solved are: 


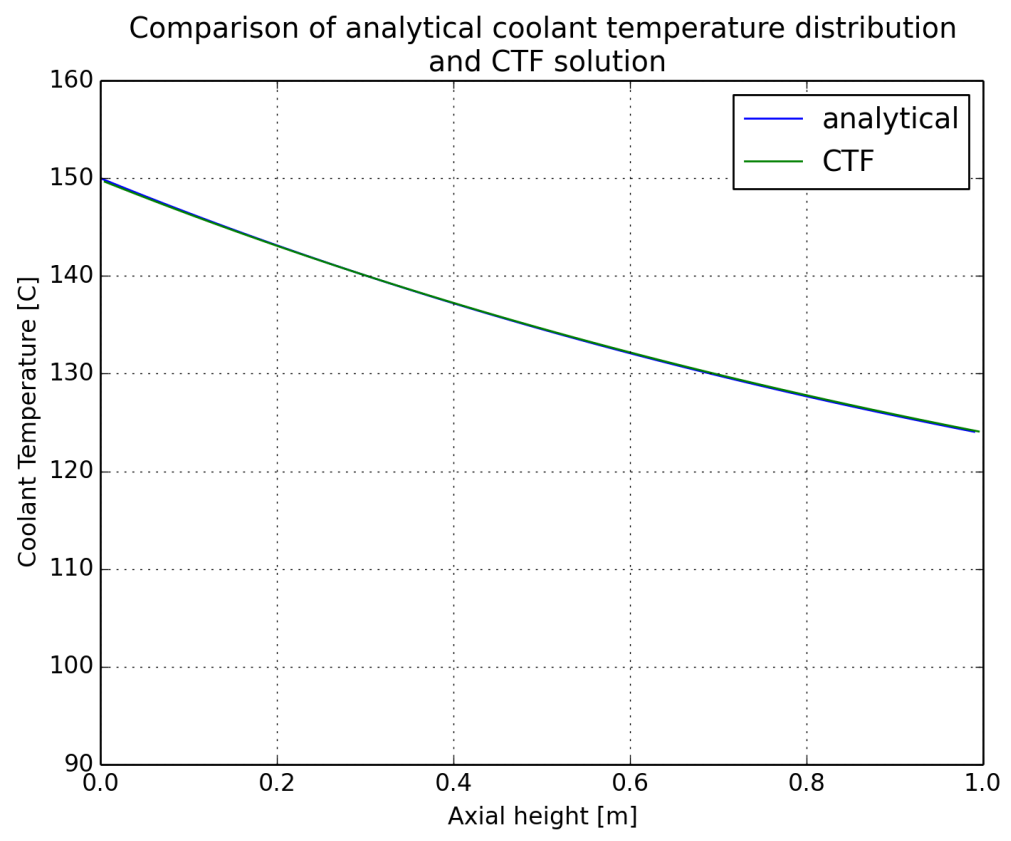

Figure 4.10: Comparison of the analytical and CTF solution for flow through a heat exchanger

$$
\begin{gathered}
V_{f} \rho_{f} c_{p f} \frac{\partial T_{f}}{\partial t}=h A\left(T_{s}-T_{f}\right) \text { and } \\
V_{s} \rho_{s} c_{p s} \frac{\partial T_{s}}{\partial t}=h A\left(T_{f}-T_{s}\right) .
\end{gathered}
$$

If the initial conditions $T_{f}(0)=T_{f 0}$ and $T_{w}(0)=T_{w 0}$ are applied, then the solutions $T_{f}$ and $T_{w}$ can be found as a function of time. To simplify the analytic solution, the thermal capacitance of $k$ as $C_{k}=V_{k} \rho_{k} c_{p k}$ is defined. Under these conditions, the analytic solution is relatively simple.

$$
\begin{aligned}
& T_{f}=\frac{1}{C_{f}+C_{s}}\left[C_{f} T_{f 0}+C_{s} T_{s 0}+\left(T_{f 0}-T_{s 0}\right) C_{s} \exp \left(-\frac{h A\left(C_{f}+C_{s}\right)}{C_{f} C_{s}} t\right)\right], \\
& T_{s}=\frac{1}{C_{f}+C_{s}}\left[C_{f} T_{f 0}+C_{s} T_{s 0}-\left(T_{f 0}-T_{s 0}\right) C_{f} \exp \left(-\frac{h A\left(C_{f}+C_{s}\right)}{C_{f} C_{s}} t\right)\right] .
\end{aligned}
$$

The parameters for this problem are defined in Table 37. Note that all quantities in Eqs. (4.10) and (4.11) can be derived from the parameters in the table. For example, the heat transfer coefficient is related to the Nusselt number via $N u=h D / k_{f}$.

The results are shown in Figure 4.11, and convergence is shown in Figure 4.12. The thermal capacitance of the solid is larger than that of the fluid, so the fluid temperature changes more over the transient. The 
Table 37: Parameters for convection verification problem

\begin{tabular}{lccc}
\hline Parameter & Symbol & Value & Units \\
\hline Initial fluid temperature & $T_{f 0}$ & 140 & ${ }^{\circ} \mathrm{C}$ \\
Initial solid temperature & $T_{s 0}$ & 160 & ${ }^{\circ} \mathrm{C}$ \\
Fluid density & $\rho_{f}$ & 900 & $\mathrm{~kg} / \mathrm{m}^{3}$ \\
Solid density & $\rho_{s}$ & 900 & $\mathrm{~kg} / \mathrm{m}^{3}$ \\
Fluid specific heat & $c_{p f}$ & 4.25 & $\mathrm{~kJ} / \mathrm{kg} / \mathrm{K}$ \\
Solid specific heat & $c_{p s}$ & 4.25 & $\mathrm{~kJ} / \mathrm{kg} / \mathrm{K}$ \\
Fluid thermal conductivity & $k_{f}$ & 1 & $\mathrm{~W} / \mathrm{m} / \mathrm{K}$ \\
Nusselt number & $N u$ & 10 & - \\
Control volume height & $\Delta x$ & 0.1 & $\mathrm{~m}$ \\
Flow area & $A_{f}$ & 0.0001 & $\mathrm{~m}^{3}$ \\
Wetted perimeter & $P_{w}$ & 0.04 & $\mathrm{~m}$ \\
Rod diameter & $D_{r}$ & 0.05 & $\mathrm{~m}$ \\
Surface area & $A$ & $3.142 \times 10^{-3}$ & $\mathrm{~m}^{2}$ \\
\hline
\end{tabular}

point-wise error is shown, and all spatial points appear to display first-order convergence. The error between the code solution and the analytic solution converges at approximately $p=1.06$ for both the solid and fluid solutions. Therefore, the code displays the expected first-order convergence for the solid-to-liquid coupling.

\subsubsection{Pipe boiling}

Once water is saturated, additional heat contributes to the transition of saturated water to saturated steam (latent heat). It is important to thoroughly test this phenomenon to ensure that energy is conserved, even in a two-phase mixture. This problem verifies that latent heat properly conserves energy and does not degrade the expected order of accuracy.

Problem Description Saturated water enters a pipe at the inlet, and heat is added directly to the fluid as it flows. The water is initially saturated, so all energy is added as latent heat, which increases the amount of steam.

First, the following assumptions are made to allow the CTF energy equations to be approximated using mixture equations: (1) there is not a significant amount of droplets, and (2) the liquid and steam are in mechanical and thermal equilibrium. Under these conditions, the mixture energy equation is solved as follows.

$$
\frac{\partial \rho_{m} h_{m}}{\partial t}+\frac{\partial \rho_{m} u_{m} h_{m}}{\partial x}=\dot{q}+\alpha \frac{\partial P}{\partial t}
$$

The following assumptions further simplify the solved equation: (1) the heat flux is small enough that $P$, $\rho_{m}$, and $u_{m}$ are constant throughout the domain, and (2) the heat flux is constant in time and has a cosine shape in space. 


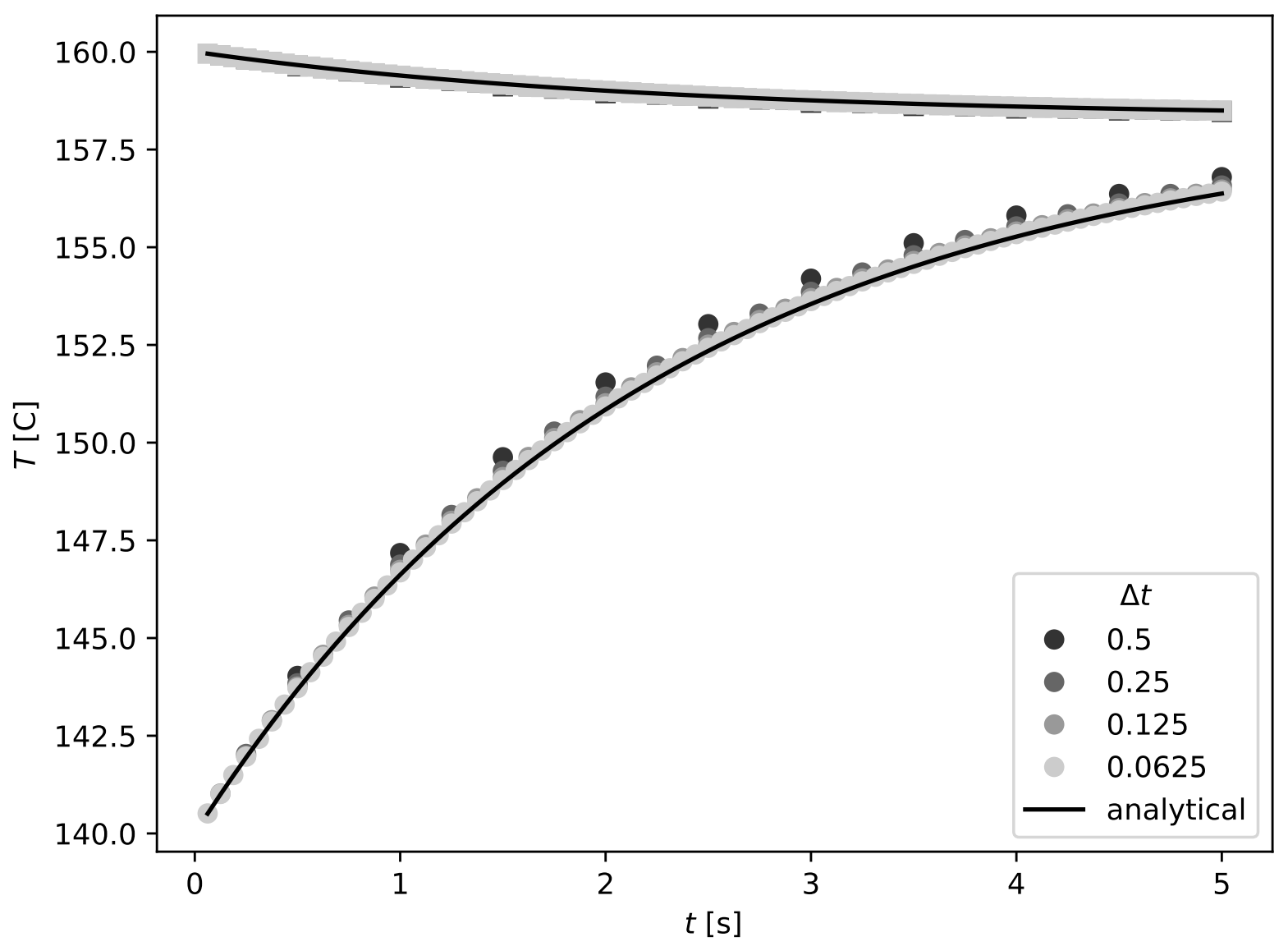

Figure 4.11: Zero dimensional convection temperature vs. time. 


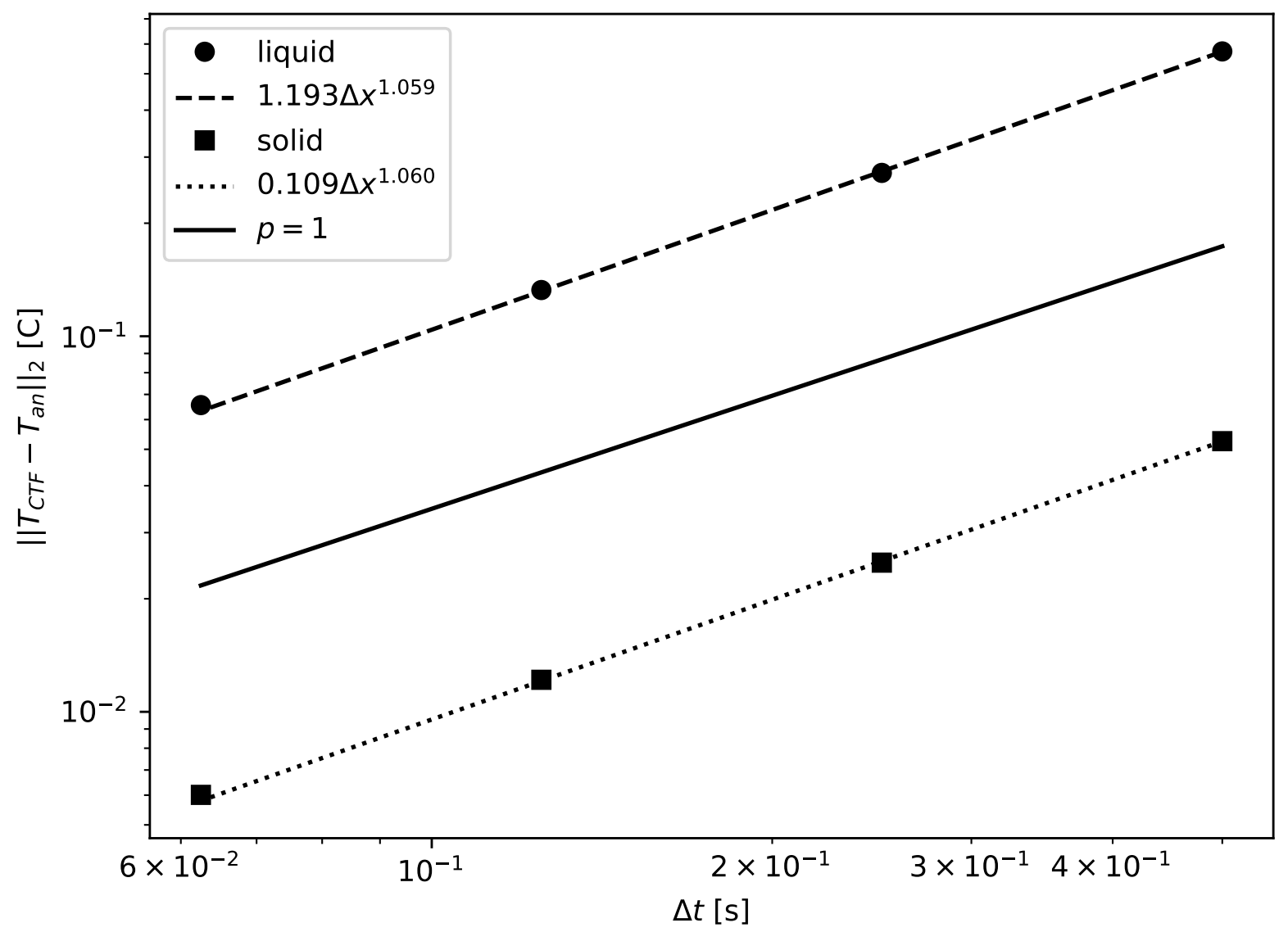

Figure 4.12: Convergence for zero dimensional convection problem. 


$$
\rho_{m} \frac{\partial h_{m}}{\partial t}+\rho_{m} u_{m} \frac{\partial h_{m}}{\partial x}=\frac{q_{o}^{\prime}}{A_{f}} \sin \left(\pi \frac{x}{L}\right)
$$

The inlet energy boundary condition is set so that the flow is fully saturated water: $h(x=0)=h_{o}$. The initial condition sets the entire domain to the same enthalpy: $h(t=0)=h_{o}$. Under these conditions, the analytic solution for enthalpy as a function of time and space can be found.

$$
h(x, t)=h_{o}+\frac{q_{o}^{\prime} L}{\dot{m} \pi}\left[\cos \left(\pi \frac{u t-x}{L}\right)-\cos \left(\pi \frac{x}{L}\right)+2 H[u t-x] \sin ^{2}\left(\pi \frac{u t-x}{2 L}\right)\right] .
$$

Here, $H[\phi]$ indicates a heavyside function:

$$
H[\phi]=\left\{\begin{array}{ll}
0, & \phi<0 \\
1, & \phi>0
\end{array} .\right.
$$

All parameters necessary to fully define this problem are shown in Table 38. Note that the inlet/initial enthalpies are calculated using a direct call to CTF property tables.

Table 38: Parameters for pipe boiling verification problem

\begin{tabular}{lccc}
\hline Parameter & Symbol & Value & Units \\
\hline Flow area & $A$ & 0.0001 & $\mathrm{~m}^{2}$ \\
Pipe length & $L$ & 1 & $\mathrm{~m}$ \\
Inlet mass flow & $\dot{m}_{\text {in }}$ & 0.1 & $\mathrm{~kg} / \mathrm{s}$ \\
Inlet enthalpy & $h_{o}$ & 1630.554 & $\mathrm{~kJ} / \mathrm{kg}$ \\
Outlet pressure & $P_{\text {out }}$ & 155 & $\mathrm{bar}$ \\
Initial enthalpy & $h_{o}$ & 1630.554 & $\mathrm{~kJ} / \mathrm{kg}$ \\
Maximum heat rate & $q_{o}^{\prime}$ & 10.0 & $\mathrm{~kW} / \mathrm{m}$ \\
\hline
\end{tabular}

CTF Model Description Four details should be discussed about the modeling of this problem in CTF.

1. The wall heat flux is deposited directly into the fluid using the DHFRAC input in Card 1.2. This bypasses the conduction solution and calculation of the heat transfer coefficient.

2. The cosine shape of the wall heat flux is achieved using a table in Card 11.4. It has 13 digits of accuracy and is defined at each grid location used in the verification study.

3. Wall friction is disabled using vuq-param.txt, and gravity is disabled using vuq_mult.txt.

4. Interfacial friction is many orders of magnitude larger than usual, which approximates mechanical equilibrium between the two phases. 


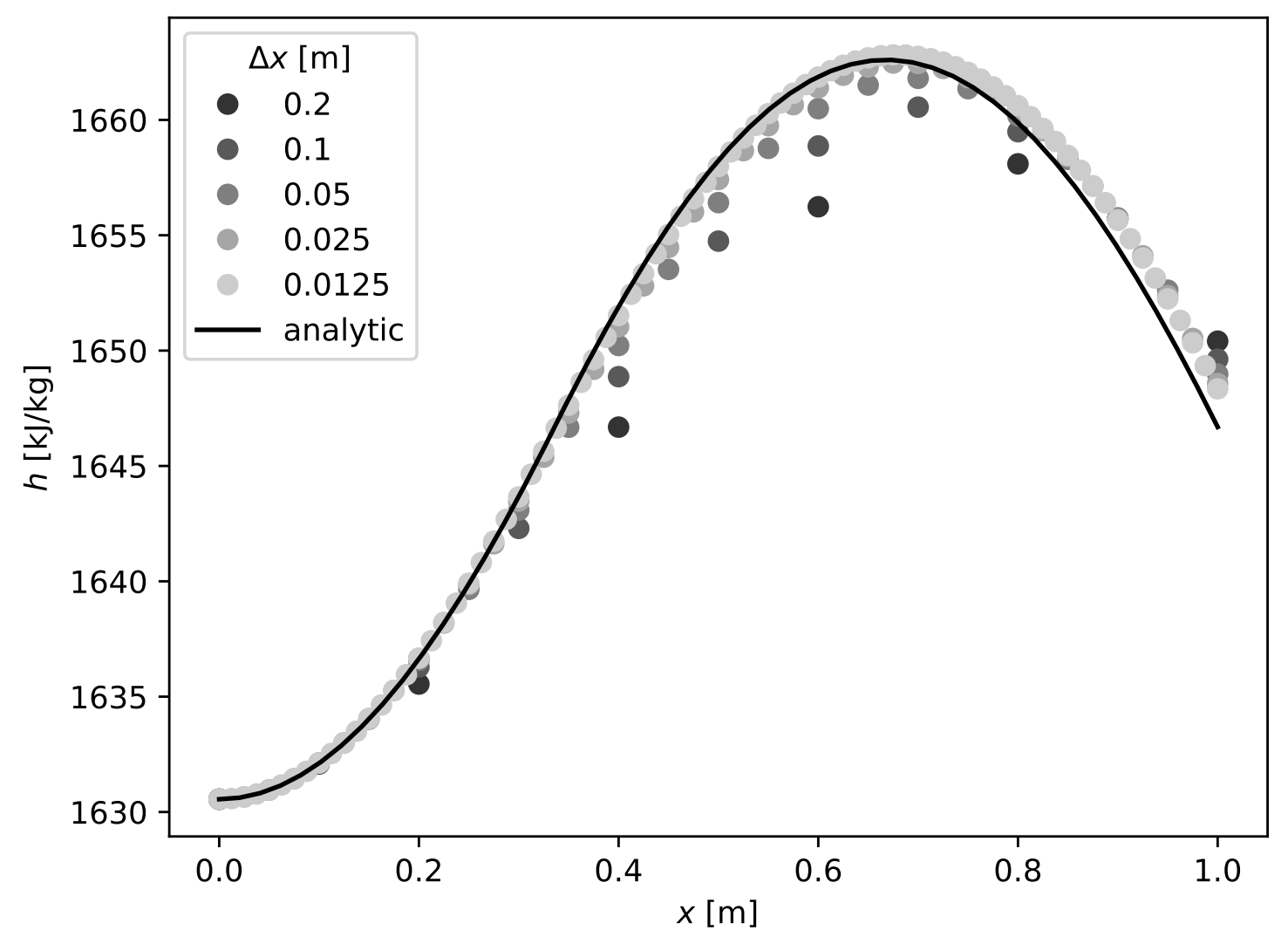

Figure 4.13: Results for the pipe boiling verification problem

Even so, the analytic solution uses HEM assumptions to find the enthalpy distribution in the pipe. This is not quite equivalent to the CTF two-fluid model used for this problem. Therefore, a small bias caused by these model mismatches is expected.

Results The CTF results are shown in Figure 4.13 and Figure 4.14. The enthalpy distribution at $t=0.2 \mathrm{~s}$ is shown for different choices of mesh. It appears that the solution is approaching the analytic solution, but with a small bias. This is confirmed in the convergence plot, which indicates that the CTF solution is approaching a constant error as the mesh is refined. To account for this in the calculation of the observed order of accuracy, a constant error model is used: $\varepsilon=\varepsilon_{o}+c h^{p}$. Using this error model, the order of accuracy is approximately $p=0.96$. This is close enough to unity to be considered first order. 


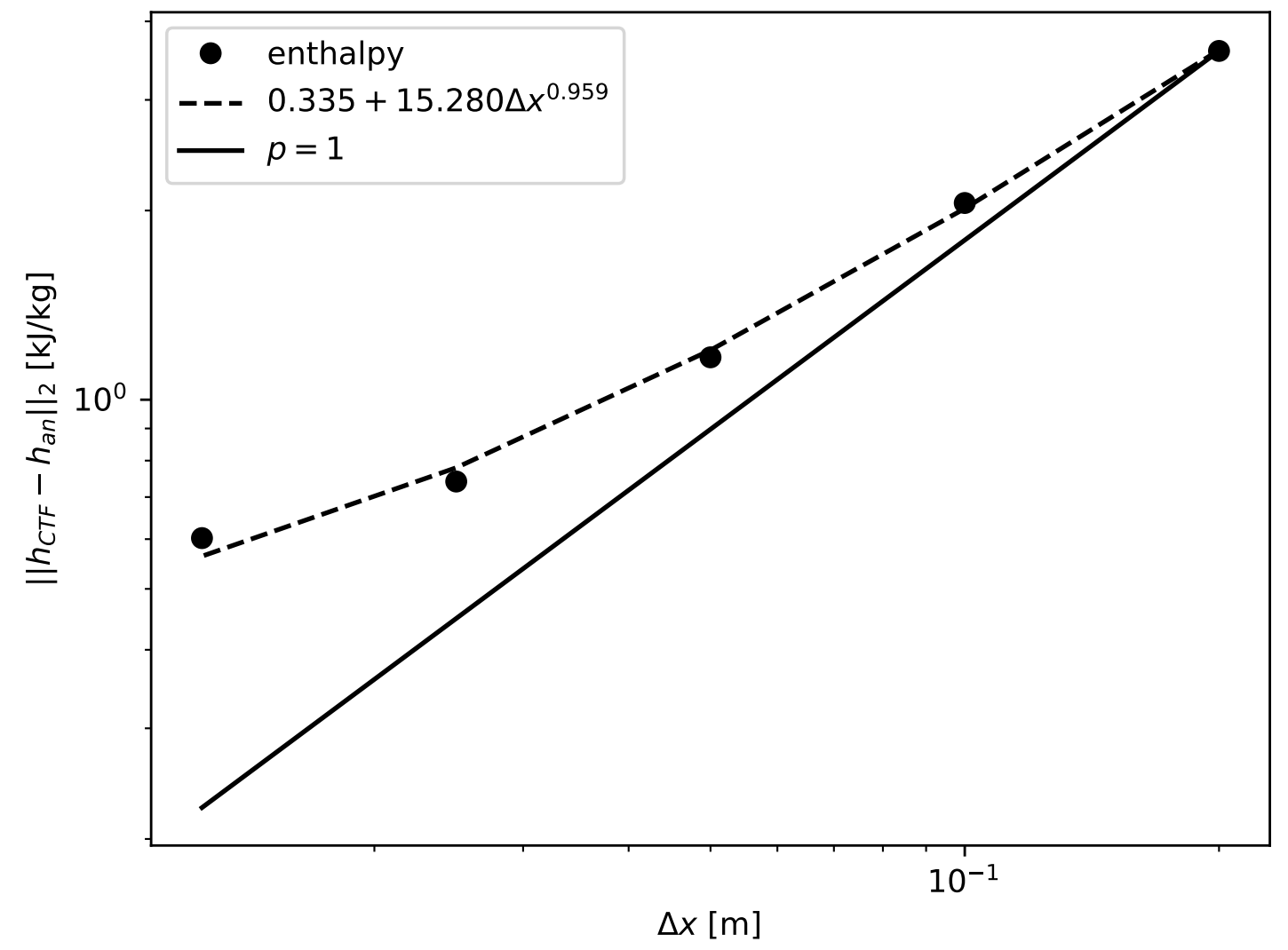

Figure 4.14: Convergence for pipe boiling verification problem 


\section{Spacer Grid Models}

CTF uses spacer grid models to capture the turbulence-enhancing effect of spacer grids in the flow, which is not otherwise captured in CTF due to the lack of a turbulence model and the meshing approach employed. Spacer grid models are currently applied to the single-phase convective heat transfer and TKE calculated by CTF, as discussed in the Theory Manual.

\subsection{Yao-Hochreiter-Leech}

In CTF, the Yao-Hochreiter-Leech (YHL) correlation is used to calculate an exponentially decaying heat transfer and turbulent kinetic energy (TKE) enhancement factor downstream of spacer grids. The following test is a grid refinement code verification study on the single-phase heat transfer and turbulent kinetic energy enhancement due to a single, simple (swirl factor set to zero) spacer grid in an axial channel with a heated rod. The expected Nusselt number and turbulent kinetic energy enhancement factor obtained by the YHL correlation are tested with the CTF prediction for the rod wall temperature and TKE for first order convergence.

Problem Description For single-phase axial channel flow with constant axial heat flux boundary condition, the CTF energy equation in its complete form can be simplified as follows:

$$
\frac{\partial}{\partial x}(\rho h u)=q_{w}^{\prime \prime \prime}
$$

Assuming constant thermophysical properties, Eq. (5.1) can be integrated from inlet to outlet for constant mass flux, as follows:

$$
\dot{m}_{l} C_{p}\left(T_{\text {liq,out }}-T_{\text {liq,in }}\right)=Q_{T}
$$

The terms $C_{p}$ and $Q_{T}$ represent the specific heat capacity at constant pressure and the total heat added to liquid from the heated rod, respectively. For the heated rod, the local wall temperature can be obtained by the solid-liquid interface boundary condition, as follows: 


$$
q_{w}^{\prime \prime}=N u_{0} k_{f} \frac{\left(T_{\mathrm{w}, \mathrm{x}}-T_{\mathrm{liq}, \mathrm{x}}\right)}{D_{h}} .
$$

The terms $q_{w}^{\prime \prime}, N u_{0}, k_{f}$, and $D_{h}$ represent the constant wall heat flux, the base Nusselt number, the thermal conductivity of the liquid, and the hydraulic diameter, respectively. In the current study, the base Nusselt number, $N u_{0}$ is set as a constant. The YHL Nusselt number enhancement factor with zero swirl factor is as follows:

$$
E F=1+5.55 \epsilon^{2} \exp \left[-0.13 \frac{\left(x-x_{\text {grid }}\right)}{D_{h}}\right]
$$

The terms $\epsilon, x_{\text {grid }}$, and $E F$ represent the spacer grid blockage, the axial location of the grid spacer, and the grid spacer enhancement factor, respectively. It must be noted that upstream of the spacer grid, the enhancement factor is set to zero in CTF, and downstream of the spacer grid, the enhancement factor at a node is calculated by numerical integration of Eq. (5.4) across the cell. Finally, the enhanced Nusselt number, $N u$, can be calculated from the base Nusselt number and the enhancement factor, as follows:

$$
\frac{N u}{N u_{0}}=E F
$$

The physics-based model which is implemented in CTF to correlate TKE to the wall shear stress is discussed in detail in [35]. Using a constant friction factor, the wall shear stress can be obtained using the DarcyWeisbach equation, as follows:

$$
\tau_{w}=\frac{f \rho u^{2}}{8} .
$$

The terms $\tau_{w}$ and $f$ represent the wall shear stress and the friction factor, respectively. Using Eq. (5.6), the base TKE (upstream of the grid spacer) can be calculated using the relationship discovered by Bradshaw [35], as follows:

$$
K_{0}=\frac{\tau_{w}}{0.297 \rho}
$$

The term $K_{0}$ represents the turbulent kinetic energy. Finally, the enhanced TKE, $K$, can be obtained from the base TKE and the enhancement factor defined in Eq. (5.4), as follows:

$$
\frac{K}{K_{0}}=E F .
$$

CTF Input Model Description The channel dimensions are set in a manner similar to the dimensions of a PWR subchannel, with a mass source inlet boundary condition of $0.3 \mathrm{~kg} \mathrm{~s}^{-1}$ and an outlet pressure of 155 bar using the following constant thermophysical properties for water: density of $1000 \mathrm{~kg} \mathrm{~m}^{-3} \mathrm{~s}^{-1}$, thermal 


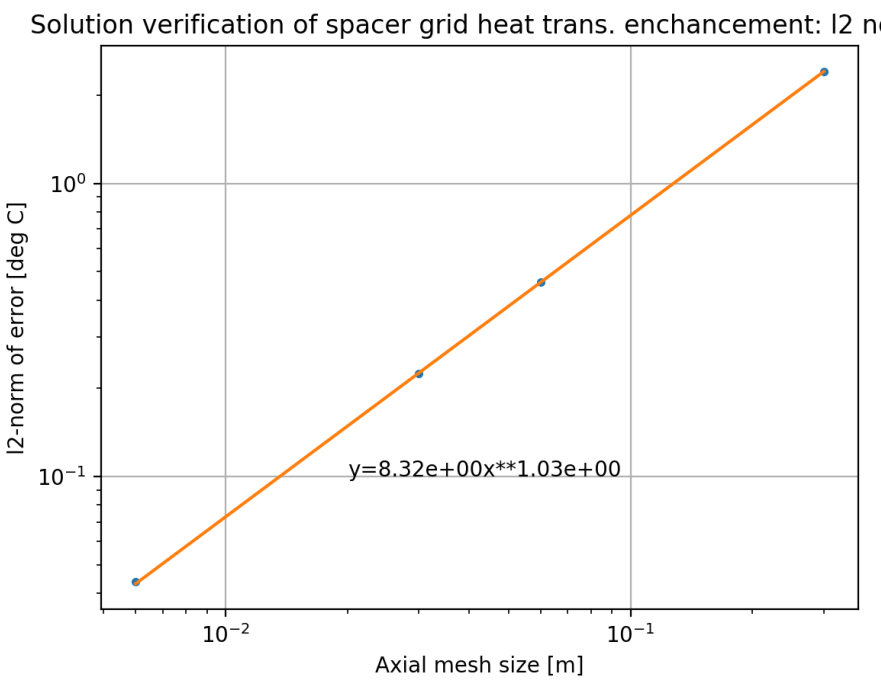

Figure 5.1: CTF-prediction and curve fit of $L_{2}$ norm of the wall temperature for successive mesh refinements.

conductivity of $0.65 \mathrm{~W} \mathrm{~m}^{-1} \mathrm{~K}^{-1}$, specific heat capacity of $4.15 \mathrm{~kJ} \mathrm{~kg}^{-1} \mathrm{~K}^{-1}$, and viscosity of $7 \times 10^{-4} \mathrm{Pas} \mathrm{s}^{-1}$. The base Nusselt number is set to 50. The spacer grid with a blockage of 0.2 is located at the center of the rod. A form loss coefficient of 1.0 is used for the spacer grid. The friction factor is set to a constant of 0.02 . The axial mesh is successively refined with the coarsest mesh having a uniform size of $0.3 \mathrm{~m}$ and the finest mesh with a size of $0.006 \mathrm{~m}$, for a channel length of $3 \mathrm{~m}$.

Discussion of Results Figure 5.1 shows first-order convergence based on the $L_{2}$ norm of the CTF predicted and expected wall temperature for a series of successive mesh refinements. Similarly, Figure 5.2 shows first-order convergence based on the $L_{2}$ norm of the CTF predicted and expected TKE for a series of successive mesh refinements. Figure 5.3 shows the expected and CTF predicted liquid axial temperature profiles, and Figure 5.4 shows the comparison between CTF and predicted axial wall temperature profiles. Finally, Figure 5.5 shows the comparison between CTF and predicted axial TKE profiles for the finest mesh. The $L_{2}$ norm for the finest mesh for the wall temperature and TKE is on the order of 1e-4 and 1e-5, respectively, which demonstrates the good agreement between CTF prediction and expected heated wall temperature and TKE.

Conclusions Single-phase heat transfer and TKE enhancement study of an axial channel with a heated rod and a single spacer grid was conducted for a series of mesh refinements. The liquid temperature profile, wall temperature profile, and TKE profile were compared with the expected solution in the axial direction. CTF-predicted wall temperature and TKE match the expected results, with an $L_{2}$ norm on the order of $1 \mathrm{e}-4$ and 1e-5, respectively. This study demonstrates the proper implementation of the YHL model for spacer grids. 


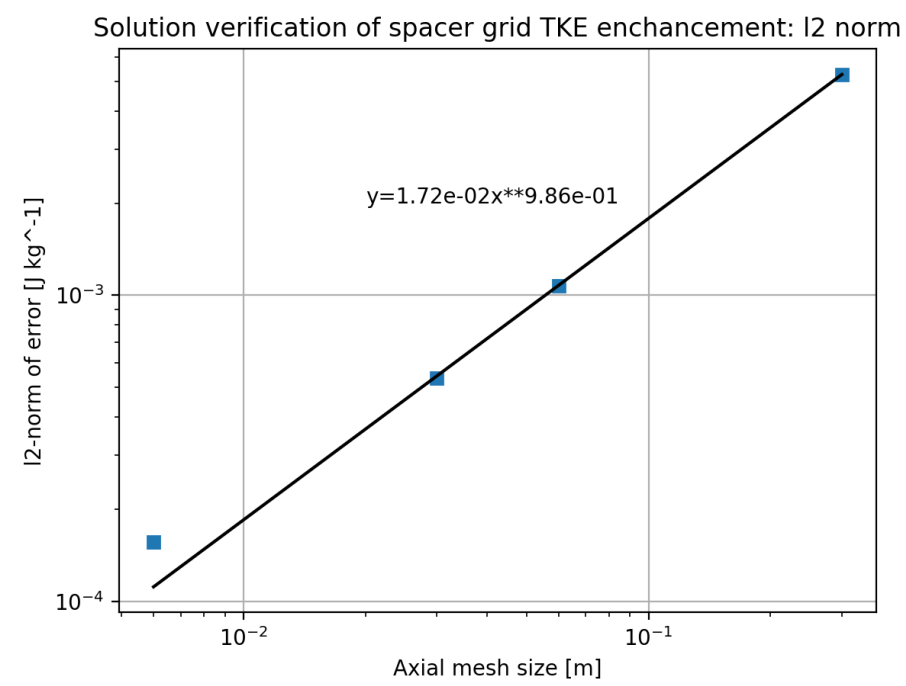

Figure 5.2: CTF-prediction and curve fit of $L_{2}$ norm of the wall temperature for successive mesh refinements.

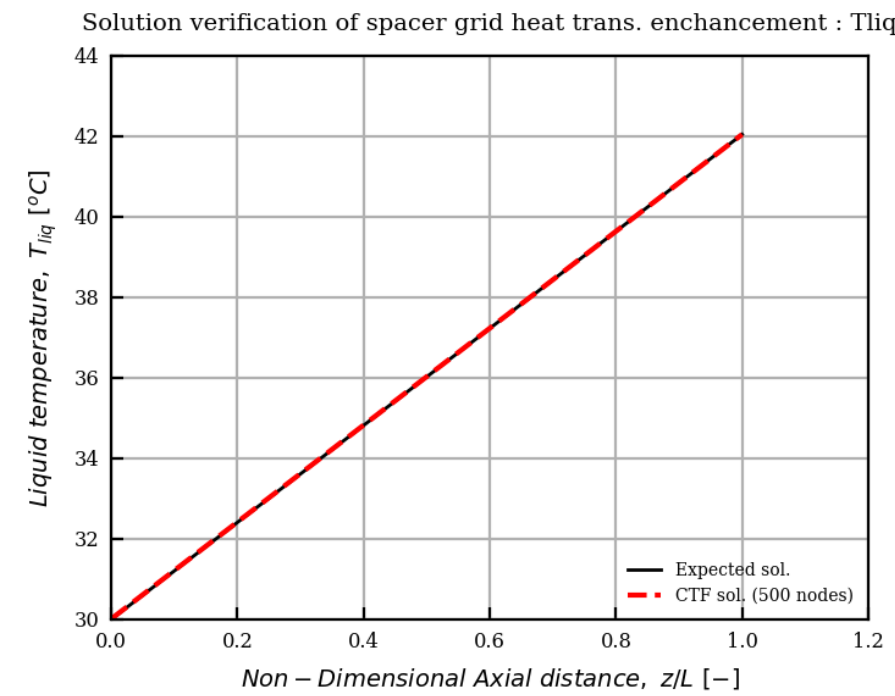

Figure 5.3: CTF-prediction and expected axial liquid temperature profile of the channel. 
Solution verification of spacer grid heat trans. enchancement: Tw

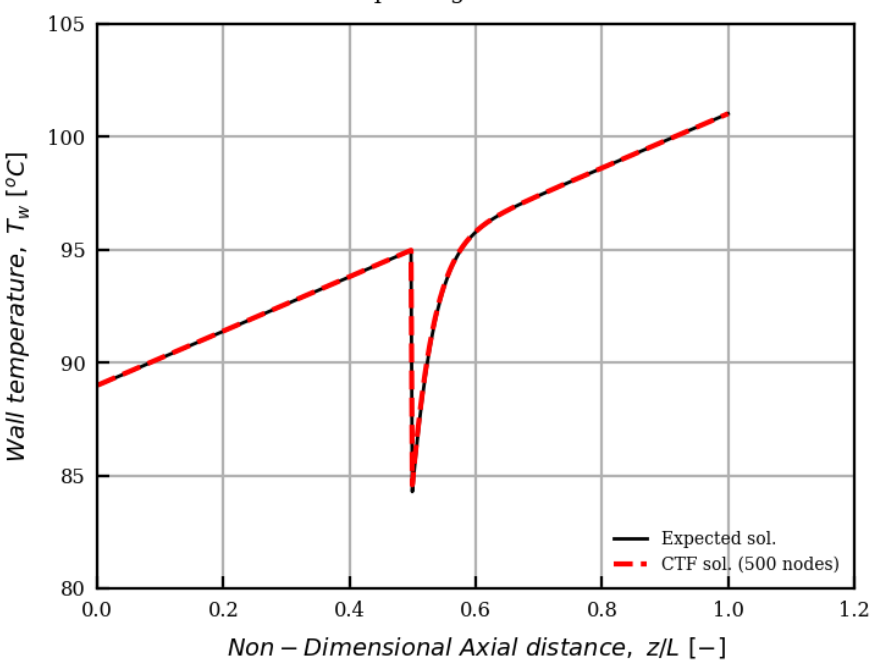

Figure 5.4: CTF-prediction and expected wall temperature with a spacer grid.

Solution verification of spacer grid TKE enchancement

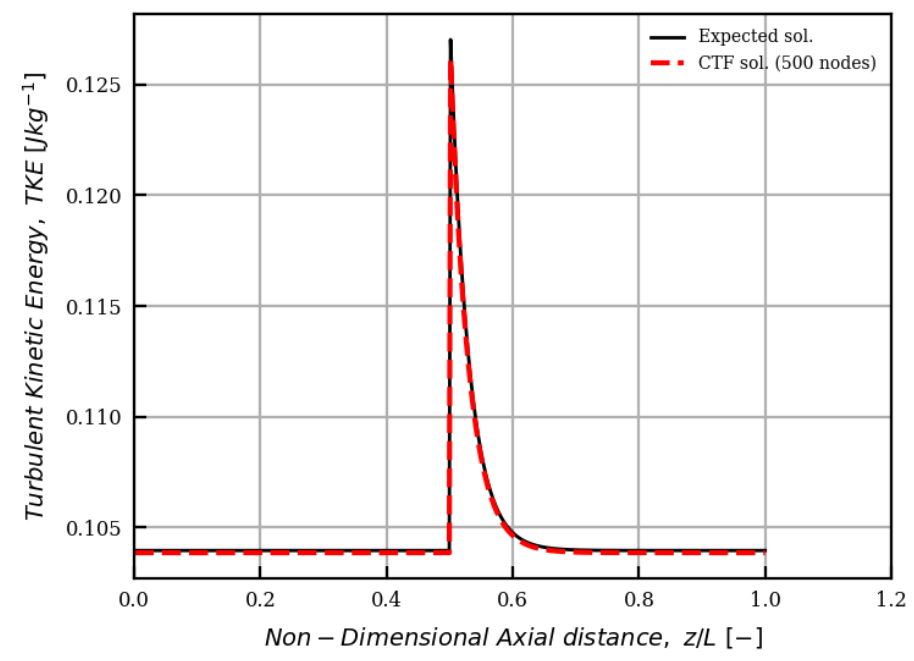

Figure 5.5: CTF-prediction and expected wall temperature with a spacer grid. 


\section{Pressure loss}

\subsection{Pressure drop in CTF}

Prior to discussing results, a discussion of CTF-calculated pressure drop is included. Some of the experimental results present only a component of pressure drop (e.g. frictional and acceleration pressure drop, having the gravitational component subtracted off of measured results during experimentation). CTF only solves for a single, total pressure in the model, but will print out the pressure drop components (gravitational, frictional, and acceleration) to an output file. The individual components of pressure drop are calculated upon completion of the simulation. Considering this, it is prudent to discuss the calculation of these terms prior to their use.

The acceleration pressure drop is calculated as the change in the momentum flux across a scalar mesh cell in the code.

$$
\Delta P_{\mathrm{accel}}=\left(\frac{G_{m}^{2}}{\rho_{m}^{+}}\right)_{\mathrm{out}}-\left(\frac{G_{m}^{2}}{\rho_{m}^{+}}\right)_{\mathrm{in}}
$$

The momentum flux term is calculated as:

$$
\frac{G_{m}^{2}}{\rho_{m}^{+}}=\rho_{v} \alpha_{v} v_{v}^{2}+\rho_{l} \alpha_{l} v_{l}^{2}+\rho_{l} \alpha_{d} v_{d}^{2}
$$

Here, $\alpha$ is the phase volume fraction, $v$ is the phase velocity, and $\rho$ is the phase density. The subscripts, $v$, $l$, and $d$ stand for vapor, liquid, and droplets, respectively. In CTF, the momentum flux is calculated at the boundary of each mesh cell. Therefore, by taking the difference between momentum fluxes at two adjacent axial locations, we obtain the acceleration pressure drop over that corresponding scalar mesh cell.

The gravitational pressure drop is calculated for the scalar mesh cell as follows:

$$
\Delta P_{\text {gravity }}=\rho_{\text {mix }} g \Delta X
$$

Here, $\Delta X$ is the axial length of the scalar cell, $g$ is the acceleration due to gravity, and $\rho_{\text {mix }}$ is the mixture density, which is calculated as follows: 


$$
\rho_{\text {mix }}=\rho_{v} \alpha_{v}+\rho_{l}\left(\alpha_{l}+\alpha_{d}\right)
$$

Finally comes the frictional pressure drop. It is simply calculated by subtracting the gravitational and acceleration losses from the total pressure drop calculated by CTF.

$$
\Delta P_{\text {friction }}=\Delta P_{\text {total }}-\Delta P_{\text {accel }}-\Delta P_{\text {gravity }}
$$

A final note on the calculation of pressure drop terms; these results were averaged across the whole crosssection of the model for a given axial level using an area-weighting approach.

\subsection{Single-phase pressure drop}

\subsubsection{Validation}

\subsubsection{BFBT}

The CTF-predicted pressure drops are plotted against experimental ones in Figure 6.1. Note that the specification presented these pressure drop results as frictional loss only; the gravitational loss between pressure taps was subtracted off of the total loss before reporting data. Since CTF gives total pressure drop, it was necessary to subtract the gravitational component from the total loss between pressure tap locations.

These results show that the lower Reynolds test cases are generally underpredicted by CTF. As Reynolds increases past 100,000, the data seem to cluster better around the measurements. 


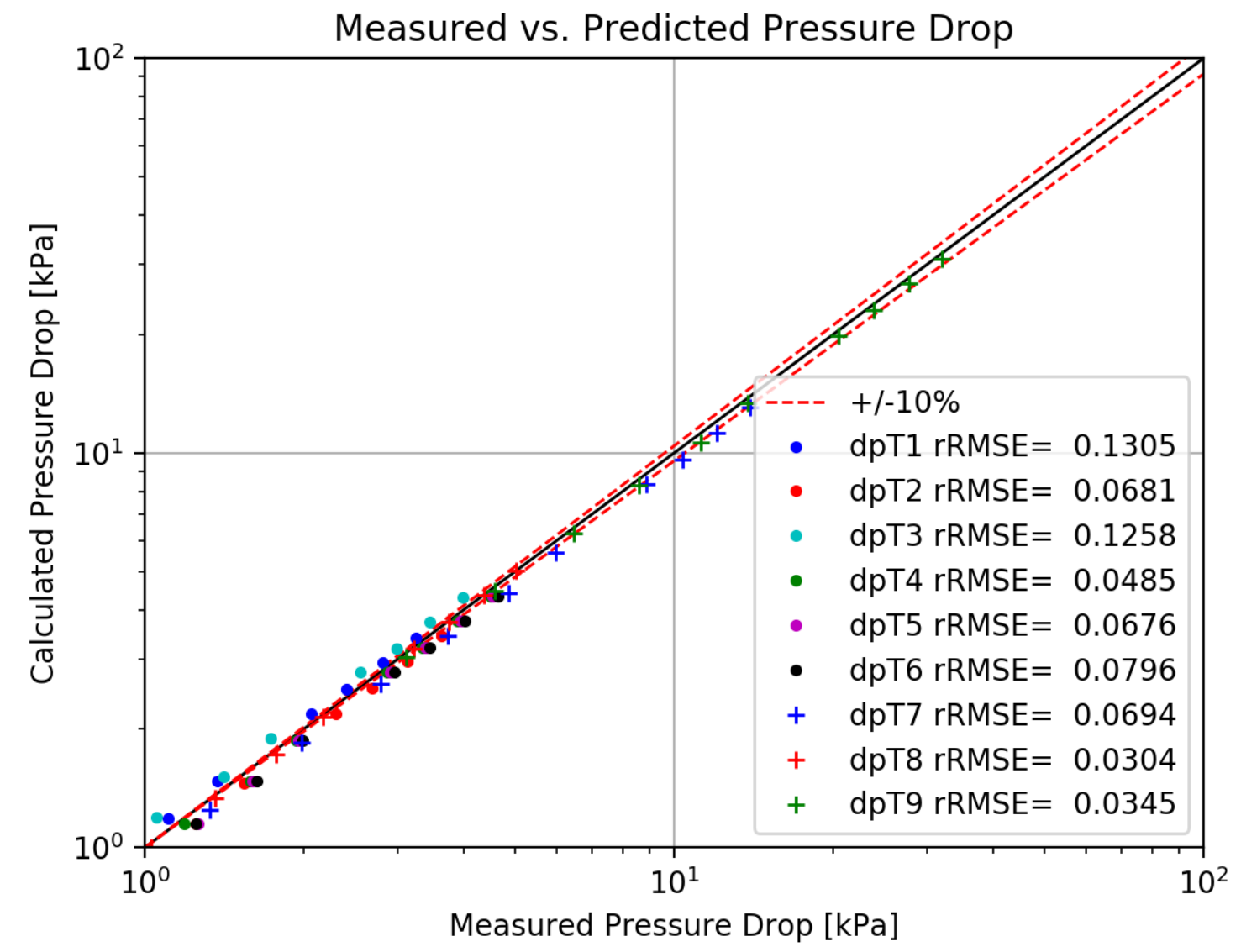

Figure 6.1: BFBT single-phase pressure drop predictions (Series 7) 


\subsubsection{Nikuradse}

The Nikuradse test section is used as a separate effects validation test for single-phase wall shear in CTF $[25$, 26]. Of the 487 tests run by Nikuradse, 398 are selected for simulation in CTF. The results are compared in Figure 6.2, which combines results of both smooth and rough pipes.

It is clear from the simulation results that the original CTF $(\mathrm{IRFC}=1)$ and McAdams $(\mathrm{IRFC}=2)$ correlations underperform for both smooth and rough pipes. The newer CTF options, IRFC $=3$ and IRFC $=4$, perform much better. 


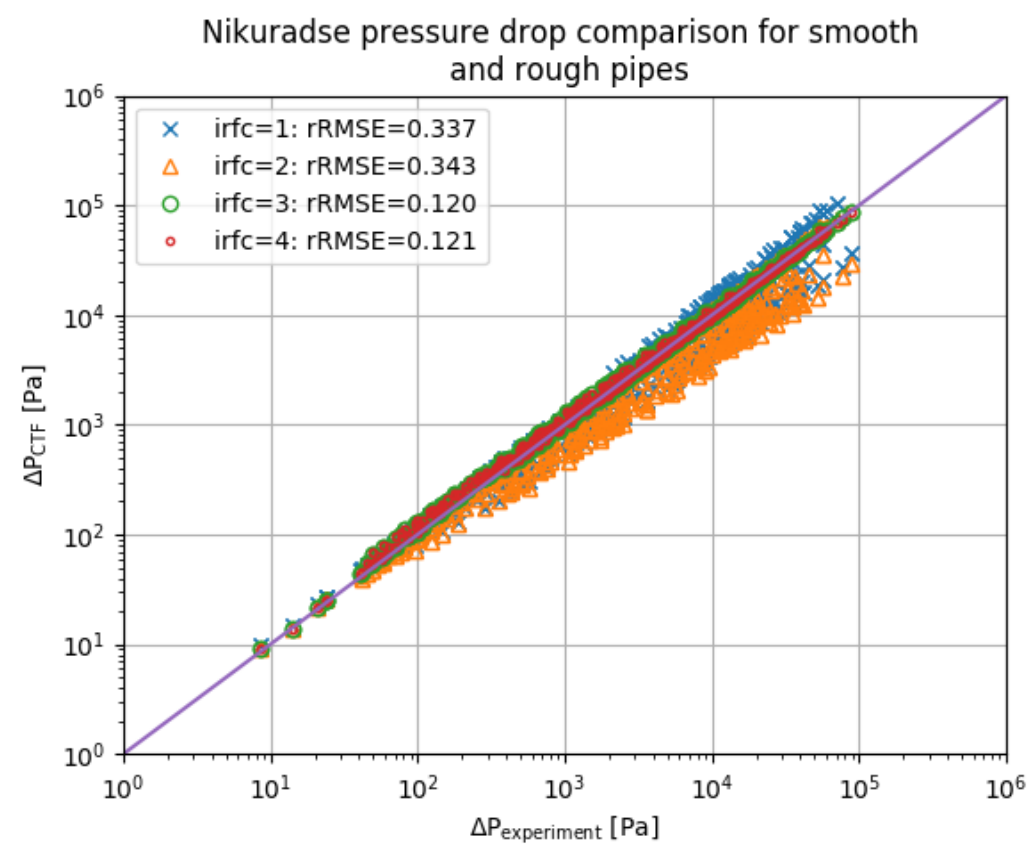

Figure 6.2: Results for Nikuradse smooth and rough pipes

\subsubsection{Verification}

\subsubsection{Single-Phase Friction Flow Split}

In CTF, there are three mechanisms for lateral flow between channels: pressure differences, turbulent-mixing, and void-drift. The user has the option to shut off the turbulent-mixing and void-drift independently of the pressure-driven cross-flow effects. If the turbulent-mixing and void-drift models are disabled, flow redistribution can only occur due to lateral pressure differences in the model. Lateral pressure gradients can arise due to lateral density differences (due to non-uniform radial heating in the model), due to non-uniform inlet boundary conditions, or due to lateral boundary conditions. If we create an unheated model with uniform inlet boundary conditions, lateral pressure gradients will form due to differences in pressure drops in adjacent channels.

In CTF, pressure drop is a combination of gravitational, acceleration, form, and friction losses. This problem is built such that wall friction becomes the only driver for cross-flow and, thus, determines the flow distribution between channels. An analytical solution is formed to determine this ideal flow split and the $\mathrm{CTF}$ results are compared to the analytical solution.

Problem Description A model of two channels connected by a gap is created. One channel has a larger hydraulic diameter than the other. The inlet velocity is uniform in the two channels, the case is unheated, and the coolant is single-phase and highly subcooled. This creates a difference in Reynolds number at the 
inlet of the two channels, which creates different frictional pressure drops in the two channels, as the friction model is Reynolds dependent.

The different frictional pressure drops create a lateral pressure gradient that drives flow from the higher resistance channel to the lower resistance channel. Moving up the channels, velocity grows larger in the low-resistance channel, which increases frictional pressure drop in that channel. Simultaneously, velocity decreases in the high-resistance channel, which decreases frictional pressure drop. This continues until the frictional pressure drop is the same in both channels, at which point cross-flow ceases. At this point, the channels are said to be in mechanical equilibrium.

An analytical solution is derived for this point of mechanical equilibrium. We consider a control volume in each channel at this level where equilibrium has been reached. It is safe to neglect the lateral momentum equation because cross-flow has stopped. An axial momentum equation can be formed for each channel control volume. The general axial momentum equation is shown below.

$$
\rho\left[\frac{\partial \boldsymbol{V}}{\partial t}+u \frac{\partial \boldsymbol{V}}{\partial x}+v \frac{\partial \boldsymbol{V}}{\partial y}+w \frac{\partial \boldsymbol{V}}{\partial z}\right]=\rho g-\nabla p+\nabla \dot{\tau}_{i j}
$$

The density is removed from the left-hand side terms since it is assumed constant in the problem. The bracketed terms include: 1) time-change of momentum, 2) axial (x) advection of momentum, 3) lateral (y) advection of momentum, and 4) lateral $(z)$ advection of momentum. The three right-hand side terms are the relevant force terms, including: 1) gravity, 2) pressure, and 3) shear.

This equation can be significantly reduced considering

1. the case is steady-state, eliminating the temporal term;

2. there is no cross-flow, eliminating lateral convection terms; and

3. the axial velocity distribution in this control volume is constant, as density is constant and there is no cross-flow, meaning the axial momentum convection term can be eliminated.

This eliminates the entire left-hand side of the equation and leaves the following equation for an individual subchannel, where $x$ is taken as the axial direction:

$$
\frac{d P}{d x}=\rho g+\frac{d \tau_{w}}{d x}
$$

Because the two channels are in mechanical equilibrium, the pressure drops in the channels are equal, allowing us to equate the right-hand side of each individual channel equation. Note that the gravity head is identical in the two channels, allowing the term to be cancelled. Finally, integrating over the control volume height, $d x$, allows us to obtain the final relation between the two channels.

$$
\tau_{w, 1}=\tau_{w, 2}
$$


The wall drag, $\tau_{w}$, is determined from the CTF friction model, which is substituted into Equation 6.8 to produce the following expansion:

$$
\frac{f_{1} u_{1}^{2}}{2 D_{h, 1}}=\frac{f_{2} u_{2}^{2}}{2 D_{h, 2}}
$$

The terms, $f, u$, and $D_{h}$, represent the Darcy friction factor, liquid velocity, and channel hydraulic diameter, respectively. The subscripts indicate which channel the term represents. The CTF friction factor model is used in the problem to calculate $f$ as a function of Reynolds number. It has the following form:

$$
f=C_{1} R e^{C_{2}}
$$

$C_{1}$ and $C_{2}$ are model coefficients. Expanding the Reynolds number and substituting this into Equation 6.9 yields the following relationship between channel velocities:

$$
\frac{C_{1}\left(\frac{\rho u_{1} D_{h, 1}}{\mu}\right)^{C_{2}} u_{1}^{2}}{D_{h, 1}}=\frac{C_{1}\left(\frac{\rho u_{2} D_{h, 2}}{\mu}\right)^{C_{2}} u_{2}^{2}}{D_{h, 2}}
$$

Canceling terms and reducing leads to the following form:

$$
\left(\frac{u_{1}}{u_{2}}\right)^{2+C_{2}}=\left(\frac{D_{h, 2}}{D_{h, 1}}\right)^{C_{2}-1}
$$

The hydraulic diameters of the two channels are defined by the model geometry. For CTF, $C_{2}$ is -0.2 . If we also consider the mass conservation equation, which tells us that the sum of the outlet mass flow rates is equal to the inlet mass flow rate, we can obtain the expected solutions for the absolute outlet mass flow rate of each channel. First, Equation 6.12 is set in terms of mass flow rate to produce the following:

$$
\left(\frac{\dot{m}_{1}}{\dot{m}_{2}} \frac{A_{2}}{A_{1}}\right)^{2+C_{2}}=\left(\frac{D_{h, 2}}{D_{h, 1}}\right)^{C_{2}-1}
$$

Next, the mass conservation equation is used to relate the mass flow rates in the individual channels to the total mass flow rate in the system:

$$
\begin{aligned}
& \dot{m}_{\text {in }}=\dot{m}_{1}+\dot{m}_{2} \\
& \dot{m}_{\text {in }}=\dot{m}_{2}\left(1+\left(\frac{D_{h, 2}}{D_{h, 1}}\right)^{\frac{C_{2}-1}{2+C_{2}}} \frac{A_{1}}{A_{2}}\right)
\end{aligned}
$$




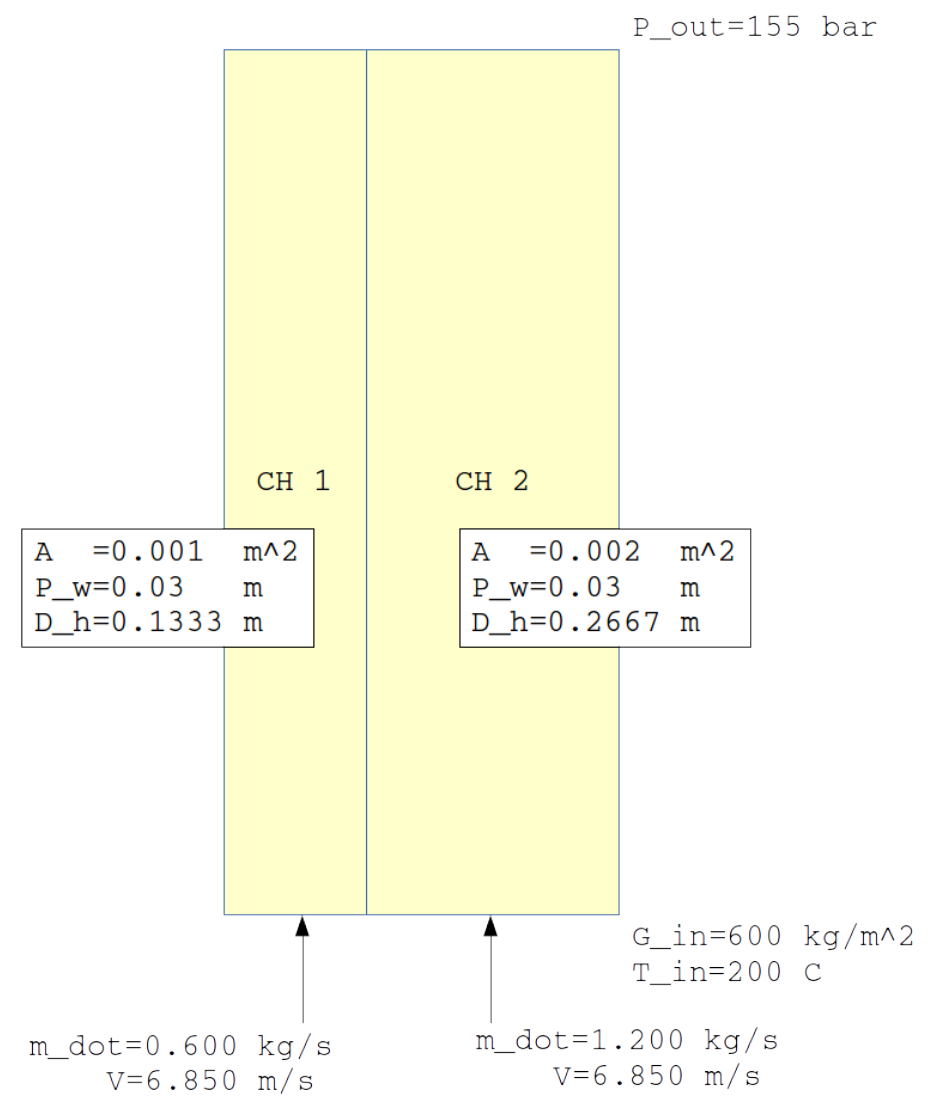

Figure 6.3: Diagram of the two-channel flow split problem.

CTF Model Description Channel 2 has a hydraulic diameter that is twice the size of the Channel 1 hydraulic diameter. The area and wetted perimeter of Channel 1 are set to values close to those expected of typical PWR rod-lattice geometry. The outlet pressure is 155 bar and inlet mass flux is $3500 \mathrm{~kg} \mathrm{~m}^{-2} \mathrm{~s}^{-1}$. The inlet temperature is set to $200^{\circ} \mathrm{C}$ to keep the model sufficiently subcooled, and the case is unheated. Turbulent mixing and void drift is disabled so that pressure is the only driver for cross-flow. A diagram of this model is shown in Figure 6.3. The length of the model is set to $10 \mathrm{~m}$ to allow the flow to completely redistribute within the $\mathrm{CTF}$ solution space.

The axial mesh is set to different sizes, including $2.54 \mathrm{~cm}, 5.08 \mathrm{~cm}$, and $10.16 \mathrm{~cm}$; however, it is found that axial mesh size has no impact on the axial mass flow rate profiles. With the geometry defined, Equation 6.15 is used to calculate that the expected outlet mass flow rates in Channels 1 and 2. 


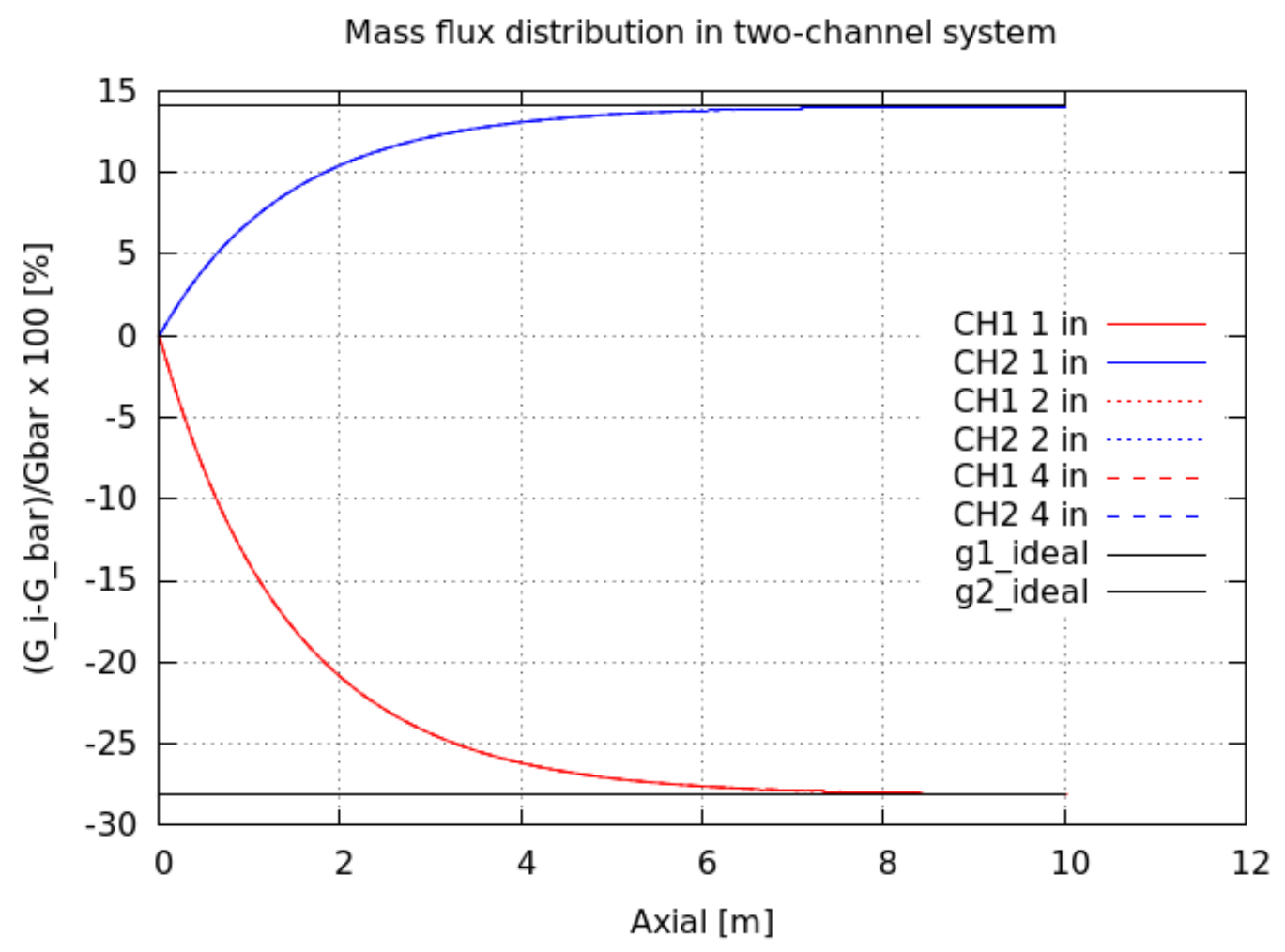

Figure 6.4: CTF-predicted axial mass flux distribution in two-channel system compared with analytical solution.

Discussion of Results The CTF solution includes the axial mass flow rate distribution in each channel. The analytical solution only gives us the expected flow rate distribution at the exit. Therefore, we cannot compare the CTF axial flow distribution to the analytical solution, but we can at least guarantee that CTF achieves the correct flow split when mechanical equilibrium is achieved. The mass flux in each channel is normalized before plotting using the following relationship:

$$
G_{i, \mathrm{norm}}=\frac{G_{i}-\bar{G}}{\bar{G}} \times 100
$$

$\bar{G}$ is the average mass flux between channels, which is equal to the inlet mass flux. Therefore, the normalized mass flux in each channel is zero at the inlet and then re-distributes due to frictional resistance. Figure 6.4 shows the CTF-predicted flow distribution in the two-channel system. The correct analytical flow split is shown with the dashed lines.

These results demonstrate that CTF predicts the expected flow split between the two channels at about $7 \mathrm{~m}$ from the inlet. 


\subsubsection{Friction Model Verification}

A suite of tests is defined in this section for verifying that the various friction factor models are correctly implemented in CTF. CTF has four pre-defined friction factor models; two assume all surfaces are smooth and the other two consider the effect of surface roughness. The code also includes a fifth option for the user to specify a custom friction correlation. This set of tests considers various permutations of a $3 \times 3$ rod bundle. Permutations include varying the size of the center guide tube, changing the roughness of the rods, and adding a wall around the bundle.

In considering the effects of surface roughness, CTF considers an area-weighted average of the surface roughness in each channel. Therefore, it should be expected that the meshing of the problem will lead to different results (i.e., a rod-centered subchannel model will experience slightly different frictional losses than a channelcentered subchannel model). Therefore, the $3 \times 3$ bundle is modeled in two ways: 1) using a rod-centered approach, and 2) using a channel-centered approach. Each of these sub-series of tests activates each of the four pre-defined friction models in CTF and uses multiple permutations of bundle geometry. The custom friction model is tested by specifying the Blasius correlation as input.

Problem Description A suite of channel-centered and rod-centered models is defined to adequately test the friction models of CTF for several geometries of interest. Figure 6.5 shows the difference between the rod-centered and channel-centered modeling approaches for the $3 \times 3$ bundle. The blue shaded region shows the definition of a channel in the CTF model; as a result, the rod-centered model has 9 channels and the channel-centered model has 16 channels. The blue rod is the guide tube.

The channel-centered model characteristics are shown in Table 39. The rod-centered model characteristics are shown in Table 40. In each test name, the letter suffix (ranging from "a" - "e") represents the friction model being employed in the study. The number following "test" represents the test series; the geometry is varied slightly between test series. The main perturbations are to vary the guide tube size, the rod roughness, and the presence of a wall around the bundle.

Varying the guide tube size will cause flow area of the channels touching the guide tube to change, resulting in a flow redistribution. Likewise, increasing guide tube roughness causes greater flow resistance in the center of the bundle and causes flow to migrate to the boundary channels. The channel-centered models investigate the effect of including a wall around the bundle. Due to the implementation of the surface-roughness models in CTF, the wall is assumed smooth unless the wall is explicitly modeled as a heated or unheated conductor in the model. Including the wall in the verification tests is necessary to ensure that this intended behavior is correct.

The proper implementation of the friction models is determined by calculating the expected flow split and then comparing this to the CTF simulation results. It is difficult to directly calculate the expected pressure drop in each channel of the model because wall shear is a function of velocity, which changes along the axial length as the flow redistributes. The approach for determining the flow split is similar to Section 6.2.2.1. In these problems, the friction factor correlation for some of the models is much more complicated than the form given in Equation 6.10, so it is not possible to directly solve for the expected velocity distribution in each channel type. Rather, an iterative solution is required to calculate the friction factor using a guessed velocity, then use the friction factor to obtain a more accurate guess of the flow distribution, and so on.

The approach is slightly different depending on whether the model is channel-centered or rod-centered. A 

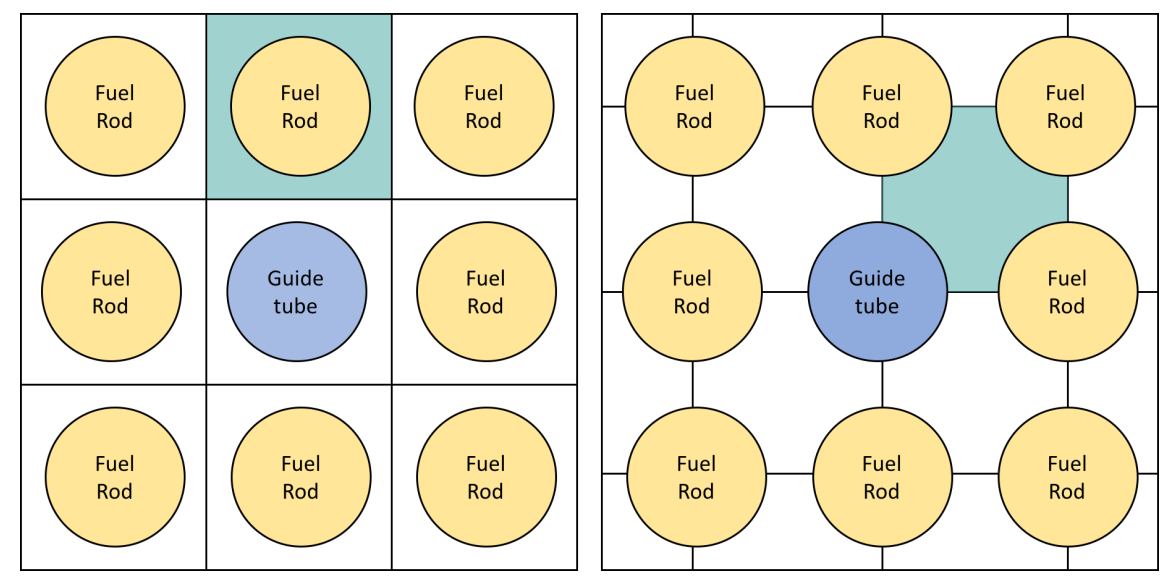

Figure 6.5: $3 \times 3$ rod bundle geometry with (a) rod-centered and (b) channel-centered subchannels.

Table 39: Summary of characteristics of channel-centered friction verification tests

\begin{tabular}{crrrrrr}
\hline Test & $\mathrm{d}_{\text {rod }}[\mathrm{mm}]$ & $\mathrm{d}_{\mathrm{gt}}[\mathrm{mm}]$ & $\epsilon_{\text {rod }}[\mu \mathrm{m}]$ & $\epsilon_{\text {gt }}[\mu \mathrm{m}]$ & Wall & IRFC \\
\hline cc_test1a & 9.5 & 9.5 & 0.0 & 0.0 & No & 1 \\
cc_test1b & 9.5 & 9.5 & 0.0 & 0.0 & No & 2 \\
cc_test1c & 9.5 & 9.5 & 0.0 & 0.0 & No & 3 \\
cc_test1d & 9.5 & 9.5 & 0.0 & 0.0 & No & 4 \\
cc_test1e & 9.5 & 9.5 & 0.0 & 0.0 & No & 5 \\
\hline cc_test2a & 9.5 & 11.22 & 0.0 & 0.0 & No & 1 \\
cc_test2b & 9.5 & 11.22 & 0.0 & 0.0 & No & 2 \\
cc_test2c & 9.5 & 11.22 & 0.0 & 0.0 & No & 3 \\
cc_test2d & 9.5 & 11.22 & 0.0 & 0.0 & No & 4 \\
cc_test2e & 9.5 & 11.22 & 0.0 & 0.0 & No & 5 \\
\hline cc_test3a & 9.5 & 11.22 & 0.0 & 10.0 & No & 1 \\
cc_test3b & 9.5 & 11.22 & 0.0 & 10.0 & No & 2 \\
cc_test3c & 9.5 & 11.22 & 0.0 & 10.0 & No & 3 \\
cc_test3d & 9.5 & 11.22 & 0.0 & 10.0 & No & 4 \\
cc_test3e & 9.5 & 11.22 & 0.0 & 10.0 & No & 5 \\
\hline cc_test4a & 9.5 & 9.5 & 0.0 & 0.0 & Yes & 1 \\
cc_test4b & 9.5 & 9.5 & 0.0 & 0.0 & Yes & 2 \\
cc_test4c & 9.5 & 9.5 & 0.0 & 0.0 & Yes & 3 \\
cc_test4d & 9.5 & 9.5 & 0.0 & 0.0 & Yes & 4 \\
cc_test4e & 9.5 & 9.5 & 0.0 & 0.0 & Yes & 5 \\
\hline cc_test5a & 9.5 & 9.5 & 10.0 & 10.0 & Yes & 1 \\
cc_test5b & 9.5 & 9.5 & 10.0 & 10.0 & Yes & 2 \\
cc_test5c & 9.5 & 9.5 & 10.0 & 10.0 & Yes & 3 \\
cc_test5d & 9.5 & 9.5 & 10.0 & 10.0 & Yes & 4 \\
cc_test5e & 9.5 & 9.5 & 10.0 & 10.0 & Yes & 5 \\
\hline
\end{tabular}


Table 40: Summary of characteristics of rod-centered friction verification tests

\begin{tabular}{crrrrrr}
\hline \multicolumn{1}{c}{ Test } & $\mathrm{d}_{\text {rod }}[\mathrm{mm}]$ & $\mathrm{d}_{\mathrm{gt}}[\mathrm{mm}]$ & $\epsilon_{\text {rod }}[\mu \mathrm{m}]$ & $\epsilon_{\mathrm{gt}}[\mu \mathrm{m}]$ & Wall & IRFC \\
\hline rc_test1a & 9.5 & 9.5 & 0.0 & 0.0 & No & 1 \\
rc_test1b & 9.5 & 9.5 & 0.0 & 0.0 & No & 2 \\
rc_test1c & 9.5 & 9.5 & 0.0 & 0.0 & No & 3 \\
rc_test1d & 9.5 & 9.5 & 0.0 & 0.0 & No & 4 \\
\hline rc_test2a & 9.5 & 11.22 & 0.0 & 0.0 & No & 1 \\
rc_test2b & 9.5 & 11.22 & 0.0 & 0.0 & No & 2 \\
rc_test2c & 9.5 & 11.22 & 0.0 & 0.0 & No & 3 \\
rc_test2d & 9.5 & 11.22 & 0.0 & 0.0 & No & 4 \\
\hline rc_test3a & 9.5 & 9.5 & 0.0 & 10.0 & No & 1 \\
rc_test3b & 9.5 & 9.5 & 0.0 & 10.0 & No & 2 \\
rc_test3c & 9.5 & 9.5 & 0.0 & 10.0 & No & 3 \\
rc_test3d & 9.5 & 9.5 & 0.0 & 10.0 & No & 4 \\
\hline
\end{tabular}

rod-centered model has two unique channel types that must be considered: 1) the center channel and, 2) the boundary channels. The system of equations that must be solved for the rod-centered model are:

$$
\begin{aligned}
u_{b, k+1} & =\sqrt{\frac{f_{c}}{f_{b}} u_{c, k}^{2} \frac{D h_{b}}{D h_{c}}} \\
u_{c, k+1} & =\frac{\dot{m}-8 \rho u_{b, k+1} A_{b}}{\rho A_{c}}
\end{aligned}
$$

The subscripts $b$ and $c$ represent "boundary channel" and "center channel", respectively. The subscripts $k$ and $k+1$ represent the current and next iterations in the iterative solution scheme. The friction factor is denoted with $f$, the channel velocity with $u$, the channel flow areas with $A$, liquid density with $\rho$, the total bundle mass flow rate with $\dot{m}$, and the hydraulic diameters with $D h$. The fluid properties are taken directly from the CTF fluid property tables for consistency. The flow areas and hydraulic diameters are calculated from the bundle geometry, which is representative of PWR geometry. The new iteration boundary channel velocity, $u_{b, k+1}$, is relaxed using logarithmic interpolation as shown in Equation 6.19. This is done to smoothly converge on the correct flow distribution and prevent numerical oscillations. The relaxed $u_{b}$ term is used in the mass conservation equation to calculated $u_{c, k+1}$.

$$
u_{b, k+1}=u_{b, k+1}^{0.1} u_{b, k}^{0.9}
$$

The friction factor is calculated from one of the four pre-defined CTF friction factor correlations. They are shown in Equations 6.20-6.23. Note that all correlations return the Darcy friction factor except for the Churchill correlation (Equation 6.23). The Churchill correlation returns the Fanning friction factor, which is four times smaller than the Darcy friction factor. The friction factor correlations are dependent on fluid velocity, which is why an iterative approach is required. The Blasius correlation is used to test the custom input option. It is shown in Equation 6.24. 


$$
\begin{gathered}
f=\max \left(1.691 R e^{-0.43}, 0.117 R e^{-0.14}\right) \\
f=0.204 R e^{-0.2} \\
f=\frac{1}{\sqrt{f}}=-2.0 \log _{10}\left(\frac{\epsilon}{3.7 D}+\frac{2.51}{R e}\left[1.14-2 \log _{10}\left(\frac{\epsilon}{D}+\frac{21.25}{R e^{0.9}}\right)\right]\right) \\
f_{w}=2\left[\left(\frac{8}{R e}\right)^{12}+\frac{1}{(a+b)^{3 / 2}}\right]^{1 / 12}, \text { where: } \\
\left.a=\left(2.475 \ln \left[\frac{1}{\left(\frac{7}{R e}\right.}\right)^{0.9}+0.27\left(\frac{\epsilon}{D_{h}}\right)\right]\right)^{16} \\
b=\left(\frac{3.753 x 10^{4}}{R e}\right)^{16} \\
f=0.316 R e^{-0.25}
\end{gathered}
$$

In the channel-centered system, there are three unique channel types: 1) corner channel, 2) side channel, and 3) inner channel. The system of equations for the channel-centered model is as follows:

$$
\begin{aligned}
u_{c, k+1} & =\frac{\dot{m}}{4 \rho A_{c}+8 \sqrt{\frac{f_{c}}{f_{s}} \frac{D h_{s}}{D h_{c}}} \rho A_{s}+4 \sqrt{\frac{f_{c}}{f_{i}} \frac{D h_{i}}{D h_{c}}} \rho A_{i}} \\
u_{s, k+1} & =\sqrt{\frac{f_{c}}{f_{s}} u_{c, k+1}^{2} \frac{D h_{s}}{D h_{c}}} \\
u_{i, k+1} & =\sqrt{\frac{f_{s}}{f_{i}} u_{s, k+1}^{2} \frac{D h_{i}}{D h_{s}}}
\end{aligned}
$$

The subscripts $c, s$, and $i$ represent the "corner", "side", and "inner" type channels, respectively. All other nomenclature is the same as it was for the rod-centered approach. The $u_{c, k+1}$ velocity is also relaxed after each iteration, similar to what was shown in Equation 6.19. The relaxed term is used in the calculation of $u_{s, k+1}$ and $u_{i, k+1}$.

Python scripts are created for the purpose of performing the iterative solution for each of the tests outlined in Tables 39 and 40. Additionally, the scripts read CTF output files and extract the flow distribution for comparison with the analytical solutions. The scripts can be found in the test directory in the COBRA-TF repository. 
CTF Input Model Description The test sections are made sufficiently long so that an equilibrium flow distribution is achieved by the exit of the bundle. Axial meshing is set to $0.2 \mathrm{~m}$, as it was shown in Section 6.2.2.1 that axial mesh size has no impact on the flow redistribution. Outlet pressure is set to 155.13 bar and inlet temperature is set to $292.78^{\circ} \mathrm{C}$. Inlet mass flow rate is set to about $3 \mathrm{~kg} \mathrm{~s}^{-1}$, which corresponds to around $3500 \mathrm{~kg} \mathrm{~m}^{-2} \mathrm{~s}^{-1}$. The test cases are unheated and turbulent mixing and void drift are disabled so that the ideal flow distribution may be achieved.

Discussion of Results Table 41 shows the comparison between CTF-predicted exit velocities and the corresponding expected results. Results are shown for each channel type along with the relative error between CTF-predicted results and expected results. Relative error is always less than $0.10 \%$. Table 42 shows a comparison between the linear pressure drop in the top level of the CTF model and the expected linear pressure drop using the expected velocity distribution and relevant friction correlation. At the exit of the CTF model, the flow is in mechanical equilibrium, and so the pressure drop is constant everywhere in the radial direction. Agreement between expected and CTF results is also good; with answers being identical to three decimal places and relative error staying below $0.15 \%$.

Results for the rod-centered models are shown in Table 43 for velocity and in Table 44 for linear pressure drop. Similar to the channel-centered results, comparison with expected results is good. 
Table 41: Comparison of CTF-predicted and expected velocity for channel-centered model

\begin{tabular}{|c|c|c|c|c|c|c|c|c|c|}
\hline \multirow[b]{2}{*}{ Test } & \multicolumn{3}{|c|}{ Corner } & \multicolumn{3}{|c|}{ Side } & \multicolumn{3}{|c|}{ Inner } \\
\hline & $\begin{array}{r}\text { Expected } \\
{[\mathrm{m} / \mathrm{s}]}\end{array}$ & $\begin{array}{l}\mathrm{CTF} \\
{[\mathrm{m} / \mathrm{s}]}\end{array}$ & $\begin{array}{r}\text { Error } \\
{[\%]}\end{array}$ & $\begin{array}{r}\text { Expected } \\
{[\mathrm{m} / \mathrm{s}]}\end{array}$ & $\begin{array}{l}\mathrm{CTF} \\
{[\mathrm{m} / \mathrm{s}]}\end{array}$ & $\begin{array}{r}\text { Error } \\
{[\%]}\end{array}$ & $\begin{array}{r}\text { Expected } \\
{[\mathrm{m} / \mathrm{s}]}\end{array}$ & $\begin{array}{r}\mathrm{CTF} \\
{[\mathrm{m} / \mathrm{s}]}\end{array}$ & $\begin{array}{r}\text { Error } \\
{[\%]}\end{array}$ \\
\hline cc_test1a & 4.735 & 4.737 & 0.03 & 5.090 & 5.090 & 0.00 & 5.104 & 5.104 & -0.00 \\
\hline cc_test1b & 4.708 & 4.709 & 0.04 & 5.092 & 5.092 & 0.00 & 5.108 & 5.107 & -0.00 \\
\hline cc_test1c & 4.716 & 4.718 & 0.04 & 5.091 & 5.091 & 0.00 & 5.106 & 5.106 & -0.00 \\
\hline cc_test1d & 4.717 & 4.719 & 0.05 & 5.091 & 5.091 & 0.00 & 5.106 & 5.106 & -0.01 \\
\hline cc_test1e & 4.683 & 4.686 & 0.06 & 5.094 & 5.094 & 0.00 & 5.111 & 5.111 & -0.01 \\
\hline cc_test2a & 5.071 & 5.072 & 0.02 & 5.450 & 5.450 & 0.00 & 5.063 & 5.063 & -0.00 \\
\hline cc_test $2 b$ & 5.055 & 5.057 & 0.03 & 5.468 & 5.468 & -0.01 & 5.047 & 5.047 & 0.01 \\
\hline cc_test2c & 5.060 & 5.062 & 0.04 & 5.462 & 5.462 & -0.01 & 5.052 & 5.053 & 0.01 \\
\hline cc_test $2 \mathrm{~d}$ & 5.061 & 5.063 & 0.04 & 5.462 & 5.461 & -0.01 & 5.053 & 5.053 & 0.01 \\
\hline cc_test2e & 5.042 & 5.045 & 0.06 & 5.484 & 5.483 & -0.03 & 5.033 & 5.034 & 0.03 \\
\hline cc_test3a & 5.071 & 5.072 & 0.02 & 5.450 & 5.450 & 0.00 & 5.063 & 5.063 & -0.00 \\
\hline cc_test $3 b$ & 5.055 & 5.057 & 0.03 & 5.468 & 5.468 & -0.01 & 5.047 & 5.047 & 0.01 \\
\hline cc_test3c & 5.264 & 5.266 & 0.05 & 5.682 & 5.681 & -0.02 & 4.766 & 4.767 & 0.02 \\
\hline cc_test3d & 5.277 & 5.279 & 0.05 & 5.694 & 5.693 & -0.02 & 4.750 & 4.751 & 0.02 \\
\hline cc_test3e & 5.042 & 5.045 & 0.06 & 5.484 & 5.483 & -0.03 & 5.033 & 5.034 & 0.03 \\
\hline cc_test4a & 3.164 & 3.166 & 0.05 & 4.285 & 4.285 & 0.01 & 6.253 & 6.252 & -0.01 \\
\hline cc_test $4 b$ & 3.029 & 3.031 & 0.06 & 4.213 & 4.214 & 0.03 & 6.355 & 6.353 & -0.02 \\
\hline cc_test $4 \mathrm{c}$ & 3.042 & 3.044 & 0.07 & 4.232 & 4.234 & 0.04 & 6.332 & 6.330 & -0.03 \\
\hline cc_test $4 \mathrm{~d}$ & 3.045 & 3.047 & 0.07 & 4.235 & 4.237 & 0.05 & 6.329 & 6.326 & -0.04 \\
\hline cc_test $4 \mathrm{e}$ & 2.913 & 2.917 & 0.11 & 4.148 & 4.152 & 0.09 & 6.444 & 6.440 & -0.07 \\
\hline cc_test5a & 3.164 & 3.166 & 0.05 & 4.285 & 4.285 & 0.01 & 6.253 & 6.252 & -0.01 \\
\hline cc_test5b & 3.029 & 3.031 & 0.06 & 4.213 & 4.214 & 0.03 & 6.355 & 6.353 & -0.02 \\
\hline cc_test $5 \mathrm{c}$ & 3.303 & 3.304 & 0.04 & 4.399 & 4.399 & 0.01 & 6.109 & 6.108 & -0.01 \\
\hline cc_test5d & 3.300 & 3.302 & 0.04 & 4.396 & 4.397 & 0.01 & 6.112 & 6.111 & -0.01 \\
\hline cc_test5e & 2.913 & 2.917 & 0.11 & 4.148 & 4.152 & 0.09 & 6.444 & 6.440 & -0.07 \\
\hline
\end{tabular}


Table 42: Comparison of CTF-predicted and expected linear pressure drop for channel-centered model

\begin{tabular}{lrrr}
\hline Test & $\begin{array}{r}\text { Expected } \\
{[\mathrm{bar} / \mathrm{m}]}\end{array}$ & $\begin{array}{r}\text { CTF } \\
{[\mathrm{bar} / \mathrm{m}]}\end{array}$ & $\begin{array}{r}\text { Error } \\
{[\%]}\end{array}$ \\
\hline cc_test1a & 0.147 & 0.147 & -0.09 \\
cc_test1b & 0.117 & 0.117 & -0.13 \\
cc_test1c & 0.104 & 0.104 & -0.11 \\
cc_test1d & 0.102 & 0.102 & -0.07 \\
cc_test1e & 0.094 & 0.094 & -0.10 \\
cc_test2a & 0.167 & 0.167 & -0.12 \\
cc_test2b & 0.133 & 0.133 & -0.08 \\
cc_test2c & 0.118 & 0.118 & -0.07 \\
cc_test2d & 0.116 & 0.116 & -0.10 \\
cc_test2e & 0.107 & 0.107 & -0.09 \\
cc_test3a & 0.167 & 0.167 & -0.12 \\
cc_test3b & 0.133 & 0.133 & -0.08 \\
cc_test3c & 0.127 & 0.127 & -0.06 \\
cc_test3d & 0.125 & 0.125 & -0.06 \\
cc_test3e & 0.107 & 0.107 & -0.09 \\
cc_test4a & 0.215 & 0.215 & -0.11 \\
cc_test4b & 0.173 & 0.173 & -0.10 \\
cc_test4c & 0.154 & 0.154 & -0.06 \\
cc_test4d & 0.151 & 0.151 & -0.05 \\
cc_test4e & 0.141 & 0.141 & -0.03 \\
cc_test5a & 0.215 & 0.215 & -0.11 \\
cc_test5b & 0.173 & 0.173 & -0.10 \\
cc_test5c & 0.220 & 0.220 & -0.09 \\
cc_test5d & 0.218 & 0.218 & -0.11 \\
cc_test5e & 0.141 & 0.141 & -0.03 \\
\hline
\end{tabular}


Table 43: Comparison of CTF-predicted and expected linear pressure drop for rod-centered model

\begin{tabular}{lrrrrrrrr}
\hline & \multicolumn{3}{c}{ Boundary } & & \multicolumn{3}{c}{ Center } \\
\cline { 2 - 3 } \cline { 7 - 8 } Test & $\begin{array}{r}\text { Expected } \\
{[\mathrm{m} / \mathrm{s}]}\end{array}$ & $\begin{array}{r}\text { CTF } \\
{[\mathrm{m} / \mathrm{s}]}\end{array}$ & $\begin{array}{r}\text { Error } \\
{[\%]}\end{array}$ & $\begin{array}{r}\text { Expected } \\
{[\mathrm{m} / \mathrm{s}]}\end{array}$ & $\begin{array}{r}\text { CTF } \\
{[\mathrm{m} / \mathrm{s}]}\end{array}$ & $\begin{array}{r}\text { Error } \\
{[\%]}\end{array}$ \\
\hline rc_test1a & 4.987 & 4.987 & 0.00 & & 4.987 & 4.987 & 0.00 \\
rc_test1b & 4.987 & 4.987 & 0.00 & & 4.987 & 4.987 & 0.00 \\
rc_test1c & 4.987 & 4.987 & 0.00 & & 4.987 & 4.987 & 0.00 \\
rc_test1d & 4.987 & 4.987 & 0.00 & & 4.987 & 4.987 & 0.00 \\
rc_test2a & 5.281 & 5.281 & 0.00 & & 3.800 & 3.801 & 0.01 \\
rc_test2b & 5.290 & 5.290 & 0.01 & & 3.698 & 3.699 & 0.01 \\
rc_test2c & 5.288 & 5.288 & 0.01 & & 3.720 & 3.721 & 0.02 \\
rc_test2d & 5.288 & 5.288 & 0.01 & & 3.724 & 3.724 & 0.02 \\
rc_test3a & 4.987 & 4.987 & 0.00 & & 4.987 & 4.987 & 0.00 \\
rc_test3b & 4.987 & 4.987 & 0.00 & & 4.987 & 4.987 & 0.00 \\
rc_test3c & 5.089 & 5.090 & 0.01 & & 4.165 & 4.166 & 0.02 \\
rc_test3d & 5.092 & 5.093 & 0.01 & & 4.143 & 4.144 & 0.02 \\
\hline
\end{tabular}

Table 44: Comparison of CTF-predicted and expected linear pressure drop for rod-centered model

\begin{tabular}{lrrr}
\hline Test & $\begin{array}{r}\text { Expected } \\
{[\text { bar/m] }}\end{array}$ & $\begin{array}{r}\text { CTF } \\
{[\text { bar } / \mathrm{m}]}\end{array}$ & $\begin{array}{r}\text { Error } \\
{[\%]}\end{array}$ \\
\hline rc_test1a & 0.141 & 0.141 & -0.11 \\
rc_test1b & 0.112 & 0.112 & -0.06 \\
rc_test1c & 0.100 & 0.100 & -0.12 \\
rc_test1d & 0.098 & 0.098 & -0.12 \\
rc_test2a & 0.157 & 0.157 & -0.06 \\
rc_test2b & 0.125 & 0.124 & -0.09 \\
rc_test2c & 0.111 & 0.111 & -0.08 \\
rc_test2d & 0.109 & 0.109 & -0.12 \\
rc_test3a & 0.141 & 0.141 & -0.11 \\
rc_test3b & 0.112 & 0.112 & -0.06 \\
rc_test3c & 0.104 & 0.103 & -0.08 \\
rc_test3d & 0.102 & 0.101 & -0.11 \\
\hline
\end{tabular}


Conclusions An extensive set of tests is applied to CTF in this study. The four pre-defined friction factor models and their implementation are verified. A $3 \times 3$ bundle with guide tube in the center is used as the base model. Several perturbations of this model are made by changing guide tube dimensions, rod roughness, and the presence of a rod bundle wall. All of these changes cause changes to the flow distribution due to frictional pressure drop. Because void drift and turbulent mixing are disabled, the flow distribution is solely defined by the frictional losses. The ideal flow split is analytically calculated for the test suite in this study and results are compared to CTF predictions. CTF-predicted velocity matches expected results within $0.1 \%$ relative error for both rod-centered and channel-centered models. CTF-predicted linear pressure drop matches expected results within $0.15 \%$ for all models. This study indicates proper implementation of the friction factor models in CTF.

\subsubsection{Single-Phase Axial and Lateral Pressure drop Verification}

In CTF, single-phase pressure drop can arise due to form loss (spacer grids, orifice plates, and/or any other geometrical obstructions) and wall friction/shear, acceleration/deceleration, and gravity. In lateral pipe flow, pressure drop caused by gravity, as well as pressure drop due to turbulent mixing, are negligible for the geometry under consideration. As stated previously, it is possible to disable modeling parameters in CTF to switch on and test individual pressure-drop components.

This set of tests is used to verify that (a) the code converges within zeroth order for axial pipe flow with only form loss turned on, and (b) horizontal/lateral pipe flow with zero total, form loss, and friction loss. Although convergence is not checked for the lateral pipe flow tests, it is expected that the code will converge within first order for wall friction and zeroth order for form loss and acceleration/deceleration pressure drops.

Problem Description Two sets of tests are performed: axial pipe flow and horizontal pipe flow. In the axial pipe flow test, as previously stated, the total pressure drop (inlet-outlet) can be obtained by integrating the steady-state axial momentum equation in CTF as:

$$
\Delta P_{\text {total, axial }}=\Delta P_{\text {friction }}+\Delta P_{\text {accel }}+\Delta P_{\text {gravity }}
$$

For purely axial pipe flow with wall friction and gravity turned off, the only contribution towards the pressure drop comes from form loss, so the total pressure drop can be calculated as follows:

$$
\Delta P_{\text {total,axial }}=\frac{k \rho u^{2}}{2}
$$

The term $k$ represents the form loss coefficient, and the term $u$ represents liquid velocity. For the lateral pipe flow tests, the steady-state lateral momentum equation can be integrated to obtain the lateral pressure drop (ignoring gravity):

$$
\Delta P_{\text {total, lateral }}=\Delta P_{\text {friction }}+\Delta P_{\text {accel }}
$$

The frictional pressure drop is subdivided into wall friction and form loss. Pressure drop due to form loss 
can be obtained using Eq. (6.29), whereas the wall friction/wall drag can be obtained using Eq. (6.9), as follows:

$$
\Delta P_{\text {frictional, lateral }}=\frac{f \rho u^{2} L}{2 D_{h}} .
$$

The terms $f, u$, and $D_{h}$ represent the Darcy friction factor, liquid velocity, and channel hydraulic diameter, respectively. By referring to Eq. (6.1), for the case of a mass flow rate inlet boundary condition in which the inlet momentum flux is zero, the acceleration pressure drop can be obtained as follows:

$$
\Delta P_{\text {accel,lateral }}=\left(\frac{G_{m}^{2}}{\rho_{m}^{+}}\right)_{\text {out }} .
$$

For decelerating flow, the inlet momentum flux in Eq. (6.1) is non-zero, while the outlet momentum flux is zero, leading to a rise in pressure. In the lateral pipe flow case, with mass flow rate inlet and pressure outlet boundary conditions and zero frictional pressure drop, the acceleration pressure drop should match the deceleration pressure drop, giving a net pressure drop of zero within numerical error. In order to isolate the deceleration pressure drop component, a mass source boundary condition is defined in the first lateral momentum cell, along with a pressure outlet. Similarly, a pressure sink can be defined in the last lateral momentum cell, along with a mass flow inlet to isolate the acceleration pressure drop.

CTF Input Model Description For the axial pipe flow, the pipe dimensions are set in a manner similar to that used for a PWR subchannel, with a mass source inlet boundary condition of $0.62395 \mathrm{~kg} \mathrm{~s}^{-1}$, and an outlet pressure of 150 bar using a constant density of $700 \mathrm{~kg} \mathrm{~m}^{-3} \mathrm{~s}^{-1}$ for water. Form loss is specified at one location ( $5^{\text {th }}$ node), with the form loss coefficient set to 0.5 , and the friction factor and gravity set to zero. The axial mesh is successively refined, with the coarsest mesh having an uniform size of $1 \mathrm{~m}$, and the finest mesh with a size of $0.02 \mathrm{~m}$.

For the lateral pipe flow case, four tests are performed: zero pressure drop, form loss pressure drop, frictional pressure drop, and deceleration pressure drop. For the first three cases, a mass flow rate inlet of $100.0 \mathrm{~kg} \mathrm{~s}^{-1}$ is specified, with an outlet pressure of 100 bar. For the deceleration pressure drop case, a mass source of $100.0 \mathrm{~kg} \mathrm{~s}^{-1}$ is defined in the first lateral momentum cell with an outlet pressure of 100 bar. A form loss coefficient of 1.0 is used for the form loss pressure drop test, and a constant friction factor of 0.5 is used for the frictional pressure drop test. The density of water is set to $900.0 \mathrm{~kg} \mathrm{~s}^{-1}$ for both the form loss and frictional pressure drop cases, and it is set to $1000.0 \mathrm{~kg} \mathrm{~s}^{-1}$ for the deceleration pressure drop case. For all four cases, one axial node is used with 10 channels (9 gaps), with a gap width of $0.1 \mathrm{~m}$, and axial mesh size of $0.1 \mathrm{~m}$.

Discussion of Results Figure 6.6 shows zeroth order convergence for series of successive mesh refinements, and Figure 6.6 shows the $L_{\text {inf }}$ norm between the expected pressure drop of approx 0.18 bar and the calculated pressure drop for series of successive mesh refinements. The $L_{\text {inf }}$ norm is on the order of $1 \mathrm{E}-4$, which demonstrates the good agreement between CTF prediction and expected pressure drop.

Table 45 shows the difference between the expected and CTF-predicted pressure drops for the four lateral flow cases: zero pressure drop $\left(z e r o \_d p\right)$, form loss pressure drop $($ form_d $d p)$, frictional pressure drop $($ fric_dp $)$, and 


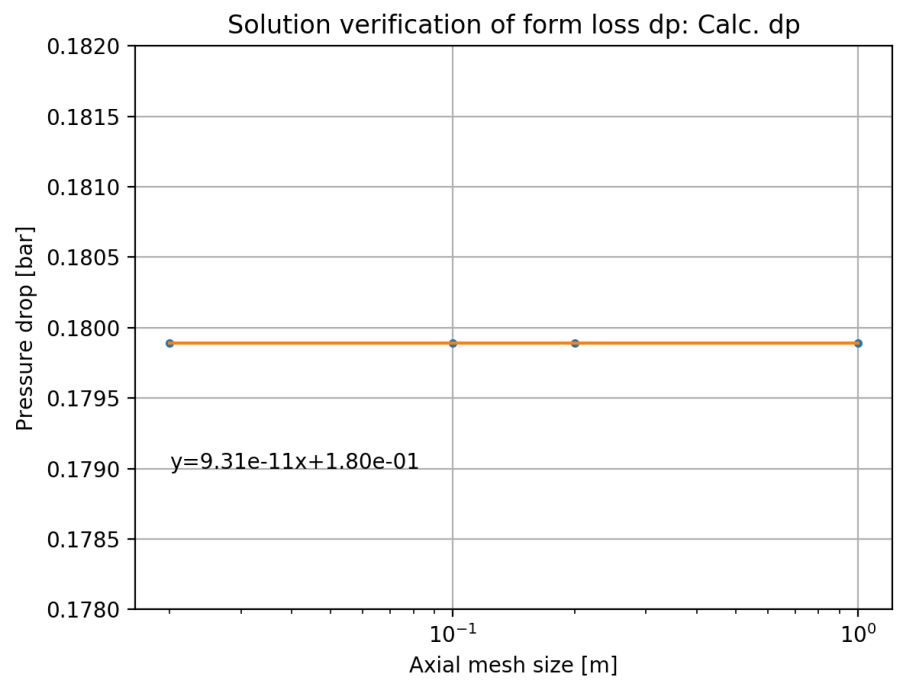

Figure 6.6: CTF-prediction and curve fit of pressure drop for axial pipe flow for successive mesh refinements.

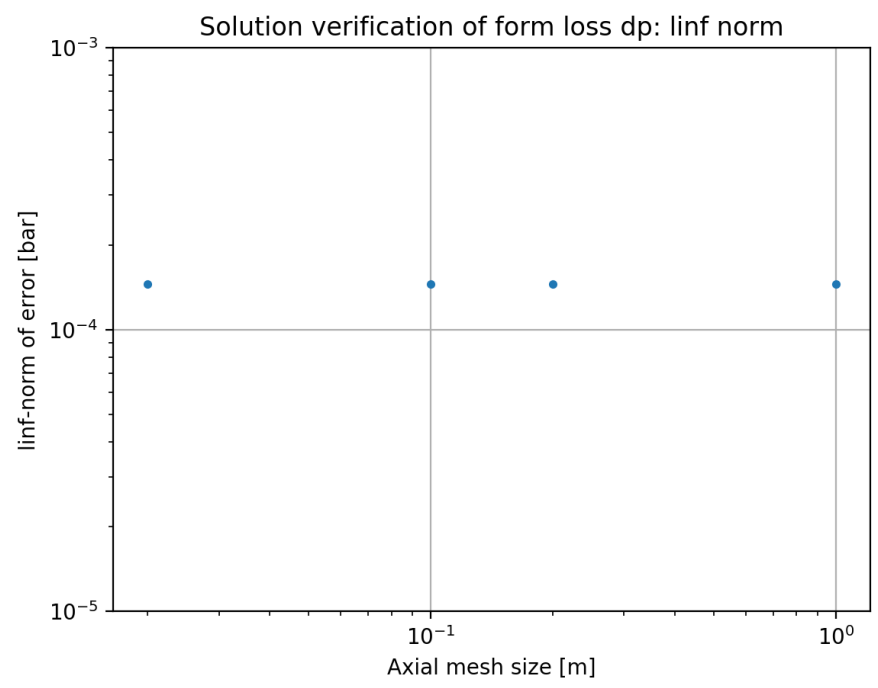

Figure 6.7: $L_{\text {inf }}$ norm of CTF-prediction and expected pressure drop for axial pipe flow for successive mesh refinements. 
Table 45: Comparison of CTF-predicted and expected pressure drop for the lateral flow case

\begin{tabular}{lrrrr}
\hline Test & $\begin{array}{r}\text { Expected } \\
{[\text { bar }]}\end{array}$ & $\begin{array}{r}\text { CTF } \\
{[\text { bar }]}\end{array}$ & $\begin{array}{r}\text { Abs. Error } \\
{[\text { bar }]}\end{array}$ & $\begin{array}{r}\text { Rel. error } \\
{[-]}\end{array}$ \\
\hline zero_dp & 0.0000 & $1.46 \mathrm{E}-9$ & $1.46 \mathrm{E}-9$ & NA \\
form_dp & 5.0000 & 4.9959 & $4.03 \mathrm{E}-3$ & $8.06 \mathrm{E}-4$ \\
fric_dp & 1.3889 & 1.3878 & $1.10 \mathrm{E}-3$ & $8.06 \mathrm{E}-4$ \\
decc_dp & 1.0000 & 0.9991 & $8.80 \mathrm{E}-4$ & $8.78 \mathrm{E}-4$ \\
\hline
\end{tabular}

deceleration pressure drop $\left(d e c c_{-} d p\right)$. The relative errors for all cases are within $1 \mathrm{E}-3$, demonstrating that CTF predicts the expected pressure drop.

Conclusions An extensive set of pressure drop tests were conducted with CTF in this study to test different single-phase pressure drop components for axial and lateral pipe flow geometries. Form loss for both axial and lateral pipe flow, as well as frictional pressure drop and deceleration pressure drops, are verified with the expected solutions, thus indicating their proper implementation within CTF.

\subsubsection{Flow expansion}

The flow expansion problem involves axial flow expanding into a more open cross sectional area. This feature can be used for capturing variation in axial rod and channel geometry. To capture this effect, the model is set up using a flow area and wetted perimeter axial modification table in CTF. This test ensures that CTF correctly predicts the deceleration of the fluid, frictional pressure drop in lower and upper sections of the model, form loss pressure drop, and deceleration pressure rise. The model is set up for single-phase flow to ensure that an analytical solution can easily be calculated.

Problem Description The model includes two channels connected to one rod. The channel area and wetted perimeter increase at one axial location and then again at a second location. A schematic of the model is shown in Figure 6.8. The model length is $2.0 \mathrm{~m}$, with the first variation occurring at $1.0 \mathrm{~m}$ from the inlet $\left(L_{1}=1.0 \mathrm{~m}\right)$ and the second variation occurring at $1.6 \mathrm{~m}\left(L_{2}=0.6 \mathrm{~m}\right)$. The inlet boundary conditions are as follows: a total mass flow rate of $0.9227 \mathrm{~kg} \mathrm{~s}^{-1}$, an inlet temperature of $200{ }^{\circ} \mathrm{C}$, and an outlet pressure of 150 bar. The model is unheated. The nominal flow area of each channel is $1.2332 \times 10^{-4} \mathrm{~m}^{2}$, and the wetted perimeter of each channel is $5.2723 \times 10^{-2} \mathrm{~m}$. The diameter of the rod is $9.5 \times 10^{-3} \mathrm{~m}$. The width of the gap between the two channels is $3.1 \times 10^{-3} \mathrm{~m}$, and its length from channel to channel is $0.0126 \mathrm{~m}$. A lateral form loss coefficient of 0.5 is applied in the gap. A form loss coefficient of 1.0 is also applied at the location of both expansions.

To make an analytical solution possible, the liquid density is set to a constant value of $900 \mathrm{~kg} \mathrm{~m}^{-3}$, and the friction factor is set to a constant of 0.1. As mass flow rate will be conserved over the geometric expansion, the velocity in the expanded region can be related to the inlet velocity as follows: 


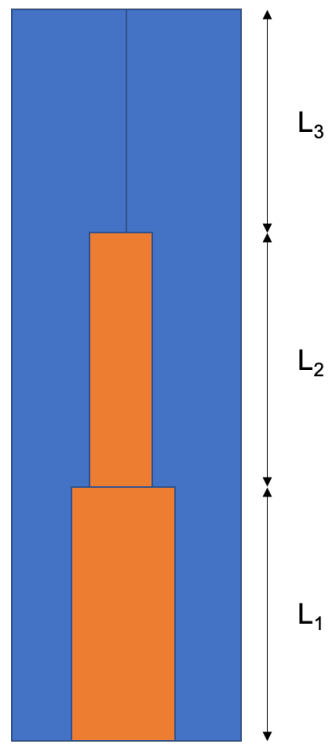

Figure 6.8: Example of flow expansion model geometry.

$$
\begin{aligned}
\dot{m}_{\text {in }} & =\dot{m}_{\text {out }} \\
\rho v_{\text {in }} A_{\text {in }} & =\rho v_{\text {out }} A_{\text {out }} \\
v_{\text {out }} & =\frac{A_{\text {in }}}{A_{\text {out }}} v_{\text {in }}
\end{aligned}
$$

In the equation, "in" and "out" refer to before and after an expansion, $\rho$ is the liquid density, $A$ is the cross sectional flow area, and $v$ is the liquid velocity. The frictional form loss is calculated as follows:

$$
\frac{d P}{d z f}_{f}=\frac{1}{2 D_{h}} f \rho v^{2} .
$$

In the equation, $f$ is the Darcy friction factor, $D_{h}$ is the hydraulic diameter, and $\frac{d P}{d z}$ is the pressure drop per unit length. As the velocity will be one of two values, there will be different frictional pressure drops in the lower and upper sections of the model. The pressure change over the expansion will include effects of the form loss and the deceleration of the fluid. These effects are calculated as follows.

$$
\begin{aligned}
d P_{\text {loss }} & =\frac{1}{2} \rho k v_{\text {in }}^{2} \\
d P_{\text {acc }} & =\frac{1}{2} \rho\left(v_{\text {out }}^{2}-v_{\text {in }}^{2}\right)
\end{aligned}
$$




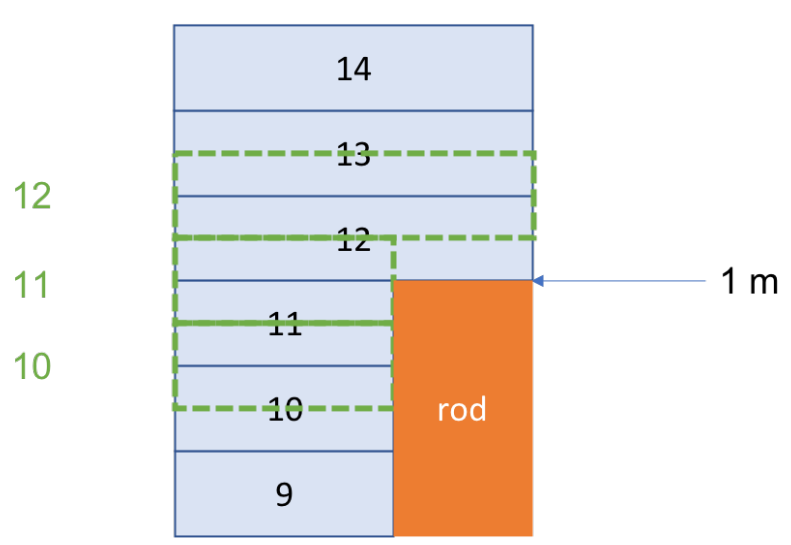

Figure 6.9: CTF staggered mesh for flow expansion test near point of expansion.

The first equation presents the pressure drop due to form loss, and the second is the pressure drop due to the slowing down of the fluid, where $k$ is the form loss coefficient.

CTF Input Model Description The axial mesh is uniform, with each cell being $0.1 \mathrm{~m}$. The inlet region has a multiplier of 1.0 applied for both the area and the wetted perimeter. The second region $\left(L_{2}\right)$ has multipliers of 1.2874 and 0.71695 applied for area and wetted perimeter (calculated based on rod diameter being reduced to zero). The third region has arbitrary multipliers of 1.5 and 0.5 applied, meaning that the area will be 1.5 times the nominal inlet area, and the wetted perimeter will be 0.5 times the nominal inlet wetted perimeter. The first expansion is modeled using a set of area and wetted perimeter modification tables that apply the change in channel geometry at axial scalar and momentum level 12 in the model, which corresponds to $1 \mathrm{~m}$. The reason that this is applied to level 12 can be visualized in Figure 6.9, which shows the CTF staggered mesh around the expansion.

Each cell in CTF is $0.1 \mathrm{~m}$. Because the first cell is a ghost cell, it is not counted when placing the location of the expansion. The top of CTF scalar cell 11 is therefore located at $1.0 \mathrm{~m}$, which is the desired point of expansion. Scalar level 12 will be the first scalar cell where the area is opened. The momentum mesh index leads the scalar index by one because the first momentum level occurs at the top of the first scalar level. Momentum level 11 will retain the inlet area, which is consistent with the geometry being modeled. Momentum level 12 will be the first momentum level to have an expanded flow area. The form loss, however, occurs at momentum cell 11, which is the top of the rod. The second expansion is applied in the same manner, but for axial level 18 instead of 12 . To further simplify the analysis, the gravity is disabled in the CTF model, so gravitational head loss does not need to be considered.

Discussion of Results The predicted and expected velocity profile in the model match nicely as indicated in Figure 6.10. The pressure drop is plotted as the total pressure drop per momentum cell. The analytical solution is calculated for each momentum cell individually. The momentum levels not affected by the form or expansion losses are simply the frictional loss using the velocity in that level. The form losses occur at momentum levels 11 and 17. The acceleration loss is spread over two momentum levels in CTF because the flow slows down over scalar level 12 (it is at the inlet value at the inlet of scalar level 12 and at the outlet value at the exit of level 12). Therefore, the deceleration drop is considered to occur between scalar levels 


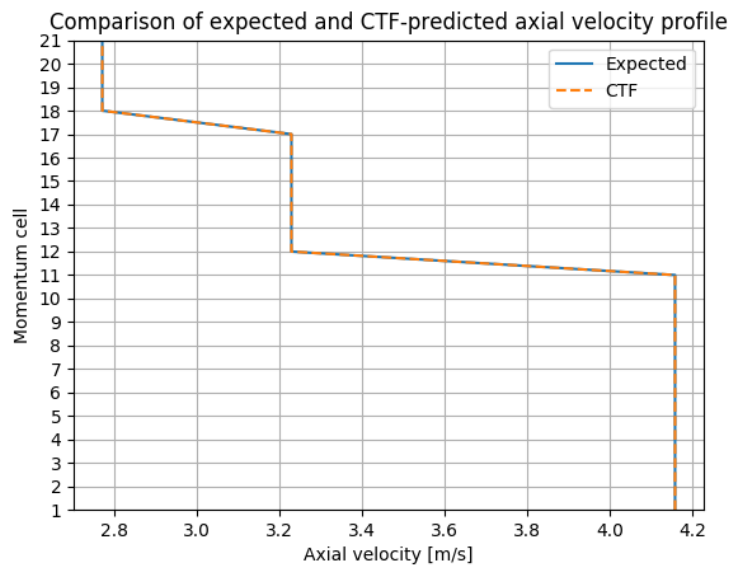

Figure 6.10: Comparison of expected and calculated velocity in expansion verification test.

11 and 13. For simplicity, half of the expected value is added to each momentum cell (11 and 12). Similarly, the deceleration for the second loss occurs over momentum cells 17 and 18. The expected and predicted pressure drop over each cell is shown in Figure 6.11.

Conclusions This demonstrates that CTF can be used to model a flow expansion and capture the deceleration and form loss components of pressure drop. This problem provides guidance on how to correctly place the form loss coefficient and expansion of area and contraction of wetted perimeter when modeling this geometry. It was discovered that the acceleration pressure drop occurs over two levels, which may require further investigation in the future. 


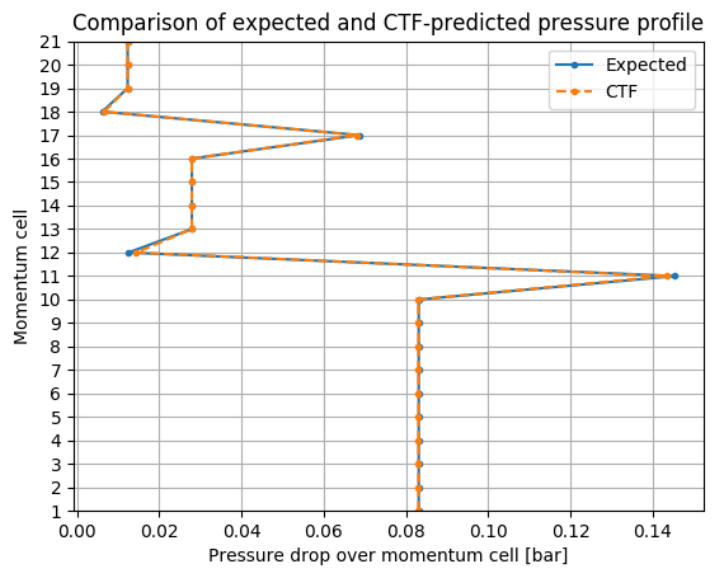

Figure 6.11: Comparison of expected and calculated pressure in expansion verification test.

\subsubsection{Single-Phase Axial Pressure Drop Verification}

In this section, the pressure distribution in CTF is verified, focusing on the friction and gravity components. The following conditions are assessed: (1) steady-state and one-dimensional flow, (2) constant density and velocity, (3) pipe geometry with a constant cross section, (4) constant friction factor, and (5) isothermal flow. Under these conditions, the mass and energy equation are eliminated, and the momentum equation is

$$
\frac{\partial P}{\partial x}-\rho g=f \frac{\rho u^{2}}{2 D} .
$$

The equation is integrated, and an outlet boundary condition $P(L)=P_{\text {out }}$ is applied. In this case, the analytic solution becomes

$$
P(x)=P_{\text {out }}-\left[f \frac{\rho u^{2}}{2 D_{h}}+\rho g\right](L-z) .
$$

A CTF input deck is created that satisfies this analytic solution with the parameters defined in Table 46. Note that the gravitational acceleration is calculated from CTF's value of $32.2 \mathrm{ft} / \mathrm{s}^{2}$.

The results are shown in Figure 6.12, and the errors are plotted in Figure 6.13. First, note that the pressure distribution is extremely accurate. In this particular case, the pressure drop is constant $(\partial P / \partial x=$ constant $)$. Under these conditions, the linear trucation error (LTE) being assessed by the convergence study identically cancels. Therefore, it is expected that CTF exactly calculates the pressure distribution, regardless of the choice of mesh. However, it appears that there is a small bias in the CTF results that is revealed because the level of numerical error is so small.

The specific cause of this bias is currently unknown. One possible cause could be inconsistent conversions between pounds-feet and pounds-mass $(32.2,32.174$, or 32.1850394$)$. However, the bias is extremely small- 
Table 46: Parameters for friction and gravity verification problem

\begin{tabular}{lccc}
\hline Parameter & Symbol & Value & Units \\
\hline Gravitational acceleration & $g$ & 9.81456 & $\mathrm{~m} / \mathrm{s}^{2}$ \\
Density & $\rho$ & 1000 & $\mathrm{~kg} / \mathrm{s}$ \\
Pipe diameter & $D$ & 0.1 & $\mathrm{~m}$ \\
Pipe length & $L$ & 1 & $\mathrm{~m}$ \\
Mass flow rate & $\dot{m}$ & 0.1 & $\mathrm{~m} / \mathrm{s}$ \\
Inlet temperature & $T_{\text {in }}$ & 200 & ${ }^{\circ} \mathrm{C}$ \\
Outlet pressure & $P_{\text {out }}$ & 150 & $\mathrm{bar}$ \\
\hline
\end{tabular}

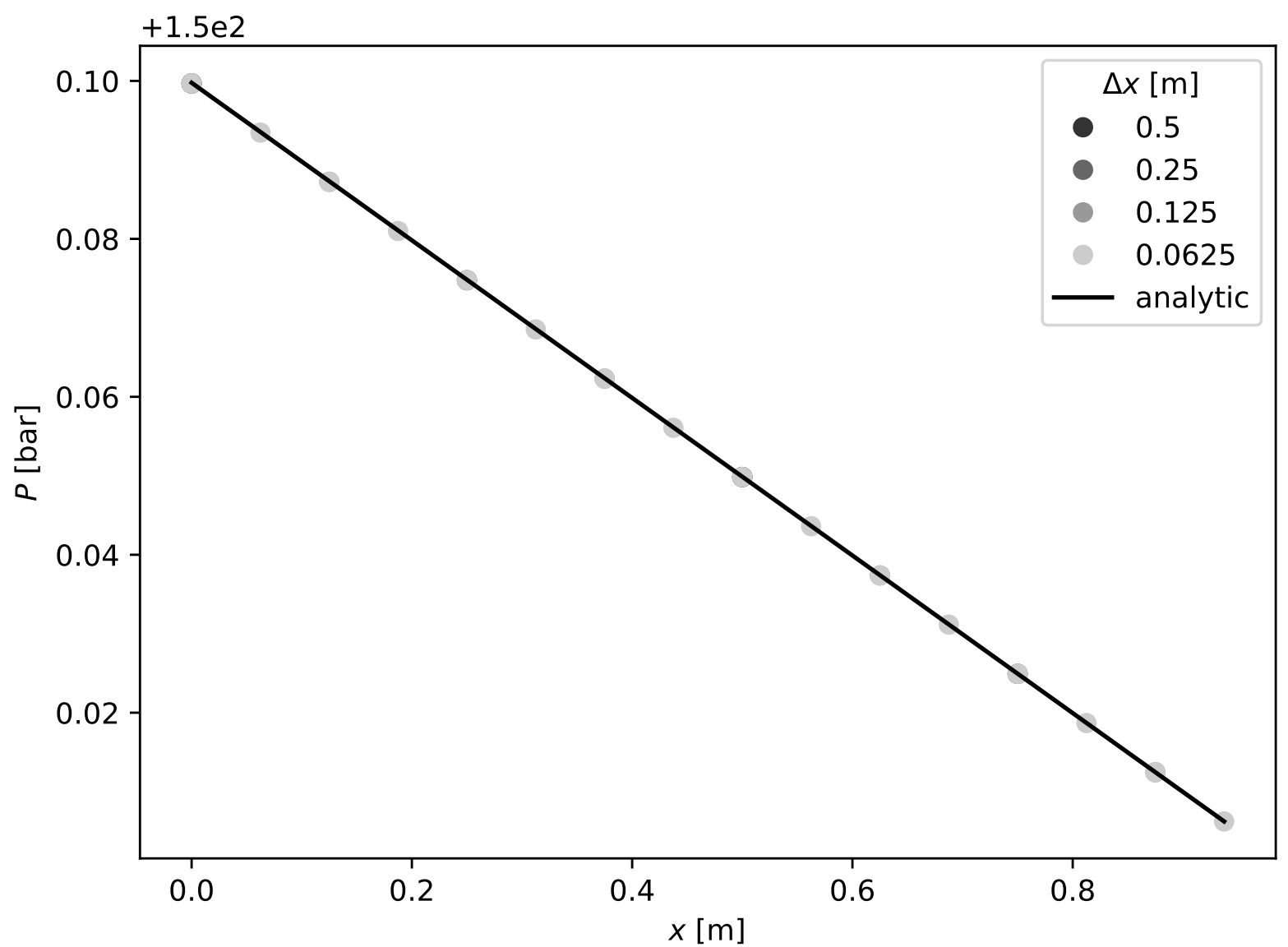

Figure 6.12: Verification results for single phase pressure drop with friction and gravity 


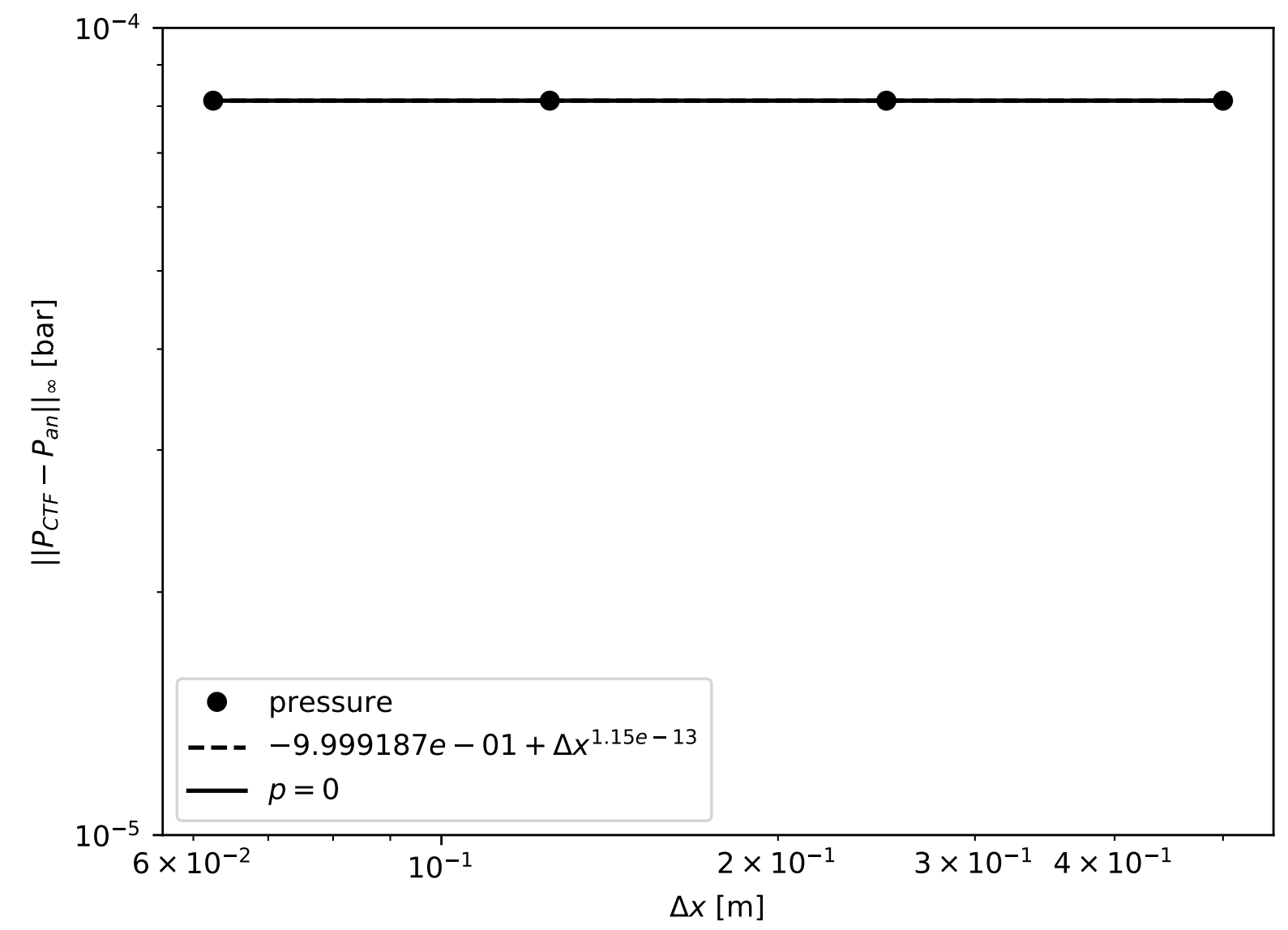

Figure 6.13: Convergence for single phase pressure drop with friction and gravity 
approximately $8 \mathrm{~Pa}$ for this case - and it results in an acceptably small error. 


\subsection{Two-phase pressure drop}

\subsubsection{Validation}

\subsubsection{BFBT}

The ratio of measured-to-predicted pressure drop is shown in Figure 6.14 with respect to bundle-averaged exit quality. The higher disagreement of Pressure taps 1, 2, and 3 become very evident in this figure and it seems there is very little correlation between the discrepancy and test quality.

The average bundle-exit quality was also measured in the experiments. This result was also compared to CTF predictions. A figure comparing the measured to predicted values for the modeled tests is shown in Figure 6.15. Dashed lines are provided in the figure to act as a guide-post of sorts, showing where $\pm 5 \%$ lies in the figure; the experimental uncertainty of the exit quality was not explicitly stated in the specification. The RMSE for the exit quality for all tests was $0.05 \%$, which indicates very good agreement with the experimental conditions. 


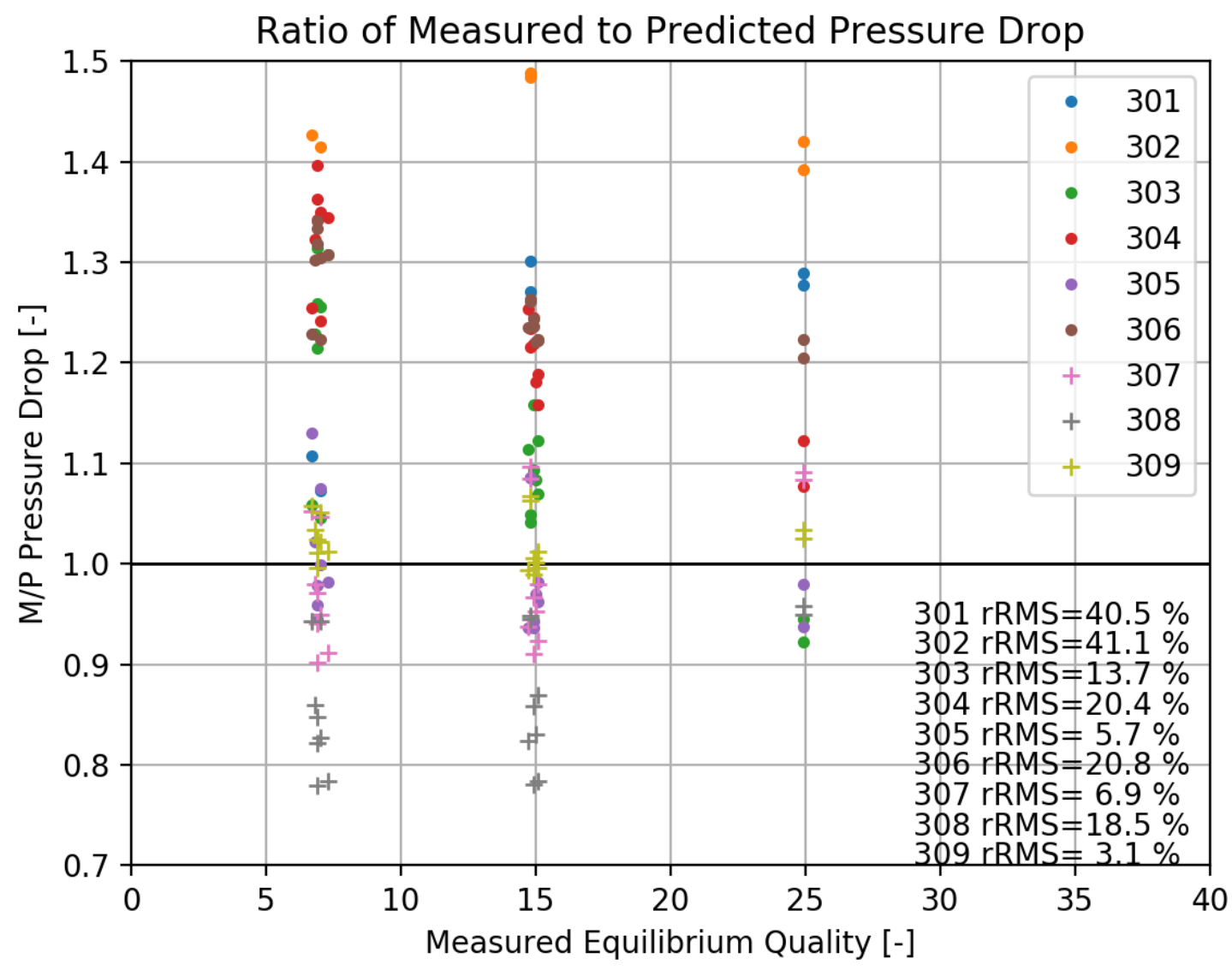

Figure 6.14: Ratio of measured-to-predicted pressure drop compared to bundle-average exit quality for BFBT two-phase tests (Series P6) 


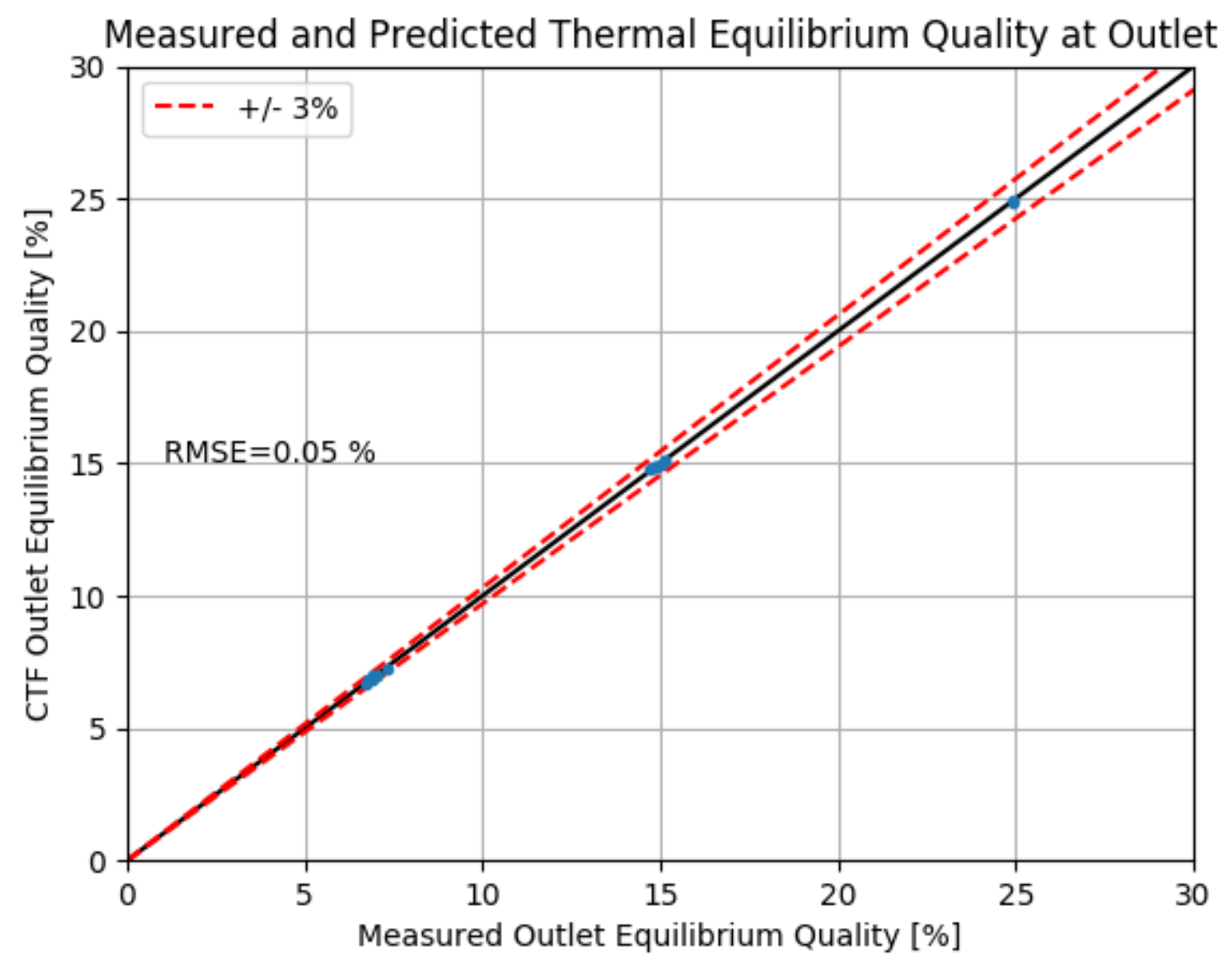

Figure 6.15: Comparison of measured and predicted bundle-averaged exit quality for BFBT P6 Series 


\subsubsection{FRIGG}

Cumulative pressure drop was broken up into three components in the experiment report: acceleration pressure drop, friction pressure drop, and gravitational pressure drop.

The comparison of the three pressure drop terms are shown in Figure 6.16. It is useful to note that pressure is shown in a cumulative fashion. In other words, the acceleration pressure drop profile includes both acceleration and gravitational pressure drop. Further, the friction pressure drop includes the effects of all three components. So, in reality, what is shown as the friction pressure drop is also the total pressure drop of the system. As this figure shows, CTF was able to match both the magnitude and behavior of each of the three components, in addition to the overall pressure drop of the system.

It should be noted, however, that the quantities used to plot experimental pressure drops were obtained from the original report using a digitizer which will contain a certain level of unknown error. Furthermore, the authors of the specification were not clear about how the components of the total pressure drop were calculated. Because of these factors, only the total pressure drop was considered in performing quantitative analysis. It can be observed that the pressure drop behavior at the bottom of the facility is not captured well in CTF, likely due to some entrance effects being missed in the CTF model. 


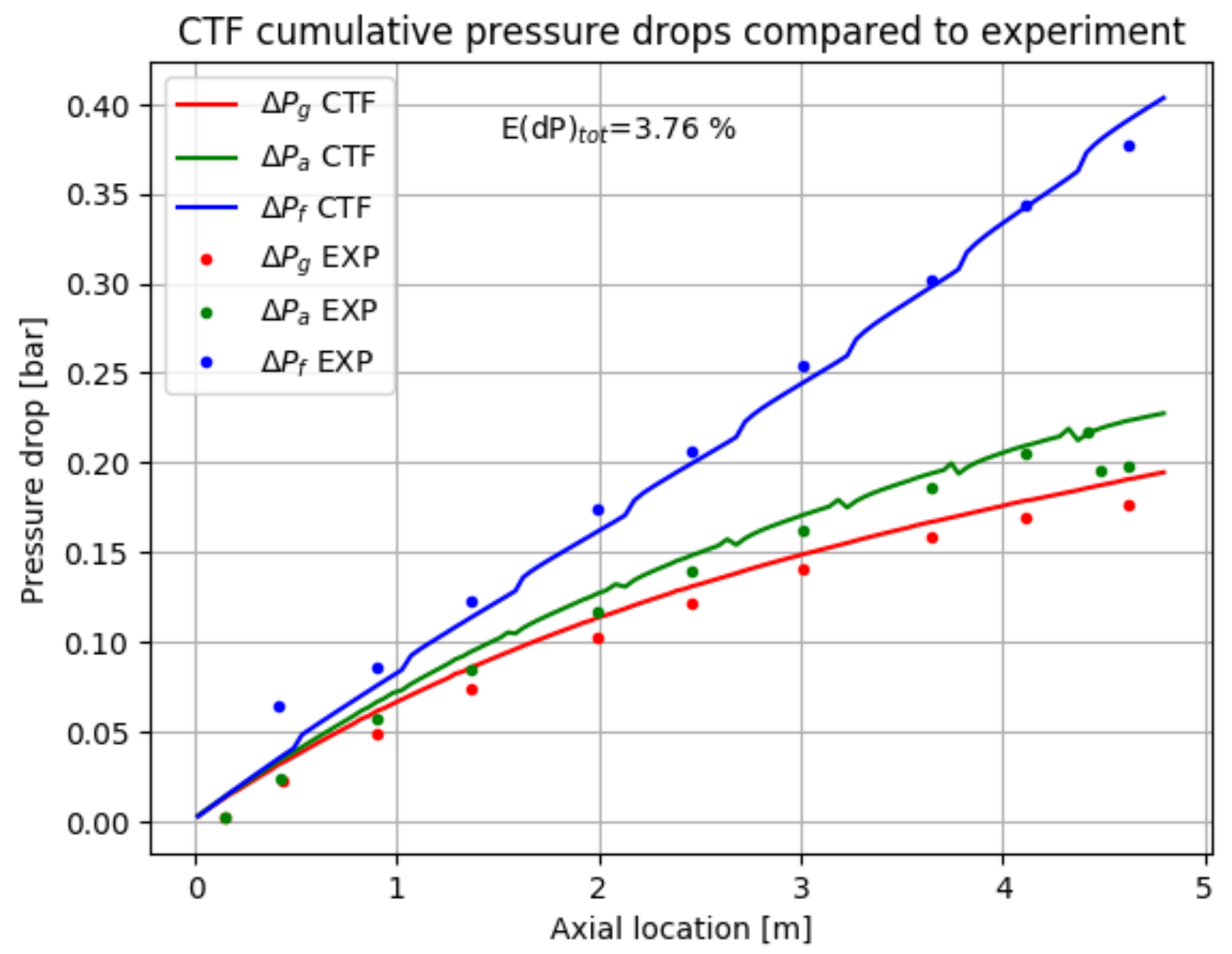

Figure 6.16: Comparison of experimental and predicted components of pressure drop 


\subsubsection{Ris $\varnothing$}

Figure 6.17 shows a comparison between CTF and the experiment for the drop in total pressure per unit length across the top $1 \mathrm{~m}$ of the test section. CTF predicts roughly three times the rate of pressure drop as was found in the experiment, despite close agreement in flow quality.

The Ris $\varnothing$ tests are more of a separate effects test because they include no form losses, no gaps, no turbulent mixing or void drift, and the high-void flow regime is maintained over the pressure drop measurement region. This is unlike the BFBT tests, where the high void regions occur only in the upper-most pressure taps and there is a form loss effect that hides the friction over-prediction. The over-prediction observed in these tests has been determined to be caused by inaccuracies in the annular-mist flow regime closure models [36] by implementing a more physical set of closure models discussed in [37]. Further analyses of the pressure drop predictions can be found in [38]. 


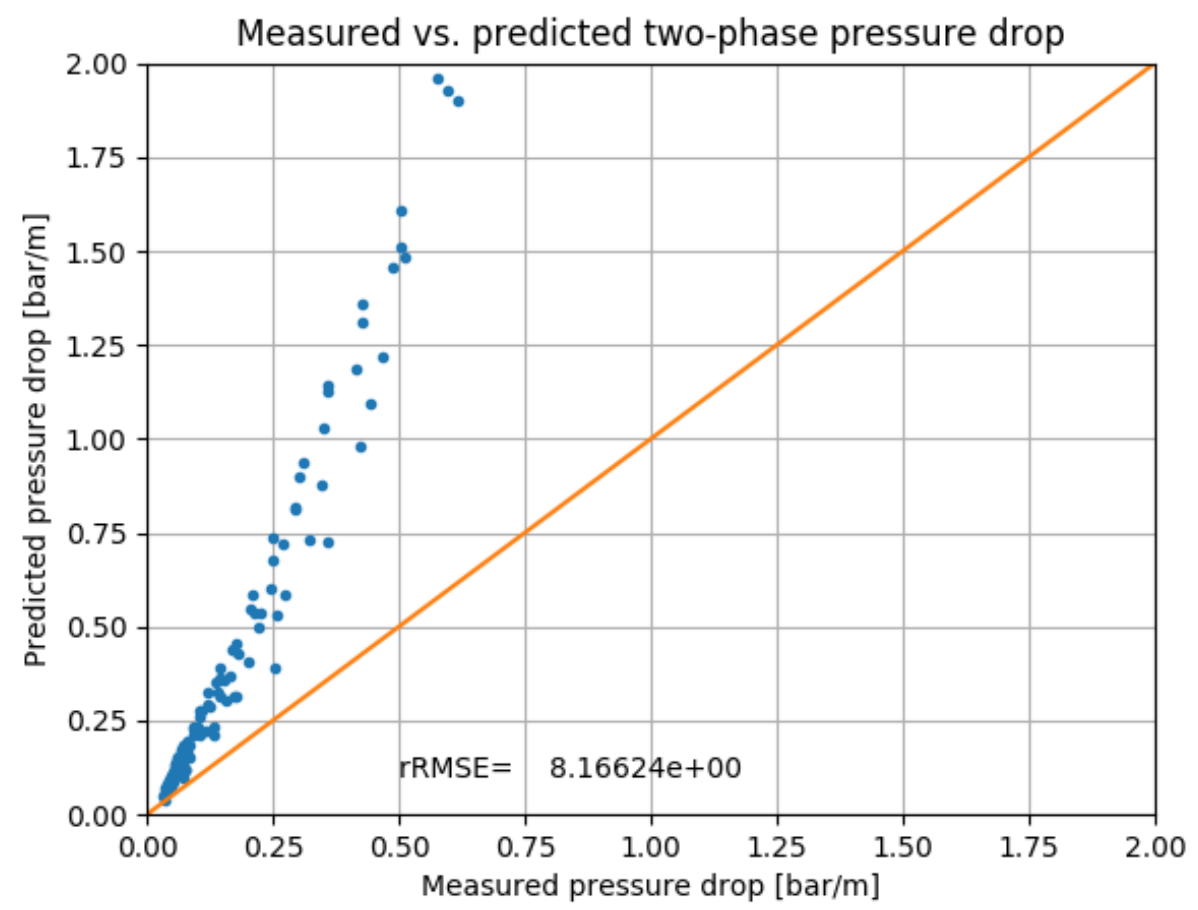

Figure 6.17: Measured vs. predicted total pressure drop per unit length at the outlet 


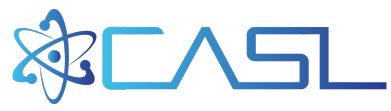

CTF V\&V

\section{Single-Phase Turbulent Mixing}

\section{$7.1 \quad$ Validation}

\subsubsection{CE 5x5}

The CE 5x5 tests were performed for the purpose of assessing heat transfer models. Test conditions were run such that all regions of the boiling curve were experienced from single-phase convection up to Critical Heat Flux (CHF). Temperature measurements were made on the rod surfaces via thermocouples attached to the inside of the heater tubes. However, it is also possible to assess coolant mixing between channels because thermocouples were also placed at the outlet of the test section in the center of each of the 36 coolant channels.

Test 74 was simulated with CTF, which was actually a collection of over 70 separate experiments with different operating conditions that led to different heat transfer mechanisms. The primary operating condition that was modified was the test heat flux. Each individual test was simulated with CTF, then the predicted outlet temperature of each channel was compared to the measured outlet temperature for that channel in the bundle. This was done for all tests in the series and then a mean difference between predicted and measured values was obtained for each channel.

It is important to note that, prior to doing any data comparison on the outlet temperatures, outlier data points were removed. You can see points for three low heat flux cases that are clearly outliers from the rest of the experimental data. To prevent such data from polluting the summary statistics, any data that led to a discrepancy of more than $50 \mathrm{C}$ between predicted and measured results was eliminated from the analysis.

Figure 7.1 is useful in demonstrating two things: first, the mean discrepancies fall between $\pm 5 \mathrm{C}$ and, second, the largest errors seem to occur in the corner and side channels of the bundle. A more quantitative view of the data is presented in Figure 7.2. This figure shows a few important things. First, the data is organized by channel type: red dots represent the side-type channels, green dots represent the inner-type channels, and blue dots represent the corner type channels. Additionally, the data are plotted against channel index on the x-axis. The indexing scheme is such that channel numbers start at the north-west corner of the bundle and increase going west-to-east and north-to-south. What we see in this figure is similar to what we saw in Figure 7.1; the corner and side channel discrepancies are generally further from the experimental results than the inner-type channels.

Additionally, this plot also shows the mean discrepancy (flow-weighted bundle-average predicted outlet 
temperature minus measured experimental outlet temperature) with the horizontal blue line. We can see that, in general, CTF correctly capturing the energy input into the facility.

Finally, this plot also shows the $2 \sigma$ variation in experimental temperature measurements for each thermocouple. Note, the error bars are not showing the standard deviation in the mean discrepancy that is shown. For example, for Channel 5, we see the discrepancy is about $0.5 \mathrm{C}$, but the error bars show that the experimental measurements varied by about $0.25 \mathrm{C}$ during successive measurements taken in the calibration phase of the tests. So the actual discrepancy can be anywhere between 0.25 and $0.75 \mathrm{C}$ for this thermocouple. The average error in predicted exit temperature (straight average the difference between CTF and experiment at each thermocouple location) is $1.8 \mathrm{C}$. 


\section{Difference between COBRA-TF and Experimental Results} Channel Liquid Exit Temperatures

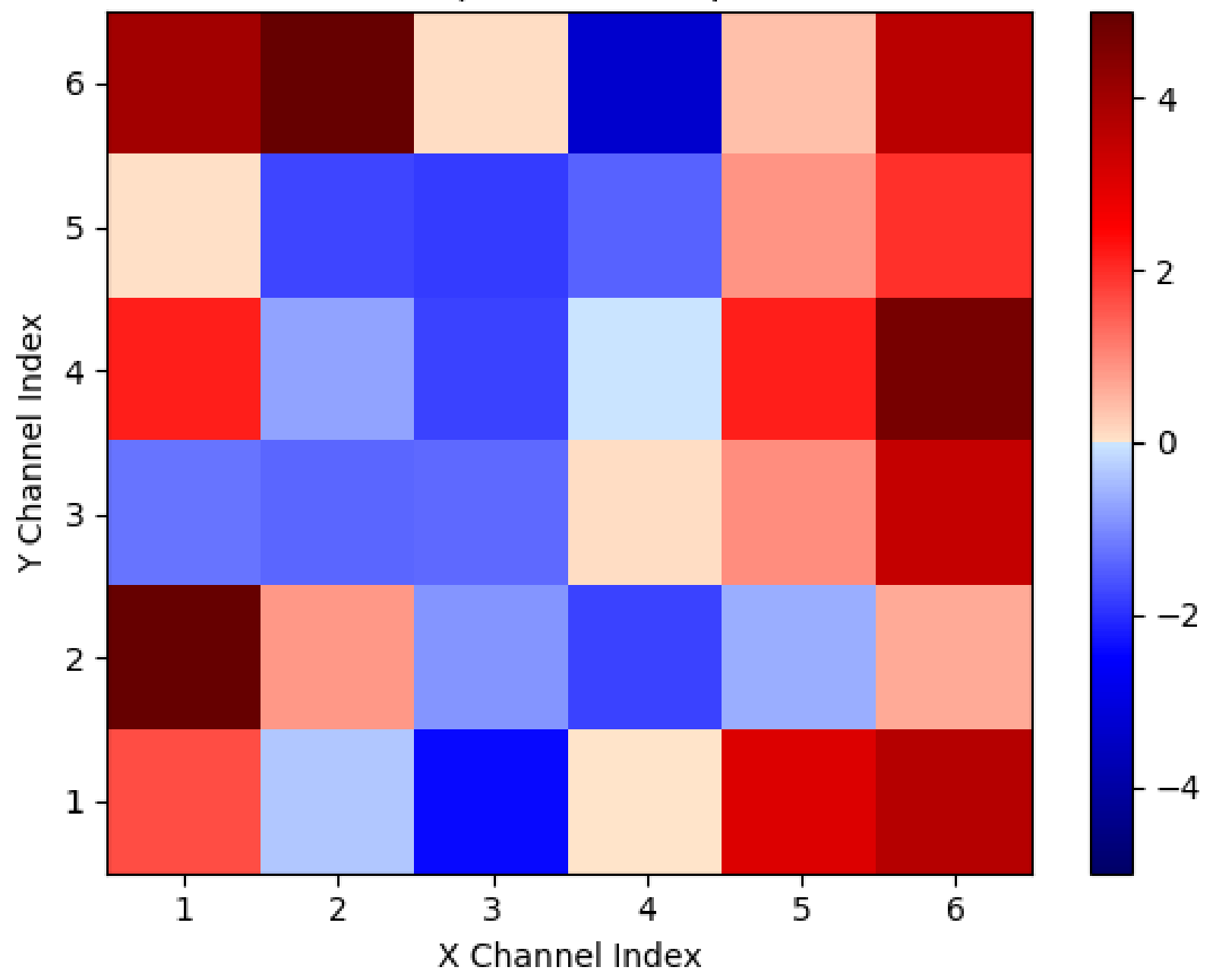

Figure 7.1: Average difference between CTF predicted channel exit temperatures and experimental values for all tests in CE $5 \times 5$ Series 74 


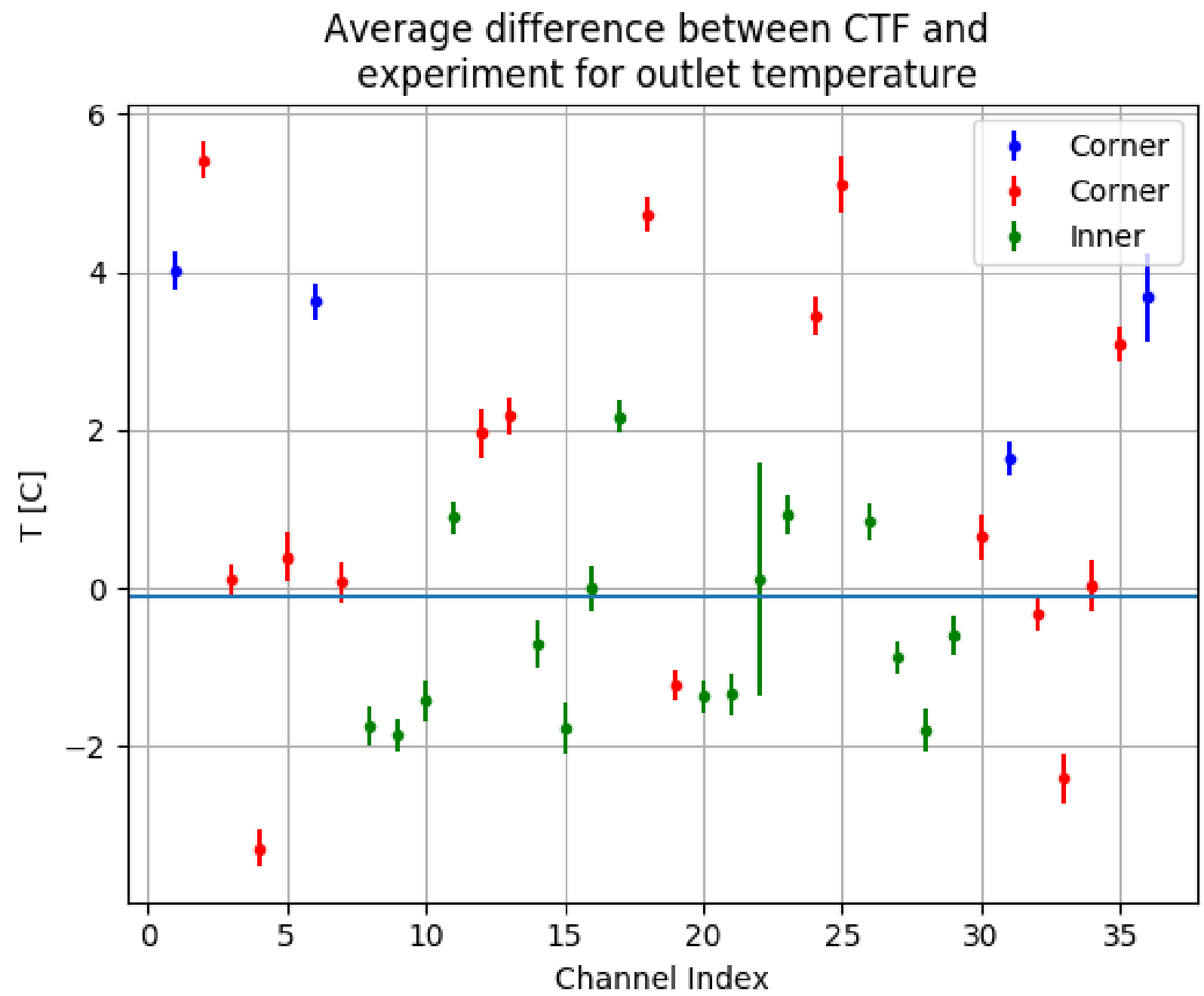

Figure 7.2: Average difference between CTF predicted channel exit temperatures and experimental values with measurement error and mean discrepancy 


\subsubsection{Kumamoto University $2 \times 3$}

Results are shown as the nondimensional mixing rate, $W_{i j}^{\prime} / \mu$, vs. the two-channel Reynolds number. The two-channel Reynolds number is calculated as follows:

$$
R e_{i j}=\frac{\rho u_{i j} D_{e, i j}}{\mu}
$$

Where the average two-channel velocity, $u_{i j}$, is an area weighted average of the velocities in the two adjacent channels, $i$ and $j$. The hydraulic diameter, $D_{e, i j}$, is the hydraulic diameter of the two-channel system, $\rho$ is density, and $\mu$ is dynamic viscosity. This is the way in which the author presents mixing results. A mixing rate is given for each unique channel connection, which includes: 1) inner-to-inner, 2) side-to-inner, and 3) side-to-side for each of the four single phase tests. Figure 7.3 shows the CTF results compared with the experimental results using the Rogers and Rosehart correlation [2] to predict single-phase mixing. The Blasius friction factor correlation is used in this case.

Two types of data points are shown in the plot: pluses represent the CTF predictions and circles represent the experimental measurements. There are three colors of the data points: red represents the inner-to-inner connection, green represents the side-to-side connection, and blue represents the side-to-inner connection. Ideally, a "plus" and a "circle" data point should sit in a vertical column; this would mean that the $i j$ Reynolds number of CTF matches the experimental value exactly. Looking at the figure, it is evident this is not the case. The CTF-predicted Reynolds number tends to be higher than its experimental counterpart in every case.

Likely, there are some differences in steam properties and inlet mass flow rate that lead to this discrepancy. However, it is evident that there is a near-linear trend for nondimensional mixing with respect to Reynolds number that we can use for comparison. The results of Figure 7.3 indicates that the Rogers and Rosehart correlation over-predicts the mixing rate observed in this facility substantially.

The study is re-run with a user-set, constant single-phase mixing coefficient of 0.004. A mixing-coefficient optimization study done using the CE $5 \times 5$ facility found that a value of 0.0044 was optimum for that configuration [39], so 0.004 is considered to be a lower bounding value. Figure 7.4 shows the results of changing the mixing coefficient to 0.004 .

Results indicate that this mixing coefficient underpredicts the mixing in the facility. As it turns out, a mixing coefficient of about 0.007 tends to lead to the best agreement, as shown in Figure 7.5. The choice of friction factor correlation has little impact on the predicted mixing rates. Figure 7.6 shows the results using $\beta=0.004$ with the friction factor correlation set to the CTF correlation instead of the Blasius correlation.

It is important to note that the mixing coefficient is simply a tuning parameter that will be dependent on the actual geometry of the facility being modeled. This facility is a square lattice, but the geometry is much larger than typical PWR or BWR rod-lattice geometry. This study is useful for showing that CTF is capable of predicting the correct mixing rate if $\beta$ is tuned correctly to the facility. Furthermore, it offers a range of values from which to select the mixing coefficient.

The physical relevance of the mixing rate is not immediately obvious. It is better to observe the impact of the term on simulation parameters that affect the solution. The CASL Problem 7 challenge problem (quarter symmetry model of Watts Bar Unit 1) is modeled using a power distribution from a coupled MPACT/CTF 

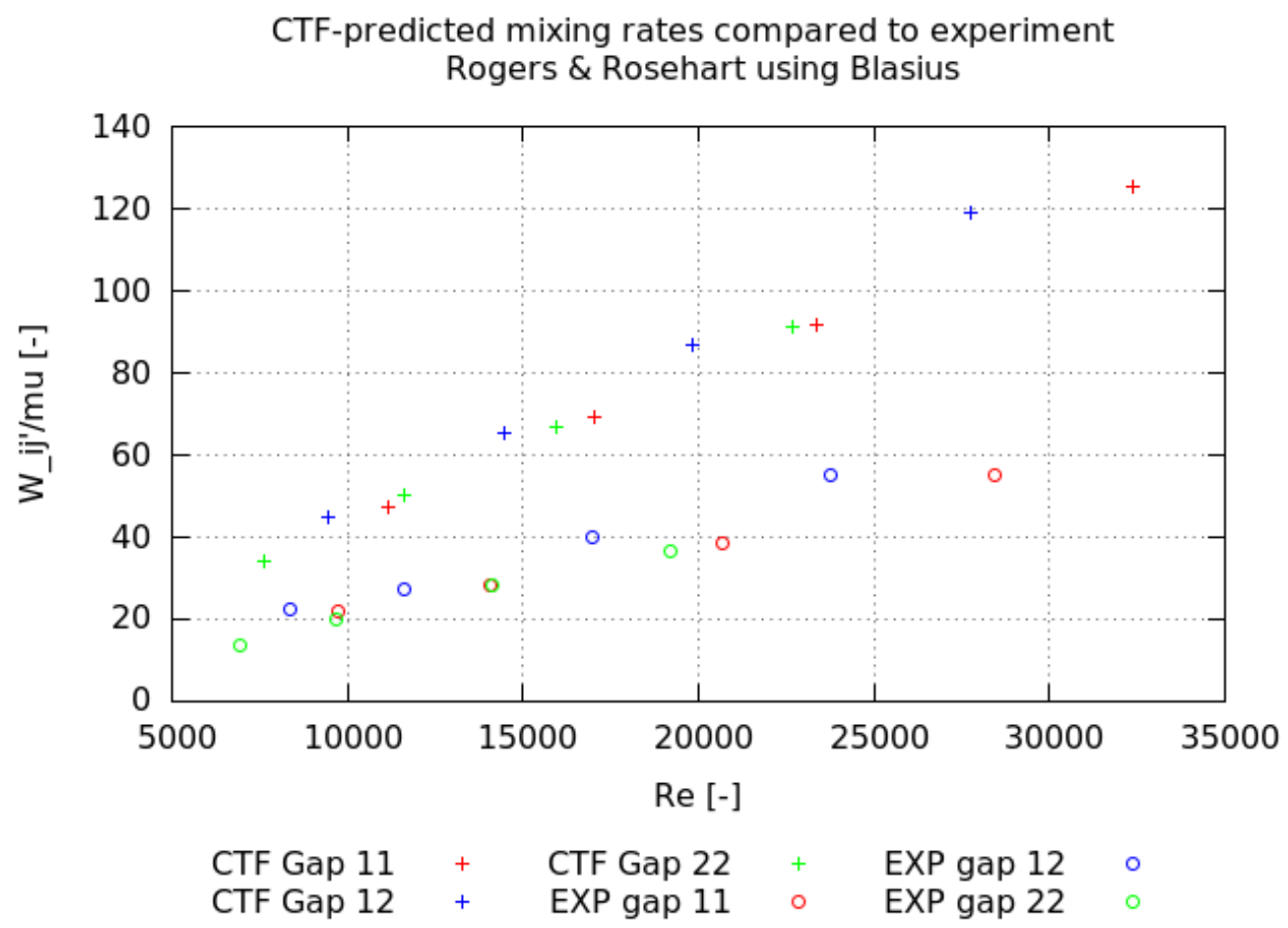

Figure 7.3: Comparison of CTF-predicted mixing rates and experimental measured rates from $2 \times 3$ facility using Rogers and Rosehart for $\beta$ and the Blasius friction correlation. 


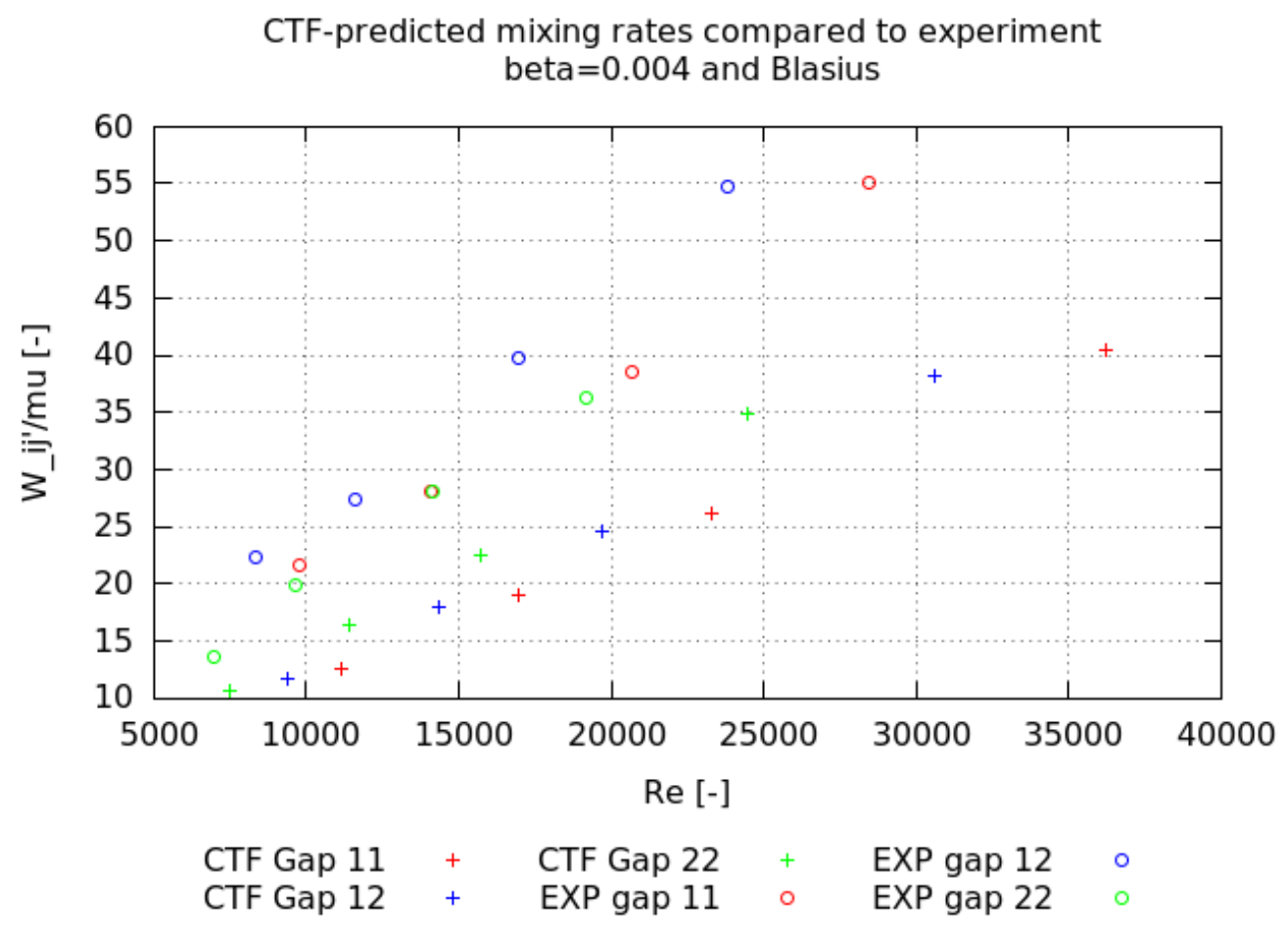

Figure 7.4: Comparison of CTF-predicted mixing rates and experimental measured rates from $2 \times 3$ facility using $\beta=0.004$ and the Blasius friction correlation. 


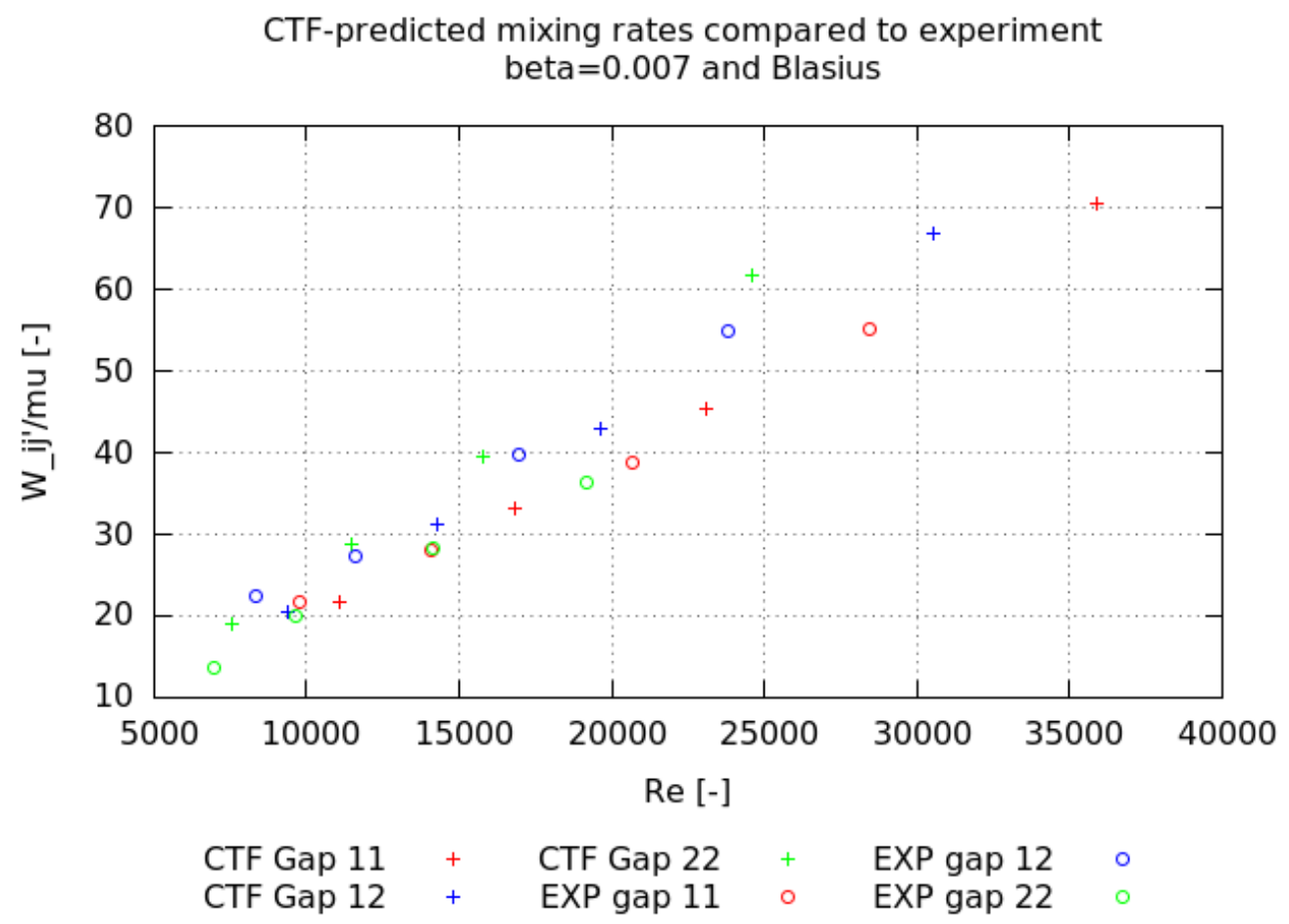

Figure 7.5: Comparison of CTF-predicted mixing rates and experimental measured rates from $2 \times 3$ facility using $\beta=0.007$ and the Blasius friction correlation. 


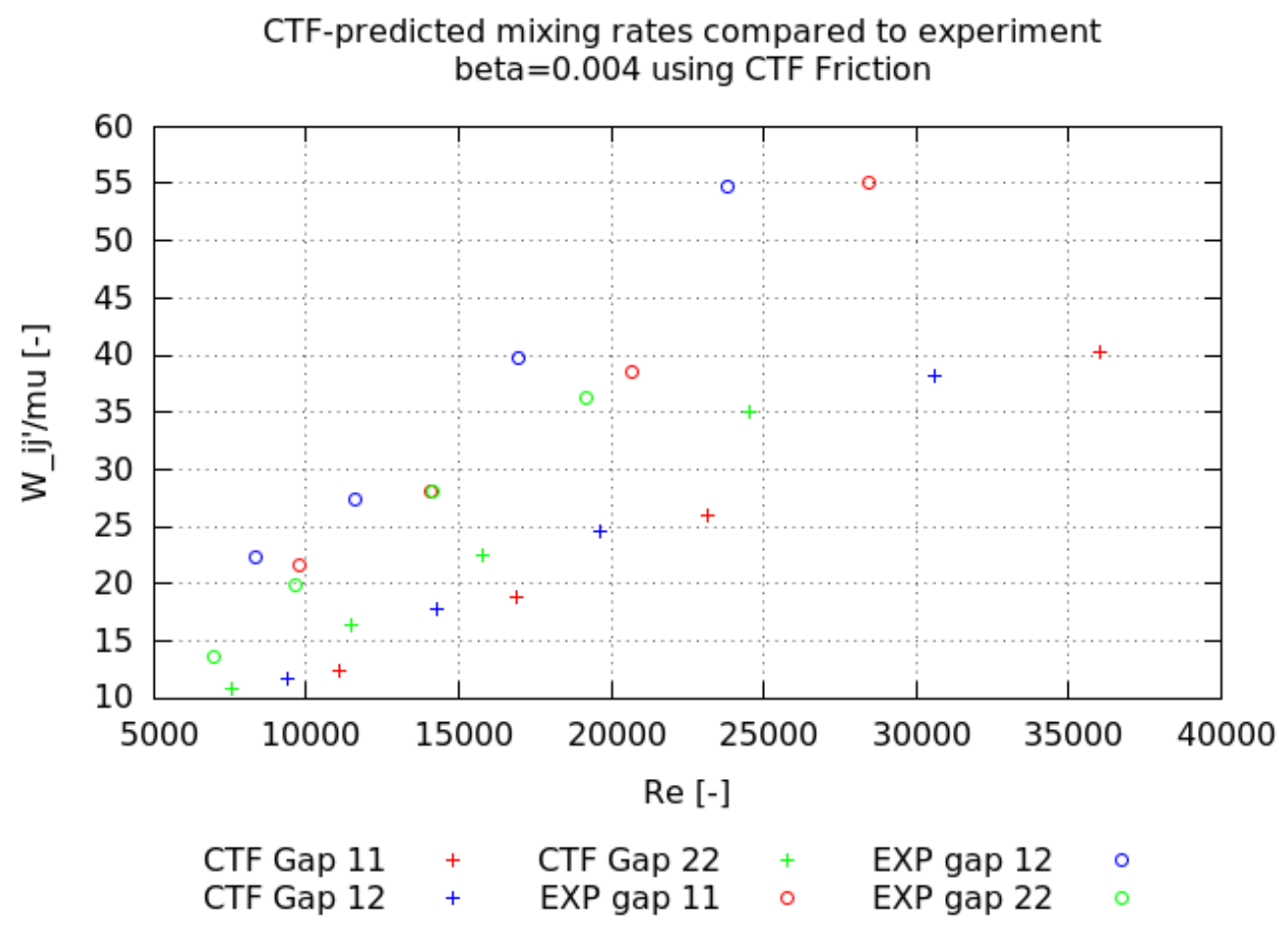

Figure 7.6: Comparison of CTF-predicted mixing rates and experimental measured rates from $2 \times 3$ facility using $\beta=0.007$ and the CTF friction correlation. 


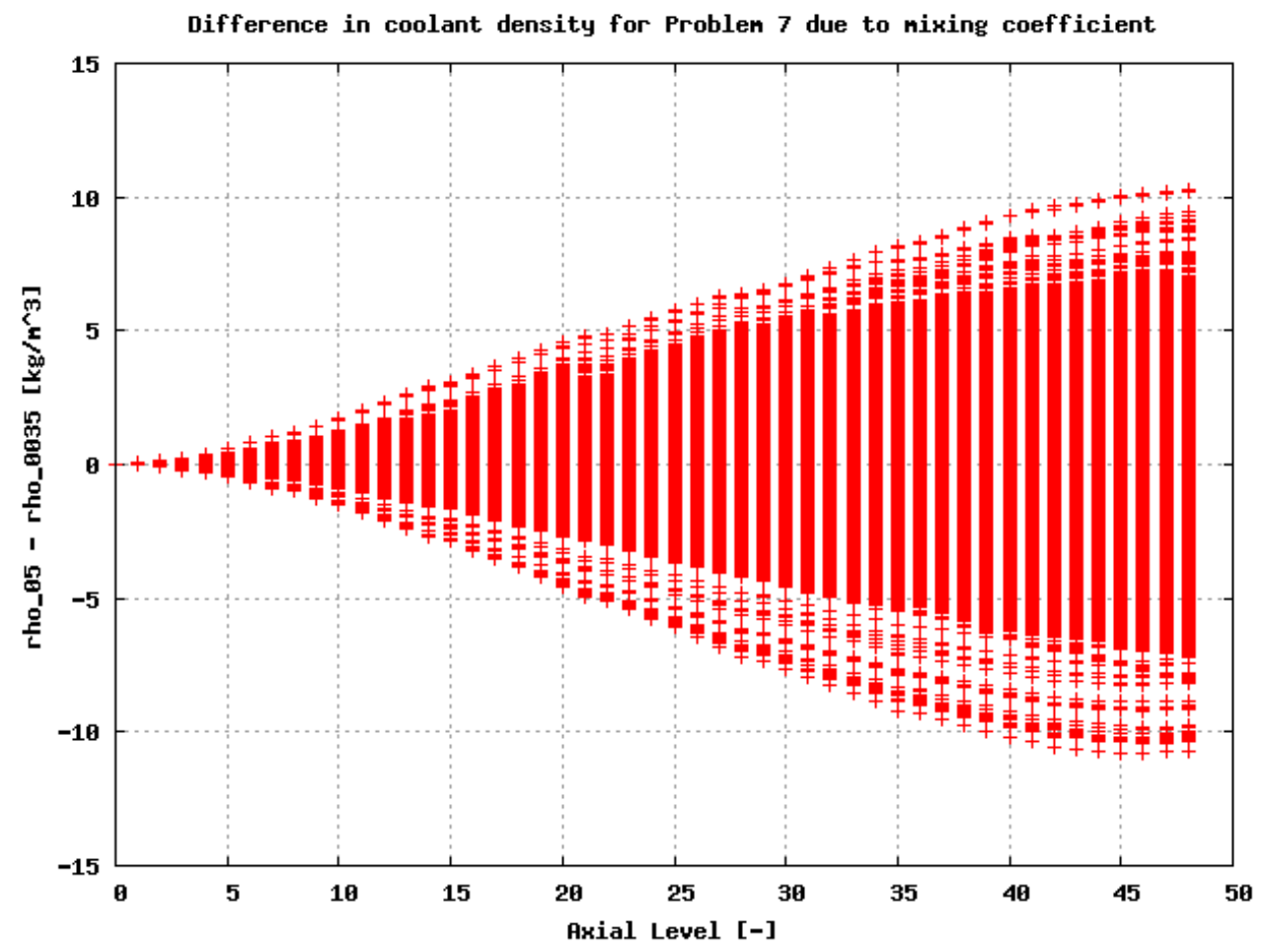

Figure 7.7: Variation of local liquid density in CTF simulation of Watts Bar Unit 1 when $\beta$ is changed from 0.0035 to 0.05 .

solution of the facility. The mixing coefficient is changed from 0.0035 to 0.05 , with 0.05 being much greater than the value predicted by Rogers and Rosehart. The impact of changing this parameter on local predicted liquid density is shown in Figure 7.7. The results are presented as density in a cell when $\beta$ is 0.05 minus density in the cell when $\beta$ is 0.0035 . This calculation is made in each of the roughly 500,000 computational cells of the model and presented in the figure. The results show that differences increase to a maximum at the outlet of the facility and reach as much as $0.01 \mathrm{~g} \mathrm{~cm}^{-3}$, which will have a small, but noticeable impact on reactivity in those locations.

\subsubsection{RPI $2 \times 2$}

In this step of the validation, we are concerned only with correctly predicting the single-phase flow distribution. Therefore, only the two single-phase tests are modeled (Tests 1 and 2). The ideal flow distribution is determined similar to how it is in Section 6.2.2.1; the momentum equation is used to relate velocity in two individual subchannels, and the mass conservation equation is used to link all channels together. In this case, we have two unique momentum equations and one mass equation with three unknown variables. The coefficients for the CTF friction correlation are used in the momentum equation, leading to an exponent of $2 / 3$ on the ratio of hydraulic diameters. 


$$
\begin{aligned}
\frac{u_{\text {corner }}}{u_{\text {side }}} & =\left(\frac{D h_{\text {corner }}}{D h_{\text {side }}}\right)^{2 / 3} \\
\frac{u_{\text {corner }}}{u_{\text {inner }}} & =\left(\frac{D h_{\text {corner }}}{D h_{\text {inner }}}\right)^{2 / 3} \\
\dot{m}_{\text {tot }} & =4 \rho u_{\text {corner }} A_{\text {corner }}+4 \rho u_{\text {side }} A_{\text {side }}+\rho u_{\text {inner }} A_{\text {inner }}
\end{aligned}
$$

Solving this system of equations gives an ideal flow split for each individual channel type:

$$
\begin{gathered}
\dot{m}_{\text {corner }}=\frac{\dot{m}_{\text {tot }}}{C}\left(\frac{D h_{\text {corner }}}{D h_{\text {inner }}}\right)^{2 / 3}\left(\frac{A_{\text {corner }}}{A_{\text {inner }}}\right) \\
\dot{m}_{\text {side }}=\frac{\dot{m}_{\text {tot }}}{C}\left(\frac{D h_{\text {side }}}{D h_{\text {inner }}}\right)^{2 / 3}\left(\frac{A_{\text {side }}}{A_{\text {inner }}}\right) \\
\dot{m}_{\text {inner }}=\frac{\dot{m}_{\text {tot }}}{C} \text {, where } \\
C=4\left(\frac{D h_{\text {corner }}}{D h_{\text {inner }}}\right)^{2 / 3} \frac{A_{\text {corner }}}{A_{\text {inner }}}+4\left(\frac{D h_{\text {side }}}{D h_{\text {inner }}}\right)^{2 / 3} \frac{A_{\text {side }}}{A_{\text {inner }}}+1 .
\end{gathered}
$$

Each single-phase case is first run with turbulent mixing disabled, so as to see that CTF predicts the correct single-phase flow distribution. Results show that CTF does predict the correct flow distribution; however, not within the axial length of the test section, which is $1 \mathrm{~m}$. The CTF model is extended to $7 \mathrm{~m}$ to show that the correct flow split is eventually achieved. Figures 7.8 and 7.9 show the results of running CTF with no turbulent mixing for Case 1 and 2 of the $2 \times 2$ facility, respectively.

The channel mass flux results are normalized as shown in Equation 6.16. The figures show four important pieces of information:

1. The CTF normalized channel mass fluxes are shown for corner, side, and inner type channels (red, blue, and green) using the solid lines,

2. The analytical solution for the flow split (obtained using Equations 7.5, 7.6, and 7.7) is shown with the three horizontal dashed lines using the same color scheme to denote channel types,

3. The experimental measurements are shown with the dot-dash lines using the same color scheme for denoting channel type, and

4. The shaded regions show the maximum experimental measurement uncertainty for channel mass flux $(5 \%)$, as quoted in the $2 \times 2$ technical report [14].

The figures shows that the trend for flow to migrate into the lower resistance inner channel and out of the higher resistance corner channel is correctly predicted. The CTF channel flows hit the expected values at 


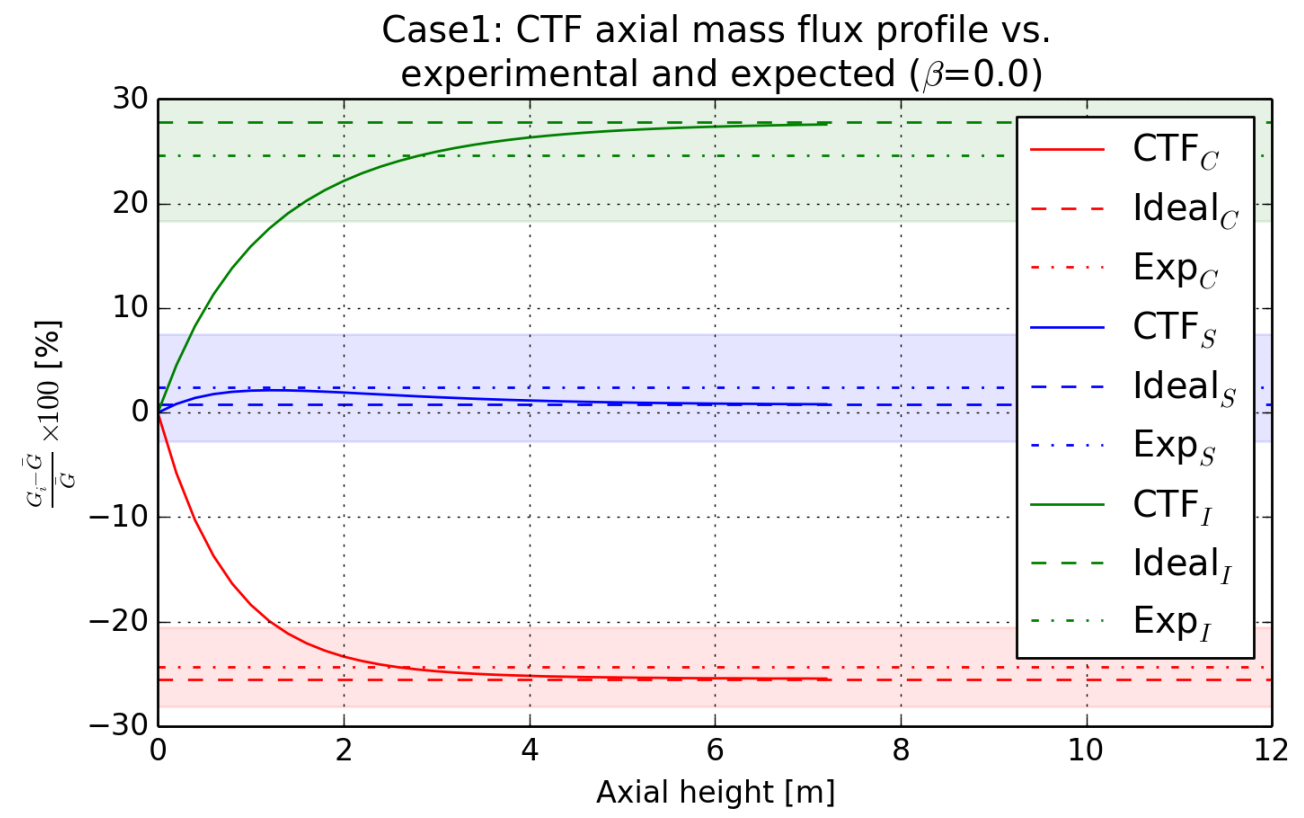

Figure 7.8: $\mathrm{CTF}$-predicted flow split (no mixing) for $2 \times 2$ Case 1 single-phase test compared with experimental values and ideal flow split.

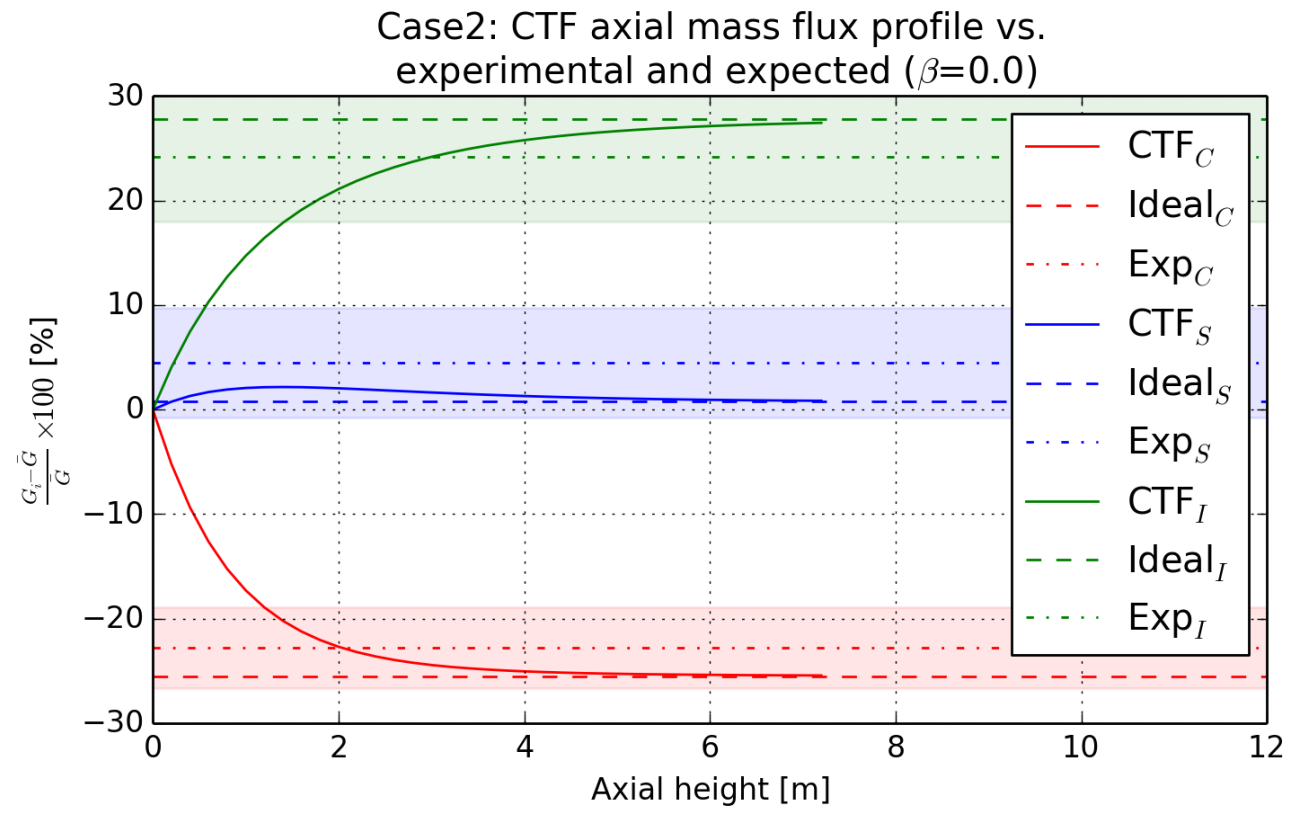

Figure 7.9: CTF-predicted flow split (no mixing) for $2 \times 2$ Case 2 single-phase test compared with experimental values and ideal flow split. 
about $6 \mathrm{~m}$. The experimental results are not exactly the same as the theoretical values since turbulent mixing drives momentum from the higher velocity inner channel back to the corner and side channels. However, note that the experimental results are obtained within $1 \mathrm{~m}$ of test section length.

Figures 7.10 and 7.11 show the same results with turbulent mixing enabled in CTF and the single-phase turbulent mixing coefficient, $\beta$, set to 0.007 (the ideal value discovered in Section 7.1.2).

Enabling turbulent mixing leads to a new mechanical equilibrium point in CTF. The inner channel flow does not go as high and corner channel flow does not go as low. The results seem to indicate that the CTF mixing coefficient may be slightly too high for this experimental facility. The CTF inner channel mass flux is lower than the experimental inner channel measurement and the CTF corner channel mass flux is higher the than the experimental corner measurement. The corner channel prediction is just outside of the measurement uncertainty bands.

Adjusting the mixing coefficient down to $\beta=0.0035$ leads to a more favorable prediction of the flow split, as shown in Figures 7.12 and 7.13.

Similar to the cases without mixing, the flow takes about 5-6 $\mathrm{m}$ to reach equilibrium distribution in the CTF model. Since Case 2 has a mass flux that is twice that of Case 1, it appears that the magnitude of flow has no significant effect on the distance to reach equilibrium flow distribution. 


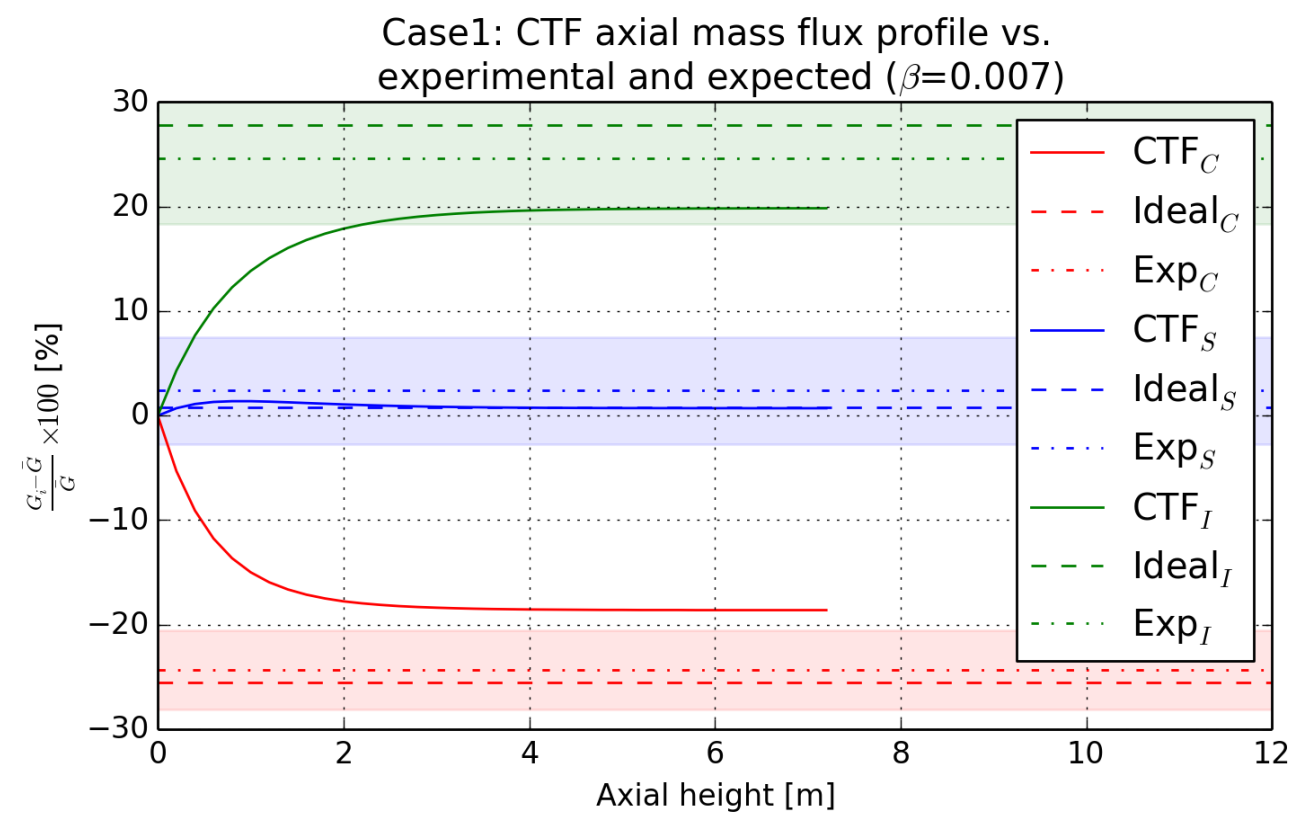

Figure 7.10: CTF-predicted flow split (with $\beta$ set to 0.007 ) for $2 \times 2$ Case 1 single-phase test compared with experimental values and ideal flow split.

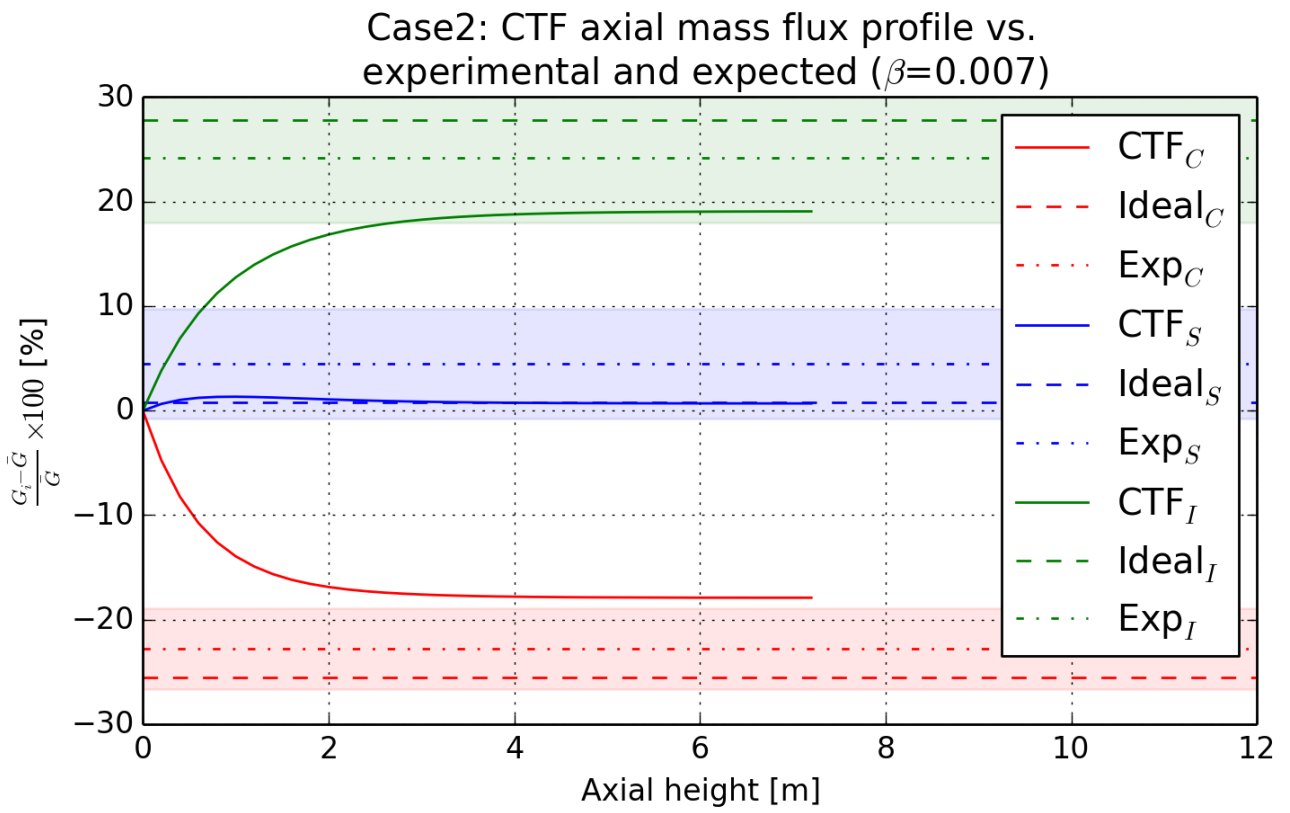

Figure 7.11: CTF-predicted flow split (with $\beta$ set to 0.007 ) for $2 \times 2$ Case 2 single-phase test compared with experimental values and ideal flow split. 


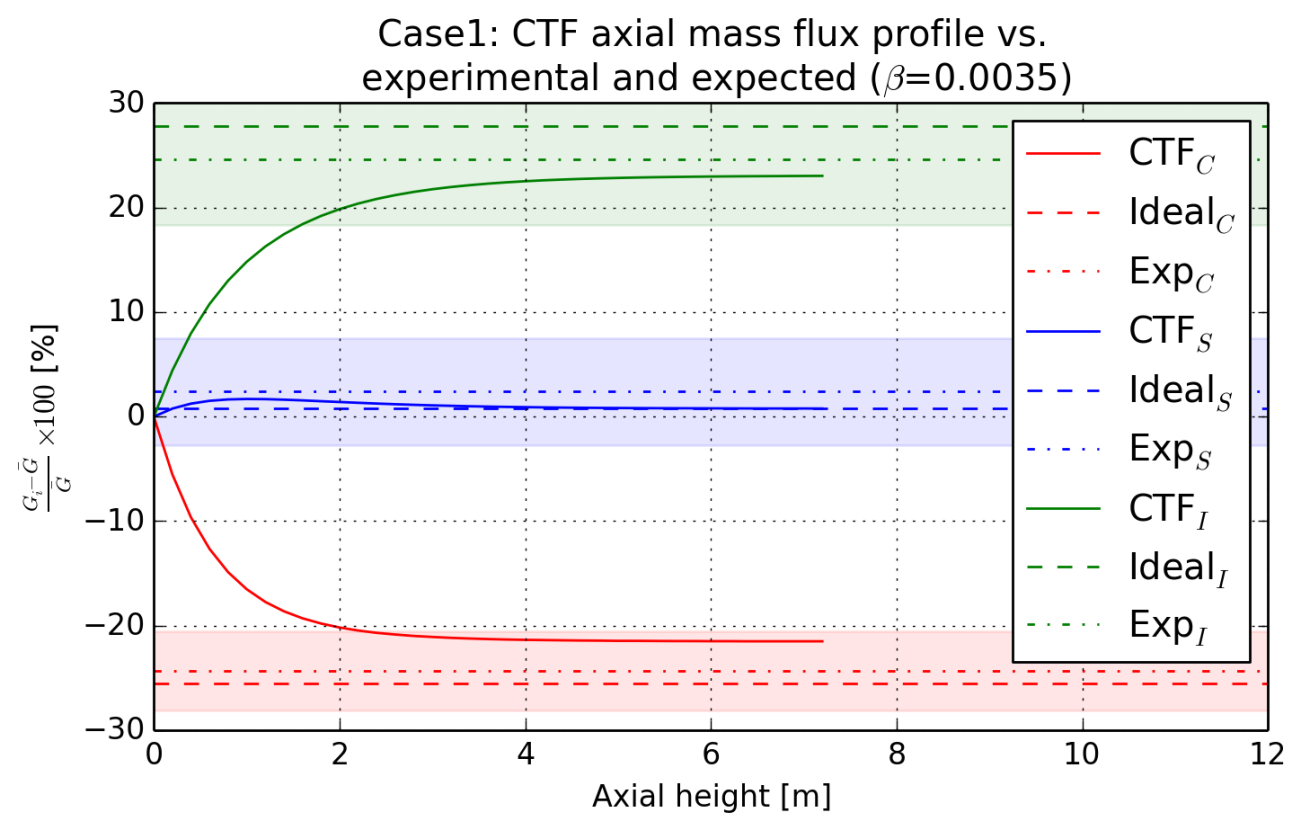

Figure 7.12: CTF-predicted flow split (with $\beta=0.0035$ ) for $2 \times 2$ Case 1 single-phase test compared with experimental values and ideal flow split.

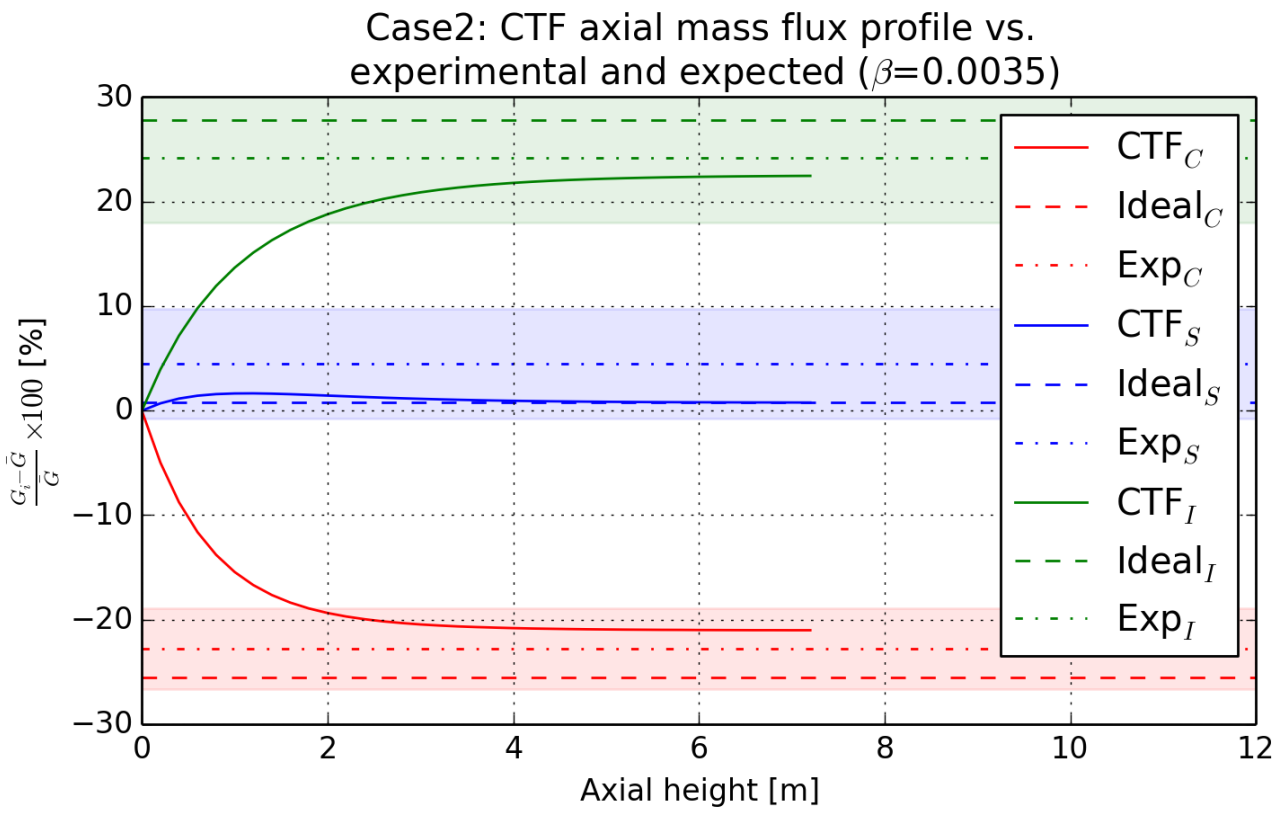

Figure 7.13: CTF-predicted flow split (with $\beta=0.0035$ ) for $2 \times 2$ Case 2 single-phase test compared with experimental values and ideal flow split. 


\subsubsection{GE $3 \times 3$}

Because this section of the study is reviewing the ability to predict correct single-phase flow distribution, only the four single-phase cases $(1 \mathrm{~B}, 1 \mathrm{C}, 1 \mathrm{D}$, and $1 \mathrm{E})$ are run. Considering the difficulty CTF exhibited in correctly predicting the flow split for the $2 \times 2$ facility, it is prudent to assess the flow-distribution prediction for the GE $3 \times 3$ case before analyzing the two-phase void drift cases.

Results are shown in Figures 7.14-7.17. The solid lines are the CTF prediction of normalized mass flux in each channel type. In addition, the "ideal" flow split (calculated using the approach outlined in Section 6.2.2.1) and the experimental results are shown as horizontal lines in the plot. Horizontal lines are plotted because values are only available at the outlet.

All single-phase predicted outlet mass fluxes are compared with their measured counterparts in Figure 7.18. Each color represents a unique channel type: red for corner, blue for side, and green for inner. Inner- and side-predicted mas fluxes match experimental values closely, having rRMS (Equation 1.5) values that are close to experimental measurement uncertainty. Corner results vary from experimental values by a much larger degree. 


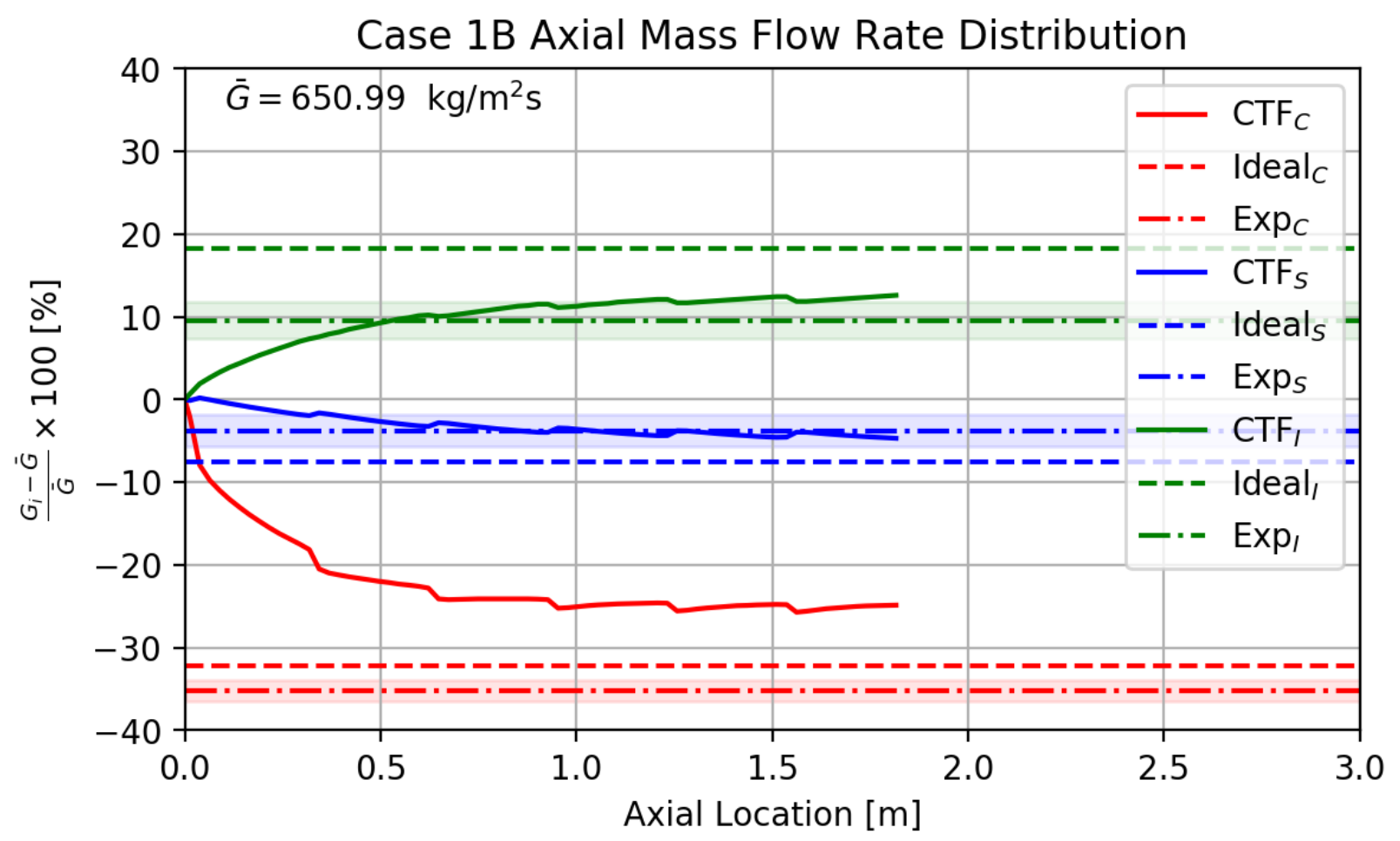

Figure 7.14: CTF-predicted flow split compared with ideal and measured results for GE $3 \times 3$ Case $1 \mathrm{~B}$ (with $\beta=0.007$ ). 


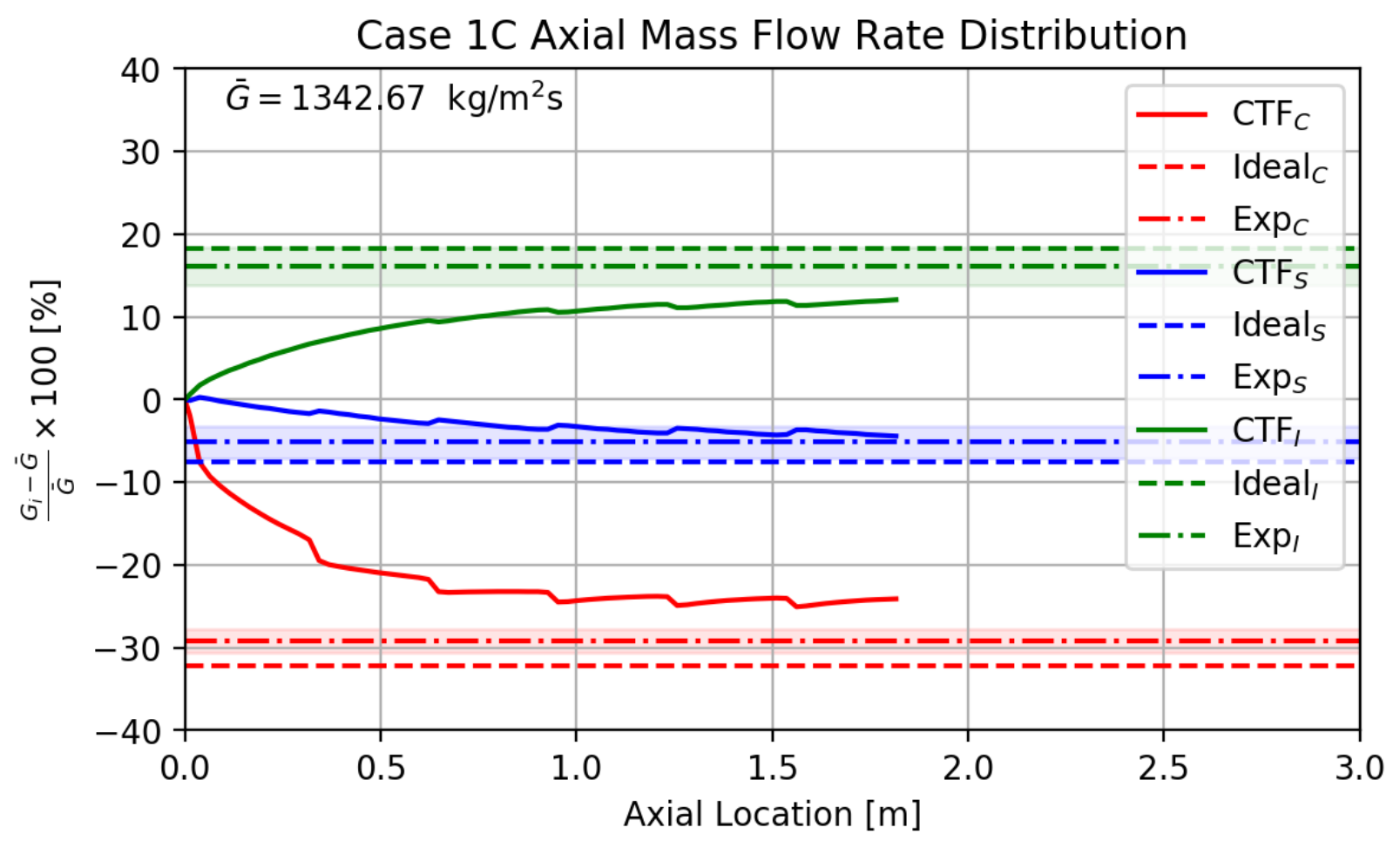

Figure 7.15: CTF-predicted flow split compared with ideal and measured results for GE $3 \times 3$ Case $1 \mathrm{C}$ (with $\beta=0.007$ ). 


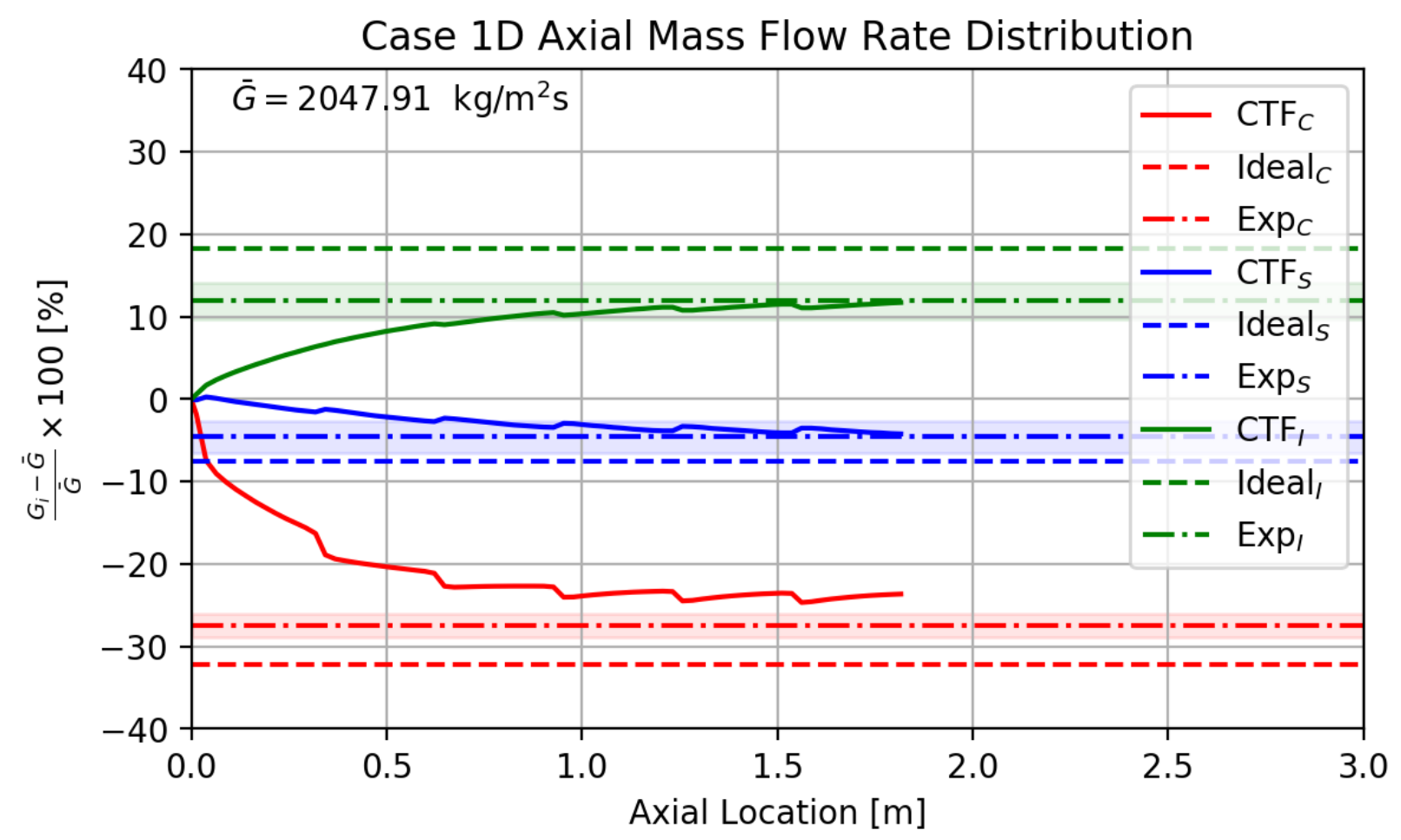

Figure 7.16: CTF-predicted flow split compared with ideal and measured results for GE $3 \times 3$ Case $1 \mathrm{D}$ (with $\beta=0.007$ ). 


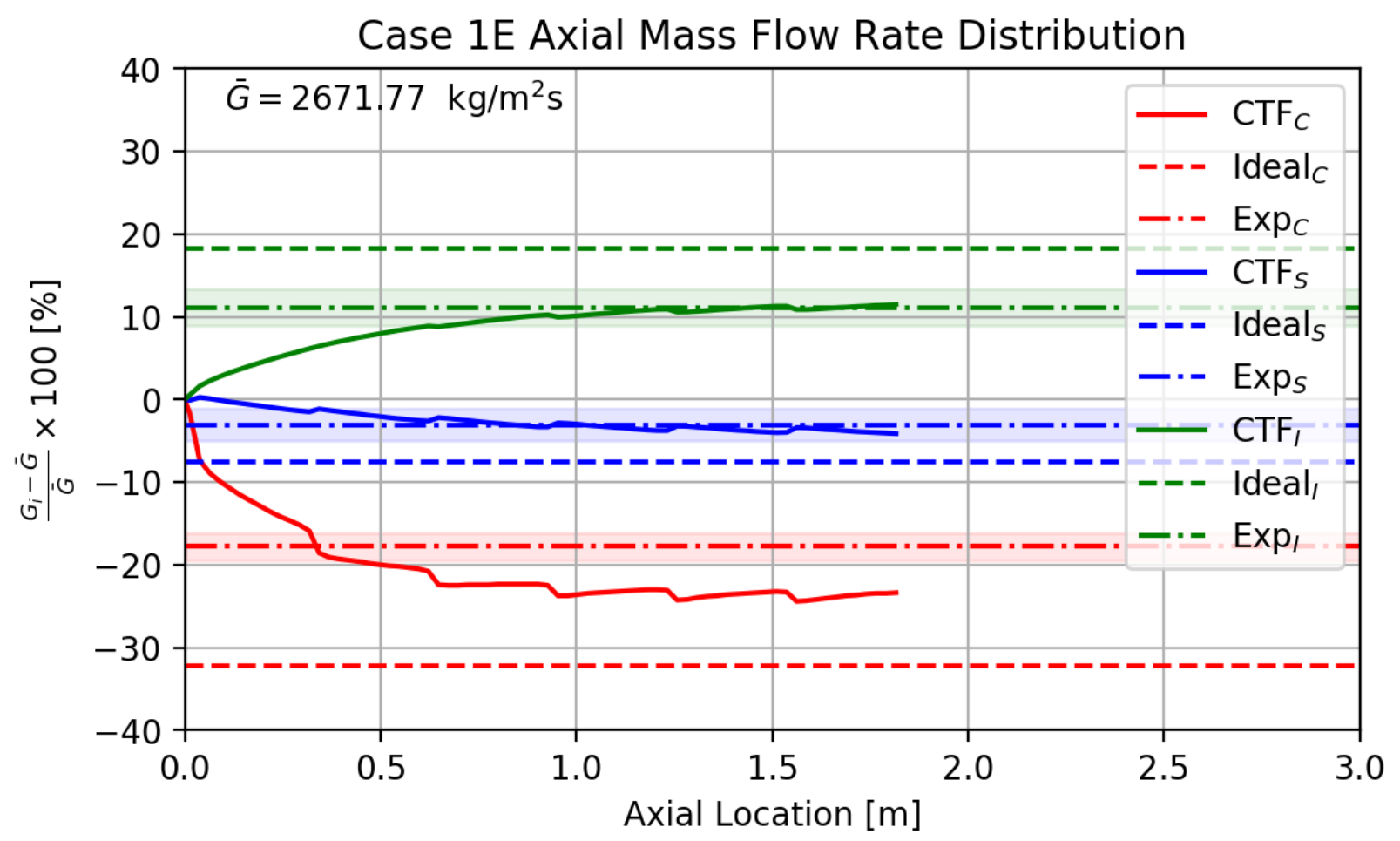

Figure 7.17: CTF-predicted flow split compared with ideal and measured results for GE $3 \times 3$ Case $1 \mathrm{E}$ (with $\beta=0.007$ ). 


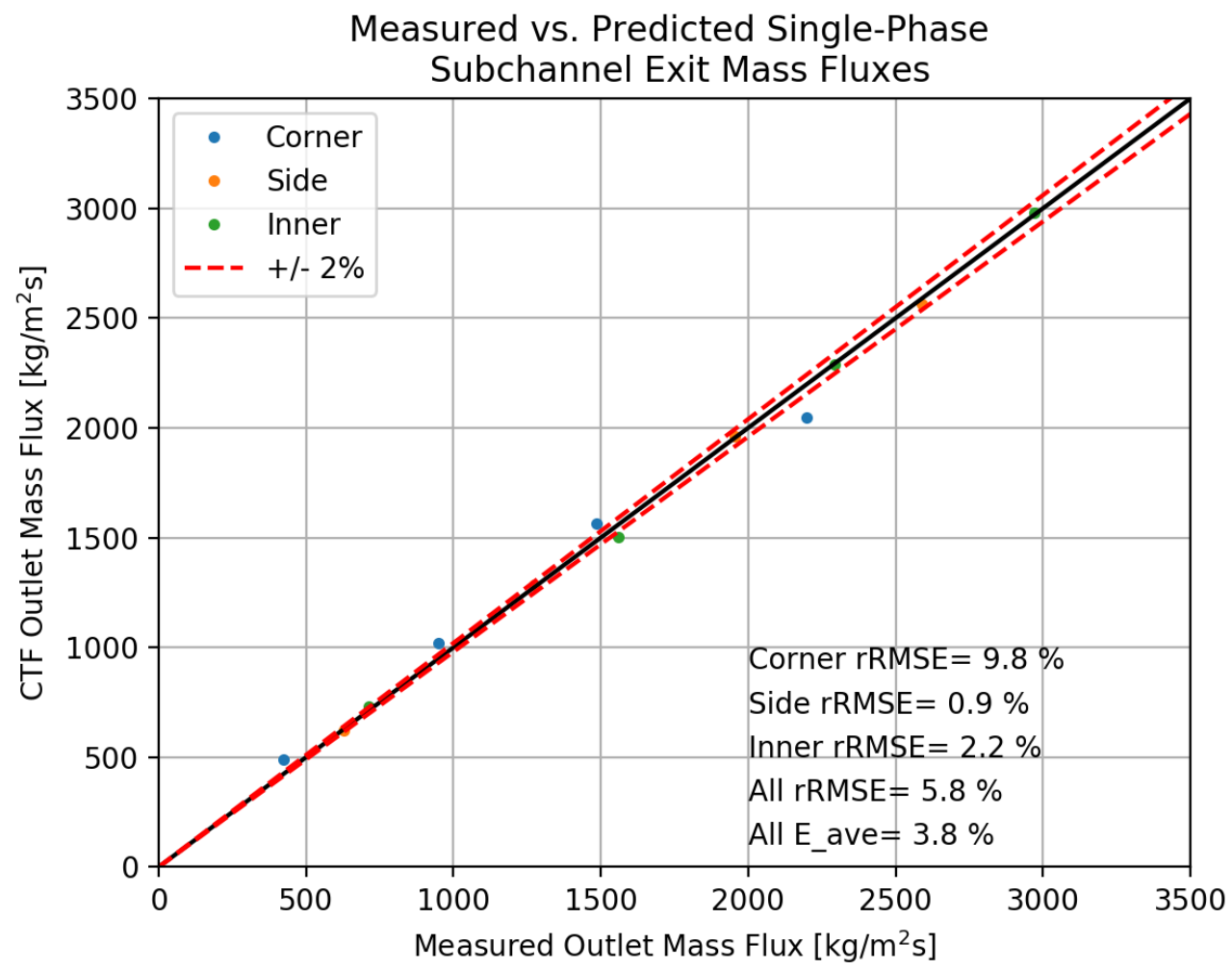

Figure 7.18: Comparison of predicted and measured exit mass flux for GE $3 \times 3$ single-phase cases using $\beta=0.007$. 


\subsection{Verification}

\subsubsection{Single-Phase Two-Channel}

Problem Description The problem consists of two channels connected by a gap. Because the CTF model for turbulent mixing is gradient-driven, it is necessary to make a gradient in either energy or momentum. Because there is no net transfer of mass due to turbulent mixing in single phase, unheated flows, it is not possible to analyze mass transfer in this case. Forming an analytical solution requires us to form and solve the relevant governing equations for the system.

If we choose to look at turbulent mixing of momentum, we will need to set velocity of one channel higher than the other. The result will be migration of velocity due to pressure-driven directed cross-flow (due to higher frictional pressure drop in the high velocity channel) as well as turbulent mixing of the momentum. We wish to verify that the turbulent mixing model works as expected without interference from other effects. We can disable the friction model to stop the pressure-driven directed cross-flow. However, because the axial velocity profile will not be constant in the channel, the convective terms of the momentum equation cannot be eliminated, which requires a complicated solution of the equations.

The energy equation can be solved much more easily as long as we disable the temperature-dependent density in CTF. With this disabled, the velocity profile will be constant, as the turbulent mixing model for energy does not actually move mass from one channel to another; it captures the effect of mixing on the migration of energy from one channel to the other.

This problem is a modification of Example 6-1 in Todreas and Kazimi Volume II [40]. The problem in the textbook uses the concept of tracer dyes to demonstrate mixing. In place of looking at mixing of a dye, the mixing of enthalpy is observed in this problem.

The design of the system is shown in Figure 7.19. The geometry of the two channels is identical in this case, which should eliminate any pressure-driven directed cross-flow. Channel geometry is based on typical PWR rod-lattice geometry. To activate the turbulent-mixing model, the temperature of one channel is raised $10^{\circ} \mathrm{C}$ over the second channel. The "vuq_param.txt" file is used to set a constant liquid density in the system.

For this case, we can set up an energy equation for each channel. The CTF energy equation is as follows:

$$
\begin{gathered}
\frac{\partial}{\partial t}\left(\alpha_{k} \rho_{k} h_{k}\right)+\frac{\partial}{\partial x}\left(\alpha_{k} \rho_{k} h_{k} u_{k}\right)+\frac{\partial}{\partial y}\left(\alpha_{k} \rho_{k} h_{k} v_{k}\right)+\frac{\partial}{\partial z}\left(\alpha_{k} \rho_{k} h_{k} w_{k}\right)= \\
\Sigma_{\text {gap }=1}^{N G A P} q_{k, \text { gap }}^{T^{\prime \prime \prime}}+\Gamma^{\prime \prime \prime} h+q_{w k}^{\prime \prime \prime}+\alpha_{k} \frac{\partial P}{\partial t}
\end{gathered}
$$

The left-hand side terms include: (1) time-change of energy, (2) axial $(x)$ advection of energy, 3) lateral $(y)$ advection of energy, and 4) lateral ( $z$ ) advection of energy. The terms, $\alpha, \rho, h$, and $u$, represent the volume fraction, density, enthalpy, and velocity, respectively. The subscript, $k$, indicates the field; liquid, vapor, or droplet. The right-hand side terms include: 1) turbulent mixing of energy (lateral direction only), 2) the implicit heat transfer, 3) energy entering the volume from the wall, and 4) the pressure-work on the volume over time. The term, $\Gamma^{\prime \prime \prime}$ represents the volumetric evaporation rate (transfer of mass from the liquid phase to the vapor phase). 


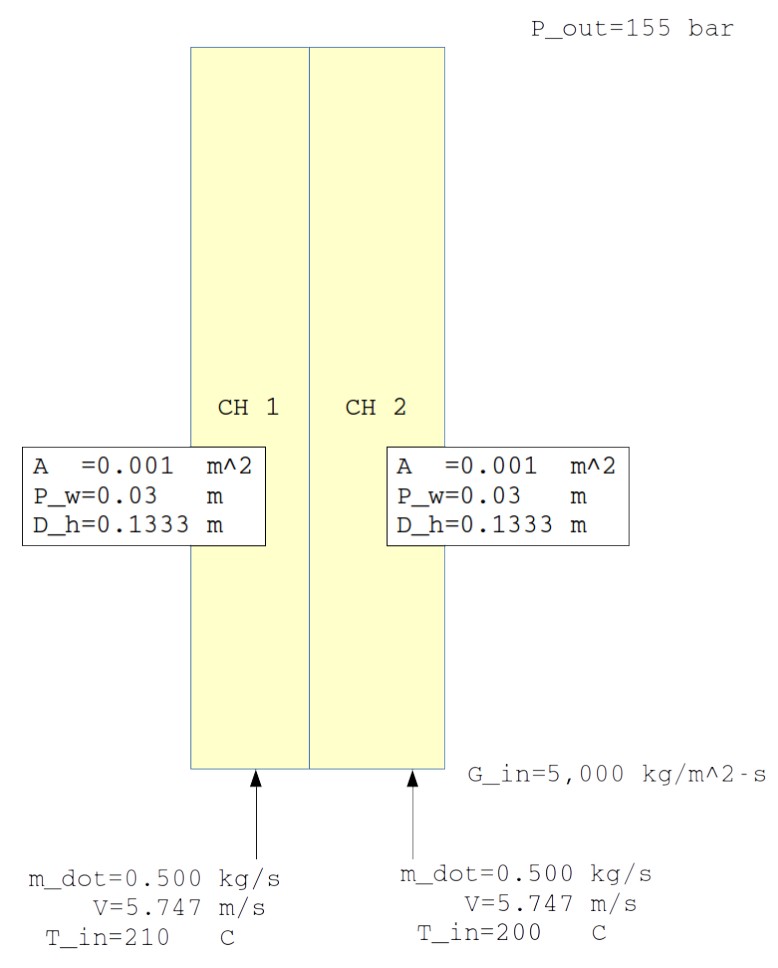

Figure 7.19: Model of problem for testing single-phase turbulent mixing of enthalpy. 
We can make the following assumptions about this case:

1. The case is steady-state, eliminating the transient change in energy and the pressure work term.

2. The case is single-phase, eliminating all $k$ phase subscripts, void fractions, and the mass transfer term.

3. The case is unheated.

4. Because the case is set up so there is no lateral directed cross-flow, only the axial convective term remains.

The simplified equation, with $x$ being the axial direction, becomes

$$
\frac{\partial}{\partial x}(\rho h u)=q_{y}^{T^{\prime \prime \prime}}
$$

The density and velocity can be removed from the derivative because they are constants in the solution. This is applied directly to our problem of interest by formulating it for each channel in the model. Substituting the lateral transport of enthalpy due to turbulent mixing $\left(q_{y}^{T}\right)$ with the CTF form of the model yields the following set of equations:

$$
\begin{aligned}
& \dot{m}_{1} \frac{d}{d x} h_{1}+W_{1 \rightarrow 2}^{\prime}\left(h_{1}-h_{2}\right)=0 \\
& \dot{m}_{2} \frac{d}{d x} h_{2}-W_{1 \rightarrow 2}^{\prime}\left(h_{1}-h_{2}\right)=0
\end{aligned}
$$

Each equation is multiplied by the cross-sectional area to convert the velocity to mass flow rate and the volumetric mixing rate to a linear one. The $W_{1 \rightarrow 2}^{\prime}$ term represents the mixing rate of energy from Channel 1 to Channel 2. It has units of $\mathrm{kg} \mathrm{m}^{-1} \mathrm{~s}^{-1}$ and is defined as

$$
W_{1 \rightarrow 2}^{\prime}=\beta S_{12} \bar{G}
$$

The $\beta$ term is the turbulent mixing coefficient; it is the "tuning parameter" for the mixing model. Physically, it is a non-dimensional coefficient that represents the ratio of the lateral mass flux due to mixing to the axial mass flux. The other terms, $S_{12}$ and $\bar{G}$, are the gap width between Channels 1 and 2 (in $\mathrm{m}$ ) and the areaweighted average mass flux between the two channels (in $\mathrm{kg} \mathrm{m}^{-2} \mathrm{~s}^{-1}$ ). For this case, we set $\beta$ to a "typical" value of 0.0035 , the gap thickness based on problem geometry $(0.003 \mathrm{~m})$, and the mass flux based on problem operating conditions.

Returning to Equations 7.11 and 7.12 , we can solve the enthalpy distribution in a channel by relating the enthalpy in the two channels at any axial level, as follows:

$$
\dot{m}_{1} h_{1}+\dot{m}_{2} h_{2}=\dot{m}_{1} h_{1, \text { in }}+\dot{m}_{2} h_{2, \text { in }}
$$


Because the mass flow rates in the channels are identical, this term cancels out and we are left with a relationship between the enthalpy in Channels 1 and 2 and the known inlet enthalpy boundary conditions. This is substituted into Equation 7.11 to develop a first-order, linear, ordinary differential equation that describes the enthalpy profile in Channel 2:

$$
\frac{d}{d x} h_{2}+\frac{2 W_{1 \rightarrow 2}^{\prime}}{\dot{m}} h_{2}-\frac{W_{1 \rightarrow 2}^{\prime}}{\dot{m}}\left(h_{1, \text { in }}+h_{2, \text { in }}\right)=0
$$

The solution of this equation is

$$
h_{2}=\frac{1}{2}\left(h_{1, \text { in }}+h_{2, \text { in }}\right)+C \exp \left(\frac{-2 W_{1 \rightarrow 2}^{\prime}}{\dot{m}} x\right) .
$$

Using the inlet enthalpy boundary conditions, the value of the constant, $C$ is determined. The same process is repeated for the first channel, leading to the following final solutions for enthalpy distribution in the system due to turbulent mixing.

$$
\begin{aligned}
& h_{1}=\frac{1}{2}\left(h_{1, \text { in }}+h_{2, \text { in }}\right)-\frac{1}{2}\left(h_{2, \text { in }}-h_{1, \text { in }}\right) \exp \left(\frac{-2 W_{1 \rightarrow 2}^{\prime}}{\dot{m}} x\right) \\
& h_{2}=\frac{1}{2}\left(h_{1, \text { in }}+h_{2, \text { in }}\right)+\frac{1}{2}\left(h_{2, \text { in }}-h_{1, \text { in }}\right) \exp \left(\frac{-2 W_{1 \rightarrow 2}^{\prime}}{\dot{m}} x\right)
\end{aligned}
$$

CTF Model Description The CTF input deck is set up from Figure 7.19. Axial meshing is set to $2.54 \mathrm{~cm}$. The gap thickness is set to $0.003 \mathrm{~m}$ and its length is set to $0.0126 \mathrm{~m}$, which would result from a PWR lattice with $12.6 \mathrm{~mm}$ pitch and $9.5 \mathrm{~mm}$ rod outside diameter. The turbulent mixing model is set so that a single-phase turbulent-mixing parameter, $\beta$, could be set equal to the value used in the analytical solution. The liquid density is set to a constant value of $700 \mathrm{~kg} \mathrm{~m}^{-3}$. Note, however, that density does not appear in the analytical solution and, thus, has no impact on the CTF results in terms of turbulent mixing of enthalpy.

Discussion of Results The case is run to steady-state in CTF and the axial enthalpy profile is extracted for each channel. Figure 7.20 shows results for the two channels compared with the analytical solution that is calculated above. The figure demonstrates excellent agreement between code and expected results.

As noted above, the turbulent mixing model is defined so that it predicts net transfer of the parameter of interest due to turbulent exchange between channels. This transfer may or may not include mass transfer. For a single-phase, unheated case, turbulent exchange leads to zero net mass transfer because each unit of liquid volume that moves from Channel 1 to 2 is replaced with an equal volume that moves from Channel 2 to 1. The exchange does cause enthalpy to be moved, as is observed in Figure 7.20. A check is made on the axial mass flow rate profile in each of the channels in Figure 7.21.

It is noted that there is a slight discrepancy in the axial mass flow rate profile. Ideally, the normalized mass flux should be zero everywhere; however, the plot indicates that it varies by as much as $0.13 \%$ from the 


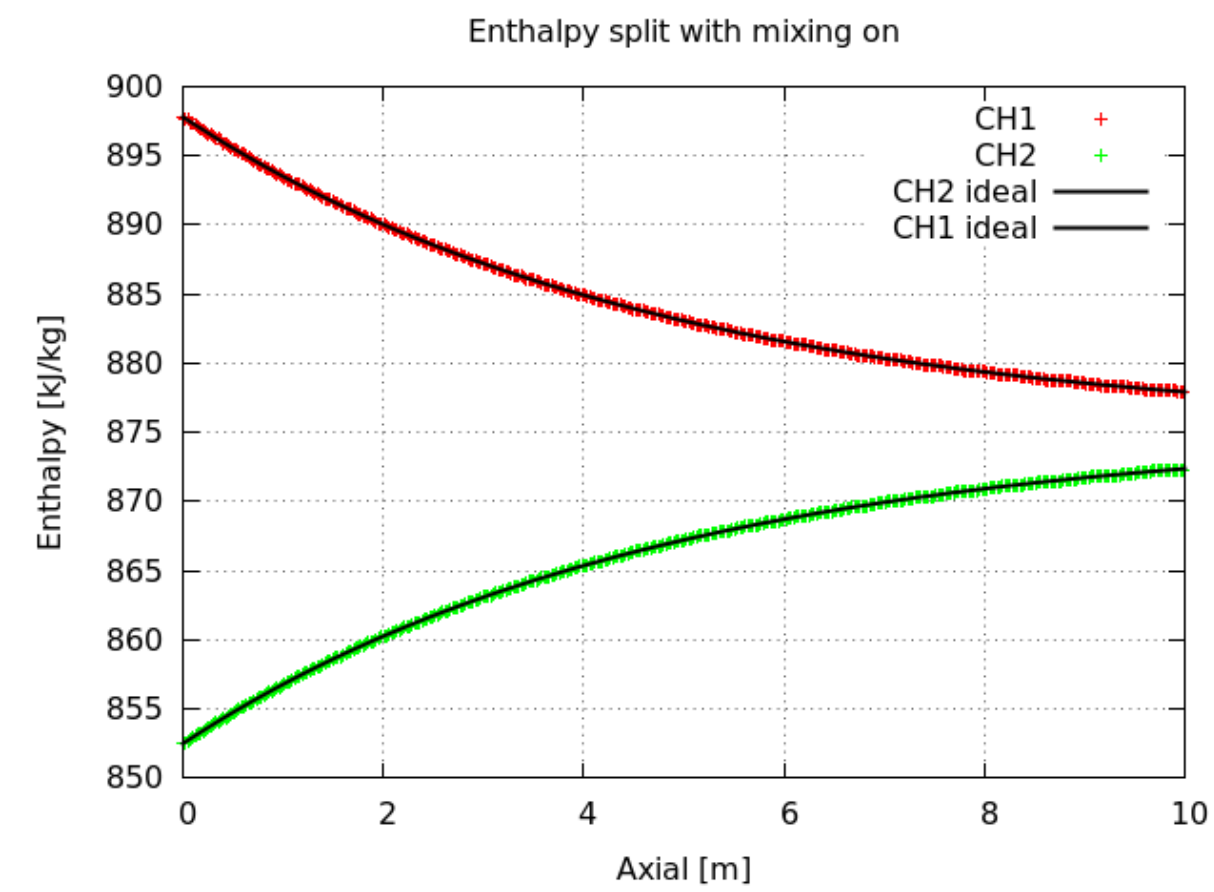

Figure 7.20: Comparison of CTF-predicted liquid enthalpy in channels and the analytical solution.

average mass flux. This discrepancy is further investigated by disabling the turbulent-mixing and void drift, leading to the flow redistribution shown in Figure 7.22.

The magnitude of the flow redistribution is small; however, results indicate that something in the solution is causing a slight drift in mass flux despite the channels being geometrically identical. 


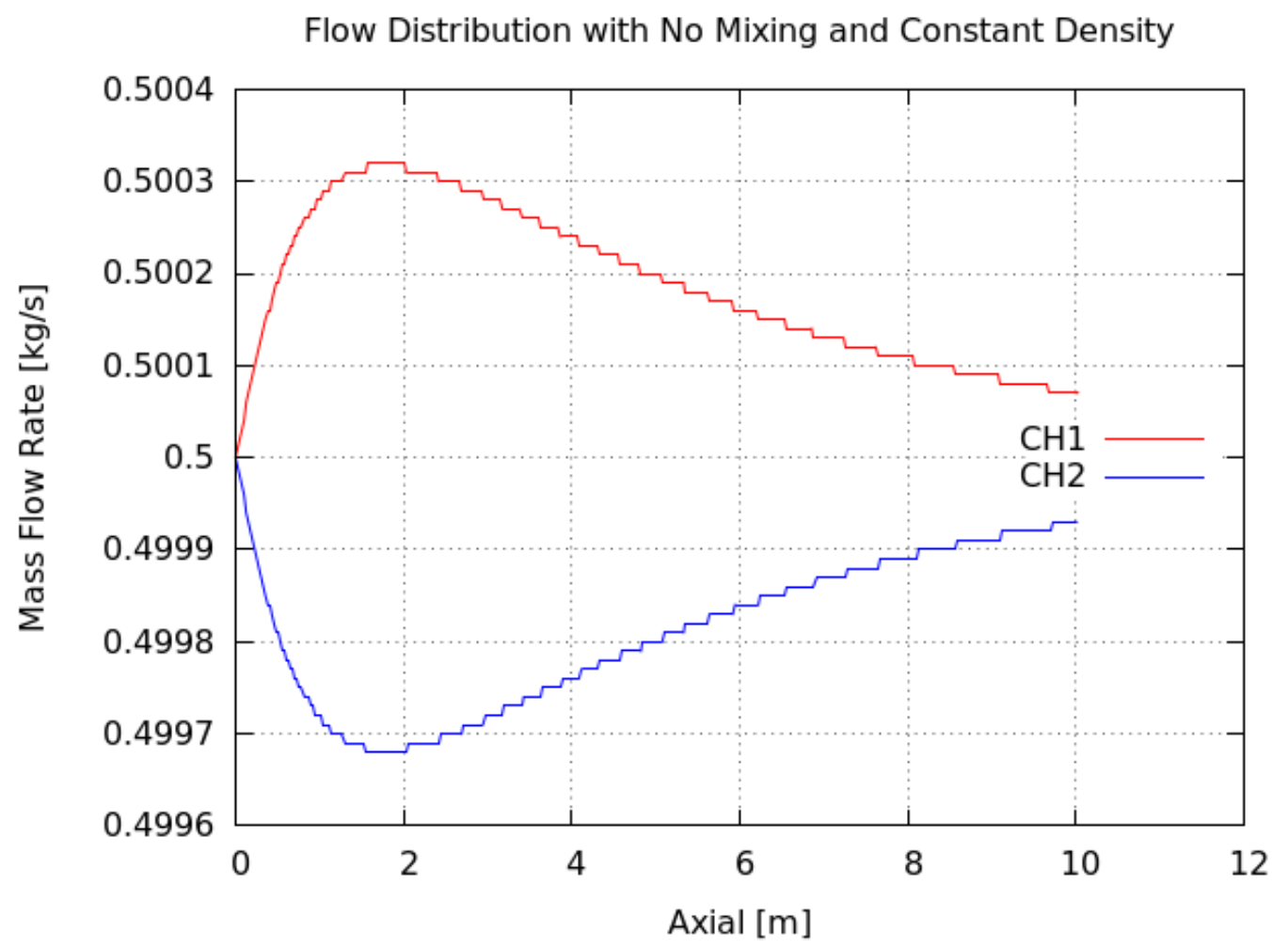

Figure 7.21: CTF-predicted axial mass flow rate profile in the channels. 


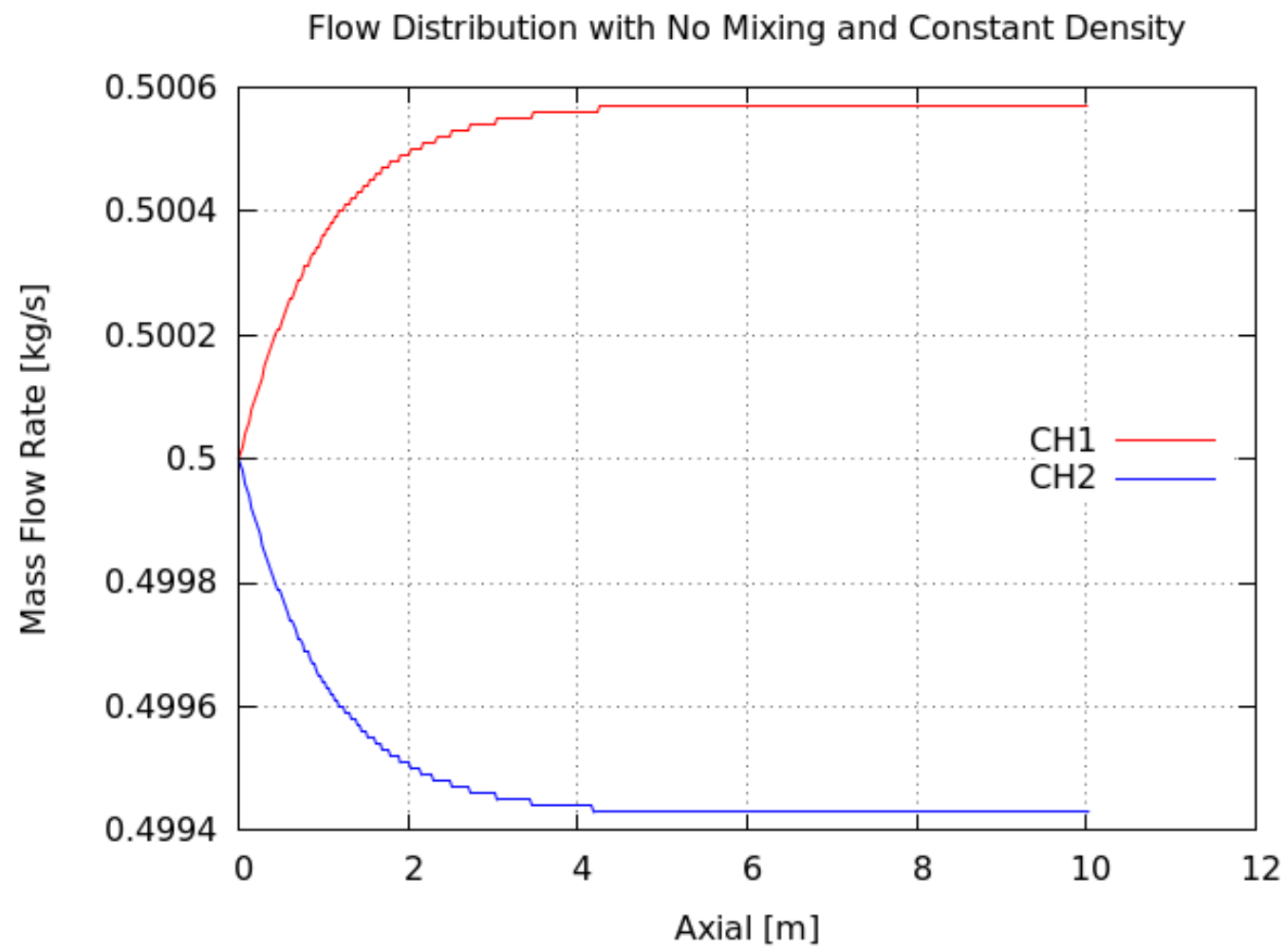

Figure 7.22: CTF-predicted axial mass flow rate profile in the channels with turbulent-mixing disabled. 


\section{\&્子约}

CTF V\&V

\section{Void Content}

\subsection{PSBT}

\subsubsection{Single Subchannel Facility}

Void measurements were taken at $1.4 \mathrm{~m}$ from channel bottom in the single subchannel facility. Although the initially reported measurement uncertainty was $3 \%$, it was later pointed out in the PSBT Benchmark results[41] that due to the low fidelity of current CT scan technology along with concerns on shifting of the CT images, the actual experimental uncertainty should be around $6 \%$. In CTF, Thom heat transfer correlation is used for predicting void generation rate in nucleate boiling region. The predicted void fraction results for all four test series are compared against experimental data in Figures 8.1, 8.2, 8.3, and 8.4. In these figures, the dashed black lines depict the experimental uncertainty of $6 \%$. One can notice that most test points fall within these dashed boundaries. The statistics (average absolute mean errors displayed on the figures) further confirm the close agreement between CTF and measured void results. 


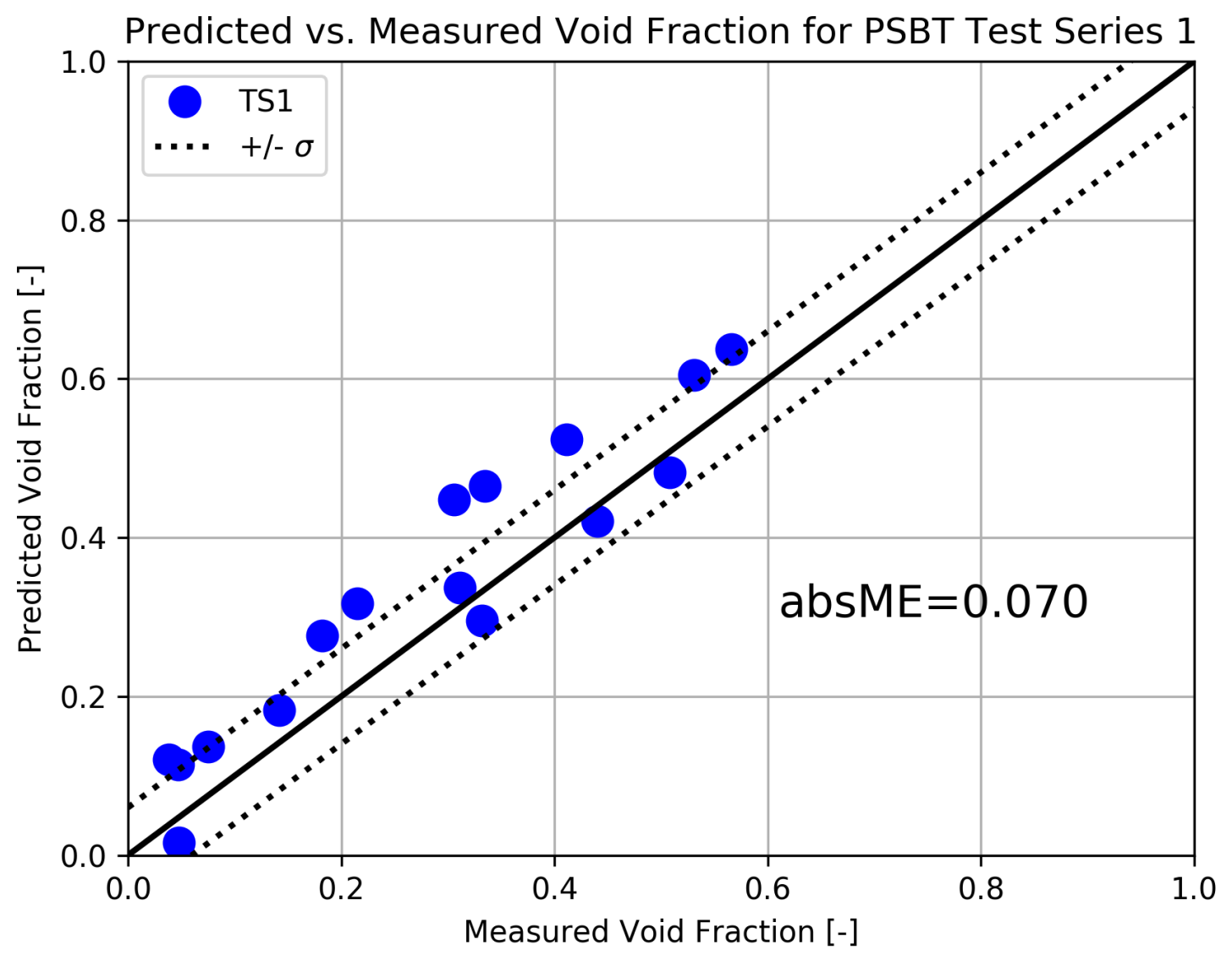

Figure 8.1: PSBT Series 1 predicted versus measured void fraction 


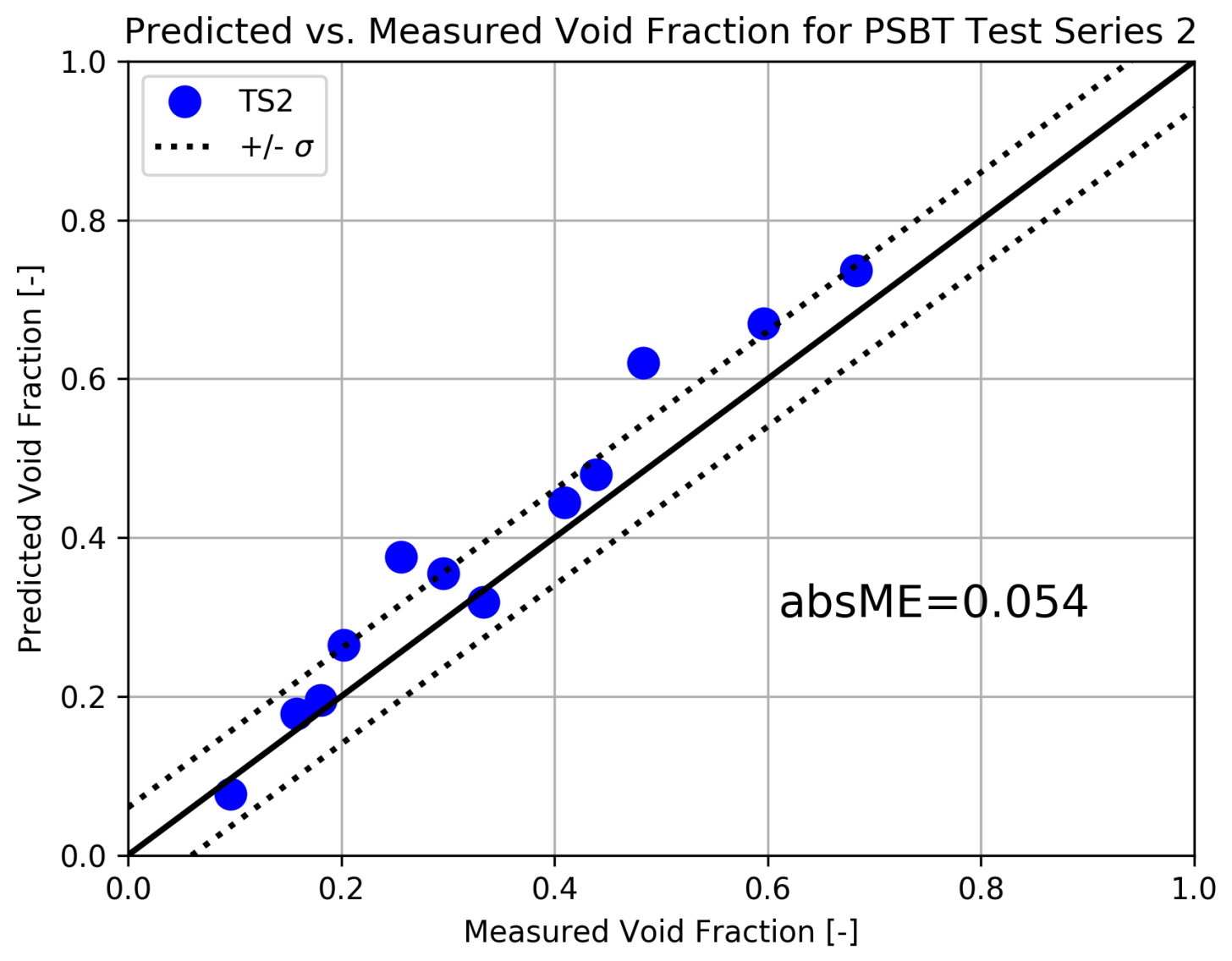

Figure 8.2: PSBT Series 2 predicted versus measured void fraction 


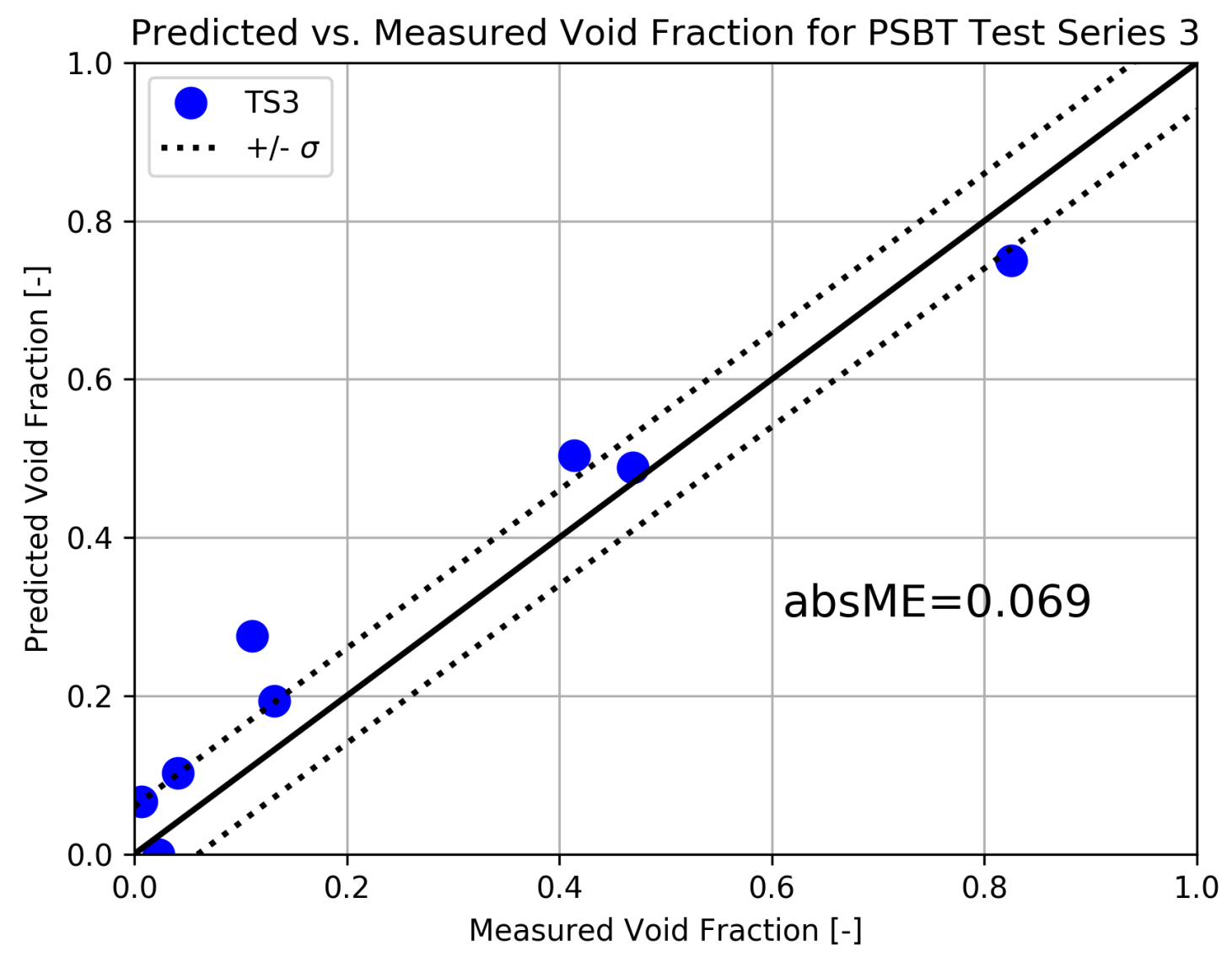

Figure 8.3: PSBT Series 3 predicted versus measured void fraction 


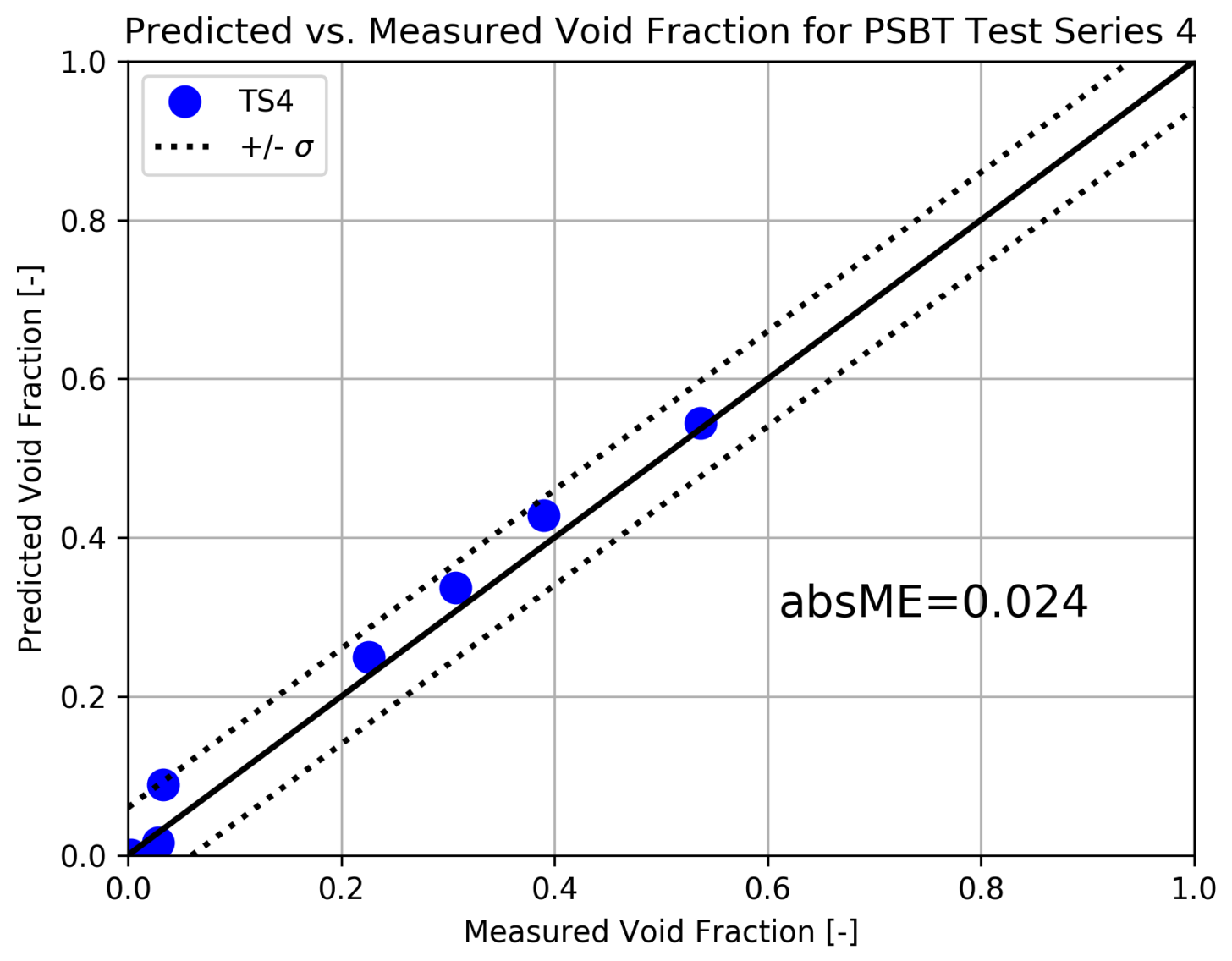

Figure 8.4: PSBT Series 4 predicted versus measured void fraction 


\subsubsection{Rod Bundle Facility}

Void measurements were taken at three axial locations in the rod-bundle facility. The measurements represent the average void of the four subchannels surrounding the central rod. Table 2.4.1 of the specification[3] states that the rod-bundle void measurement uncertainty is $4 \%$. To demonstrate CTF's capability to predict the void content in the flow, the predicted void (y-axis) is compared to measured void (x-axis) for the three test series. Thom correlation is used as the nucleate boiling heat transfer model. Results are shown in Figures 8.5, 8.6, and 8.7. In these figures, the dashed black lines depict the experimental uncertainty of $4 \%$. Data that fall within these dashed black boundaries are in agreement with experimental results to within experimental uncertainty.

Generally, the agreement with measured data is satisfactory. For Test Series 7 though, there is a tendency for CTF to over-predict void (in particular for lower and middle locations). 


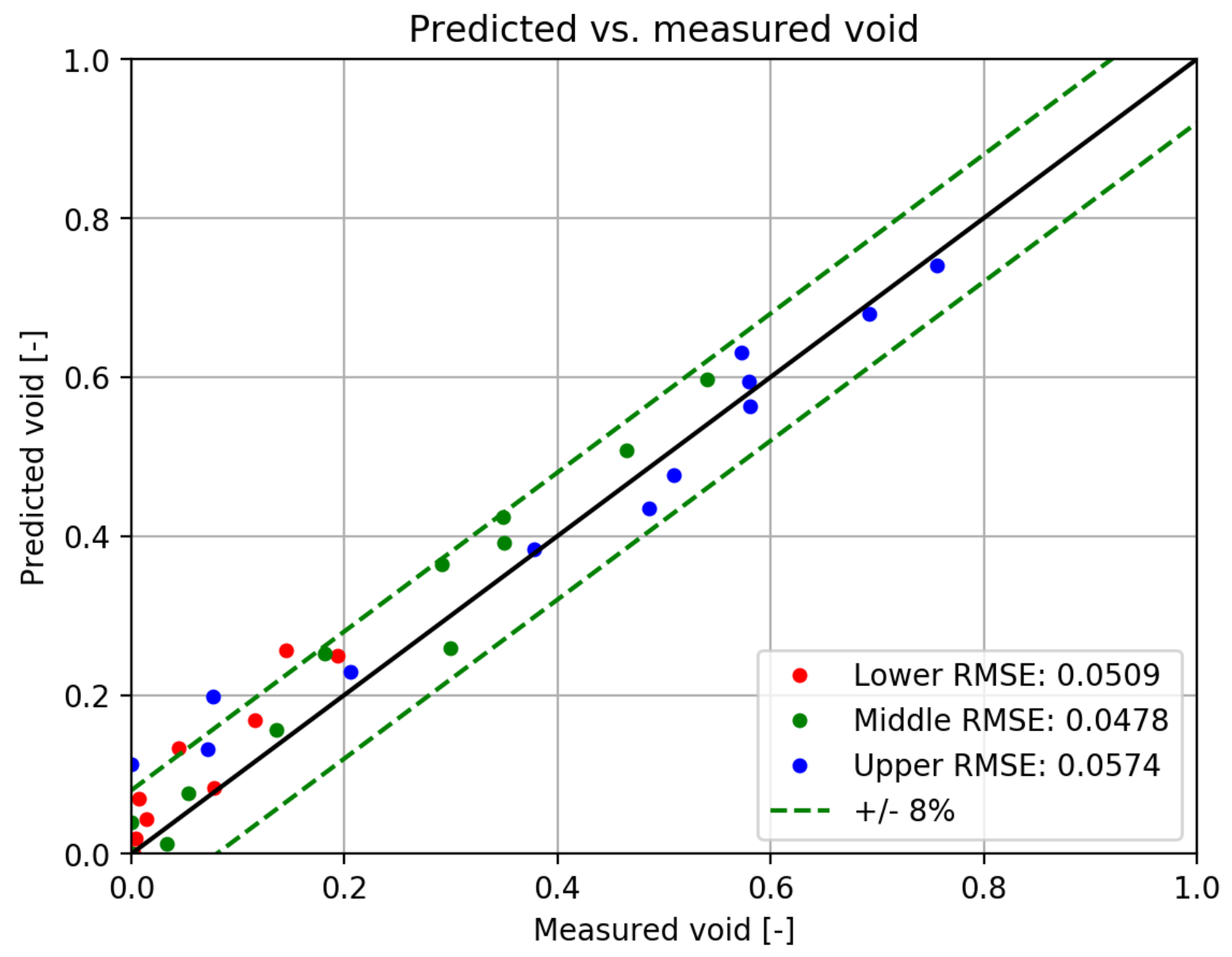

Figure 8.5: PSBT Series 5 predicted versus measured void around central rod in bundle 


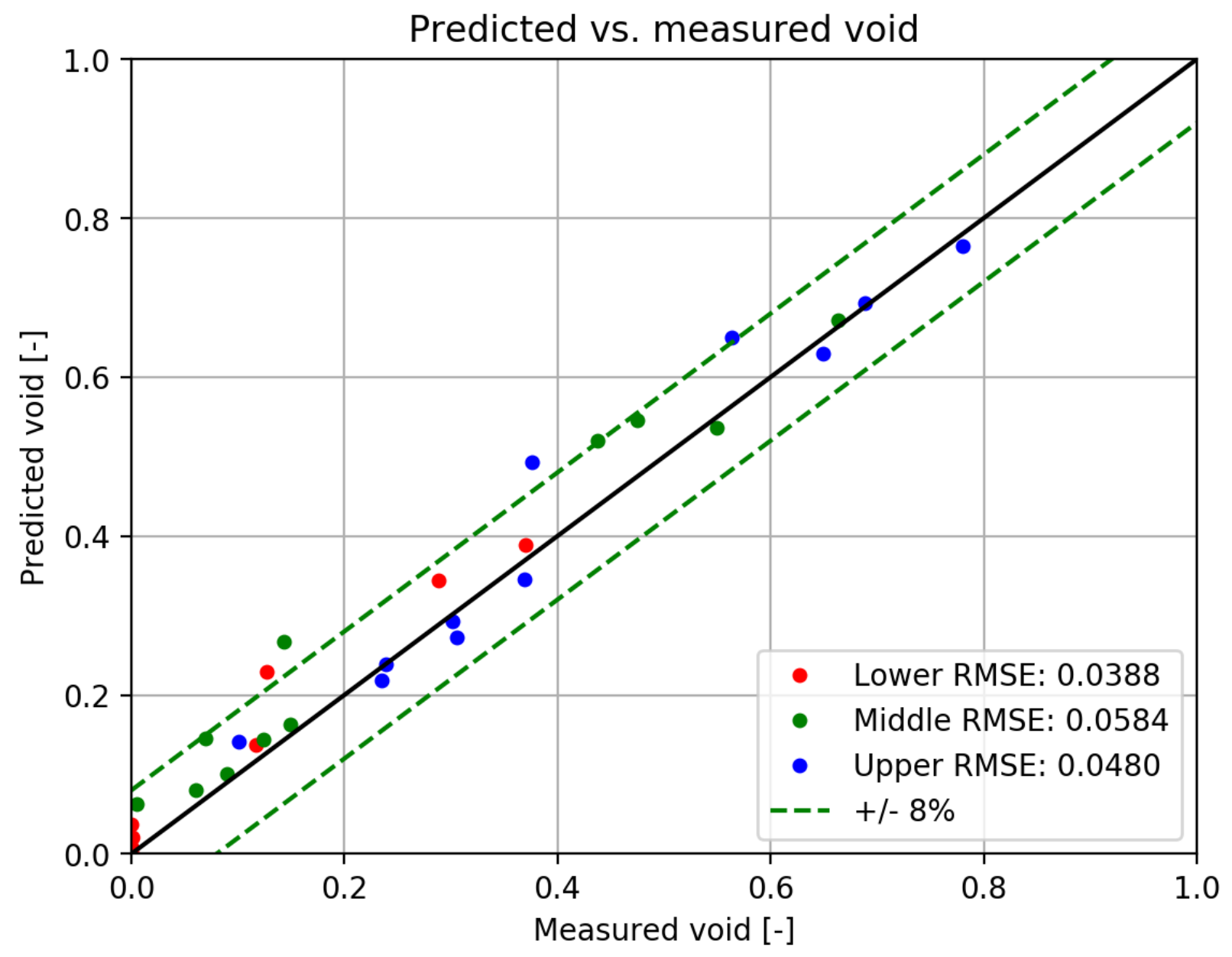

Figure 8.6: PSBT Series 6 predicted versus measured void around central rod in bundle 


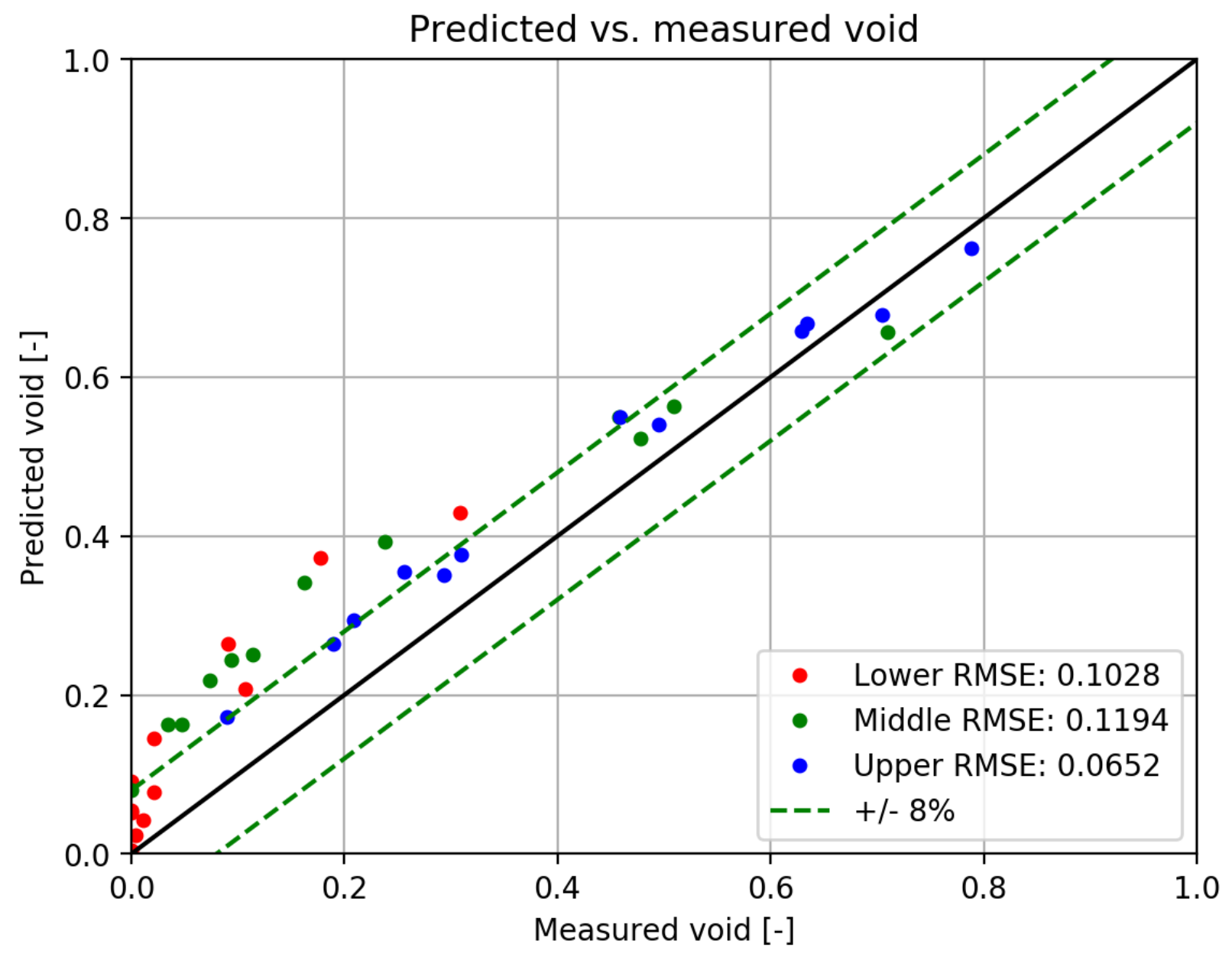

Figure 8.7: PSBT Series 7 predicted versus measured void around central rod in bundle 


\subsection{FRIGG Facility}

The CTF prediction of the bundle-averaged void profile in the FRIGG facility is shown in Figure 8.8. The RMSE was calculated from all of the points that were sampled from the experimental results (summarized in Table 47).

\subsection{Bartolomei}

CTF Model Experimental data used for this study include 27 conditions from the two tubular geometry test sections. A script is used to generate the CTF input files automatically for cases 13-38, whereas the input file for Case 1 is manually generated. The CTF models are created using internal flow in a single channel, with the inlet mass flux and inlet temperature used to set the inlet boundary conditions, and system pressure used for the outlet boundary condition. Uniform heat flux is provided at the outer heater wall surface with the linear heat rate calculated to correspond to the experimental heat flux at the inner heater surface/outer internal tube surface. The $15.4 \mathrm{~mm}$ and $12.0 \mathrm{~mm}$ diameter test sections are both divided into uniformly spaced axial nodes with a nodal size of $25.0 \mathrm{~mm}$ (roughly twice the hydraulic diameter), which is consistent with the typically recommended mesh size for CTF calculations. The Bartolomei data was presented in a way that did not associate measured void values with specific test sections or measurement locations. The actual measurement locations were unknown. However, the void measurement was given as a function of local quality, and the boundary conditions (i.e., inlet subcooling, inlet mass flux, heat flux, and outlet pressure) were known for the different test geometries. Since the exact tube lengths are unknown for cases 13-38, a fixed tube length of $1.5 \mathrm{~m}$ is used which corresponds to a total of 60 nodes. The CTF void predictions were then interpolated as a function of equilibrium quality to match the experimental values. The total number of nodes for Case 1 is 80 .

Results of Case 1 are shown in Figure 8.9. CTF captures the overall trend in the axial void development and liquid temperature profile very well. However, CTF predicts the location of onset of nucleate boiling (ONB) at the very first node in comparison to the experimental data, which predict ONB further downstream. Furthermore, the liquid temperature measurement close to the exit exceeds the saturation temperature according to the IF97 steam-water properties used in CTF. The experimental void fraction plateaus at the exit, whereas the CTF predicted void fraction continues to rise as the liquid temperature plateaus, giving a constant temperature difference between the heater surface and the bulk liquid.

Table 47: Summary of void fractions sampled from FRIGG data report

\begin{tabular}{|c|c|}
\hline Axial Location $(\mathrm{m})$ & Measured Void $(-)$ \\
\hline 0.824 & 0.252 \\
1.291 & 0.304 \\
1.921 & 0.469 \\
2.388 & 0.618 \\
2.972 & 0.607 \\
3.567 & 0.676 \\
4.034 & 0.696 \\
\hline
\end{tabular}

Consortium for Advanced Simulation of LWRs 


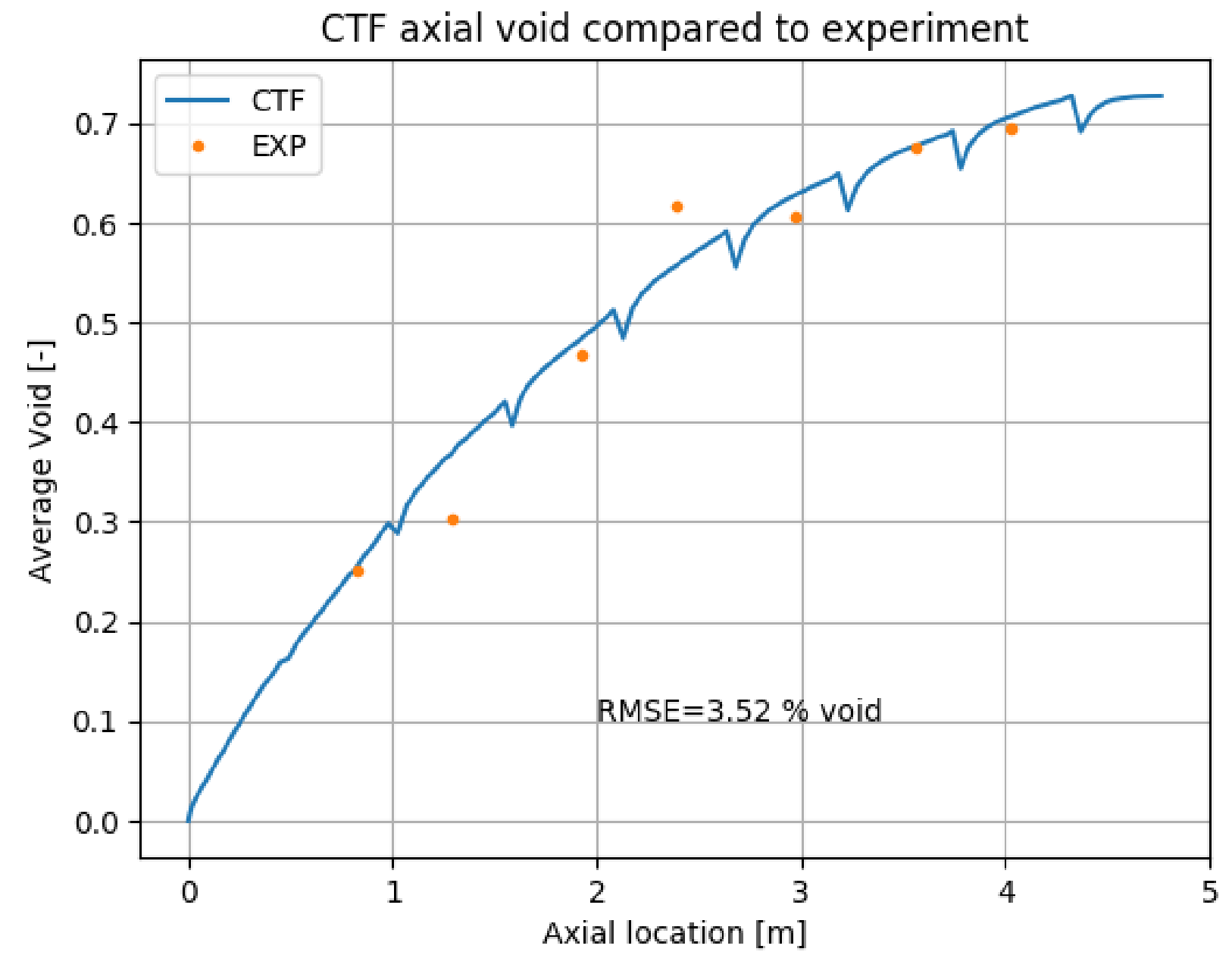

Figure 8.8: Predicted and measured axial bundle-averaged void profile in FRIGG facility 


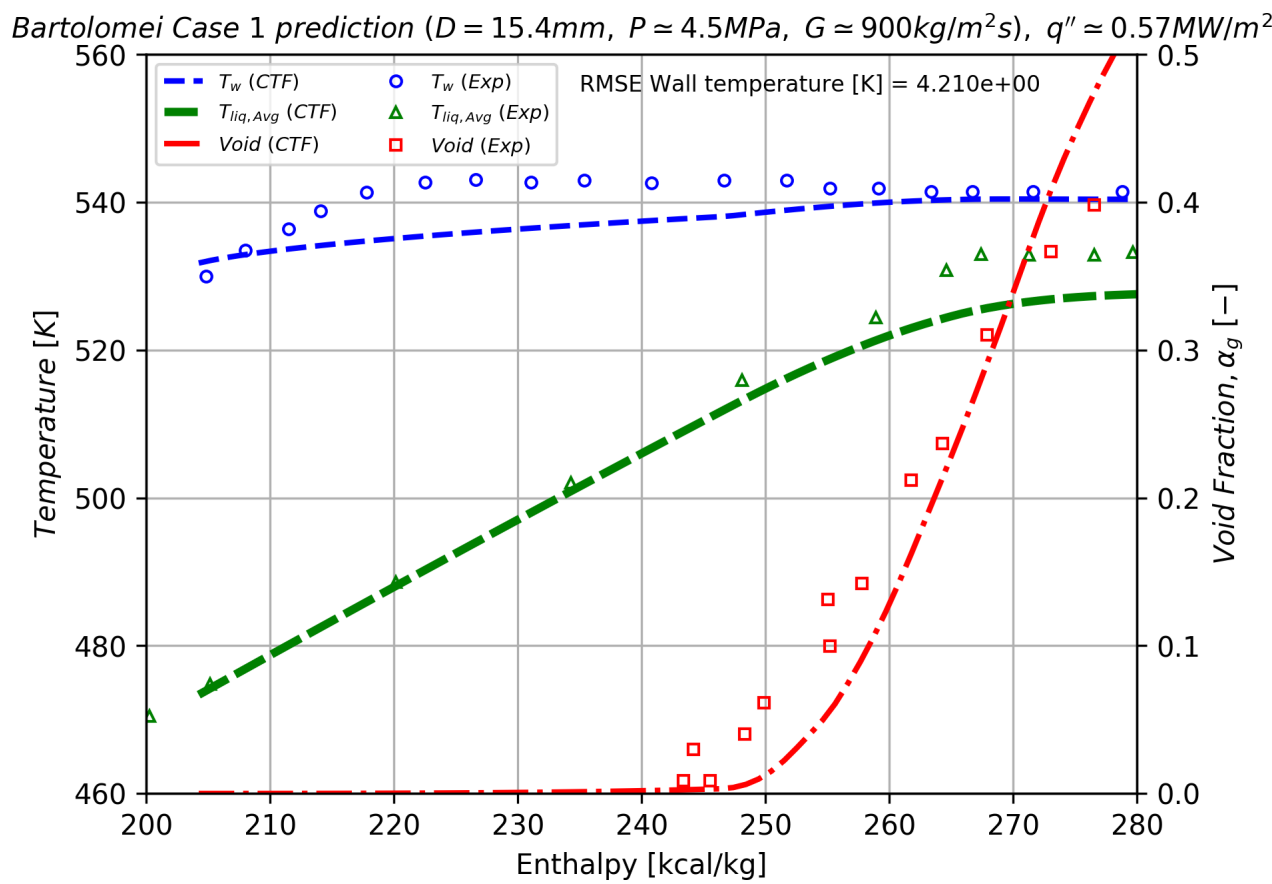

Figure 8.9: Comparison of CTF and experimental data of wall temperature, mean liquid temperature, and void fraction for case 1. 


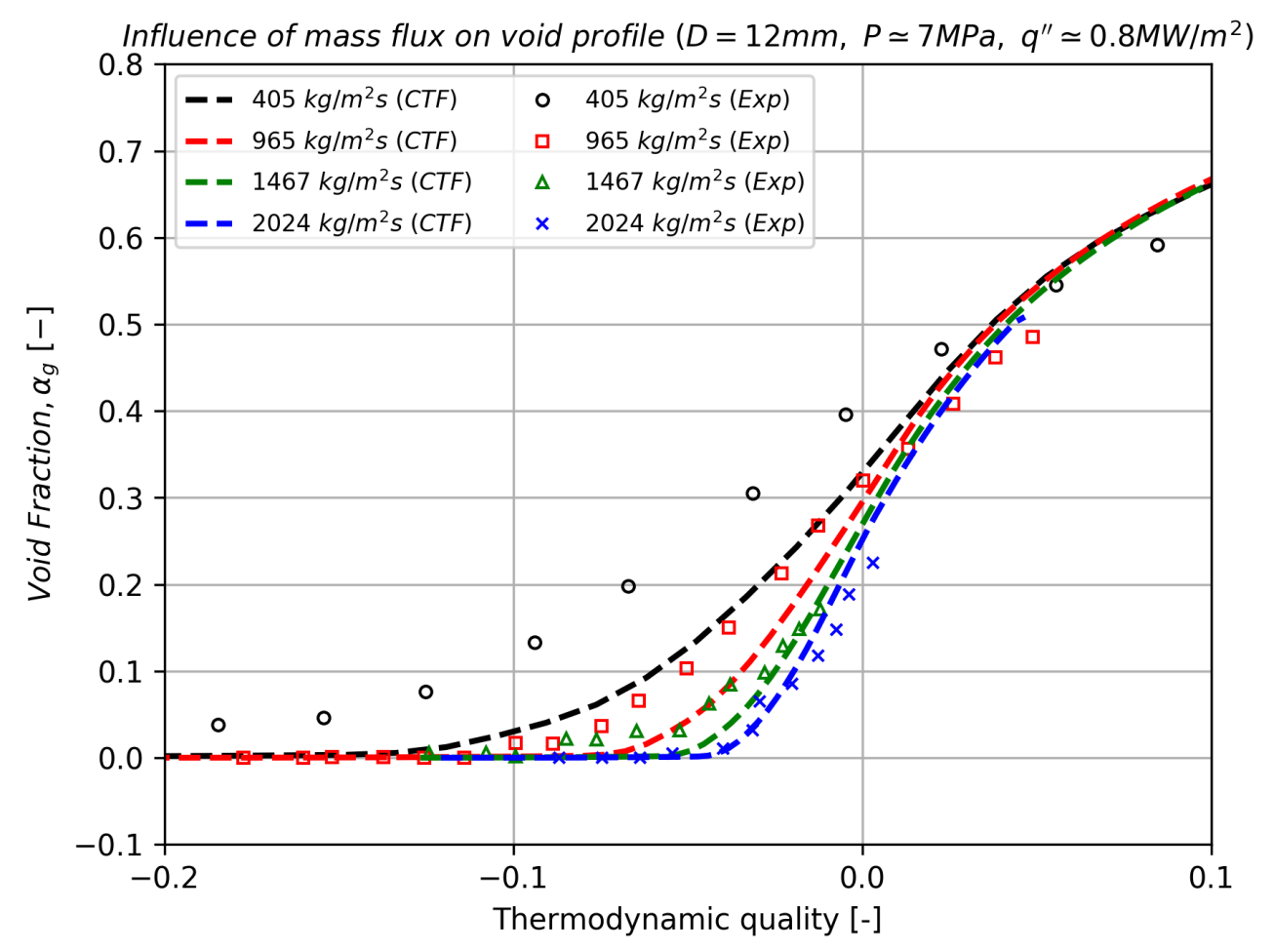

Figure 8.10: Comparison of CTF and experimental data for variation in mass flux at 70.0 bar and heat flux of $0.8 \mathrm{MW} / \mathrm{m}^{2}$.

Results of parametric variation of key experimental system parameters from select cases are shown in the following four figures: mass flux variation in Figure 8.10, heat flux variation at 70.0 bar in Figure 8.11, and at 150.0 bar in Figure 8.12, and pressure variation in Figure 8.13. Qualitatively, CTF void fraction predictions are better at higher mass fluxes and higher heat fluxes, and they worsen at lower mass fluxes. At both 70.0 bar and at 150.0 bar for varying heat fluxes and at moderate to high mass fluxes, the CTF predictions capture the void fraction development with underpredictions at low void fractions and overpredictions at high void fractions. The effect of pressure shown in Figure 8.13 is more pronounced in CTF in comparison to the experimental data in terms of the ONB location and void fraction development.

The overall void fraction comparison between the experimental data and CTF are shown in Figure 8.14. These results use the default subcooled wall boiling model of Thom. The CTF comparison using the subcooled wall boiling of Chen is shown in Figure 8.15. For the other benchmarking studies in this chapter, the model of Chen overpredicts the wall temperature and subsequently the void fraction. For this dataset, the model of Chen does better with the wall temperature prediction for case 1. Overall, the model of Chen predicts the void fraction more accurately at low voids with a consistent overprediction at higher void fractions. The model of Thom has considerable scatter on either side of the 1:1 line at lower void fractions, with a consistent overprediction at higher void fractions, similar to the other benchmarking studies in this chapter. 


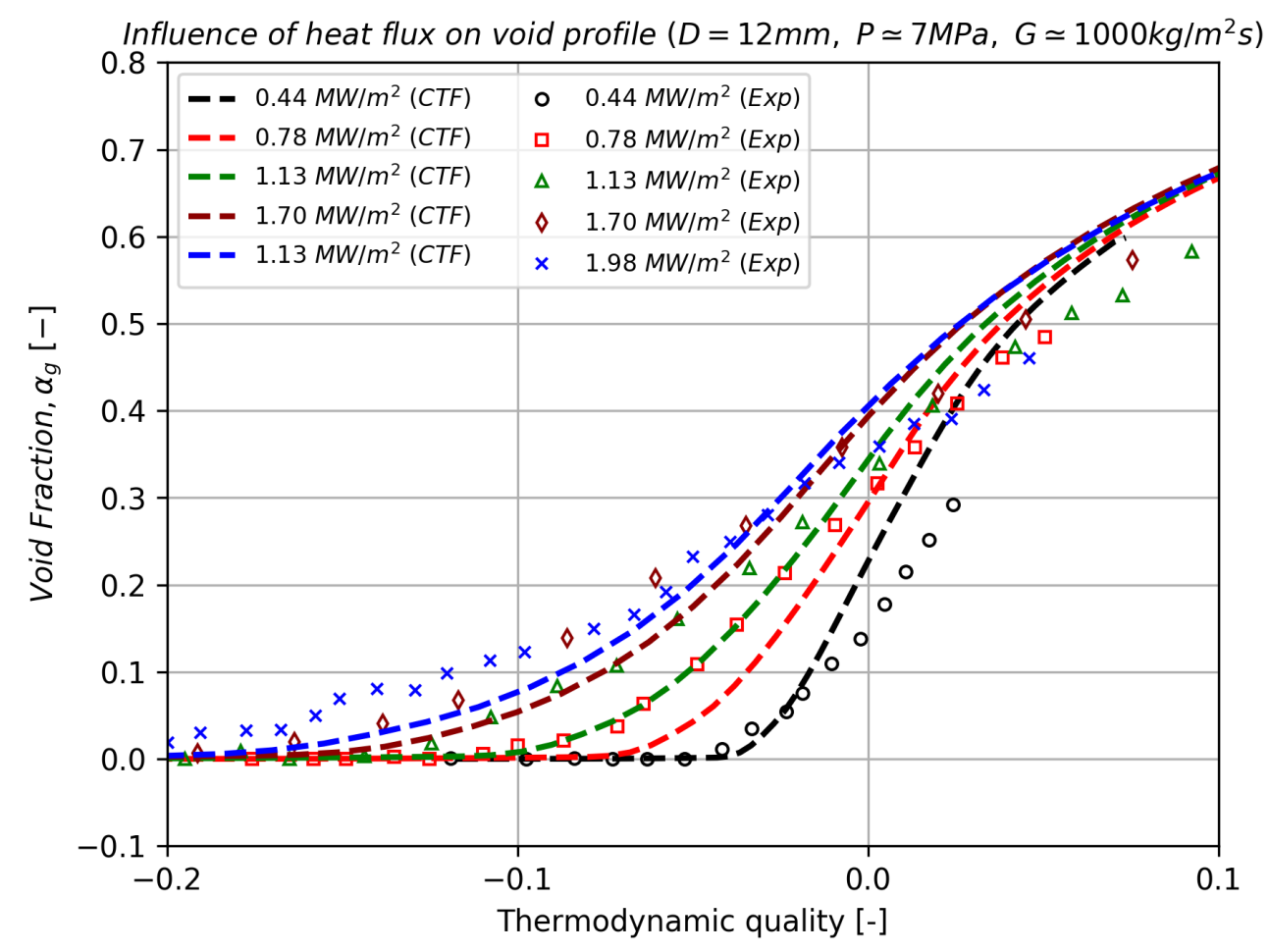

Figure 8.11: Comparison of $\mathrm{CTF}$ and experimental data for variation in mass flux at 70.0 bar and mass flux of $1000.0 \mathrm{~kg} / \mathrm{m}^{2}$-s. 


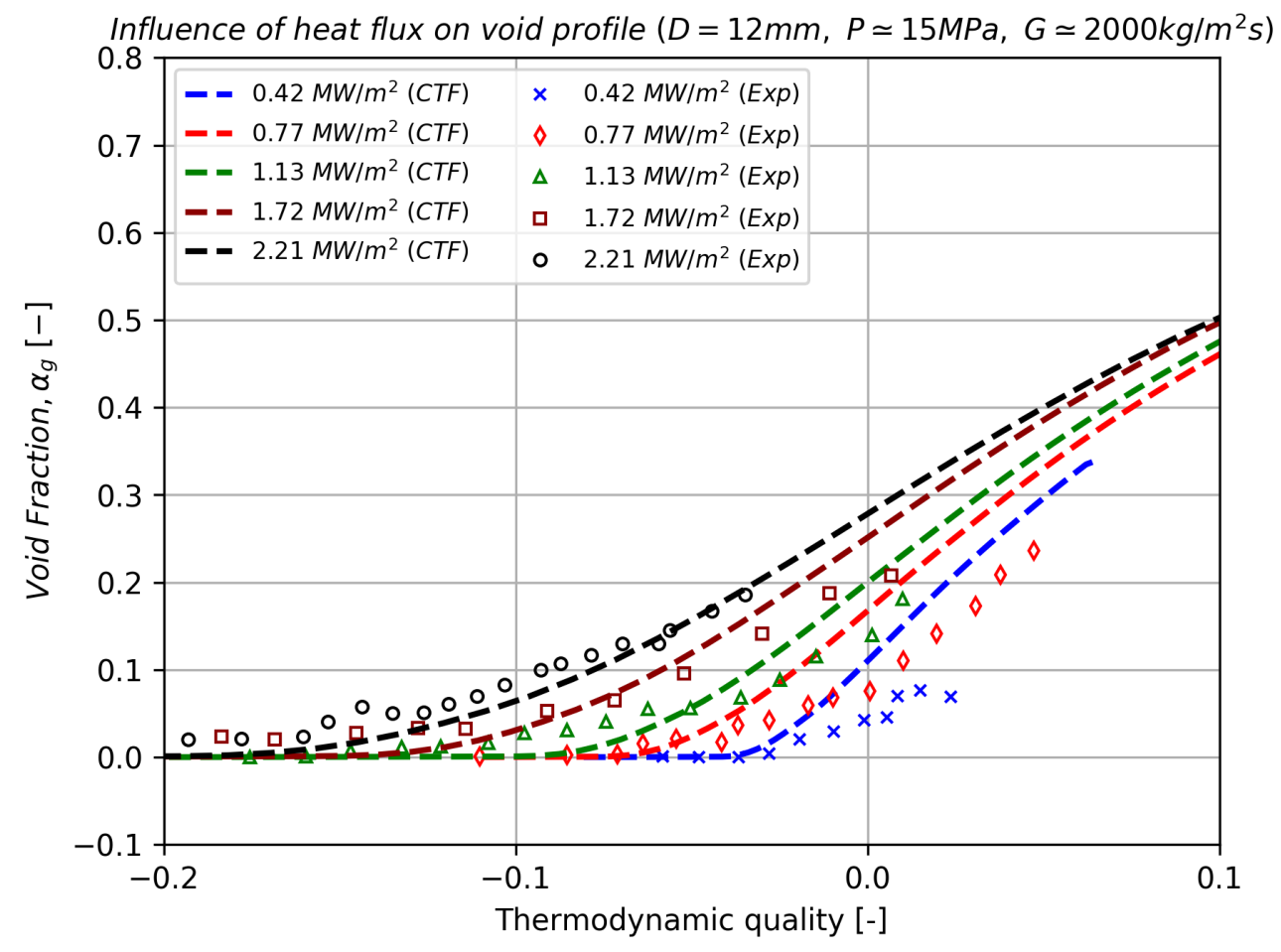

Figure 8.12: Comparison of CTF and experimental data for variation in mass flux at 150.0 bar and mass flux of $2000.0 \mathrm{~kg} / \mathrm{m}^{2}-\mathrm{s}$. 


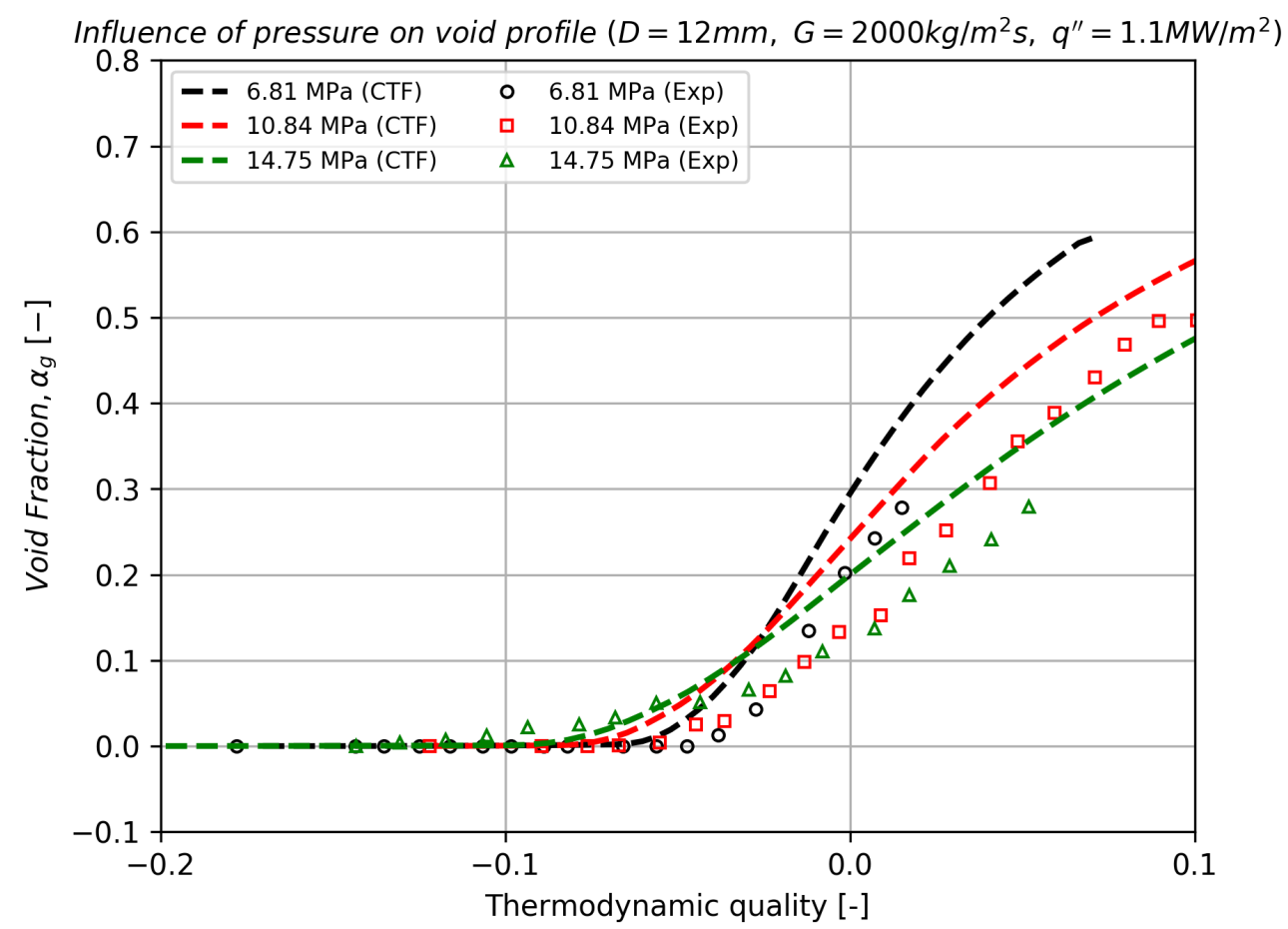

Figure 8.13: Comparison of $\mathrm{CTF}$ and experimental data for variation in system pressure at mass flux of $2000.0 \mathrm{~kg} / \mathrm{m}^{2}$-s and heat flux of $1.1 \mathrm{MW} / \mathrm{m}^{2}$. 


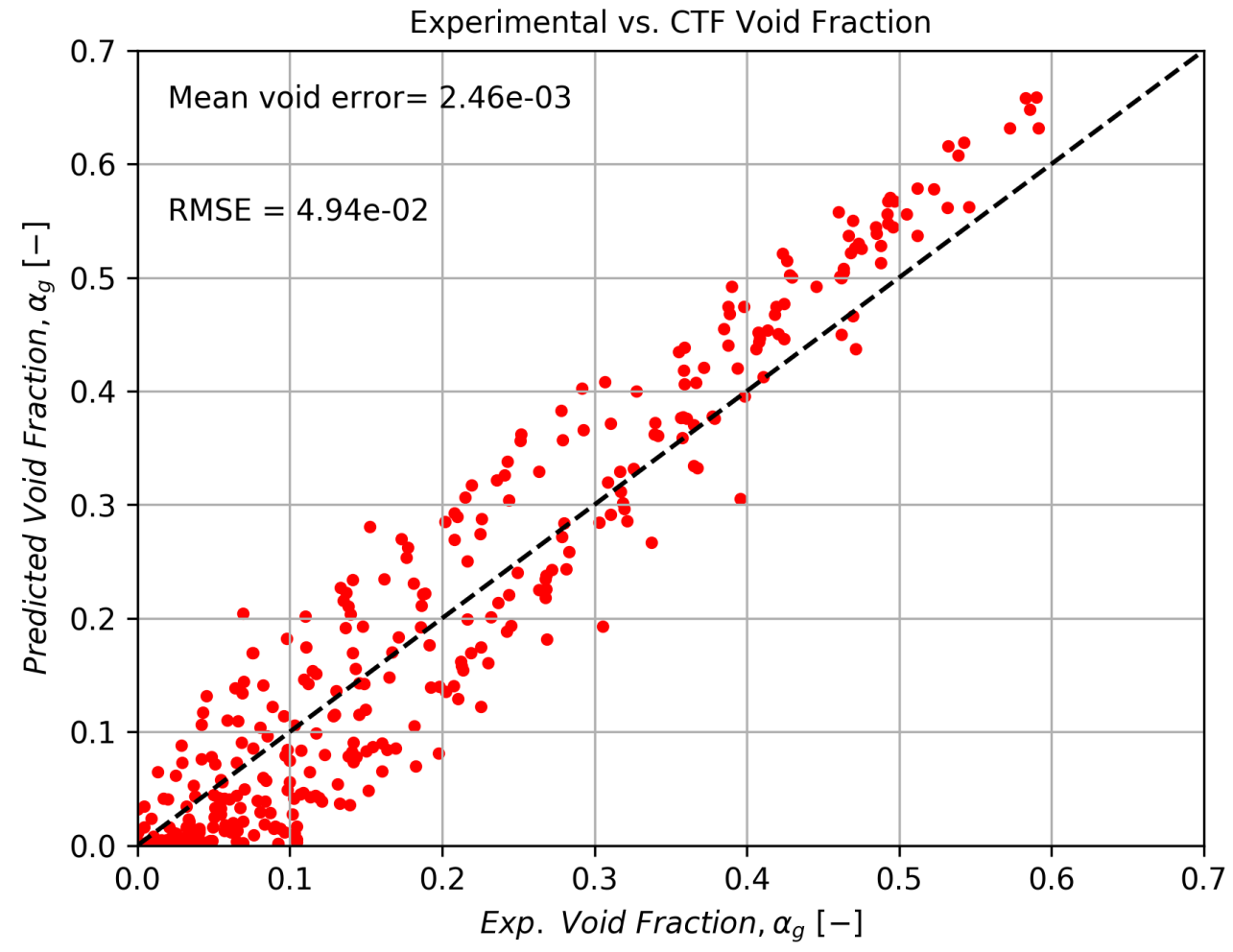

Figure 8.14: Overall comparison of CTF versus experimental void fractions using the subcooled boiling model of Thom. 


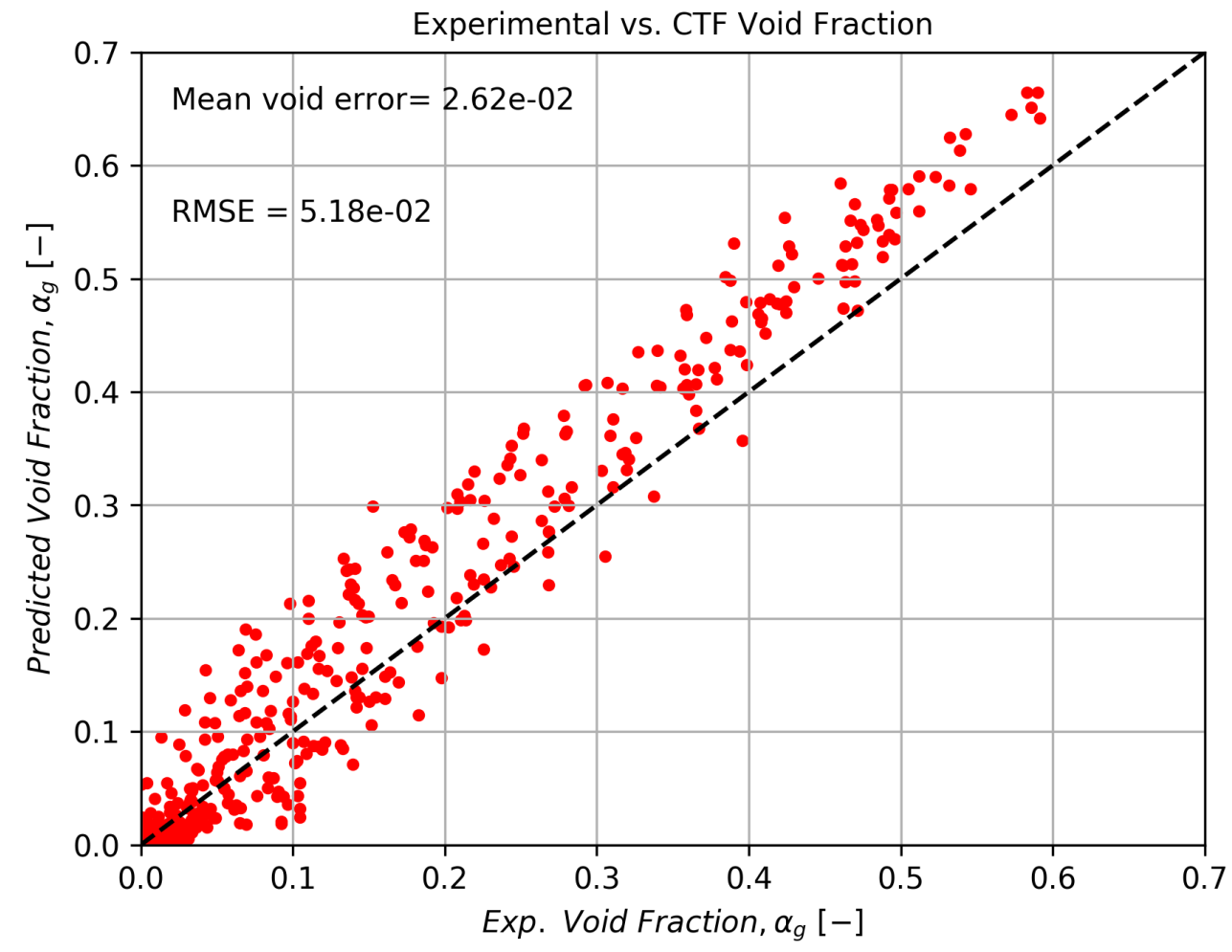

Figure 8.15: Overall comparison of CTF versus experimental void fractions using the subcooled boiling model of Chen. 


\section{Two-Phase Turbulent Mixing and Void Drift}

\subsection{Validation}

\subsubsection{GE 3x3}

A comparison of the measured and predicted exit equilibrium qualities is provided in Figure 9.1 for the base case model, which uses the void drift model with $K_{a}$ set to 1.4 , the turbulent mixing model with $\beta_{\mathrm{sp}}$ set to 0.007 , and $\Theta_{M}$ set to 5.0. Figure 9.1 shows that most predicted exit qualities fall within experimental uncertainty. However, the values that seem to vary furthest from measured results are qualities in the corner type subchannel.

In addition to the exit equilibrium quality, the authors also measured the exit mass flux of each individual subchannel. Results for the two-phase experiments are shown in Figure 9.2. 


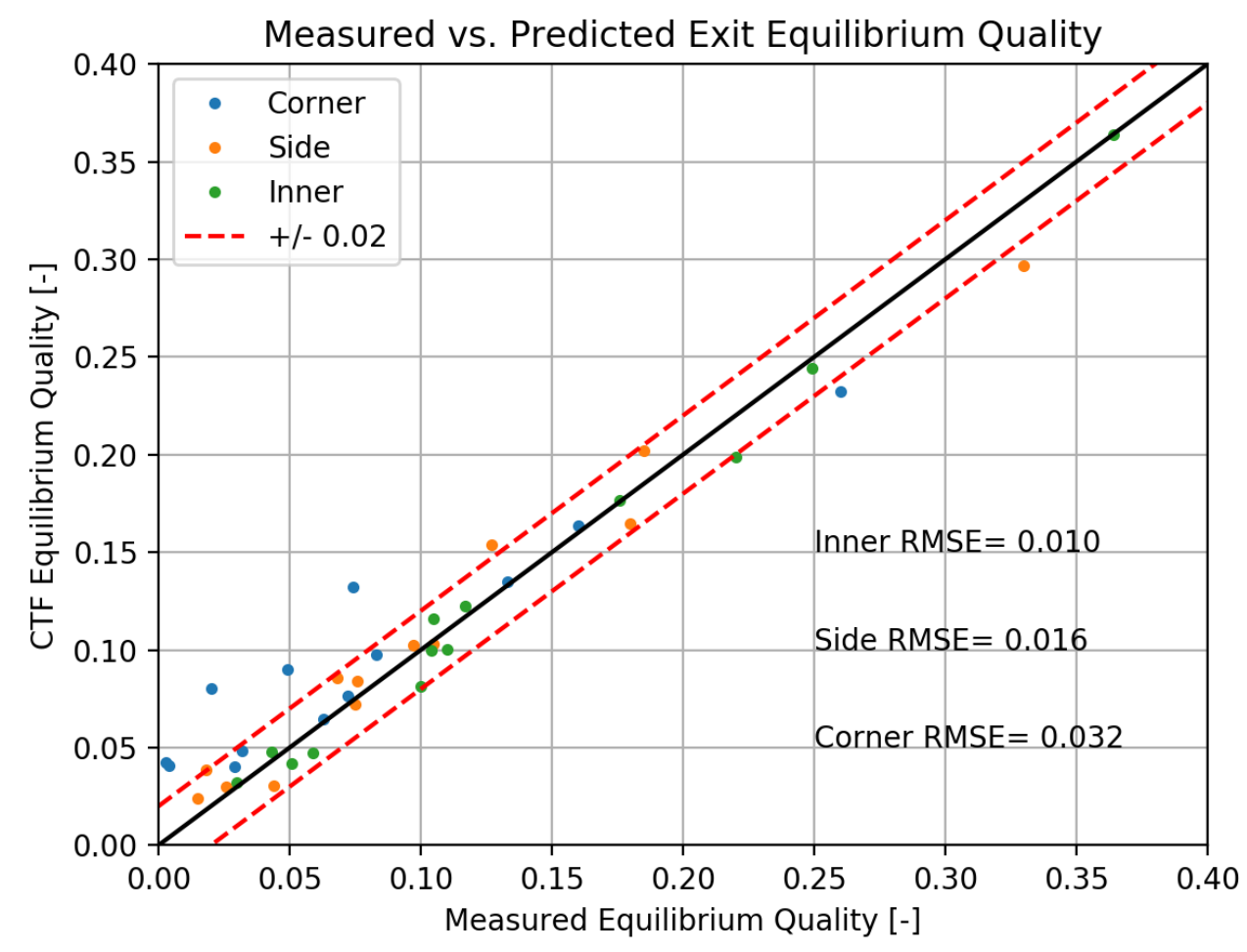

Figure 9.1: Comparison of predicted and measured exit equilibrium quality for GE $3 \times 3$ two-phase cases. 


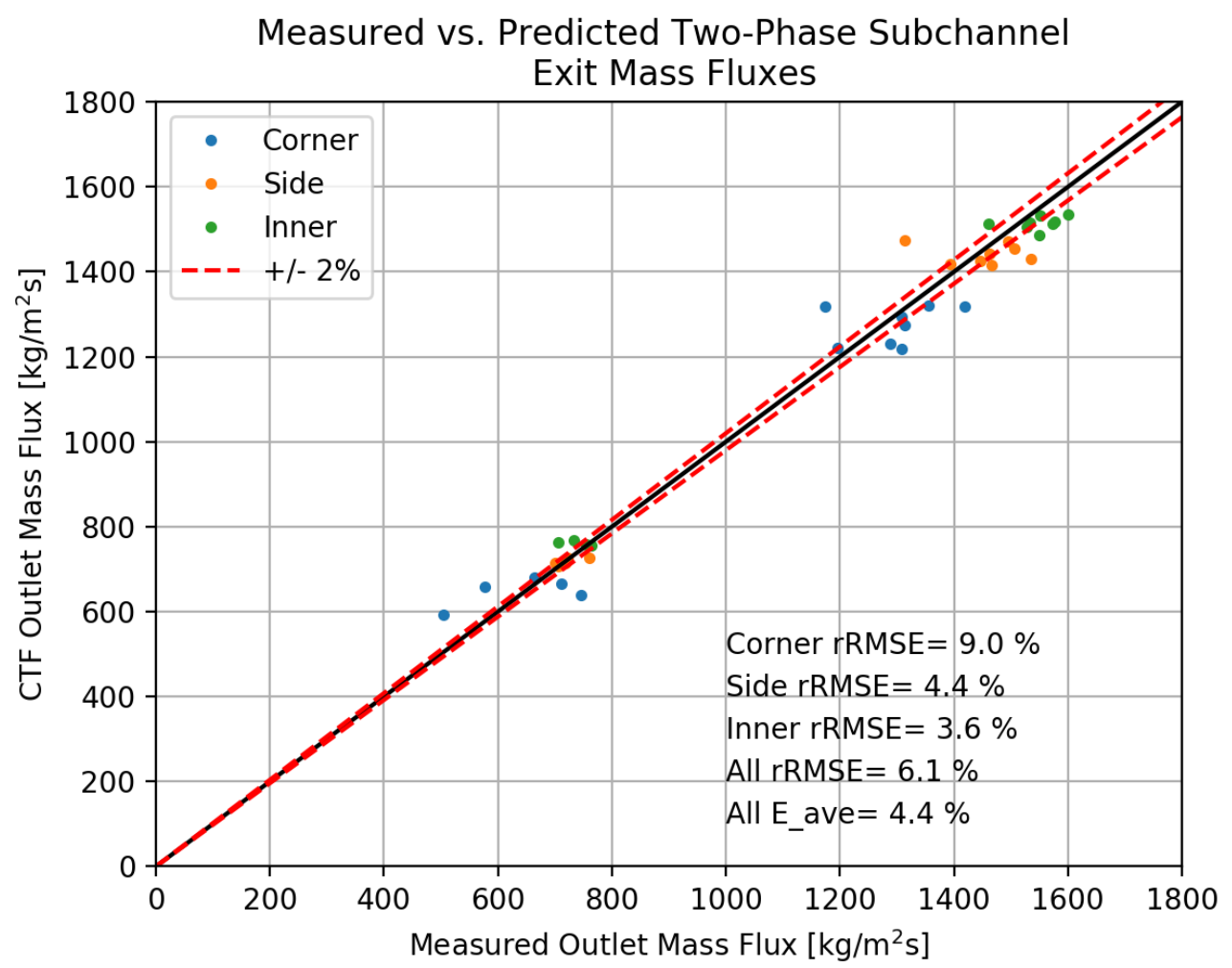

Figure 9.2: Comparison of predicted and measured exit mass flux for GE $3 \times 3$ two-phase cases. 


\subsubsection{BFBT $8 \times 8$}

It has been found that the stopping-criteria for these terms need to be substantially relaxed compared to single-phase cases, which tend to converge much more tightly. In particular, the droplet velocity stopping criteria has to be set to a fairly large number; however, because we do not actually model the droplet entrainment and de-entrainment (meaning the droplet void stays insignificant throughout the simulation), this is considered acceptable.

As a first step, CTF-predicted bundle average void at the outlet and thermal equilibrium quality is compared with the experimental results. The thermal equilibrium quality is calculated from the flow-weighted mixture enthalpy of individual channels. The exit void is calculated from the area-weighted void of individual channel values. The comparison of CTF-predicted and measured bundle average outlet void is shown in Figure 9.3 and the comparison of CTF-predicted and measured bundle average thermal equilibrium quality is shown in Figure 9.4.

There is a tendency for CTF to consistently overpredict the bundle-average void. The overprediction is more severe for the cases in bubbly flow with lower amounts of void (about 40\%). At outlet void of about $80 \%$, the prediction almost matches the experimental values. If the same amount of energy is entering the CTF model as is entering the experimental facility, the mixture enthalpy at the outlet should be identical to the experiment (barring steam table differences); however, phase slip differences can lead to discrepancies between the predicted and experimental void.

Figure 9.4 shows that exit equilibrium quality agrees with experimental values to within $+/-3 \%$ for most cases. One low quality case experiences a substantial discrepancy, which currently has no explanation. The system power has been checked for this case and it agrees favorably with the specified power in the BFBT specification.

Figure 9.5 gives an overview of the measured versus predicted subchannel void measurements for all tests. The data is grouped by assembly type, so multiple experiments are included in each dataset. The data shows that there is an overprediction of void; data is frequently over the $+10 \%$ line but rarely falls below the $-10 \%$ line. Also, the data appears to form into horizontal "stripes." This is due to the asymmetric behavior and spread of the experimental data compared with the symmetric behavior of the CTF predictions.

More insight is gained by breaking the data comparison into different categories as shown in Figure 9.6. This is shown in Figures 9.7-9.11.

The worst predictions are for the channels that touch unheated elements. CTF tends to overpredict the void in these channels. The inner channels, on the other hand, almost always fall within the $\pm 10 \%$ bands in the plot. As was observed in the GE $3 \times 3$ validation study, the error in the boundary channels tends to be higher than normal inner channel types. However, it was also found in the GE $3 \times 3$ study that the corner channel exhibited the largest error compared with experimental data, but this is not the case for BFBT.

To observe void distribution trends, void data is visualized over the diagonals of each assembly. Figure 9.12 gives an example of the two diagonal lines that are struck through the assemblies. The example is given for Assembly Type 0-1, but the process is the same for all five assembly types. Note that the red line goes from the northwest to the southeast, and the blue line goes from the southwest to the northeast. The experimental data along these lines will be shown with dots, and the CTF predictions will be shown with lines. The red dots and lines will be for the northwest to southeast diagonal, and the blue dots and lines will be for the southwest to northwest diagonal. Figures 9.13-9.22 show the radial void profile results on the diagonals for 


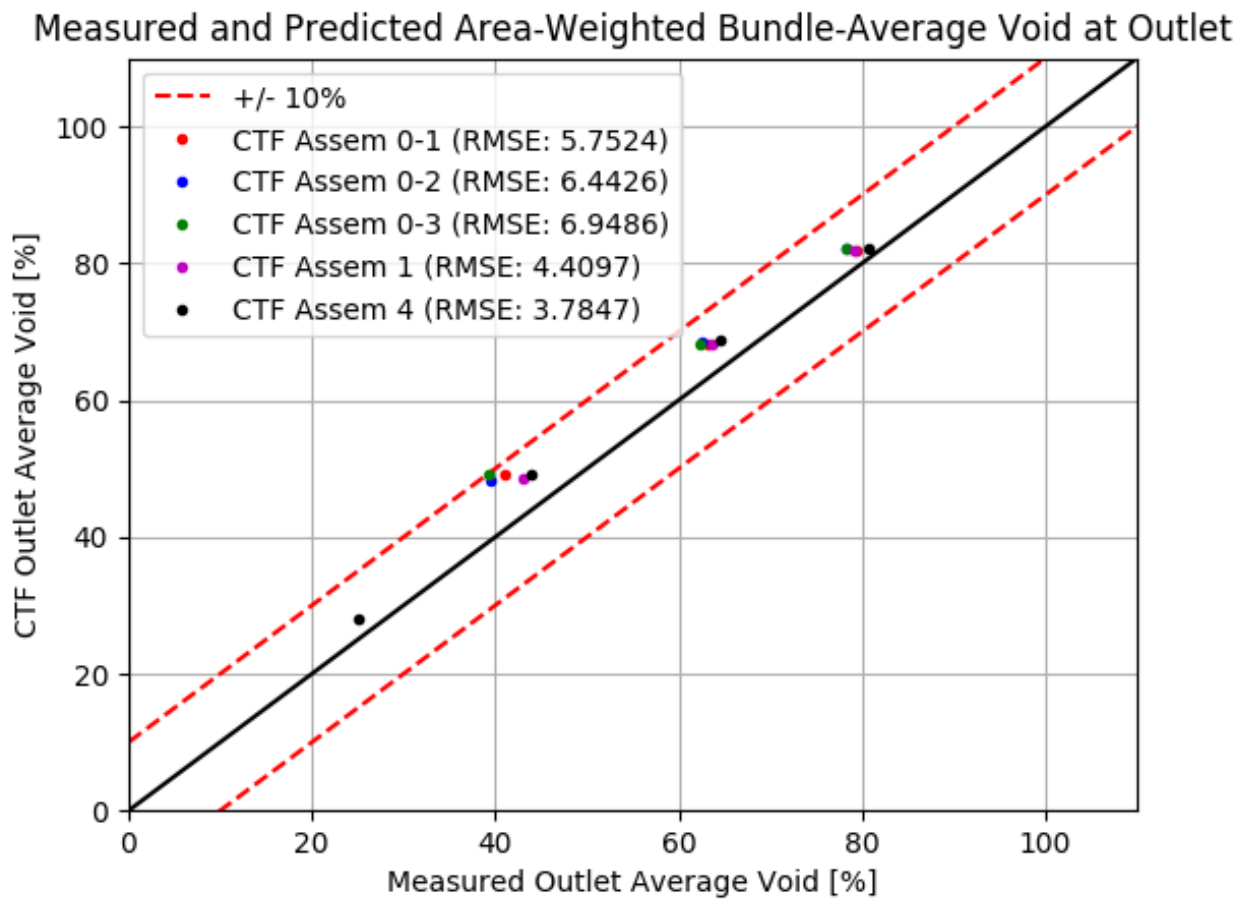

Figure 9.3: Comparison of measured and predicted bundle-averaged outlet void. 


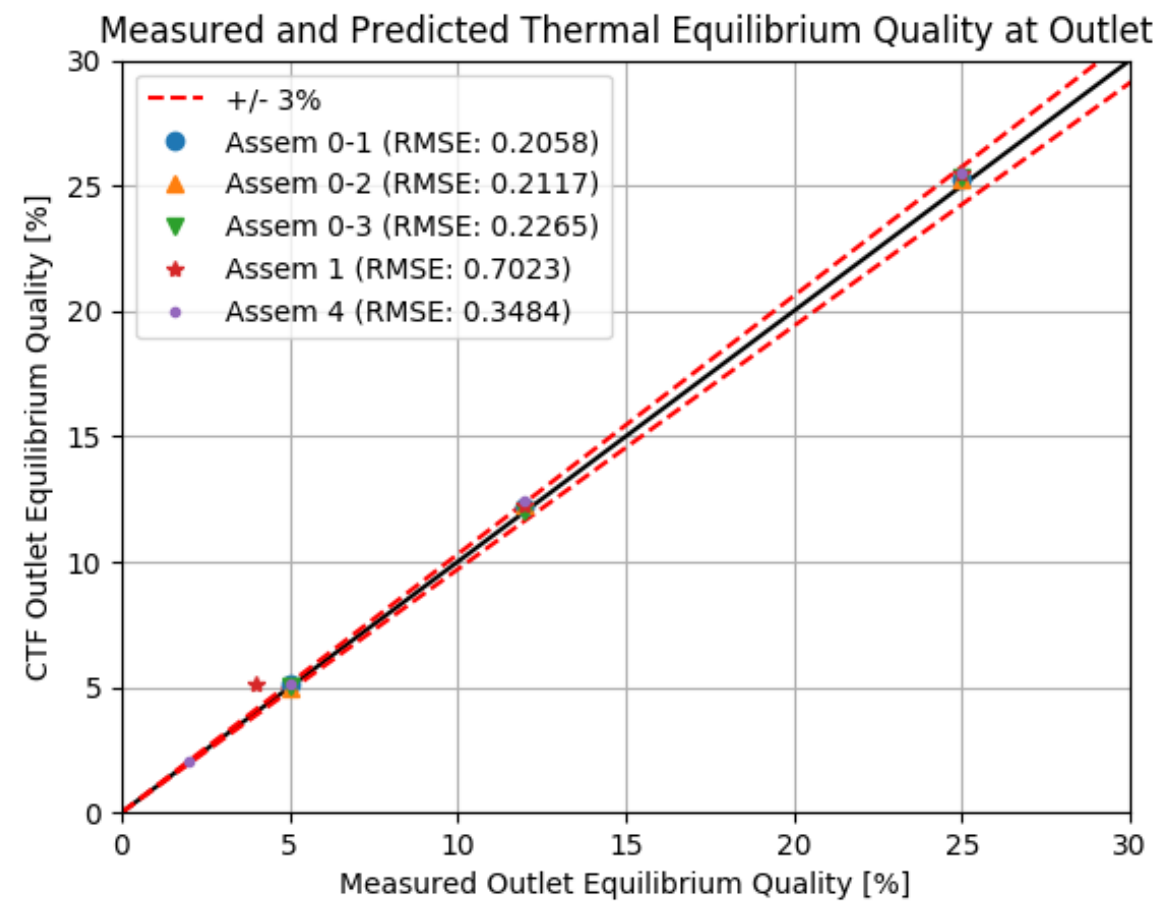

Figure 9.4: Comparison of measured and predicted bundle-averaged outlet thermal equilibrium quality. 


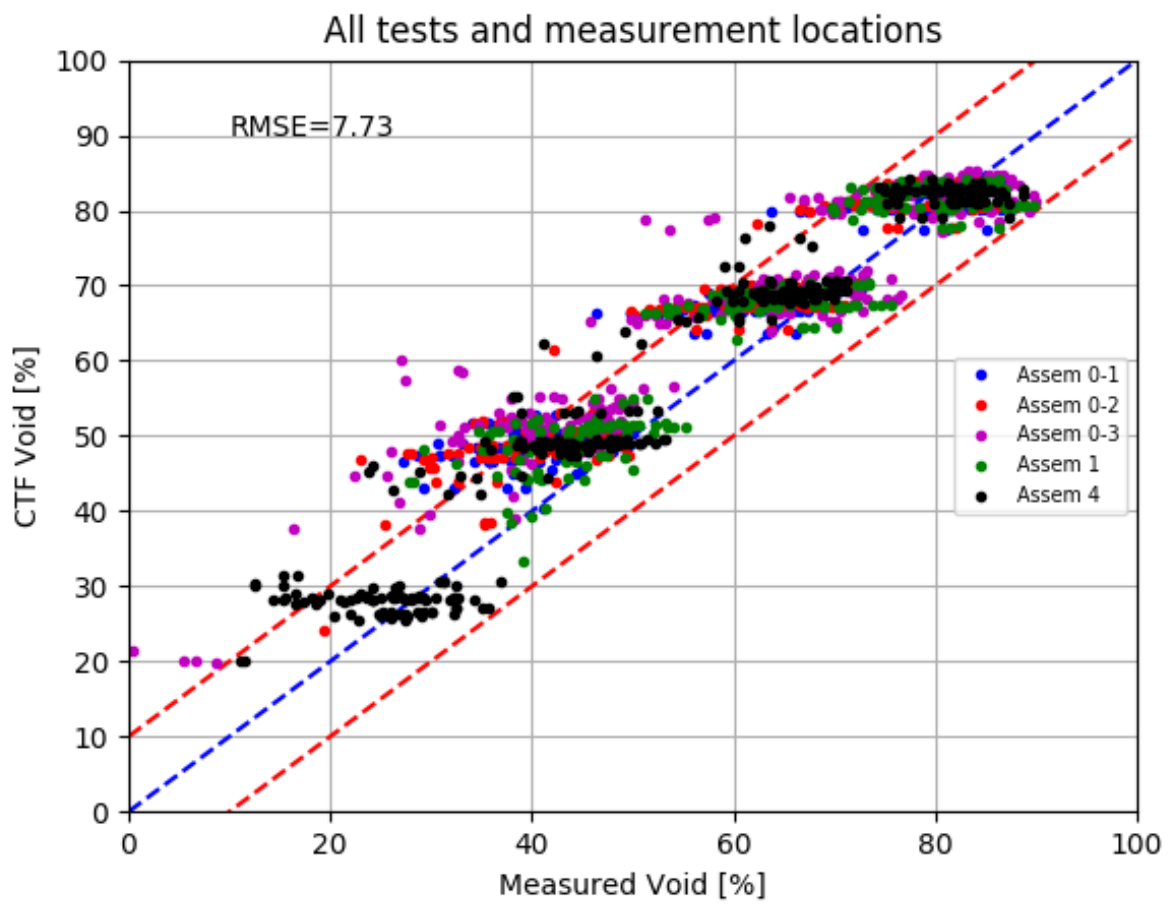

Figure 9.5: Comparison of CTF predictions and measurements of subchannel void for all BFBT void distribution tests. 


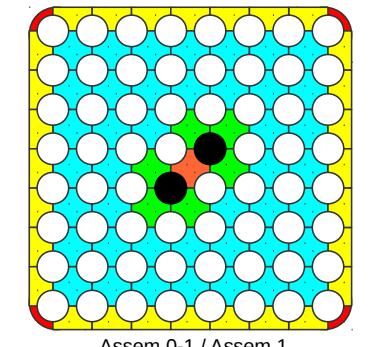

Assem 0-1 / Assem 1

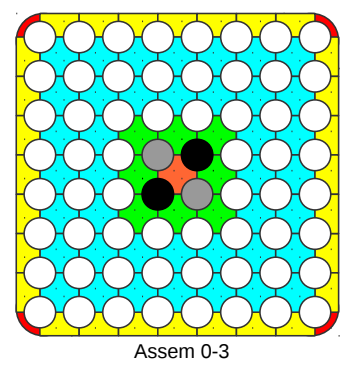

Side channel

\section{Inner channel}

Touch unheated

In unheated

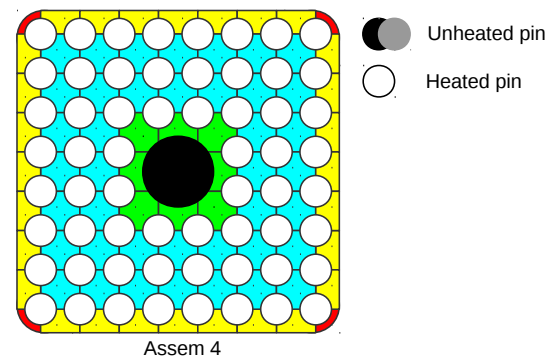

Figure 9.6: Channel grouping for modeled BFBT cases

each case. The tendency to over-predict void in the central region is observed in these figures. Additionally, it is also observed that lower void cases tend to experience more of a radial gradient in void, whereas radial void gradient is more uniform for the higher void cases. 


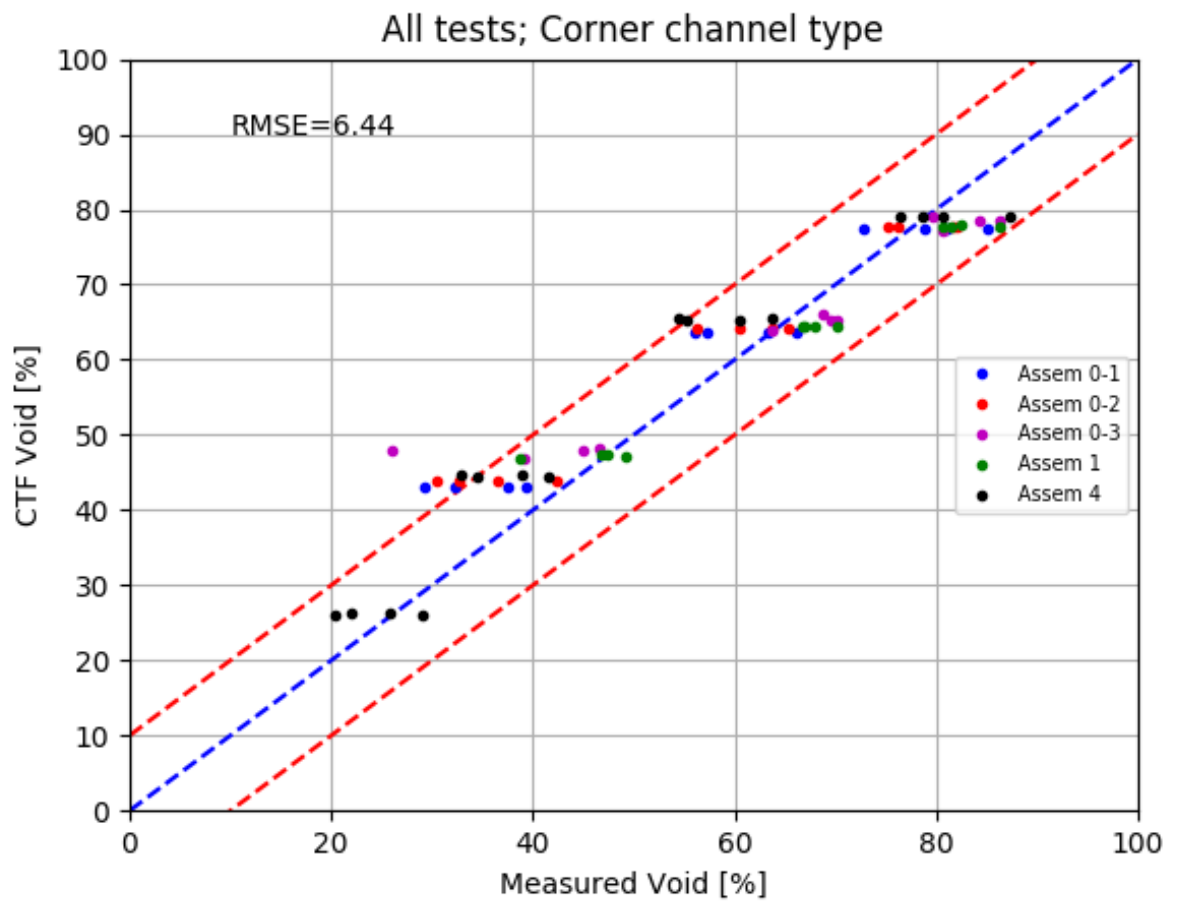

Figure 9.7: Comparison of CTF predictions and measurements of subchannel void for corner-type channels in all modeled BFBT void distribution tests. 


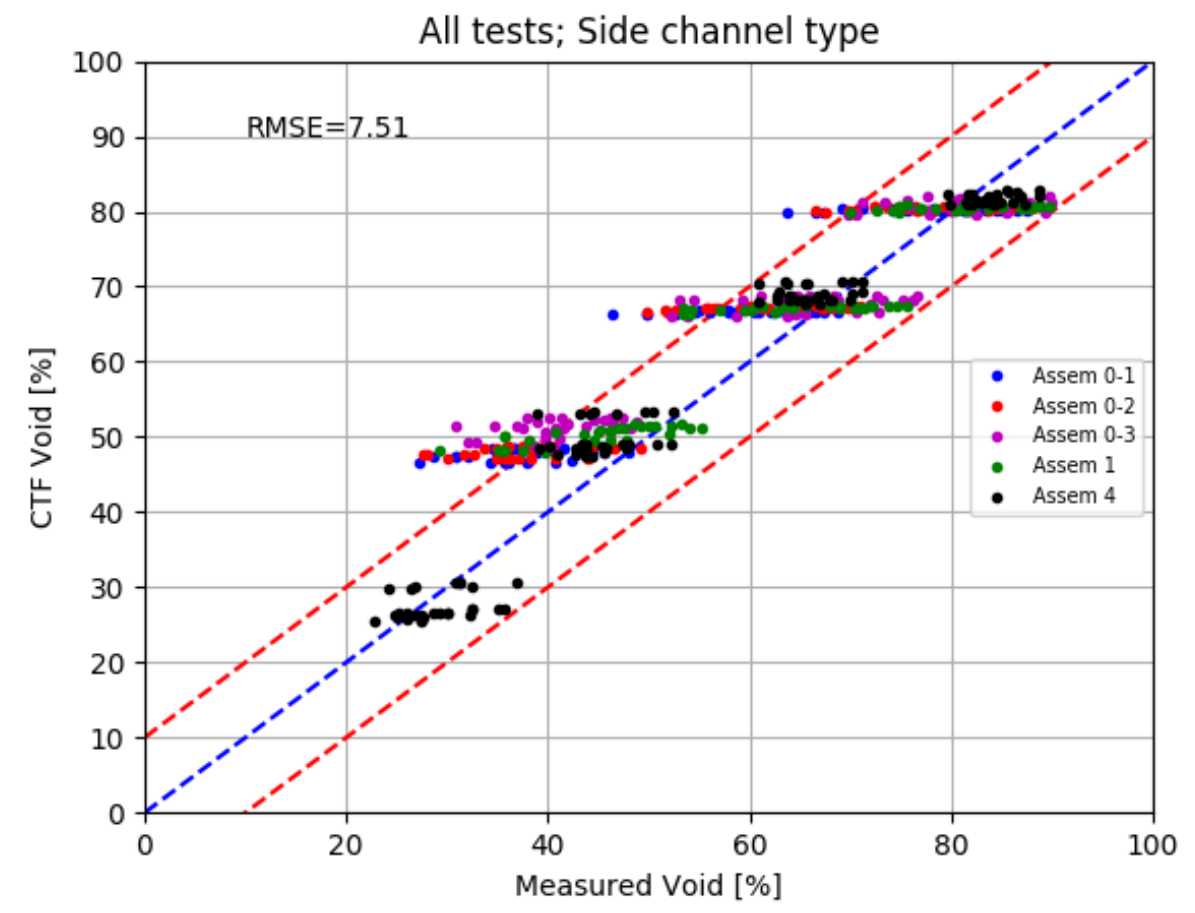

Figure 9.8: Comparison of CTF predictions and measurements of subchannel void for side-type channels in all modeled BFBT void distribution tests. 


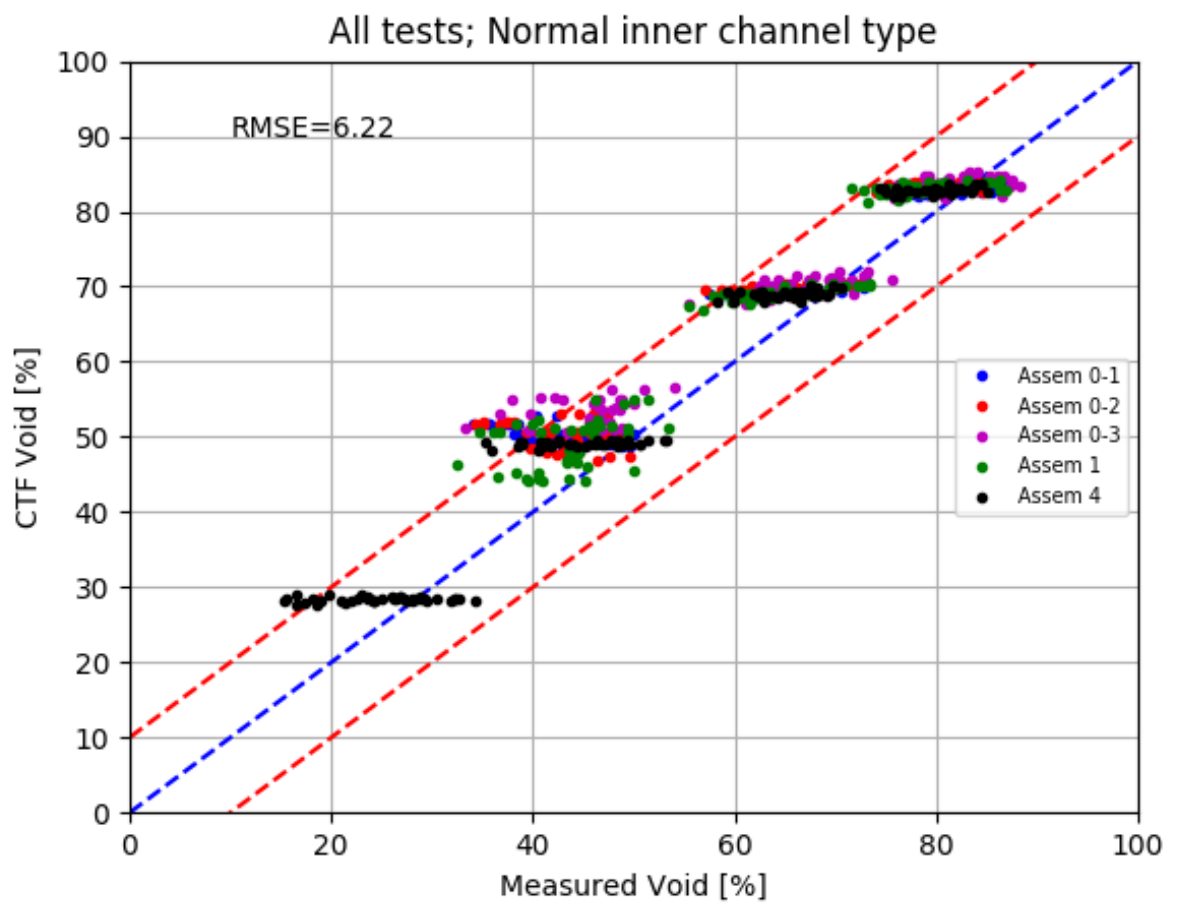

Figure 9.9: Comparison of CTF predictions and measurements of subchannel void for inner-type channels in all modeled BFBT void distribution tests. 


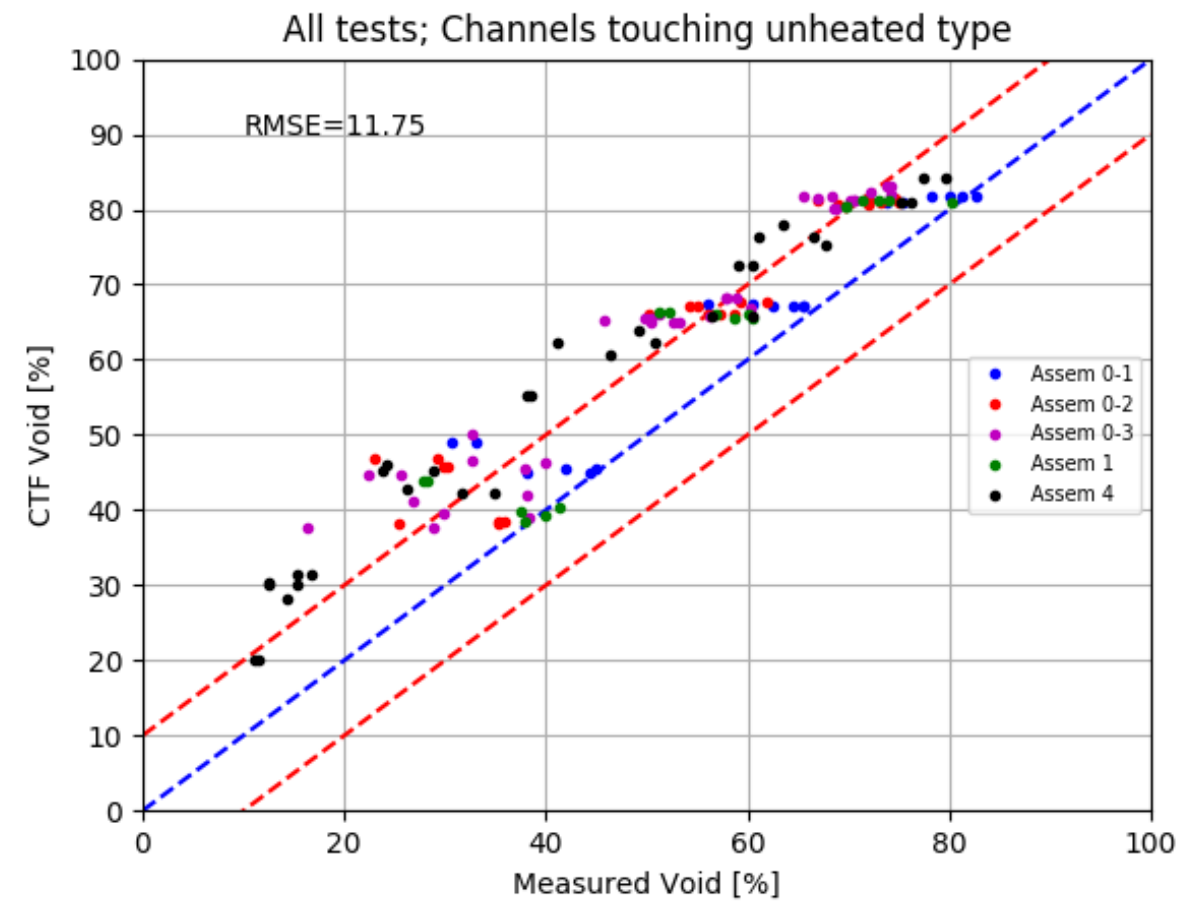

Figure 9.10: Comparison of CTF predictions and measurements of subchannel void for channels touching an unheated element in all modeled BFBT void distribution tests. 


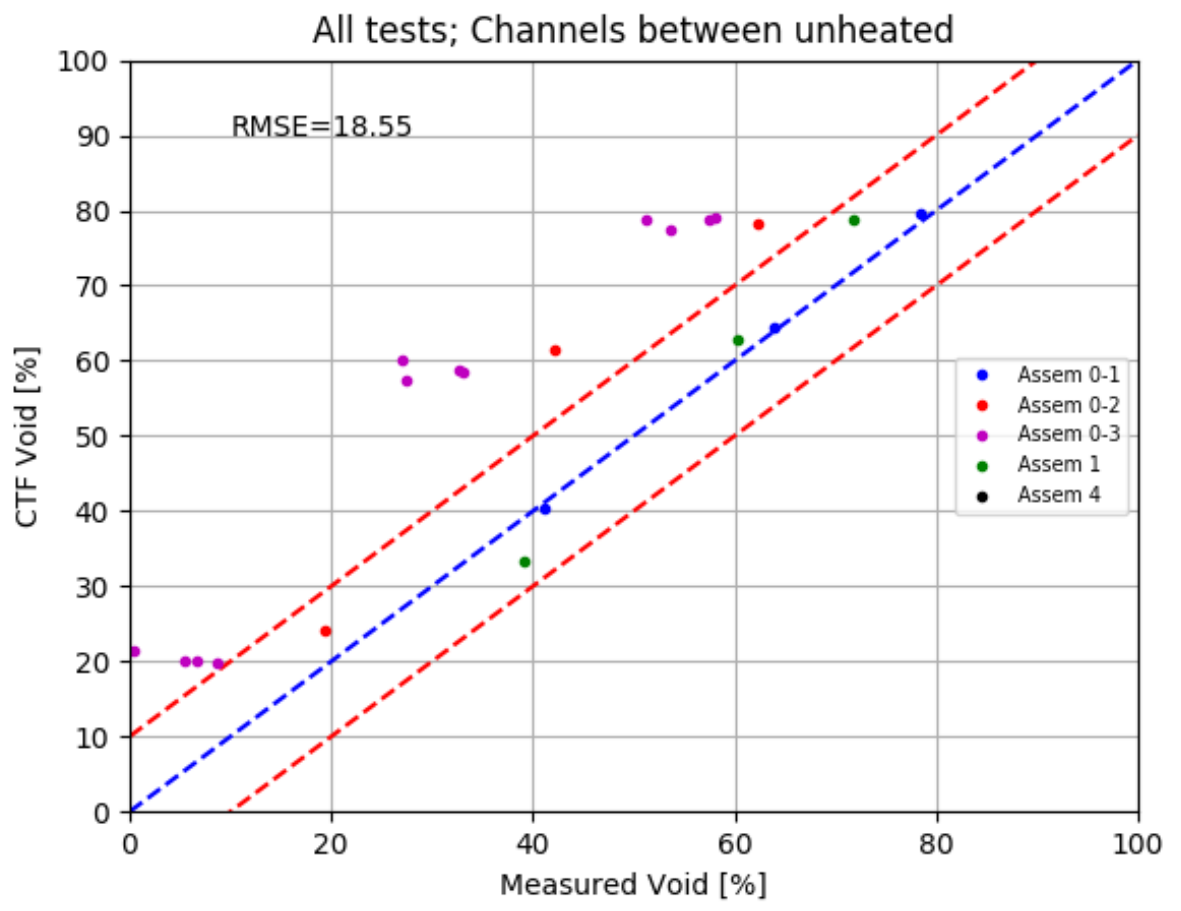

Figure 9.11: Comparison of CTF predictions and measurements of subchannel void for channels between unheated elements in all modeled BFBT void distribution tests. 


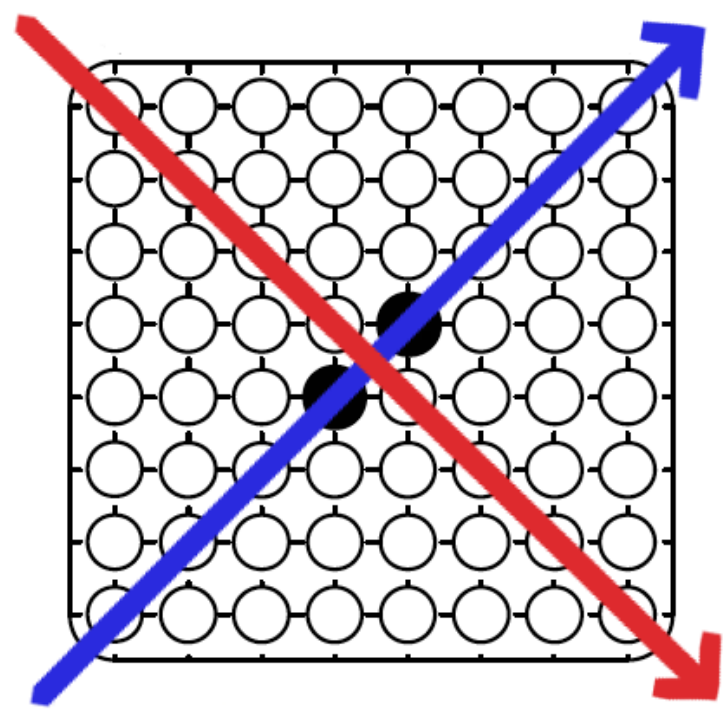

Figure 9.12: Example of diagonal lines from where subchannel void data is extracted.

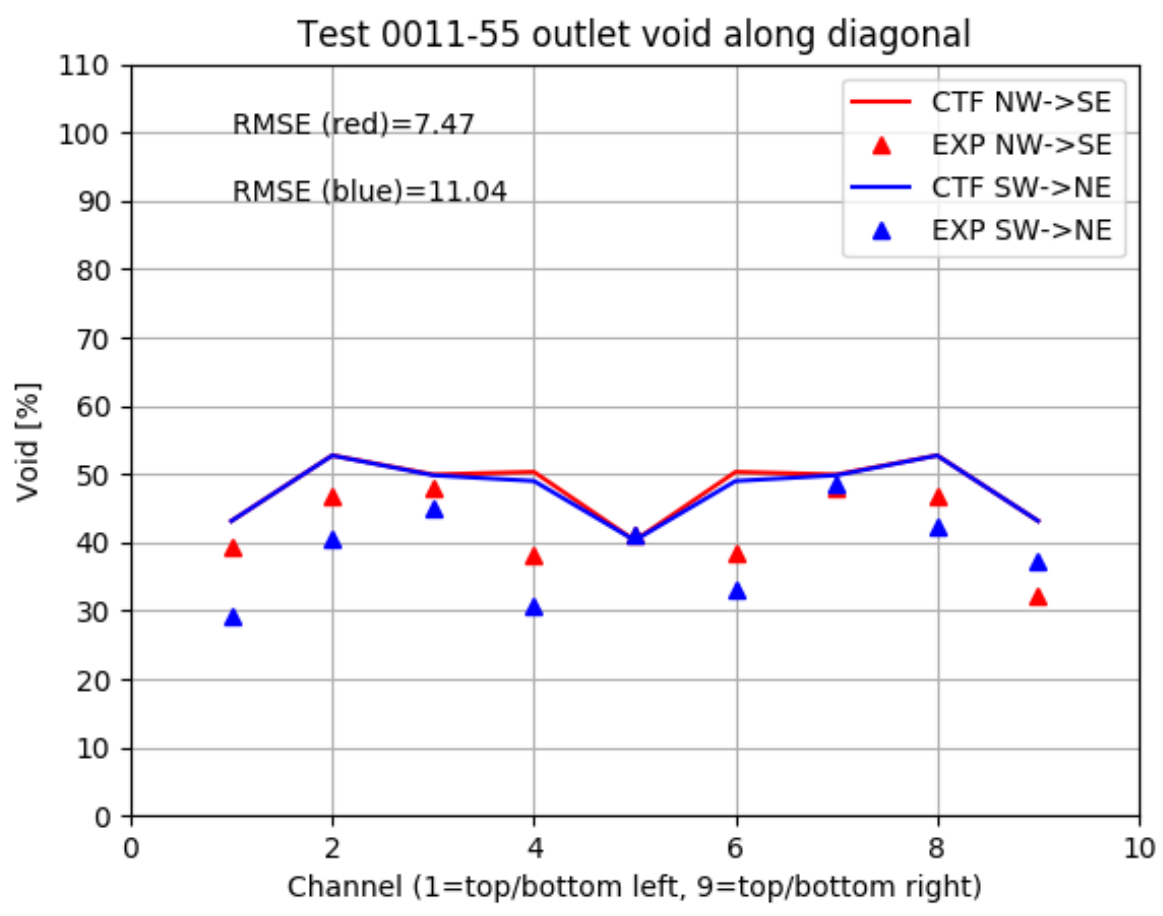

Figure 9.13: Measured and predicted void along diagonals of Test 0011-55. 


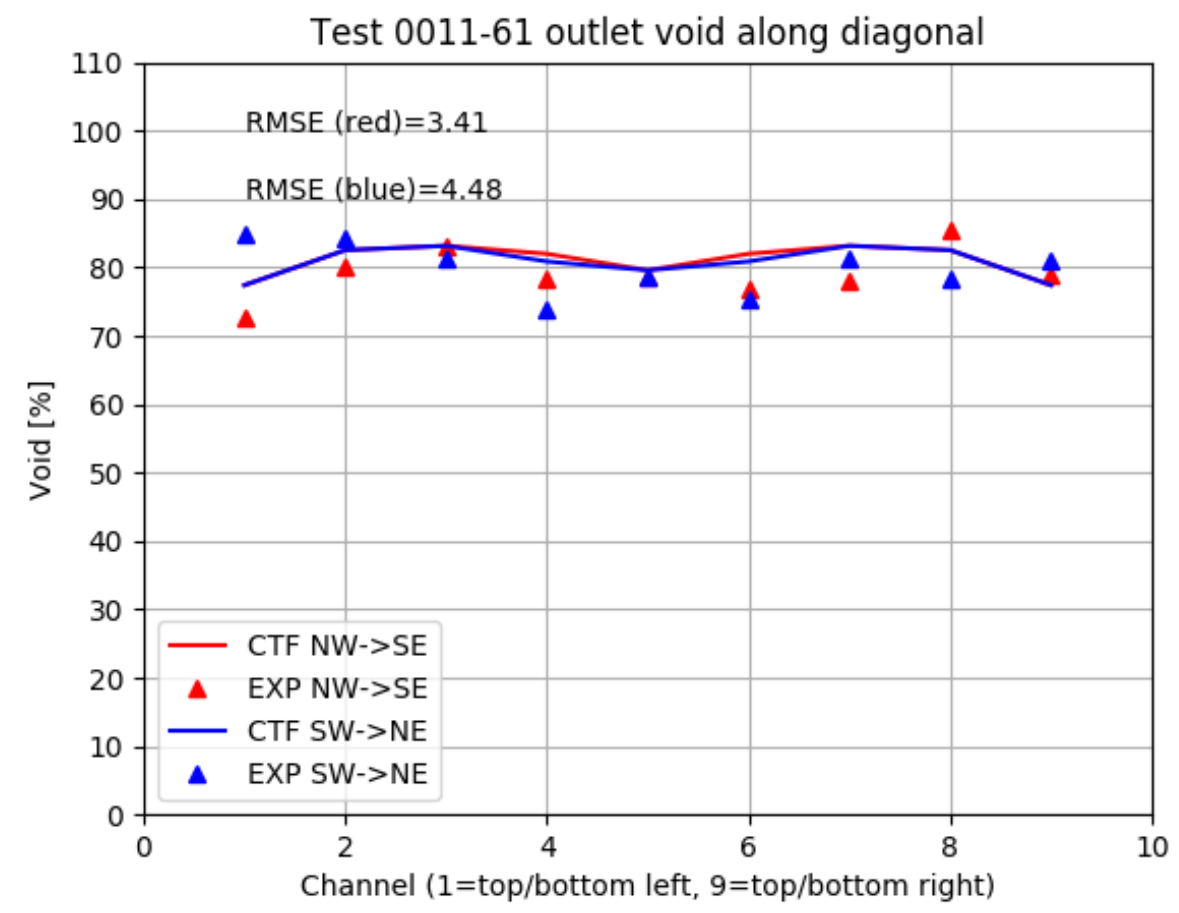

Figure 9.14: Measured and predicted void along diagonals of Test 0011-61. 


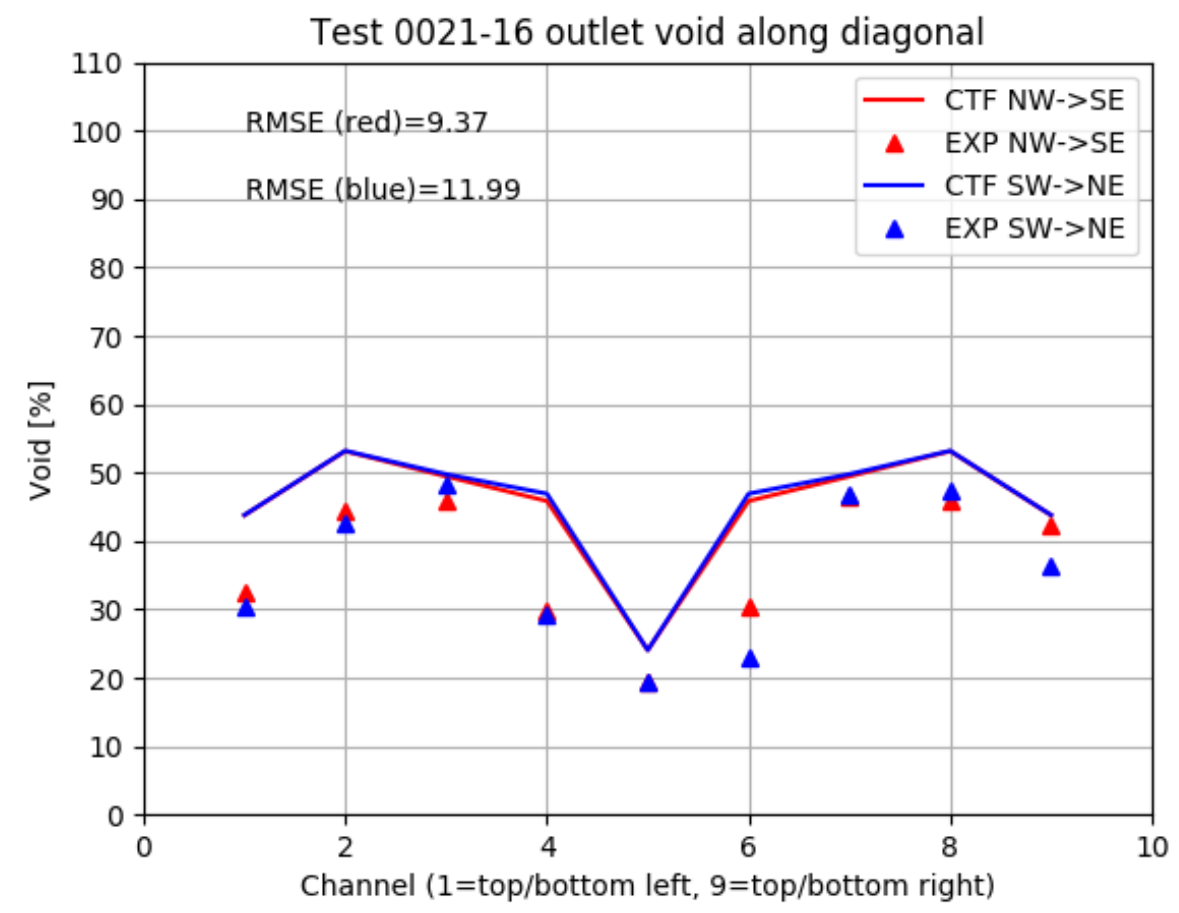

Figure 9.15: Measured and predicted void along diagonals of Test 0021-16. 


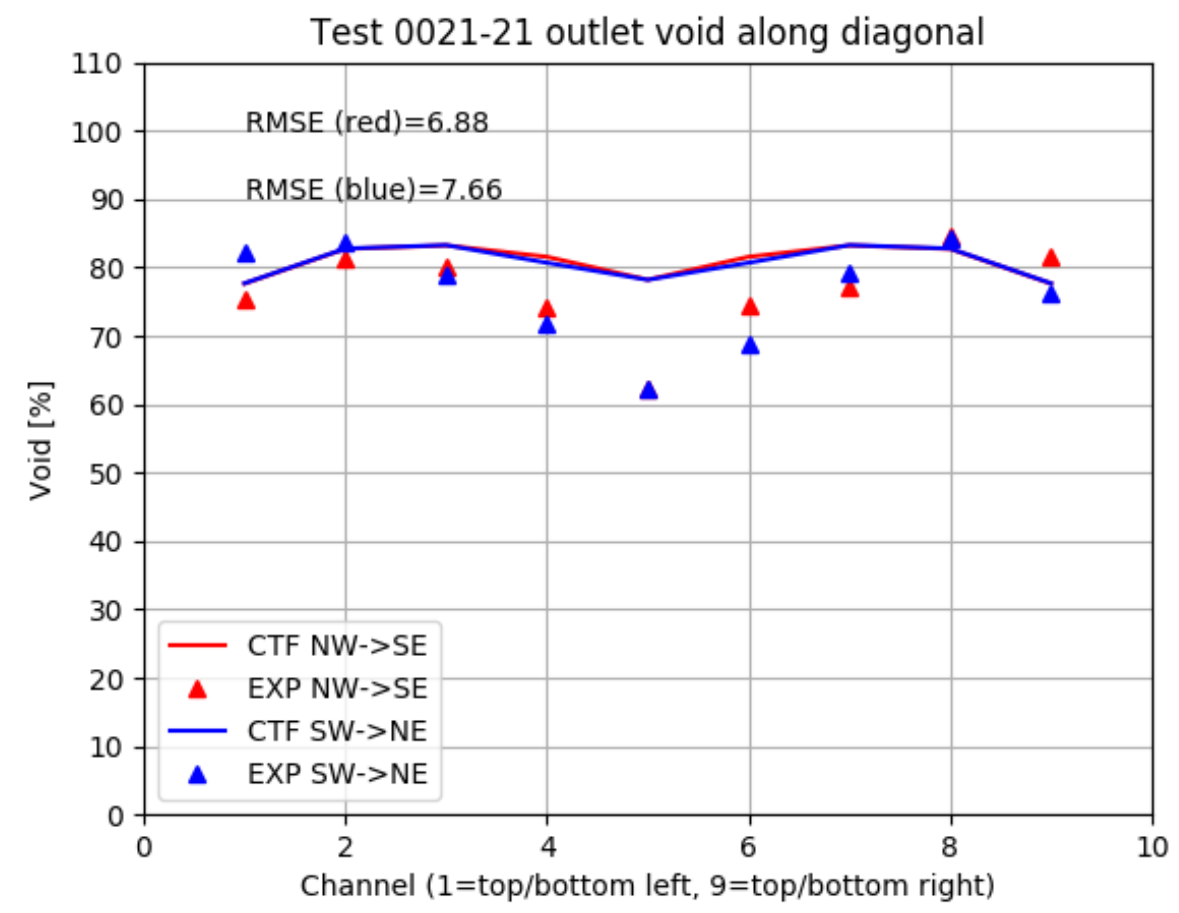

Figure 9.16: Measured and predicted void along diagonals of Test 0021-21. 


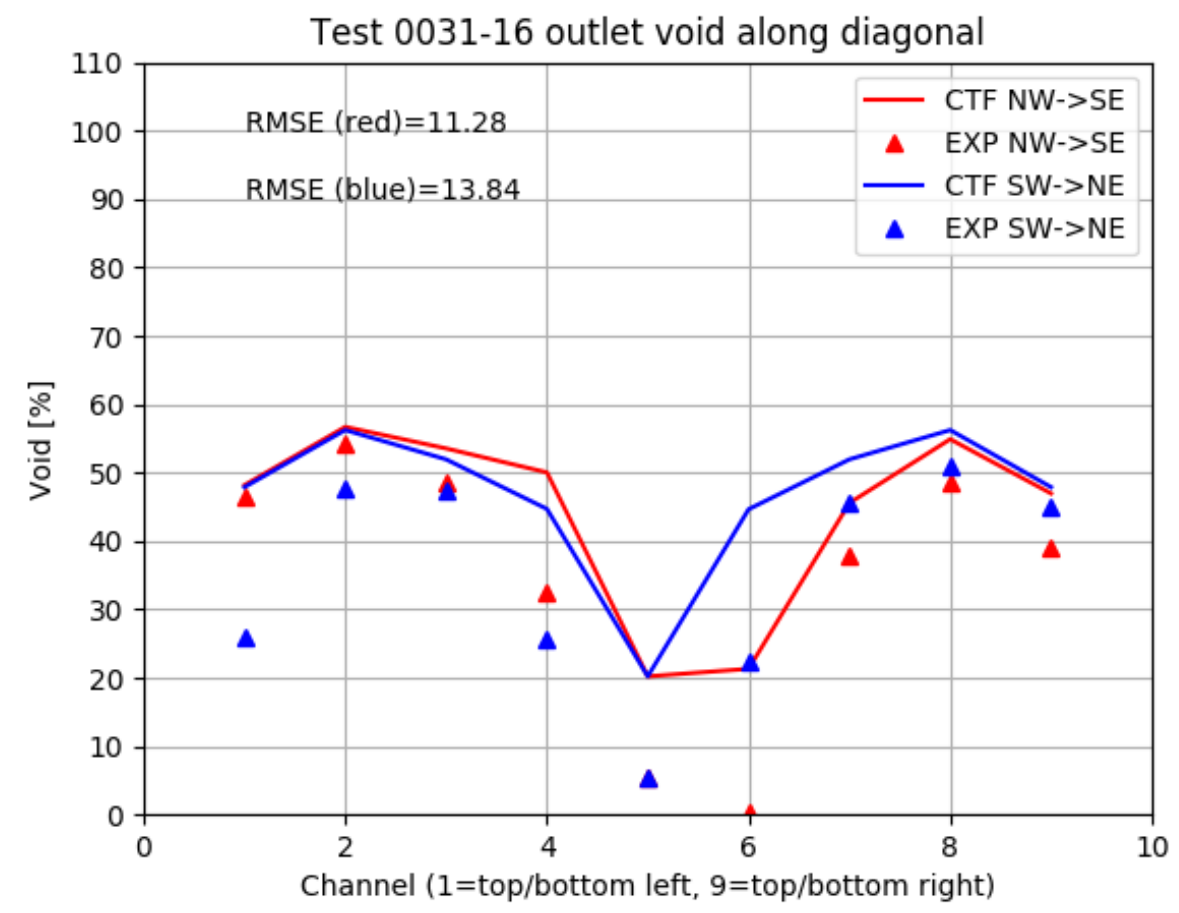

Figure 9.17: Measured and predicted void along diagonals of Test 0031-16. 


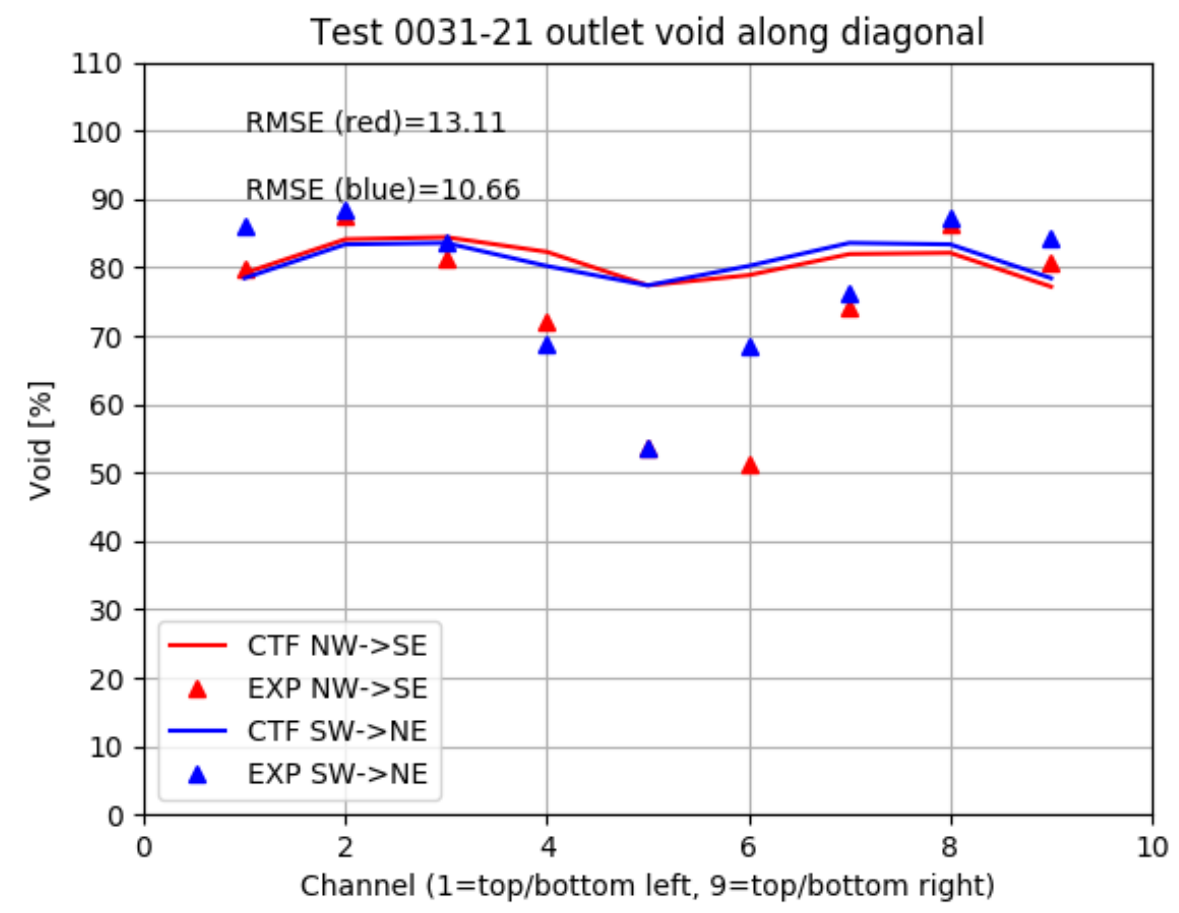

Figure 9.18: Measured and predicted void along diagonals of Test 0031-21. 


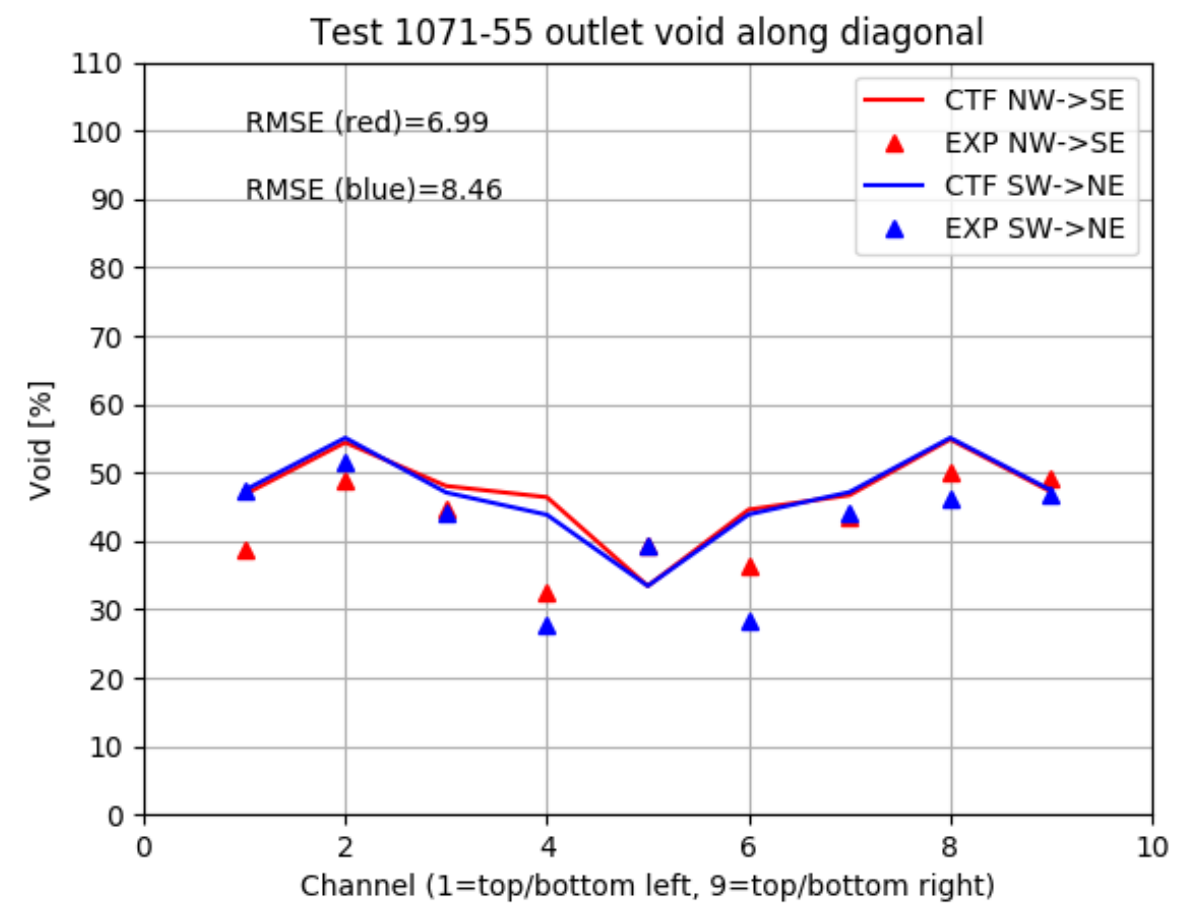

Figure 9.19: Measured and predicted void along diagonals of Test 1071-55. 


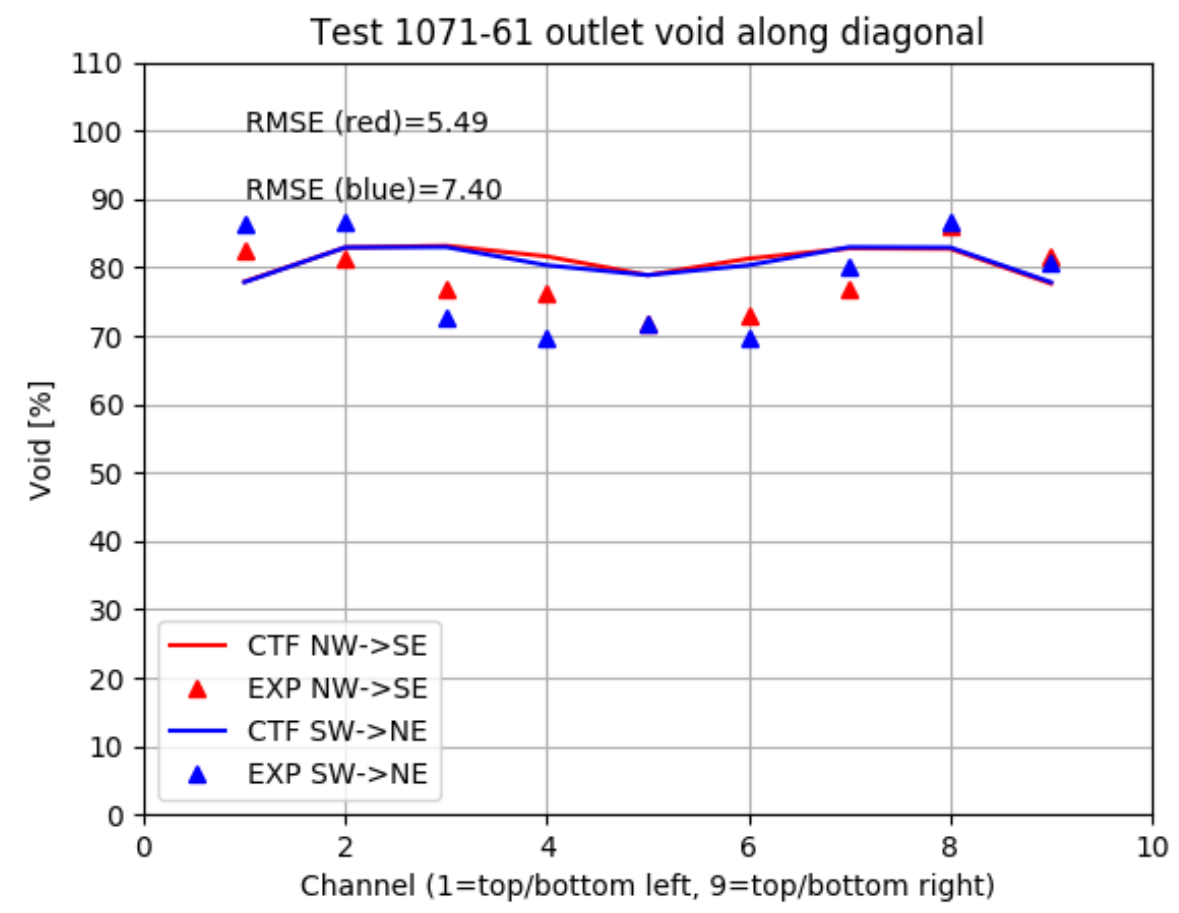

Figure 9.20: Measured and predicted void along diagonals of Test 1071-61. 


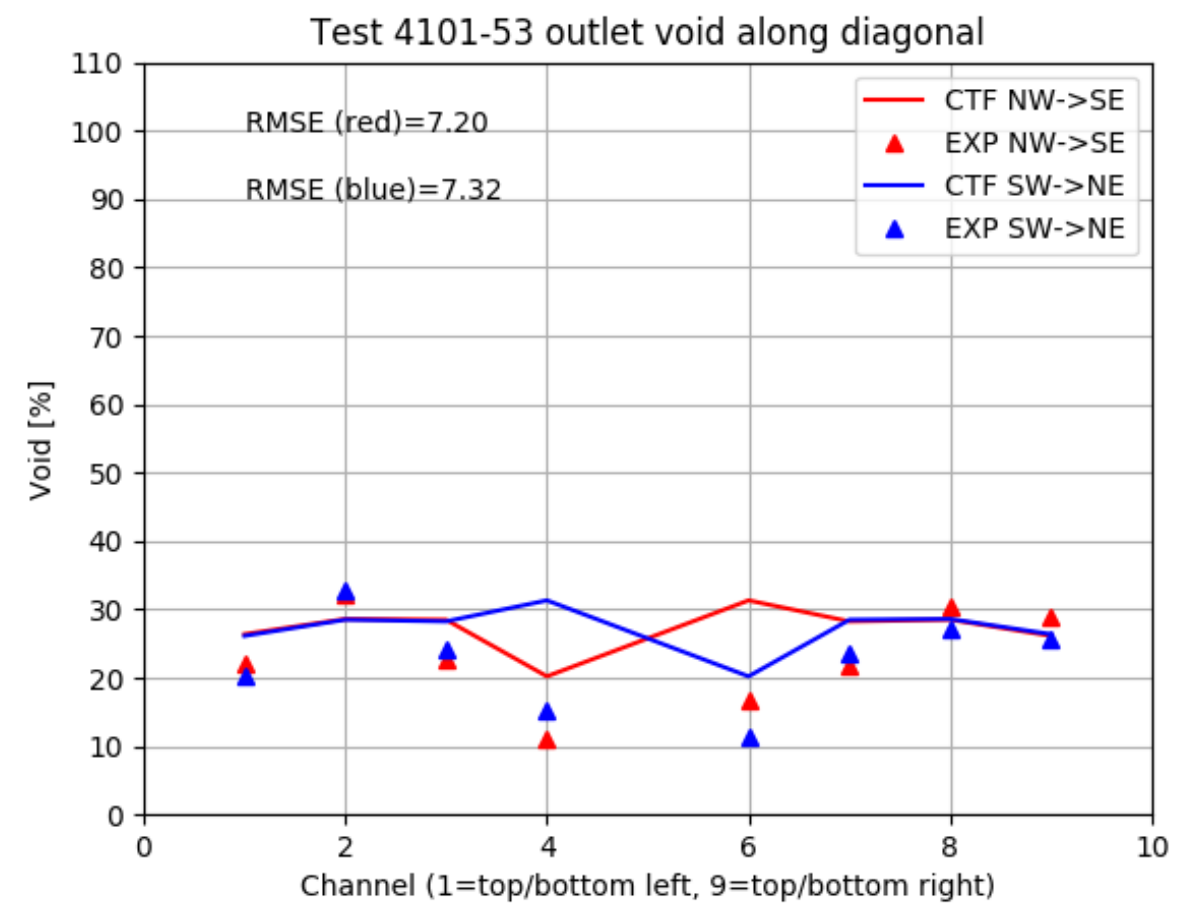

Figure 9.21: Measured and predicted void along diagonals of Test 4101-53. 


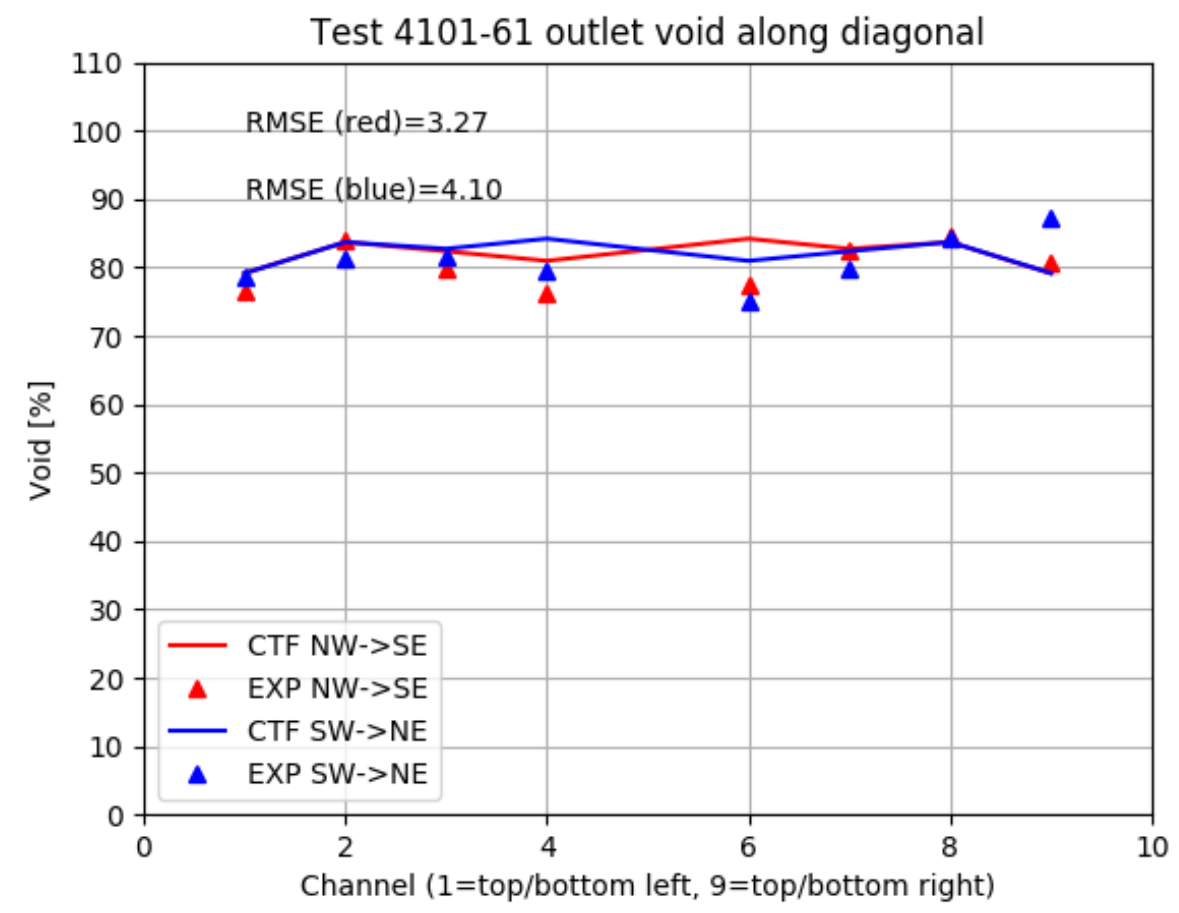

Figure 9.22: Measured and predicted void along diagonals of Test 4101-61. 


\section{Dryout}

\section{$10.1 \quad$ Validation}

Prediction of CHF in CTF primarily depends on the CHF model employed which, for these tests, was the "standard" model. This model considers three possible scenarios that can lead to CHF: pool-boiling, forcedconvection boiling, and annular-film dryout. The means for selecting which scenario prevails is detailed in Section 4.4.2.1 of the CTF Theory Manual[2]. If in pool-boiling or annular film dryout, a modified-Zuber equation will be used. For forced convection, the Biasi equation is used. The critical heat flux ( $\left.q_{\mathrm{chf}}^{\prime \prime}\right)$ will be calculated at each timestep during the rod solution. The wall temperature required to make the nucleate boiling heat flux match the critical heat flux is calculated in an iterative fashion; this temperature is known as the CHF temperature $\left(T_{\text {chf }}\right)$. When the actual wall temperature of the rod exceeds this $T_{\text {chf }}$ value, a post-CHF heat transfer regime is entered and DNB occurs.

With this behavior, we can see that the CTF prediction of CHF will be affected by the CHF correlation employed and the heat transfer correlations employed (both single-phase convection and boiling).

\subsubsection{Harwell Facility}

The QoI for these tests is the location where dryout occurs. The experimenters reported the tube surface temperatures recorded by thermocouples attached to the outside of the test tube at discrete axial locations. A steep increase in temperature at a given thermocouple indicates a drop in heat transfer due to vapor blanketing of the tube surface (CHF). This location was selected in a subjective way by choosing the thermocouple reporting a steep temperature increase.

Similarly, dryout was determined in CTF by looking at the tube surface temperatures reported in the results output file. A significant increase in temperature indicates a change in the heat transfer regime. Conveniently, the CTF output file also prints the heat transfer regime; a nucleate boiling heat transfer regime is pre-CHF and a transition boiling heat transfer regime is post-CHF.

A large set of test cases were modeled, 13 in total, in order to cover a wide range of operating conditions and generate good statistics. The predicted dryout location is plotted against the experimental observed dryout location in Figure 10.1. A note about the error bars on the data is in order for this figure. The experimental uncertainty comes from the fact that the thermocouples are placed at discrete locations. If a thermocouple reads an elevated temperature due to vapor blanketing, it does not necessarily mean that CHF occurred 
at exactly that axial location. Rather, CHF could have occurred at any axial location upstream of that thermocouple, up to the next thermocouple which is not reading an elevated temperature. The experimental uncertainty bars represent this distance to the next upstream thermocouple.

The error bars on the CTF predicted location represent the fact that CTF did not give a single location for CHF. Rather, for some test cases, the tube surface temperature shot up in one axial level, then dropped back down in the next axial level, then shot up in the level above that, and so on. Some test cases gave a single axial location where temperature increased, but others saw two or three oscillations in temperature with increasing height before the temperature became continuously elevated. In the event of such oscillations, the point where temperature became continuously elevated was selected as the predicted dryout location. The error bars on the CTF predictions then wrap any oscillations in temperature that occur upstream of that location. As shown in Figure 10.1, there are two oscillation regions were relatively small compared to experimental uncertainty. Furthermore, in no case did the these oscillations result in the CTF predictions falling in the range of experimental dryout location.

The discrepancy between calculated and measured dryout location is capture with the RMSE statistic. As stated before, the CTF dryout location is chosen as the axial location where the wall temperature becomes constantly elevated. The measured dryout location is set at the thermocouple where temperature is observed to rapidly increase, even though the actual dryout location may occur further upstream. From Figure 10.1, we can see that there are two outliers that will skew that statistic significantly. 


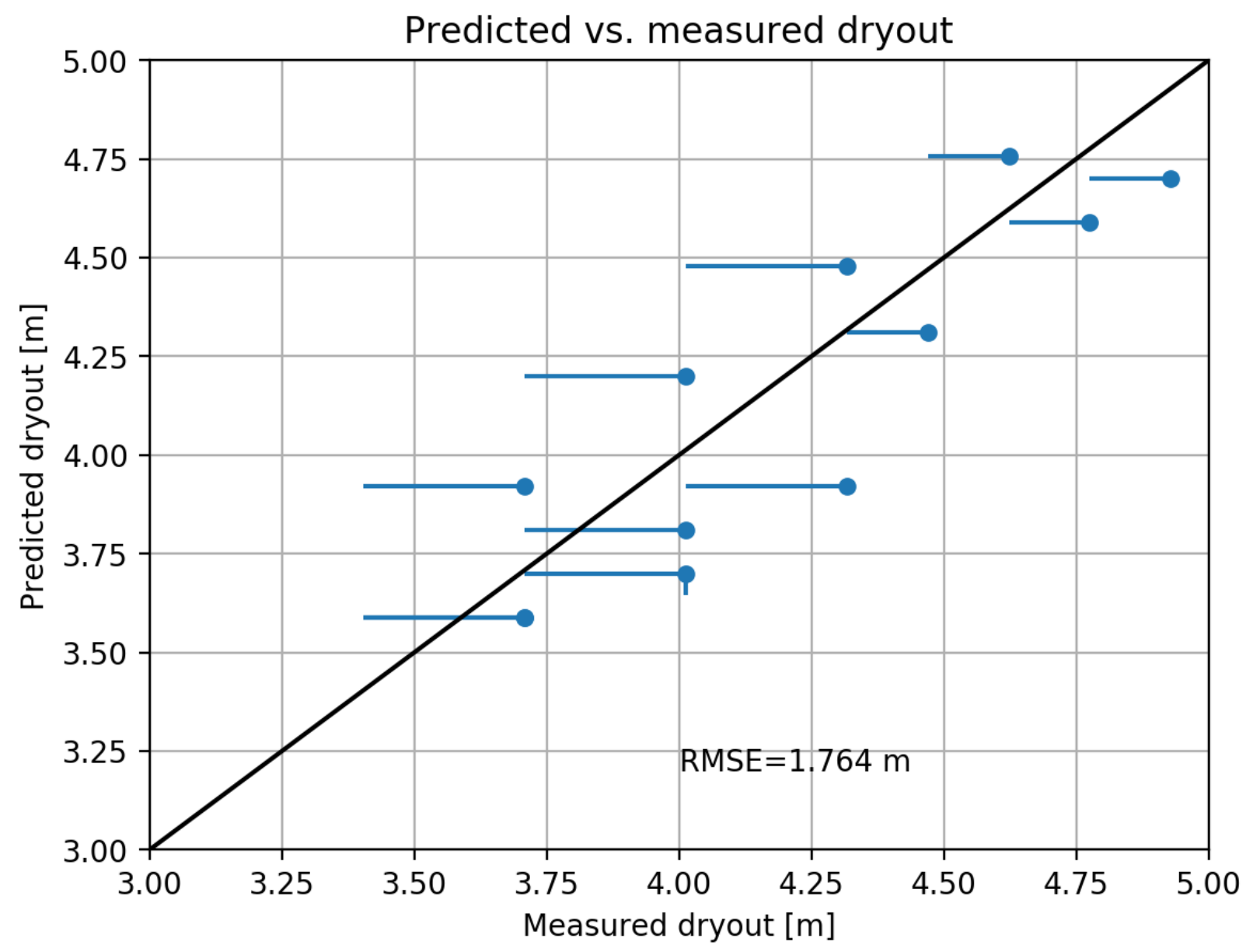

Figure 10.1: Summary of predicted and experimental dryout locations for Harwell test cases modeled by $\mathrm{CTF}$ 


\subsubsection{Takahama Tests}

Additional validation of the DNB models of CTF were performed using the Takahama tests[39]. This facility has not been described in Chapter 3 due to its proprietary nature. Additionally, it is not possible to include the specific results of the study in this open document, but mention is made of these tests and the general conclusions are discussed. A more detailed analysis can be found in the technical report, Application of Multi-Scale Thermal-Hydraulic Models to DNB Analysis[39].

In the analysis, CTF was used to model the Japanese Atomic Energy Research Institute (JAERI) sponsored Takahama tests, which simulate the RIA transient. These tests used actual nuclear fuel rod segments having burnups between 40 and $50 \mathrm{GWD} / \mathrm{MTU}$ in a specially designed test facility. A short pulse was produced in the rods, representative of the conditions of a RIA. This led to the occurrence of DNB in all tests, with most specimens surviving the transient, but some failing. CTF was able to successfully simulate this fast transient for all modeled test cases. The code correctly predicted the occurrence of DNB compared to experimental

observations. Furthermore, the failure mechanism for the test specimens that experience failure was also correctly predicted.

\subsection{Verification}

\subsubsection{Bowring Correlation}

The Bowring correlation [42] is encoded into CTF as an additional option for determining the CHF. This correlation was developed with a wide range of mass-flux and pressure values.

Code Models Assessed Assesses the CHF modeling capability of CTF with the Bowring correlation. This is performed with a unit test designed from a textbook end-of-chapter problem and with a regression test.

Problem Description The unit test portion was taken from Todreas \& Kazimi [43] end-of-chapter problem 13.5 on pg. 818. The input values from the problem are:

- Pressure $=6.89 \mathrm{MPa}$

- Inlet temperature $=204{ }^{\circ} \mathrm{C}$

- Mass flux $=2000 \mathrm{~kg} / \mathrm{m}^{2} \mathrm{~s}$

- Tube diameter $=0.01 \mathrm{~m}$

- Heated length $=3.66 \mathrm{~m}$

- Sat. liquid enthalpy $=1.2617 \times 10^{6} \mathrm{~J} / \mathrm{kg}$

- Sat. vapor enthalpy $=2.7740 \times 10^{6} \mathrm{~J} / \mathrm{kg}$ 
- Inlet enthalpy $=8.7249 \times 10^{6} \mathrm{~J} / \mathrm{kg}$

The enthalpies were determined using the inlet temperature and pressure, and the equilibrium quality and latent heat of vaporization were determined using these enthalpies. According to the problem statement, the result using the Bowring correlation should be $q_{c h f}^{\prime \prime}=1.41 \times 10^{6} \mathrm{~W} / \mathrm{m}^{2}$.

The regression test problem is a $3 \times 3$ model with a central water rod. A cosine shaped axial power shape with a corner peaked radial power profile was used. The model is intended to surpass saturation near the exit, which verifies that the Bowring function does not break under two-phase considerations.

CTF Input Model Description The Bowring single unit test is in Test Group "Bowring CHF Correlation" and is called "Test bowring". It is performed in CTF by passing the state point parameters to the Bowring correlation function. The results are then verified to match the expected values.

The regression test has been added to the automated regression suite as COBRA_TF_run_bowring_verify_3x3 using the model mentioned above.

Discussion of Results The unit test results match the textbook problem.

Conclusions The solution of these tests show that the Bowring correlation is correctly encoded into CTF.

\subsubsection{Groeneveld Look-up Table}

The Groeneveld look-up table [44] of CHF values were developed from curve fits to available experimental data. The CHF look-up tables are encoded into CTF as a $15 \times 21 \times 23$ three-dimensional array of CHF values in pressure, mass-flux and equilibrium quality and normalized to $8 \mathrm{~mm}$ tubes. Using the Groeneveld look-up tables required an appropriate interpolation scheme to obtain a single CHF value at a state point.

The correlation is also accompanied with several form or "K" factors [45]. The definitions of the K-factors have changed over time, but the most recent are being used. Each is applied to the interpolated CHF, and serves to account for additional physical effects in the assembly. Of the eight available $\mathrm{K}$ factors proposed by Groeneveld, three are used in CTF:

- $K_{1}$, Channel geometry factor

- $K_{4}$, Heated length factor

- $K_{5}$, Axial heat flux distribution factor

Code Models Assessed Assesses the Groeneveld CHF interpolation scheme and available K-factors calculations in CTF. 
Problem Description The unit test problem was developed from example problem 13.6 from Todreas \& Kazimi [43] on pg. 802, which is used to verify the calculation of $K_{1}$ and $K_{5}$. The interpolation scheme and $K_{4}$ were verified against a hand calculation. The input to the example problem is as follows:

- Pressure $=15.51 \mathrm{MPa}$

- Mass flux $=3807 \mathrm{~kg} / \mathrm{m}^{2} \mathrm{~s}$

- Sat. liquid density $=594.1 \mathrm{~kg} / \mathrm{m}^{3}$

- Sat. vapor density $=102.1 \mathrm{~kg} / \mathrm{m}^{3}$

- Equilibrium quality $=-0.059$

- Rod diameter $=0.0095 \mathrm{~m}$

- Hydraulic diameter $=0.0118 \mathrm{~m}$

- Heated length $=3.658 \mathrm{~m}$

- Axial location $=1.0 \mathrm{~m}$

- Gap width between rods $=0.0031 \mathrm{~m}$

Note that only $K_{1}$ and $K_{5}$ are used from the example problem for comparison. $K_{4}$ is determined in the example problem using a negative homogeneous equilibrium void fraction, $\alpha_{H E M}$, which is impossible. The version encoded into CTF only calculates $\alpha_{H E M}$ for non-negative equilibrium quality, ensuring that $\alpha_{H E M} \geq 0$ always. $K_{4}$ is then verified by hand calculation.

The hand interpolation of the CHF value begins by reducing the $15 \times 21 \times 23$ CHF array to a $2 \times 2 \times 2$ array that bounds the state points. Interpolation is then performed in pressure, mass flux and finally in equilibrium quality to return the interpolated CHF value. Using the pressure, mass-flux and equilibrium quality values, the bounds of this $2 \times 2 \times 2$ array are as follows from the Groeneveld look-up tables:

$$
\begin{aligned}
-0.10 & \leq x_{e} \leq-0.05 \\
14 \mathrm{MPa} & \leq p \leq 16 \mathrm{MPa} \\
3,500 \mathrm{~kg} / \mathrm{s} \cdot \mathrm{m}^{2} & \leq G \leq 4,000 \mathrm{~kg} / \mathrm{s} \cdot \mathrm{m}^{2}
\end{aligned}
$$

The resulting $2 \times 2 \times 2$ array and linear interpolation scheme can be represented in Figure 10.2. The order of interpolation has no impact as it will return the same value. The verification value, $q_{c h f}^{\prime \prime}=0.919 \times 10^{6}$ $\mathrm{BTU} / \mathrm{hr}^{-\mathrm{ft}^{2}}$, is the product of the interpolated CHF from the hand calculation (converted to US units) and the applied K-factors.

The regression test is a $3 \times 3$ model with a central water rod. The axial power profile was cosine shaped with a corner peaked rod. The model is intended to surpass saturation near the exit, which verifies that the Groeneveld function does not break under two-phase considerations. 

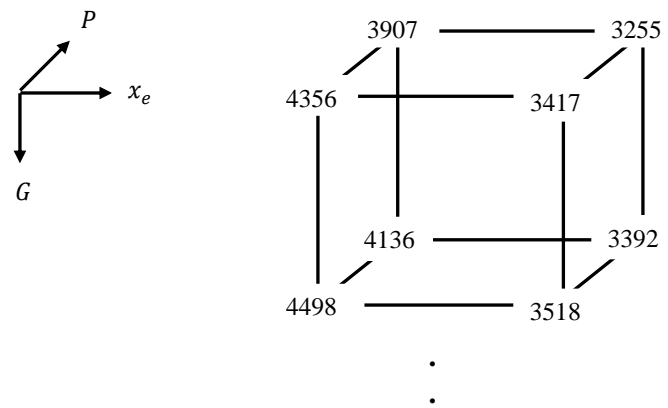

Interpolate in Pressure

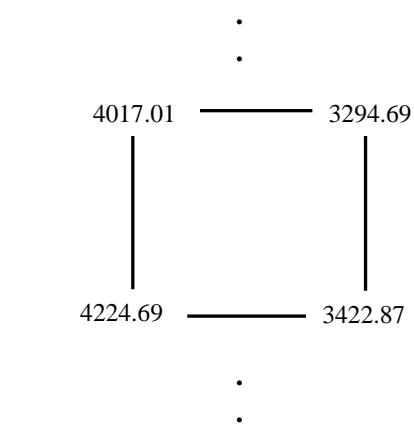

Interpolate in Mass-flux

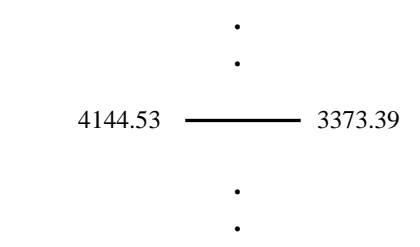

Interpolate in Equilibrium quality

$3512.20 \mathrm{~kW} / \mathrm{m}^{2}$

Figure 10.2: Groeneveld Hand Interpolation Scheme 
CTF Input Model Description The unit test for the Groeneveld correlation is in the "Groeneveld CHF Correlation" test group and is called "Test groeneveld". The test is performed in CTF by passing the state point parameters to the Groeneveld correlation function. The results are then verified to match the expected values.

The regression test has been added to the automated regression suite as COBRA_TF_run_groeneveld_verify_3x3 using the model mentioned above.

Discussion of Results The unit test results match the result of the textbook example.

Conclusions The positive result of the Groeneveld verification test shows that the portion tested is correctly encoded. The example problem used miscalculates $K_{4}$ by using a negative value for $\alpha_{H E M}$. This was user corrected by calculating $K_{4}$ with $\alpha_{H E M}=0$. This test also uses a uniform axial power profile, which initializes $K_{5}$ to unity. An additional unit test should be developed to verify $K_{5}$ for a non-uniform heating. 


\section{Solid Structure Models}

\subsection{Inside Tube Flow}

The inside tube flow feature allows CTF to model flow inside solid structures. Experiments with tubular test sections can be accurately modeled by placing the connection to the fluid channel on the inside surface of a heated tube. This feature also allows for multiple channel connections to the inside of a heater rod. An example of where this would be used is the housing of a small rod bundle during a reflood experiment where a quench front would be tracked on the housing using the fine-mesh rezoning model.

Three types of tests were used to verify the correct functionality of this feature:

- Direct Heat Input $(\mathrm{NC}=0)$

- Radial Conduction $(\mathrm{NC}=1)$

- Multiple Inside Channels

All three sets of tests were based on the tube geometry and boundary conditions of the Bennett Tube Test \#5273. However, only the first 20 inches of the test section was modeled using a total of five axial levels. This limited the heat transfer regime to that of subcooled boiling. Also, the very small time steps used in the original Bennett model were increased to make this a fast running problem.

Currently, this feature does not allow for simultaneous fluid connections to the interior and exterior surfaces of a heater rod. This capability will be implemented in a future update. An example of where this capability would be used is for a BWR canister during a LOCA where the fine-mesh rezoning model would be used to track the quench front.

\subsubsection{Direct Heat Input}

Code Models Assessed Assesses the tube inside flow modeling capability of CTF.

Problem Description The objective was to demonstrate the correct heat input from the rod to an interior fluid channel. The conduction model was turned off by setting the flag $\mathrm{NC}=0$. 
CTF Input Model Description Two cases were created: one for inside flow and one for outside flow. The wetted perimeter of the cases was matched so that results were the same between the cases.

The outside flow is called COBRA_TF_run_inflow_1_out. The tube geometry is that of the original Bennett input model with the fluid channel connected to the exterior surface of the tube. The result is used as a base case for other test cases in this series.

The inside flow case is called COBRA_TF_run_inflow_1_in. The tube geometry was modified to match that of the actual experiment, that is, the inside diameter was set to 0.497 in $(1.2624 \mathrm{~cm})$ and the outside diameter was set to 0.625 in $(1.5875 \mathrm{~cm})$. The fluid channel connection was set to the interior surface of the tube. All calculated fluid conditions (void fraction, vapor generation rate, etc.) and wall temperatures should be identical to those of the base case (COBRA_TF_run_inflow_1_out).

Discussion of Results Fluid solution results were identical between the two tested cases, as expedted.

Conclusions In this study, the exterior flow capability (channel connected to the outside of a rod) was used to generate a "gold" result file. The insie flow feature was used for the same conditions and was shown to produce identical results to the exterior flow case, thus verifying that the inside flow feature is properly functioning for a single channel in the tube.

\subsubsection{Radial Conduction Test}

Code Models Assessed Assesses the tube inside flow modeling capability of CTF and the radial conduction equations of CTF.

Problem Description Two separate input models were used in this test series as described below. Both tests use the radial conduction model $(\mathrm{NC}=1)$. The purpose was to make sure that the wall heat transfer boundary condition were applied to the correct surface and that the wall temperatures were calculated correctly. The number of radial nodes in the heat structure was increased from 2 to 5 to allow for a more accurate calculation of the temperature drop across the tube wall.

CTF Input Model Description Two cases were created: one for inside flow and one for outside flow. The wetted perimeter of the cases was matched so that results were the same between the cases.

COBRA_TF_run_inflow_2_in is the name of the model with flow on the inside of the tube. The tube geometry matches that of Bennett experiment with fluid connection on the inside tube surface. Thermal conductivity of the tube wall was set to a constant value of $20(\mathrm{~W} / \mathrm{m}-\mathrm{K}$ to enable comparison to analytical solution. Calculated fluid conditions and inside wall temperature should match those of the base case. The wall temperature drop should be equal to that of the analytical solution for a tube with uniform thermal conductivity and internal heat generation.

COBRA_TF_run_inflow_2_out is the name of the model with flow on the outside of the tube. The connection to the fluid channel was made to the outside surface of the tube as in the base case. The outside tube diameter 
was set to the ID of the Bennett tube (i.e., 0.497 in $[1.2624 \mathrm{~cm}]$ ). Calculated fluid conditions and outside wall temperature should match those of the base case. The wall thickness, and hence inside diameter, were set so that the temperature drop across the tube wall would match that of the case COBRA_TF_run_inflow_2_in. Specifically, the tube wall thickness was set to $1.49806 \mathrm{~mm}$.

Discussion of Results For the inside flow case, the analytical result is given by:

$$
T_{0}-T_{i}=\left(\frac{q^{\prime}}{4 \pi k}\right)\left[\frac{2 r_{0}^{2}}{\left(r_{0}^{2}-r_{i}^{2}\right)} \ln \left(\frac{r_{0}}{r_{i}}\right)-1\right]
$$

For the conditions of this experiment, namely a linear heat generation rate of $36.494 \mathrm{~kW} / \mathrm{m}$, the temperature difference across the tube wall should be $35.8057^{\circ} \mathrm{C}$. The calculated value was $35.85{ }^{\circ} \mathrm{C}$ and approached the correct value as the number of nodes increased.

For the outside flow case, the analytical solution is given by:

$$
T_{0}-T_{i}=\left(\frac{q^{\prime}}{4 \pi k}\right)\left[1-\frac{2 r_{i}^{2}}{\left(r_{0}^{2}-r_{i}^{2}\right)} \ln \left(\frac{r_{0}}{r_{i}}\right)\right]
$$

For the chosen dimensions, this gives a value of $35.8057{ }^{\circ} \mathrm{C}$. The calculated value was $35.73{ }^{\circ} \mathrm{C}$ and approached the correct value as the number of nodes increased. Figure 11.1 shows the CTF predicted wall temperature drops for increasing levels of radial mesh refinement compared to the analytical solution for both inside flow and outside flow. Both modeling approaches approach the analytical solution as the mesh is refined.

Conclusions This case shows that the inside-flow feature works when radial conduction is enabled in the tube. Additionally, it shows that the conduction equation solution in the tube matches an analytical solution to within $0.1{ }^{\circ} \mathrm{C}$. The solution improves as the mesh is refined.

\subsubsection{Multiple Inside Channels}

Code Models Assessed Assesses the tube inside flow modeling capability of CTF. Particularly, tests the capability to have multiple fluid channels on the inside of a tube instead of only one.

Problem Description The input model of COBRA_TF_run_inflow_2_in was modified for this test and is labeled COBRA_TF_run_inflow_3. Specifically, the original one fluid channel on the inside of the tube was replaced by two channels. Each of these half-channels had half the flow area and wetted perimeter of the original one channel. Likewise, they connected to half of the heater perimeter of the tube inside wall. No gaps were provided between these two channels so they were simply 1-D pipes connected to half of the tube wall.

Both half-channels should have identical results and be equal to the calculated fluid parameters of the base case with two exceptions. That exception is that the flow rates and vapor generation rates should 


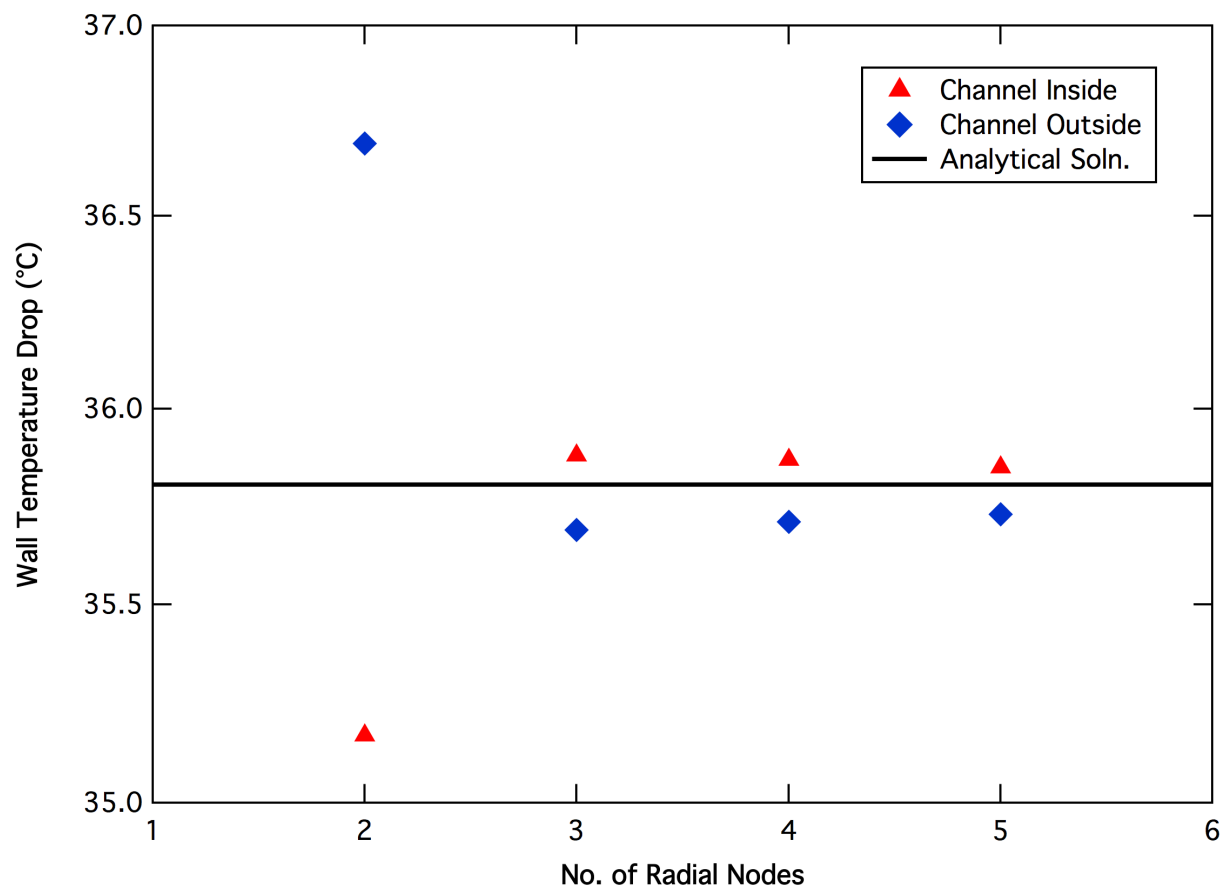

Figure 11.1: CTF predicted wall temperature drop compared to analytical solution for channel on outside versus channel on inside 
be exactly one-half of those in the base case. The wall temperature solution should match that of the COBRA_TF_run_inflow_2_in case.

\section{CTF Input Model Description}

Discussion of Results The wall temperature solution matched the COBRA_TF_run_inflow_2_in case. The flow rates and vapor generation rates in the inside channels were half that of the COBRA_TF_run_inflow_2_in case, as expected.

Conclusions This verification study showed that CTF is capable of modeling multiple channels inside of a tube. Furthermore, the fluid results match the base case, COBRA_TF_run_inflow_2_in. 


\section{Natural Circulation}

Natural circulation does not classify as an "effect" like the previous sections; it is really a state of operation that has its own effects. However, because it may be of special interest to the user to see how CTF performs when modeling buoyancy-driven natural-circulation flows, it has been given its own section. The primary test of interest here is the PNNL 2x6 case, though the reader should be aware that the FRIGG facility, discussed in Sections 6.3.1.2 and 8.2 was also operated at natural circulation conditions.

\subsection{PNNL 2x6 Tests}

The facility was operated in two different scenarios; steady state and transient conditions. The steadystate results are discussed here. Two types of measurements were made in the facility: LDA local-velocity measurements and subchannel-center thermocouple measurements. Both of these measurement types were taken at five discrete axial locations in the bundle. The LDA measurements were taken along a single 1-D line, or "rake", in each of the three rows of subchannels. The thermocouple measurements were taken along a single 1-D rake in one of the rows of subchannels (the center row). The main difference between the two measurement types was that the LDA measurements were taken in finer increments, allowing for a mapping of the velocity profile within a subchannel, whereas the thermocouple measurements were only taken at subchannel centers.

The experimental results of these tests do not lend themselves as well to quantitative comparison with $\mathrm{CTF}$ predictions as well as for other effects that were discussed. This has to do with the fundamental differences between what the measurements and predictions mean. Concerning the velocity measurements, the values were local, so a velocity measurement taken at the center of the subchannel is much higher than that taken in the gap region between rods, where wall drag and viscous effects have a much larger impact on the fluid, leading to slower flows. While this appears in the measurements, the CTF prediction is one averaged velocity that includes these wall effects and smears them across the entire cross section of the subchannel.

One workaround to this problem is to average the LDA measurements, but this requires that the measurements be taken at equal intervals, so as not to weight some measurements more than others. This was not the case during the experiments. Additionally, the actual velocity values were not made available in the report but, rather, values were extracted from figures using a digitizer. A second option is to fit a correlation to each subchannel velocity profile and then integrate that curve over the bounds of the subchannel. Even this approach wouldn't be entirely accurate, however, as the subchannel dimensions where the LDA rake was made does not represent the geometry of the channel if we were to move the rake line closer or farther 


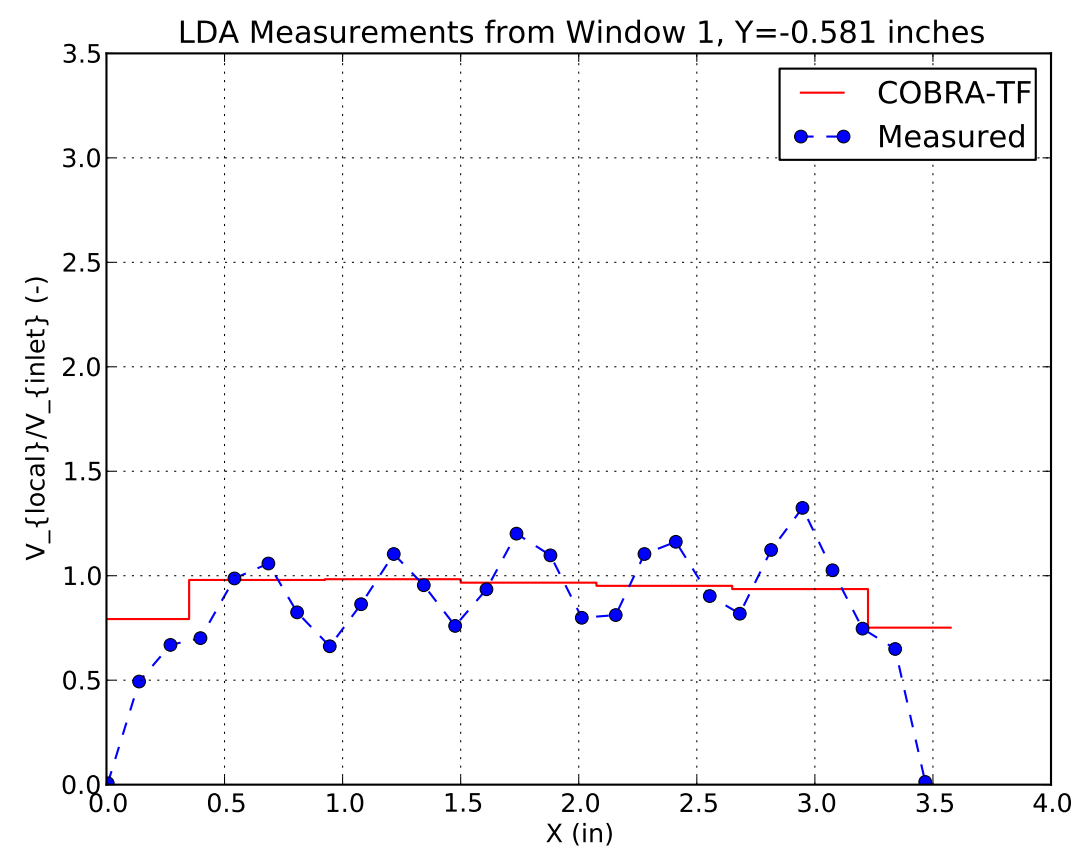

Figure 12.1: Predicted and measured subchannel velocities for Window 1 at Rake Location $Y=-0.581$ in in PNNL 2x6

from us, the observer. In lieu of these considerations, the figures showing predicted and measured values are simply presented here for each window and rake location. The LDA results are presented in Figures 12.1 through 12.11.

In these figures, the LDA location in the rod bundle is shown on the $x$-axis. The $y$-axis of the figure shows a non-dimensionalized velocity at that measurement location (see Figure 3.19 for the meaning of the $\mathrm{X}$ and $\mathrm{Y}$ dimensions of the test assembly). Note in the results figures how the measured velocities will move above and below CTF values in many cases, which is expected, as the CTF values are a smeared, averaged value for the entire channel. The important thing to look for in these figures is that CTF is capturing the behavior of the velocity distribution, which should be for "left-side" velocities to be higher than "right-side" velocities due to the fact that only the "left-side" rods are heated. This uneven heating creates thermal plumes that drive the flow upwards on the left-side of the bundle.

CTF generally captures this behavior for all axial levels for rake locations at $\mathrm{Y}=0.0$ inch and $\mathrm{Y}=0.581$ inch. CTF tends to over-predict velocities for the $\mathrm{Y}=-0.581$ inch rake location. However, the fact that measurements at rake location $\mathrm{Y}=-0.581$ inch disagree with those at rake location $\mathrm{Y}=0.581$ inch is a cause for concern because the measurements should agree due to symmetry of the test conditions. It was noted by the authors that there was a misalignment of the flow housing which accounts for this discrepancy. Since we do not capture this effect in $\mathrm{CTF}$, we can disregard the disagreement of measured and predicted results for the $\mathrm{Y}=-0.581$ inch rake location.

Figures 12.12 through 12.16 provide the comparison of measured and predicted temperature results for the 


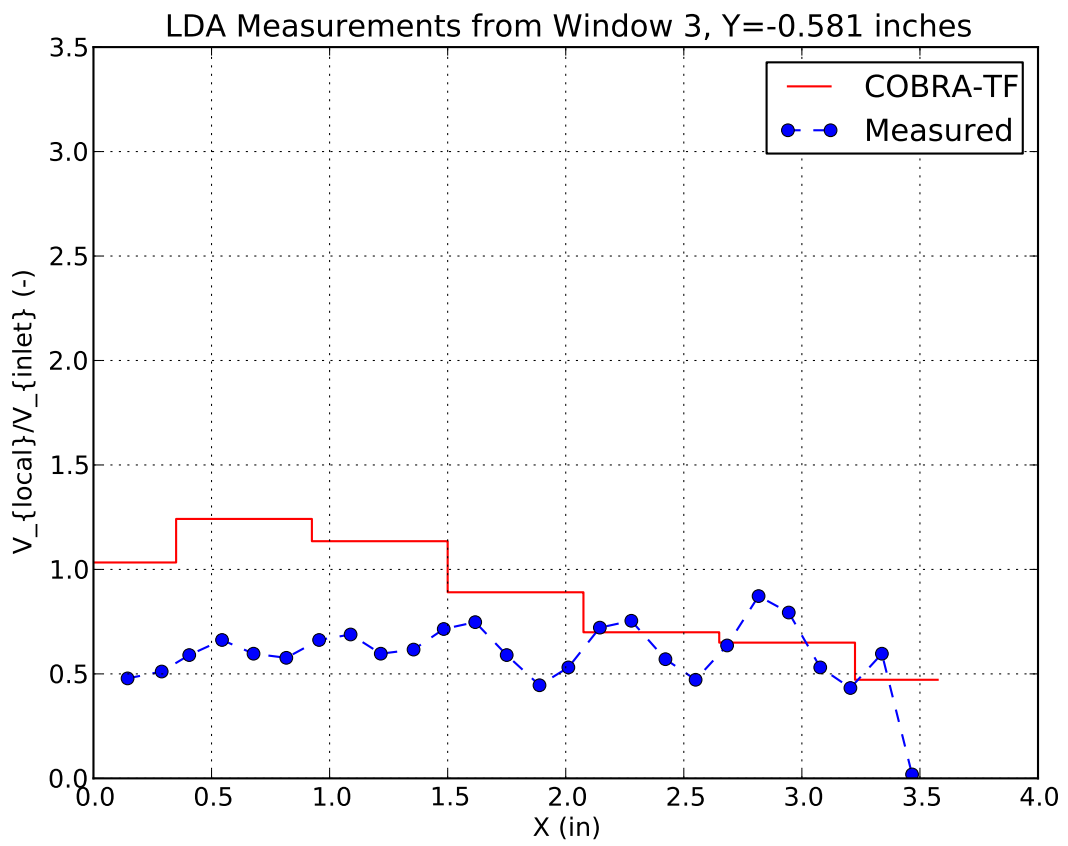

Figure 12.2: Predicted and measured subchannel velocities for Window 3 at Rake Location $Y=-0.581$ in in PNNL 2x6 


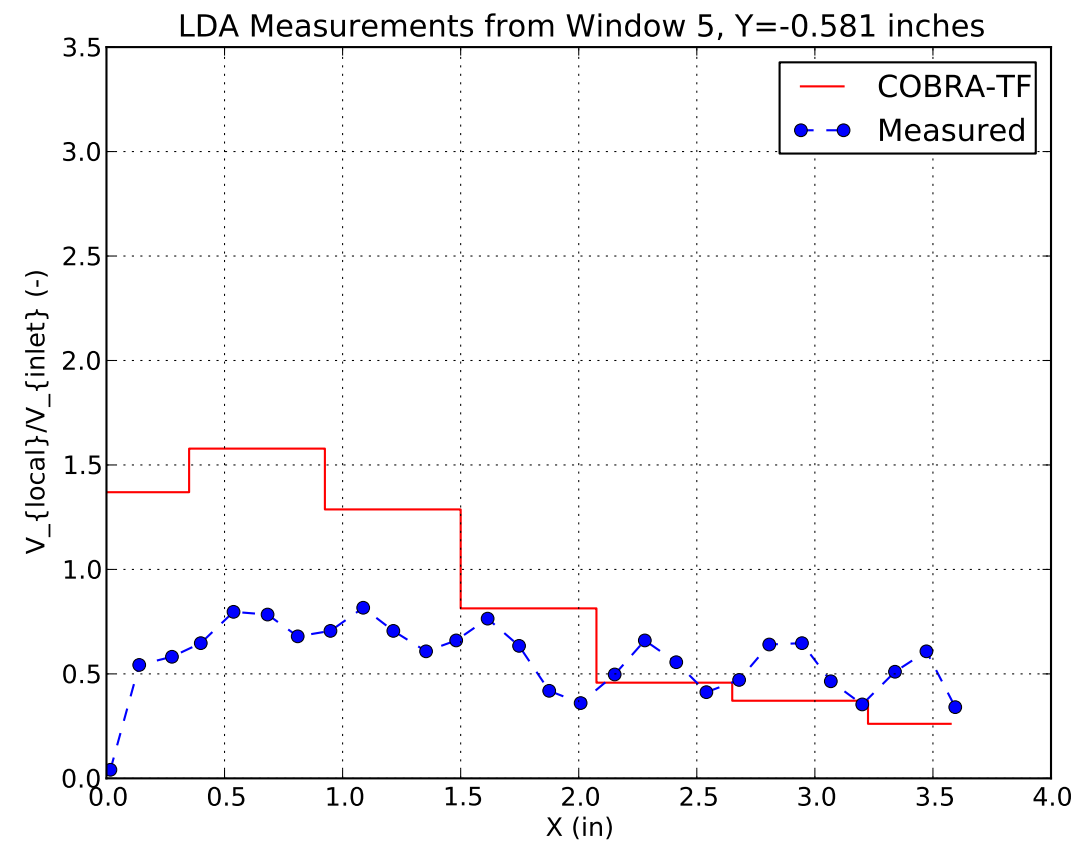

Figure 12.3: Predicted and measured subchannel velocities for Window 5 at Rake Location $Y=-0.581$ in in PNNL 2x6 


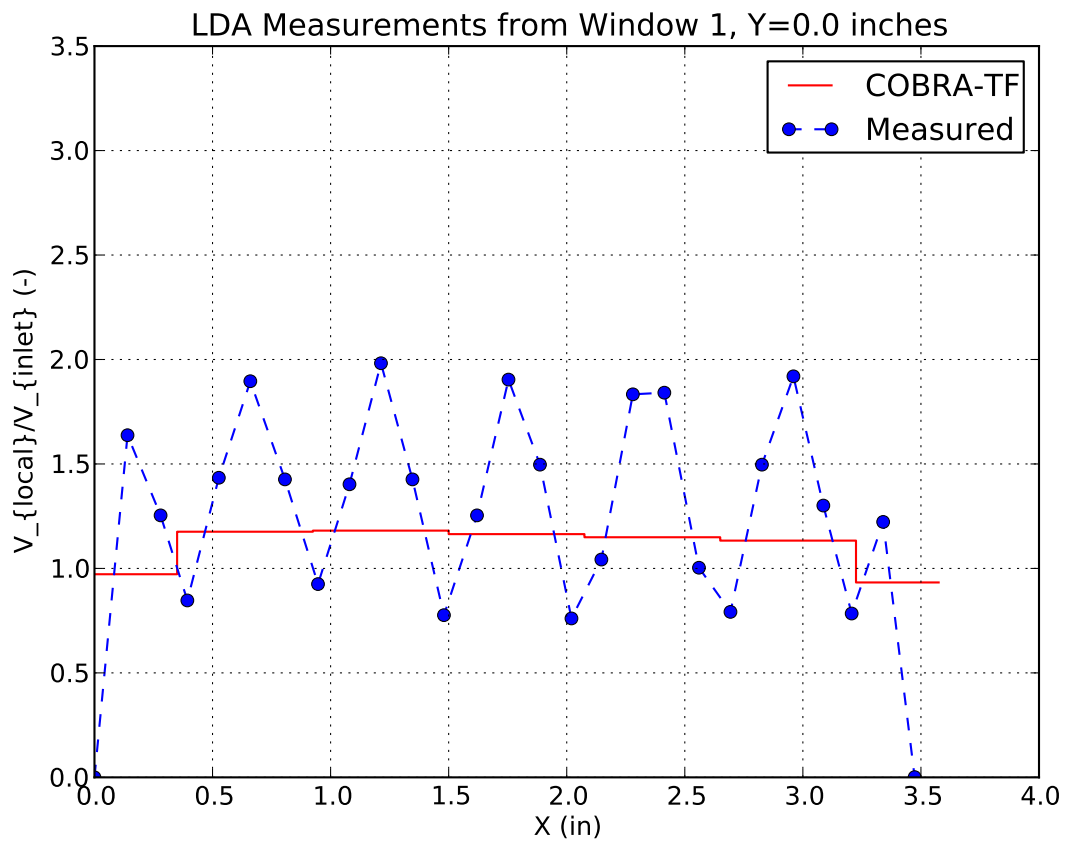

Figure 12.4: Predicted and measured subchannel velocities for Window 1 at Rake Location $Y=0.0$ in in PNNL 2x6 


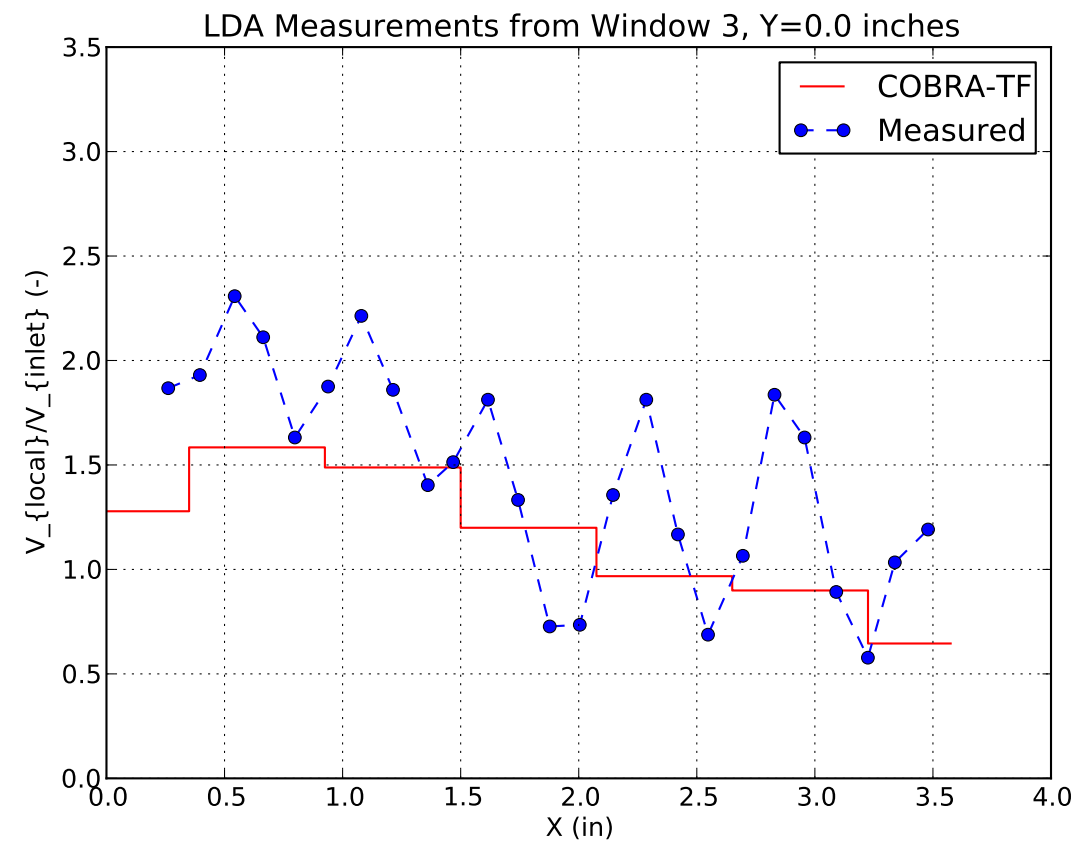

Figure 12.5: Predicted and measured subchannel velocities for Window 3 at Rake Location $Y=0.0$ in in PNNL 2x6 


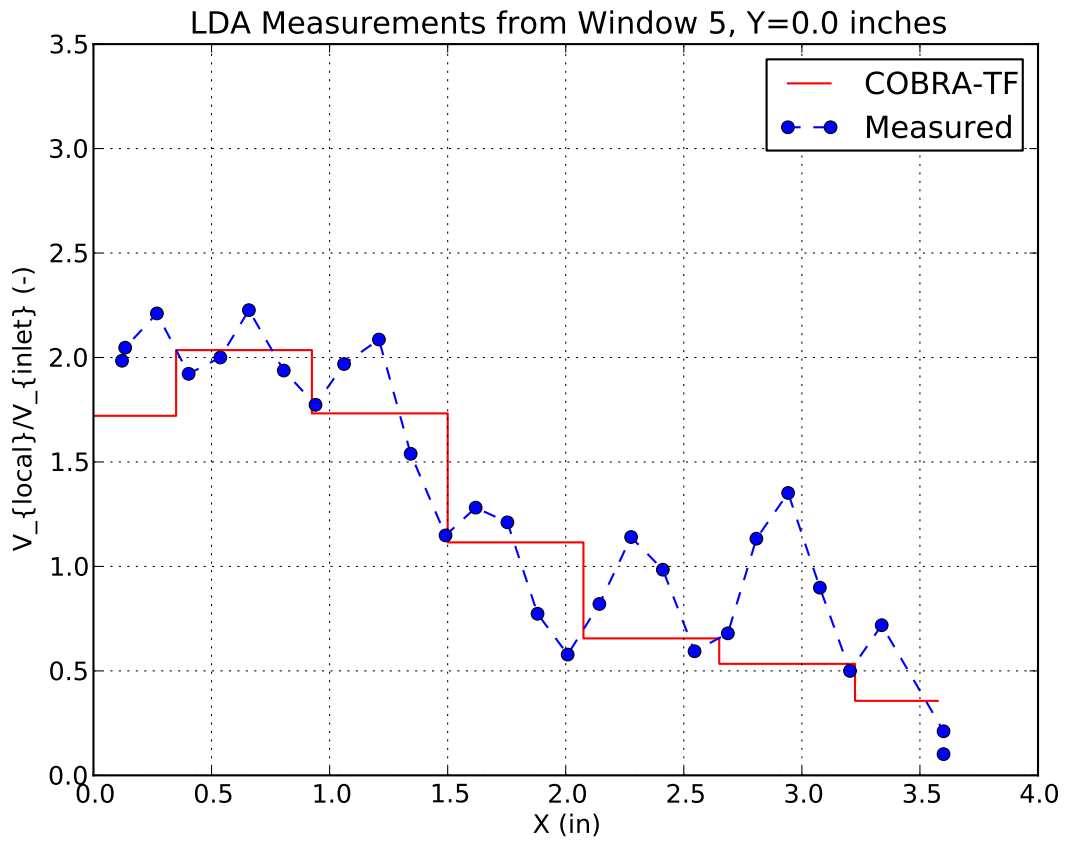

Figure 12.6: Predicted and measured subchannel velocities for Window 5 at Rake Location $Y=0.0$ in in PNNL 2x6 


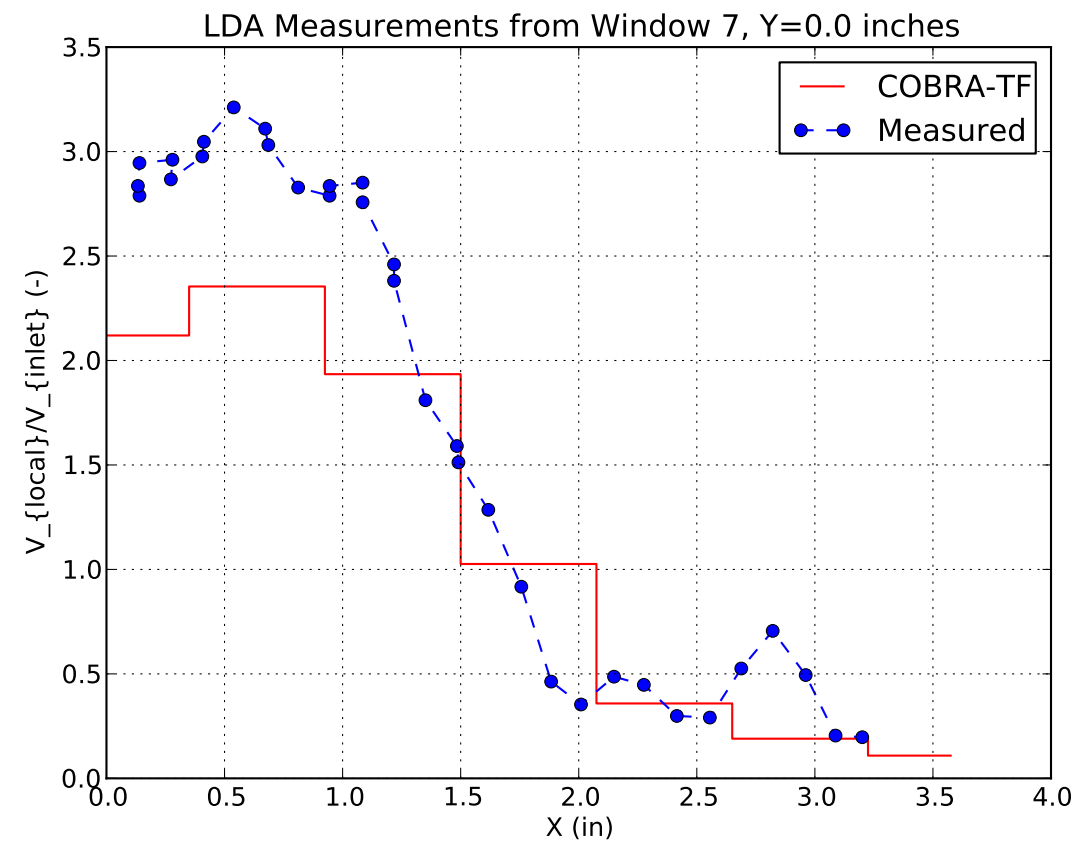

Figure 12.7: Predicted and measured subchannel velocities for Window 7 at Rake Location $Y=0.0$ in in PNNL 2x6 


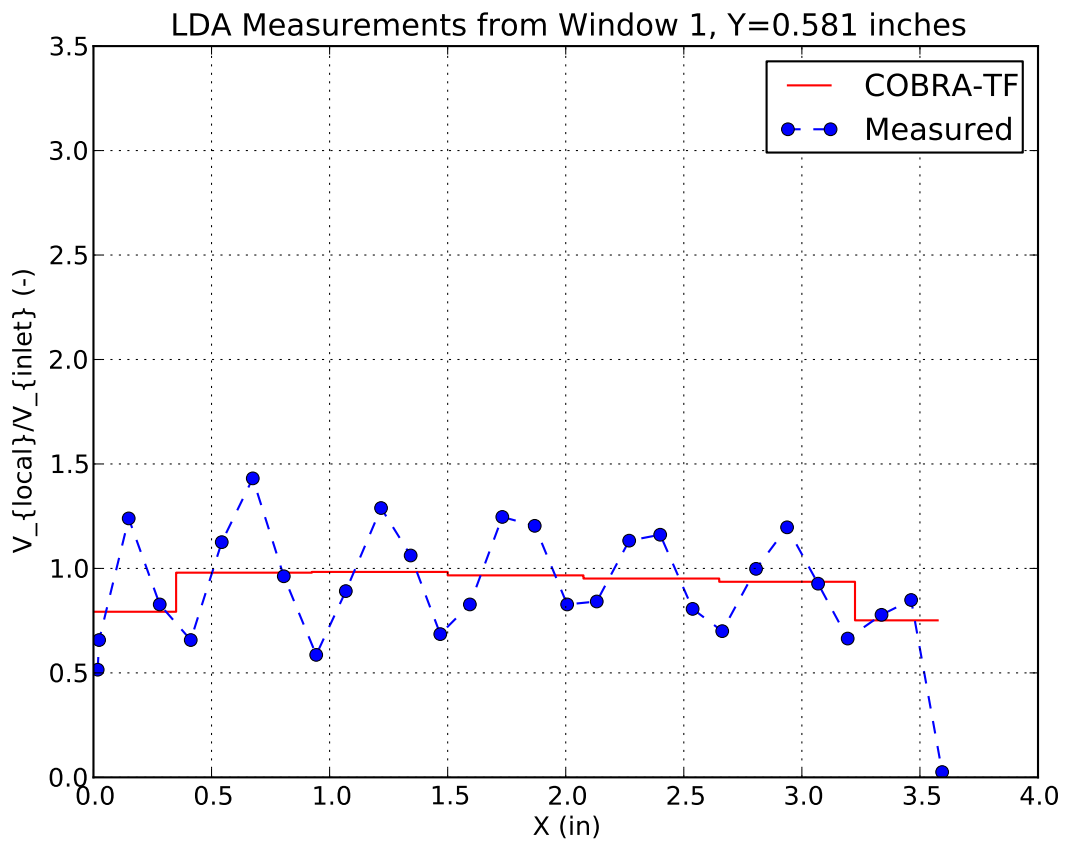

Figure 12.8: Predicted and measured subchannel velocities for Window 1 at Rake Location $Y=0.581$ in in PNNL 2x6 


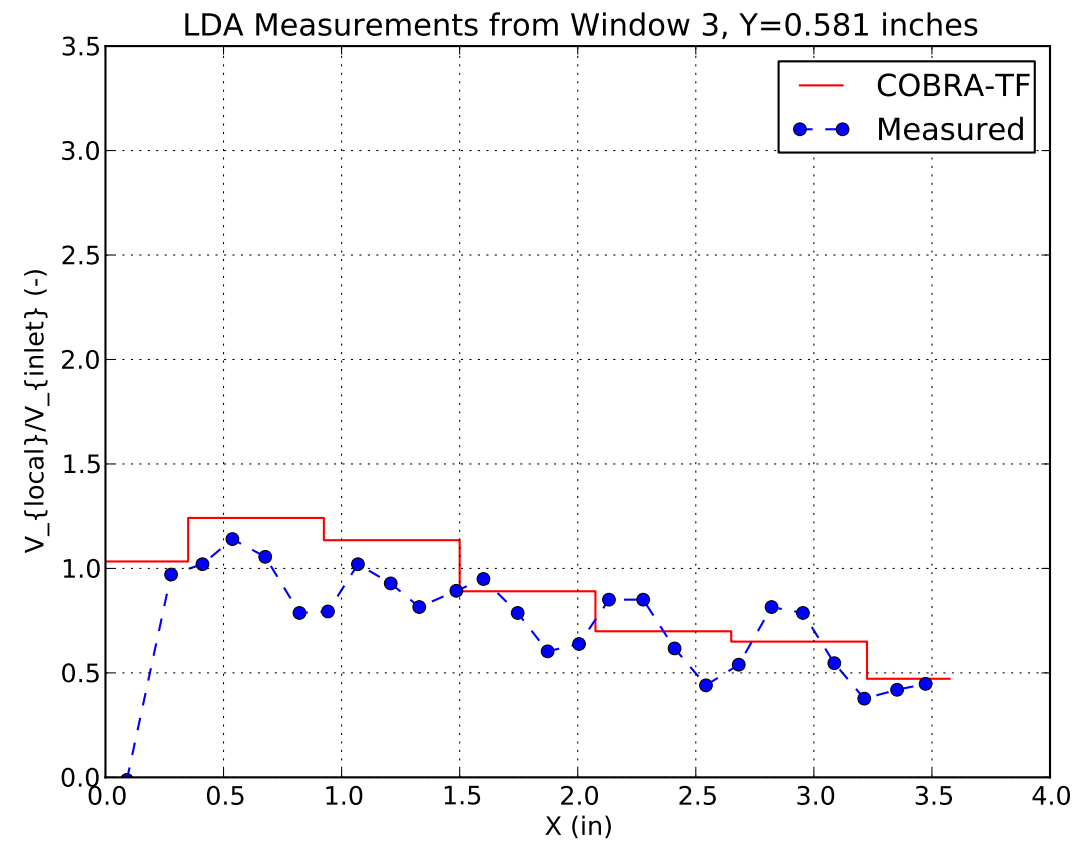

Figure 12.9: Predicted and measured subchannel velocities for Window 3 at Rake Location $Y=0.581$ in in PNNL 2x6 


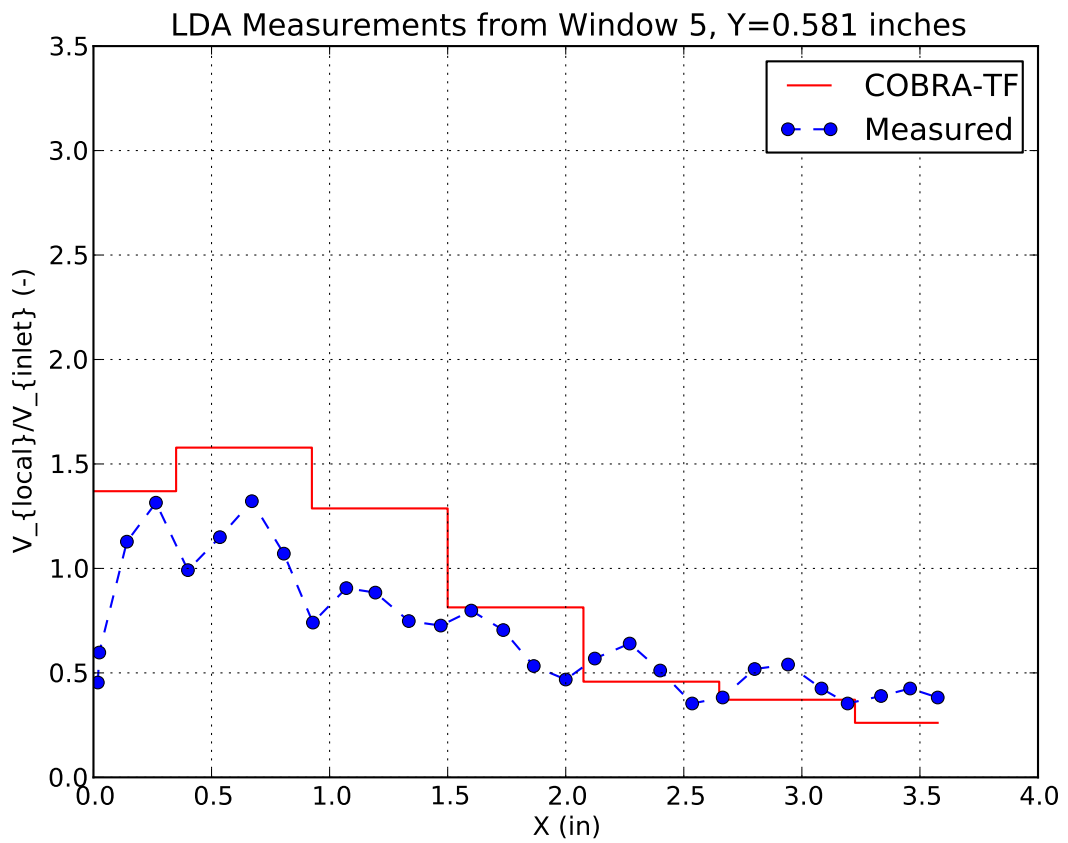

Figure 12.10: Predicted and measured subchannel velocities for Window 5 at Rake Location $Y=0.581$ in in PNNL 2x6 


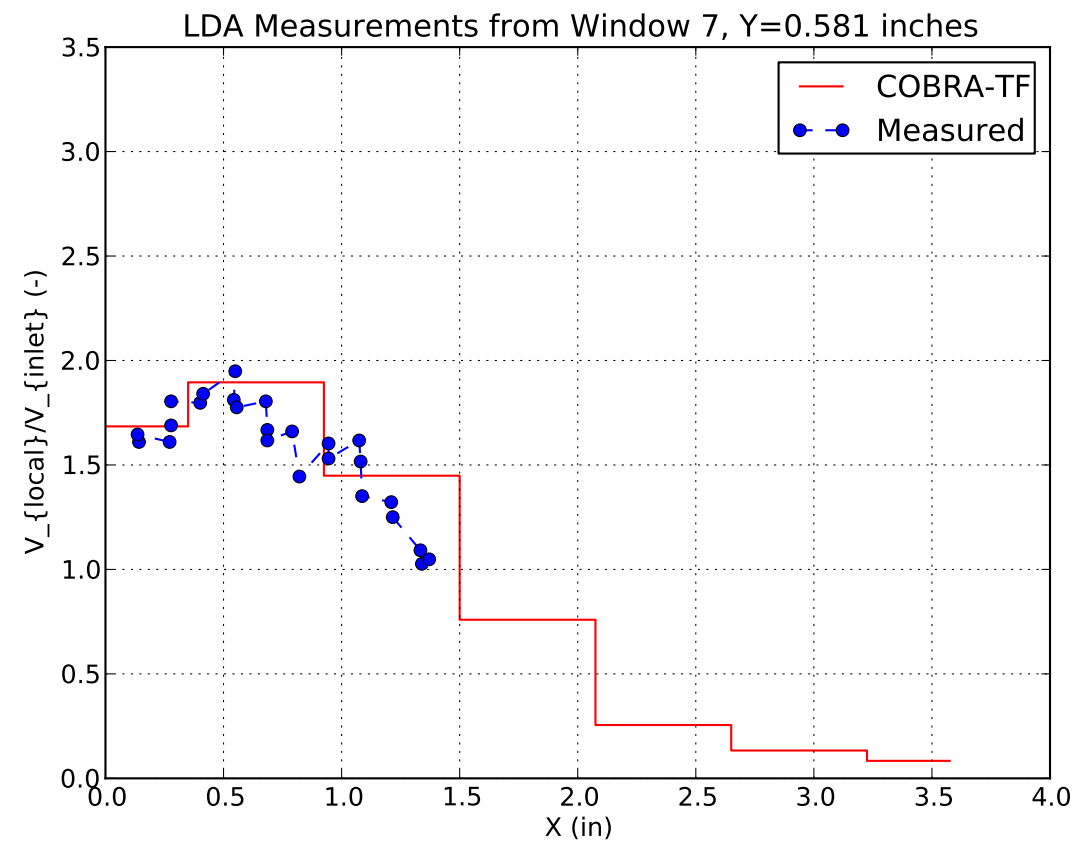

Figure 12.11: Predicted and measured subchannel velocities for Window 7 at Rake Location $Y=0.581$ in in PNNL 2x6 
TC Measurements from Window 1

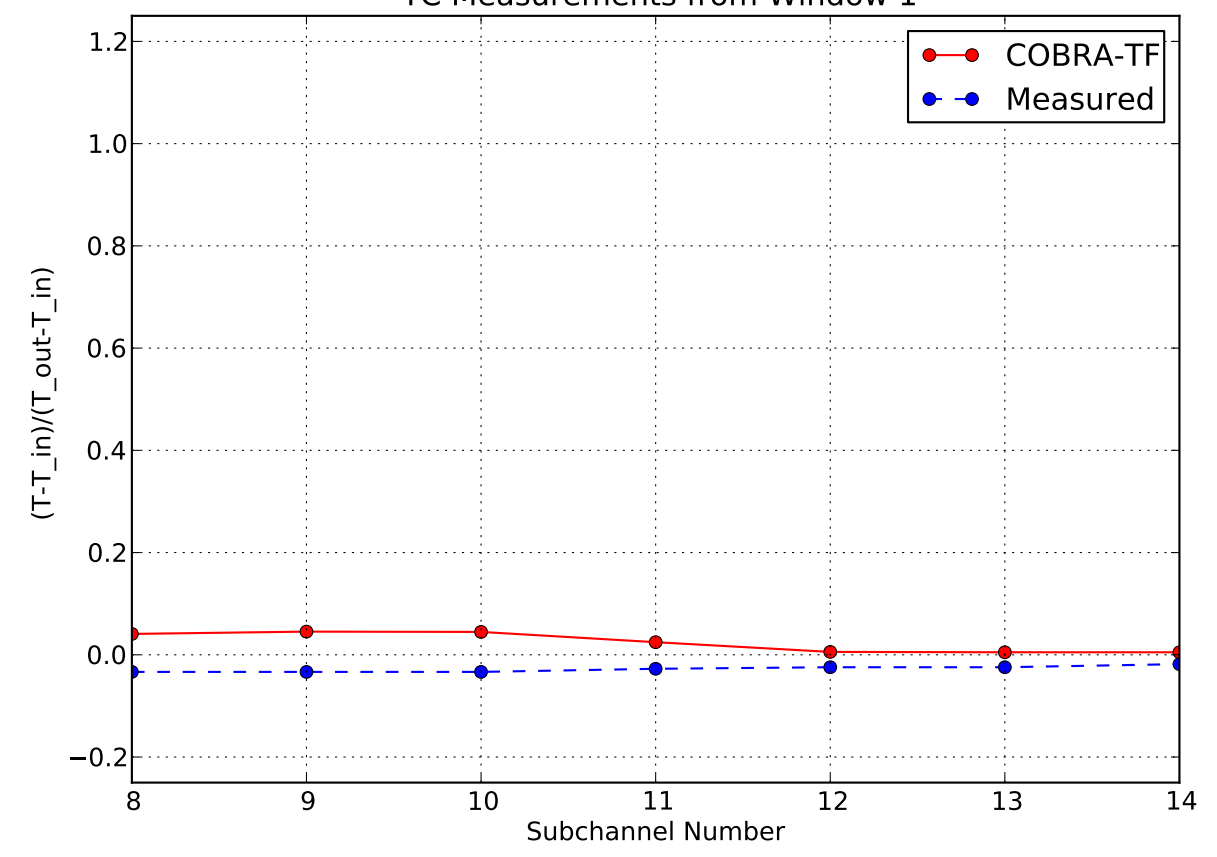

Figure 12.12: Predicted and measured subchannel-center temperatures for Window 1 in PNNL 2x6

9 axial locations. These results will naturally suffer from the same problem as the velocity results. Here we are comparing a measurement of temperature taken at the center of the subchannel with a predicted value that is a smeared average over the whole subchannel. Being that most of these axial measurement locations are taken in a laminar flow, we expect very steep gradients in temperature as we move away from the wall. This accounts for the fact why we see an over-prediction of temperatures by CTF in many cases. However, note how this over-prediction only occurs in the lower axial regions of the bundle. When we move to the higher windows (7 and 9), the results match quite well. The reason for this is likely that the thermal plumes surrounding the rods have merged and led to a turbulent flow in the upper section of the bundle. The increased turbulence acts to mix the fluid and reduce the temperature gradient across the subchannel. Data in this turbulent region will, naturally, match the volume-average results of CTF better.

Again, we need to concern ourselves with CTF's capability to match the behavior of the data. This is mostly the case except for the far-left side of the plots, where we see a drastic drop in measured temperatures. Since the entire left side of the bundle is heated, this drop in temperature is also unexpected. The predicted results are the behavior we would expect; temperatures should rise all the way up to the left side of the bundle. The authors noted this anomalous behavior, but did not provide a reason for its why it occurred. 
TC Measurements from Window 3

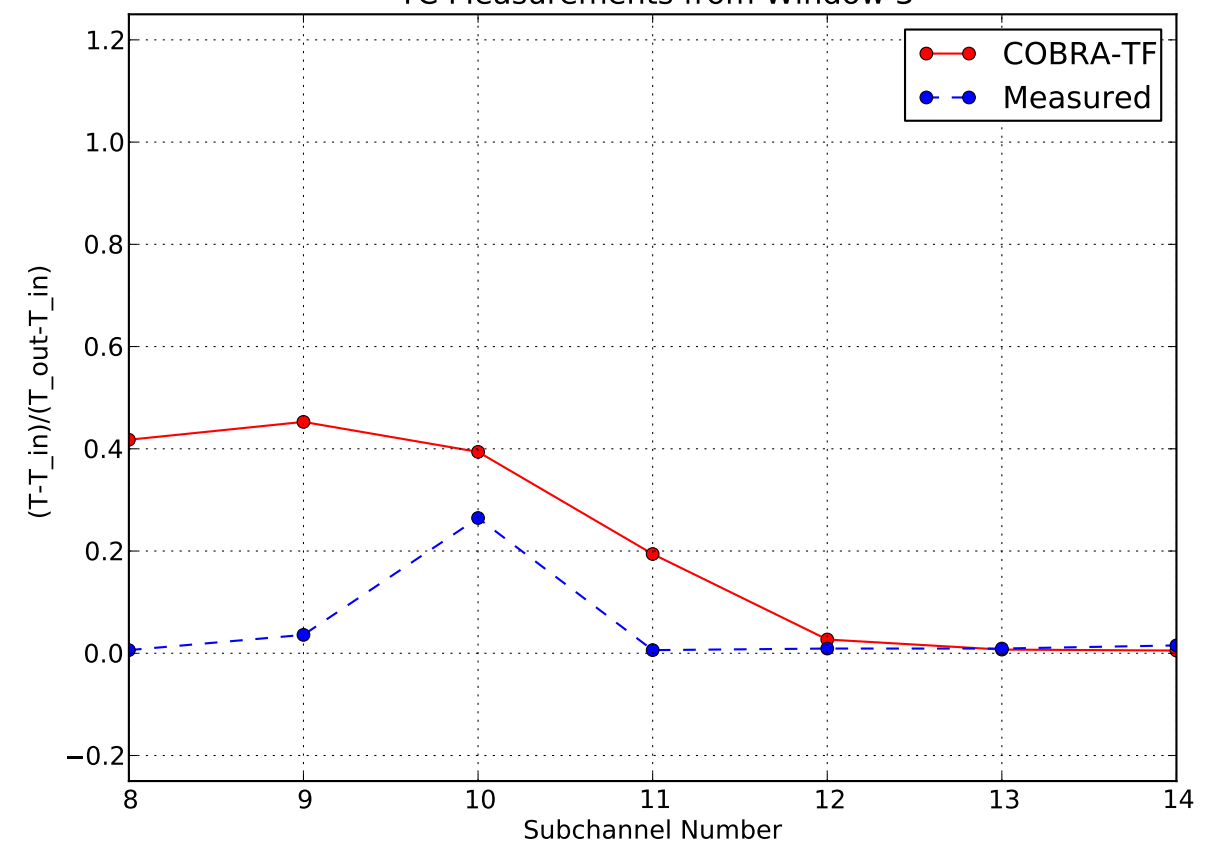

Figure 12.13: Predicted and measured subchannel-center temperatures for Window 3 in PNNL $2 x 6$ 


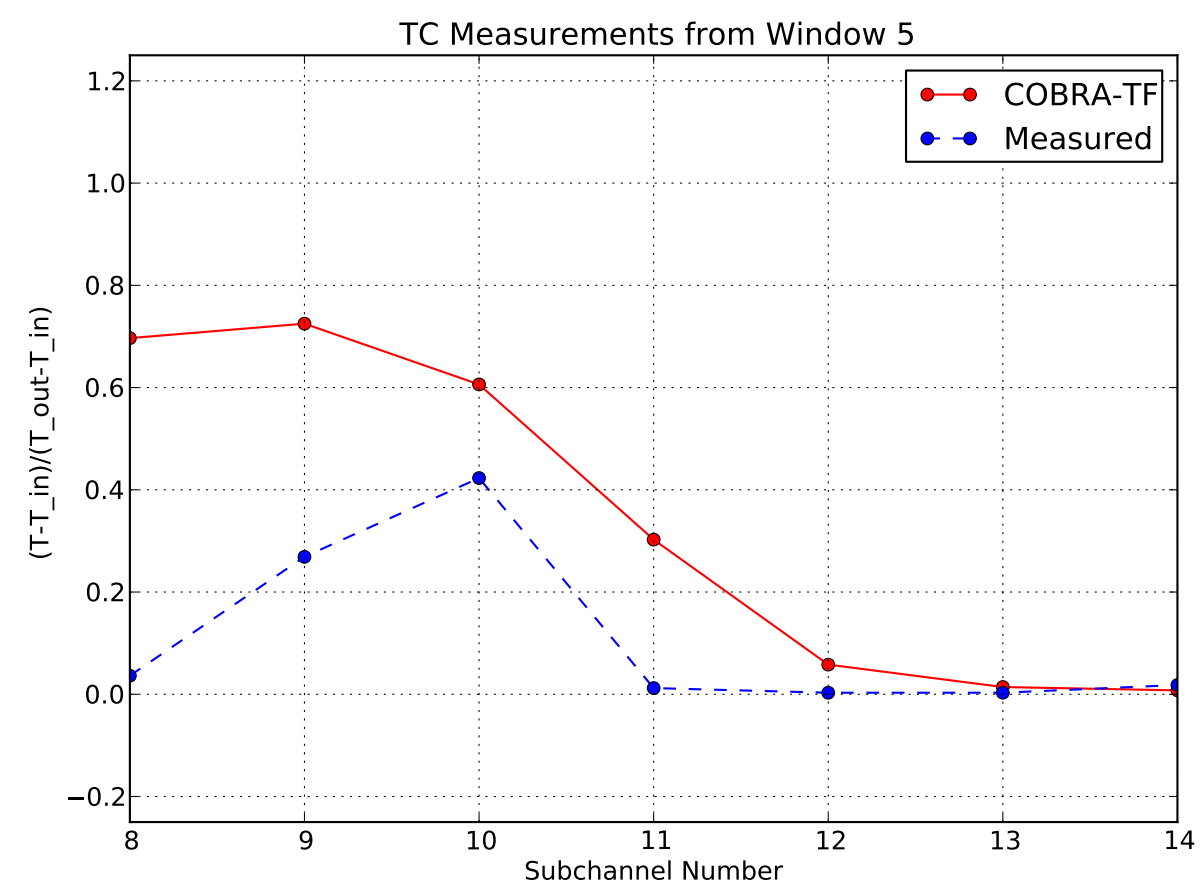

Figure 12.14: Predicted and measured subchannel-center temperatures for Window 5 in PNNL 2x6 
TC Measurements from Window 7

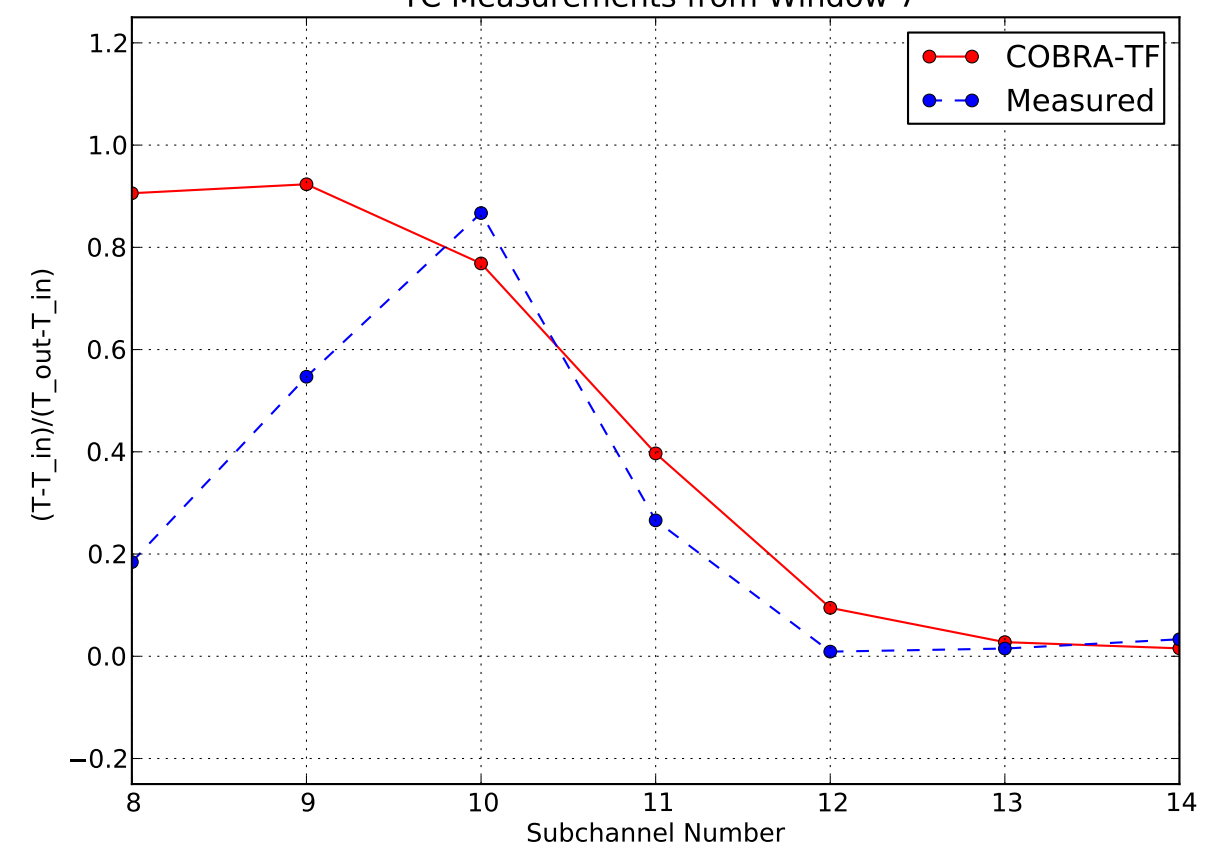

Figure 12.15: Predicted and measured subchannel-center temperatures for Window 7 in PNNL $2 \times 6$ 


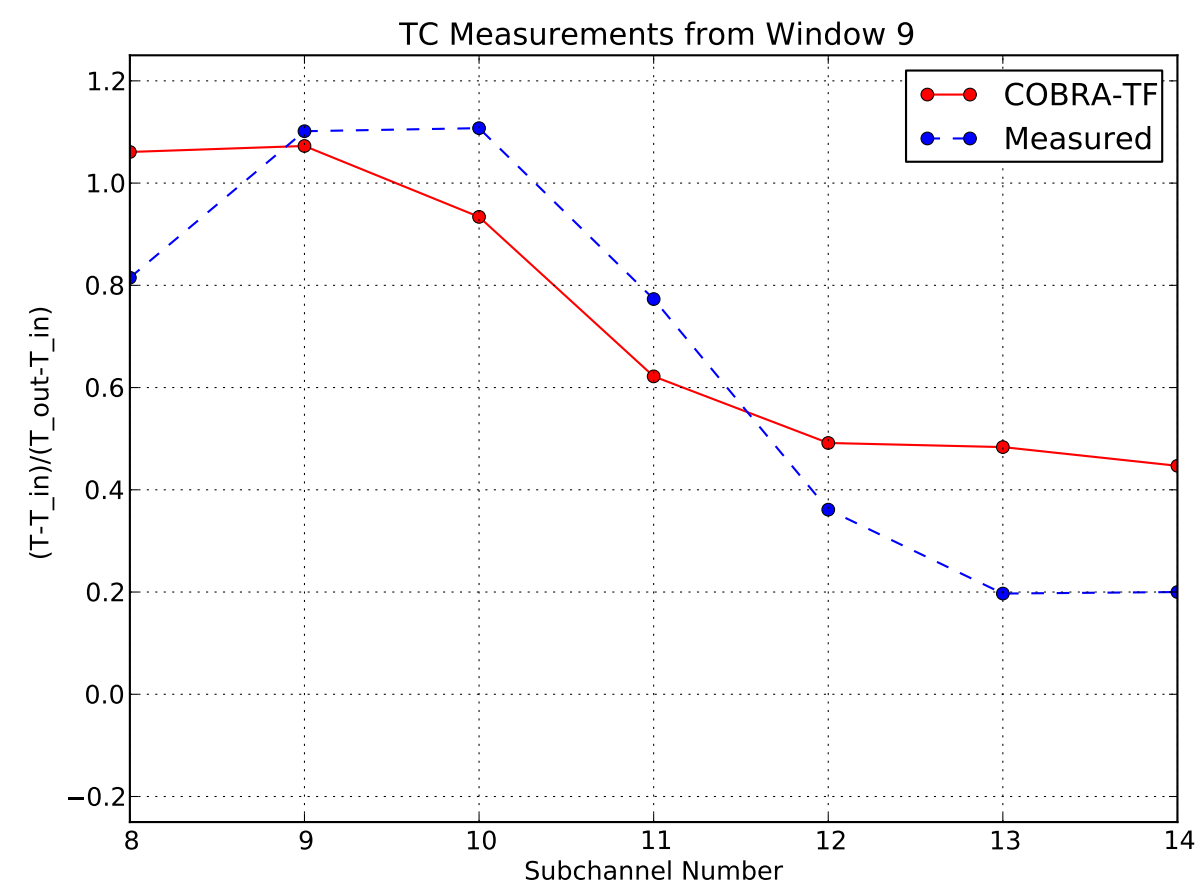

Figure 12.16: Predicted and measured subchannel-center temperatures for Window 9 in PNNL 2x6 


\section{Fuel Temperature}

\subsection{Fuel Temperature in CTF}

Prior to discussing results, a discussion of CTF-calculated fuel temperatures is included. Most of the experimental results present only fuel centerline temperature. CTF solves fuel temperature profiles in the rod; clad outside temperature, clad inside temperature, fuel pellet surface temperature, and fuel centerline temperature. Considering this, it is prudent to discuss the calculation of these terms prior to their use.

The temperature drops being added from the coolant side inward. An illustration of the radial fuel temperature profile is shown in Figure 13.1.

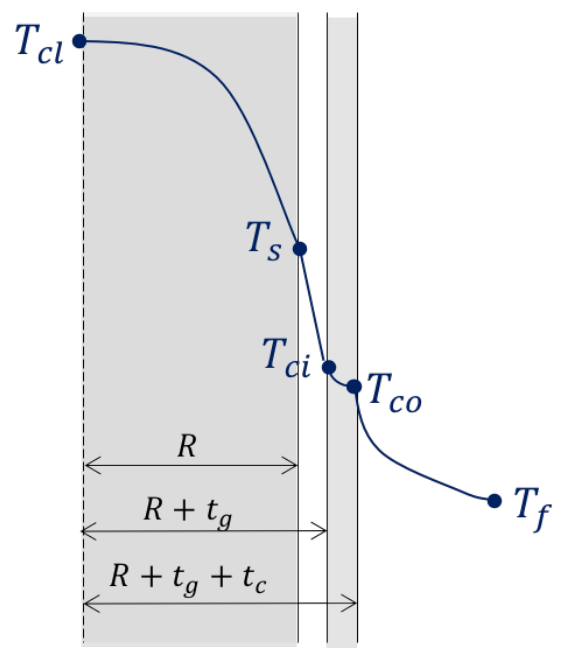

Figure 13.1: Illustration of the fuel rod and fuel temperature profiles.

The cladding outside temperature $T_{c o}$ is calculated as:

$$
T_{c o}=T_{f}+\frac{q^{\prime}}{h 2 \pi\left(R+t_{g}+t_{c}\right)}
$$

Here, $q^{\prime}$ is the linear power rate, $T_{f}$ is the bulk fluid temperature, $h$ is the convective heat transfer coefficient, 
$R$ is the fuel rod diameter, $t_{g}$ is the gap thickness, and $t_{c}$ is the cladding thickness. $h$ is calculated through the Dittus-Boelter correlation.

The cladding inside temperature $T_{c i}$ is calculated as:

$$
T_{c i}=T_{c o}+\frac{q^{\prime} \ln \left(\frac{R+t_{g}+t_{c}}{R+t_{g}}\right)}{2 \pi k_{c}}
$$

Here, $k_{c}$ is the cladding thermal conductivity.

The fuel surface temperature $T_{s}$ is calculated as:

$$
T_{s}=T_{c i}+\frac{q^{\prime}}{2 \pi R h_{g a p}}
$$

Here, $h_{\text {gap }}$ is the gap conductance. CTF uses two options for gap conductance specification; user-defined gap conductance radially/axial and dynamic calculation of the gap conductance. The dynamic gap conductance model simply calculates the gap conductance as sum of solid-solid conductance (through contact), conduction through the fill gas and radiation, where the convective heat transfer in the gap is ignored due to thin gap approximation.

The fuel centerline temperature $T_{c l}$ is calculated as:

$$
\int_{T_{s}}^{T_{c l}} k_{f} d T=\frac{f q^{\prime}}{4 \pi}
$$

Here, $k_{f}$ is the fuel thermal conductivity, and $f$ is the flux depression factor. The typical values of the flux depression factor is $0.92<f<1.00$. For simplicity, $f$ is set to 1.0 in this study. Also, the fuel thermal conductivity is calculated in two different ways: 1) the user can specify a constant fuel thermal conductivity, and 2) the thermal conductivity is calculated by CTF. In CTF, there are six pre-defined fuel thermal conductivity models. The default model is only temperature dependent. The rest takes into account burnup, $\mathrm{Gd} /$ plutonium $(\mathrm{Pu})$ content in addition to the temperature.

\subsection{Verification}

To test the implementation of the CTF conduction solution, a series of defect tests (subsection 13.2.1) and a code verification study (subsection 13.2.2) are included in the regression testing suite.

\subsubsection{Defect Testing}

A suite of tests is defined in this section to be used to verify that the various fuel thermal conductivity models are correctly implemented in CTF. There are six predefined fuel thermal conductivity models: three options for $\mathrm{UO}_{2}$ fuel and the other three for the MOX fuel. CTF uses a temperature-dependent correlation for fuel thermal conductivity in default. With the implemented correlations which account for temperature, burnup and $\mathrm{Gd} / \mathrm{Pu}$ content in calculation of the fuel thermal conductivity, temperature predictions can be estimated properly. 
Problem Description This study assesses the prediction of CTF fuel temperature predictions. The proper implementation of the fuel thermal conductivity models is determined by calculating fuel temperatures and then, comparing this to the CTF simulation results. Two ways are considered as follows:

1) Setting a constant fuel thermal conductivity and then, Equation 13.4 reduces to the following form for the fuel centerline temperature

$$
T_{c l}=T_{s}+\frac{f q^{\prime}}{4 \pi k_{f}}
$$

Several perturbations are made by changing parameters affecting the temperature profiles: mass flux, heat flux, rod dimensions, fuel thermal conductivity and gap conductance. The test conditions are selected with perturbed parameters as tabulated in Table 48.

2) CTF calculates the fuel thermal conductivity; an iterative scheme is used. Equation 13.4 is formed as

$$
\int_{0}^{T_{c l}} k_{f} d T=\int_{0}^{T_{s}} k_{f} d T+\frac{f q^{\prime}}{4 \pi}
$$

The integration of the fuel thermal conductivity correlations in Equation 13.6 are much more complicated. Therefore, an iterative solution is used for calculation of the fuel centerline temperature as:

i. The integral $\int_{0}^{T_{s}} k_{f} d T$ is calculated using trapezoidal rule from 0 to known fuel pellet surface temperature.

ii. The integral $\int_{0}^{T_{c l}} k_{f} d T$ is calculated similarly using the trapezoidal rule in a iterative method. $T_{c l}$ is set to the fuel surface temperature plus a step-size (i.e., $T_{c l}=T_{s}+0.01^{\circ} \mathrm{K}$ ). The numerical solution converges when Left-Hand Side (LHS) equals to Right-Hand Side (RHS) in Equation 13.6 with a predefined tolerance.

The fuel thermal conductivity models are tabulated in Table 49. Default fuel thermal conductivity model is MATPRO-11 correlation for $\mathrm{UO}_{2}$ fuel, which is only temperature dependent. The burnup dependent models are specified by IMOX (see CTF User's Manual). The models for $\mathrm{UO}_{2}$ fuel are IMOX $=1,2$ in addition to MATPRO-11 model (IMOX $=0$ ), while the models for MOX fuel are IMOX $=3,4,5$. The burnup dependent models are corrected with

$$
K=K_{95} \cdot 1.0789 \cdot\left(\frac{T D}{1.0+0.5(1.0-T D)}\right)
$$

Here, $K_{95}$ is the thermal conductivity of unirradiated $95 \%$ theoretical density (TD) fuel. Several perturbations are made by changing burnup and Gd content. The test conditions are tabulated in Table 50. 
Table 48: Test conditions

\begin{tabular}{|c|c|c|c|c|c|c|c|c|}
\hline $\begin{array}{l}\text { Test } \\
\text { No. }\end{array}$ & $\begin{array}{c}\dot{m} \\
(\mathrm{~kg} / \mathrm{s})\end{array}$ & $\begin{array}{c}q^{\prime} \\
(\mathrm{kW} / \mathrm{m})\end{array}$ & $\begin{array}{c}t_{g} \\
(\mu m)\end{array}$ & $\begin{array}{c}t_{c} \\
(\mathrm{~cm}) \\
\end{array}$ & $\begin{array}{c}D_{f} \\
(\mathrm{~cm})\end{array}$ & $\begin{array}{c}\text { NFUL } \\
(-) \\
\end{array}$ & $\begin{array}{c}k_{f} \\
(\mathrm{~W} / \mathrm{m}-\mathrm{K})\end{array}$ & $\begin{array}{c}h_{g a p} \\
\left(\mathrm{~W} / \mathrm{m}^{2}-\mathrm{K}\right) \\
\end{array}$ \\
\hline 1 & 0.30 & 10.00 & 65 & 0.57 & 8.24 & 3 & 16.0 & 3000 \\
\hline 2 & 0.30 & 10.00 & 65 & 0.57 & 8.24 & 6 & 16.0 & 3000 \\
\hline 3 & 0.30 & 10.00 & 65 & 0.57 & 8.24 & 12 & 16.0 & 3000 \\
\hline 4 & 0.30 & 10.00 & 65 & 0.57 & 8.24 & 24 & 16.0 & 3000 \\
\hline 5 & 0.30 & 10.00 & 65 & 0.57 & 8.24 & 48 & 16.0 & 3000 \\
\hline 6 & 0.10 & 10.00 & 65 & 0.57 & 8.24 & 24 & 16.0 & 3000 \\
\hline 7 & 0.20 & 10.00 & 65 & 0.57 & 8.24 & 24 & 16.0 & 3000 \\
\hline 8 & 0.30 & 10.00 & 65 & 0.57 & 8.24 & 24 & 16.0 & 3000 \\
\hline 9 & 0.40 & 10.00 & 65 & 0.57 & 8.24 & 24 & 16.0 & 3000 \\
\hline 10 & 0.50 & 10.00 & 65 & 0.57 & 8.24 & 24 & 16.0 & 3000 \\
\hline 11 & 0.30 & 5.0 & 65 & 0.57 & 8.24 & 24 & 16.0 & 3000 \\
\hline 12 & 0.30 & 7.5 & 65 & 0.57 & 8.24 & 24 & 16.0 & 3000 \\
\hline 13 & 0.30 & 24.5 & 65 & 0.57 & 8.24 & 24 & 16.0 & 3000 \\
\hline 14 & 0.30 & 15.0 & 65 & 0.57 & 8.24 & 24 & 16.0 & 3000 \\
\hline 15 & 0.30 & 20.0 & 65 & 0.57 & 8.24 & 24 & 16.0 & 3000 \\
\hline 16 & 0.30 & 30.0 & 65 & 0.57 & 8.24 & 24 & 16.0 & 3000 \\
\hline 17 & 0.30 & 10.00 & 20 & 0.57 & 8.24 & 24 & 16.0 & 3000 \\
\hline 18 & 0.30 & 10.00 & 30 & 0.57 & 8.24 & 24 & 16.0 & 3000 \\
\hline 19 & 0.30 & 10.00 & 40 & 0.57 & 8.24 & 24 & 16.0 & 3000 \\
\hline 20 & 0.30 & 10.00 & 50 & 0.57 & 8.24 & 24 & 16.0 & 3000 \\
\hline 21 & 0.30 & 10.00 & 60 & 0.57 & 8.24 & 24 & 16.0 & 3000 \\
\hline 22 & 0.30 & 10.00 & 65 & 0.20 & 8.24 & 24 & 16.0 & 3000 \\
\hline 23 & 0.30 & 10.00 & 65 & 0.30 & 8.24 & 24 & 16.0 & 3000 \\
\hline 24 & 0.30 & 10.00 & 65 & 0.40 & 8.24 & 24 & 16.0 & 3000 \\
\hline 25 & 0.30 & 10.00 & 65 & 0.60 & 8.24 & 24 & 16.0 & 3000 \\
\hline 26 & 0.30 & 10.00 & 65 & 0.70 & 8.24 & 24 & 16.0 & 3000 \\
\hline 27 & 0.30 & 10.00 & 65 & 0.80 & 8.24 & 24 & 16.0 & 3000 \\
\hline 28 & 0.30 & 10.00 & 65 & 0.57 & 7.00 & 24 & 16.0 & 3000 \\
\hline 29 & 0.30 & 10.00 & 65 & 0.57 & 7.50 & 24 & 16.0 & 3000 \\
\hline 30 & 0.30 & 10.00 & 65 & 0.57 & 8.00 & 24 & 16.0 & 3000 \\
\hline 31 & 0.30 & 10.00 & 65 & 0.57 & 8.50 & 24 & 16.0 & 3000 \\
\hline 32 & 0.30 & 10.00 & 65 & 0.57 & 9.00 & 24 & 16.0 & 3000 \\
\hline 33 & 0.30 & 10.00 & 65 & 0.57 & 8.24 & 24 & 5.0 & 3000 \\
\hline 34 & 0.30 & 10.00 & 65 & 0.57 & 8.24 & 24 & 7.5 & 3000 \\
\hline 35 & 0.30 & 10.00 & 65 & 0.57 & 8.24 & 24 & 10.0 & 3000 \\
\hline 36 & 0.30 & 10.00 & 65 & 0.57 & 8.24 & 24 & 17.5 & 3000 \\
\hline 37 & 0.30 & 10.00 & 65 & 0.57 & 8.24 & 24 & 20.0 & 3000 \\
\hline 38 & 0.30 & 10.00 & 65 & 0.57 & 8.24 & 24 & 25.0 & 3000 \\
\hline 39 & 0.30 & 10.00 & 65 & 0.57 & 8.24 & 24 & 16.0 & 1000 \\
\hline 40 & 0.30 & 10.00 & 65 & 0.57 & 8.24 & 24 & 16.0 & 1500 \\
\hline 41 & 0.30 & 10.00 & 65 & 0.57 & 8.24 & 24 & 16.0 & 2000 \\
\hline 42 & 0.30 & 10.00 & 65 & 0.57 & 8.24 & 24 & 16.0 & 2500 \\
\hline 43 & 0.30 & 10.00 & 65 & 0.57 & 8.24 & 24 & 16.0 & 3000 \\
\hline 44 & 0.30 & 10.00 & 65 & 0.57 & 8.24 & 24 & 16.0 & 4000 \\
\hline
\end{tabular}




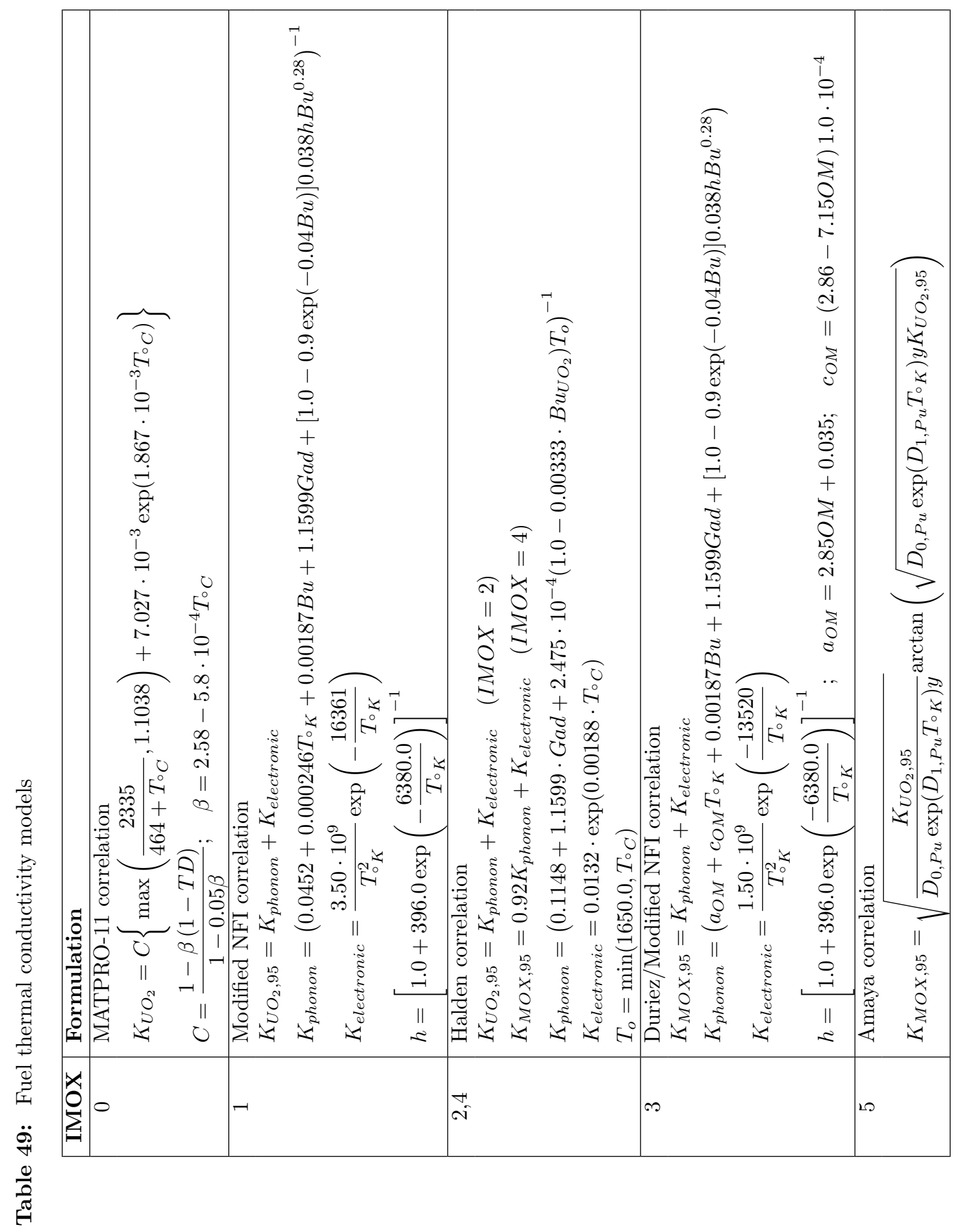


Table 50: Test descriptions

\begin{tabular}{|c|c|c|}
\hline Test No. & Bu(GWD/MTU) & Gad (-) \\
\hline a, g, m & $0.0,10.0,50.0$ & 0.000 \\
b, h, n & $0.0,10.0,50.0$ & 0.025 \\
c, i, o & $0.0,10.0,50.0$ & 0.050 \\
d, j, p & $0.0,10.0,50.0$ & 0.075 \\
e, k, r & $0.0,10.0,50.0$ & 0.100 \\
\hline
\end{tabular}

Python scripts are created for the purpose of performing the comparison of the analytical solution versus the CTF simulation results. The scripts read CTF output files and extract the fuel temperature profiles to compare with the computed analytical solutions. The scripts can be found in the test directory in the CTF repository.

CTF Input Model Description A single subchannel with a rod-centered configuration is used as the base geometry. A constant linear power rate is assumed along the fuel total length. The parameters are selected to be representative of a typical PWR subchannel. Outlet pressure is set to 155.13 bar and inlet temperature is set to $292.78^{\circ} \mathrm{C}$. Inlet mass flow rate is set to $3.0 \mathrm{~kg} / \mathrm{s}$.

Discussion of Results Table 51 shows the comparison between CTF-predicted fuel pellet surface and fuel centerline temperatures and the corresponding expected results with the relative error between the CTFpredicted results and the expected results. Test cases $1-5$, number of radial nodes in the fuel (NFUL) is perturbed to study its impacts on fuel centerline temperature. CTF computes the fuel centerline temperature by extrapolating the temperature in the closest radial node to centerline. Therefore, the fuel centerline temperature will be sensitive to the NFUL. The number of radial nodes in the fuel is varied, NFUL=3, 6 , 12, 24 and 48. The optimum value of NFUL is found to be 24 . And, the relative error is always less than $0.03 \%$ in Table 51. This indicates CTF is capable of predicting fuel surface temperature accurately once it is informed correctly. This is important for coupled mutli-physics calculations such as thermal-hydraulic and fuel performance codes coupling.

In case of fuel thermal conductivity models, the ring sensitivity study is repeated. All thermal conductivity models are compared for the case with zero burnup and zero Gd/Pu content. The CTF predicted fuel centerline temperatures are tabulated in Table 52 for each model. The optimized value of NFUL is found to be 24 as well and it is kept constant for the rest of study. It is important to note that the expected values are obtained from the iterative solution, therefore, it is numerical model dependent. This leads to have slightly different expected values between the models.

Test cases are created according to Table 50 by basically varying the burnup and $\mathrm{Gd} / \mathrm{Pu}$ content for the fuel thermal conductivity models, and CTF-predicted and expected results are plotted in Figure 13.2. The maximum relative error is found for test condition with the highest burnup and $\mathrm{Gd} / \mathrm{Pu}$ content. The discrepancy between the implementations and the expected solutions is the consequence of using the trapezoidal rule (simple numerical integration model with low precision degree). With increased degree of precision, the discrepancy will reduce significantly, but it will introduce more complexities. For the scope of this study, trapezoidal rule is used for its simplicity. 
Table 51: Results for fuel centerline temperature

\begin{tabular}{|c|c|c|c|c|c|c|}
\hline $\begin{array}{l}\text { Test } \\
\text { No. }\end{array}$ & $\begin{array}{c}\mathbf{C T F} \\
T_{s}(\mathbf{K})\end{array}$ & $\begin{array}{c}\text { True } \\
T_{s}(\mathbf{K})\end{array}$ & $\epsilon_{r e l}(\%)$ & $\begin{array}{c}\mathbf{C T F} \\
T_{c l}(\mathbf{K})\end{array}$ & $\begin{array}{c}\text { True } \\
T_{c l}(\mathbf{K})\end{array}$ & $\epsilon_{r e l}(\%)$ \\
\hline 1 & $\begin{array}{l}717.1 \\
\end{array}$ & $\begin{array}{l}717.1 \\
\end{array}$ & 0.00 & $\begin{array}{l}766.8 \\
\end{array}$ & $\begin{array}{l}762.3 \\
\end{array}$ & -0.60 \\
\hline 2 & 717.1 & 717.1 & 0.00 & 766.8 & 765.4 & -0.19 \\
\hline 3 & 717.1 & 717.1 & 0.00 & 766.8 & 766.4 & -0.06 \\
\hline 4 & 717.1 & 717.1 & 0.00 & 766.8 & 766.7 & -0.02 \\
\hline 5 & 717.1 & 717.1 & 0.00 & 766.8 & 766.8 & 0.00 \\
\hline 6 & 731.1 & 731.1 & 0.00 & 780.8 & 780.7 & $\begin{array}{l}-0.02 \\
\end{array}$ \\
\hline 7 & 720.9 & 720.9 & 0.00 & 770.6 & 770.5 & -0.02 \\
\hline 8 & 717.1 & 717.1 & 0.00 & 766.8 & 766.7 & -0.02 \\
\hline 9 & 715.1 & 715.1 & 0.00 & 764.8 & 764.7 & -0.02 \\
\hline 10 & 713.8 & 713.8 & 0.00 & 763.5 & 763.4 & -0.02 \\
\hline 11 & 641.5 & 641.6 & 0.00 & 666.4 & 666.4 & $\begin{array}{l}-0.01 \\
\end{array}$ \\
\hline 12 & 679.3 & 679.3 & 0.00 & 716.6 & 716.5 & -0.01 \\
\hline 13 & 935.7 & 935.8 & 0.00 & 1057.6 & 1057.3 & -0.03 \\
\hline 14 & 792.6 & 792.6 & 0.00 & 867.2 & 867.0 & -0.02 \\
\hline 15 & 868.0 & 868.0 & 0.00 & 967.4 & 967.2 & -0.03 \\
\hline 16 & 1018.5 & 1018.5 & 0.00 & 1167.7 & 1167.4 & -0.03 \\
\hline 17 & 717.5 & 717.5 & 0.00 & 767.2 & 767.1 & -0.02 \\
\hline 18 & 717.4 & 717.4 & 0.00 & 767.1 & 767.0 & -0.02 \\
\hline 19 & 717.3 & 717.3 & 0.00 & 767.0 & 766.9 & -0.02 \\
\hline 20 & 717.2 & 717.2 & 0.00 & 767.0 & 766.8 & -0.02 \\
\hline 21 & 717.1 & 717.1 & 0.00 & 766.9 & 766.7 & -0.02 \\
\hline 22 & 711.4 & 711.4 & 0.00 & 761.2 & 761.0 & $\begin{array}{l}-0.02 \\
\end{array}$ \\
\hline 23 & 713.0 & 713.0 & 0.00 & 762.7 & 762.6 & -0.02 \\
\hline 24 & 714.5 & 714.5 & 0.00 & 764.3 & 764.1 & -0.02 \\
\hline 25 & 717.5 & 717.5 & 0.00 & 767.3 & 767.1 & -0.02 \\
\hline 26 & 719.0 & 719.0 & 0.00 & 768.7 & 768.6 & -0.02 \\
\hline 27 & 720.4 & 720.4 & 0.00 & 770.2 & 770.1 & -0.02 \\
\hline 28 & 745.8 & 745.9 & 0.00 & 795.6 & 795.5 & $\begin{array}{l}-0.02 \\
\end{array}$ \\
\hline 29 & 733.2 & 733.2 & 0.00 & 782.9 & 782.8 & -0.02 \\
\hline 30 & 722.0 & 722.0 & 0.00 & 771.7 & 771.6 & -0.02 \\
\hline 31 & 712.1 & 712.1 & 0.00 & 761.8 & 761.7 & -0.02 \\
\hline 32 & 703.2 & 703.2 & 0.00 & 753.0 & 752.9 & -0.02 \\
\hline 33 & 717.1 & 717.1 & 0.00 & 876.2 & 875.8 & -0.05 \\
\hline 34 & 717.1 & 717.1 & 0.00 & 823.2 & 822.9 & -0.03 \\
\hline 35 & 717.1 & 717.1 & 0.00 & 796.7 & 796.5 & -0.03 \\
\hline 36 & 717.1 & 717.1 & 0.00 & 762.6 & 762.4 & -0.01 \\
\hline 37 & 717.1 & 717.1 & 0.00 & 756.9 & 756.8 & -0.01 \\
\hline 38 & 717.1 & 717.1 & 0.00 & 748.9 & 748.8 & -0.01 \\
\hline 39 & 974.6 & 974.6 & 0.00 & 1024.4 & 1024.2 & -0.01 \\
\hline 40 & 845.9 & 845.9 & 0.00 & 895.6 & 895.5 & -0.01 \\
\hline 41 & 781.5 & 781.5 & 0.00 & 831.2 & 831.1 & -0.01 \\
\hline 42 & 742.8 & 742.8 & 0.00 & 792.6 & 792.5 & -0.02 \\
\hline 43 & 717.1 & 717.1 & 0.00 & 766.8 & 766.7 & -0.02 \\
\hline 44 & 684.9 & 684.9 & 0.00 & 734.6 & 734.5 & -0.02 \\
\hline
\end{tabular}


Table 52: Comparison of the CTF predicted fuel centerline temperature with analytical results for $B u=0.0^{\mathrm{GWD}} / \mathrm{MTU}$ and $\mathrm{Gad}=0.0$.

\begin{tabular}{|c|cccccc|}
\hline \multirow{2}{*}{ IMOX } & \multicolumn{5}{|c|}{ Fuel centerline temperature, $\mathbf{T}_{C L}\left({ }^{\circ} \mathbf{K}\right)$} \\
& $\mathbf{3}$ & $\mathbf{6}$ & $\mathbf{1 2}$ & $\mathbf{2 4}$ & $\mathbf{4 8}$ & Expected \\
\hline 1 & 582.9 & 583.4 & 583.5 & 583.5 & 583.6 & 588.9 \\
& $1.02 \%$ & $0.93 \%$ & $0.92 \%$ & $0.92 \%$ & $0.90 \%$ & - \\
\hline 2 & 583.0 & 583.4 & 583.6 & 583.7 & 583.7 & 589.0 \\
& $1.02 \%$ & $0.95 \%$ & $0.92 \%$ & $0.90 \%$ & $0.90 \%$ & - \\
\hline 3 & 583.4 & 583.8 & 584.0 & 584.1 & 584.1 & 589.8 \\
& $1.09 \%$ & $1.02 \%$ & $0.98 \%$ & $0.97 \%$ & $0.97 \%$ & - \\
\hline 4 & 583.5 & 584.1 & 584.2 & 584.3 & 584.3 & 590.2 \\
& $1.14 \%$ & $1.03 \%$ & $1.02 \%$ & $1.00 \%$ & $1.00 \%$ & - \\
\hline 5 & 587.9 & 588.7 & 589.0 & 589.1 & 589.1 & 588.9 \\
& $0.17 \%$ & $0.03 \%$ & $0.02 \%$ & $0.03 \%$ & $0.03 \%$ & - \\
\hline
\end{tabular}

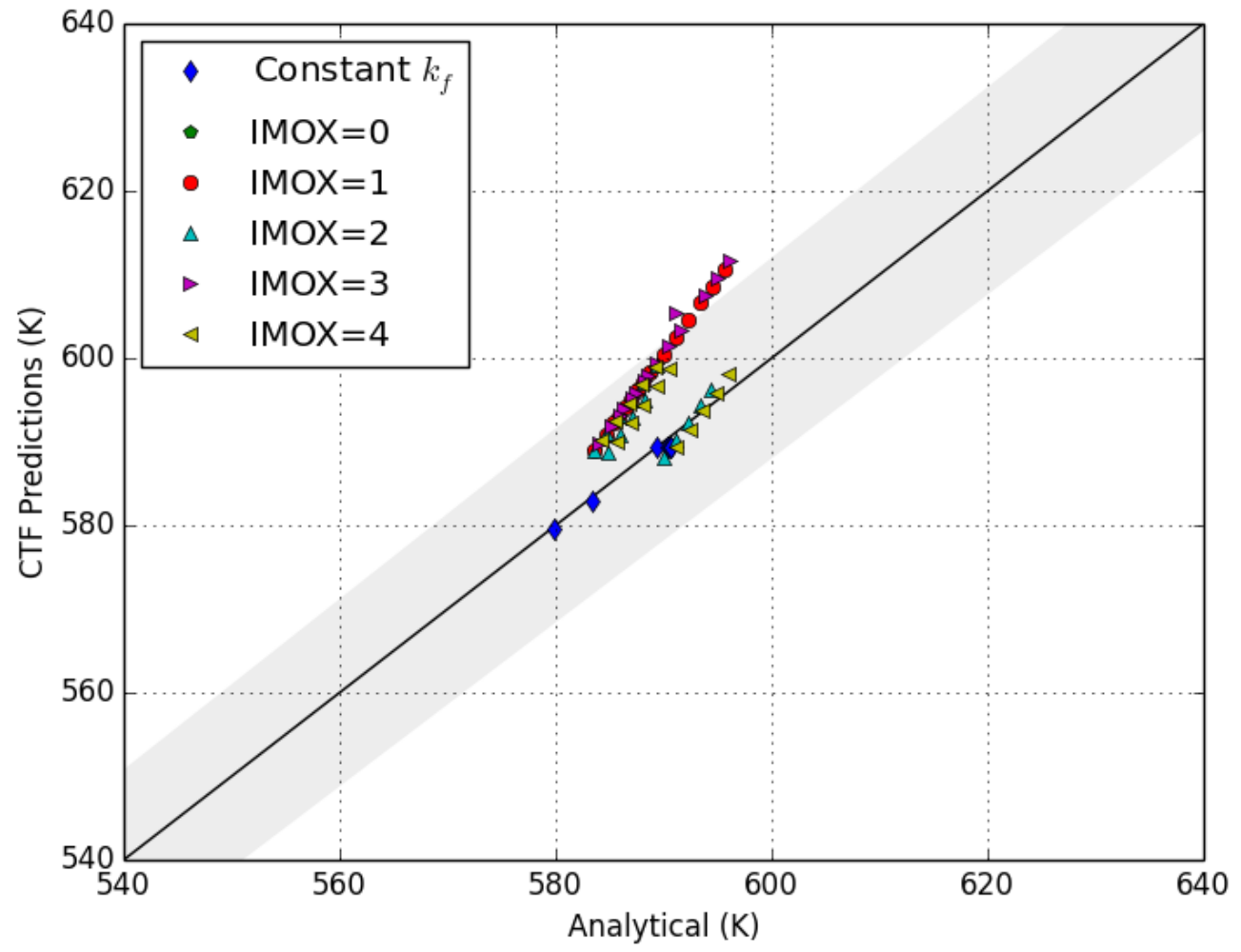

Figure 13.2: Comparison of CTF predictions vs. analytical results for fuel centerline temperatures. Grey areas indicate $\pm 2 \%$ error margin. 
Table 53: Settings for the fuel temperature code verification problem

$\begin{array}{cc}r_{f} & 0.5430 \mathrm{~cm} \\ r_{c i} & 0.6370 \mathrm{~cm} \\ r_{c o} & 0.6402 \mathrm{~cm} \\ k_{f} & 7 W / m / K \\ k_{c} & 7 W / m / K \\ h_{g} & 1000 W / m^{2} / K \\ T_{c o} & 300 \mathrm{~K} \\ q^{\prime} & 18.29943 \mathrm{~kW} / \mathrm{m}\end{array}$

Conclusions An extensive set of tests is applied to CTF in this study. The six predefined fuel thermal conductivity models and their implementations are verified. A single rod-centered channel is used as the base geometry. Several perturbations are made by changing parameters affecting the temperature profiles. Also, prediction of the temperature improves as the number of radial nodes increases. The optimal value of the number of radial nodes in the fuel is found to be 24 . This study indicates the proper implementation of the fuel thermal conductivity models in CTF.

\subsubsection{Convergence Behavior}

The CTF conduction solution uses a second-order central difference scheme. This section presents verification that the temperature distribution in the pin converges second order to an analytic solution. This study is a modification of the verification work performed in [46].

Problem Description The simulated problem for this code verification study is very similar to the constant thermal conductivity problems in the previous section. If all properties are constant, then the solution of the conduction solution is relatively simple. In cylindrical geometry with boundary conditions, $T\left(r=r_{f}\right)=T_{f}$ and $T(r \rightarrow 0)=$ finite, this solution is

$$
T(r)=T_{f}+\frac{q^{\prime}}{4 \pi k_{f}}\left(1-\frac{r^{2}}{r_{f}^{2}}\right)
$$

There is an additional temperature difference over the fuel surface $\left(r_{f}\right)$ and the outer cladding surface which is found using thermal resistances:

$$
T_{f}-T_{c o}=\frac{q^{\prime}}{2 \pi r_{f} h_{g a p}}+\frac{q^{\prime}}{2 \pi k_{c}} \ln \left(\frac{r_{c o}}{r_{c i}}\right)
$$

The required settings are described in Table 53.

CTF Input Description The CTF input is created using CTF's stand-alone fuel solver, CTFFuel [46]. All input configuration is straightforward. The cladding and gap discretization is fixed in CTF, so only the 
temperature distribution inside the fuel pellet is analyzed for convergence. All inputs are the same except for the number of radial rings in the model.

Discussion of Results The temperature distribution results are shown in Figure 13.3. The convergence behavior of these results is shown in Figure 13.4 as a function of $\Delta r$. As expected, the conduction solve is second order.

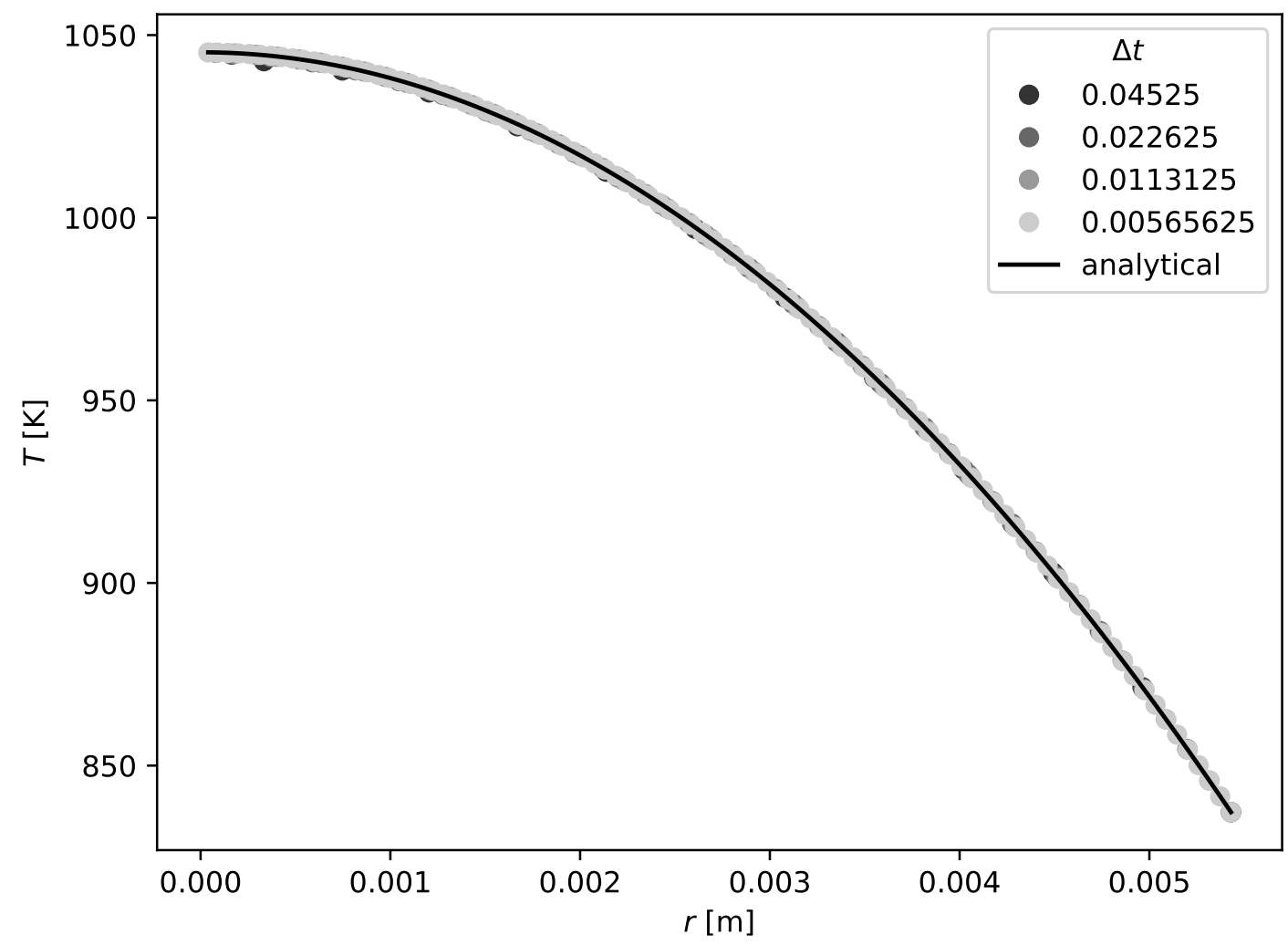

Figure 13.3: Comparison of CTF predictions with analytic solution for various $\Delta r$ choices.

\subsection{Validation}

CTF's fuel temperature predictions are improved by incorporating fuel performance models in CTF. The previous CTF fuel model did not account for the irradiation effects, which diminishes the accuracy of CTF when used for obtaining thermal feedback in cycle depletion calculations. It interests to the user to see how CTF predicts fuel temperatures, it has been given its own section. The primary test of interest here is the Halden IFA cases, though the reader should be aware that the test facility, discussed in Sections 3. 


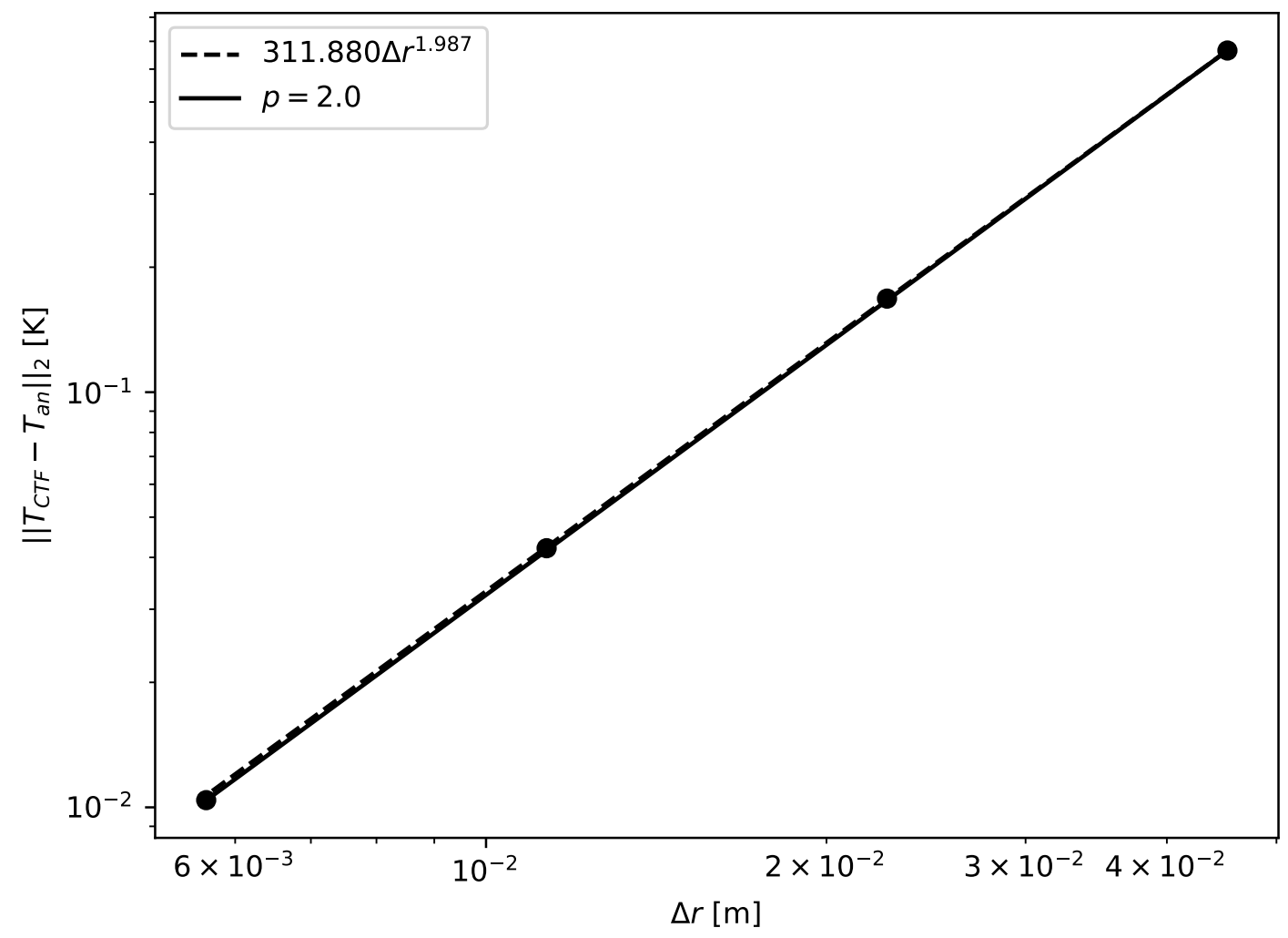

Figure 13.4: Convergence behavior of CTF conduction solution. 


\subsubsection{Halden IFA Cases}

\subsubsection{Assessment of Fuel Centerline Temperature Predictions at $\mathrm{UO}_{2}$ Fuel}

IFA432 at BOL Conditions The BOL fuel centerline temperature predictions are compared against the measurements taken during first ramp to power for IFA432 Rod-1 from Halden experiments [20]. First ramp to power takes place during the first 1 or 2 days of operation. Since this is a short time period, initial fuel rod dimensions will still be valid because there will be no time to change in dimensions due to fission gas release, fuel densification, swelling, cladding creeps, or corrosion [20]. There will be only thermal expansion due to temperature increase.

IFA432 Rod-1 is selected from FRAPCON-3.4 Integral Assessment study for comparison of the fuel centerline temperatures. Fuel centerline temperatures predicted by CTF are compared against FRAPCON-3.4 predictions and the experimental data. Figures 13.5 and 13.6 - for the measurements at lower and upper thermocouple positions - show the comparison of fuel centerline temperature predicted by CTF, FRAPCON-3.4, and the measured data for IFA432 Rod-1 at BOL. FRAPCON-3.4 underestimates the temperature predictions, whereas CTF's estimates are in good agreement with the measurements.

IFA432 Exposed up to 30GWD/MTU The assessment of fuel centerline temperature predictions by CTF is performed using IFA432 Rod-1 exposed $\mathrm{UO}_{2}$ with burnup of $30 \mathrm{GWD} / \mathrm{MTU}$ from Halden reactor test assemblies [20] to evaluate CTF's ability to account for the fuel thermal conductivity degradation with burnup.

Figure 13.7 shows the fuel centerline temperature profile vs. the rod average burnup for the measured data, FRAPCON-3.4 and CTF predictions. It is observed in Figure 13.7 that CTF gives good agreement with both the data and the FRAPCON-3.4 predictions.

\subsubsection{Assessment of Fuel Centerline Temperature Predictions for $\mathrm{UO}_{2}+2 \% \mathrm{Gd}_{2} \mathrm{O}_{3} \mathrm{Fuel}$}

IFA681 Exposed up to 23GWD/MTU The assessment of fuel centerline temperature predictions by CTF is performed using IFA681 Rod-2 $\mathrm{UO}_{2}+2 \% \mathrm{Gd}_{2} \mathrm{O}_{3}$ fuel with a burnup of $23 \mathrm{GWD} / \mathrm{MTU}$ rod from Halden reactor test assemblies [18] to evaluate CTF's ability to account for the fuel thermal conductivity degradation with burnup and Gd concentration. IFA681 Rod-2 is selected for comparison of the analysis because it is a solid rod and has $2 \% \mathrm{Gd}$ that consists of standard $\mathrm{Gd}\left({ }^{155} \mathrm{Gd}\right.$ or $\left.{ }^{157} \mathrm{Gd}\right)$. This allows investigation of the degradation of fuel thermal conductivity due to Gd and also the effect of neutron absorption by Gd atoms on the radial power profile.

Figure 13.8 shows the comparison of fuel centerline temperature predicted by FRAPCON-3.4 and CTF predictions against the measured data as a function of rod average burnup. It is observed in Figure 13.8 that FRAPCON-3.4 underestimates the fuel centerline temperature during first rise up to around 5GWD/MTU. The reason for the underprediction in this region is that there is uncertainty in the measured rod power as Gd burns out. After all the Gd burns out the effect of Gd on the rod power decreases, and the only effect would be on the thermal conductivity. After 5GWD/MTU, FRAPCON-3.4 starts overestimating the fuel centerline temperature. The FRAPCON-3.4 predictions lie within a 5\% error band. 


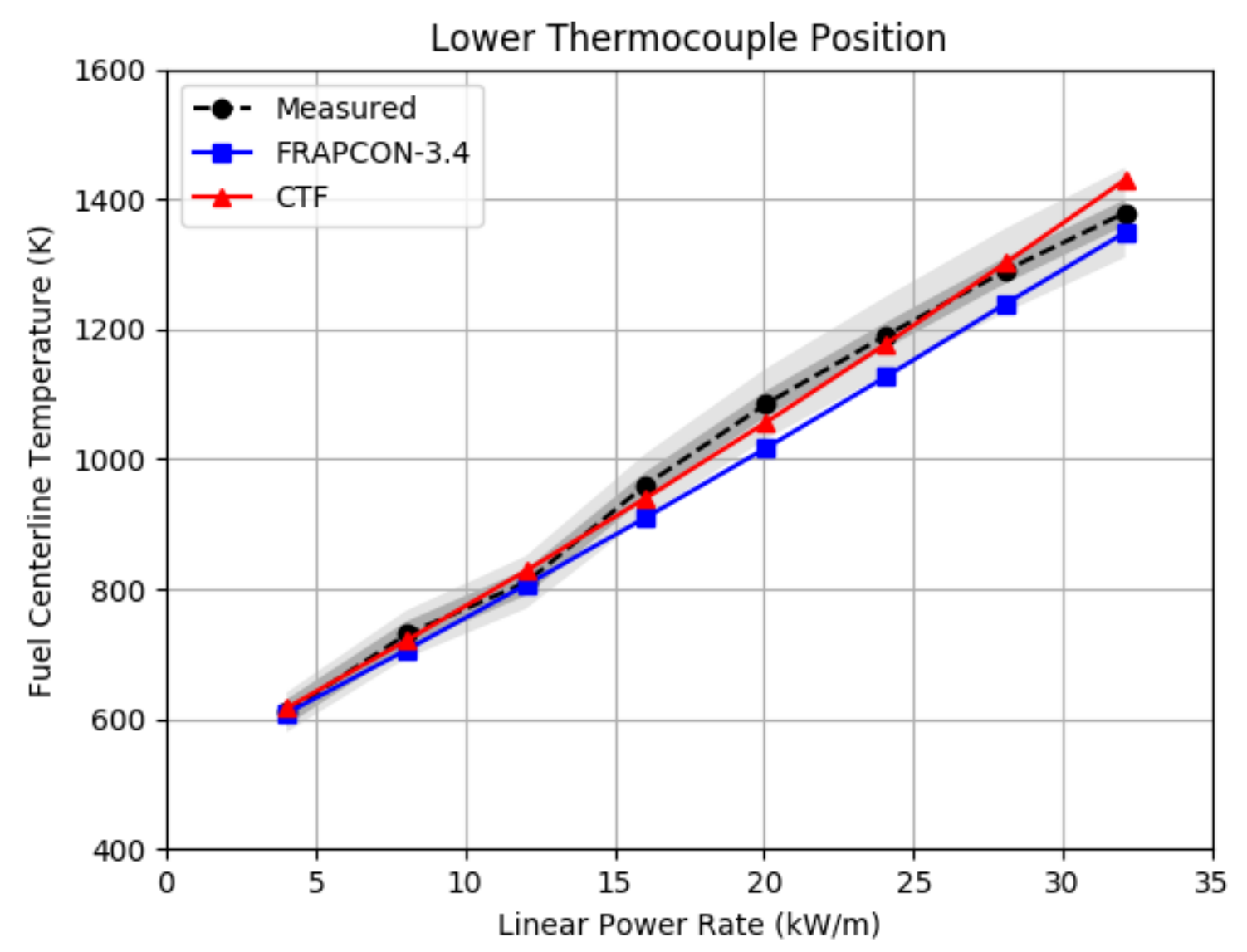

Figure 13.5: Predicted and measured fuel centerline temperature predictions for IFA432 Rod- 1 at BOL at lower thermocouple. The light and dark gray areas in the plot correspond to $\pm 5 \%$ relative error and $\pm 20^{\circ} \mathrm{K}$ around the measured data, respectively. 


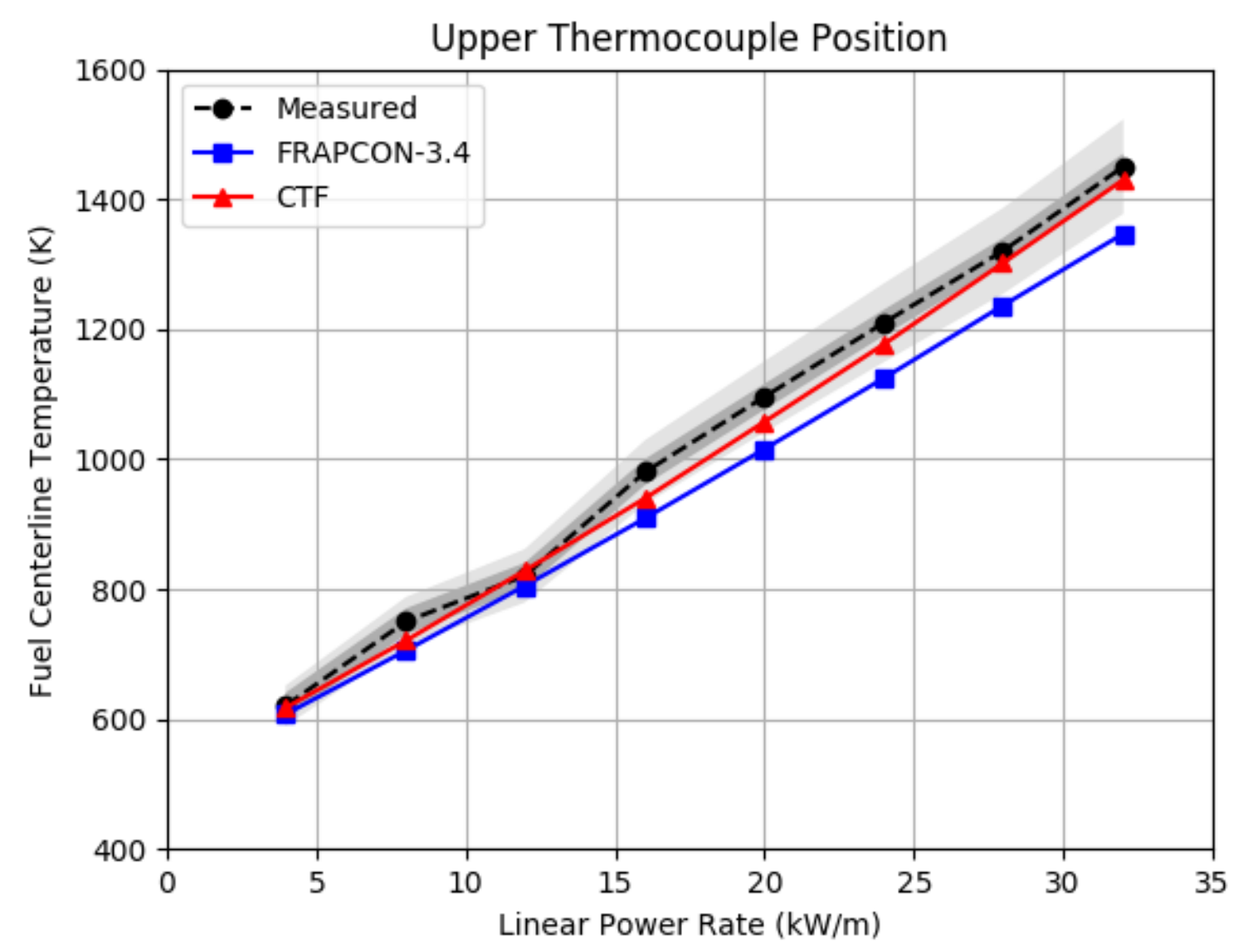

Figure 13.6: Predicted and measured fuel centerline temperature predictions for IFA432 Rod- 1 at BOL at upper thermocouple. The light and dark gray areas in the plot correspond to $\pm 5 \%$ relative error and $\pm 20^{\circ} \mathrm{K}$ around the measured data, respectively. 


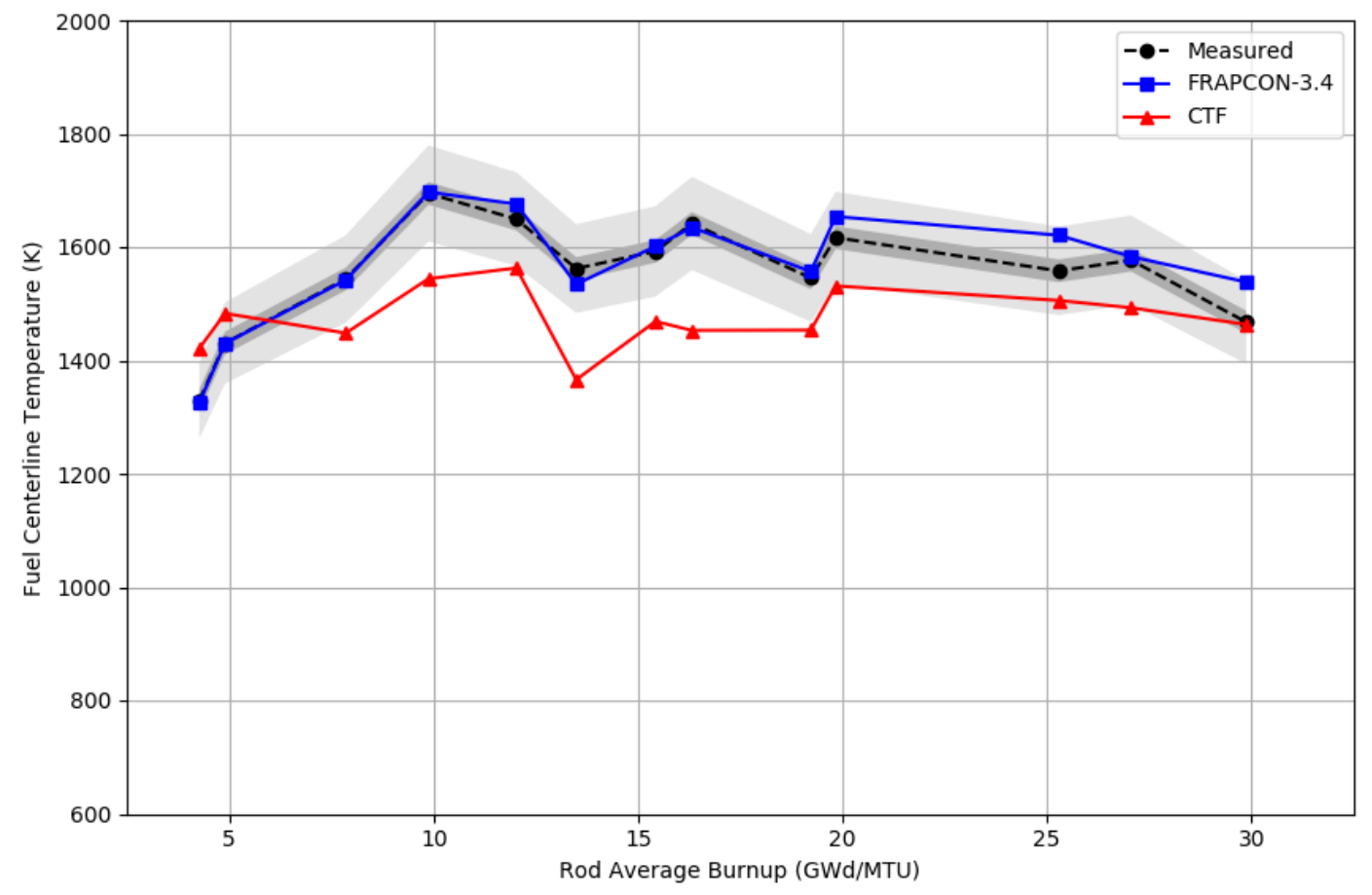

Figure 13.7: Predicted and measured fuel centerline temperature predictions for IFA432 Rod-1 (at lower thermocouple position). The light, dark gray areas in the plot correspond to $\pm 5 \%$ relative error and $\pm 20^{\circ} \mathrm{K}$ around the measured data, respectively. 


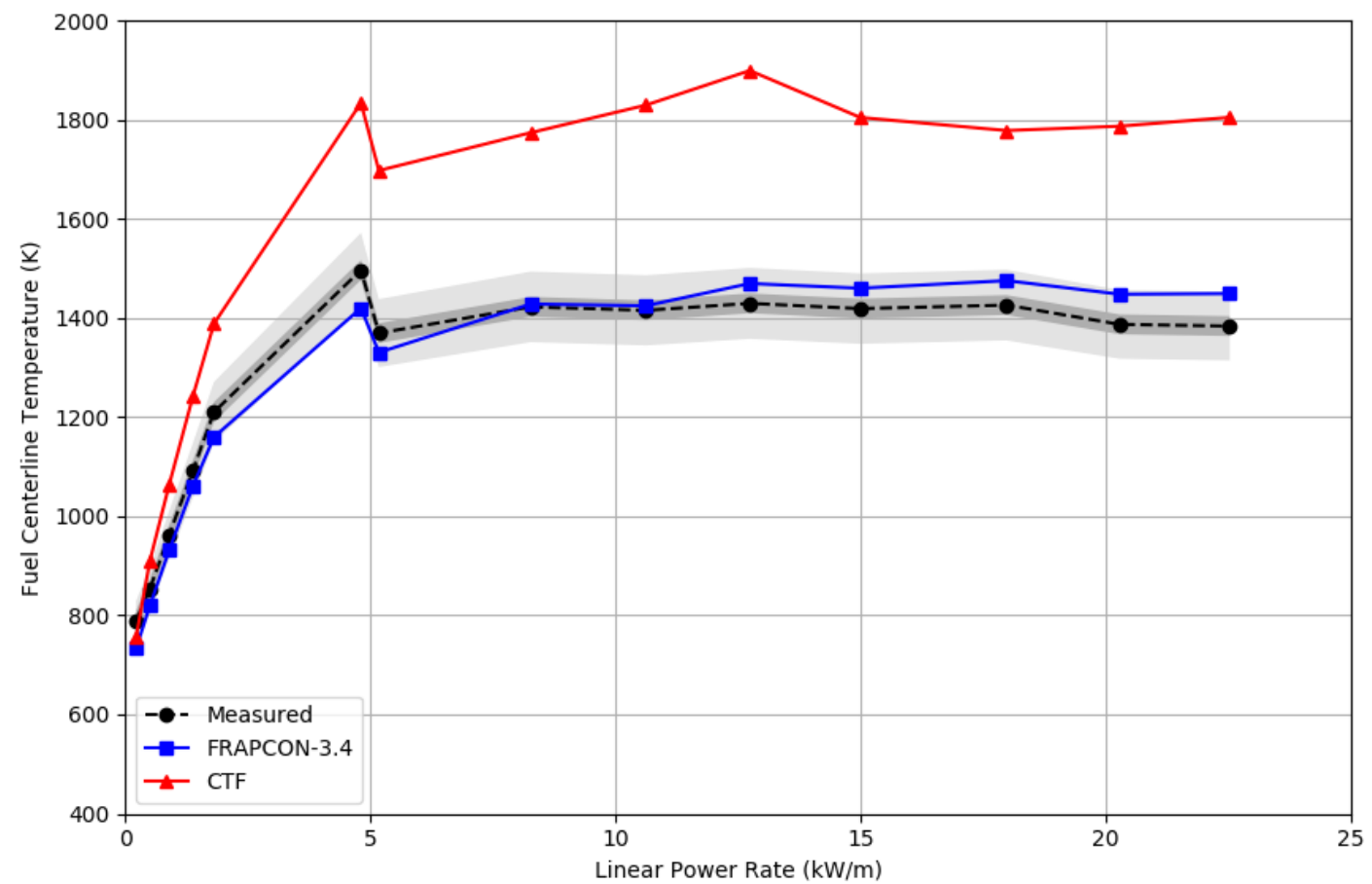

Figure 13.8: Predicted and measured fuel centerline temperature predictions at lower thermocouple position for IFA681 Rod-2 (at lower thermocouple position). The light, dark gray areas in the plot correspond to $\pm 5 \%$ relative error and $\pm 20^{\circ} \mathrm{K}$ around the measured data, respectively. 


\subsubsection{Assessment of Fuel Centerline Temperature Predictions for MOX Fuel}

IFA610 Exposed up to $58 \mathrm{GWD} / \mathrm{MTU}$ The assessment of fuel centerline temperature predictions by CTF for MOX fuel rod is performed using IFA610 Rod-2 from Halden reactor test assemblies [21]. IFA610 Rod-2 is base irradiated for four cycles in the French Gravelines- 4 reactors to burnup level of $55 \mathrm{GWD} / \mathrm{kgM}$ and then it is refabricated and instrumented with a centerline thermocouple to be used for cladding liftoff experiments in Halden reactor $([20],[21])$.

Figure 13.9 shows fuel centerline temperature predicted by CTF, FRAPCON-3.4 and the experimental data as function of measurement time. It is observed in Figure 13.9 that CTF predictions show a good agreement with the experimental data. CTF temperature predictions are around $10 \%$ relative error while the FRAPCON-3.4 gives an excellent agreement with the measured data. The default thermal conductivity model in CTF underestimates the fuel centerline temperatures significantly as compared to measured data. With the newly implemented models, CTF's temperature predictions get better. However, there needs to be improvement in CTF fuel temperature predictions.

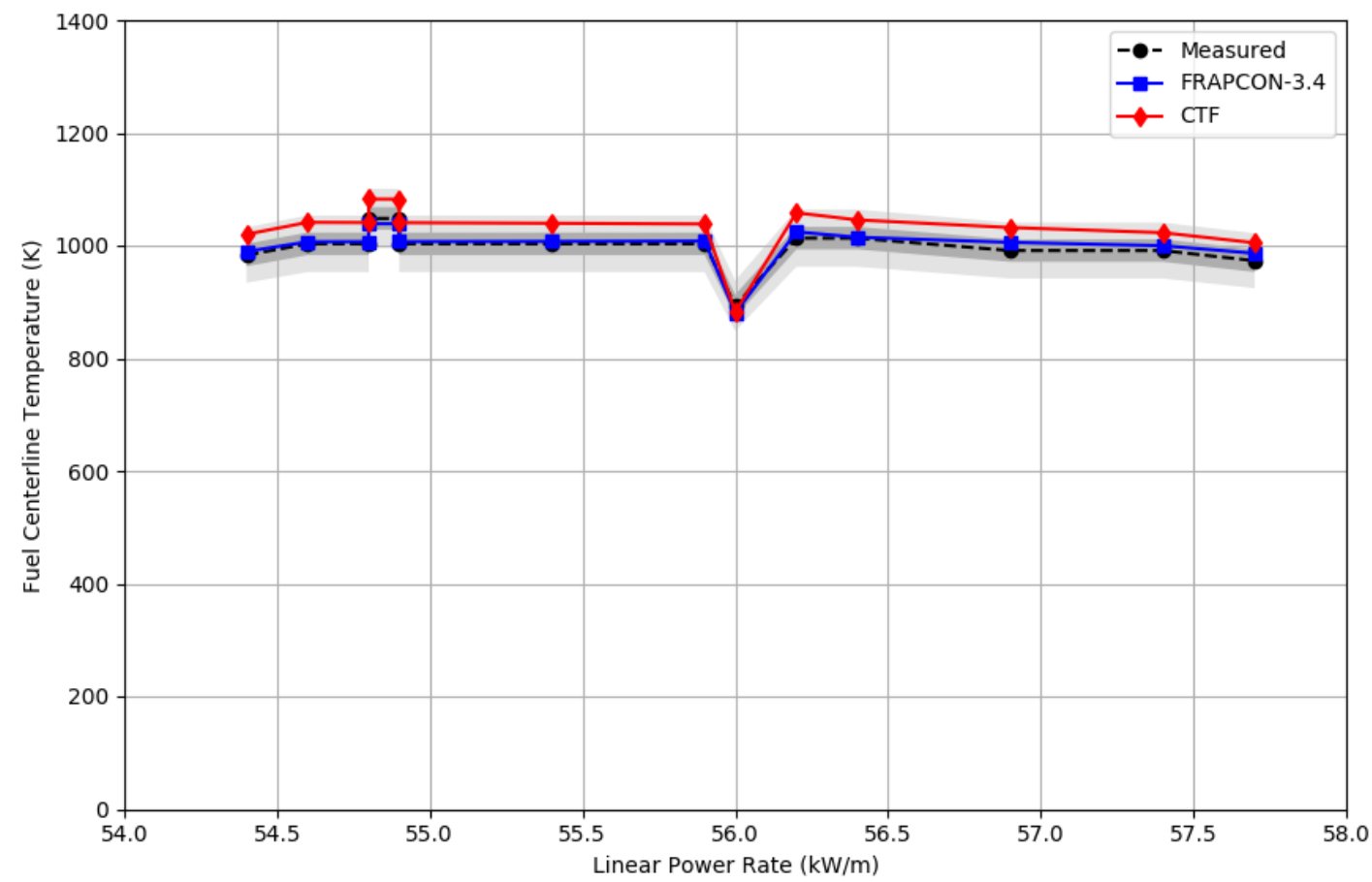

Figure 13.9: Predicted and measured fuel centerline temperature predictions for IFA610 Rod-2 (at lower thermocouple position). The light, dark gray areas in the plot correspond to $\pm 5 \%$ relative error and $\pm 20^{\circ} \mathrm{K}$ around the measured data, respectively.

This study is performed referring to [19]. For the simulations, in-pellet power distributions, rod-average burnup and Gd content values are obtained by FRAPCON-3.4 simulations in [19] for the CTF simulations 
and compared to the measured data [19]. The discrepancies between measured and predicted temperatures might be consequences of input deck preparation etc in [19]. These studies will be repeated by CTF/MPACT coupled analysis to read the in-pellet power distribution, burnup and Gd content, and pass to CTF. By this way, any uncertainties could be minimized, and calculations will be automated. Then, the CTF predictions will be compared to the measured data.

Additionally, CTF's dynamic gap conductance model is being improved to reflect the irradiation effects on the thermal properties in the fuel rod. It is already proved that CTF is capable of predicting the fuel temperatures correctly with a relative error less than $0.03 \%$ (see Section 13.2) once CTF is informed correctly. This is important for the multi-physics coupled code calculations such as thermal-hydraulics and fuel performance coupled code analysis. 


\section{Droplet Entrainment}

\subsection{Validation}

\subsubsection{Ris $\varnothing$}

A comparison of film and droplet flow rates (as a fraction of the total flow rate) between CTF and the experimental data is shown in Figures 14.1 and 14.2, respectively. In general, CTF tends to predict similar film and droplet flow fractions relative to the experimental data for cases with high outlet quality, but tends to over-predict the film flow fraction (under-predict the droplet flow fraction) relative to the experimental data for cases with low outlet quality.

A more in-depth analysis of results, including sensitivity studies and comparison with other subchannel codes, can be found in [38]. 


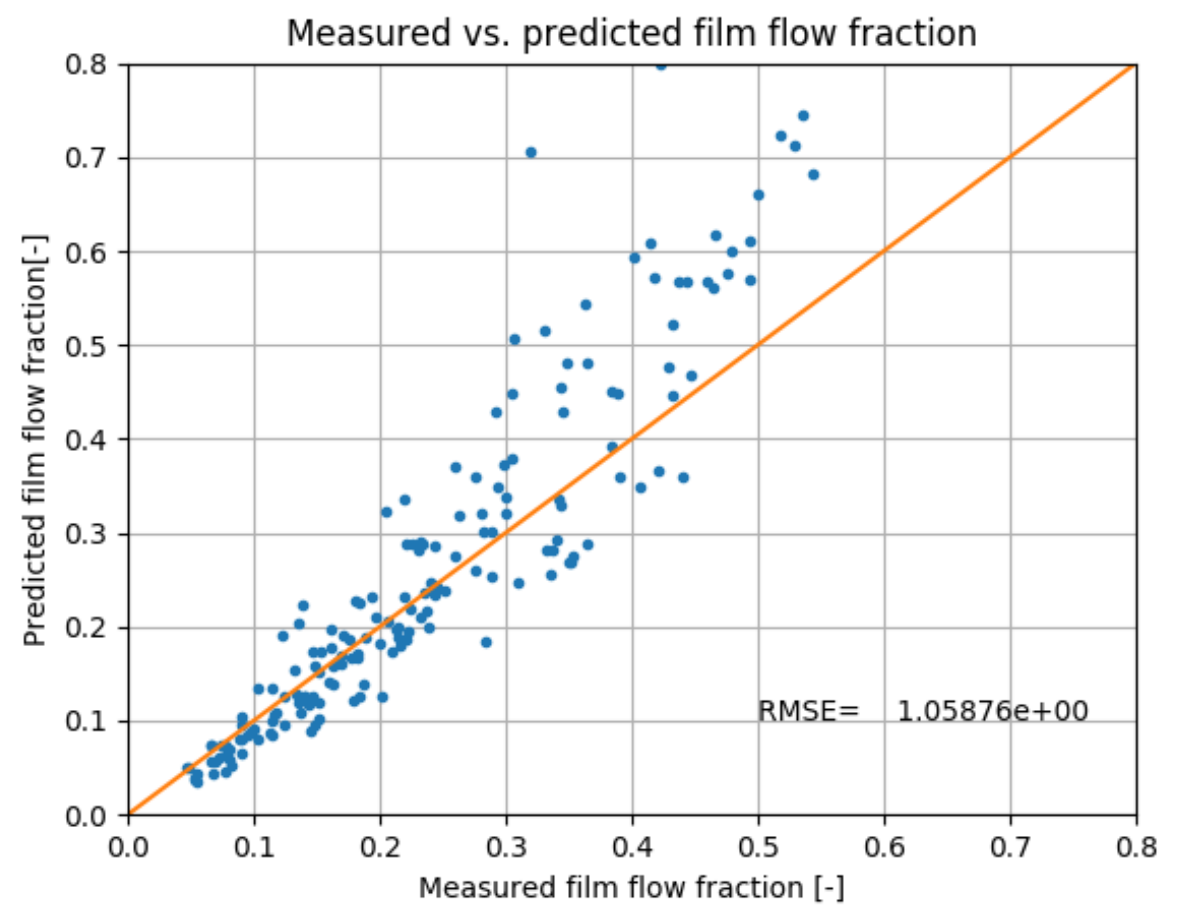

Figure 14.1: Comparison of CTF and experimental results for film flow rate at the outlet as a fraction of total flow. 


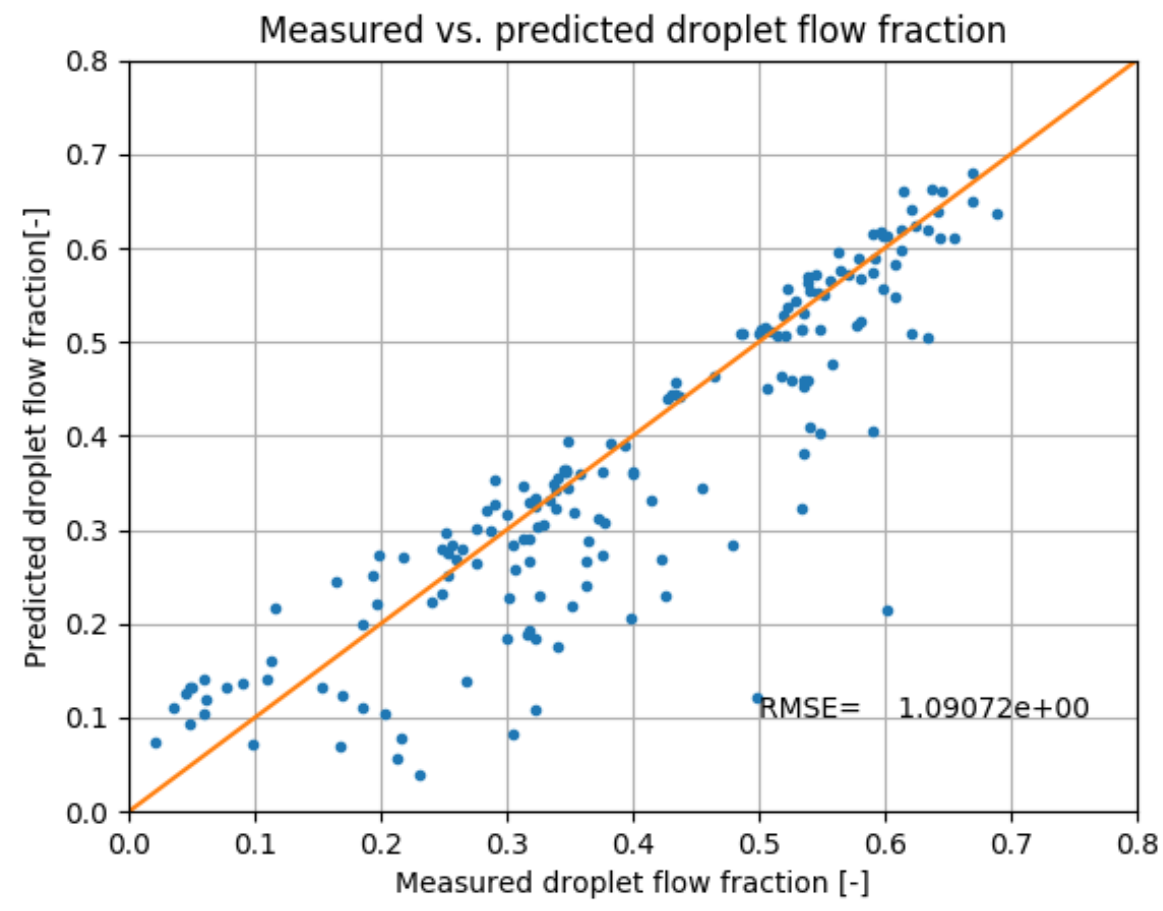

Figure 14.2: Comparison of $\mathrm{CTF}$ and experimental results for droplet flow rate at the outlet as a fraction of total flow. 


\section{Miscellaneous Problems}

\subsection{Water Faucet Problem}

Problem Description The water faucet problem was introduced by Ransom [47] and constitutes a standard case for testing the implementation of the 1-D six-equation two-phase flow model and the robustness of the numerical method used. An analytical solution during the transient and at steady state [47, 48] is available and used to perform convergence studies. In this test, only the gravity force acts on the liquid and vapor phases. Thermodynamic properties of the liquid and vapor phases are obtained from the IAPWS-95 table lookup [49].

The 1-D six-equation two-phase flow model [50] is a single-pressure model that consists of a mass equation, a momentum equation and an energy equation for each phase $k=\{v, l\}$ as shown in Equation 15.1:

$$
\begin{gathered}
\partial_{t} \alpha_{k} \rho_{k}+\partial_{x} \alpha_{k} \rho_{k} u_{k}=0, \\
\partial_{t} \alpha_{k} \rho_{k} u_{k}+\partial_{x}\left(\alpha_{k} \rho_{k} u_{k}^{2}+P\right)=P_{i} \partial_{x} \alpha_{k}, \\
\partial_{t} \alpha_{k} \rho_{k} E_{k}+\partial_{x}\left[\alpha_{k} u_{k}\left(\rho_{k} E_{k}+P\right)\right]=0,
\end{gathered}
$$

where $\rho_{k}$ is the phasic density, $u_{k}$ is the phasic velocity, $\alpha_{k}$ is the phasic volume fraction, and $E_{k}$ is the phasic specific total energy. The pressure $P$ is computed from an equation of state that is a function of the phasic density and the phasic specific internal energy $e_{k}=E_{k}-0.5 u_{k}^{2}$. The temporal and spatial partial derivatives are denoted by $\partial_{t}$ and $\partial_{x}$, respectively. The IPC term is located in the righthand-side of the phasic momentum equation, Equation $15.1 \mathrm{~b}$, and is a function of an interfacial pressure $P_{i}$ and the 1-D gradient of volume fraction. The interfacial pressure could represent the effects of hydrostatics or surface tension when considering a liquid-vapor mixture, making the system behave like a two-pressure model. Its definition, though, is purely motivated from mathematical considerations and relies on the study of the eigenvalues in the incompressible limit (detailed derivations can be found in [51, 52]). Following previous works $[51,52,53]$, the following definition is used for the interfacial pressure:

$$
P_{i}=\delta \frac{\alpha_{v a p} \alpha_{l i q} \rho_{v a p} \rho_{l i q}}{\rho_{v a p} \alpha_{l i q}+\rho_{l i q} \alpha_{v a p}}\left(u_{v a p}-u_{l i q}\right)^{2} .
$$

The coefficient $\delta$ is defined as positive, and any value greater than 1 ensures real eigenvalues and thus a hyperbolic model. It is common to include a coefficient $\delta$ in the definition of the interfacial pressure to investigate the effect of the IPC term on the numerical solution. Note that the definition of the interfacial pressure proposed in Equation 15.2 was derived by considering the incompressible limit of the six-equation two-phase flow model. 


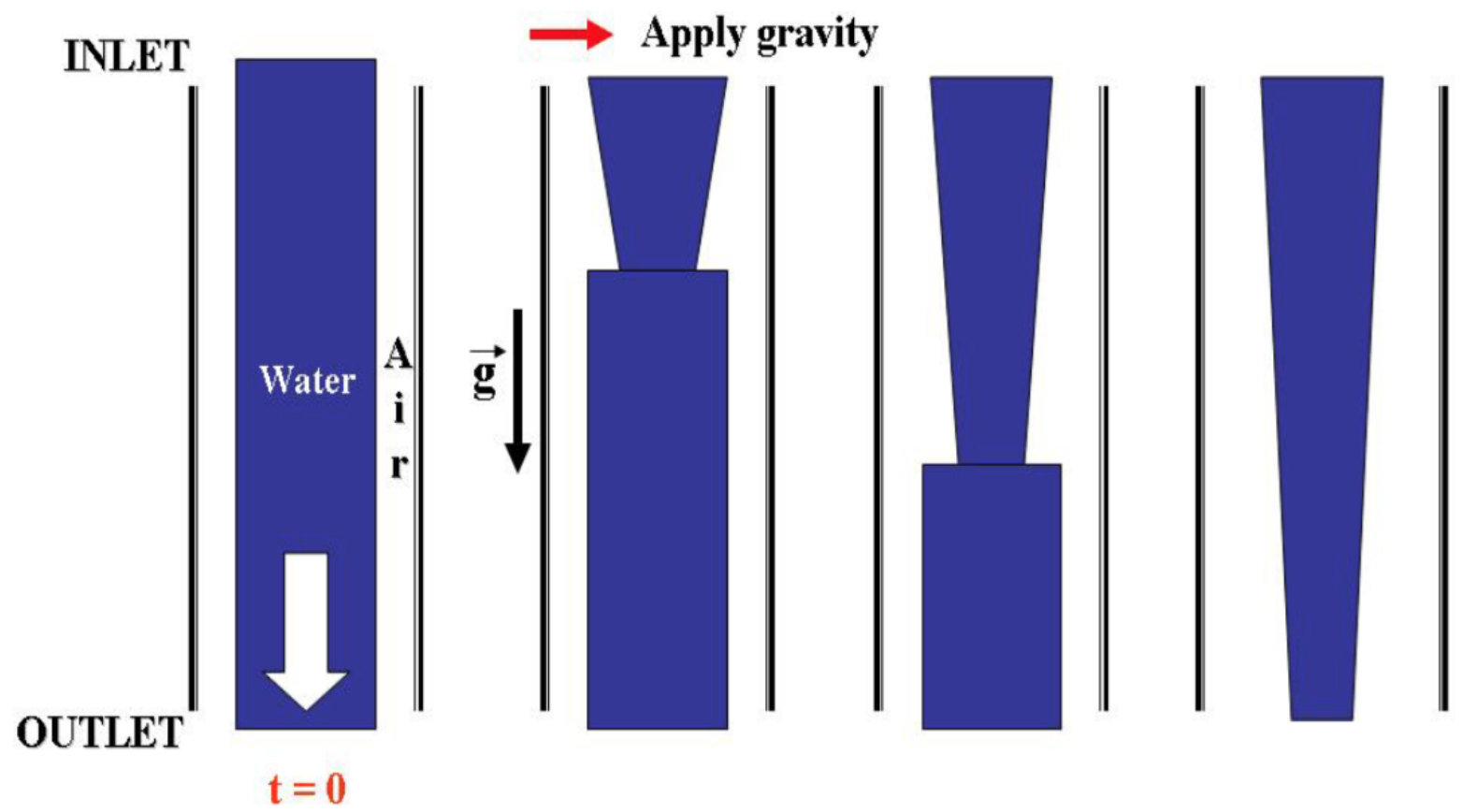

Figure 15.1: Illustration of the Water Faucet Problem.

The computational domain consists of a $1-\mathrm{D}$ vertical pipe of length $\mathrm{L}=12 \mathrm{~m}$. Initially, the liquid and vapor states are uniform, with values specified in Table 54. A pressure boundary condition is set equal to

Table 54: CTF Initial and Boundary Conditions for the Water Faucet Problem.

\begin{tabular}{llll}
\hline Initial & Value & $\begin{array}{l}\text { Boundary } \\
\text { Value }\end{array}$ & Value \\
$\alpha_{\text {vap }, 0}$ & 0.2 & $\alpha_{\text {vap }, \text { in }}$ & 0.2 \\
$u_{\text {va }, 0}$ & $0.0(\mathrm{~m} / \mathrm{s})$ & $u_{\text {vap }, \text { in }}$ & $0.0(\mathrm{~m} / \mathrm{s})$ \\
$u_{\text {liq }, 0}$ & $10.0(\mathrm{~m} / \mathrm{s})$ & $u_{\text {liq, in }}$ & $10.0(\mathrm{~m} / \mathrm{s})$ \\
$P_{0}$ & $10(\mathrm{bar})$ & $P_{\text {out }}$ & $10(\mathrm{bar})$ \\
\hline
\end{tabular}

the initial pressure, $P_{\text {out }}=P_{0}$, in the bottom of the pipe. At the inlet (top of the pipe), the vapor volume fraction $\alpha_{g, i n}$, and, the liquid and vapor velocities, $u_{g, i n}$ and $u_{l, i n}$ are specified and taken equal to the initial conditions. At time $=0 \mathrm{~s}$, the gravity is turned on $\left(\mathrm{g}=9.81 \mathrm{~m} / \mathrm{s}^{2}\right)$, and the liquid column starts thinning as the discontinuity moves towards and exits the pipe, as illustrated in Figure 15.1. After the discontinuity leaves the bottom of the pipe, the solution reaches a steady state solution.

The numerical results of the water faucet problem run with the initial and boundary conditions provided in Table 54 are showed. Profiles of the vapor volume fraction are presented during the transient and at steady state and are compared against the numerical solution recently proposed in Sections 2 and 3 of [48] by performing a convergence study when refining the mesh and keeping the Courant-Friedrichs-Lewy (CFL) number constant. CTF implements a first-order upwind scheme as a numerical method and thus can only achieve first-order accuracy at most (Note that the second order Lax-Wendroff method was previously 


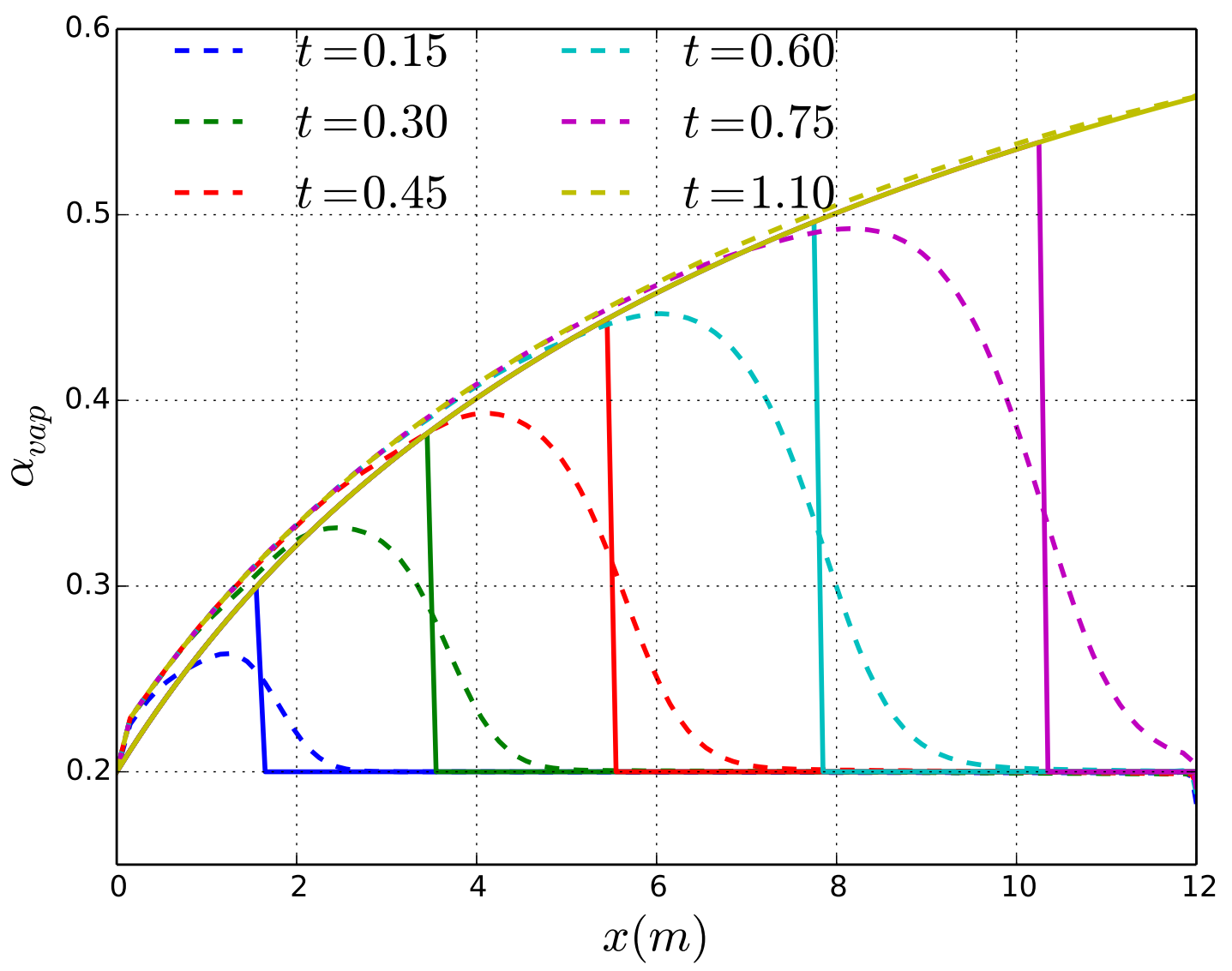

Figure 15.2: Exact and numerical solution of the vapor volume fraction during the transient and at steady state for $P_{\text {out }}=10$ bar (run with 120 cells and a CFL of 0.8 ).

implemented into CTF, but was not yet available for this study [54]). All numerical solutions presented in this section are obtained with $\delta=1.0$ in the definition of the IPC term given in Equation 15.2, unless otherwise stated. The closure model multiplier capabilities of CTF to decouple the phases (i.e., disable interfacial drag and mass transfer) was used to turn off all source terms (e.g., wall friction) except for the gravity force. Also, since CTF solves for the phasic energy equations, the phasic enthalpy variables are initialized by assuming saturation conditions computed from the outlet pressure.

Discussion of Results The numerical solution of the vapor volume fraction is plotted in Figure 15.2 at different times during the transient and at steady state, along with the exact solution. The analytical solution at different times is shown with the solid line, while the CTF solution is shown with dashed lines. Different colors denote different points in time. The location of the discontinuity is in good agreement with the analytical solution. The diffusion in the CTF solution is due to the coarseness of the grid and the numerical diffusion of the first-order upwind scheme. 


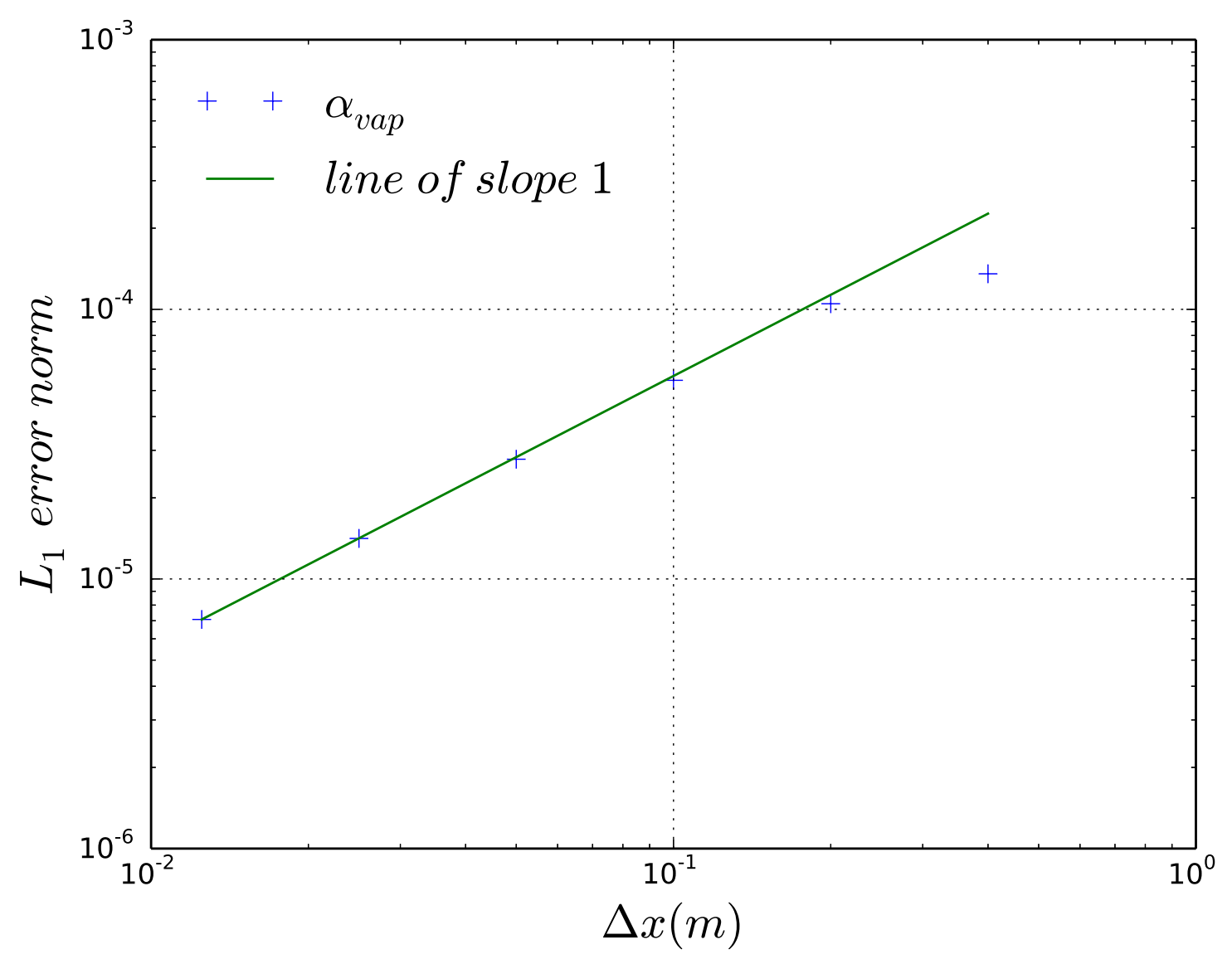

Figure 15.3: Convergence plot of the vapor volume fraction in the $L_{1}$ error norm.

A convergence study during the transient and at steady state is also performed by successively refining the mesh and keeping the CFL constant for the vapor volume fraction, the vapor velocity, and the liquid velocity. The L1 error norm between the numerical and analytical solution is computed and plotted as a function of the mesh size, along with a reference line of slope one. Plots of the convergence study for the vapor volume fraction are given in Figure 15.3 for a transient solution at time $=0.5 \mathrm{~s}$. The convergence study shows that first-order accuracy is achieved.

Effect of the mesh refinement on the numerical profile of the vapor volume fraction at $\mathrm{t}=0.4 \mathrm{~s}$ is presented in Figure 15.4. The vapor volume fraction displays a discontinuity at $\mathrm{t}=0.4 \mathrm{~s}$ that is better resolved when the mesh is refined. The numerical solution does not show any undershoot or overshoot in the vicinity of the discontinuity, even for fine meshes.

The effect of the IPC term on the vapor volume fraction profile is illustrated in Figure 15.5: the CTF sub-channel code was run with $(\delta=1)$ and without $(\delta=0)$ the IPC term until $\mathrm{t}=0.4 \mathrm{~s}$. Both runs used the same spatial and temporal discretizations. In the case of $\delta=0$ (i.e. the IPC term is turned off), the 


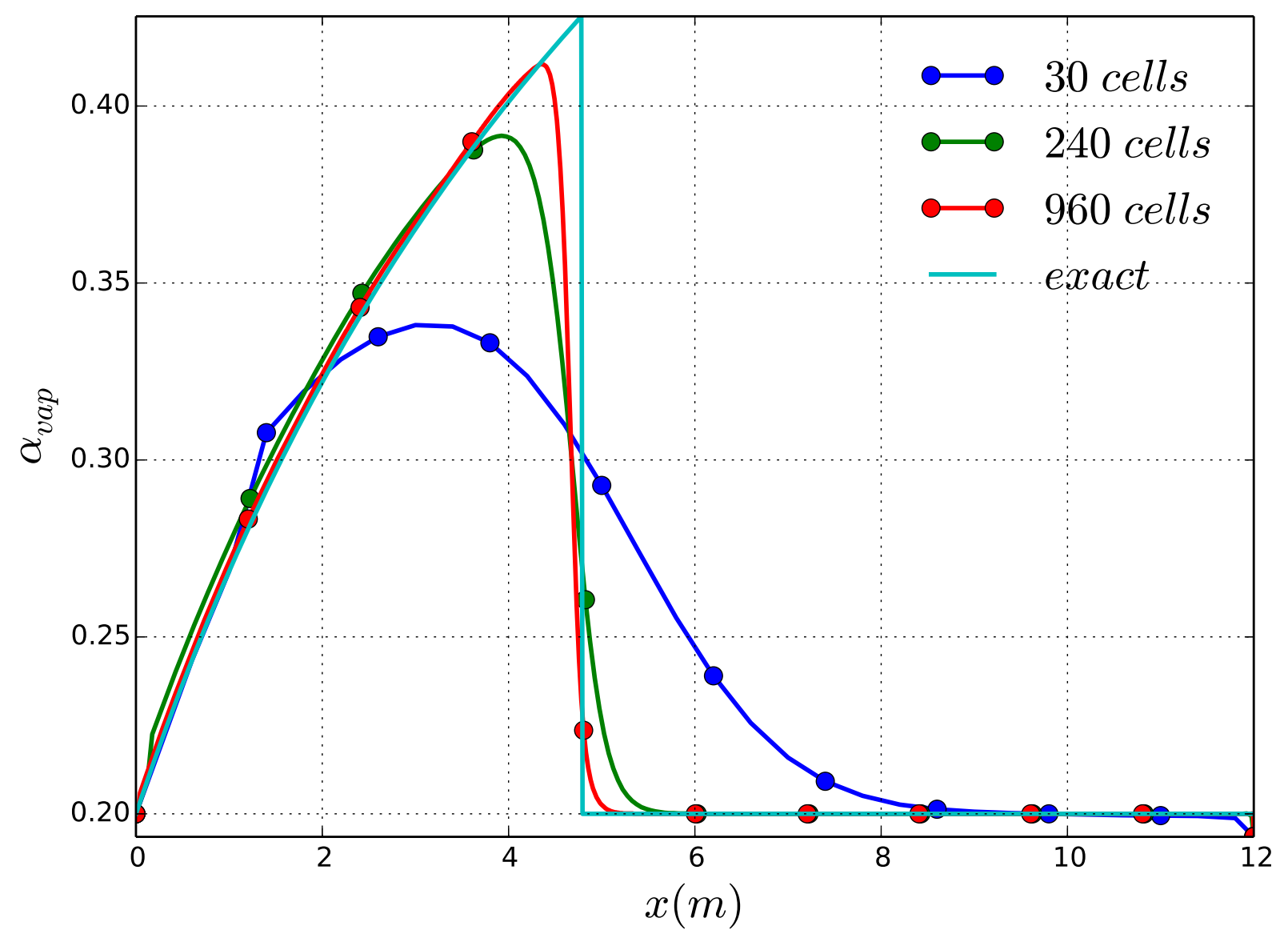

Figure 15.4: Vapor volume fraction profile for three mesh densities $(30,240$, and 960 cells $)$ at $\mathrm{t}=0.4 \mathrm{~s}$. 


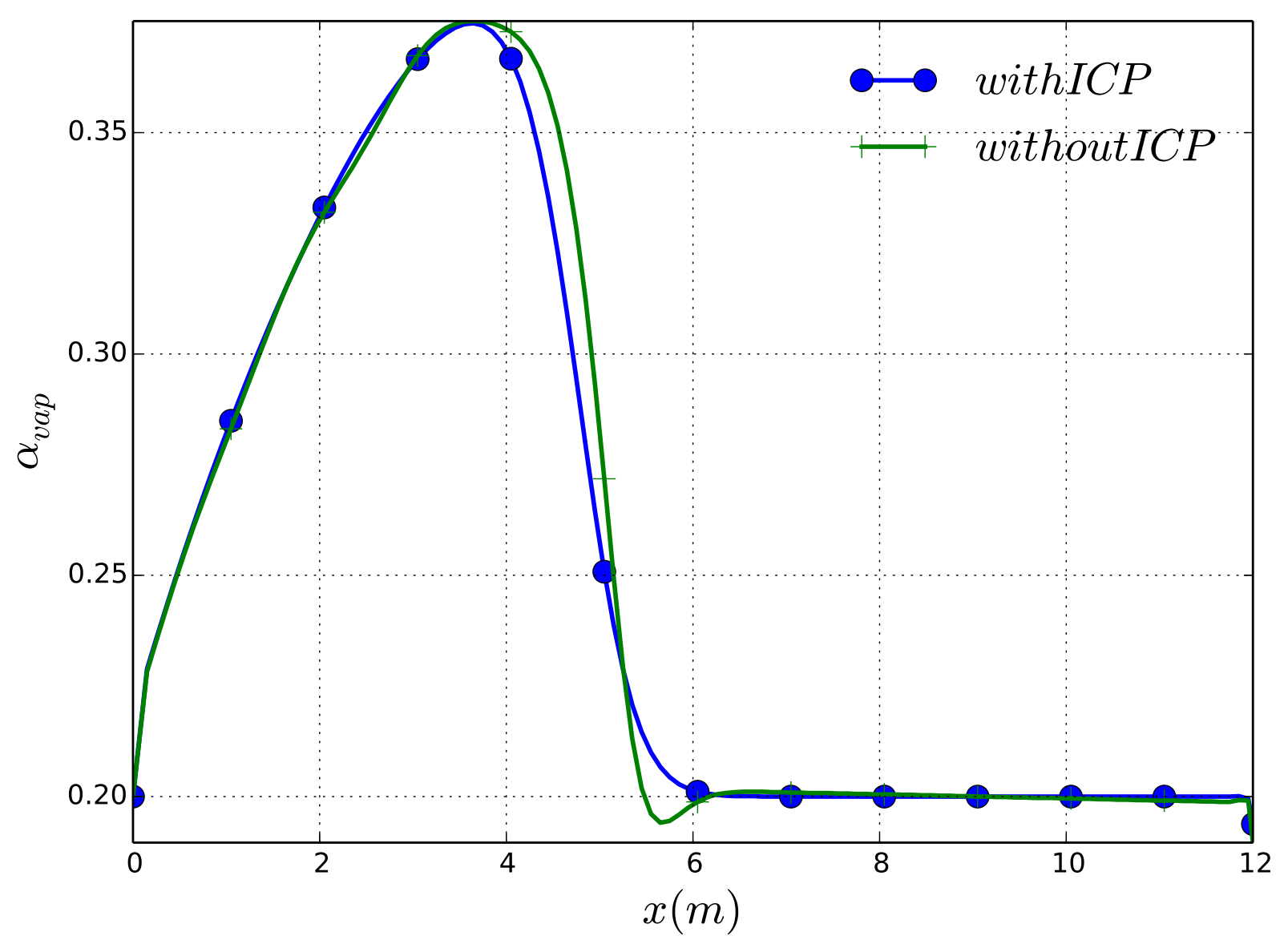

Figure 15.5: Illustration of the effect of the ICP term on the numerical solution. Vapor volume fraction profile at $\mathrm{t}=0.4 \mathrm{~s}$.

vapor volume fraction displays an undershoot in the discontinuity region around $\mathrm{x}=6 \mathrm{~m}$. This undershoot is characteristic of complex eigenvalues and the numerical instabilities they cause. CTF was able to converge because of the over-dissipative nature of the first-order upwind scheme. It was observed, however, that the code was not able to converge when run with the higher outlet pressure of $\mathrm{P}=75$ bar and with $\delta=0$.

The influence of the IPC term on the steady-state pressure profile is investigated by varying the $\delta$ coefficient in the range [0;1], and the analytical solution is used as a reference. Lou et al. [48] (see Eq. 37 in Section 3) recently proposed a new steady-state analytical solution of the pressure for the water faucet test case. The pressure is no longer constant as it was previously assumed in first approximation [47]. Results presented herein were obtained with the same initial and boundary conditions of Table 54. This particular pressure was chosen since CTF was able to converge even when setting $\delta$ to zero when the IPC term is turned off. In Figure 15.6, the steady-state pressure profile is plotted for values of $\delta$ ranging from 0 to 1 , along with the steady-state exact solution proposed by Ling et al. [48]. The steady-state exact solution of the pressure linearly increases with the axial distance. The numerical solution of the pressure predicted by CTF 


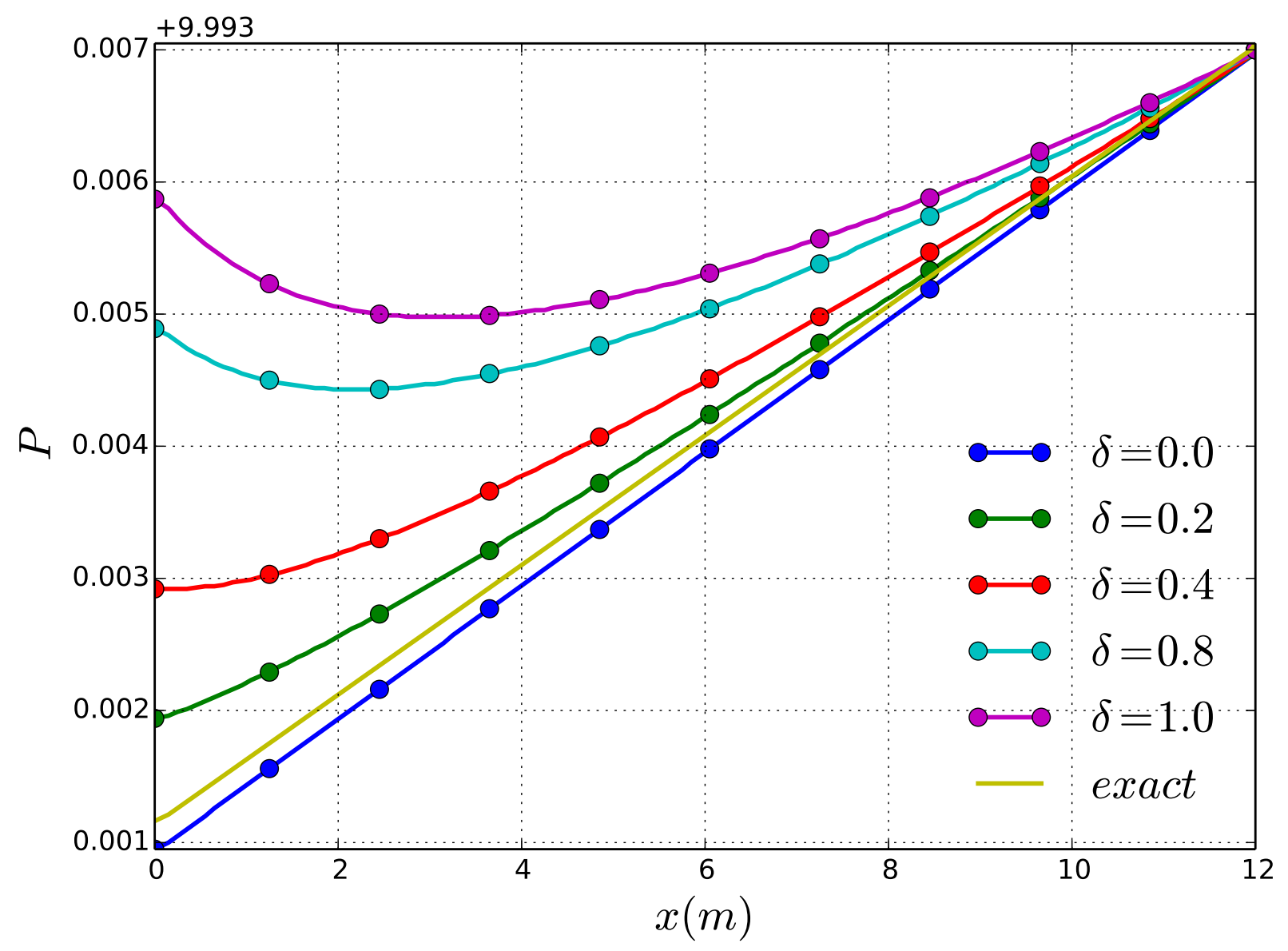

Figure 15.6: Effect of the parameter $\delta$ on the steady-state pressure profile. 
is strongly influenced by the value of $\delta$ and thus by the IPC term. It is observed that for values of $\delta$ close to zero, the numerical and exact pressure profiles are in good agreement. This effect is somehow expected as the momentum equation is modified by a IPC term proportional to the gradient of the vapor volume fraction. This effect was overlooked in the past, as the pressure was assumed constant to derive the exact solution in first approximation.

The IPC term was successfully implemented in the CTF subchannel code and tested using the water faucet problem. A convergence study was performed, and it demonstrated good agreement between the numerical solution and the exact solution during the transient and at steady state. The effect of the pressure correction term on the steady-state pressure profile was also investigated. It was shown that the numerical pressure profile only matches the analytical solution at steady state when the pressure correction term is turned off. Such effect is expected as the pressure correction term involves a gradient term that does not vanish at steady state.

This new capability is now available in CTF through a VUQ parameter. Note that the IPC term is turned off by default but can be activated by setting $k_{p c}$ to 1 in a VUQ file. 


\section{Governing Equation Verification}

\subsection{Mass and Energy Equation Advection}

This section describes a series of code order verification problems that were designed to test the advective terms in the mass and energy equations. The full work is outlined in a journal paper [55], a brief summary will be provided here. This problem is a "true" code verification test, in that it is designed to be sufficiently simple that the convergence rate of the code can be assessed.

\subsubsection{Problem Description}

This test problem is set such that:

1. There is an approximately constant velocity and pressure throughout the domain,

2. All source terms are set to zero using the exposed VUQ multipliers (including gravity), and

3. The problem is sufficiently below the saturation temperature so that it remains in single phase.

Under these conditions, the governing equations of CTF simplify significantly. The momentum equation is eliminated altogether, and the mass and energy equations simplify to a temporal and advective component.

$$
\begin{gathered}
\frac{\partial \rho}{\partial t}+u \frac{\partial \rho}{\partial x}=0 \\
\frac{\partial \rho h}{\partial t}+u \frac{\partial \rho h}{\partial x}=0
\end{gathered}
$$

With this set of governing equations, it can be shown that the formal order of accuracy for both enthalpy and density is first order [55]. The analytical solution to this problem is simply the advection of the inlet condition with the velocity $u$. Three different inlet conditions are chosen to test various behavior of the solution. The first, a square wave solution, has a large discontinuity which degrades the observed order of accuracy. The second is a cosine wave which is set continuous with the initial conditions. This has a continuous first and second derivative and is therefore first order. The final solution is a hyperbolic tangent, 
which limits to the square wave as the width approaches zero, and is therefore very smooth but similar to the square wave problem. The respective analytical solution for each of these inlet conditions is as follows:

$$
\begin{gathered}
h_{s q}= \begin{cases}h_{o}, & u t \leq x \\
h_{i n}, & u t>x\end{cases} \\
h_{\text {tanh }}=\left\{\begin{array}{l}
h_{o}, \\
\frac{1}{2}\left[\left(h_{o}+h_{\text {in }}\right)-\left(h_{o}-h_{\text {in }}\right) \tanh \left(\frac{u(t-\tau)-x}{l}\right)\right], u t>x
\end{array}\right. \\
h_{\text {cos }}=\left\{\begin{array}{l}
h_{o}, \\
\frac{1}{2}\left[\left(h_{o}+h_{\text {in }}\right)+\left(h_{o}-h_{\text {in }}\right) \cos \left(\frac{2 \pi}{p}\left(t-\frac{x}{u}\right)\right)\right], u t>x
\end{array}\right.
\end{gathered}
$$

Where all parameters of interest are defined in Table 55. The square wave and cosine wave are run for five seconds, and the hyperbolic tangent is run for ten seconds.

Table 55: Problem parameters for isokinetic advection

\begin{tabular}{lccc}
\hline Parameter & Symbol & Value & Units \\
\hline Channel Length & $L$ & 0.5 & $\mathrm{~m}$ \\
Flow Area & $A$ & 0.0001 & $\mathrm{~m}^{2}$ \\
Wetted Perimeter & $P_{w}$ & 0.040 & $\mathrm{~m}$ \\
Pressure & $P$ & 1.00 & $\mathrm{bar}$ \\
Initial Temperature & $T_{o}$ & 40 & ${ }^{\circ} \mathrm{C}$ \\
Initial Enthalpy & $h_{o}$ & 167.6 & $\mathrm{~kJ} / \mathrm{kg}$ \\
Initial Density & $\rho_{o}$ & 992.22 & $\mathrm{~kg} / \mathrm{m}^{3}$ \\
Initial Flow Rate & $\dot{m}_{o}$ & 0.005 & $\mathrm{~kg} / \mathrm{s}$ \\
Velocity & $u$ & 0.05039 & $\mathrm{~m} / \mathrm{s}$ \\
"Inlet" Temperature & $T_{i n}$ & 38 & ${ }^{\circ} \mathrm{C}$ \\
"Inlet" Enthalpy & $h_{i n}$ & 159.22 & $\mathrm{~kJ} / \mathrm{kg}$ \\
"Inlet" Density & $\rho_{i n}$ & 992.90 & $\mathrm{~kg} / \mathrm{m}^{3}$ \\
"Inlet" Flow Rate & $\dot{m}_{i n}$ & 0.05004 & $\mathrm{~kg} / \mathrm{s}$ \\
Hyperbolic Tangent Width & $l$ & 0.05 & $\mathrm{~m}$ \\
Hyperbolic Tangent Offset & $\tau$ & 5.0 & $\mathrm{~s}$ \\
Cosine Wave Period & $p$ & $L / u$ & $\mathrm{~s}$ \\
\hline
\end{tabular}

\subsubsection{Results}

Under the conditions described in the previous section, CTF follows the expected behavior with diffusive error for all types of convergence studies (constant $\Delta t$, constant $\Delta x$, and constant CFL) [55].

The constant CFL problems are chosen as regression tests because they simultaneously test both temporal and spatial error, and they do no require significant computational resources. The spatial and temporal spacings are refined at a constant rate, while all other code parameters are held constant. $\mathrm{An}_{\mathrm{L}} \mathrm{L}_{2}$ norm is used to compute the difference between the calculated and analytic solutions. 


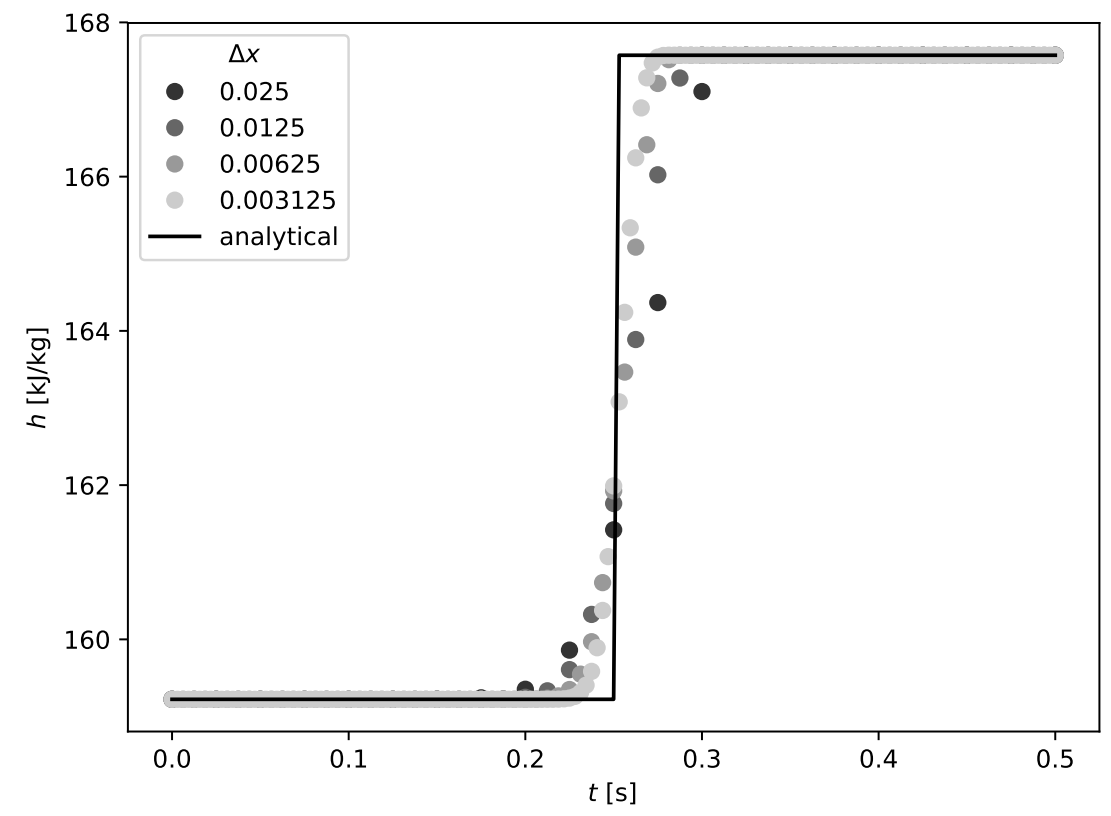

Figure 16.1: Square wave solutions

The code solutions for the square, cosine, and hyperbolic tangent waves are shown respectively in Figures 16.1, 16.2, and 16.3. The convergence is shown respectively in Figures 16.4, 16.5, and 16.6. Note that the discontinuity in the square wave degrades the order of accuracy [56], so the regression test only ensures that the code solution is convergent to the analytic. The other two wave types display first-order convergence. All three problems are automated and included as regression tests. 


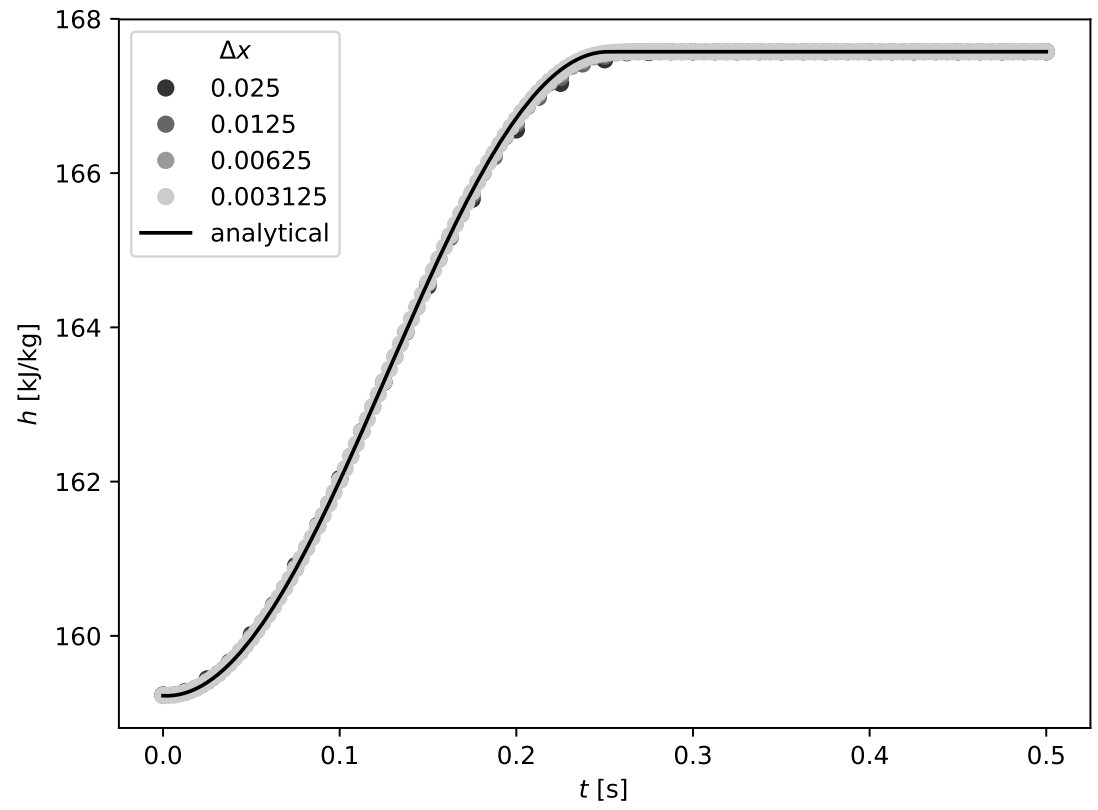

Figure 16.2: Cosine wave solutions

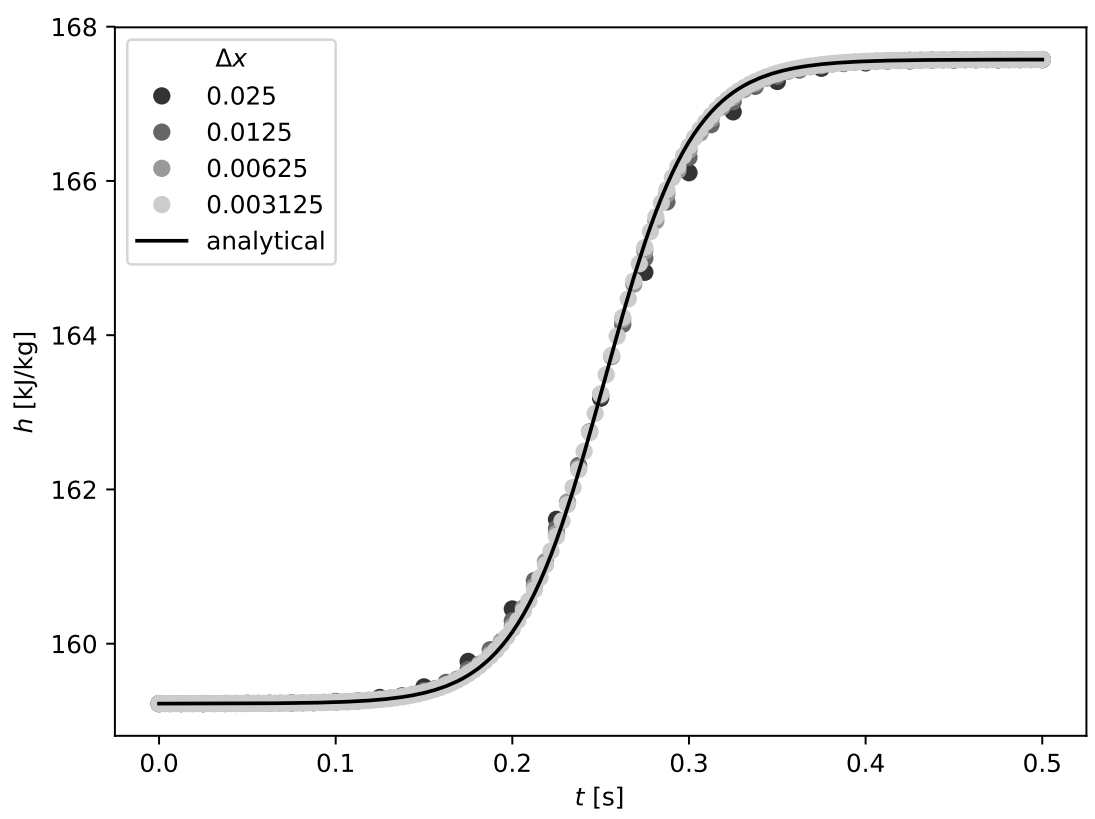

Figure 16.3: Hyperbolic tangent wave solutions 


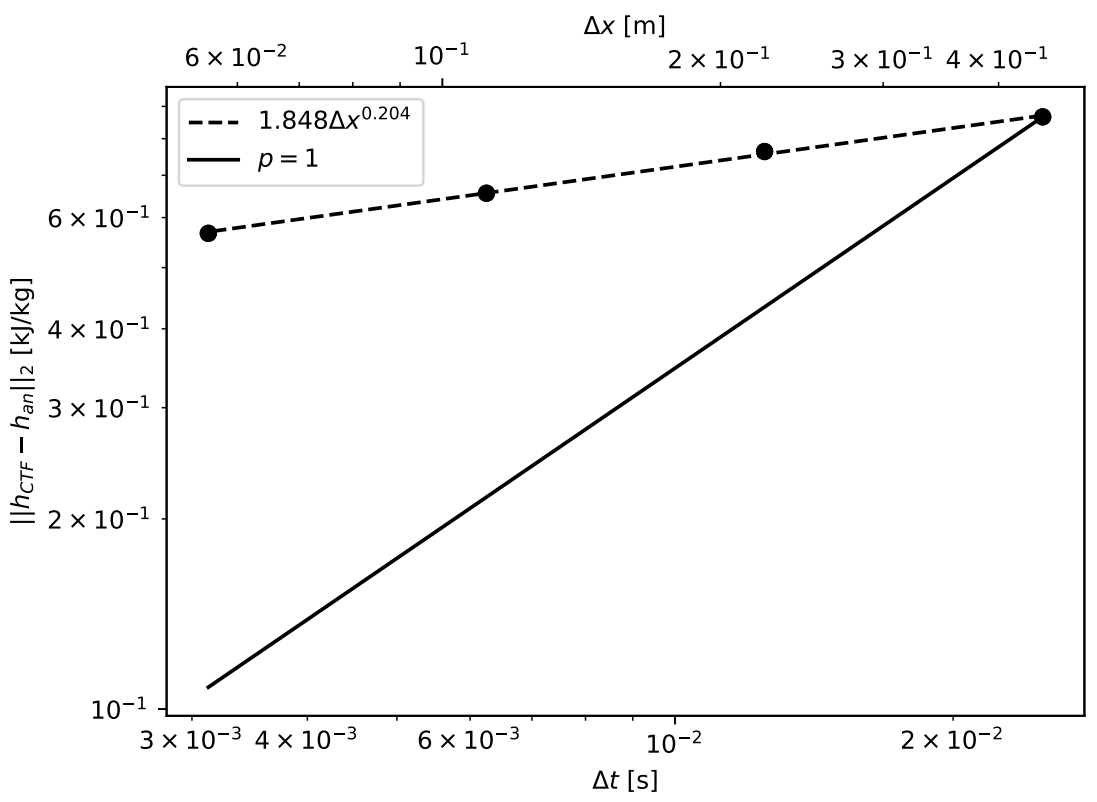

Figure 16.4: Square wave constant CFL convergence

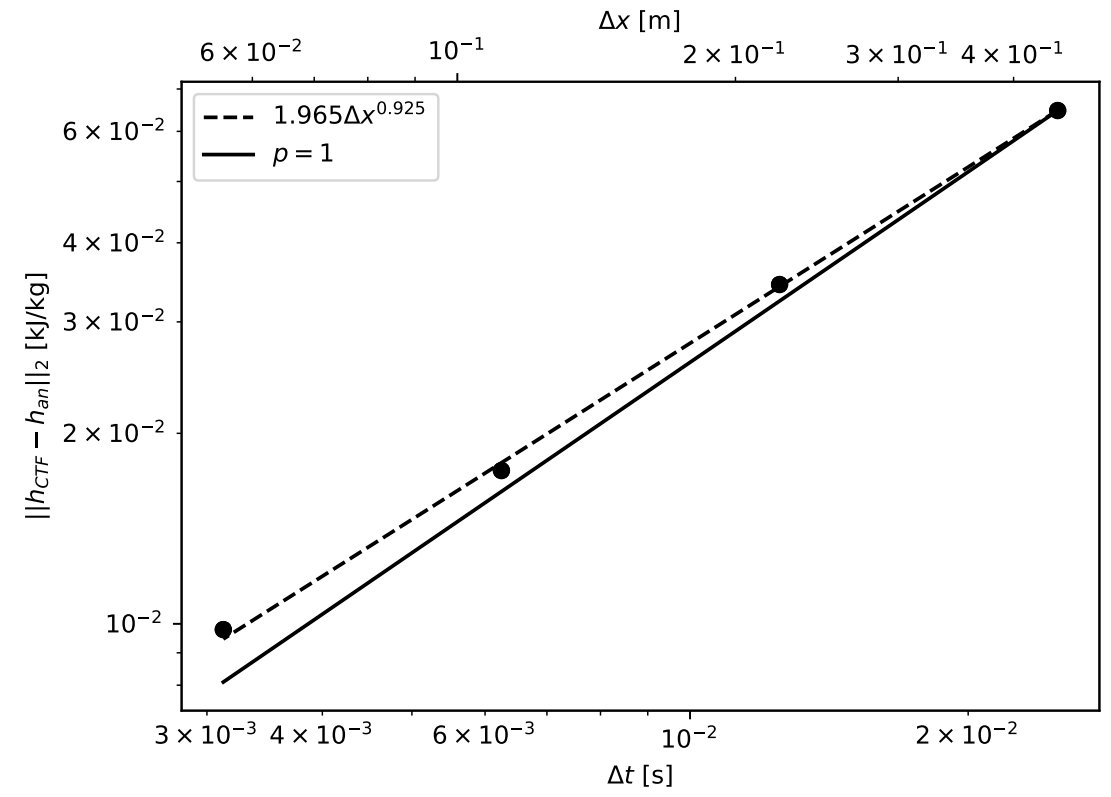

Figure 16.5: Cosine wave constant CFL convergence 


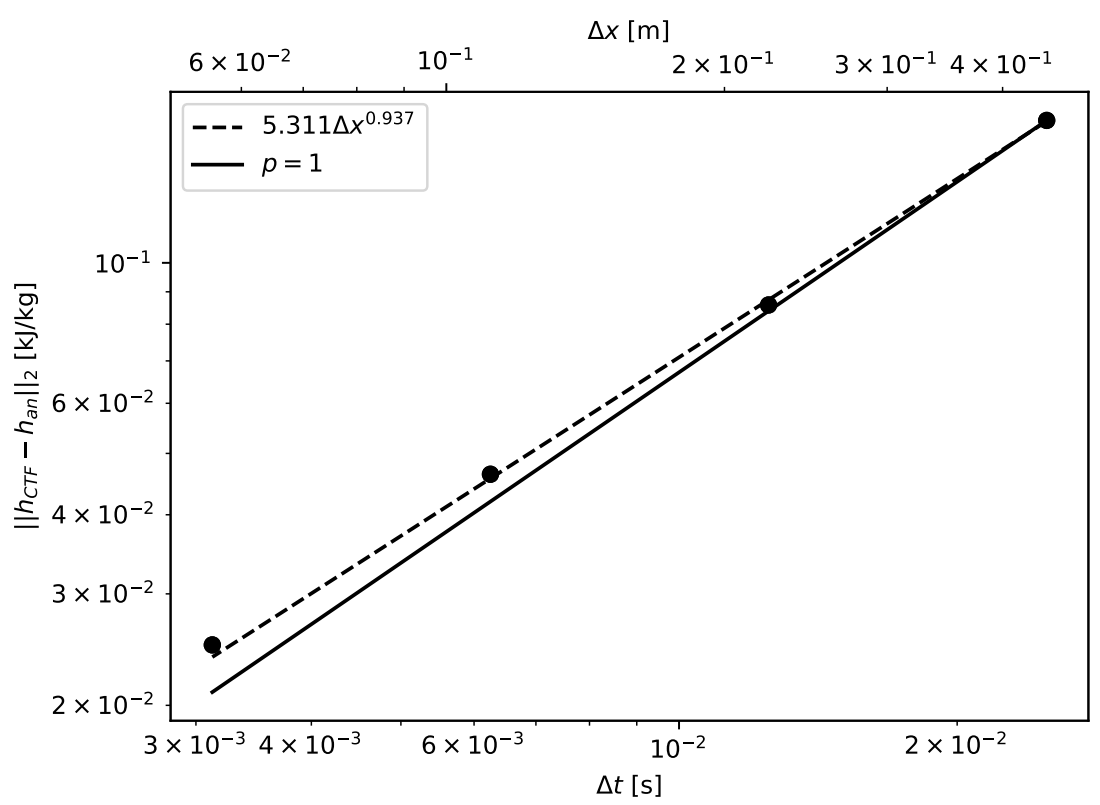

Figure 16.6: Hyperbolic tangent wave constant CFL convergence 


\section{Conclusion}

This document has been created as a means to validate CTF. It outlines the code requirements (capabilities) and then demonstrates the code's ability to to meet a significant portion of those capabilities. A variety of tests have been chosen to target the most important features of CTF, including pressure drop, void, rod and coolant temperature, and mixing prediction in rod bundle geometries. Qualitative and quantitative analysis was performed to assess CTFs ability to capture the trends and behavior of phenomena as well as to present a general measure of its accuracy.

There are some noted shortfalls in the CTF testing and documentation matrix. First, the validation studies performed and included in this document are not an exhaustive measure of the CTF capabilities; there are still more CTF features, noted in Chapter 2, that have yet to be validated. Second, the experimental data employed to validate CTF does not specifically single out individual physical models in CTF; rather, they exercise several models in combination.

This document does take great strides in improving CTF testing and validation. The tests modeled in this document act to exercise the most important code features of CTF. So long as the user is utilizing the code to model similar tests, utilizing the exercised features in this document, they can now have a greater confidence that CTF is actually capable of producing meaningful results. Furthermore, this document is meant to be a living document, giving the developers a forum for discussing future validation tasks and exercising different CTF capabilities. 


\section{Bibliography}

[1] M. N. Avramova. CTF: A Thermal Hydraulic Sub-Channel Code for LWR Transient Analyses, User's Manual. Feb. 2009.

[2] R.K. Salko and M.N. Avramova. CTF Theory Manual. The Pennsylvania State University.

[3] A. Rubin et al. OECD/NRC BENCHMARK BASED ON NUPEC PWR SUBCHANNEL AND BUNDLE TESTS (PSBT). Tech. rep. US NRC and OECD Nuclear Energy Agency, 2010.

[4] B. Neykov et al. NUPEC BWR Full-size Fine-mesh Bundle Test (BFBT) Benchmark. Tech. rep. NUCLEAR ENERGY AGENCY, 2006.

[5] B.S. Shiralkar and D.W. Radcliffe. An experimental and analytical study of the synthesis of grid spacer loss coefficients. Tech. rep. NEDE-13181. General Electric, 1971.

[6] M. Gluck. "Contributions with the Sub-Channel Code F-COBRA-TF to the NUPEC BWR FullSize Fine-Mesh Bundle Test (BFBT) Benchmark (Exercises I-1 and II-0)". In: Second Workshop on OECD/NRC Benchmark Based on NUPEC BWR Full-Size Fine-Mesh Bundle Tests (BFBT), Pisa (Italy), 26-27 April 2006. 2006.

[7] M.S. Quigley, C.A. McMonagle, and J.M. Bates. Investigation of Combined Free and Forced Convection in a $2 x 6$ Rod Bundle. Tech. rep. BNWL-2216. Battelle Pacific Northwest Laboratories, 1997.

[8] J.M. Bates and E.U. Khan. Investigation of Combined Free and Forced Convection in a $2 x 6$ Rod Bundle during Controlled Flow Transients. Tech. rep. PNL-3135. Battelle Pacific Northwest laboratories, 1980.

[9] Z. Karoutas et al. Subcooled Boiling Data from Rod Bundles. Tech. rep. 1003383. EPRI, 2002.

[10] R.T. Lahey and F.J. Moody. The Thermal Hydraulics of a Boiling Water Nuclear Reactor, Second Edition. American Nuclear Society, 1993.

[11] D. W. Radcliffe R. T. Lahey Jr. B. S. Shirlakar. Two-Phase Flow and Heat Transfer In Multirod Geometries: Subchannel and Pressure Drop Measurements in a Nine-Rod Bundle for Diabatic and Adiabatic Conditions. Tech. rep. General Electric, 1970.

[12] A.W. Bennett et al. Heat transfer to steam-water mixtures flowing in uniformly heated tubes in which the critical heat flux has been exceeded. Tech. rep. AERE-R 5373. U.K.A.E.A. Research Group, Atomic Energy Research Establishement, 1967.

[13] O. Nylund, K.M. Briker, R. Eklund, et al. FRIGG Loop Project. Tech. rep. R4-447/RTL-1007. AB Atomenergi, Stockholm, 1968.

[14] R.W. Sterner and R.T. Lahey. Air/Water Subchannel Measurements of the Equilibrium Quality and Mass Flux Distribution in a Rod Bundle. Tech. rep. Rensselaer Polytechnic Institute, 1983. 
[15] M. Sadatomi et al. "Single- and Two-Phase Turbulent Mixing Rate between Adjacent Subchanels in a Vertical $2 \times 3$ Rod Array Channel". In: International Journal of Multiphase Flow 30 (2004), pp. 481498.

[16] M. Sadatomi et al. "Flow Characteristics in Hydraulically Equilibrium Two-Phase Flows in a Vertical $2 \times 3$ Rod Bundle Channel". In: International Journal of Multiphase Flow 30 (2004), pp. 1093-1119.

[17] M. Sadatomi, Y. Sato, and S. Saruwatari. "Two-Phase Flow in Vertical Noncircular Channels". In: International Journal of Multiphase Flow 8 (1982), pp. 641-645.

[18] K. Geelhood and W. Luscher. FRAPCON-4.0: Integral Assessment Manual. 2015.

[19] Mine Ozdemir Yilmaz. "Development of Burunp Dependent Fuel Rod Model in Cobra-TF". PhD thesis. The Pennsylvania State University, Department of Mechanical and Nuclear Engineering, Dec. 2014.

[20] D.D. Lanning. Irradiation History and Final Post-Irradiation Data for IFA-432, NUREG/CR-471\%. PNL-5977. Pacific Northwest Laboratory, 1986.

[21] S. Beguin. The Lift-Off Experiment with MOX Fuel Rod in IFA-610.2 Initial Results. Tech. rep. HWR603. Pacific Northwest Laboratory, 1999.

[22] K. Geelhood, W. Luscher, and C. Beyer. FRAPCON-3.4: A Computer Code for the Calculation of Steady-State Thermal-Mechanical Behavior of Oxide Fuel Rods for High Burnup. Tech. rep. NUREG/CR7022, Vol. 1, PNNL-19418, Vol. 1. Pacific Northwest National Laboratory, 2010.

[23] K.J. Geelhood. "Material Property Correlations: Comparisons between FRAPCON-3.4, FRAPTRAN 1.4, and MATPRO, NUREG/CR-7024". In: Pacific Northwest Laboratory HWR-603 (2010).

[24] D.D.Lanning E.R. Bradley M.E. Cunningham. Final Data Report for the Instrumented Fuel Assembly (IFA)-432. Technical Report NUREG/CR-2567, PNL-4240. Pacific Northwest Laboratory, 1982.

[25] J. Nikuradse. Laws of Flow in Rough Pipes. Tech. rep. TM 1292. Translation of Stromungsgesetze in rauhen Rohren. Verein Deutscher Ingenieure-Forschungshelft, 361(4), August 1933. National Advisory Committee for Aeronautics, Nov. 1950.

[26] J. Nikuradse. Laws of Turbulent Flow in Smooth Pipes. Tech. rep. TT 359. Translation of Gesetzmassingkeiten der turbulenten Stromung in Glatten Rohren. Verein Deutscher Ingenieure-Forschungshelft, 356(3), October 1932. National Advisory Committee for Aeronautics, Oct. 1966.

[27] Jorgen Wurtz. An Experimental and Theoretical Investigation of Annular Steam-Water Flow in Tubes and Annuli at 30 to 90 bar. Tech. rep. Riso Report No. 372. Copenhagen, Denmark: Riso National Laboratory, Apr. 1978.

[28] G. G. Bartolomei and V. M. Chanturiya. "Experimental Study of True Void Fraction When Boiling Subcooled Water in Vertical Tubes". In: Thermal Engineering 14 (1967), pp. 123-128.

[29] G. G. Bartolomei et al. "An Experimental Investigation of True Volumetric Vapour Content with Subcooled Boiling in Tubes". In: Thermal Engineering 29 (1982), pp. 20-22.

[30] J. R. S. Thom et al. "Boiling in Subcooled Water During Flow Up Heated Tubes for Annuli". In: Symposium on Boiling Heat Transfer in Steam Generating Units and Heat Exchangers, Manchester, London. 1965.

[31] Electric Power Research Institute. Simulated Fuel Crud Thermal Conductivity Measurements Under Pressurized Water Reactor Conditions. Tech. rep. 1022896. EPRI, 2011.

[32] W. M. Rohsenow and J. A. Clark. Heat Transfer and Pressure Drop Data for High Heat Flux Densities to Water at High Subcritical Pressures. Tech. rep. N5ori-07827/NR035-267/DIC6627. Office of Naval Research, 1951. 
[33] W. M. Rohsenow and H. Choi. Heat, Mass, and Momentum Transfer. Englewood Cliffs, NJ: PrenticeHall, Inc., 1961.

[34] W. M. Rohsenow, J. P. Hartnett, and E. N. Ganić. Heat Transfer Fundamentals. 2nd ed. New York: McGraw-Hill, 1985.

[35] R. Salko et al. Coupling of Subchannel T/H (CTF) and CRUD Chemistry (MAMBA1D). Tech. rep. CASL-U-2015-0146-001. Consortium for Advanced Simulation of Light Water Reactors, 2015.

[36] R. Salko et al. Summary of CTF Accuracy and Fidelity Improvements in FY17. Tech. rep. CASL-U2017-1428-000. Consortium for Advanced Simulation of Light Water Reactors, 2017.

[37] J. Lane. "The Development of a Comprehensive Annular Flow Modeling Package for Two-Phase ThreeField Transient Safety Analysis Codes". PhD thesis. The Pennsylvania State University, 2009.

[38] A. Wysocki and R. Salko. Validation of CTF Droplet Entrainment and Annular/Mist Closure Models using Riso Steam/Water Experiments. Tech. rep. CASL-U-2016-1080-000. Oak Ridge National Laboratory, 2016.

[39] Y. Sung et al. Application of Multi-Scale Thermal-Hydraulic Models to DNB Analysis. Tech. rep. CASL-I-2014-0119-000. Oak Ridge National Laboratory, 2014.

[40] Neil E. Todreas and Mujid S. Kazimi. Nuclear Systems II: Elements of Thermal Hydraulic Design. Hemisphere Publishing Corporation, 1990.

[41] A. Rubin, M. Avramova, and A. Velazquez-Lozada. International Benchmark on Pressurized Water Reactor Sub-channel and Bundle Tests. Volume II: Benchmark results of phase I-Void distribution. Tech. rep. OECD Nuclear Energy Agency, 2016.

[42] R.W. Bowring. A Simple but Accurate Round Tube, Uniform Heat Flux Dryout Correlation over the Pressure Range 0.7 to 17 MPa. Tech. rep. AEE Winfrith, 1972.

[43] Neil E. Todreas and Mujid S. Kazimi. Nuclear Systems I: Thermal Hydraulic Fundamentals, $2^{\text {nd }}$ Edition. CRC press, 2011.

[44] D. C. Groeneveld et al. The 2006 CHF look-up table. Tech. rep. Nuclear Engineering and Design, 2007.

[45] D. C. Groeneveld et al. Look-Up Tables for Predicting CHF and Film Boiling Heat Transfer - Past, Present and Future (NURETH). Tech. rep. Chalk River Lab, AECL, 2003.

[46] A. Toptan et al. "A New Fuel Modeling Capability, CTF Fuel, with a Case Study on the Fuel Thermal Degradation". In: Nuclear Eng. Design 341 (), pp. 248-258.

[47] V.H. Ransom. Numerical Benchmark Tests. Washington, DC: Multiphase Science et al., 1987.

[48] L Zou, H Zhao, and H Zhang. "New analytical solutions to the two-phase water faucet problem". In: Progress in Nuclear Energy 91 (2016), pp. 389-398.

[49] IAPWS. Release on the IAPWS Formulation 2011 for the Thermal Conductivity of Ordinary Water Substance. Tech. rep. The International Association for the Properties of Water and Steam , Czech Republic, 2011.

[50] D. A. Drew and S. L. Passman. Theory of Multicomponent Fluids. New York, Berlin, Heidlberg: Applied Mathematical Sciences 135, Springer-Verlag, 1998.

[51] J. D. RAMSHAW and J. A. TRAPP. "Charesteristics, Stability, and Short- Wavelength Phenomena in Two-Phase Flow Equation Systems". In: Nucl. Sci. Eng. 66 (1978), p. 93.

[52] J. H. STUHMILLER. "The Influence of Interfacial Pressure on the Character of the Two-phase Flow Model Equations". In: Int. J. Multiphase Flow 551 (1977), p. 3. 
[53] Suneet Singh and Vincent A. Mousseau. "Modified Choke flow criterion for the two-phase two-fluid model". In: International Conference on Mathematics, Computational Methods \& Reactor Physics (MEC). 2009.

[54] K. Ren, D. Wang, and R. Salko. "Implementation of the Lax-Wendroff Method in COBRA-TF for Solving Two-Phase Flow Transport Equations". In: International Congress on Advances in Nuclear Power Plants. 2016.

[55] N. Porter, V. Mousseau, and M. Avramova. "Solution Verification of CTF and CTF-R Using Isokinetic Advection Test Problems". In: ANS MEC (Apr. 2017).

[56] J. W. Banks, T. Aslam, and W. J. Rider. "On Sub-Linear Convergence for Linearly Degenerate Waves in Capturing Schemes". In: Journal of Computational Physics 227 (2008), pp. 6985-7002. 